= Bundesministerium

Bildung, Wissenschaft und Forschung
- Bundesministerium Verkehr, Innovation und Technologie
- Bundesministerium Digitalisierung und Wirtschaftsstandort

\title{
Österreichischer Forschungs- und Technologiebericht 2019
}

Lagebericht gem. § 8 (1) FOG über die aus Bundesmitteln geförderte Forschung, Technologie und Innovation in Österreich

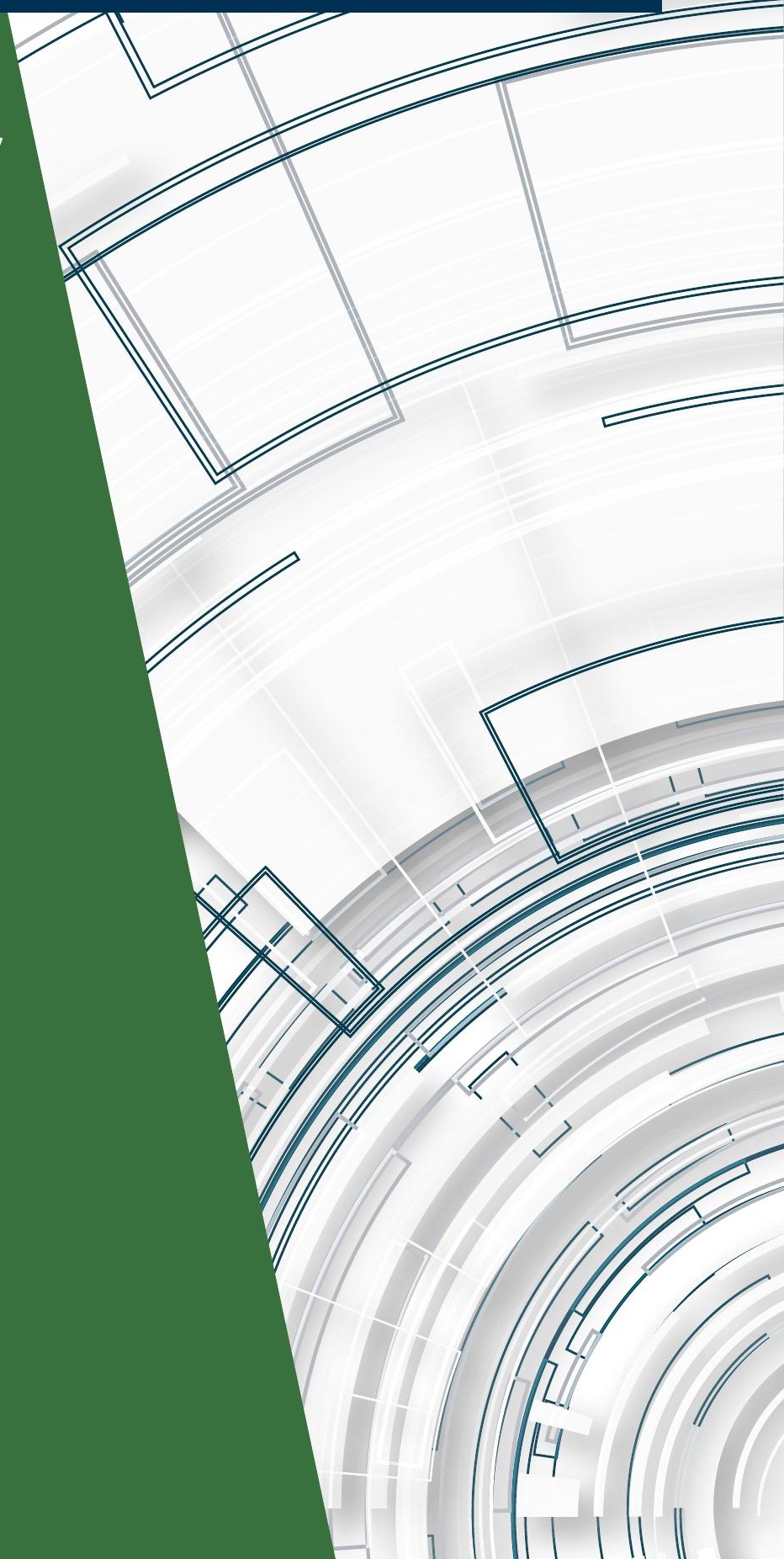


Der vorliegende Bericht ist im Auftrag der Bundesministerien für Bildung, Wissenschaft und Forschung (BMBWF), Verkehr, Innovation und Technologie (BMVIT) und Digitalisierung und Wirtschaftsstandort (BMDW) entstanden. Die Erstellung des Berichts erfolgte durch eine Arbeitsgemeinschaft, bestehend aus WPZ Research, Zentrum für Soziale Innovation (ZSI) und KMU Forschung Austria mit Unterstützung von VDI/VDE Innovation + Technik (iit), Technopolis und dem Industriewissenschaftlichen Institut (IWI).

Autorinnen- und Autorenteam: Brigitte Ecker (Koordination, WPZ Research), Philipp Brunner (IWI), Stephanie Christmann-Budian (iit), Iris Fischl (KMU Forschung Austria), Helmut Gassler (ZSI), Gerald Gogola (WPZ Research), Ernst Hartmann (iit), Eva Heckl (KMU Forschung Austria), Peter Kaufmann (KMU Forschung Austria), Stefan Krabel (iit), Katja Mayer (ZSI), Anastasia Mozhova (iit), Hans Pechar (WPZ Research), Alfred Radauer (Technopolis), Christian Reiner (WPZ Research), Sascha Ruhland (KMU Forschung Austria), Sascha Sardadvar (WPZ Research), Herwig W. Schneider (IWI), Klaus Schuch (ZSI), Dorothea Sturn (ZSI), Brigitte Tiefenthaler (Technopolis), Katharina Warta (Technopolis) und Elke Welp-Park (WPZ Research).

Impressum

Medieninhaber (Verleger):

Bundesministerium für Bildung, Wissenschaft und Forschung, 1010 Wien

Bundesministerium für Verkehr, Innovation und Technologie, 1030 Wien

Bundesministerium für Digitalisierung und Wirtschaftsstandort, 1010 Wien

Alle Rechte vorbehalten

Auszugsweiser Nachdruck nur mit Quellenangabe gestattet

Gestaltung und Produktion:

barrierefrei PDF OG, Wien

Cover: (C) stock.adobe.com

Druck:

Druckerei AV Astoria

Wien, 2019 


\section{Vorwort}

Der Blick zurück auf die FTI-Strategie 2020, die von der Bundesregierung im Jahre 2011 verabschiedet wurde, zeigt einen beeindruckenden Aufholprozess im Bereich Forschung, Technologie und Innovation. Hervorstechendes Ergebnis des Reviews, der im Rahmen des diesjährigen Forschungs- und Technologieberichts unter Einbeziehung relevanter Expertinnen und Experten erstellt wurde, ist die hohe Zahl von erfolgreich umgesetzten Maßnahmen, wenngleich ein übergeordnetes Ziel, nämlich in die Gruppe der Innovation Leader-Länder vorzustoßen, bislang nicht erreicht wurde. Die einzelnen Teilstrategien, die seit 2011 zu neuen, aktuellen Themen wie z.B. Open Innovation, Life Sciences, Kreativwirtschaft, Digitalisierung u.v.a.m. beschlossen wurden, ergänzten aus gesamtsystemischer Sicht die FTI-Strategie 2020. Insgesamt kann das akkordierte Vorgehen von Politik, den forschungsrelevanten Ministerien und Akteurinnen und Akteuren des FTI-Systems im Rahmen der FTI-Strategie als Erfolg verbucht werden.

Fortschritte gab es in allen FTI-relevanten Bereichen der Wissenschaft, der Wirtschaft und des öffentlichen Sektors. Die Position Österreichs hat sich in internationalen Rankings seit 2011 überwiegend verbessert oder stabilisiert: Österreich gehört zu den führenden Nationen im Hinblick auf seine Forschungs- und Entwicklungsausgaben und liegt europaweit hinter Schweden an zweiter Stelle und weltweit vor so innovationsstarken Ländern wie USA und China, bei Kooperationen zwischen Hochschulen und Unternehmen liegt Österreich europaweit hinter Finnland an zweiter Stelle, bei Wissenschafts-Wirtschaftskooperationen insgesamt ist Österreich europaweit führend, ebenso wie bei messbaren Leistungen seines Wissenschaftssystems wie öffentlich-privaten Ko-Publikationen, Zitationen oder bei der Entwicklung internationaler Patentanmeldungen. Damit zeigt sich Österreich im Hinblick auf steigende technische Herausforderungen in einer global vernetzten Wissenschaft und Wirtschaft gut gerüstet.
Auch die Analysen der Wettbewerbs- und Innovationsfähigkeit Österreichs anhand des Global Competitiveness Reports und des Innovationsfähigkeitsindikators des deutschen Instituts für Innovation und Technik (iit) zeigen eine überdurchschnittliche (im Vergleich zur EU-28) Positionierung Österreichs bezüglich technologischer Reife, politischer und ökonomischer Rahmenbedingungen und der Qualifizierung von Personen.

Aufholbedarf besteht neben den bekannten Schwächen im Bereich Venture Capital, wissensintensive Dienstleistungsexporte, Beschäftigung in schnellwachsenden Unternehmen und tertiäre Bildungsabschlüsse im Bereich der Digitalisierung. Daher widmet sich neben dem Review der FTI-Strategie 2020 ein weiteres Schwerpunktkapitel dem Stand des digitalen Wandels in Österreich in allen FTI-, gesellschafts-, bildungs- und verwaltungspolitischen Bereichen und gibt einen umfassenden Überblick über die zahlreichen Initiativen und Maßnahmen. Mit Schaffung eines federführenden Ressorts, einer Task Force sowie der Gründung der Digitalisierungsagentur wurden die Bemühungen um Verbesserungen von Rahmenbedingungen für die Entwicklung digitaler Technologien erheblich erweitert.

Die Vorbereitungen zur Umsetzung der mit August 2018 von der Bundesregierung beschlossenen „Zukunftsoffensive für Forschung, Technologie und Innovation" zeigen die Richtung an, die uns dem Ziel, „Innovation Leader" zu werden, näherbringt. Durch eine neue FTI-Strategie und ein Ineinandergreifen aller Teile eines Innovationssystems - vom Bildungssystem über akademische Forschungseinrichtungen über KMU bis Großunternehmen - wird größtmöglicher Output, gesellschaftlicher und wirtschaftlicher Impact längerfristig sichergestellt.

Österreich wird 2019 mit geschätzten 12,8 Mrd. € und einem Plus von nominell 554 Mio. $€$ gegenüber 
2018 einen neuen Höchstwert an Ausgaben für F\&E erreichen. Damit liegt die Steigerung der F\&E-Ausgaben mit 4,5\% über der des Bruttoinlandsprodukts von 3,8 \%. Die F\&E-Quote wird mit 3,19\% leicht über dem Wert vom Vorjahr liegen, der auf 3,17\% revidiert wurde. Der Finanzierungsanteil der Unternehmen wird mit 6,3 Mrd. $€$ voraussichtlich bei $49 \%$ liegen, gefolgt vom öffentlichen Sektor mit 4,5 Mrd. € und einem Anteil von rd. $35 \%$, dem Ausland mit rd. 2 Mrd. $€$ und einem Anteil von rd. $16 \%$ sowie dem privaten, gemeinnützigen Sektor mit rd. 0,08 Mrd. € und einem Anteil von rd. 0,6\%.

Insgesamt ist das österreichische Fördersystem gut ausgebaut und deckt mit seinen unterschiedlichen Instrumenten und Schwerpunkten der Forschungs- förderung das breite Spektrum der Fördernehmerinnen und Fördernehmer ab. Die rezente Darstellung der strategischen Förderentwicklungen der drei groBen Forschungsförderungsagenturen FWF, FFG und aws ist fixer Bestandteil des FTB und wird jährlich dargestellt.

Weitere Themen sind Beiträge zur europäischen FTIPolitik, u.a. auch die Aktivitäten im Rahmen der EURatspräsidentschaft im zweiten Halbjahr 2018, ausgewählte Evaluierungen sowie eine Analyse der Forschungsförderungen und -aufträge des Bundes 2018, die in die Bundesforschungsdatenbank eingetragen wurden. Ein umfangreicher Tabellenanhang rundet wie jedes Jahr das Bild der FTI-Aktivitäten Österreichs ab.
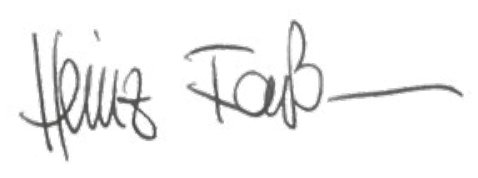

BM Univ.-Prof. Dr. Heinz Faßmann Bundesminister für Bildung,

Wissenschaft und Forschung

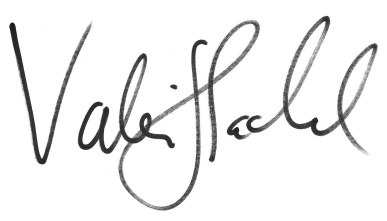

BM Dr. in Valerie Hackl

Bundesministerin für Verkehr, Innovation und Technologie

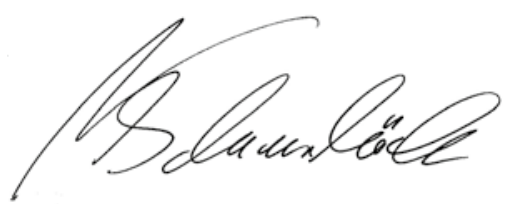

BM Dr. Margarete Schramböck Bundesministerin für Digitalisierung und Wirtschaftsstandort 


\section{Inhalt}

$\begin{array}{ll}\text { Vorwort } & 3\end{array}$

$\begin{array}{lr}\text { Executive Summary } & 8\end{array}$

1. Aktuelle Entwicklungen 17

$\begin{array}{ll}1.1 \text { Finanzierung und Durchführung von F\&E in Österreich } & 18\end{array}$

1.2 Die Position Österreichs im internationalen Vergleich 23

1.2.1 Entwicklung der Position Österreichs bei zentralen FTI-Indikatoren 25

1.2.2 Entwicklung der Position Österreichs bei der Digitalisierung 33

1.2.3 Österreichs Innovations- und Wettbewerbsfähigkeit 37

$\begin{array}{ll}\text { 1.2.4 Resümee } & 45\end{array}$

1.3 Österreich und die EU Forschungs-, Technologie- und Innovationspolitik 46

1.3.1 Aktivitäten im Rahmen der EU-Ratspräsidentschaft $\quad 47$

1.3.2 Die Performance Österreichs in Horizon 2020

1.3.3 Die Umsetzung von Horizon 2020 in Österreich $\quad 54$

1.3.4 Das neue Rahmenprogramm gemäß Vorschlag der Europäischen Kommission 57

$\begin{array}{ll}\text { 1.3.5 Resümee } & 60\end{array}$

1.4 Strategische Maßnahmen, Initiativen und Weiterentwicklungen 61

2. Die großen Förderagenturen des Bundes $\quad 72$

$\begin{array}{ll}2.1 \text { Wissenschaftsfonds (FWF) } & 74\end{array}$

$\begin{array}{ll}2.2 \text { Österreichische Forschungsförderungsgesellschaft (FFG) } & 78\end{array}$

2.3 Austria Wirtschaftsservice (aws)

3. Review FTI-Strategie $2020 \quad 90$

3.1 Einbettung der FTI-Strategie im Kontext nationaler und internationaler Entwicklungen 92

3.2 Neuerungen im Bildungssystem 94

3.2.1 Strukturreformen des Bildungssystems und verbesserte Bildungsübergänge 94

3.2.2 Qualitätsverbesserung der Hochschullehre $\quad 98$

3.2.3 Verbesserte Integrationsangebote 103

$\begin{array}{ll}\text { 3.2.4 Steigerung der Mobilität } & 105\end{array}$

3.2.5 Verbesserte Rahmenbedingungen für Forscherinnen und Forscher an Hochschulen 109

$\begin{array}{ll}\text { 3.2.6 Forcierung von Gleichstellung in der Forschung } & 115\end{array}$

3.3 Stärkung der Basis für die Wissensgesellschaft 119

$\begin{array}{ll}\text { 3.3.1 Stärkung der Grundlagenforschung } & 119\end{array}$

3.3.2 Finanzierung der Universitäten nach dem neuen Finanzierungsmodell $\quad 124$

$\begin{array}{ll}\text { 3.3.3 Ausbau der Drittmittelfinanzierung } & 127\end{array}$

3.3.4 Strukturelle Reformen der außeruniversitären Forschung 131

3.3.5 Ausbau und Weiterentwicklung von Forschungsinfrastrukturen $\quad 135$ 
3.4 Steigerung der Wissensverwertung und Wertschöpfung $\quad 139$

3.4.1 Aktivierung und Steigerung von Unternehmensforschung 140

3.4.2 Nachfrageseitige Stimulierung von Innovationen durch die öffentliche Hand 146

3.4.3 Stärkung und Ausbau von Wissenschaft-Wirtschaftskooperationen 149

3.4.4 Stärkung von Entrepreneurship an den Universitäten 153

3.4.5 Unterstützung von Unternehmensgründungen und Stärkung der Risikokapitalfinanzierung 156

$\begin{array}{ll}\text { 3.4.6 Verbesserung von wettbewerblichen Rahmenbedingungen } & 161\end{array}$

3.5 Verbesserungen in der Governance und strategische Maßnahmen $\quad 165$

3.5.1 Verbesserung von Governance-Strukturen $\quad 165$

3.5.2 Strategische, thematische Schwerpunktsetzungen 169

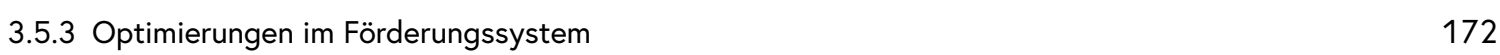

3.5.4 Stärkung der internationalen Positionierung Österreichs $\quad 175$

3.5.5 Ausbau der Verbindung Forschung und Gesellschaft $\quad 180$

3.6 Ergebnisse des OECD Reviews of Innovation Policy in Austria 189

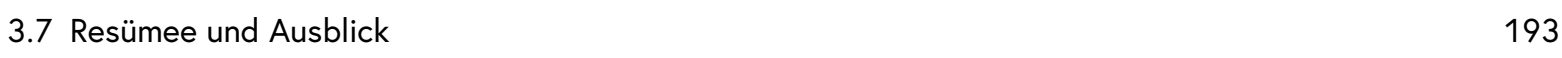

4. Digitaler Wandel 196

4.1 Status-quo der Umsetzung der Digital Roadmap Austria 197

4.2 Digitale Transformation, Digitalisierung und Industrie 4.0 in Österreichs Unternehmen 199

4.3 Fachkräfte und Ausbildungssystem für den digitalen Wandel 206

4.4 Digitalisierung im öffentlichen Sektor 211

4.4.1 Digitalisierungsstrategie, Digital Austria und Chief Digital Officers 211

4.4.2 Digitales Amt 213

4.4.3 Digitalisierung in der Verwaltung $\quad 214$

4.4.4 Digitale Entwicklungen in der öffentlichen Beschaffung 215

$\begin{array}{ll}\text { 4.4.5 Open Data } & 216\end{array}$

4.4.6 Digitalisierung von Forschungsdaten aus Archiven, Sammlungen und Museen
bzw. historischen Bibliotheken

$\begin{array}{ll}\text { 4.4.7 Resümee } & 218\end{array}$

5. FTI-Evaluierungskultur und -praxis $\quad 220$

$\begin{array}{ll}5.1 \text { Aktuelle Entwicklungen } & 221\end{array}$

5.1.1 Die Österreichische Plattform Registerforschung $\quad 221$

5.1.2 Die neuen Standards der Evaluierung in der Forschungs-, Technologie- und Innovationspolitik 222

5.1.3 Die Österreichische Ratspräsidentschaftskonferenz „Impact of R\&I Policy at the Crossroads of Policy Design, Implementation and Evaluation" 223

5.2 Ausgewählte Evaluierungen 226

5.2.1 Beyond Europe 226

5.2.2 Global Incubator Network (GIN) 229

5.2.3 Frontrunner-Initiative 230

5.2.4 Umsetzung von H2020, EUREKA, COSME, EEN und ERA in Österreich 232

5.2.5 FIT-IT und IKT der Zukunft 235 
6. Abbildungs- und Tabellenverzeichnis

8.2 Abkürzungsverzeichnis

9. Anhang II 
Summary

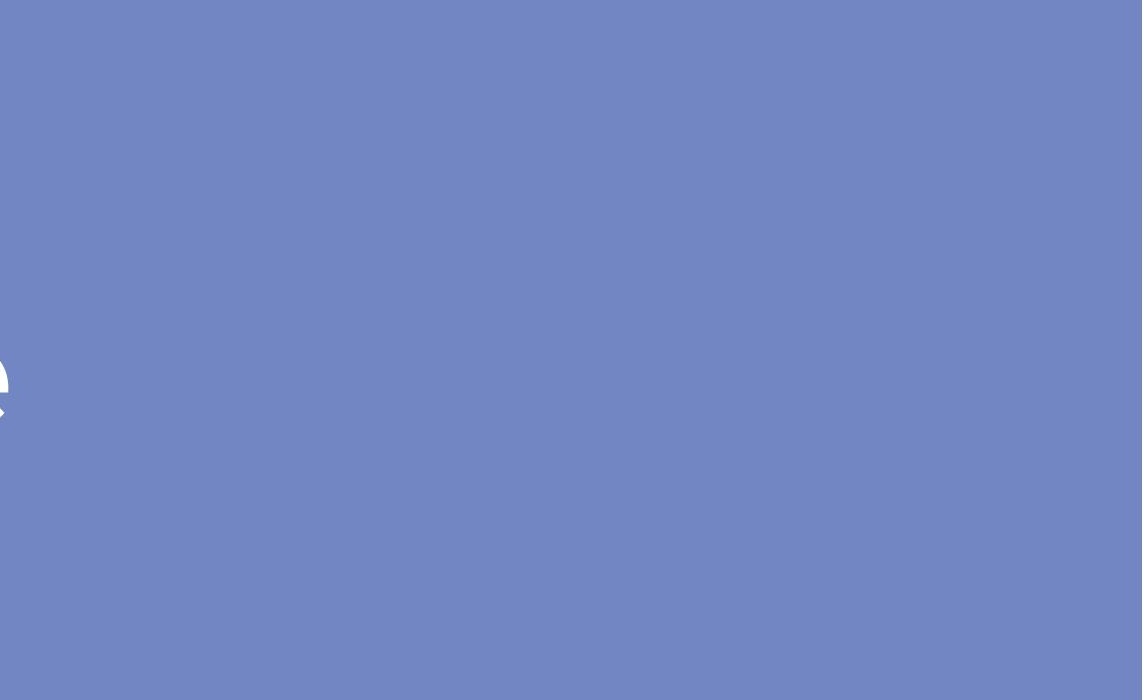


Der Forschungs- und Technologiebericht ist der Lagebericht über die aus Bundesmitteln geförderte Forschung, Technologie und Innovation in Österreich und wurde im Auftrag des Bundesministeriums für Bildung, Wissenschaft und Forschung (BMBWF), des Bundesministeriums für Verkehr, Innovation und Technologie (BMVIT) und des Bundesministeriums für Digitalisierung und Wirtschaftsstandort (BMDW) erstellt. Inhalt sind die Analyse von Daten und Befunden, die das österreichische Innovationssystem in seinen Ausprägungen wie auch im internationalen Kontext beschreiben, sowie Entwicklungstrends und ausgewählte Schwerpunktthemen.

Der Forschungs- und Technologiebericht 2019 umfasst eine Darstellung der jüngsten Globalschätzung über die Entwicklung der F\&E-Ausgaben in Österreich, die Performance des österreichischen Innovationssystems im internationalen Vergleich wie auch eine Systemanalyse hinsichtlich des Fortschritts im Bereich Digitalisierung. Zudem gibt der Bericht eine Beschreibung einer Reihe von Initiativen und strategischen Maßnahmen im Bereich Forschung, Technologie und Innovation wieder. Anlässlich der Vorbereitung der neuen FTI-Strategie steht im vorliegenden Bericht insbesondere die Umsetzung und Zielerreichung der FTI-Strategie 2020, welche im Jahr 2011 von der Bundesregierung beschlossen wurde, im Mittelpunkt der Betrachtung. Die FTI-Strategie 2020 wird dabei unter Einbeziehung externer Expertinnen und Experten einem Review unterzogen, der eine Analyse und nähere Untersuchung der Entwicklungen seit 2011 bis heute erlaubt. Ein weiterer Schwerpunkt ist dem digitalen Wandel gewidmet, der den Unternehmens-, Bildungs- wie auch den öffentlichen Sektor zunehmend erfasst. Neue Entwicklungen und aktuelle Maßnahmen und Initiativen werden auch hier für den jeweiligen Sektor aufgezeigt. Ein wesentlicher Bestandteil des österreichischen Innovationssystems ist die gut ausgebaute Forschungsförderung, welche entsprechend den aktuellen Kennzahlen und den Ansätzen zur strategischen Weiterentwicklung auf Bundesebene anhand der drei großen Förderagenturen FWF, FFG und aws dargelegt wird. Eine breit angewandte und fest verankerte Evaluierungskultur prägt die österreichische FTI-Politik. Einblicke in diese und eine Zusammenschau rezenter Evaluierungen von FTI-Programmen bilden den Abschluss des Berichts.

\section{Die Globalschätzung der F\&E-Ausgaben für 2019}

Laut der aktuellen Globalschätzung der Statistik Austria von April 2019 werden die Investitionen in Forschung und Entwicklung (F\&E) im Jahr 2019 12,8 Mrd. $€$ betragen und damit um 4,5\% über dem Wert in 2018 (12,2 Mrd. €) liegen. Die geschätzte F\&E-Quote (Anteil der Bruttoinlandsausgaben für Forschung und Entwicklung gemessen am Bruttoinlandsprodukt) beträgt 2019 voraussichtlich 3,19 \%, was einen leichten Anstieg im Vergleich zum Vorjahr (2018: 3,17\%, revidierter Wert im Vergleich zur Globalschätzung 2018) bedeutet. Österreich würde damit zum bereits sechsten $\mathrm{Mal}$ in Folge über dem europäischen Zielwert von 3 \% liegen. In den vergangenen zehn Jahren 2009-2019 erhöhten sich die F\&E-Investitionen nominell um 71,1\%, das Bruttoinlandsprodukt jedoch lediglich um 39,1\% - das Wachstum der F\&E-Investitionen lag somit deutlich über dem Wirtschaftswachstum, was zeigt, dass die österreichische Wirtschaft immer forschungsintensiver wird.

Bund und Bundesländer zusammen werden 2019 mit voraussichtlich 4,3 Mrd. $€$ einen Anteil von $33,9 \%$ der gesamten in Österreich durchgeführten F\&E finanzieren. Während das einen Anstieg um 148,4 Mio. $€$ im Vergleich zum Vorjahr bedeutet, wird der Anteil von Bund und Ländern leicht sinken (2018: 34,2 \%). Im Zehnjahreszeitraum ist dies der viertniedrigste Wert. Der größte Anteil der öffentlichen F\&E-Investitionen wird mit voraussichtlich 3,8 Mrd. $€ ~(+3,4 \%$ bzw. + 123,4 Mio. €) auf den Bund entfallen. Darunter finden sich auch Investitionen in Höhe von 138,7 Mio. € für die Nationalstiftung für Forschung, Technologie und Entwicklung und voraussichtlich 670,0 Mio. € (Schätzung BMF) für die Forschungsprämie. 
Die heimischen Unternehmen werden 2019 mit 6,3 Mrd. € oder 48,96\% knapp die Hälfte aller F\&E finanziert haben, was innerhalb der vergangenen zehn Jahre nach 2015 (49,74 \%) dem zweithöchsten Wert entspricht. Heimische Unternehmen finanzieren einen immer größeren Anteil der in Österreich durchgeführten F\&E. Im Vergleich zum Vorjahr stiegen die Investitionen um 314,1 Mio. $€$ bzw. 5,3\% an.

Rd. 2 Mrd. $€$ bzw. 15,6\% werden 2019 vom Ausland für F\&E finanziert werden, wobei dieser Betrag zum größten Teil von ausländischen Unternehmen für in Österreich durchgeführte F\&E finanziert wird, sowie Rückflüsse aus den EU-Forschungsprogrammen umfasst. Die Kategorie "Sonstige" fasst sonstige öffentliche Finanzierung und den privaten gemeinnützigen Sektor (private gemeinnützige Institutionen ohne Erwerbscharakter, deren Status ein vorwiegend privater oder privatrechtlicher, konfessioneller oder sonstiger nicht öffentlicher ist) zusammen, sie spielen mit 1,0\% bzw. 0,6\% nur eine verhältnismäßig geringe Rolle.

\section{Die großen Förderagenturen des Bundes}

Der Wissenschaftsfonds FWF ist Österreichs zentrale Einrichtung zur Förderung der Grundlagenforschung. Im Jahr 2018 wurden vom FWF 684 Projekte (2017: 642) mit einem Budget von insgesamt 230,8 Mio. $€$ (im Vergleich 2017: 217,3 Mio. €) neu bewilligt. Aufgrund eines deutlichen Anstiegs des Antragsvolumens auf rd. 950 Mio. $€$ (2017: 879,4 Mio. $€$ ) sank die gesamte Bewilligungsquote (nach Summe) dennoch leicht von $22,4 \%$ auf $22,1 \%$, während die Bewilligungsquote der Einzelprojekte mit rd. $28 \%$ gehalten werden konnte. Insgesamt konnte in 2018 die Anzahl der vom FWF finanzierten Personen auf 4.155 erhöht werden. Es handelt sich hier in erster Linie um Promovierende, die in FWF-Projekten arbeiten. Der Frauenanteil ist unter den FWFfinanzierten Forschenden im Jahr 2018 in allen Personalkategorien gestiegen. Im Jahr 2018 fanden auch zahlreiche, für den FWF zukunftsweisende Entwicklungen statt. Hierzu zählt, dass der FWF auf Basis der im Regierungsprogramm formulierten Zu- kunftsoffensive mit der Ausarbeitung einer Exzellenzinitiative für Österreich beauftragt wurde. Ebenso wurde eine Strategie für die Jahre 20192021 (FWF-Mehrjahresprogramm) ausgearbeitet, welche die drei Schwerpunkte Qualitätssicherung, Konsolidierung des Förderungsportfolios und Dialog mit der Gesellschaft in den Mittelpunkt stellt. Weiters wurde die Umsetzung der Projektförderung über Institutionen (PROFI) fortgeführt, engagierte sich der FWF stark für den Ausbau von Maßnahmen im Bereich wissenschaftlicher Ethik und Integrität, sowie verfolgte der FWF seine pro-aktive Rolle im Bereich Open Access und Open Science stetig.

Die Österreichische Forschungsförderungsgesellschaft FFG ist die nationale Förderinstitution für unternehmensnahe Forschung und Entwicklung in Österreich. Sie bietet vor allem Unternehmen, aber auch Forschungseinrichtungen und Hochschulen ein breites Portfolio an Instrumenten. Über die finanzielle Unterstützung hinaus bietet die FFG auch Dienstleistungen und Beratungen an, so fungiert die FFG beispielweise auch als Nationale Kontaktstelle für die Forschungsprogramme der Europäischen Union. Im Jahr 2018 betrugen die vertraglich zugesicherten Förderungen (inklusive Haftungen und Darlehen) 617,6 Mio. $€$, dies entspricht einem Barwert von 500,8 Mio. $€$. Im Sinne der Entwicklung neuer Förderformate wurde das FFG-Portfolio 2018 in mehrere Richtungen erweitert. Zum einen haben die beiden Piloten „Impact Innovation" und "Ideen Lab" ihren Praxistest bestanden und stehen vor der Überführung ins FFG-Portfolio. Zum anderen wurden im Schwerpunkt Digitalisierung neue Akzente Richtung Qualifikation und Diffusion gesetzt. Eine Digitalisierungsagentur wurde als eigener Bereich innerhalb der FFG eingerichtet - mit dem Ziel, Österreich als einen Standort für digitale Exzellenz und Innovation auszubauen. Erstmals finden sich damit Projekte, Kompetenzen und Akteure aus Gesellschaft, Wirtschaft und Verwaltung alle zusammen unter einem Dach.

Die Austria Wirtschaftsservice Gesellschaft aws ist die Förderbank des Bundes. Durch die Vergabe von zinsengünstigen Krediten, Zuschüssen und 
Garantien werden Unternehmen bei der Umsetzung ihrer innovativen Projekte unterstützt, insbesondere dann, wenn die erforderlichen Mittel durch sonstige Finanzierungen nicht ausreichend aufgebracht werden können. Ergänzend werden spezifische Informations-, Beratungs-, Service- und Dienstleistungen für angehende, bestehende und expandierende Unternehmen angeboten. Im Jahr 2018 lag die gesamte Finanzierungsleistung mit 2.189,5 Mio. $€$ rd. 91,2\% über dem Vergleichswert des Vorjahres, wobei vor allem temporär angebotene und breitenwirksame Zuschussprogramme (insbesondere der Beschäftigungsbonus) für den hohen Anstieg des Zuschussvolumens ausschlaggebend waren. Substanzielle Beiträge lieferten ebenfalls die Anfang 2017 gestarteten und 2018 fortgeführten Anpassungen der Förderungsbedingungen bei Garantien und Krediten. Dazu gehören beispielsweise erhöhte betragliche Obergrenzen, gesteigerte Risikobereitschaft sowie reduzierte Bearbeitungs- und Garantieentgelte oder auch die Vereinheitlichung der erp-Kreditrichtlinien in einem neuen Wachstums- und Innovationsprogramm bei gleichzeitiger Beibehaltung des Kreditzinssatzes auf einem historischen Tiefststand. Adressiert werden hiermit gleichermaßen innovative Investitionsprojekte bereits etablierter Unternehmen wie auch jene von Neugründungen und jungen Unternehmen; für letztere gelten weiterhin die Sonderkonditionen des Gründungskleinkredits mit einem Fixzinssatz von $0,5 \%$ über die gesamte Laufzeit. Weitere Änderungen umfassten die strategische Ausrichtung wie auch die kontinuierliche Weiterentwicklung des awsInstrumentenportfolios - u.a. mit den Zielen, die Wirksamkeit bei geförderten Unternehmen zu erhöhen, den Zugang zu Förderungen zu erleichtern und den administrativen Aufwand zu reduzieren.

\section{Die Position Österreichs im internationalen Vergleich}

Im internationalen Vergleich konnte Österreich seine Position bei wichtigen FTI-Indikatoren in den letzten Jahren sichtlich verbessern. In Bezug auf die Ausgaben für Forschung und Entwicklung gehört Ös- terreich zu den führenden Nationen. Mit einer F\&EQuote von 3,17\% im Jahr 2018 weist Österreich den zweithöchsten Wert innerhalb der EU-28 - hinter Schweden - auf. Österreich ist damit vor Dänemark, Deutschland, Finnland oder Belgien positioniert. Im weltweiten Vergleich liegt Österreich somit auch vor innovationsstarken Ländern wie den USA oder China. Ebenso weist Österreich bei zentralen qualitätsorientierten Parametern wie der Zitationsrate oder internationalen Patentanmeldungen bemerkenswert positive Entwicklungen auf. Europaweit führend ist Österreich bei Wissenschaft-Wirtschaftskooperationen. Bei Kooperationen zwischen Hochschulen und Unternehmen belegt Österreich unter allen europäischen Ländern Platz zwei. Österreich liegt damit nur hinter Finnland und noch vor den führenden Innovationsnationen Schweden, Dänemark, den Niederlanden oder Großbritannien. Dadurch ist Österreich auch gut positioniert, einen schnellen und effizienten Transfer für neue Verfahren, Technologien und Dienstleistungen zu gewährleisten.

Im Bereich der Digitalisierung zeigt der Index für die digitale Wirtschaft und Gesellschaft (DESI), erstellt im Auftrag der Europäischen Kommission, dass sich Österreich mit Platz 11 im Mittelfeld der EU-28 bewegt. Führend sind hier die nordischen Länder Dänemark, Schweden, Finnland und die Niederlande. Der DESI-Index bestätigt, dass noch Entwicklungspotenziale im Ausbau des Internets und der Integration der Digitaltechnik bestehen. Im Bereich Humankapital, welcher Grundkompetenzen der Internetnutzung sowie fortgeschrittene Fertigkeiten und Entwicklung umfasst, ist Österreich im internationalen Vergleich dagegen gut aufgestellt.

Was die Wettbewerbsfähigkeit Österreichs betrifft, so zeigt der "Global Competitiveness Report", dass Österreich in allen Dimensionen besser abschneidet als der Durchschnitt der EU-28. Im weltweiten Vergleich unter 140 Ländern liegt Österreich am 22. Rang. Als weltweit führende Länder werden die USA, Singapur, Deutschland, die Schweiz und Japan genannt. Besonders beim Reifegrad des Wirtschaftssystems, bei der Innovationstätigkeit und den 
institutionellen Rahmenbedingungen erzielt Österreich im Vergleich zum Durchschnitt der EU-Länder deutlich bessere Werte. Der technologische Reifegrad und die Ausbildung sind in Österreich wie auch im EU-Durchschnitt jeweils auf einem sehr hohen $\mathrm{Ni}$ veau. Entwicklungspotenziale für Österreich und den Rest der Europäischen Union werden hingegen in der Effizienz des Arbeitsmarkts und des Gütermarkts sowie in der Entwicklung des Finanzmarkts gesehen.

Insgesamt sind die erzielten Erfolge, die internationale Top-Position Österreichs bei der F\&E-Quote sowie die positiven Entwicklungen bei vielen Innovationsindikatoren ein Beleg dafür, dass es Österreich gelungen ist, sich im internationalen Wettbewerb gut zu positionieren, insbesondere wenn es darum geht, Innovationen zu entwickeln und damit auf dem globalen Markt erfolgreich zu sein.

\section{Die österreichische EU-Ratspräsidentschaft} Im zweiten Halbjahr 2018 hatte Österreich den Vorsitz des Rates der Europäischen Union über. Im Bereich der FTI-Politik konnte trotz schwieriger Rahmenbedingungen (Brexit und ausstehender Beschluss über den mehrjährigen Finanzrahmen der EU für den Zeitraum 2021-2027) in der Rekordzeit von nur fünf Monaten eine Einigung über die wesentlichen Inhalte der Horizon Europe-Verordnung, also der Verordnung über das nächste Europäische Forschungsrahmenprogramm, erzielt werden. Dies erfolgte über die Annahme der sogenannten „partial political agreement" im Rat für Wettbewerbsfähigkeit am 30.11.2018. Am 20.03.2019 haben sich die Europäische Kommission, das Europäische Parlament und der Rat, vorbehaltlich des ausständigen Formalbeschlusses, auch auf ein sogenanntes „partial political agreement" geeinigt, und am 15.04.2019 hat sich der Rat über wesentliche Teile des spezifischen Programms geeinigt, womit Horizon Europe auf die Ziellinie für einen konstruktiven Abschluss gebracht wurde. Die großen Änderungen zum laufenden Horizon 2020 Forschungsrahmenprogramm betreffen den weiteren Ausbau des Europäischen Innovationsrats, neue Governance-Prozesse und die Einführung von sogenannten "Missionen“, die in Bezug auf spezifische noch festzulegende Zielsetzungen (wie bspw. den plastikfreien Ozean) in zumeist themenübergreifenden FTI-Clustern erarbeitet werden sollen.

Nach Horizon Europe stellte die zweite Priorität im Rahmen des österreichischen Vorsitzes im europäischen Rat im FTI-Bereich die weitere Umsetzung des Europäischen Forschungsraums (ERA) dar. Auch diesbezüglich wurden am 30.11.2018 die Schlussfolgerungen, die Österreich in der Arbeitsgruppe Forschung erfolgreich über mehrere Monate verhandelt hat, von Seiten des Rats angenommen und verabschiedet. Mitte Februar 2019 wurde der Fortschrittsbericht zur Umsetzung des Europäischen Forschungsraums vorgestellt, der eine Abschwächung des Umsetzungsfortschritts in Europa und die Aufrechterhaltung groBer Disparitäten innerhalb der EU konstatiert. Für Österreich wurden positive Entwicklungen in vier der sechs Prioritätenbereiche festgestellt. Die Positionierung in der Priorität 4 („Gender equality and gender mainstreaming in research"), insbesondere der Anteil weiblicher PhD-Graduierter, wird im ERA-Vergleich hingegen weiterhin als große Schwachstelle in Österreich ausgewiesen.

Im Rahmen der österreichischen EU-Ratspräsidentschaft wurden überdies $\mathrm{rd}$. 40 Veranstaltungen durchgeführt, die auf die Ausgestaltung von Horizon Europe referenziert und wichtige Themen des Europäischen Forschungsraums behandelt haben, sowie an der Schnittstelle zwischen FTI-Politik und sektoralen Politiken angesiedelt waren.

\section{Österreich in Horizon 2020}

Im Berichtszeitraum ist es den österreichischen FTIOrganisationen gelungen, kumuliert mehr als eine Mrd. Euro Förderung durch die Europäische Kommission seit Beginn von Horizon 2020 im Jahr 2014 einzuwerben. Österreich weist damit weiterhin einen positiven Rückfluss an eingeworbenen Förderungen im Vergleich zum kalkulatorisch errechneten Einzahlungsbetrag für Horizon 2020 auf. In Österreich ansässige Institutionen bzw. Forscherinnen und Forscher nahmen an $8,82 \%$ aller 21.472 Projekte teil, 
die im Rahmen von Horizon 2020 gefördert wurden. Besonders erfreulich ist, dass Österreich mit einer Erfolgsrate von $17,87 \%$ deutlich über der durchschnittlichen Horizon 2020-Erfolgrate von 15,32\% und nach Belgien somit an zweiter Stelle unter den Mitgliedsstaaten der Europäischen Union liegt. Das spricht nicht nur für ein hohes Niveau der österreichischen Forschung, sondern auch für professionell agierende Beratungs- und Betreuungsstrukturen, die durch ihre gute Arbeit zum erfolgreichen Abschneiden in Horizon 2020 beitragen, was durch eine aktuelle Evaluierung bestätigt wurde.

Insgesamt stellt der in Österreich ansässige Unternehmenssektor die meisten Beteiligungen in Horizon 2020 (38\%). Davon sind fast zwei Drittel KMU. Danach folgen der Hochschulsektor (28\%) und der außeruniversitäre Forschungsbereich (23\%). Der Rest verteilt sich auf sonstige Einrichtungen (wie z.B. Agenturen, andere öffentliche Einrichtungen). Im Gegensatz zu anderen europäischen Ländern konnte Österreich das meiste Budget aus Horizon 2020 aus der Säule 3 "Gesellschaftliche Herausforderungen“ einwerben, in der insbesondere der Unternehmenssektor und der außeruniversitäre Sektor überdurchschnittlich erfolgreich agieren. Zu den österreichischen Stärkefeldern in Säule 3 zählen im europäischen Vergleich die thematischen Cluster „intelligenter, umweltfreundlicher und integrierter Verkehr", "integrative, innovative und reflexive Gesellschaften" und „sichere, saubere und effiziente Energie". Innerhalb der Säule 2 „Führende Rolle der Industrie" sind die thematischen Cluster "Materialien", "IKT“ sowie "Biotechnologie“ ausgewiesene Stärkefelder.

\section{Review der FTI-Strategie 2020}

Österreich hat in den vergangenen Jahren einen Aufholprozess im Bereich Forschung, Technologie und Innovation verzeichnet - das ist nicht zuletzt darauf zurückzuführen, dass der Bereich FTI zunehmend im politischen Interesse stand. Als Ausdruck dieses Interesses ist auch die FTI-Strategie, verabschiedet von der Bundesregierung 2011, zu sehen. Diese Strategie war ein unverkennbares und deutliches Com- mitment der österreichischen Bundesregierung, Forschung, Technologie und Innovation in allen relevanten Sektoren stärken zu wollen.

Die FTI-Strategie fungierte dabei als Dach für sämtliche Ziele und Maßnahmen, welcher einer Förderung, Stärkung, Unterstützung etc. bedurften. Angesichts aktueller Entwicklungen haben sich ergänzend Teil-Strategien entwickelt, die zusätzlich bestimmte ausgewählte Themen - sei es z.B. Gründungen, oder Open Innovation - unterstützen und besonders forcieren sollen. Rückblickend lässt sich konstatieren, dass die FTI-Strategie - das akkordierte Vorgehen von Politik und sämtlichen Akteuren im Innovationsystem - gelungen ist. Der Großteil der Ziele wurde erreicht, eine hohe Anzahl von Maßnahmen wurde umgesetzt. Die ganz große Vision der FTI-Strategie 2020, nämlich Österreich (d.h. im Europäischen Innovation Scoreboard) als Innovation Leader zu positionieren, konnte allerdings nicht erreicht werden.

In den vergangenen Jahren hat sich Österreich sowohl im Bereich der Wissenschaft als auch im Wirtschafts- und öffentlichen Sektor fortschrittlich entwickelt. Eine zentrale Rolle im Bündeln von Kräften spielte dabei auch die Task Force FTI, das interministerielle Gremium zur Koordination der FTI-Strategie, dessen Ziel es war bzw. ist, die Koordination der mit Forschung, Technologie und Innovation betrauten Ministerien (aktuell BMBWF, BMDW und BMVIT) unter Einbindung von BKA und BMF zu verbessern. Aktuell ist die Task Force FTI von der Bundesregierung bereits mit der Erarbeitung der neuen FTI Strategie 2030 betraut worden. Dabei wird es für die Zukunft vor allem darum gehen, bisherige Bemühungen - unter dem Blickwinkel einer gesamtsystemischen Sicht - kontinuierlich weiterzuverfolgen bzw. weiterzuentwickeln und geänderten (bzw. neuen) Rahmenbedingungen entsprechend anzupassen.

Als zentral gilt die Forderung, dass auch in Zukunft der Policy Mix in Österreich gut abgestimmt sein muss - zwischen bottom-up und top-down, wie auch zwischen direkter und indirekter Forschungsförderung. Ebenfalls ist - wie von der OECD und dem 
RFTE festgehalten - eine höhere Effizienz anzustreben (im Sinne einer Verbesserung der Relation von Input zu Output). Tatsächlich weist Österreich heute die zweithöchste Forschungsquote Europas aus, bei so manchen Output-Indikatoren liegt Österreich allerdings nach wie vor im Mittelfeld. Verbunden mit der Frage nach dem gesellschaftlichen Impact gewinnt die Diskussion um den Output damit nochmals eine neue Dimension; das bedeutet zugleich aber auch, dass diese Debatte in Zukunft noch viel differenzierter stattfinden muss als bisher.

Zu den prioritären Zielen zählt auch die Stärkung der Grundlagenforschung. Die FTI-Strategie formulierte hierzu eine Reihe von Zielen, wobei die Reformierung des Modells der Universitätsfinanzierung (unter dem Titel „kapazitätsorientierte Universitätsfinanzierung") und eine Erhöhung der im Wettbewerb einzuwerbenden Mitteln zentrale Bestandteile zur Steigerung der Investitionen in Grundlagenforschung waren. Durch die Verankerung des Ausbaus der Drittmittelfinanzierung im Gesamtösterreichischen Universitätsentwicklungsplan wurden außerdem die Universitäten angehalten, eigene Drittmittelstrategien zu entwickeln bzw. festzulegen. Zwecks Nachhaltigkeit wurde dieses Ziel auch in den universitären Entwicklungsplänen bis 2024 verankert. Des Weiteren wurden Leistungsvereinbarungen mit der Österreichischen Akademie der Wissenschaften und dem IST Austria geschlossen. Diese Maßnahmen sollen dazu beitragen, die Grundlagenforschung in Österreich zu stärken; ebenso soll sich die Forschungsförderung an den Hochschulen künftig stärker an einer kompetitiven Mittelvergabe orientieren.

Ein weiterer wichtiger Aspekt in der österreichischen FTI-Politik ist das Ziel, die Governance laufend zu verbessern bzw. anzupassen. Auch hierzu hat die OECD Empfehlungen gemacht und entsprechend soll ein FTI-Beratungsgremium der Bundesregierung in Zukunft neu gestaltet werden. Darüber hinaus soll die Governance des österreichischen Forschungssystems durch das Forschungsfinanzierungsgesetz neu geregelt bzw. optimiert werden, und zugleich den Agenturen mehr operative Autonomie zuerkennen.

\section{Digitale Transformation im öffentlichen Sektor}

Digitale Infrastrukturen, Produkte und Dienstleistungen führen zu grundlegenden Veränderungen in Wirtschaft, Wissenschaft, Gesellschaft und Politik. Insbesondere der technologische Wandel und Innovationsprozesse werden durch die Digitalisierung substanziell beschleunigt. Im Regierungsprogramm für die Jahre 2017-2022 wurde der Digitalisierung eine zentrale Bedeutung zuerkannt, sodass zur Unterstützung der Digitalisierungsinitiativen eigens eine "Digitalisierungsagentur" gegründet wurde. Weiters wurde in jedem Ministerium über einen "Chief Digital Officer" (CDO) eine Verantwortlichkeit für digitale Angelegenheiten etabliert. Die CDOs erarbeiten u.a. eine einheitliche Datenstrategie des Bundes. Dabei geht es um die Fähigkeit, Daten von Bürgerinnen und Bürgern sicher zu verwahren, Daten im Sinne des Prinzips "once only“ für Userinnen und User zu nutzen sowie qualitativ hochwertige Daten für Big Data-Auswertungen, „predictive analytics“ und Artificial Intelligence-Anwendungen zur Verfügung zu haben. Diese und weitere Maßnahmen und Initiativen haben dazu beigetragen, dass die Implementierung, von in der Digital Roadmap vorgesehenen Maßnahmen eine zunehmende Dynamik erhielt.

\section{Die Verbreitung von innovativen Technologien}

Für den Einsatz innovativer Technologien ist auch eine moderne und leistungsstarke digitale Infrastruktur von großer Bedeutung. Mit $5 G$ wird die Basisinfrastruktur für viele nachgelagerte Industrien und Dienstleistungen geschaffen, denn für Anwendungen wie Industrie 4.0, autonomes Fahren, dem flächendeckenden Einsatz von Big Data und Künstliche Intelligenz (KI) oder dem „Internet der Dinge“ wird eine leistungsfähige IT-Infrastruktur benötigt. 5G hat folglich das Potential als disruptiver Faktor in der Digitalisierung für eine weitere Beschleunigung der Prozesse zu sorgen. Mit der nationalen 5G-Strategie hat die österreichische Bundesregierung ein konkretes Arbeitsprogramm für zentrale Handlungsfelder und einen Zeit- 
plan vorgelegt, um den 5G-Standard in Österreich so rasch wie möglich flächendeckend zu ermöglichen.

$\mathrm{KI}$ und Robotik sind Zukunftsthemen, die stetig an Bedeutung gewinnen und die Industrie nachhaltig verändern werden. Aus diesem Grund präsentierte der "Österreichische Rat für Robotik und künstliche Intelligenz", der bereits 2017 vom BMVIT eingerichtet wurde, im November 2018 das Whitepaper „Die Zukunft Österreichs mit Robotik und Künstlicher Intelligenz positiv gestalten“. Es beschreibt den aktuellen Entwicklungsstand, die Chancen und Herausforderungen, sowie Handlungsfelder und -empfehlungen für Aktivitäten im Bereich KI.

Aktuell nutzen in Österreich lediglich $13 \%$ aller Unternehmen konkrete KI-Anwendungen, 29 \% entwickeln diese gerade erst. Um die Zukunft von $\mathrm{KI}$ in Österreich im breiten Konsens mitzugestalten, ist es notwendig, sowohl die Zivilgesellschaft als auch möglichst viele Akteure aus Wirtschaft und Forschung in den Strategieprozess einzubinden, damit Österreich im Wettbewerb um die KI-Technologie unter Wahrung der gesellschaftlichen und individuellen Grundrechte reüssieren kann. Zu diesem Zweck haben das BMVIT und das BMDW das Vorbereitungspapier „Artificial Intelligence Mission Austria 2030“ erstellt, um im Laufe des Jahres 2019 auf dieser Basis eine umfassende Strategie zu entwerfen.

\section{Ein digitales Kompetenzmodell für Österreich}

Der digitale Wandel beeinflusst auch die Struktur der Arbeit, und die Anforderungen an die Qualifikationsprofile der Beschäftigten ändern sich. Dabei erfordert die Digitalisierung nicht unbedingt ein formal höheres Ausbildungsniveau, sondern dass die Arbeitnehmerinnen und Arbeitnehmer über jene Kompetenzen verfügen, mit welchen sie den zukünftigen, digitalen Anforderungen gerecht werden. Die Förderung ständiger Weiterbildung, besonders wenn es um die Unterstützung beim digitalen Lernen geht, oder die Ausstattung der Schulen mit der passenden digitalen Infrastruktur, sind daher essentiell, um den digitalen Wandel in Österreich auch in der Aus- und Weiterbildung gezielt zu unterstützen.

Mit dem "Digital-Dossier 2018“ hat das BMDW eine Bestandsaufnahme über den Status-quo der Digitalisierung in Österreich erstellt. Darin wird u.a. der „Pakt für Digitale Kompetenz" als Zusammenschluss von Wirtschaft, Bildungseinrichtungen und der öffentlichen Verwaltung mit verschiedenen Teilprogrammen präsentiert. Mit einem umfassenden „Masterplan für Digitalisierung in der Bildung“ des BMBWF soll Digitale Bildung stufenweise und vor allem flächendeckend in das österreichische Bildungssystem einfließen.

Weiters wurde seitens des BMDW mit DigComp 2.2 AT ein digitales Kompetenzmodell für Österreich entwickelt, das der Einordnung und Vergleichbarkeit digitaler Kompetenzen dient und damit die Grundlage für lebenslanges Lernen, gesellschaftliche Inklusion und Beschäftigung in einer digitalisierten Gesellschaft bildet. Auf Initiative des BMDW wurde im Dezember 2018 außerdem der Verein "fit4internet“ gegründet, der als Plattform - in Zusammenarbeit mit Unternehmen, Institutionen und Organisationen - die digitalen Kompetenzen in Österreich steigern und damit die Teilhabe der gesamten Gesellschaft an Digitalisierung ermöglichen soll.

\section{Evaluierungskultur und Evaluierungspraxis}

Zur Reflexion, Bewertung und Weiterentwicklung von Maßnahmen, Instrumenten und Praktiken der FTI-Politik sind Evaluierungen ein unverzichtbarer Teil einer lernenden und strategisch orientierten FTIPolitik. Die Evaluierungspraxis im FTI-Bereich wurde im OECD-Review positiv bewertet. Im Berichtszeitraum wurden erneut einige, zum Teil größere Evaluierungen abgeschlossen. Dazu zählen die Evaluierung der Umsetzung von Horizon 2020, EUREKA, COSME, EEN und ERA in Österreich, die Evaluierung der Frontrunner-Initiative, die Evaluierung der Programme „FIT-IT“ und „IKT der Zukunft", um nur einige zu nennen. Die vorliegenden Ergebnisse fließen in die Maßnahmengestaltung und Politikentwicklung ein. So ist z.B. die Evaluierung der Umsetzung von Horizon 
2020, EUREKA, COSME, EEN und ERA in Österreich eine wichtige Evidenzgrundlage für die Neuausrichtung bzw. Anpassung der Anforderungen, die an eine zukünftige Beratungs- und Betreuungsstruktur für Horizon Europe, dem nächsten europäischen Forschungsrahmenprogramm, gestellt werden.

Ausdruck für die Weiterentwicklung der österreichischen FTI-Evaluierungskultur ist auch die Neufassung der österreichischen Evaluierungsstandards im FTI-Bereich, die von der Österreichischen Plattform für Forschungs- und Technologiepolitikevaluierung verabschiedet wurden. Diese Standards dienen dazu, beauftragenden Einrichtungen, Evaluatorinnen und Evaluatoren, sowie den Betroffenen einer Evaluierung einen Verhaltensrahmen und Handlungsanleitungen zu geben, wie "gute" Evaluierungen geplant, gemanagt, durchgeführt und genutzt werden sollen. Neuerungen betreffen u.a. eine stärkere Betonung der Genderdimension in FTI-politischen Evaluierungsprozessen, die obligatorische Veröffentlichung von Evaluierungsberichten, die Anregung zur Einführung eines Management Response-Systems, sowie zahlrei- che ethische und prozedurale Hinweise, insbesondere in Bezug auf die Gestaltung von aussagekräftigen Terms of Reference zwecks Umsetzung eines möglichst effizienten, transparenten und effektiven Evaluierungsprozesses.

Eine strukturelle Schwachstelle des österreichischen Evaluierungssystems, auf die auch der OECDReview hinweist, ist die begrenzte Verfügbarkeit bzw. Zugänglichkeit und Vernetzbarkeit statistischer Daten bei öffentlichen Stellen. Die 2018 gegründete Plattform Registerforschung setzt sich dafür ein, dass der wissenschaftlichen Forschung Zugang zu Daten aus öffentlichen Registern erleichtert wird. Dies ist jedoch weiterhin mit einer Reihe von Herausforderungen, wie der Novellierung des Bundesstatistikgesetzes bzw. im Einvernehmen zwischen dem Bundesministerium für Bildung, Wissenschaft und Forschung und den jeweils für die Register zuständigen Ministerien zu erstellenden Verordnungen für einzelne Datenbestände verbunden, die noch einer gesamtheitlichen Lösung auf legistischer, prozeduraler und instrumenteller Ebene bedürfen. 


\subsection{Finanzierung und Durchführung von F\&E in Österreich}

Mit Stand April 2019 erwartet die Statistik Austria für 2019 eine weitere Erhöhung der österreichischen F\&E-Investitionen, sowohl relativ wie absolut. Demnach werden 2019 12,8 Mrd. € für Forschung und experimentelle Entwicklung aufgewendet werden, was einer Forschungsquote (definiert als Anteil der F\&EInvestitionen am Bruttoinlandsprodukt) von 3,19\% entspräche. Das würde einer Erhöhung von 0,02 Prozentpunkten entsprechen, nachdem die Forschungsquote 2018 bei 3,17 \% lag. ${ }^{1}$

In Abbildung 1-1 wird die Entwicklung der F\&E-Investitionen seit 2008 illustriert, wobei die Balken die absoluten Ausgaben zu jeweiligen Preisen, unterteilt nach den Finanzierungsquellen, darstellen, und die durchgängige Linie die Forschungsquote darstellt. 2014 wurde erstmals der von der EU als Ziel festge- legte Wert von $3 \%$ erreicht, seither liegt die Forschungsquote in jedem Jahr über diesem Wert, bei weiterhin steigender Tendenz. Im dargestellten Beobachtungszeitraum 2008-2019 erhöhten sich die F\&E-Investitionen nominell um 69,6\%, das Bruttoinlandsprodukt jedoch lediglich um 36,4\% - das Wachstum der F\&E-Investitionen verlief somit um über $90 \%$ schneller als das gesamte Wirtschaftswachstum, was zeigt, dass die österreichische Wirtschaft immer forschungsintensiver wird.

Die öffentliche Hand wird 2019 zusammen mit rd. $35 \%$ ein gutes Drittel der gesamten in Österreich durchgeführten F\&E finanzieren (Bund: 33,9\%, Bundesländer: 4,3\%, sonstige öffentliche Finanzierung: $1,0 \%$ ). Das ist im dargestellten Beobachtungszeitraum 2009-2019 der viertniedrigste Wert, bei insgesamt fallender Tendenz. 15,6\% werden voraussichtlich vom Ausland finanziert werden, wobei dieser Betrag zum größten Teil von ausländischen Unter-

\section{Abbildung 1-1: Finanzierung der in Österreich durchgeführten Forschung und experimentellen Entwicklung}

sowie Forschungsquote, 2008-2019

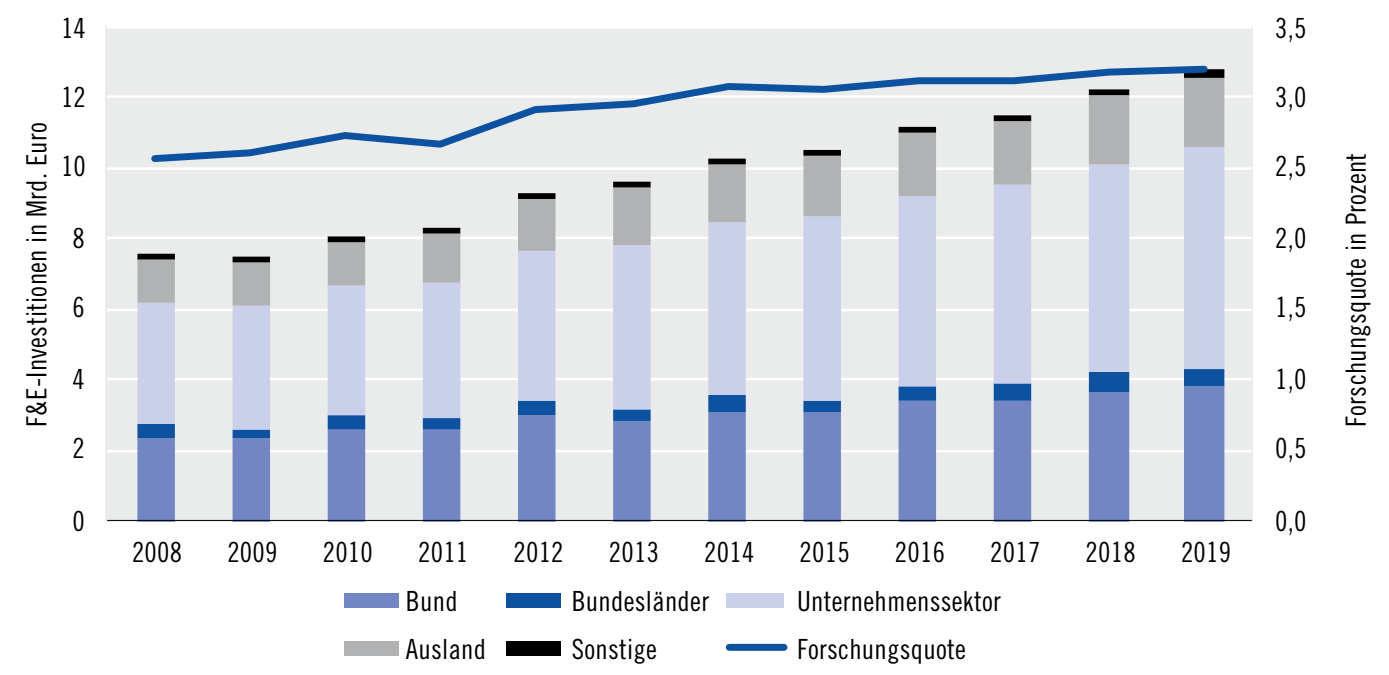

Quelle: Statistik Austria, Globalschätzung vom 11.04.2019, Darstellung: WPZ Research; die Kategorie „Sonstige“ fasst die beiden Kategorien „sonstige öffentliche Finanzierung" und „privater gemeinnütziger Sektor“ zusammen.

1 Angaben It. Pressemitteilung 12.001-067/19 der Statistik Austria. Angemerkt sei, dass im Forschungs- und Technologiebericht 2018 bereits für das Jahr 2018 eine Forschungsquote von 3,19\% erwartet wurde, die aber mit Stand April 2019 letztlich auf 3,17\% revidiert wurde. 
nehmen für ihre heimischen Töchterunternehmen finanzierte F\&E sowie Rückflüsse aus den EU-Forschungsprogrammen umfasst. Die heimischen Unternehmen werden mit 48,96 \% knapp die Hälfte aller F\&E finanziert haben, was im dargestellten Beobachtungszeitraum nach 2015 (49,74 \%) dem zweithöchsten Wert entspricht. Der private gemeinnützige Sektor (private gemeinnützige Institutionen ohne Erwerbscharakter, deren Status ein vorwiegend privater oder privatrechtlicher, konfessioneller oder sonstiger nicht öffentlicher ist) spielt für die Finanzierung mit 1,0 \% nur eine verhältnismäßig geringe Rolle. ${ }^{2}$

Innerhalb der EU liegt Österreich im Jahr 2017 dem aktuellsten Jahr, für das für alle Mitgliedstaaten Daten zur Verfügung stehen - mit einer Forschungsquote von 3,16 \% wie im Jahr davor hinter Schweden (3,40 \%) auf Rang zwei, nachdem Österreich 2015 Dänemark überholt hatte, das seither auf Rang drei liegt (Wert für 2017: 3,05 \%). ${ }^{3}$ Ansonsten erreicht auch 2017 innerhalb der EU lediglich Deutschland mit einer Forschungsquote von 3,02 \% das Drei-Prozent-Ziel der EU. Bemerkenswert ist dabei nicht nur, dass sich Österreich im Spitzenfeld etabliert hat, sondern dass der Abstand zu den folgenden Mitgliedstaaten recht groß ist: Finnland auf dem fünften Platz weist (bei fallender Tendenz) eine Forschungsquote von 2,76 \% auf, Belgien auf dem sechsten Platz eine Forschungsquote von 2,58 \%. Frankreich als letztes Land über der Zwei-Prozent-Marke erreicht 2,19\%, alle anderen Mitgliedstaaten - einschließlich der von der Europäischen Kommission ausgezeichneten „Innovation Leader" Großbritannien, Luxemburg und die Niederlande - weisen 2017 eine Forschungsquote von unter $2 \%$ auf.

In Abbildung 1-2 wird die Entwicklung der Finanzierungsanteile dargestellt, untergliedert nach öffentlichem Sektor (entspricht Bund, Bundesländer und Sonstigen in Abbildung 1-1), Unternehmenssek-

\section{Abbildung 1-2: Entwicklung der Finanzierung der in Österreich durchgeführten Forschung und experimentellen}

Entwicklung, 2008-2019 (Index, 2008=100)

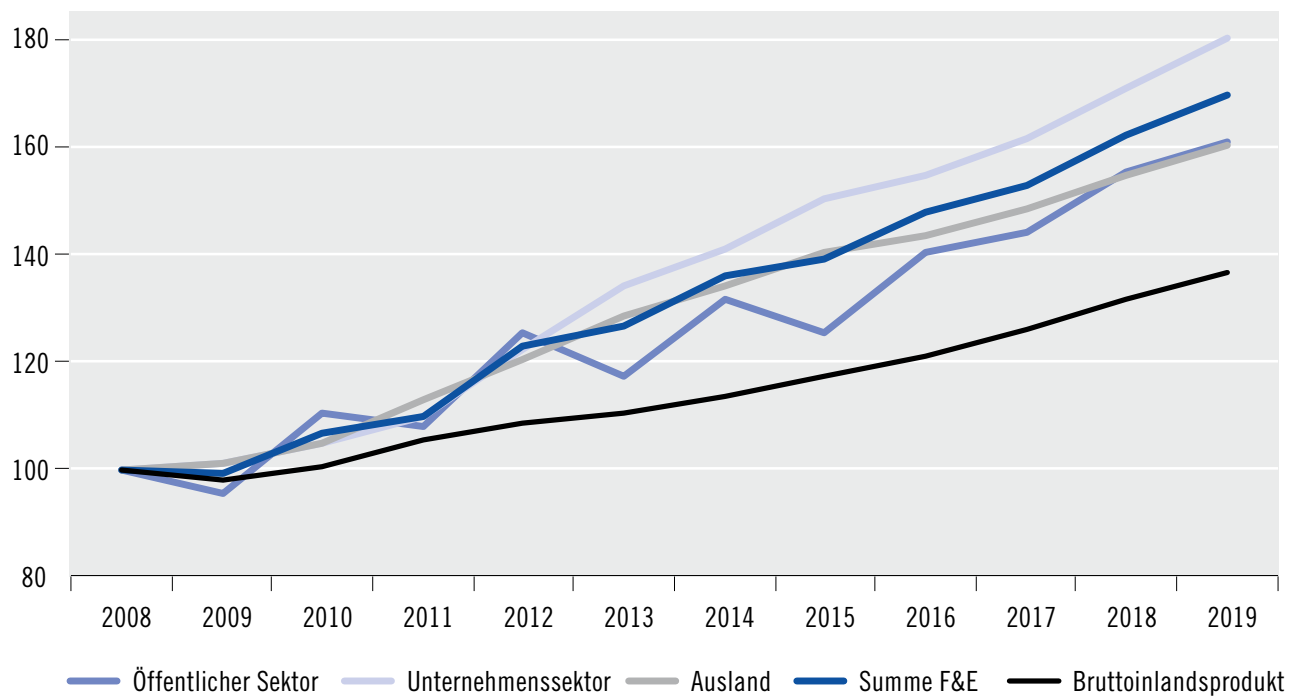

Quelle: Statistik Austria, Globalschätzung vom 11.04.2019, Berechnung und Darstellung: WPZ Research; die Kategorie „Öffentlicher Sektor“ enthält die Kategorien „Bund“, „Bundesländer“, „sonstige öffentliche Finanzierung“ und „privater gemeinnütziger Sektor“.

2 Zur besseren Darstellung werden in Abbildung 1-1 die Kategorien „sonstige öffentliche Finanzierung“ und „privater gemeinnütziger Sektor" als "Sonstige“ zusammengefasst.

3 Daten nach Eurostat, abgerufen am 11.04.2019; der Wert von 3,16 \% für Österreich weicht von 3,11 \% It. der aktuellsten Globalschätzung, auf der Abbildung 1-1 basiert, ab, da die Daten von Eurostat noch nicht entsprechend aktualisiert sind. 
tor und Ausland und verglichen mit der Entwicklung der gesamten Investitionen in F\&E sowie dem Bruttoinlandsprodukt. Wie in Abbildung 1-1 entsprechen die Werte den jeweiligen Preisen, d.h. es können keine Schlussfolgerungen hinsichtlich der realen Entwicklung gezogen werden. Allerdings erlaubt die Indizierung einen Vergleich der Entwicklung: Indem eine Kategorie von der Summe der F\&E-Investitionen nach oben oder unten abweicht, gibt die Entwicklung Aufschluss über einen steigenden oder fallenden Beitrag zur Finanzierung.

Tatsächlich liegt im dargestellten Beobachtungszeitraum 2008-2019 nur die Linie des Unternehmenssektors über jener der Summe der F\&E-Investitionen, d.h. dass die Unternehmen in diesem Zeitraum überproportional zum Wachstum und somit auch zum Erreichen des Drei-Prozent-Ziels beigetragen haben. Wie oben angemerkt enthält die Kategorie „Ausland“ großenteils Unternehmensfinanzierungen - somit sind es nicht die Unternehmen in ihrer Gesamtheit, sondern die heimischen Unternehmen, die zumindest seit 2012 überproportional zur Finanzierung in Österreich durchgeführter F\&E beitragen. Demgegenüber nimmt der Anteil des öffentlichen Sektors seither ab, wobei auch hier anzumerken ist, dass sich dieser auf den österreichischen öffentlichen Sektor bezieht, d.h. insbesondere der Finanzierungsanteil der EU ist in der Kategorie „Ausland“ enthalten.

In Abbildung 1-3 werden die Anteile der drei Kategorien öffentlicher Sektor, Unternehmenssektor und Ausland gesondert für jedes Jahr angegeben (Kategorien definiert wie in Abbildung 1-2). Hier wird besonders deutlich, wie der Beitrag der heimischen Unternehmen im Beobachtungszeitraum immer größer wird, er ist von 46,1 \% im Jahr 2008 auf für 2019 prognostizierte $49 \%$ gestiegen, was einem Wachstum des Anteils von 6,2\% im Beobachtungszeitraum entspricht. Allerdings sollte auch erwähnt werden, dass das Wachstum des Unternehmenssektors in den vorangegangenen zehn Jahren noch höher war, sein Anteil stieg von 41,7 \% im Jahr 1998 auf 46,1\% im Jahr

\section{Abbildung 1-3: Anteile der Finanzierung der in Österreich durchgeführten Forschung und experimentellen}

Entwicklung, 2008-2019

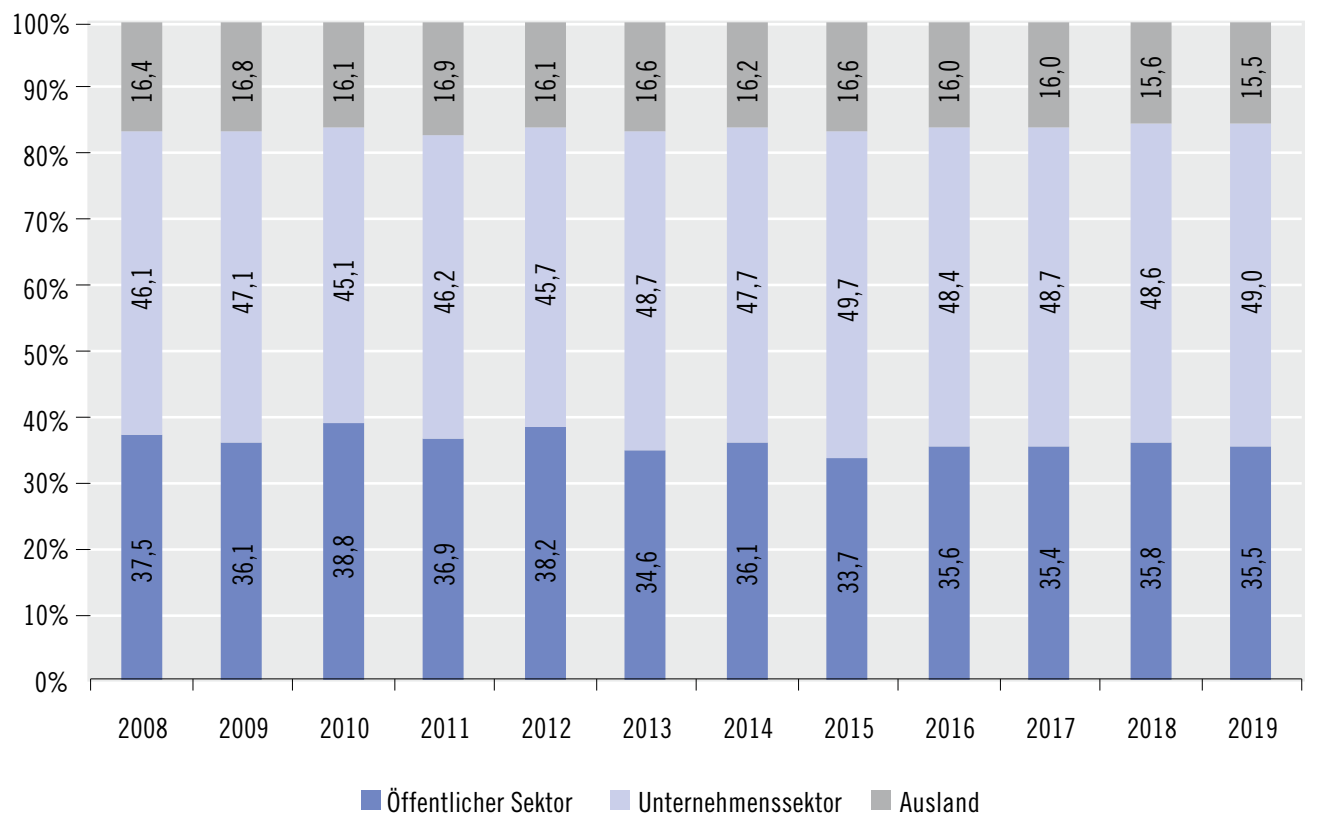

Quelle: Statistik Austria, Globalschätzung vom 11.04.2019, Berechnung und Darstellung: WPZ Research; die Kategorie „Öffentlicher Sektor" enthält die Kategorien „Bund“, „Bundesländer“, „sonstige öffentliche Finanzierung“ und „privater gemeinnütziger Sektor“. 
Abbildung 1-4: Forschungsquote in Österreich nach Finanzierung, 2008-2019

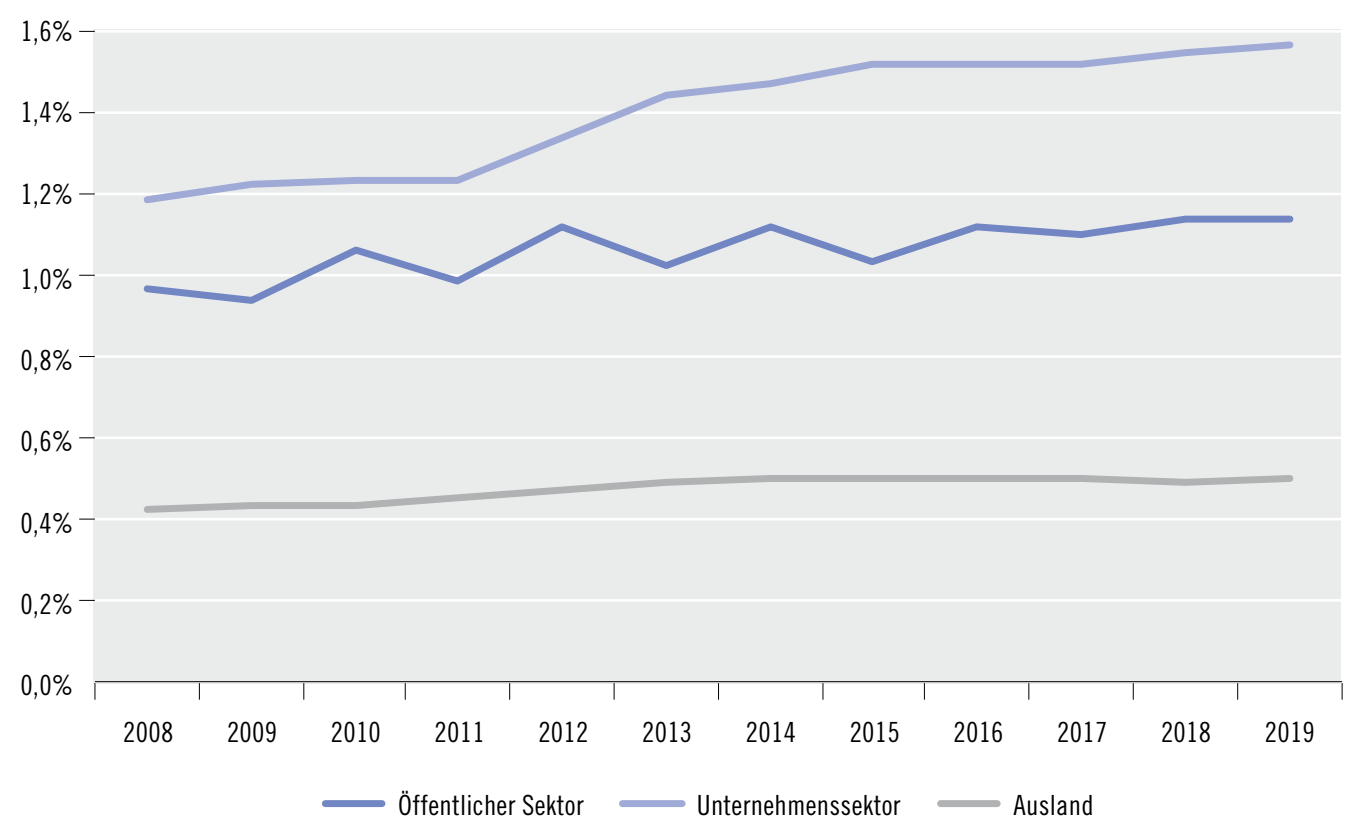

Quelle: Statistik Austria, Globalschätzung vom 11.04.2019, Berechnung und Darstellung: WPZ Research; die Kategorie „Öffentlicher Sektor" enthält die Kategorien „Bund“, „Bundesländer“, „sonstige öffentliche Finanzierung“ und „privater gemeinnütziger Sektor“.

2008, was einem Wachstum des Anteils von 10,5\% entspricht; zusätzlich war 2007 mit $48,7 \%$ bereits annähernd das heutige Niveau erreicht worden.

Durch das starke Ansteigen der Forschungsquote bei gleichzeitig steigendem Anteil des Unternehmenssektors innerhalb der F\&E-Investitionen ergibt sich nichtsdestoweniger ein kontinuierlich stark steigender Anteil der von heimischen Unternehmen finanzierten F\&E am Bruttoinlandsprodukt. In Abbildung 1-4 wird dieser Trend verdeutlicht: Der Unternehmenssektor hat seinen Anteil an der Forschungsquote von 1,18 Prozentpunkten im Jahr 2008 auf für 2019 prognostizierte 1,56 Prozentpunkte erhöht, was einem Wachstum des Anteils von $32 \%$ entspricht. Der öffentliche Sektor und der Beitrag des Auslands sind zwar auch gewachsen, aber mit 17,8 \% bzw. 17,6 \% wesentlich langsamer. Das deutet darauf hin, dass Österreich auf seinem Weg zu einem Innovationssystem, das sich durch privat finanzierte F\&E selbst trägt, Erfolge verzeichnen kann, da es offenbar immer attraktiver wird, in Österreich durchgeführte Forschung und experimentelle Entwicklung zu finanzieren. Anders formuliert: Wür- den die F\&E-Investitionen heimischer Unternehmen in Relation zum Bruttoinlandsprodukt im Jahr 2019 wie im Jahr 2008 nur 1,18 \% statt 1,56 \% betragen, dann fielen die Bruttoinlandsausgaben für F\&E 2019 um 1,52 Mrd. € niedriger aus.

In Abbildung 1-5 wird eine Übersicht über die OECD-Länder für das Jahr 2016 gegeben, sowohl hinsichtlich der F\&E-Quote als auch der Finanzierungsanteile. Die Forschungsquote Schwedens $(3,27 \%)$ als Spitzenreiter innerhalb der EU wird von Südkorea (4,23\%), Israel (4,39\%) und der Schweiz (2015: 3,37\%) noch übertroffen; hinter Japan (3,14 \%) liegt Österreich (3,13\%) innerhalb der OECD an sechster Stelle. Die Abbildung zeigt außerdem, dass Österreichs Anteil des öffentlichen Sektors (inkl. ,anderer nationaler Quellen“) mit 30,35\% knapp unter dem OECD-Durchschnitt liegt, der gewichtet 31,29\% beträgt. Der ausländisch finanzierte Anteil ist in Österreich mit 16,01\% weit über dem gewichteten OECD-Durchschnitt von 6,63\%, der (heimische) Unternehmensanteil fällt in Österreich mit 53,11 \% entsprechend niedriger aus als der OECD-Durchschnitt von $62,08 \%$. 
Bei genauerer Betrachtung zeigt sich jedoch, dass - relativ zur Forschungsquote - der Unternehmensanteil in Österreich im OECD-Vergleich niedrig, der Anteil des öffentlichen Sektors jedoch hoch ist. Der Korrelationskoeffizient des Anteils des öffentlichen Sektors und der Forschungsquote für die betrachteten Länder beträgt -0,7, d.h. tendenziell ist der Anteil des öffentlichen Sektors umso niedriger, je höher die Forschungsquote ist. Analog ist der Korrelationskoeffizient des Unternehmenssektors und der Forschungsquote mit 0,6 klar positiv, je höher also die Forschungsquote in einem Land, umso größer der Anteil heimischer Unternehmen. Dieser internationale Befund passt gut zum nationalen Befund aus $A b-$ bildung 1-1, 1-2, 1-3 und 1-4, da auch in Österreich bei steigender Forschungsquote der Finanzierungsanteil heimischer Unternehmen kontinuierlich zunimmt: sammenhang zwischen dem Finanzierungsanteil heimischer Unternehmen und der Forschungsquote, der entsprechende Korrelationskoeffizient beträgt für den Beobachtungszeitraum 2002-2019 0,6, für den Zeitraum 2008-2019, der den Abbildungen 1-1 bis 1-4 entspricht, sogar 0,8.

Zusammenfassend lässt sich somit festhalten, dass die Forschungsquote Österreichs schneller wächst als die Gesamtwirtschaft und die heimischen Unternehmen einen immer größeren Anteil der in Österreich durchgeführten F\&E finanzieren. Österreich hat bereits eine der höchsten Forschungsquoten, allerdings ist der Anteil der von heimischen Unternehmen finanzierten F\&E im internationalen Vergleich gering. Umso wichtiger ist, dass dieser Anteil weiter wächst, um Österreich als global führenden Forschungsstandort zu etablieren.

Auch für Österreich zeigt sich ein klar positiver Zu-

Abbildung 1-5: Forschungsquote und Finanzierung in OECD-Ländern, 2017

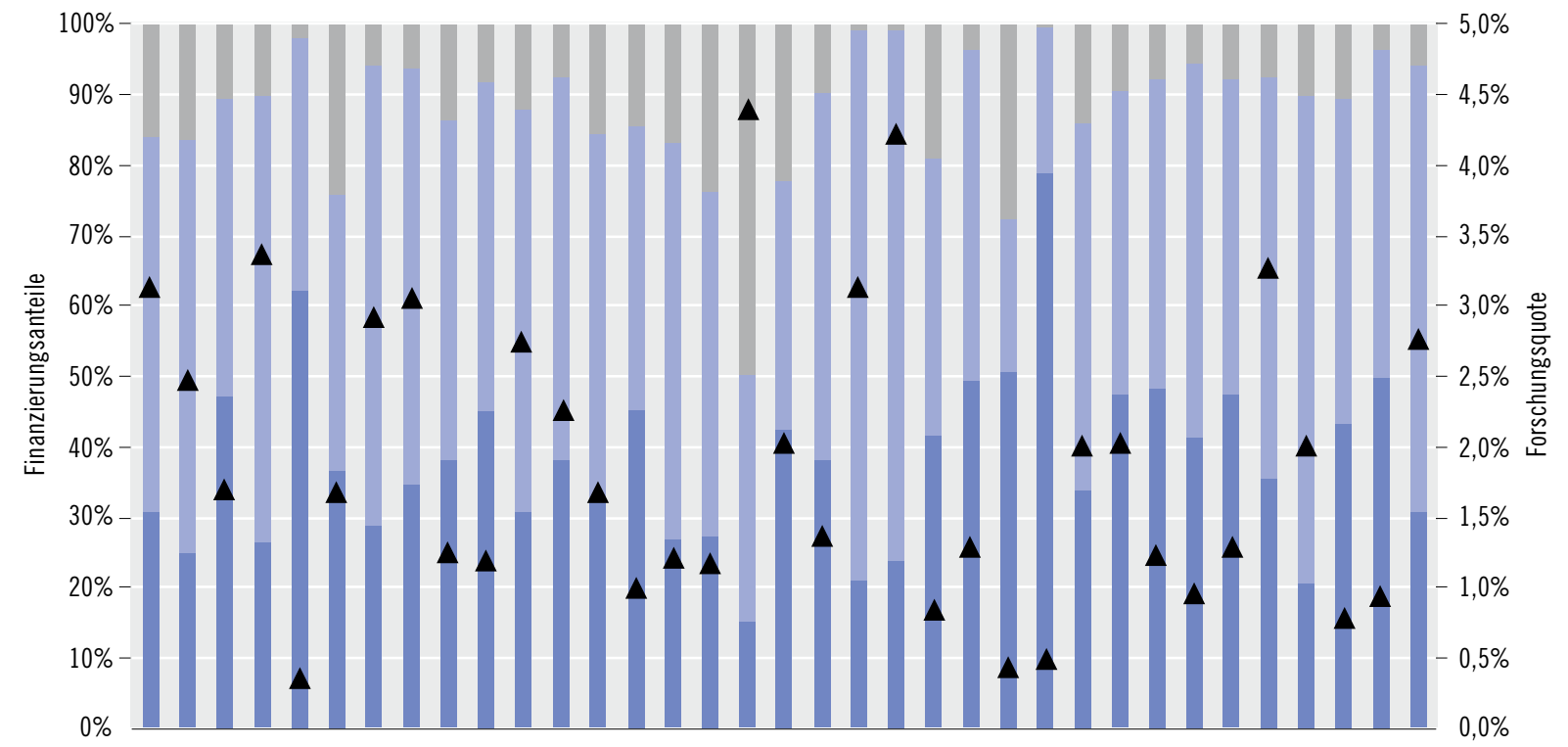

AT BE CA CH CL CZ DE DK EE ES FI FR GB GR HU IE IL IS IT JP KR LT LU LV MX NL NO NZ PL PT SE SI SK TR US

$$
\square \text { Öffentlicher Sektor } \square \text { Unternehmen } \square \text { Ausland \ Forschungsquote }
$$

Quelle: OECD, Main Science and Technology Indicators, abgerufen am 11.04.2019; Berechnung und Darstellung: WPZ Research; die Kategorie "Öffentlicher Sektor" enthält die Kategorien „government" und „other national sources", keine Finanzierungsdaten für Australien abrufbar; Daten für Belgien, Dänemark, Frankreich, Luxemburg, Neuseeland, Schweden und Schweiz für 2015; Länderkürzel nach ISO-3166-1. 


\section{Entwicklung der F\&E-Investitionen 2019 im Vergleich zum Vorjahr}

Laut der aktuellen Globalschätzung der Statistik Austria von April 2019 werden die Investitionen in F\&E im Jahr 2019 12,8 Mrd. $€$ betragen und damit um 4,5\% über dem Wert für 2018 (12,2 Mrd. €) liegen. Die geschätzte F\&E-Quote beträgt 2019 voraussichtlich 3,19\%, was einen leichten Anstieg im Vergleich zum Vorjahr (2018: 3,17 \%, revidierter Wert im Vergleich zur Globalschätzung 2018) bedeutet. Österreich würde damit zum bereits sechsten Mal in Folge über dem europäischen Zielwert von 3 \% liegen.

Bund und Bundesländer werden 2019 zusammen mit voraussichtlich 4,3 Mrd. $€$ einen Anteil von $33,9 \%$ der gesamten in Österreich durchgeführten F\&E finanzieren. Während das einen Anstieg um 148,4 Mio. $€$ bedeutet, wird der Anteil von Bund und Ländern leicht sinken (2018: 34,2\%). Der größte Anteil der öffentlichen F\&E-Ausgaben wird mit voraussichtlich 3,8 Mrd. $€(+3,4 \%$ bzw. + 123,4 Mio. $€$ ) auf den Bund entfallen. Darunter finden sich auch Ausgaben in Höhe von 138,7 Mio. $€$ für die Nationalstiftung für Forschung, Technologie und Entwicklung und voraussichtlich 670,0 Mio. $€$ (Schätzung BMF, Stand: April 2019) für die Forschungsprämie.

Die heimischen Unternehmen werden 2019 mit 6,3 Mrd. € oder 48,96\% knapp die Hälfte aller F\&E finanziert haben. Heimische Unternehmen finanzieren einen immer größeren Anteil der in Österreich durchgeführten $F \& E$, im Vergleich zum Vorjahr stiegen die Ausgaben um 314,1 Mio. $€$ bzw. $5,3 \%$ an.

Rund 2 Mrd. $€$ bzw. 15,6\% werden 2019 vom Ausland finanziert werden, wobei dieser Betrag zum größten Teil von ausländischen Unternehmen finanzierte, in Österreich durchgeführte F\&E sowie Rückflüsse aus den EU-Forschungsprogrammen umfasst. Das bedeutet einen leichten Anstieg um 76,8 Mio. $€$ bzw. 4 \%. Die Kategorie "Sonstige“ fasst sonstige öffentliche Finanzierung und den privaten gemeinnützigen Sektor (private gemeinnützige Institutionen ohne Erwerbscharakter, deren Status ein vorwiegend privater oder privatrechtlicher, konfessioneller oder sonstiger nicht öffentlicher ist) zusammen; sie spielen mit 1,0\% bzw. 0,6\% nur eine verhältnismäßig geringe Rolle.

\subsection{Die Position Österreichs im internationalen Vergleich}

Dieses Kapitel stellt die Position Österreichs in Forschung, Technologie und Innovation im internationalen Vergleich dar. Dazu werden drei Analyseschritte vorgenommen: Erstens werden Indikatoren zum aktuellen Input und Output herangezogen, um die aktuelle Leistungsfähigkeit in Forschung und Entwicklung im Ländervergleich komparativ zu analysieren. Zweitens wird die Position Österreichs bezüglich der digitalen Durchdringung in Wirtschaft und Gesellschaft betrachtet. Aufgrund des zunehmenden digitalen Wandels deuten diese Indikatoren an, inwieweit Volkswirtschaften und Gesellschaften auf eine zunehmend digitale Arbeitswelt vorbereitet sind. Und drittens werden verschiedene Aspekte und Indikatoren in den Blick genommen, von denen sich die Innovationsfähigkeit Österreichs ableiten lässt.

Die verwendeten Indikatoren basieren auf den folgenden Datenquellen ${ }^{4}$ :

- Global Innovation Index 20185: Im Global Innovation Index (GII) werden insgesamt 80 Indikatoren zu 126 Ländern erhoben. Mit Hilfe der 80 Indikatoren wird eine Rangliste aller untersuchten Länder veröffentlicht, die die Innovationsfähigkeit einzelner Länder darstellt. Die Rangliste wird in einem jährlichen Intervall von der französischen Business

4 Anmerkung: Der vorliegende Bericht geht nicht auf den European Innovation Scoreboard ein, da seit dem FTB 2018 keine neuen Daten vorliegen.

5 Vgl. https://www.globalinnovationindex.org/Home, zuletzt geprüft am 15.01.2019 
School INSEAD, der Cornell University und der Weltorganisation für geistiges Eigentum (WIPO) der Vereinten Nationen herausgegeben. In dieser Untersuchung werden der Gesamtindex sowie Informationen zur Nutzung und zum Zugang von Informations- und Kommunikationstechnologien untersucht.

- Global Competitiveness Report 20186: Im Global Competitiveness Report (GCR) werden 140 Volkswirtschaften analysiert und eine Rangliste der Volkswirtschaften mit den höchsten Wachstumschancen erstellt. Der Bericht wird vom Weltwirtschaftsforum (WEF) herausgegeben. Die Einstufung basiert auf der Auswertung öffentlich zugänglicher Primärdaten und Befragungen von Wirtschaftsführern. In dieser Untersuchung werden der Gesamtindex sowie Informationen zum Humankapital und zur Fähigkeit, technologische Entwicklungen in Innovationen umzusetzen, untersucht.

- Readiness for the Future of Production Report 2018: Das Weltwirtschaftsforum gibt den Bericht "Readiness for the Future of Production Report" heraus, in dem eine vergleichende Analyse von 100 Ländern durchgeführt wird. Mit Hilfe von 59 Indikatoren wird darin die Produktionsbasis analysiert und werden Schlüsseltechnologien, sowie die Fähigkeit, diese zu nutzen und Produktionssysteme zu verändern, vergleichend analysiert.

- OECD - Main Science and Technology Indicators: Die Organisation für wirtschaftliche Zusammenarbeit und Entwicklung (OECD) veröffentlicht in ihrer Datenbank ${ }^{8}$ wichtige Indikatoren zu einem breiten Spektrum von Themen, u.a. zu Wirtschaft, Bildung, Energie, Verkehr sowie Forschung und Entwicklung. Die Datenbank enthält Daten für alle
OECD-Länder, ergänzt durch den jeweiligen OECD-Gesamtwert, den Euroraum und die Europäische Union sowie - falls verfügbar - Daten für Brasilien, China, Indien, Indonesien, die Russische Föderation und Südafrika. Für den vorliegenden Bericht werden insbesondere die Daten zu Ausgaben in Forschung und Entwicklung und F\&E-Personal verwendet.

- Digital Economy and Society Index (DESI) Report 20189: In diesem Report führt die Europäische Kommission die Ergebnisse des Index für die digitale Wirtschaft und Gesellschaft (DESI) 2018 an, mit dem die Fortschritte der Mitgliedstaaten bei der Internetanbindung, der Anwendung digitaler Kompetenzen im Internet, der Digitalisierung der Unternehmen und digitalen öffentlichen Diensten beobachtet werden. Dabei werden einzelne Unterindikatoren fünf übergeordneten Themenberichten zugeordnet, die fünf zugehörigen Indikatoren zu den Themenbereichen werden infolge ausgewertet.

- Innovationsindikator: Der Innovationsindikator wird durch das Fraunhofer Institut für Systemund Innovationsforschung (ISI) und das LeibnizZentrum für Europäische Wirtschaftsforschung $\mathrm{GmbH}$ Mannheim (ZEW) für den Bundesverband der deutschen Industrie (BDI) erstellt. ${ }^{10}$ Dieser Kompositindikator für die Bemessung nationaler Innovationspotentiale setzt sich aus insgesamt 38 In- und Output-Indikatoren zusammen, die wiederum auf die fünf Subindikatoren Bildung, Wissenschaft, Wirtschaft, Staat und Gesellschaft aufgeteilt sind. ${ }^{11}$

In diesem Kapitel werden nachfolgend Indikatoren innerhalb der 28 EU-Staaten vergleichend ausgewertet. Zusätzlich werden die Schweiz sowie Südafrika,

6 Vgl. http://www3.weforum.org/docs/GCR2018/05FullReport/TheGlobalCompetitivenessReport2018.pdf, zuletzt geprüft am 02.05 .2019

7 Vgl. http://www3.weforum.org/docs/FOP_Readiness_Report_2018.pdf, zuletzt geprüft am 15.01.2019

8 Vgl. http://stats.oecd.org/, zuletzt geprüft am 15.01.2019

9 Vgl. https://ec.europa.eu/digital-single-market/en/desi, zuletzt geprüft am 15.01.2019

10 Zuvor wurde der Innovationsindikator außer für den BDI auch für die Deutsche Akademie der Technikwissenschaften (Acatech) und für die Deutsche Telekom AG erstellt.

11 Vgl. http://www.innovationsindikator.de/, zuletzt geprüft am 15.01.2019 
die USA, China, Singapur, Brasilien und Australien als Referenzländer betrachtet, sofern vergleichbare Daten zu den jeweiligen Indikatoren vorliegen. Letztere Länder stehen stellvertretend für ihren Kontinent als die größten Volkswirtschaften gemessen an der Bruttowertschöpfung. Mit dieser Auswahl lässt sich der Abstand Österreichs zu wirtschaftlich und innovationspolitisch weltweit bedeutenden Ländern anhand verschiedener Aspekte untersuchen.

Der Rest dieses Kapitels gliedert sich wie folgt: In Abschnitt 1.3.1 werden Indikatoren zu Input und Output in Forschung und Technologie betrachtet, um die aktuelle Position Österreichs bewerten zu können. In Abschnitt 1.3.2 werden explizit Indikatoren zur digitalen Wirtschaft in den Blick genommen, da die Nutzung digitaler Technologien bei der zukünftigen Wettbewerbstätigkeit sowohl innerhalb als auch außerhalb der Landesgrenzen von besonderer Bedeutung ist. Abschließend werden in Abschnitt 1.3.3 Indikatoren zur Innovationsfähigkeit von Ländern untersucht, wobei die Methodik des Innovationsfähigkeitsindikators des Instituts für Innovation und Technik ${ }^{12}$ adaptiert wird.

\subsubsection{Entwicklung der Position Österreichs bei zentralen FTI-Indikatoren}

In diesem Abschnitt wird die Position Österreichs im internationalen Vergleich anhand unterschiedlicher zentraler Indikatoren aus Forschung, Technologie und Innovation dargestellt. Den Anfang nimmt dabei eine Auswahl an klassischen Indikatoren zu In- und Output des FTI-Systems im internationalen Vergleich. Wie oben erwähnt erfolgt bei den Einzelindikatoren die ländervergleichende Analyse in der Regel auf der Auswahl der 28 EU-Staaten sowie den folgenden einzelnen Nationen: China, Singapur für Asien, Brasilien und USA, sowie Südafrika und Australien als relevan- te, innovationsdynamische Vertreter der weiteren vier Kontinente. Hinzu kommt außerdem die Schweiz als weiterer wichtiger Akteur unter den globalen Wissenschafts- und Innovationsnationen.

Abbildung 1-6 bietet zunächst einen Überblick über die Entwicklung der F\&E-Quote Österreichs im internationalen Vergleich, das heißt die nationalen Ausgaben für Forschung und Entwicklung gemessen am Bruttoinlandsprodukt gemäß den OECD Main Science and Technology Indicators. Die sogenannte F\&E-Quote (Gross Expenditure for Research and Development - GERD in Prozent des Bruttoinlandsprodukts (GDP)) ist ein zentraler Indikator zur Beschreibung der F\&E-Aktivitäten eines Landes in einem abgegrenzten Zeitraum (i.d.R. Kalenderjahr) und umfasst alle Ausgaben für $\mathrm{F} \& \mathrm{E}$, die in einer nationalen Volkswirtschaft durchgeführt werden. Somit beinhaltet die F\&E-Quote auch die im Inland erbrachten Mittel, die aus anderen Ländern finanziert werden (jedoch nicht im Ausland erbrachte F\&E-Leistungen). Die F\&E-Quote ist der wichtigste Indikator für den internationalen Vergleich der F\&EAktivitäten. ${ }^{13}$

Die F\&E-Quote Österreichs hat sich 2017 mit Blick auf das Vergleichsjahr 2010 von 2,73\% auf $3,16 \%{ }^{14}$ stark gesteigert. Österreichs F\&E-Quote liegt damit innerhalb der 28 EU-Länder an zweiter Stelle hinter Schweden, dessen Quote sich von 2010 bis 2017 erneut von 3,21 \% auf 3,33 \% erhöht hat. Während sich die durchschnittlichen, proportionalen F\&E-Ausgaben der EU-28 in Höhe von zuletzt 1,96 \% (2017) ungefähr auf Augenhöhe mit investitionsstarken Nationen wie der VR China $(2,13 \%)$ oder Singapur $(2,16 \%)$ befinden, hat Österreich dieses Niveau somit bereits seit Jahren hinter sich gelassen. Nach einem ungefähren Gleichstand mit den USA um 2010 (2,74 \%) weist die F\&E-Quote Österreichs 2017 bereits einen großen Abstand

12 Vgl. https://www.iit-berlin.de/de/indikator/laenderanalyse, zuletzt geprüft am 25.01.2019

13 Vgl. OECD (2015).

14 Vgl. OECD Main Science and Technology Indicators, https://stats.oecd.org/Index.aspx?DataSetCode=MSTI_PUB, zuletzt geprüft am 01.03.2019. Dieser Wert der OECD weist eine geringfügige Differenz zu Berechnungen von Eurostat (für Österreich werden hier 2016 3,13\% ausgewiesen) und der Globalschätzung von Statistik Austria (2016: 3,15\%) auf. 


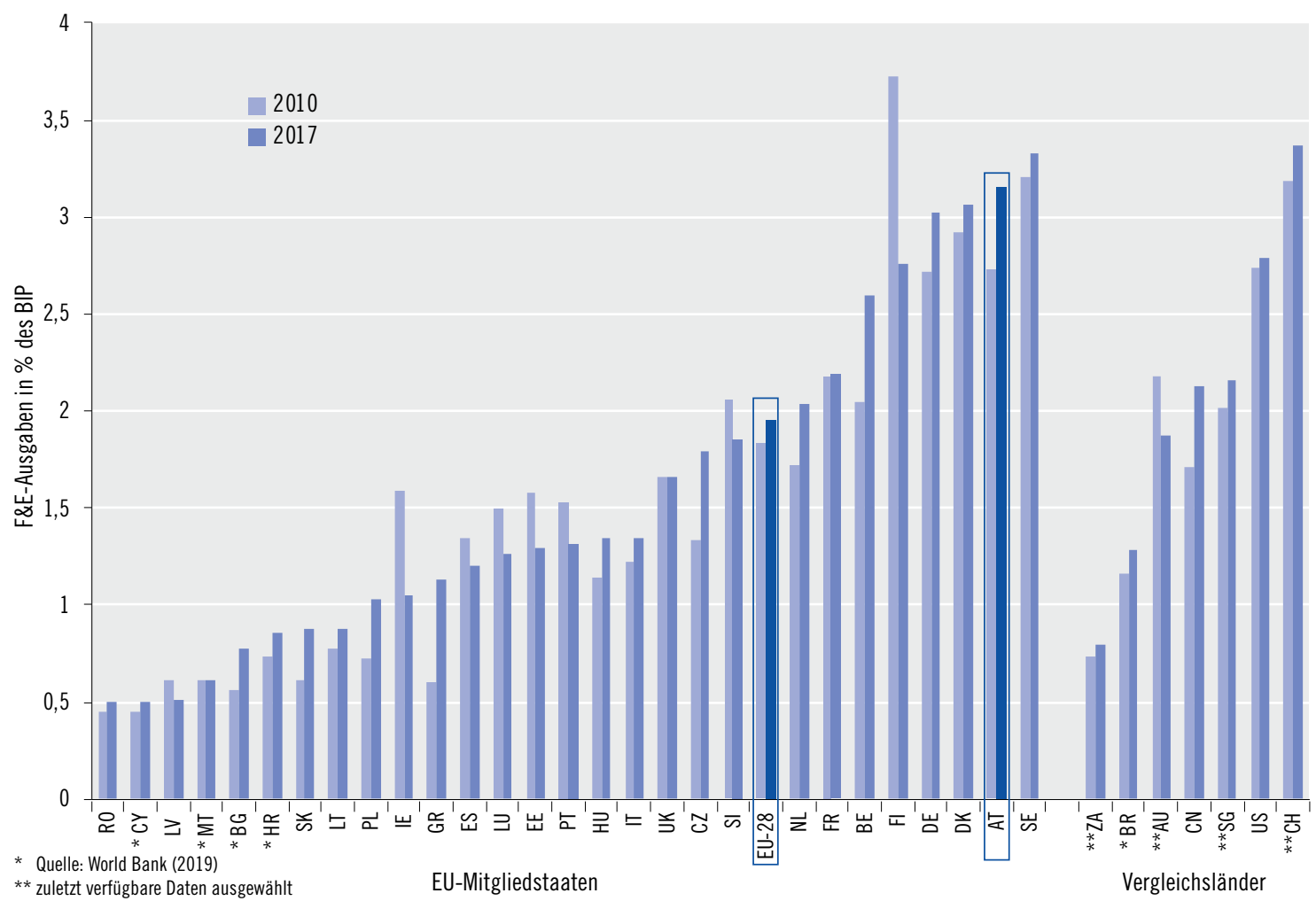

Quelle: OECD (2018b): MSTI, ergänzt mit Daten aus der World Bank (2019).

zum Wert der USA auf, deren F\&E-Quote zwischen 2010 und 2017 (mit geringfügigen Schwankungen) fast stagnierte. Unter allen Referenzländern führend ist 2016 die Schweiz mit 3,37\%.

Die Werte der F\&E-Quote Österreichs im internationalen Vergleich sowie mit Blick auf die zeitliche Entwicklung verdeutlichen somit ein insgesamt intensives finanzielles Engagement im Forschungs- und Entwicklungsbereich, das in Bezug auf das nationale BIP im Vergleich zu den weltweit führenden Nationen gehört.

In Europa weisen 2017 neben Österreich und dem hierin EU-weit führenden Schweden (3,33 \%) noch Dänemark (3,06 \%), Deutschland (3,02\%), Finnland $(2,76 \%)$ sowie Belgien (2,60\%) vergleichbar hohe Quoten auf.

\section{Patentanmeldungen}

Die OECD definiert "Triade-Patente" als eine Reihe von Patenten, die zum Schutz derselben Erfindung beim Europäischen Patentamt (EPA), dem Japanischen Patentamt (JPO) und dem Patent- und Markenamt der Vereinigten Staaten (USPTO) angemeldet wurden. Dieses Konzept ermöglicht es auch, internationale Vergleiche durchzuführen: Patenterfindungen in mehreren Ländern können als Indikator für die Qualität von Erfindungen angesehen werden und somit der Abschätzung des Innovationspotentials dienen.

Abbildung 1-7 zeigt die Triade-Patentintensität nach Herkunftsland für die Jahre 2012 und 2016 ${ }^{15}$. Es wird die Anzahl der Patente je 1.000 Beschäftigte in Forschung und Entwicklung dargestellt. Durch die

15 Für die Normierung der absoluten Anzahl von Triade-Patenten je Land über die Anzahl der F\&E-Beschäftigten wurden für den letzteren Parameter jeweils die Werte im Abstand von zwei Jahren zugrunde gelegt (2014 und 2010). Damit wurde der üblichen Antrags- und Registrierungsdauer der Patente Rechnung getragen. 
Abbildung 1-7: Patentintensität nach Herkunftsland (Triade-Patente, 2012 und 2016)*

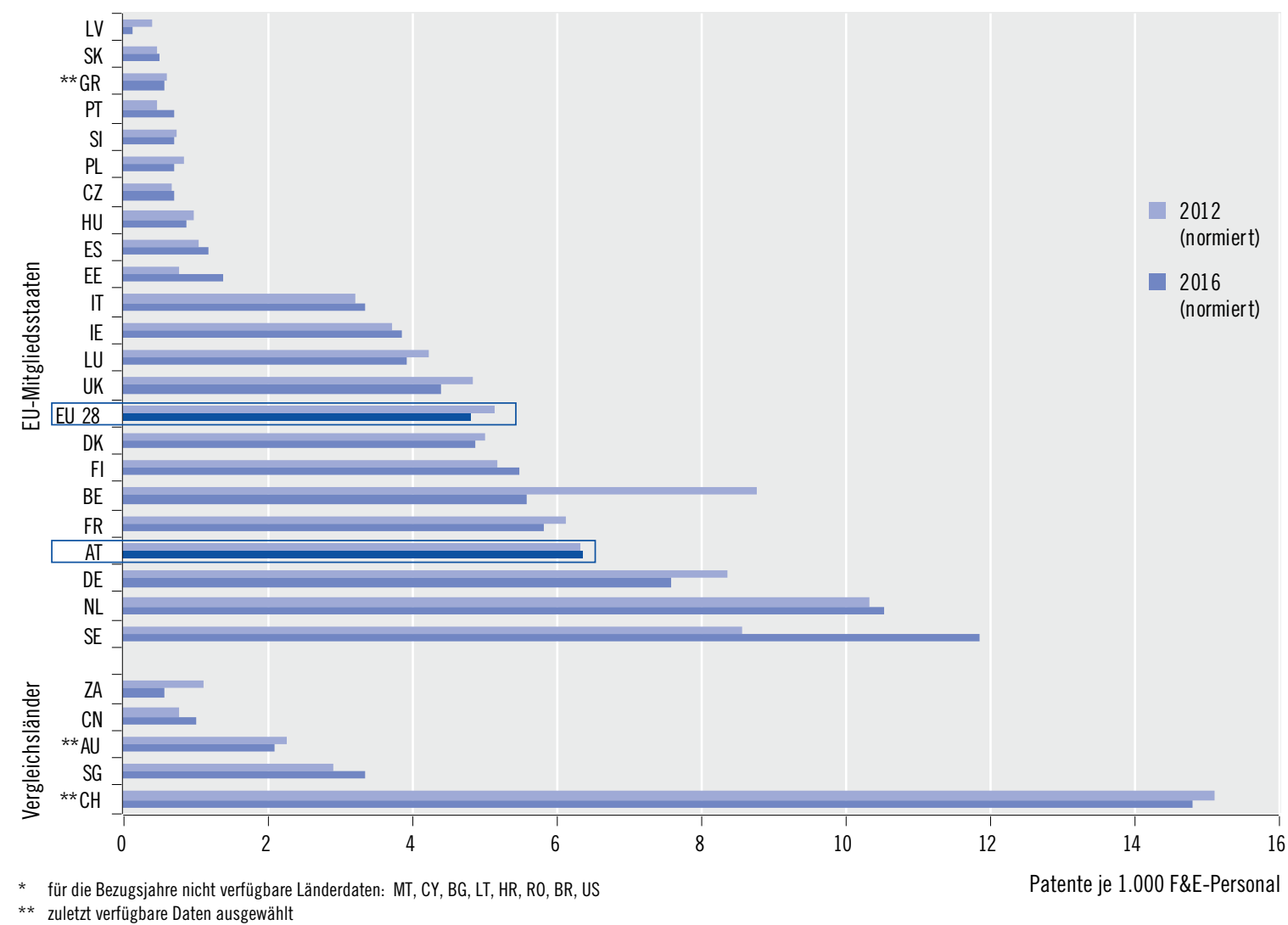

Quelle: OECD (2018b): MSTI.

Konzentration auf das F\&E-Personal soll die Effektivität des wissenschaftlichen Systems in Bezug auf sein ökonomisches Anwendungspotenzial eines Landes aufgezeigt werden.

Im Jahr 2012 wurden in Österreich 6,3 Patente pro 1.000 Beschäftigte in Forschung und Entwicklung angemeldet. Vier Jahre später hat sich die Anzahl der Patente leicht auf 6,4 erhöht. Somit konnte sich die Position Österreichs vom fünften auf den vierten Rang in der Patentintensität verbessern. Bei den anderen, in der Patentintensität führenden Ländern ist das Bild uneinheitlich: Während die Anzahl in den Niederlanden leicht (von 10,3 auf 10,5) und in Schweden stark (von 8,6 auf 11,8) zunahm, hat sich die Anzahl der Patente in Deutschland von 8,4 auf 7,6 verringert.

Sowohl 2012 als auch 2016 liegt die Patentintensität Österreichs deutlich über dem Durchschnittswert der EU-28-Länder. Dieser hat sich in diesem Zeitraum von 5,2 auf 4,8 verschlechtert. Auch im Vergleich mit führenden Volkswirtschaften der anderen Kontinente liegt Österreich deutlich vorne, während beispielsweise das in allen Innovationsbereichen dynamische Singapur hier mit 3,6 Patenten pro 1.000 F\&E-Beschäftigten im Jahr 2016 proportional wesentlich schwächer abschneidet. Führend auch in dieser Kategorie ist die Schweiz mit zuletzt 14,8 Patenten je 1.000 Beschäftigte in 2016.

\section{Österreichs internationale Position in Bezug auf wissenschaftliche Publikationen}

Weitere relevante Indikatoren zur Bewertung wissenschaftlich-technologischer Leistung bietet die Analyse wissenschaftlicher Publikationen. Hier werden in der Regel zunächst die quantitativen Werte auf nationaler Ebene betrachtet, in denen aktive Nationen 


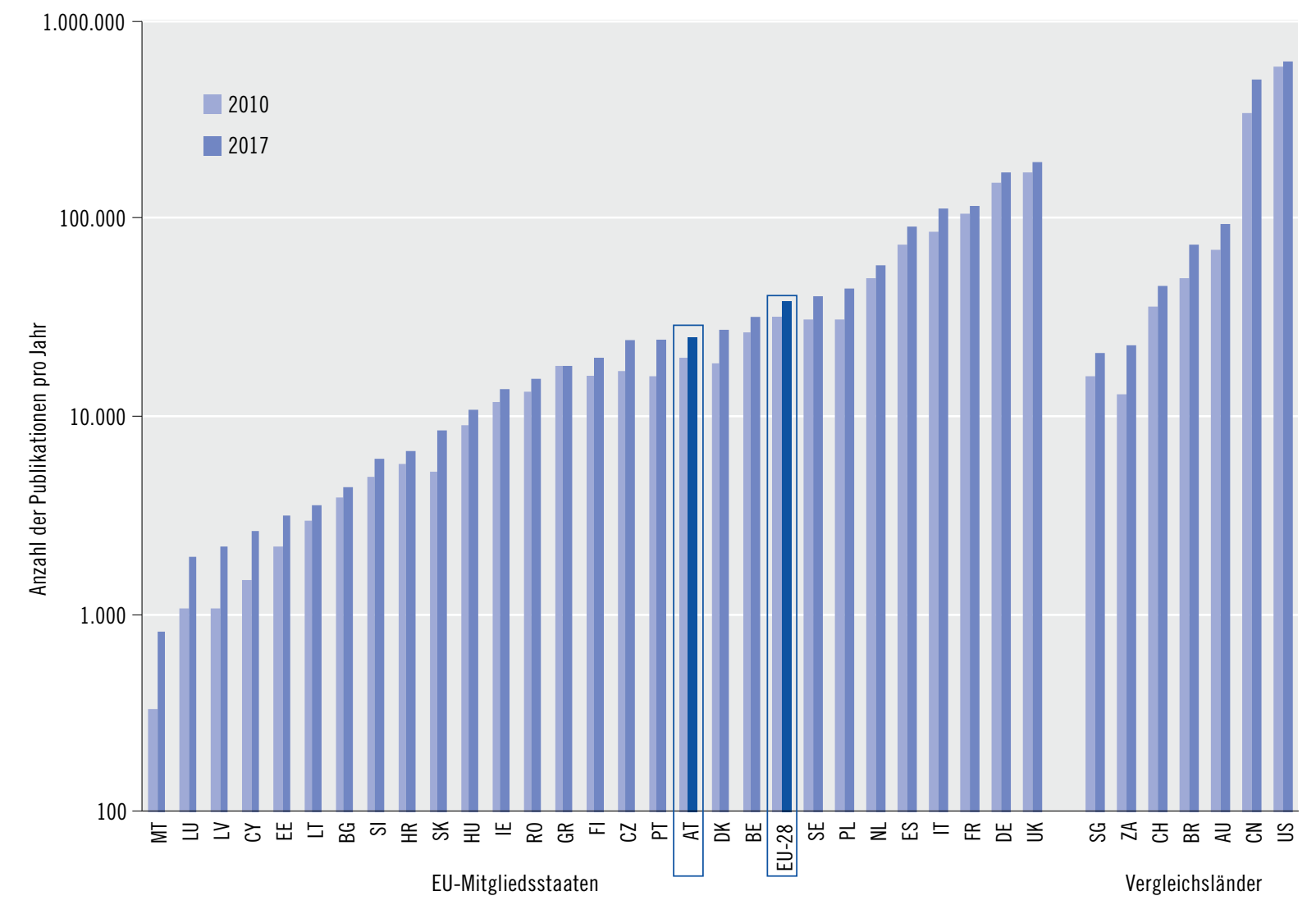

Quelle: SCImago (2018).

mit größeren Populationen naheliegenderweise die vorderen Plätze einnehmen.

Auf Basis der Publikationsdaten von $\mathrm{SClmago}^{16}$ ergibt sich diesbezüglich zunächst folgendes Bild: Die USA führen 2017 wie gewohnt mit der höchsten Anzahl an Publikationen weltweit (und somit auch unter den Referenzländern) mit 626.403 erfassten Publikationen. China folgt auf dem zweiten Rang mit in 2017 erneut gesteigerten Werten in Höhe von 508.654 Publikationen.

Österreich liegt im Hinblick auf die Gesamtzahl seiner wissenschaftlichen Publikationen im Jahr 2017 im Vergleich der EU-28-Länder im Mittelfeld auf dem 11. Rang. Im Vergleich zum Jahr 2010 wird in der Darstellung die insgesamt für alle Länder zunehmende Publikationsstärke als anhaltender globaler Trend deutlich. Hierbei fallen bei einigen traditionell publikationsstarken Nationen wie den USA, UK, Deutschland oder auch Österreich die Steigerungen im Vergleich zu aufstrebenden Ländern wie China oder auch Brasilien geringer aus.

Ergänzend wird daher das Leistungspotential Österreichs im internationalen Vergleich anhand der wissenschaftlichen Publikationen im Verhältnis zur jeweiligen Bevölkerungsanzahl der Länder (erneut vergleichend für die Jahre 2017 und 2010) betrachtet. Die Position Österreichs ändert sich im europäischen Vergleich aufgrund dieser Normierung nicht. Allerdings weisen die in absoluten Publikationszahlen global führenden Nationen unter den außereuropäischen Vergleichsländern im Hinblick auf die ProKopf-Verteilung des wissenschaftlichen Outputs ins-

16 SClmago Journal \& Country Rank bezieht seine Daten aus der Publikationsdatenbank Scopus des Elsevier-Verlags. https://www. scimagojr.com/, zuletzt geprüft am 25.01.2019 
Abbildung 1-9: Anzahl der Artikel im Bereich Wissenschaft und Technik normiert mit der Länderpopulation, 2010 und $2017^{*}$

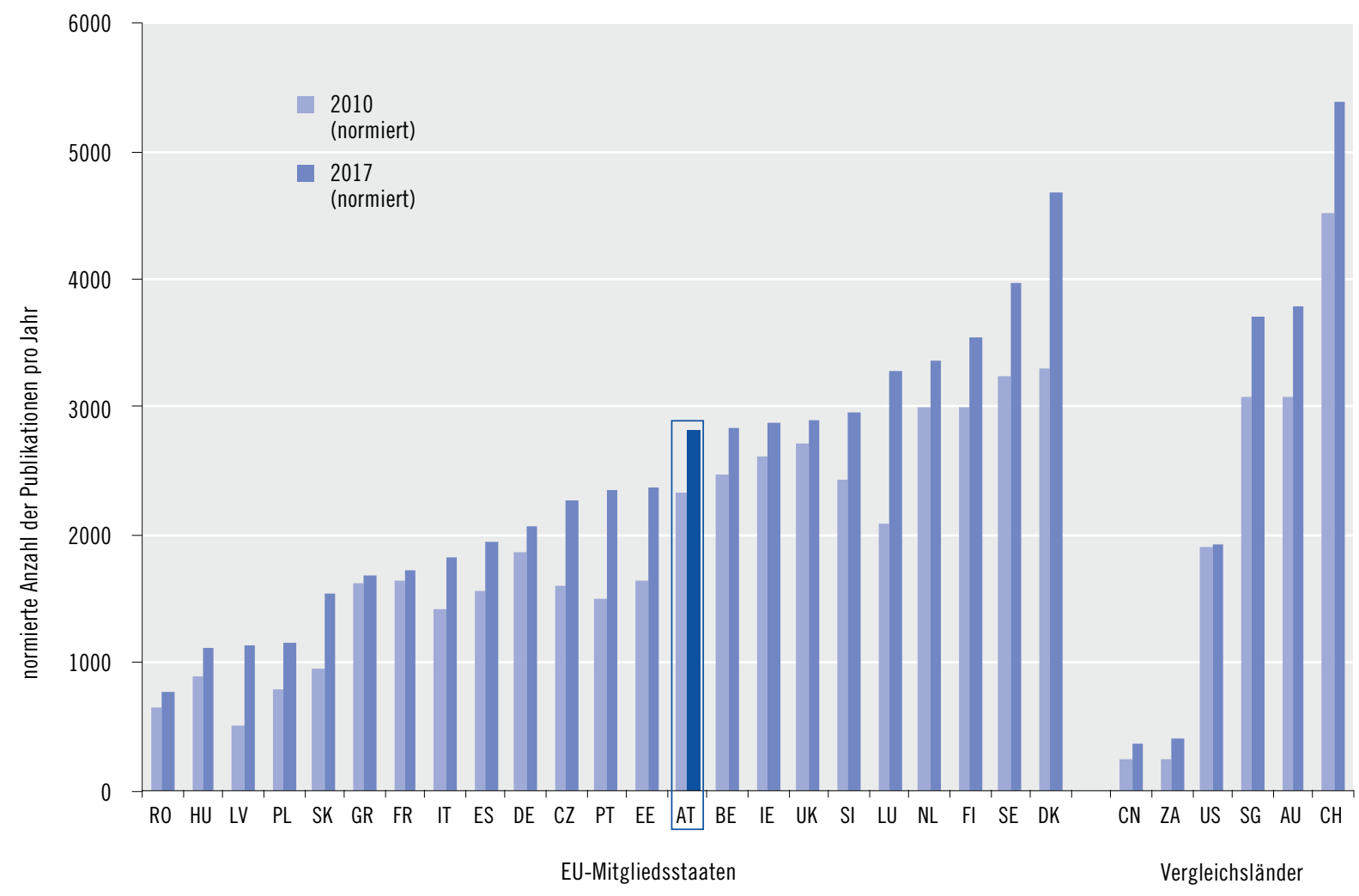

* für die Bezugsjahre nicht verfügbare Länderdaten: BG, BR, CY, HR, LT, MT Quelle: SClmago (2018).

gesamt schwächere Werte auf. Die Schweiz sticht in dieser Gruppe als mit Abstand führende Nation hervor. Die vergleichsweise hohe Effizienz der EU-Region in dieser Kategorie wird in der Gesamtschau deutlich, was als positiver Faktor auch für Österreich als Teil der European Research Area zu werten ist (siehe Abbildung 1-9). Nichtsdestotrotz sind die in dieser Kategorie führenden nordeuropäischen Nationen wie Dänemark, Schweden oder Finnland als anschauliche Beispiele für zusätzliches Verbesserungspotenzial anzusehen.

Ebenfalls basierend auf Scopus-Daten (über SClmago, s.o.) bietet Abbildung 1-10 eine zusätzliche Sichtweise auf die wissenschaftliche Leistung Österreichs im internationalen Vergleich: Mithilfe der Zitationsrate werden in der Bibliometrie die Wahrnehmung bzw. weitere Nutzung von Publikationen als Ansatz einer qualitativ vertiefenden Bewertung in- nerhalb der Publikationsanalyse verwendet. Da auch dieser Indikator jedoch nicht frei von Beeinflussungsrisiken ist, wird hier vergleichend neben der Rate aller Zitationen auf der Gesamtheit der Publikationen eines Landes noch eine Rate der Zitationen abzüglich der Selbstzitationen von Autorinnen und Autoren erstellt. Insbesondere bei der Zitationsrate ohne Selbstzitationen zeigt sich erneut Österreichs relativ starke Position mit dem Wert 0,61 auf dem achten Platz im europäischen Vergleich (2017). Auch unter den Vergleichsländern liegt nur Singapur bei diesem Leistungsindikator vor Österreich mit 0,65. EU-weit und im Vergleich mit den außereuropäischen Referenzländern führend sind Dänemark mit 0,71, die Niederlande mit 0,70 und Belgien mit 0,69.

China als derzeit aufstrebende Wissenschaftsund Innovationsnation, das in absoluten Publikationswerten in den letzten Jahren aufsehenerregende 
Abbildung 1-10: Zitationsraten mit und ohne Selbstzitation, 2017

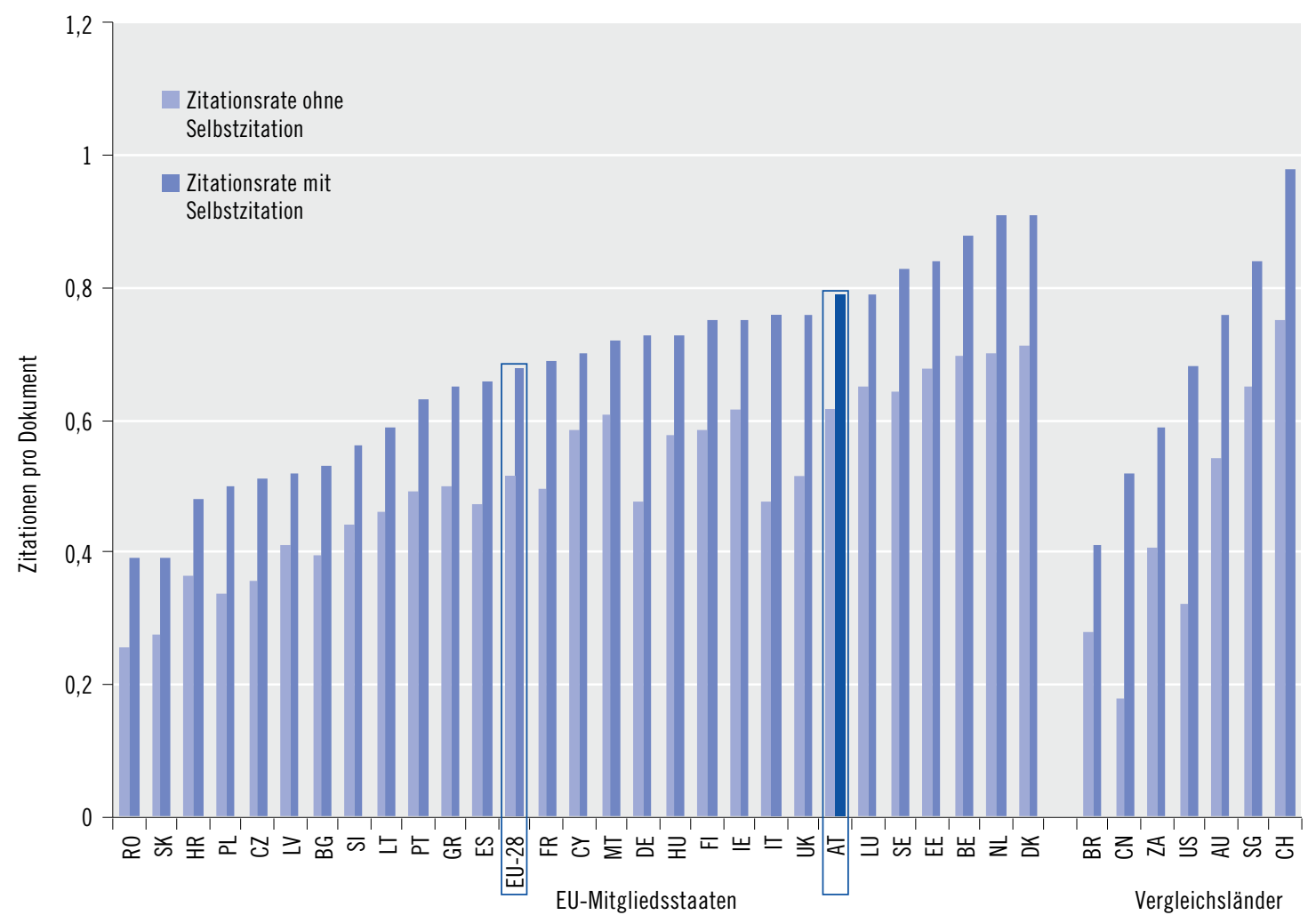

Quelle: SCImago (2018).

Leistungen darstellen konnte und auch in der allgemeinen Zitationsrate starke Fortschritte erzielt hat, weist bei der Zitationsrate abzüglich der Selbstzitationen jedoch schwache Ergebnisse auf (0,17 im Jahr 2017). Auch die in der Regel in Bezug auf Publikationsdaten starken USA weisen im Vergleich zur allgemeinen Zitationsrate mit 0,68 im Jahr 2017 bei Abzug der Selbstzitationen lediglich eine Rate von 0,32 auf.

Der letztgenannte Indikator (Zitationsrate ohne Selbstzitationen) stellt eine qualitätsorientierte Vertiefung der Publikationsanalysen dar. Dieser rundet den Gesamteindruck Österreichs internationaler Position im Bereich Forschung, Technologie und Innovation insofern ab, als dass die wissenschaftlich meßbare Leistung von Österreich sich nicht nur im zeitlichen Vergleich kontinuierlich gesteigert hat, sondern dieser positive Trend angesichts der Wahrnehmung durch die jeweilige Wissenschaftscommunity (Zitationsrate) auch auf eine nachhaltige qualitative Ent- wicklung verweist (hier 2017 zu 2010). Dennoch befindet sich Österreich in allen publikationsbezogenen Indikatoren noch im vorderen Mittelfeld, so dass eine Fortsetzung und weitere Intensivierung der Bemühungen auch in diesem Bereich weiterhin notwendig erscheint, um zu den führenden Nationen aufzuschließen.

\section{Österreichs internationale Position aus der Sicht globaler Innovationsrankings}

Für eine Einordnung der Position Österreichs im internationalen Vergleich kann eine Verwendung von Kompositindikatoren, die unterschiedliche einzelne Indikatoren zu einer Kennzahl bündeln, zusätzliche Erkenntnisse liefern. Sie ermöglichen eine Informationsverdichtung und erleichtern somit einen globalen Vergleich der Volkswirtschaften. Unterschiedliche Kompositindikatoren stehen für verschiedene Ansätze der Innovationspotentialanalyse, womit sich auch tendenziell divergierende Gesamtergebnisse ablei- 
Tabelle 1-1: Internationale Position Österreichs anhand einschlägiger Innovationsindikatoren

\begin{tabular}{|c|c|c|c|}
\hline $\begin{array}{l}\text { Position Österreichs } \\
\text { in internationalen } \\
\text { Kompositindikatoren }\end{array}$ & $\begin{array}{r}\text { Global Competitiveness } \\
\text { Report (GCR) } 2018\end{array}$ & $\begin{array}{r}\text { Global Innovation Index } \\
\text { (GII) } 2018\end{array}$ & Innovationsindikator \\
\hline Wert & 76 (von 100) & 51,32 (von 100) & 50 (von 100) \\
\hline Rang & 22 & 21 & 11 \\
\hline Anzahl der Länder & 140 & 126 & 35 \\
\hline Anzahl der Einzelindikatoren & 98 & 80 & 38 \\
\hline
\end{tabular}

Quelle: Darstellung: iit; Anmerkung: eigene Darstellung auf Grundlage der Quellen von GCR, GII, Innovationsindikator (s.o.).

ten. Ebenso kann durch die Auswahl und Gewichtung der Einzelindikatoren und der Vergleichsländer die Platzierung innerhalb eines Rankings beeinflusst werden. Für ein umfassendes Bild zur Positionierung Österreichs bezüglich der Innovationsleistung ist es daher zielführend, verschiedene Kompositindikatoren zu betrachten. Zu den geläufigsten Kompositindikatoren, die eine weltweite Analyse liefern, gehören der Global Innovation Index (GII), der Global Competitiveness Index $(\mathrm{GCl})$ sowie der Innovationsindikator (Fraunhofer ISI, ZEW). ${ }^{17}$ Insgesamt zeigt das Bild eine durchgehend vordere Position Österreichs im ersten Drittel der jeweils analysierten Staaten bzw. Nationalen Innovationssysteme (NIS).

Im Rahmen des Global Innovation Index (GII), eines jährlich aktualisierten Innovationsrankings, wird die Innovationsleistung von Ländern anhand unterschiedlicher Felder, wie Institutionenumfeld, Humankapital und Forschung, Infrastruktur, Markt- und Unternehmensentwicklung, Wissens-, Technologie und Kreativoutputs dargestellt. Dafür werden 80 Einzelindikatoren für 126 Länder verwendet. Für das Jahr 2018 befindet sich Österreich auf dem Platz 21 (Wert: 51,32 von 100) des Global Innovation Indexes, mit einer leichten Abschwächung seit 2017 (Rang 20). Die in diesem Innovationsindex führenden fünf Volkswirtschaften sind die Schweiz $(68,4)$, die Niederlande (63,32), Schweden $(63,08)$, Großbritannien $(60,13)$ und Singapur $(59,83)$.

Der Global Competitiveness Index (GCl) 2018 misst für 140 Volkswirtschaften Faktoren, die langfristiges Wachstum und Wohlstand fördern. Wettbe- werbsfähigkeit wird dabei als die Gesamtheit von Institutionen, politischen Maßnahmen und Faktoren, die das Produktivitätsniveau eines Landes bestimmen, definiert. Der $\mathrm{GCl}$ umfasst derzeit 98 Indikatoren, die für Produktivität und langfristigen Wohlstand relevant sind. Dabei werden Felder wie Institutionen, Infrastruktur, makroökonomisches Umfeld, Gesundheit, Bildung, Arbeitsmarkteffizienz, Finanzmarktentwicklung, technologische Einsatzbereitschaft, Marktgröße, Geschäftsentwicklung und Innovationen betrachtet. Österreich befindet sich im Global Competitiveness Index für das Jahr 2018 mit Rang 22 von 140 (Wert: 76 von 100) auf einer vorderen Position. Die ersten fünf Volkswirtschaften sind die USA (Wert: 85,6), Singapur (83,5), Deutschland $(82,8)$, die Schweiz $(82,6)$ und Japan $(82,5)$.

Grundlage des Innovationsindikators (Fraunhofer ISI, ZEW, s.o.) bildet der Innovationssystemansatz, unter dem klassische, sozusagen „harte“ Innovationsindikatoren wie F\&E-Ausgaben oder Forschungspersonal mit "weichen" Parametern (in der Regel basierend auf Befragungen, wie zum Beispiel zu gesellschaftlichen Einstellungen) zu einem ganzheitlichen Bild des jeweiligen Innovationssystems zusammengeführt werden. Insgesamt verwendet der Innovationsindikator 38 Input- und Output-Indikatoren zum Innovationssystem, die auf die fünf Teilbereiche Wirtschaft, Wissenschaft, Bildung, Staat und Gesellschaft aufgeteilt sind. Diese werden auf eine internationale Auswahl von 35 innovationsdynamischen Volkswirtschaften vergleichend angewendet.

Im Gesamtindex des jüngsten Innovationsindika-

17 Wie bereits zuvor angemerkt sind für den vorliegenden Bericht noch keine aktuellen Daten über den European Innovation Scoreboard verfügbar. 
Abbildung 1-11: Innovationsindikator "Gesamt", 2017*

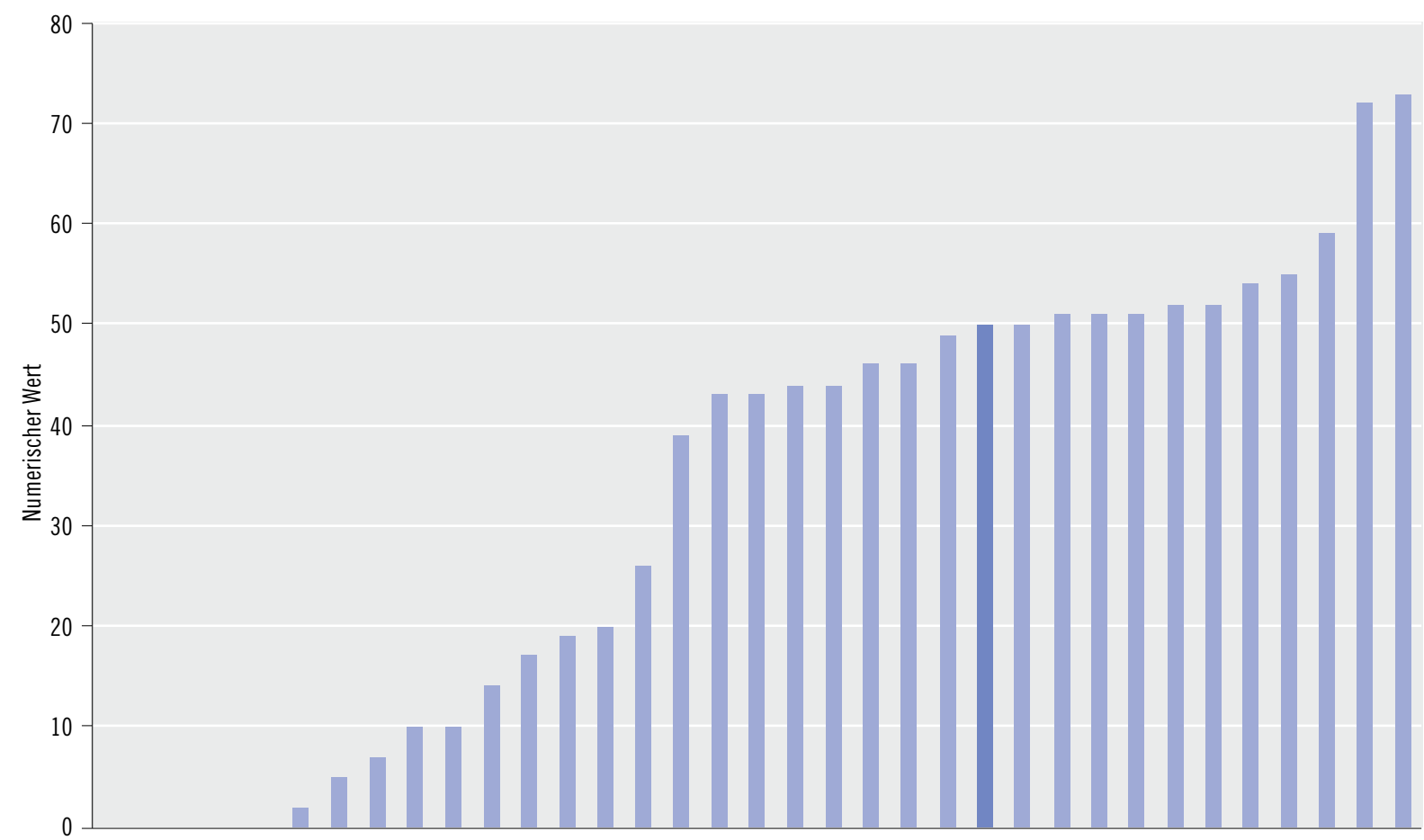

BR IN MX TR ZA ID GR RU HU PL CN IT ES PT CZ JP CA AU IL NO FR TW FI AT NL DK IE KR US UK SE DE BE CH SG Referenzländer des Innovationsindikators

Quelle: Innovationsindikator 2018.

tors von 2017 belegt Österreich den elften Rang mit 50 von 100 Punkten. Führend ist in diesem Jahr Singapur mit der Höchstpunktzahl von 73. Ihm folgen der bisherige langjährige Rangführer im Innovationsindikator, die Schweiz, mit 72 Punkten, sowie mit gröBerem Abstand Belgien (59) und Deutschland (55 Punkte). Österreich hatte im vorhergehenden Jahr noch den zehnten Rang inne sowie 2015 den neunten Platz, so dass sich in diesem Innovationsindikator ein Abwärtstrend für das Land andeutet.

Im Subindikator Wissenschaft des Innovationsindikators nahm Österreich 2017 den neunten Platz ein (Rangführer Singapur, 99 Punkte), im Subindikator Wirtschaft den zwölften Platz und im Subindikator Bildung den elften Rang. Im Subindikator Staat befindet sich Österreich 2017 auf Platz 13 mit 49 Punkten, wohingegen Singapur erneut auf Platz eins die volle Punktzahl von 100 erreicht. Im Subindikator Gesellschaft erreicht Österreich nur den 14. Rang. In dieser Kategorie des Innovationsindikators führen Australien (82 Punkte), Großbritannien (80 Punkte) sowie Finnland (73 Punkte) $)^{18}$.

Im Auswertungsbericht des Innovationsindikators wird Österreichs Entwicklung wie folgt kommentiert: „Für Österreich scheint der Aufholprozess der letzten Jahre zunächst gestoppt. (...) In Bezug auf die Subsysteme weist Österreich eine ähnliche Struktur wie Deutschland auf, da es in allen Subsystemen solide aber keine Spitzenwerte erzielt."19 Die starken Bemühungen und vor allem Investitionen, die in den letzten Jahren in Österreich im F\&E-Bereich erfolgt sind, werden in der Analyse konstatiert. Doch bleibe

18 Vgl. http://www.innovationsindikator.de/mein-indikator/, zuletzt aktualisiert am 11.12.2018, zuletzt geprüft am 25.01.2019

19 Vgl. Bundesverband der Deutschen Industrie e.V. (BDI) (2018), S. 6. 
der Output bisher noch hinter dem Input Österreichs (wie z.B. bei den Patentwerten und Publikationen oder auch im Innovationsbereich) zurück, so der Innovationsindikator-Bericht 2018.

\subsubsection{Entwicklung der Position Österreichs bei der Digitalisierung}

Um die Position Österreichs bei der Digitalisierung im internationalen Vergleich einzuordnen, werden für die Analyse infolge Informationen aus dem „Digital Economy and Society Index" (DESI) und dem „Global Innovation Index" verwendet.

Mit Hilfe des Indikators „Digital Economy and Society Index" (DESI) der Europäischen Kommission ${ }^{20}$ wird die Position Österreichs innerhalb der 28 Staaten der Europäischen Kommission verglichen. Dieser Indikator umfasst insgesamt fünf Dimensionen: Konnektivität, Humankapital, Internetnutzung, Integration der Digitaltechnik sowie digitale öffentliche Dienste. Die fünf Dimensionen und deren Zusammensetzung nach verschiedenen Unterindikatoren sind in der nachfolgenden Box dargestellt. Insgesamt liegt der maximal mögliche Wert des Indexes bei $100 \%$.

\section{Indikatoren des „Digital Economy and Society Index" (DESI)}

\section{- Indikator 1: Konnektivität}

Die Konnektivität wird berechnet als gewichteter Mittelwert der fünf Subindikatoren: FestnetzBreitband (20\%), Mobilfunk-Breitband (30\%), schnelles Breitband (20\%), ultraschnelles Breitband $(20 \%)$ und Preise (10\%).

\section{- Indikator 2: Humankapital}

Das Humankapital wird berechnet als gewichteter Mittelwert von zwei Subindikatoren: Grundkompetenzen der Internetnutzung (50\%) und fortgeschrittene Fertigkeiten und Entwicklung (50\%).

- Indikator 3: Internetnutzung

Die Internetnutzung wird berechnet als gewichte- ter Mittelwert der drei Subindikatoren: Nutzung von Inhalten (33,3\%), Kommunikation (33,3\%) und Online-Transaktion durch die Bürgerinnen und Bürger (33,3\%).

\section{- Indikator 4: Integration der Digitaltechnik:} Die Integration der Digitaltechnik wird berechnet als gewichteter Mittelwert der zwei Subindikatoren: Digitalisierung von Unternehmen (60\%) und elektronischer Handel (40\%).

\section{- Indikator 5: Digitale öffentliche Dienste:}

Diese Dimension umfasst elektronische Behördendienste (100\%).

Anhand dieser fünf Dimensionen lässt sich ein Ländervergleich der 28 EU-Länder darstellen (siehe Abbildung 1-12).

Die Ergebnisse zeigen, dass sich Österreich auf dem elften Platz im Ländervergleich befindet, wenn die Länder bezüglich des kumulierten Ergebnisses aller fünf Indikatoren verglichen werden. Führend sind die nordischen Länder Dänemark, Schweden, Finnland und die Niederlande. Innerhalb dieser Länder sind die Unterschiede im Index nur sehr gering. Von diesen Ländern ist Österreich im Index über zehn Prozentpunkte entfernt, der Abstand zum fünftplatzierten Land Luxemburg ist hingegen nur sehr gering. Auffällig ist allerdings, dass Österreich u.a. hinter Belgien und Spanien liegt, die aktuell geringere Bruttoinlandsprodukte pro Kopf aufweisen. Bei der Betrachtung des angeführten Indexes ist zudem festzuhalten, dass Österreich sich u.a. bezüglich der Konnektivität und der Integration der Digitaltechnik nicht vom Durchschnitt der 28 EU-Länder abhebt. Insofern deuten die Ergebnisse Entwicklungspotenziale im Ausbau des Internets und der Integration der Digitaltechnik an.

Um einen detaillierteren Einblick über Österreichs Stand in der Informations- und Kommunikationstechnologie zu erhalten, werden im Folgenden zwei Einzelindikatoren "Nutzung von Informations- und Kommunikationstechnologien" (siehe Abbildung 1-13) und „Verfügbarkeit von Informations- und Kommunikati-

20 Vgl. https://ec.europa.eu/digital-single-market/en/desi, zuletzt geprüft am 25.01.2019 
Abbildung 1-12: Index für die digitale Wirtschaft und Gesellschaft (DESI, 2018)

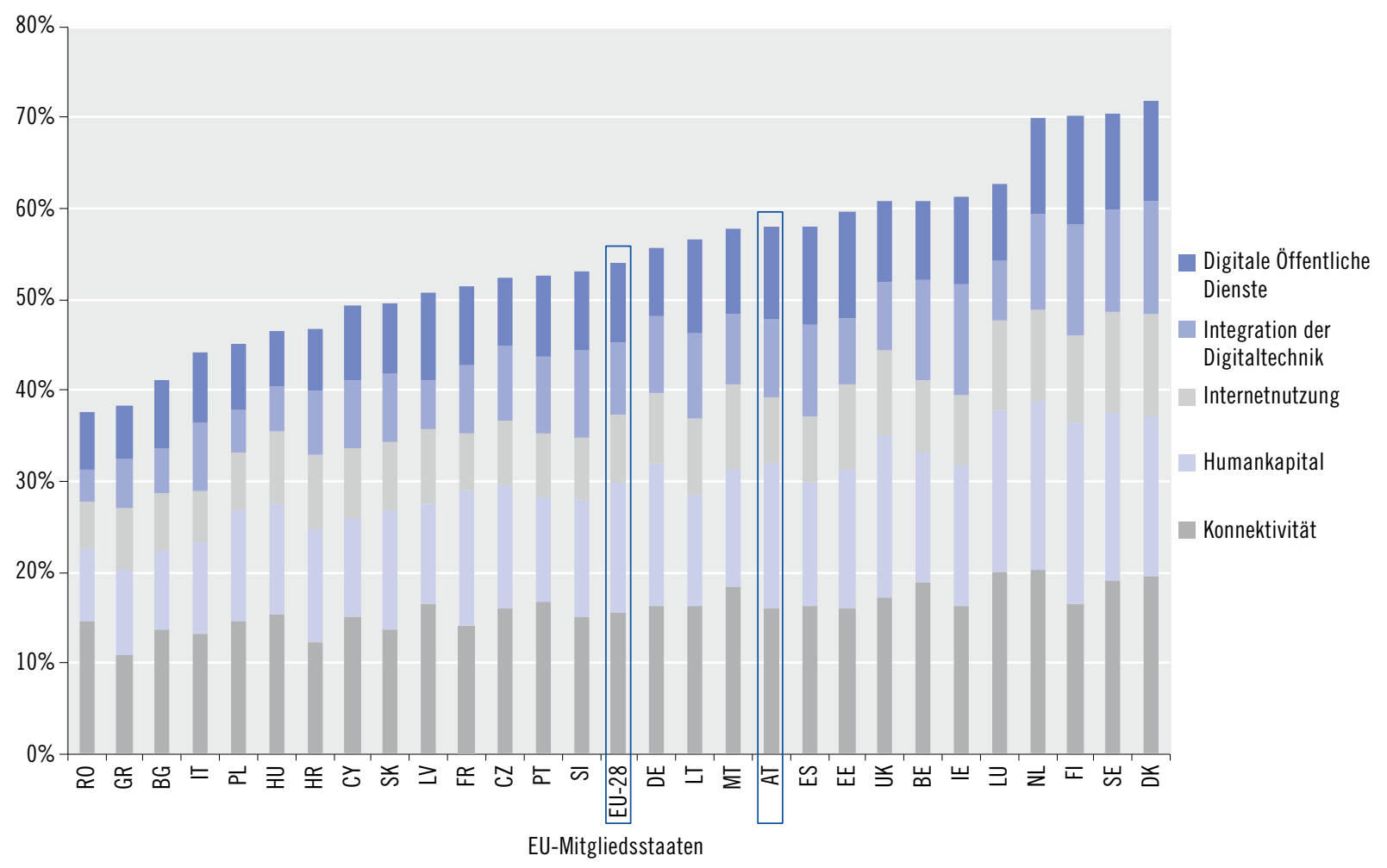

Quelle: Europäische Kommission (2019b).

Abbildung 1-13: Indexwert „Nutzung von Informations- und Kommunikationstechnologien” im Vergleich, 2018

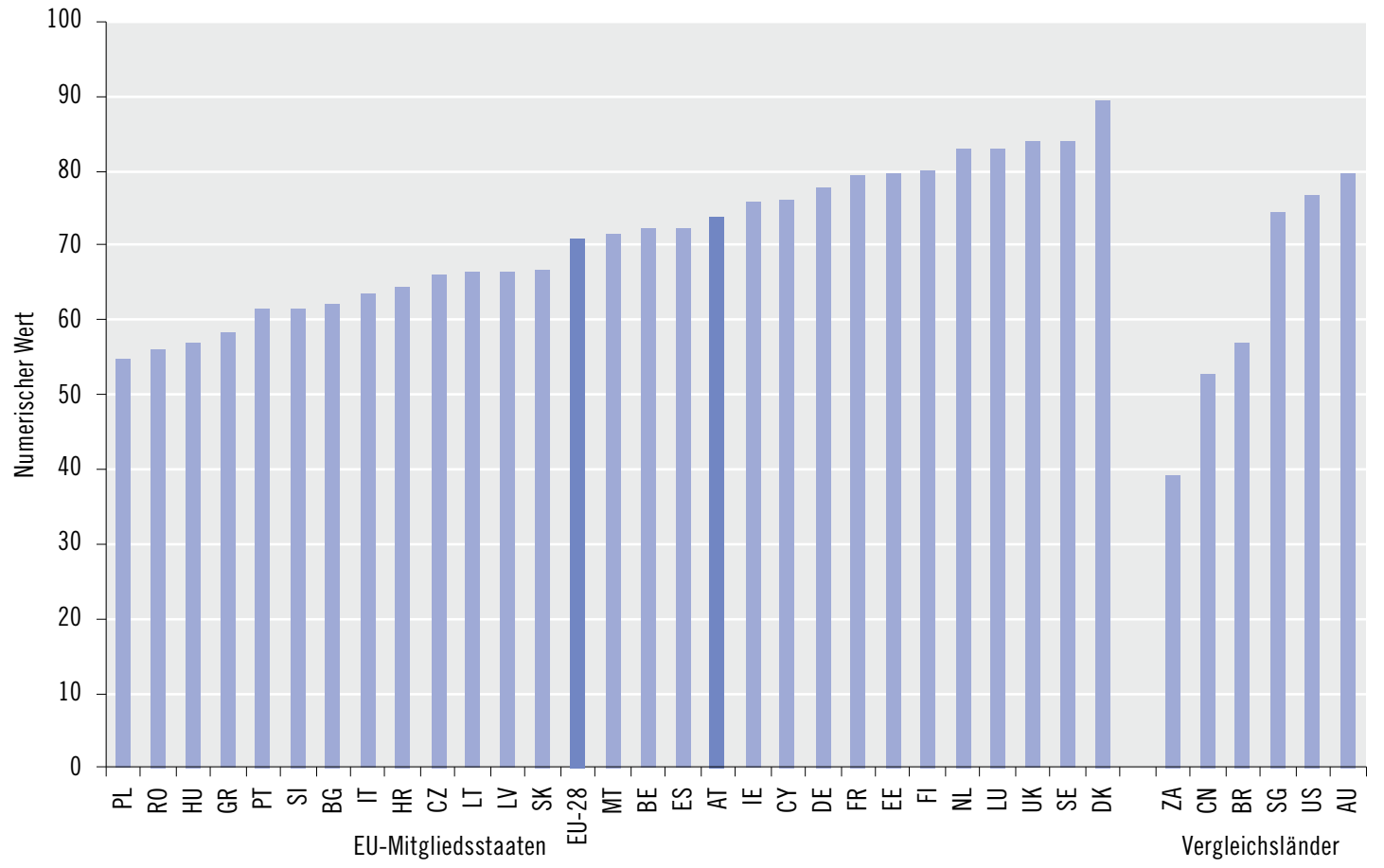

Quelle Cornell College et al. (2018). 
onstechnologien“ (siehe Abbildung 1-14) aus dem Global Innovation Index dargestellt. Die beiden angeführten Indikatoren beruhen auf Erhebungen der International Telecommunication Union, die 2017 im Bericht "Measuring the Information Society 2017" präsentiert wurden. Bei beiden Indikatoren liegt der maximal mögliche Wert des Indexes bei $100 \%$.

Die Ergebnisse zeigen, dass Österreich bei der Nutzung und der Verfügbarkeit von Informationsund Kommunikationstechnologien innerhalb der 28 EU-Staaten im Mittelfeld zu finden ist und sich jeweils hinter den Ländern Schweden, Dänemark, Luxemburg, den Niederlanden, Deutschland und dem Vereinigten Königreich einordnet. Bezüglich der Nutzung von Informations- und Kommunikationstechnologien (IKT) liegt Österreich im Vergleich der EUStaaten auf Platz zwölf und bei der Verfügbarkeit von IKT nimmt Österreich im entsprechenden Ländervergleich Platz neun ein.

\section{Indikatoren zur Nutzung von IKT im „Global}

\section{Innovation Index"}

- Indikator 1: Nutzung von Informations- und

\section{Kommunikationstechnologien}

Die Nutzung von Informations- und Kommunikationstechnologien wird berechnet als gewichteter Mittelwert der drei Subindikatoren: Anteil von Personen, die das Internet nutzen (33,3\%), FestnetzBreitband-Anschlüsse pro 100 Einwohnerinnen bzw. Einwohner (33,3\%), sowie aktive, angemeldete mobile Breitband-Anschlüsse pro 100 Einwohnerinnen und Einwohner (33,3\%).

- Indikator 2: Verfügbarkeit von Informationsund Kommunikationstechnologien

Die Verfügbarkeit von Informations- und Kommunikationstechnologien wird berechnet als gewichteter Mittelwert der fünf Subindikatoren: Festnetz-Telefonanschlüsse pro 100 Einwohnerinnen und Einwohner, Mobiltelefon-Verträge pro 100 Einwohnerinnen und Einwohner (20\%), Datenübertragung in andere Länder (International Bandwidth) in bit/s (20\%), Anteil an Haushalten, in denen mindestens ein Computer vorhanden ist (20\%), sowie Anteil an Haushalten mit Zugang zum Internet (20\%).

Da die angeführten Indikatoren größtenteils auf Subindikatoren beruhen, die auf die Anzahl der Einwohnerinnen und Einwohner normiert sind, sind die Daten und Indikatoren auch zwischen Ländern unterschiedlicher Größe vergleichbar. Insofern kann man festhalten, dass Österreich bezüglich dieser Indikatorik einen Mittelfeldplatz in Europa einnimmt, der aber ausbaufähig ist. Insbesondere beim Vergleich mit der Nutzung von Informations- und Kommunikationstechnologien wäre ein mögliches Ziel, zu den davorliegenden Ländern aufzuschließen - was durch eine stärkere und flächendeckende Nutzung von schnellem Internet erreichbar ist. Allerdings bleibt ebenfalls festzuhalten, dass Österreich im Vergleich eine stärkere Nutzung und Verfügbarkeit von Informations- und Kommunikationstechnologien aufweist als jeweils der Durchschnitt der 28 EU-Länder und der Durchschnitt der nicht-europäischen Vergleichsländer.

Abschließend wird noch ein "Gesamtindex zu Informations- und Kommunikationstechnologien" im Global Innovation Index betrachtet, der die beiden angeführten Indikatoren zur Nutzung und zur Verfügbarkeit sowie weitere Informationen zum eGovernment (elektronische Behördendienste) berücksichtigt. In Abbildung 1-15 ist der Vergleich Österreichs bezüglich dieses Indexes sowie der in Abbildung 1-13 und Abbildung 1-14 angeführten Indikatoren dargestellt, wobei Österreich mit dem Durchschnittswert der 28 EU-Staaten und dem Durchschnittswert der Vergleichsländer Brasilien, USA, Südafrika, China, Singapur und Australien verglichen wird.

Die Auswertung zeigt, dass Österreich im Vergleich eine stärkere Nutzung und Verfügbarkeit von Informations- und Kommunikationstechnologien aufweist als jeweils der Durchschnitt der 28 EU-Länder und der Durchschnitt der nicht-europäischen Vergleichsländer. Bei der Betrachtung des Gesamtindexes zu IKT liegt Österreich ebenfalls oberhalb des Durch- 
Abbildung 1-14: Indexwert „Verfügbarkeit von Informations- und Kommunikationstechnologien“ im Vergleich, 2018

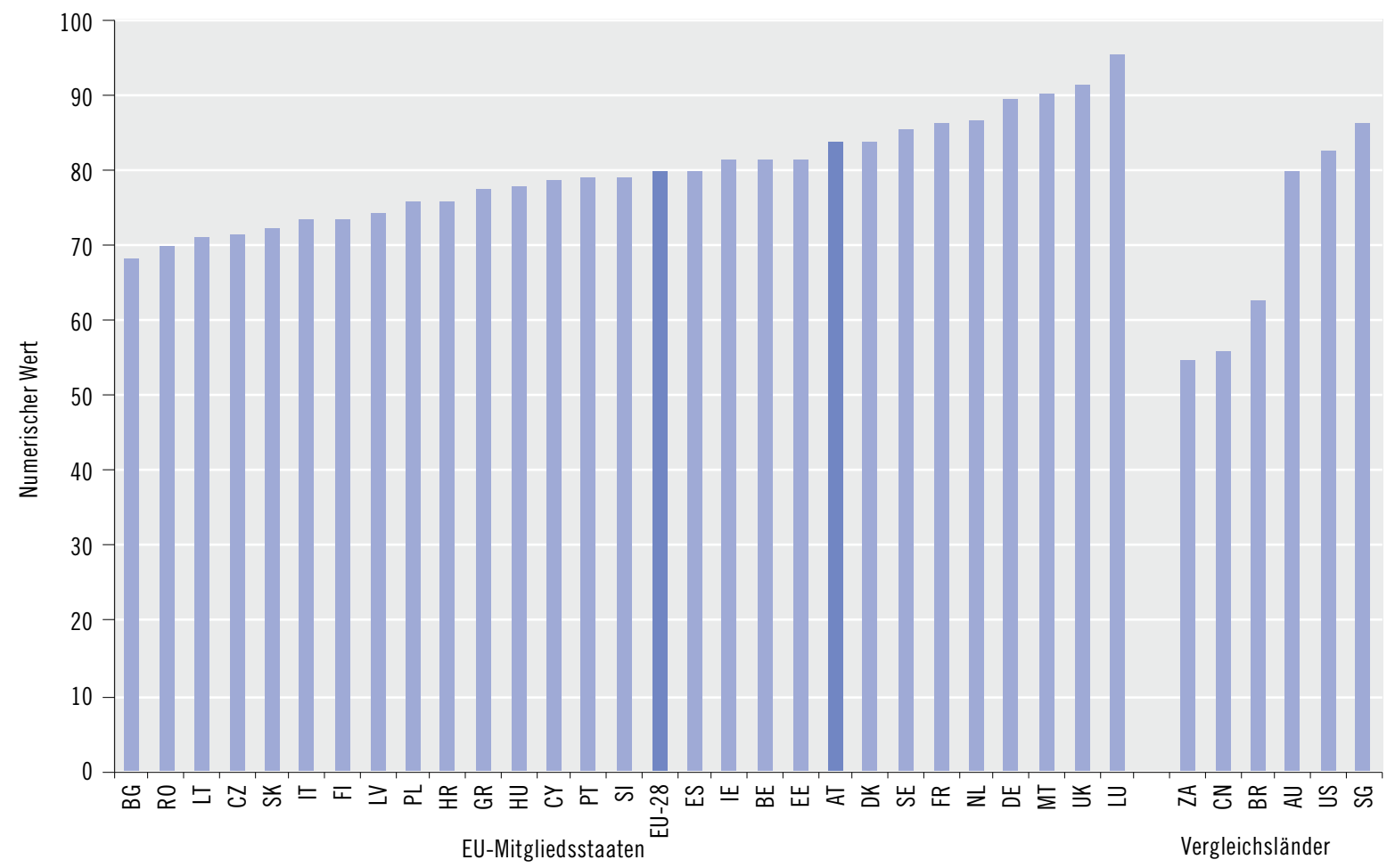

Quelle: Cornell College et al. (2018).

Abbildung 1-15: Position Österreichs im Ländervergleich - Übersicht über Ergebnisse im Global Innovation Index

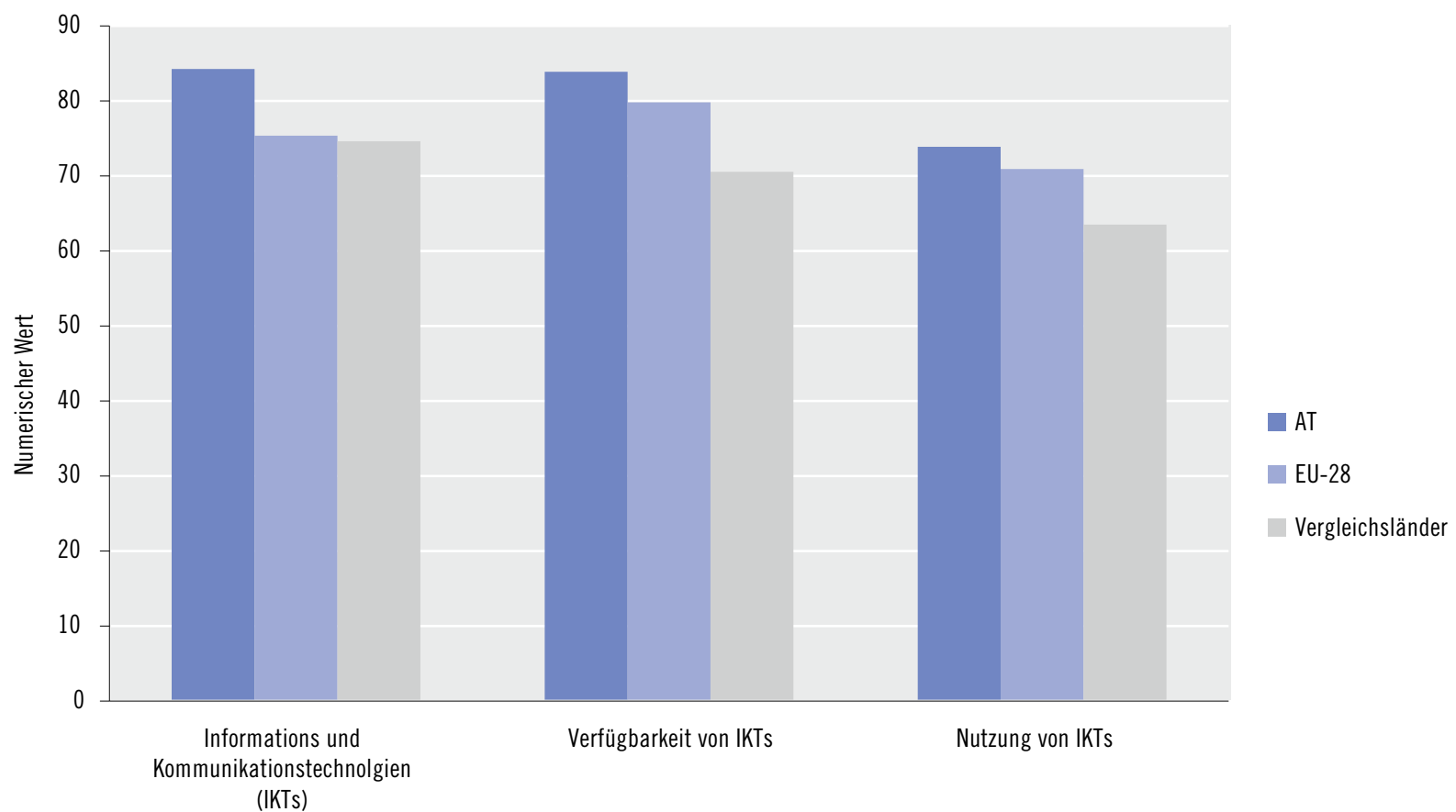

Quelle: Cornell College et al. (2018). 
schnitts der 28 EU-Länder und des Durchschnitts der nicht-europäischen Vergleichsländer (siehe Abbildung 1-15). Es bestehen somit gute Aussichten, dass Österreich auch im Bereich IKT und deren Nutzung für seine FTI-Performance - insbesondere bei einer engen strategischen Verknüpfung der Politikfelder Wissenschaft und Technologie - mit der internationalen Entwicklung Schritt hält. Allerdings zeigt auch ein Vergleich des Global Innovation Index 2018 mit dem Global Innovation Index 2017, dass sich die Position Österreichs in den hier angeführten Indikatoren leicht verschlechtert hat - von Position 12 auf Position 16 im Ranking (bei der Betrachtung des Gesamtindex IKT).

Die in diesem Abschnitt angeführten Darstellungen bilden nur einen kleinen Teil der Fähigkeit $a b$, digitale Transformationsprozesse in der Wirtschaft zu gestalten. Weiterführende und feingliedrigere Informationen zu unternehmensspezifischen und arbeitsmarktpolitischen Maßnahmen sind notwendig, um ein umfassenderes Bild zur digitalen Leistungsfähigkeit in Wirtschaft und Gesellschaft zu zeichnen.

Trotz dieser Einschränkungen lässt sich jedoch festhalten, dass die Position Österreichs bei der Nutzung digitaler Verbindungen international im oberen Mittelfeld liegt. Allerdings deuten die Resultate auch an, dass es Entwicklungspotentiale gibt, insbesondere beim Ausbau von Breitbandanschlüssen sowie deren flächendeckende Nutzung.

\subsection{3 Österreichs Innovations- und Wettbewerbsfähigkeit}

In diesem Kapitel werden die Innovations- und Wettbewerbsfähigkeit Österreichs näher betrachtet. Dabei werden insbesondere Faktoren untersucht, die Ausgangssituation bzw. Rahmenbedingungen für innovative Tätigkeiten darstellen und somit die Fähig- keit zur zukünftigen Innovationstätigkeit und Wettbewerbsfähigkeit im Sinne der Nachhaltigkeit abbilden. Insofern deuten die Ergebnisse dieses Abschnittes an, welche Grundlagen für eine zukünftige Rolle als Technologieführer in Österreich bereits gegeben sind und welche Stellschrauben noch adjustiert werden müssen, um eine solche Rolle erfüllen zu können.

\section{Wettbewerbsfähigkeit}

Hierzu wird zunächst die Wettbewerbsfähigkeit des "Global Competitiveness Report" dargestellt. Dieser Bericht ${ }^{21}$ vergleicht insgesamt 137 Länder anhand von 114 Indikatoren, die zwölf übergeordneten Säulen bzw. Dimensionen zugeordnet sind. Für die zwölf übergeordneten Dimensionen werden dabei Kompositindikatoren errechnet und dokumentiert, mit Hilfe deren auch Ländervergleiche möglich sind. Diese umfassen:

- Institutionen (Institutions),

- Infrastruktur (Infrastructure),

- gesamtwirtschaftliche Rahmenbedingungen (Macroeconomic environment),

- Gesundheit und primäre Ausbildung (Health and primary education),

- Hochschul- und Berufsbildung (Higher education and training),

- Effizienz des Gütermarkts (Goods market efficiency),

- Effizienz des Arbeitsmarkts (Labor market effiency),

- Entwicklung des Finanzmarkts (Financial market development),

- Technologischer Reifegrad (Technological readiness),

- Marktgröße/Marktvolumen (Market size),

- Reifegrad des Wirtschaftssystems (Business sophistication) und

- Innovation (Innovation).

21 Die Analyse in diesem Abschnitt basiert auf einer Vorversion des Competitiveness Reports 2018, https://www.weforum.org/ reports/the-global-competitiveness-report-2017-2018, zuletzt geprüft am 15.01.2019. Mittlerweile ist - wie zuvor in Tabelle 1-1 dargestellt - eine aktuellere Fassung verfügbar, welche das Gesamtergebnis der in diesem Abschnitt dargelegten Analyse jedoch de facto nur unerheblich beeinflusst. 
Abbildung 1-16: Dimensionen des Global Competitiveness Report: Position Österreichs im Vergleich zum Durchschnitt der 28 EU-Mitgliedsstaaten

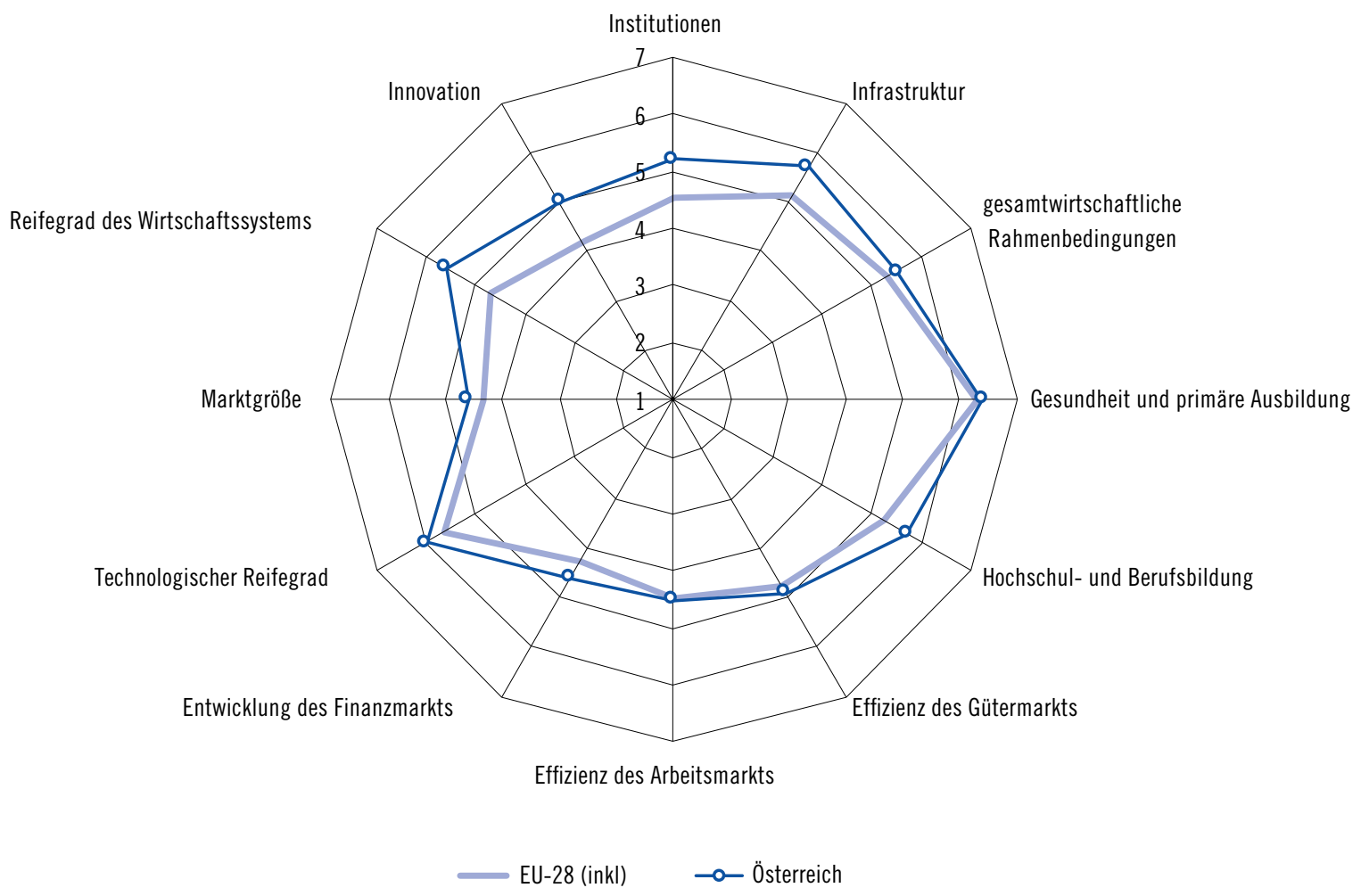

Quelle: Weltwirtschaftsforum (2017).

Die Kompositindikatoren im Global Competitiveness Report werden so errechnet, dass sie für jede Dimension Werte zwischen 1 und 7 annehmen können. Je höher der Wert, desto besser schneidet ein Land bzw. eine Volkswirtschaft in dieser Dimension $a b$. Als Datengrundlage werden verschiedene Informationsquellen genutzt, u.a. Daten vom Internationalen Währungsfonds und von der Weltbank, Daten von den Vereinten Nationen, UNESCO und der Weltgesundheitsorganisation, sowie Informationen aus der Führungskräfteumfrage des Weltwirtschaftsforums. In Abbildung 1-16 ist die Position Österreichs im Vergleich zum Durchschnitt der 28 EU-Länder angegeben.

Insgesamt muss bei der Auswertung des Rankings berücksichtigt werden, dass die Werte der Kompositindikatoren eher im Vergleich zu anderen Volkswirtschaften als in ihrer absoluten Höhe aussagekräftig sind. Nichtsdestotrotz lassen sich grobe Aussagen über Stärken und Schwächen der Volkwirtschaften ableiten. Die Formulierung konkreter Handlungsempfehlungen ist aufgrund der zusammengesetzten Indikatorik allerdings nicht valide möglich.

Trotz dieser Einschränkungen deuten die Ergebnisse daraufhin, dass Österreich in allen Dimensionen besser abschneidet als der Durchschnitt der 28 EU-Länder. Besonders beim Reifegrad des Wirtschaftssystems, Innovationstätigkeit und den institutionellen Rahmenbedingungen erzielt Österreich im Vergleich zum Durchschnitt der EU-Länder deutlich bessere Werte. Der technologische Reifegrad und die Ausbildung sind in Österreich sowie im EUDurchschnitt jeweils auf einem sehr hohen Niveau. Ferner deuten die Ergebnisse an, dass die berichtete Effizienz des Arbeitsmarkts, des Gütermarkts sowie die Entwicklung des Finanzmarkts Entwicklungspotenziale darstellen - für Österreich wie für den Rest der Europäischen Union. 


\section{Innovationsfähigkeit}

Die folgende Analyse der Innovationsfähigkeit basiert konzeptionell auf dem Ansatz des Innovationsfähigkeitsindikators des Instituts für Innovation und Technik $^{22}$. Die Innovationsfähigkeit wird im iit-Innovationsfähigkeitsindikator als die Fähigkeit betrachtet, Neues zu generieren und in konkurrenzfähige Produkte, Prozesse und Dienstleistungen zu übersetzen. Sowohl das vorhandene Wissen als auch die Fähigkeit, unterschiedliche Wissensbestände zusammenzubringen, werden daher berücksichtigt. Der iit-Innovationsfähigkeitsindikator erfasst dabei die folgenden vier Themenbereiche bzw. „Säulen“:

- Humankapital: Die Aus- und Weiterbildung und das lebenslange Lernen der Beschäftigten,

- Komplexitätskapital: Die Vielfalt an nützlichem Wissen, die es erlaubt, komplexe Produkte herzustellen,

- Strukturkapital: Die Fähigkeit, Wissen innerhalb von Unternehmen zusammenzubringen,

- Beziehungskapital: Die Fähigkeit, Wissen über Organisationsgrenzen hinweg zusammenzubringen.

Auf der Grundlage des theoretischen Gerüsts und der Datenstruktur des iit-Innovationsfähigkeitsindikators wird dieser für die folgende Analyse erstmals um den außereuropäischen Raum erweitert, um auf globaler Ebene vergleichende Aussagen über Österreichs Innovationsfähigkeit treffen zu können. Die Erweiterung auf den außereuropäischen Raum ist allerdings insofern eingeschränkt, als dass Datenerhebungen im europäischen Raum besonders geeignet sind, um das Struktur- sowie das Beziehungskapital adäquat auszuwerten. Daher werden das Humankapital und das Komplexitätskapital für alle Vergleichsländer untersucht, während sich die Analyse des Struktur- und des Beziehungskapitals auf den europäischen Bereich beschränkt.

\section{Humankapital}

Das Humankapital im Sinne des Bestands an qualifizierten Beschäftigten eines Landes spielt eine zent- rale Rolle im Kontext der Innovationsfähigkeit: Erst durch gut ausgebildetes Personal werden Innovationen generiert, die zur Wertschöpfung in einer Volkswirtschaft wichtige Beiträge leisten können. Entsprechend bildet Humankapital auch eine weitere Dimension im Konzept des Innovationsfähigkeitsindikators, der hier erneut auf die globale Ebene erweitert angewendet werden soll. Für eine Einschätzung des Humankapitals Österreichs werden in diesem Bericht deshalb internationale Daten aus dem letzten OECDBericht „Education at a Glance“ (2018) hinzugezogen.

In Bezug auf das Humankapital vermittelt Abbildung 1-17 zunächst einen Gesamteindruck zum Bildungsniveau der jeweiligen Landesbevölkerung im global ausgerichteten Gesamtvergleich der Referenzländer. Österreichs Position befindet sich in Bezug auf die breite Bevölkerung aller Personen im arbeitsfähigen Alter (25-64 Jahre) mit 32 \% im unteren mittleren Bereich und etwas unter dem Durchschnitt der hier erfassten 22 EU-Länder (34\%). Schwellenländer wie China und Südafrika liegen weit unter diesen Werten mit jeweils $9 \%$ und $6 \%$, und auch Brasilliens Position ist hier mit einem geschätzten Gesamtwert von $15 \%$ Hochschulqualifizierten noch stark ausbaufähig. Hohe Werte mit Blick auf das formale Bildungsniveau der aktuellen Bevölkerungssituation erzielen dagegen die USA, Australien und die Schweiz. In der Tertiärquote von $32 \%$ sind avancierte berufsbildende Abschlüsse (Meister-, Werkmeister-, Bauhandwerker-Qualifikationen) bereits inkludiert.

Qualitative Besonderheiten und Unterschiede der jeweiligen Bildungssysteme wurden in dieser Gesamtschau jedoch nicht berücksichtigt. So ist bei der Interpretation der Daten zu beachten, dass Staaten mit einer langen Tradition der dreigliedrigen Architektur der Universitätsabschlüsse (Bachelor als Erstabschluss, Master als Folgeabschluss) traditionell höhere Abschlussquoten im akademischen Sektor aufweisen als Staaten, in denen es etwa längere Diplomstudiengänge gab. Zudem besteht in Staaten, die

22 Vgl. Institut für Innovation und Technik (iit) (2018). 
Abbildung 1-17: Prozentualer Anteil 25 bis 64-Jähriger mit einem Abschluss im Tertiärbereich, 2017*

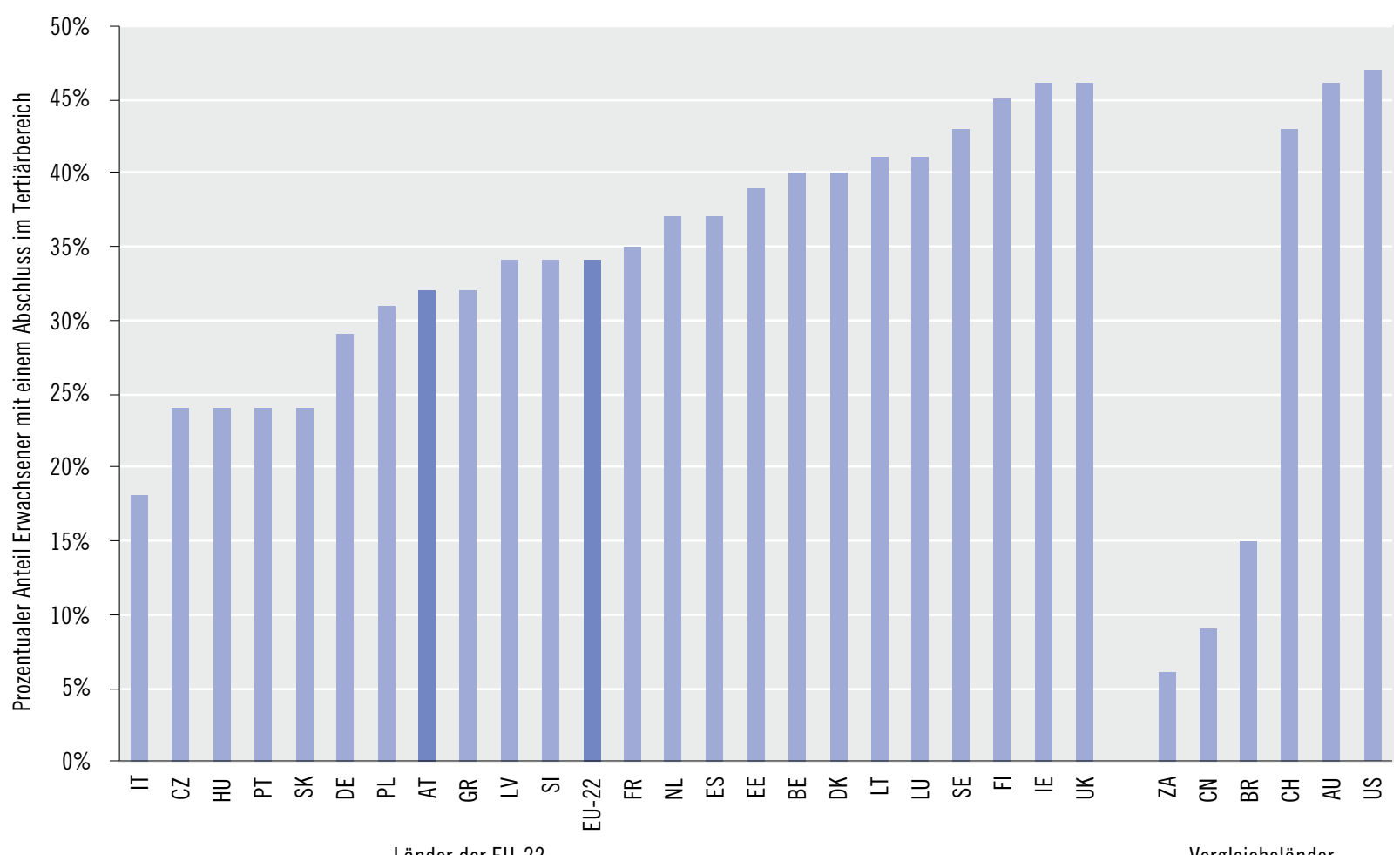

Länder der EU-22

Vergleichsländer

* für das Bezugsjahr nicht verfügbare Länderdaten: SG

Quelle: OECD (2018b), Berechnungen iit.

kein ausgebautes System der Berufsbildung auf Ebene der Sekundarstufe haben, eine stärkere Tendenz, formale berufliche Qualifikationen in Bildungsgängen auf postsekundarer Ebene zu vermitteln (sei es im Rahmen avancierter Berufsbildung oder durch Bachelorstudiengänge).

Zusammenfassend ist somit festzuhalten, dass der aktuelle Bevölkerungsanteil mit Hochschulabschluss in Österreich mit Blick auf die Innovationsfähigkeit des Landes zwar noch ausbaufähig ist, der unterdurchschnittliche Wert der Tertiärquote Österreichs lässt sich aber auch durch die im letzten Abschnitt dargestellten Besonderheiten des österreichischen Bildungssystems erklären. Darüber hinaus zeigen weitere Daten aus derselben Quelle der OECD, die auf die jüngere Altersgruppe von 25-34 Jahren fokussieren, jedoch für das Jahr 2017 für Österreich bereits $40 \%$ mit Hochschulabschluss, sodass ein Aufwärtstrend ersichtlich ist.
Ein anderes Bild des Humankapitals insbesondere in Bezug auf die zukünftigen Perspektiven ergibt sich, wenn die Daten zu Studienabsolventinnen und -absolventen dieser Zeit betrachtet werden (Abbildung 1-18). Gerade in den MINT-Fächern sowie den Abschlüssen im IKT-Bereich kommt Österreich im internationalen Vergleich zu den erfassten Referenzländern auf die zweithöchste Position hinter Deutschland (Österreich: 31 \% aller Hochschulabsolventinnen und -absolventen, Deutschland: $36 \%$ ). Diese beiden Länder sind jeweils insbesondere durch hohe Anteile von Absolventinnen und Absolventen der Ingenieurswissenschaften unter allen Absolventinnen und Absolventen geprägt (Deutschland: $22 \%$, Österreich: $21 \%$ ). Mit Blick auf das technologiebasierte Innovationspotential Österreichs und die zukünftige Innovationsfähigkeit des Landes verspricht dieser hohe Wert nachhaltig positive Aussichten, vorausgesetzt, dass das Innovations- 


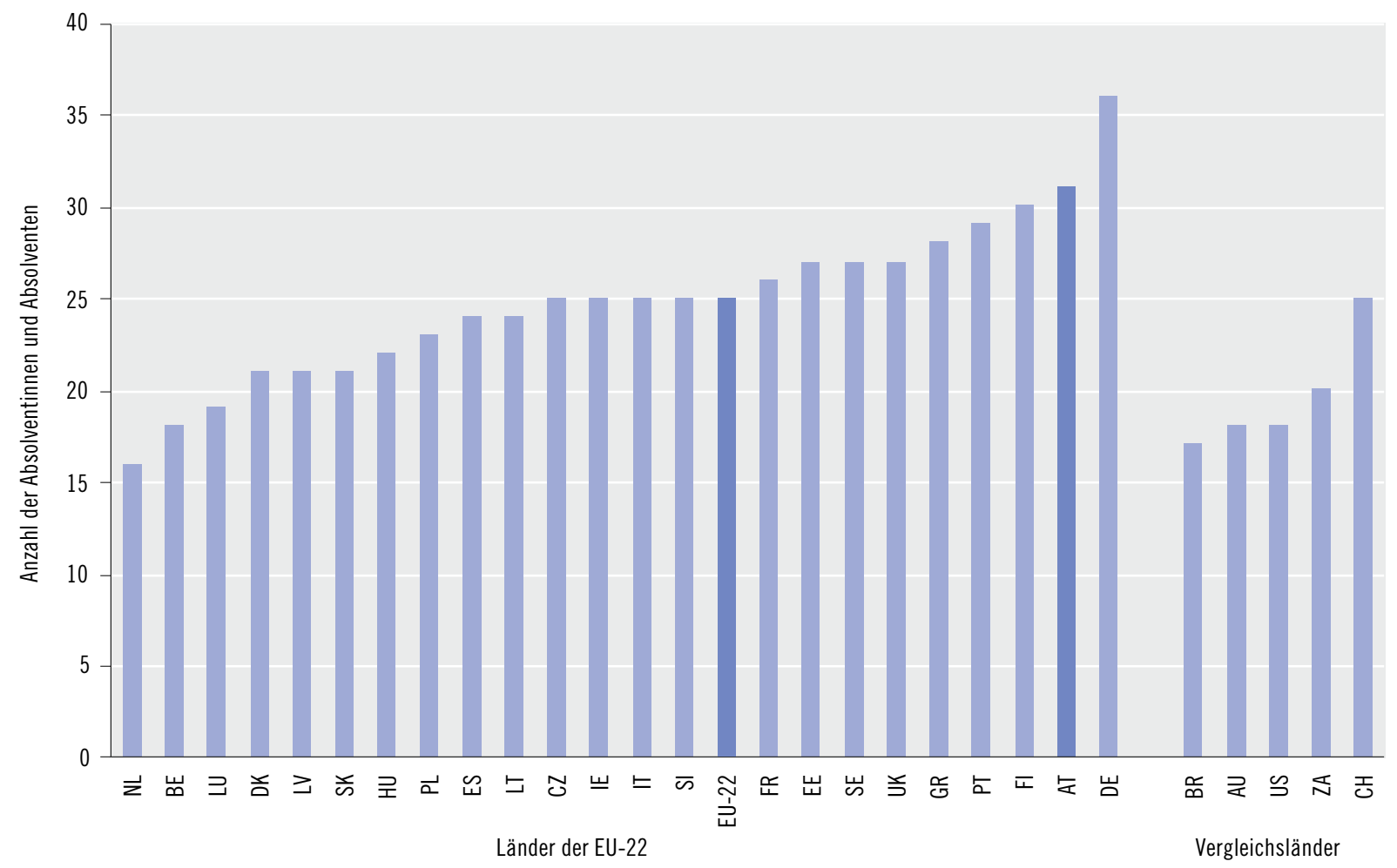

* für das Bezugsjahr nicht verfügbare Länderdaten: CN, SG Quelle: OECD (2018b), Berechnungen iit.

system Österreichs in der Lage ist, seine Talente im Land zu halten und entsprechende Entfaltungsmöglichkeiten zu bieten. Insgesamt erweist sich Europa in Bezug auf das Humankapital an technologisch-orientierten Hochschulqualifikationen als gut aufgestellt mit durchschnittlich $25 \%$ der Hochschulabsolventinnen und -absolventen aus den Bereichen MINT und IKT (bei 22 EU-Ländern). Die Aussichten für Österreich und Europa im Kontext der Innovationsfähigkeit sind aus Sicht der Dimension des Humankapitals derzeit somit grundsätzlich positiv. Für die künftige Entwicklung ist jedoch wichtig, dass sie mit den, voraussichtlich in MINT und IKT weiterhin steigenden Bedarfen am Arbeits- markt ${ }^{23}$ Schritt hält und sich nicht mit dem aktuellen Stand begnügt.

\section{Komplexitätskapital}

Aus der Innovationsforschung ist bekannt, dass für die Innovationsfähigkeit neben der Qualifizierung bzw. dem Humankapital auch die Intensität und die Vielfalt des (nützlichen) Wissens entscheidend sind. Diese Heterogenität des Wissens ist beispielsweise auch für die Innovationsfähigkeit in Regionen und Clustern ausschlaggebend, sie lässt sich aber auch auf ganze Volkswirtschaften ausweiten. Die vorhandene Vielfalt an nützlichem Wissen wird nach dem Konzept des Innovationsfähig- 
Abbildung 1-19: Ökonomische Komplexität - Position Österreichs im internationalen Vergleich, 2016*

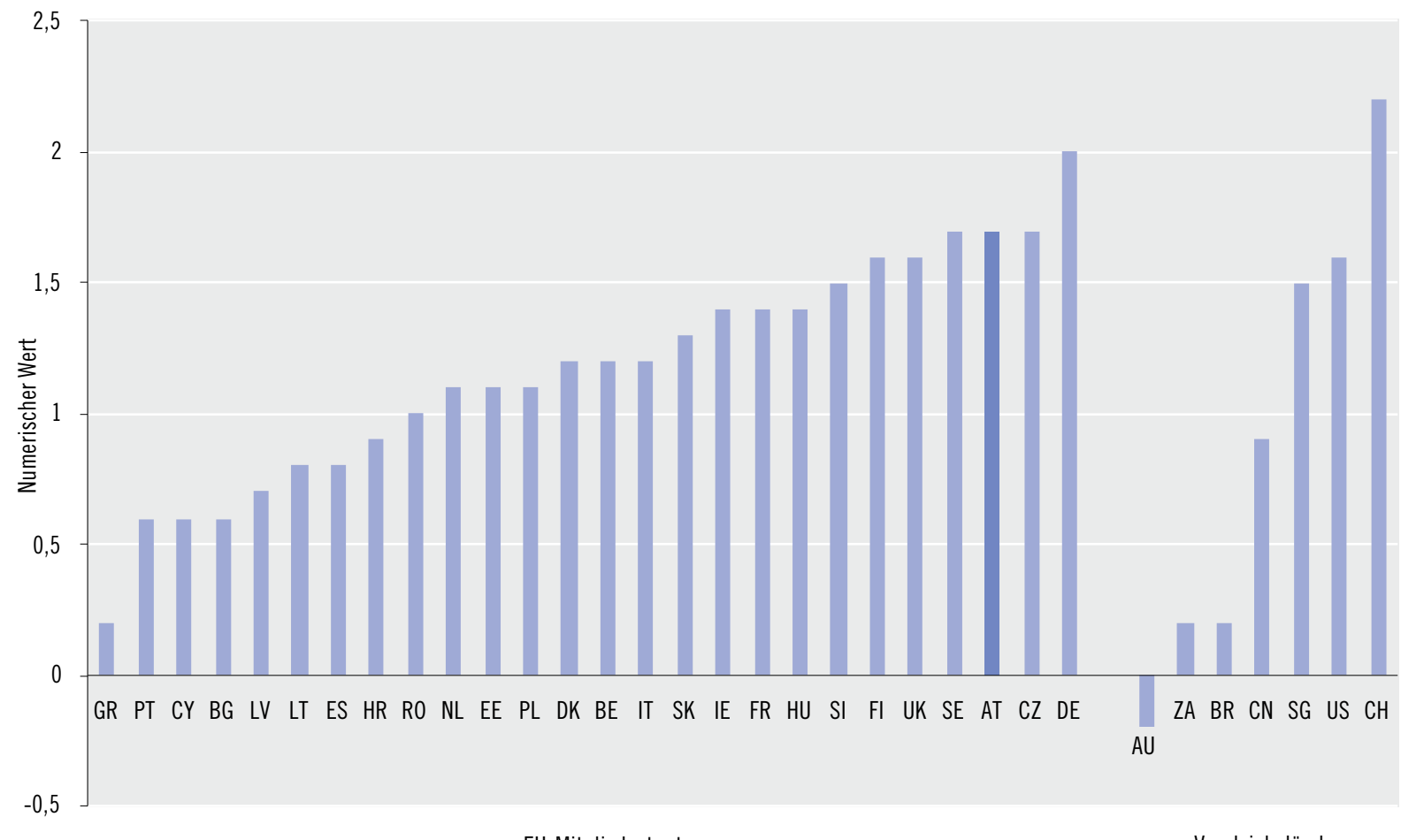

EU-Mitgliedsstaaten

Vergleichsländer

* für das Bezugsjahr nicht verfügbare Länderdaten: LU, MT

Quelle: Weltwirtschaftsforum (2017).

keitsindikators als Komplexitätskapital bezeichnet.

Die Daten für das Komplexitätskapital stammen aus dem „Economic Complexity Index" (Index der wirtschaftlichen Komplexität), der auf einem von Forscherinnen und Forschern des MIT und der Harvard University entwickelten Konzept basiert. ${ }^{24}$ Die wirtschaftliche Komplexität wird in diesem Indikator anhand der Diversifikation des Güterexportes verschiedener Volkswirtschaften abgebildet. Dies bedeutet, dass eine Volkswirtschaft dann eine hohe wirtschaftliche Komplexität erreicht, wenn es eine große Vielfalt von verschiedenartigen Gütern an eine möglichst diversifizierte Anzahl von Handelspartnern exportiert. Die notwendigen Informationen für die Erfassung der ökonomischen Komplexität stammen aus Daten zum Außenhandel der Länder.
In Abbildung 1-19 ist die Position Österreichs im internationalen Vergleich dargestellt.

Die Ergebnisse zeigen, dass Österreich einen hohen Wert im Indikator der ökonomischen Komplexität innehat. Im internationalen Vergleich erzielt Österreich den dritten Platz innerhalb der 28 EU-Länder und liegt auch im Vergleich mit den in diesem $A b-$ schnitt betrachteten weiteren Vergleichsländern nur hinter der führenden Schweiz und noch vor den USA. Die vordere Platzierung Österreichs bei der wirtschaftlichen Komplexität ist konform mit den o.a. Ergebnissen zur Wettbewerbsfähigkeit. Insgesamt lässt sich damit festhalten, dass Österreich gut aufgestellt ist, um zukünftige Innovationen nicht nur zu erzeugen, sondern sie auf einem weltweiten Markt auch erfolgreich zu positionieren.

24 Vgl. Harvard University (2018). 
Abbildung 1-20: Indikator „Lernförderliche

Arbeitsorganisation“, 2018

\begin{tabular}{|c|c|c|c|}
\hline Rang & Kategorie & Wert & Graph \\
\hline 1 & Norwegen & 0,88 & \\
\hline 2 & Finnland & 0,83 & \\
\hline 3 & Schweden & 0,83 & \\
\hline 4 & Dänemark & 0,81 & \\
\hline 5 & Estland & 0,81 & \\
\hline 6 & Niederlande & 0,78 & \\
\hline 7 & Vereinigtes Königreich & 0,76 & \\
\hline 8 & Belgien & 0,73 & \\
\hline 9 & Österreich & 0,73 & 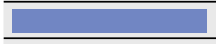 \\
\hline 10 & Polen & 0,73 & \\
\hline 11 & Rumänien & 0,73 & \\
\hline 12 & Slowenien & 0,73 & \\
\hline 13 & Frankreich & 0,72 & \\
\hline 14 & Irland & 0,72 & \\
\hline 15 & Litauen & 0,72 & \\
\hline 16 & Deutschland & 0,7 & $\bar{t}$ \\
\hline 17 & Spanien & 0,7 & 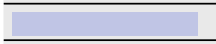 \\
\hline 18 & Lettland & 0,69 & $\overline{7}$ \\
\hline 19 & Tschechische Republik & 0,69 & \\
\hline 20 & Italien & 0,68 & \\
\hline 21 & Griechenland & 0,66 & \\
\hline 22 & Portugal & 0,66 & \\
\hline 23 & Ungarn & 0,65 & \\
\hline 24 & Slowakei & 0,64 & \\
\hline 25 & Bulgarien & 0,63 & \\
\hline
\end{tabular}

Quelle: Institut für Innovation und Technik (iit) (2018).

\section{Strukturkapital}

Die Dimension des Strukturkapitals steht für sämtliche Strukturen und Prozesse, die das verteilte Wissen in Unternehmen und Institutionen zusammenführen und so deren Innovationsfähigkeit beeinflussen. Somit kommt über die Dimension des Strukturkapitals zum Wissen einzelner Personen noch die strukturelle Lernfähigkeit der Organisationen dazu. Dazu gehören F\&E-Organisationseinheiten oder auch "lernförderliche" Organisationsformen.

Die Daten zur lernförderlichen Arbeitsorganisation im Innovationsfähigkeitsindikator stammen aus dem „EWCS - European Working Condition Survey“25. Diese werden mangels vergleichbarer außereuropäischer Daten für die Analyse herangezogen, um Rückschlüsse auf Österreichs Strukturkapital ziehen zu können.
Der umfragebasierte Indikator umfasst unterschiedliche Facetten der lernförderlichen Arbeitsorganisation, wie bspw. Autonomie bei der inhaltlichen und organisatorischen Ausgestaltung der Arbeit oder die Möglichkeit, neue Dinge zu erlernen. Von den 25 betrachteten europäischen Ländern nimmt Österreich den neunten Platz ein (Wert 0,73 von 1). Die fünf führenden europäischen Länder sind Norwegen $(0,88)$, Finnland $(0,83)$, Schweden $(0,83)$, Dänemark $(0,81)$ und Schweden $(0,81)$. Trotz des insgesamt guten Ergebnisses in dieser Dimension der Innovationsfähigkeit besteht für Österreich noch Weiterentwicklungspotenzial bezüglich der lernförderlichen Arbeitsorganisation. Österreichs Stärken bei der lernförderlichen Arbeitsorganisation liegen bspw. in den Angeboten der Berufsbildung weiterbildender Unternehmen oder der Komplexität kognitiver Aufgabenstellungen. Schwächen zeichnen sich in diesem Kontext hingegen zum Beispiel im Bereich themenspezifische Weiterbildungsangebote in der Informatik ab (EWCS, Bezugsjahr 2015).

\section{Beziehungskapital}

Schließlich wird als vierte „Säule“ der Innovationsfähigkeit das Beziehungskapital betrachtet. Beziehungen - also Netzwerke und Kooperationen von Unternehmen mit externen Partnern - spielen für den Innovationsprozess eine entscheidende Rolle. Sowohl für die Wissensgenerierung als auch für den Wissensund Technologietransfer können durch das Zusammenspiel von Unternehmen und Forschungseinrichtungen neue Produkte und Prozesse entwickelt werden, die ohne Kollaboration von Forschung und Industrie nicht entstanden wären. Sowohl aufgrund der Komplexität von (insbesondere technischen) Innovationen als auch aus Kostengründen sind Netzwerke und Kooperationen ein maßgeblicher Faktor, um Forschungseffizienz zu erhöhen und die Entwicklungszeit von neuen bzw. verbesserten Produkten (Technologien) zu beschleunigen.

25 Es handelt sich dabei um die letzte EWCS-Umfrage der EU-Agentur Eurofound aus dem Jahr 2015. Diese Umfrage wird alle fünf Jahre durchgeführt. 


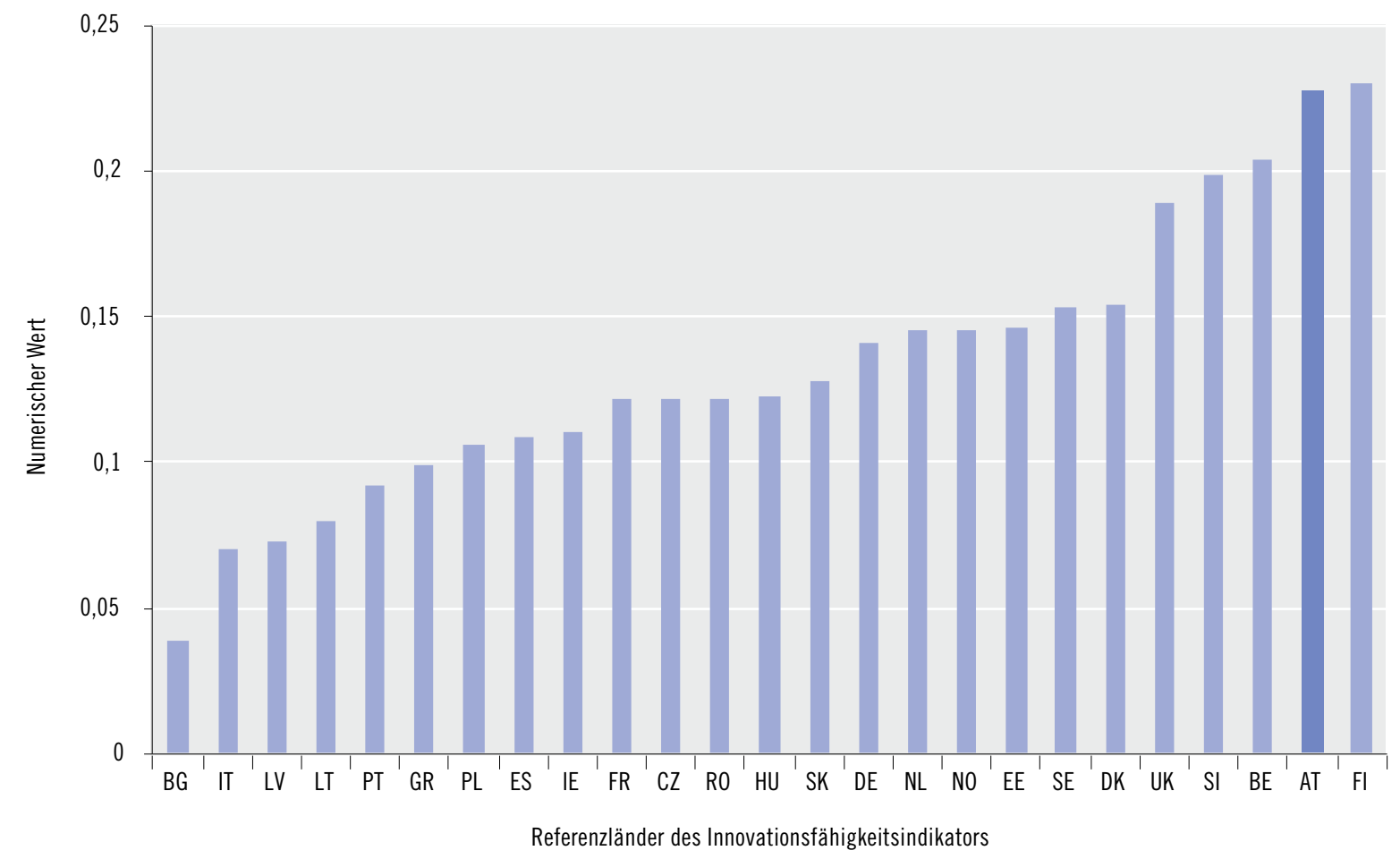

* für das Bezugsjahr nicht verfügbare Länderdaten: SE

Quelle: Institut für Innovation und Technik (2018), basierend auf Ergebnissen des Community Innovation Survey 2014.

Das Beziehungskapital wird in diesem Teilabschnitt anhand des Grades der Kooperationen und des Austauschs zwischen Unternehmen und einzelnen Forschungseinheiten untersucht. Dabei wird der Grad der Kooperationen zwischen Unternehmen und Hochschulen (siehe Abbildung 1-21) sowie der Grad der Kooperationen zwischen Unternehmen und öffentlichen oder privaten Forschungseinrichtungen betrachtet (siehe Abbildung 1-22). Die Auswertungen basieren dabei auf den Daten des Community Innovation Survey (CIS). Der CIS ist eine harmonisierte Erhebung der Innovationsaktivitäten, die in EUMitgliedsländern und einigen weiteren europäischen Ländern durchgeführt wird. Mit der harmonisierten Erhebung werden Informationen über das Innovationsverhalten der Unternehmen sowie verschiedene Aspekte der Innovationsentwicklung untersucht.

Der Grad der Kooperationen wurde als Anteil normiert, der einen Wert zwischen 0 und 1 annehmen kann. Dies bedeutet, dass ein Wert von 0,23 (für Ös- terreich) bei der Betrachtung der Kooperationen mit sich bringt, dass $23 \%$ der Unternehmen mit technologischen Innovationstätigkeiten mit Hochschulen kooperieren, um Innovationen zu generieren (siehe Abbildung 1-21). Der Wert von 0,12 bei Österreich in Abbildung 1-22 bedeutet, dass $12 \%$ der österreichischen Unternehmen mit technologischen Innovationstätigkeiten mit Forschungseinrichtungen kooperieren (bezüglich wichtiger Innovationsaktivitäten). Dieser Anteil liegt im europäischen Vergleich im vorderen Mittelfeld.

Die Ergebnisse zeigen, dass Österreich europaweit führend ist, wenn das Ausmaß der Kooperationen zwischen Unternehmen und Hochschulen (siehe Abbildung 1-21) betrachtet wird. Österreich belegt hierbei im europaweiten Vergleich den zweiten Platz hinter Finnland. Bei der Betrachtung des Ausmaßes der Zusammenarbeit zwischen Unternehmen und öffentlichen wie privaten - Forschungseinrichtungen (siehe Abbildung 1-22) belegt Österreich den sechs- 
Abbildung 1-22: Grad der Kooperationen zwischen Unternehmen und Forschungseinrichtungen*

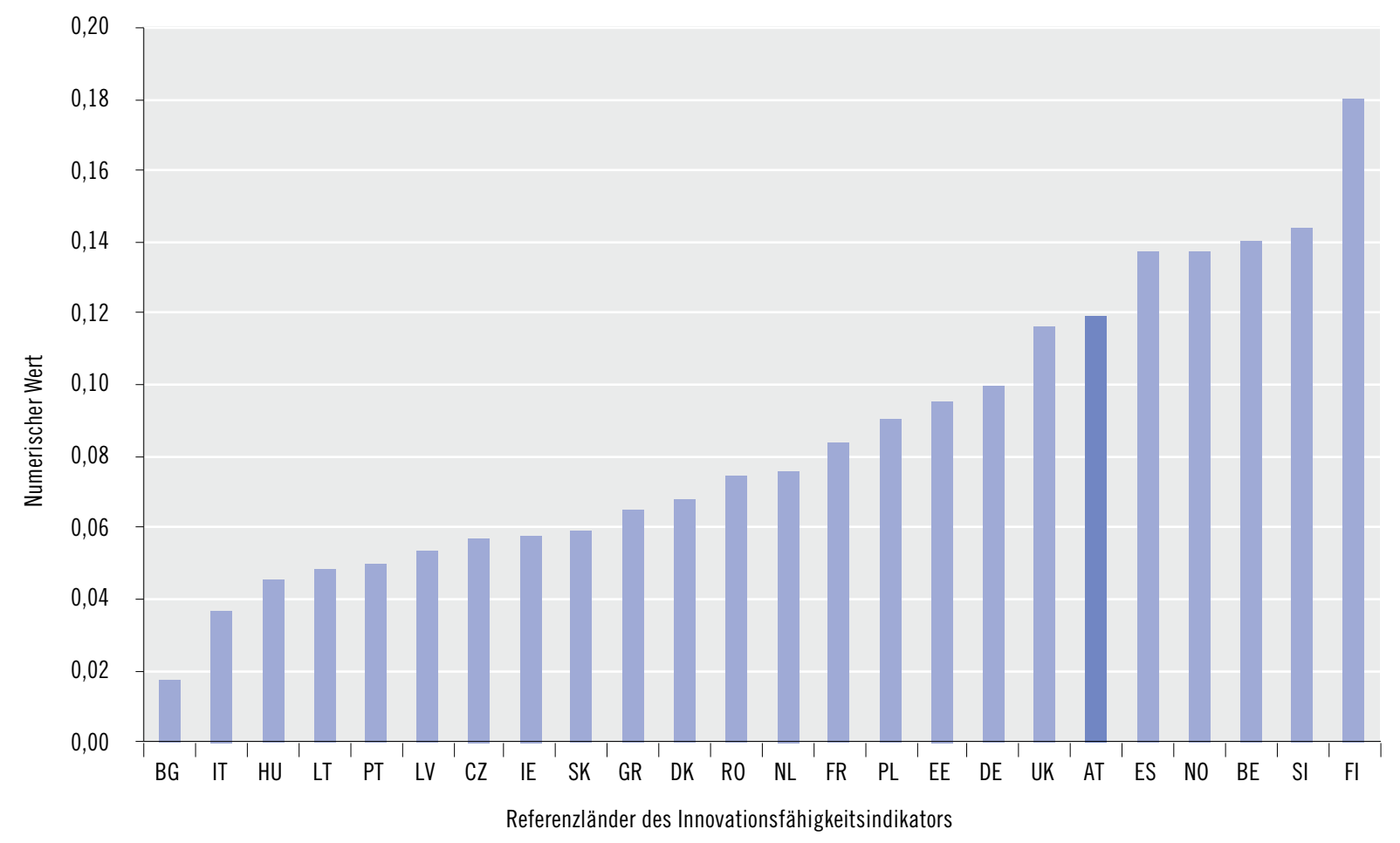

* für das Bezugsjahr nicht verfügbare Länderdaten: SE

Quelle: Institut für Innovation und Technik (2018), basierend auf Ergebnissen des Community Innovation Survey 2014.

ten Platz im europäischen Vergleich und nimmt damit auch eine führende Platzierung ein.

Insgesamt deuten diese Ergebnisse an, dass die Zusammenarbeit von Unternehmen mit Hochschulen sowie Forschungseinrichtungen in Österreich relativ stark ausgeprägt ist. Damit ist Österreich gut positioniert, um einen schnellen und effizienten Transfer für neue Verfahren, Technologien und Dienstleistungen einerseits und gemeinsame Innovationsaktivitäten zwischen Forschung und Industrie andererseits durchzuführen. Insbesondere vor dem Hintergrund steigender technischer Herausforderungen, einer global vernetzten Wissenschaft und finanzieller Ressourcen wird die Bedeutung von Kooperationen zwischen Unternehmen und Forschungseinrichtungen in Zukunft vermutlich noch größer werden. Wie die hier angegebenen Auswertungen zeigen, hat Österreich ein stark ausgeprägtes „Beziehungskapital“, von dem zukünftige Innovationstätigkeiten profitieren können und werden.

\subsubsection{Resümee}

Im siebten Jahr nach Veröffentlichung der letzten Strategie der Bundesregierung für Forschung, Technologie und Innovation hat Österreich auch im internationalen Vergleich seine Position sichtlich verbessern können. Österreich gehört zu den führenden Nationen in Bezug auf seine Ausgaben für Forschung und Entwicklung. Auch in den global üblichen Einzelindikatoren für FTI hat sich Österreich überwiegend gesteigert. So weist Österreich durchgehend Verbesserungen oder stabile Werte auf, was die messbaren Leistungen seines Wissenschaftssystems betrifft. Dies bezieht sich insbesondere auf qualitätsorientierte Parameter wie beispielsweise die Performance bei der Zitationsrate zu wissenschaftlichen Leistungen. Die Position Österreichs im Bereich Digitalisierung ist nicht herausragend, aber auch nicht zurückgefallen. Der Bezug des vorläufigen Status-quo im Kontext von IKT zu den anderen (unmittelbaren) Entwicklungsfeldern im Bereich Forschung, Technologie 
und Innovation ist zudem nicht eindeutig. Bei der Betrachtung der Nutzung und Verfügbarkeit von IKT ist Österreich im internationalen Vergleich mittelmäBig platziert, die Ergebnisse deuten dabei mögliche Lücken an systemischen Schnittstellen, wie z.B. im Kontext der Integration der Digitaltechnik, an.

Ferner wurde im vorliegenden Kapitel die Wettbewerbs- und Innovationsfähigkeit Österreichs untersucht. Die Analyse der Wettbewerbsfähigkeit Österreichs erfolgte dabei anhand des Global Competitiveness Reports. Eine Auswertung dieses Berichts zeigt, dass Österreich in allen untersuchten Dimensionen - u.a. bezüglich der technologischen Reife, der politischen und ökonomischen Rahmenbedingungen und der Qualifizierung der Personen sehr gut abschneidet und jeweils besser positioniert ist als der Durchschnitt der 28 EU-Länder. Auch in der Analyse der Innovationsfähigkeit erzielt Österreich gute bis sehr gute Ergebnisse. Den ausgewerteten Indikatoren zufolge ist die österreichische Wirtschaft in der Lage, komplexe Produkte und Prozesse zu generieren und auf einem globalen Markt zu etablieren, die Bevölkerung ist gut ausgebildet und Unternehmen kooperieren im internationalen Vergleich relativ häufig mit Hochschulen und Forschungseinrichtungen.

Wie dargestellt wurde, betonen darüber hinaus die gängigen Innovationsrankings unterschiedliche Schwerpunkte und verwenden variierende Parameter in ihren internationalen Vergleichsanalysen. So wird u.a. in manchen Quellen ${ }^{26}$ der noch ausbleibende Impact des Engagements gerade im Innovationsbereich angemerkt. Insofern sollten die Kompositindikatoren nur vorsichtig interpretiert werden. In der Gesamtzusammenschau zeigen sowohl die Kompositindikatoren als auch die "harten" Indikatoren in Abschnitt 1.3.1, dass Österreich gute Aussichten hat, Innovationen zu entwickeln und damit auf dem globalen Markt erfolgreich zu sein.

\section{3 Österreich und die EU Forschungs-, Technologie- und Innovationspolitik}

In Kapitel 1.4 werden die folgenden vier Aspekte näher beleuchtet:

1. Das Ergebnis der österreichischen Bemühungen als Vorsitzland im Rahmen der EU-Ratspräsidentschaft in Bezug auf die Entwicklung des nächsten Europäischen FTI-Rahmenprogramms;

2. Das Abschneiden österreichischer Institutionen und Forscherinnen und Forscher im laufenden Europäischen FTI-Rahmenprogramm Horizon 2020 zwei Jahre vor dessen Beendigung;

3. Die Umsetzung von Horizon 2020 in Österreich durch die beauftragten operativen und strategischen Betreuungs- und Unterstützungsstrukturen;

4. Eine Vorausschau auf die Struktur und die Neuerungen von Horizon Europe, dem nächsten Europäischen FTI-Rahmenprogramm.

In der zweiten Jahreshälfte 2018 hatte Österreich zum dritten Mal in seiner Geschichte den Vorsitz des Rates der Europäischen Union inne. Die wichtigsten Meilensteine im FTI-Bereich zur Entwicklung des nächsten Europäischen Forschungsrahmenprogramms, die unter dem österreichischen Ratsvorsitz in Abstimmung mit den europäischen Mitgliedsstaaten, dem Europaparlament und der Europäischen Kommission stattgefunden haben, werden in Abschnitt 1.4.1 systematisch zusammengefasst. Danach werden die Schlussfolgerungen des Rates zur Weiterentwicklung des Europäischen Forschungsraums (ERA) erläutert und wird über die FTI-Aktivitäten des österreichischen EU-Ratsvorsitzes zur Unterstützung sektoraler Politiken berichtet.

Das Abschneiden österreichischer Institutionen und Forscherinnen und Forscher im laufenden europäischen FTI-Rahmenprogramm Horizon 2020 wird im Abschnitt 1.4.2 im Detail analysiert. Es werden die Bereiche des Europäischen Forschungsrahmenpro- 
gramms herausgearbeitet, in denen Österreich besonders intensiv teilnimmt, und die u.a. dazu geführt haben, dass die Milliarden Euro-Benchmark an eingeworbenen Mitteln aus dem EU-Budget überschritten werden konnte.

Im Abschnitt 1.4 .3 werden die Aktivitäten und Maßnahmen zur Umsetzung von Horizon 2020 in Österreich analysiert. Augenmerk wird dabei erstens auf die Beratung und Betreuung von Horizon 2020 durch die FFG, zweitens auf das ebenfalls von der FFG durchgeführte EU-Performance Monitoring und drittens auf das ERA-Observatorium, das einen übergeordneten Rahmen für die Integration einzelner Aufgabenstellungen im Bereich der Governance der EU-FTI-Politik in Österreich bietet, gelegt.

Schließlich wird in Abschnitt 1.4.4 die Grundstruktur des neuen Europäischen Forschungsrahmenprogramms Horizon Europe skizziert und werden die damit einhergehenden beabsichtigten Neuerungen erläutert.

\subsubsection{Aktivitäten im Rahmen der EU-Ratspräsidentschaft}

Mit 01.07.2018 hat Österreich den Vorsitz des Rates der Europäischen Union von Bulgarien übernommen und das Trio-Ratspräsidentschaftsprogramm weitergeführt. Das Trio von drei aufeinanderfolgenden Ratspräsidentschaften bestand aus Estland (Präsidentschaft im zweiten Halbjahr 2017), Bulgarien (erstes Halbjahr 2018) und Österreich (zweites Halbjahr 2018). Der österreichische Vorsitz im Rat der Europäischen Union dauerte bis zum 31.12.2018 und wurde danach von Rumänien übernommen, das gemeinsam mit Finnland (zweites Halbjahr 2019) und Kroatien (erstes Halbjahr 2020) das derzeitige Trio des Ratsvorsitzes bildet.

Das offizielle Programm der österreichischen Präsidentschaft des Rates der Europäischen Union stand unter dem Motto „Ein Europa, das schützt“. Diese bis- lang dritte österreichische EU-Ratspräsidentschaft war insofern speziell, da Europa vor einer Reihe paralleler Herausforderungen stand, die im Rahmen der österreichischen Präsidentschaft zu berücksichtigen waren:

- Der Vorschlag für den mehrjährigen Finanzrahmen der Europäischen Union für den Zeitraum 20212027 wurde knapp vor Beginn der österreichischen EU-Ratspräsidentschaft am 02.05.2018 veröffentlicht. ${ }^{27}$ In diesem Vorschlag hat die Kommission 97,6 Mrd. $€$ für „Horizon Europe“ und weitere 2,4 Mrd. € für das Euratom-Forschungs- und Ausbildungsprogramm bereitgestellt, was einen Gesamtbetrag von $100 \mathrm{Mrd}$. $€$ ergibt.

- Der Vorschlag für „Horizon Europe“, das Nachfolgeprogramm von „Horizon 2020“, wurde am 07.06.2018 von der Europäischen Kommission vorgelegt. $^{28}$

- Die zweite Hälfte des Jahres 2018 und die ersten Monate des Jahres 2019 wurden von den BrexitVerhandlungen bestimmt.

- Die Legislaturperiode des Europäischen Parlaments endet mit der konstituierenden Sitzung des neu gewählten Parlaments im Juli 2019; die Wahlen zum Europäischen Parlament erfolgten im Mai 2019; eine neue Kommission wird im November 2019 eingesetzt.

Die Prioritäten des österreichischen Vorsitzes im Bereich Wissenschaft, Forschung und Innovation waren folgende:

- Verhandlungen über „Horizon Europe“, das 9. EURahmenprogramm für Forschung und Innovation (RPQ);

- Schlussfolgerungen des Rates zum Europäischen Forschungsraum (ERA) gestützt auf die Überprüfung der ERA-Beratungsstruktur durch ERAC (European Research Area and Innovation Committee);

- Unterstützung sektoraler Politiken (z.B. Verteidigungsforschung, Hochschulnetzwerkinitiative usw.). 
Für diese Prioritäten wurden auf Ministerinnen- und Ministerebene (siehe Tabelle 8-1 in Anhang I) sowie auf höherer Beamtinnen- und Beamtenebene intensive Gespräche und Verhandlungen vorbereitet und geführt, Schlussfolgerungen und Dokumente erarbeitet und Veranstaltungen (siehe Tabellen 8-2, 8-3 und 8-4 in Anhang I) organisiert.

\section{Priorität 1: Das nächste europäische}

\section{Rahmenprogramm für Forschung und Innovation}

Den Verhandlungen über „Horizon Europe“, dem nächsten und damit neunten europäischen Rahmenprogramm für Forschung und Innovation (RP9), liegt ein Paket zugrunde, das aus mehreren Legislativdokumenten besteht. Das wichtigste dieser Dokumente ist die Horizon Europe-Verordnung, in der die Struktur, Ziele und Hauptmerkmale des Rahmenprogramms für die Jahre 2021-2027 festgelegt sind. Im Gegensatz zu seinen Vorgängern enthält die Horizon Europe-Verordnung die Regeln für die Teilnahme und die Verbreitung der Ergebnisse. Darüber hinaus sind alle Einzelheiten zum Inhalt und zur Durchführung des Programms in einem spezifischen Programm festgelegt. Weitere Dokumente im Bereich der Forschung fallen in den Zuständigkeitsbereich des EURATOMVertrags.

Ein Höhepunkt der österreichischen EU-Ratspräsidentschaft im Bereich Wissenschafts-, Forschungs- und Innovationspolitik war die Einigung über die wesentlichen Inhalte der Horizon EuropeVerordnung. ${ }^{29}$ Am 30.11.2018 erreichte der Rat für Wettbewerbsfähigkeit dazu eine sogenannte „partielle allgemeine Ausrichtung“ („Partial General Approach" oder PGA). Der PGA, bei dem bindende Aussagen zur Budgetverteilung fehlen, da der von der Europäischen Kommission vorgeschlagene mehrjährige Finanzrahmen noch nicht beschlossen ist, wurde mit einer überaus breiten Mehrheit angenommen (es gab nur eine Gegenstimme). Darüber hinaus setzte der Ratsvorsitz die Verhandlungen über das spezifische Programm von „Horizon Euro- pe" fort, was kurz vor Ende 2018 zu einem zweiten Kompromisstext des Ratsvorsitzes führte.

Die „partielle allgemeine Ausrichtung“ für das weltgrößte Förderungsprogramm für Forschung und Innovation, namens „Horizon Europe“, wurde in einer Rekordzeit von nur fünf Monaten ausgehandelt und vereinbart. Dem Vorschlag der Europäischen Kommission zufolge soll von 2021-2027 ein Betrag von 100 Mrd. € zur Förderung exzellenter Forschung, für Mobilität und Ausbildung von Forscherinnen und Forschern sowie für Forschungsinfrastrukturen zur Verfügung stehen. Des Weiteren werden wichtige gesellschaftliche und wirtschaftliche Herausforderungen in themenübergreifenden Clustern aufgegriffen und unter anderem durch die sogenannten „Missionen" zu spezifischen Problemstellungen mithilfe eines Portfolios von Forschungs- und Innovationsmaßnahmen umgesetzt, die innerhalb von zehn Jahren zu konkreten Ergebnissen und Effekten führen sollen. Ebenso soll ein neu gegründeter „Europäischer Innovationsrat" bahnbrechende Innovationen fördern, die neue Märkte, Arbeitsplätze und Wohlstand in Europa schaffen können. Schließlich wird sich ein Teil von „Horizon Europe“ auf die Unterstützung der Strukturreformen der Mitgliedstaaten im Europäischen Forschungsraum konzentrieren, wobei der Schwerpunkt auf den Ländern mit geringer F\&E-Leistung liegt, nicht zuletzt um die Innovationslücke in Europa zu verringern. Eine weitere wichtige Neuerung ist ein neuer Governance-Ansatz, der sich durch Haushaltsflexibilität und einen umfassenden strategischen Planungsprozess auszeichnet. (Zur Ausgestaltung von „Horizon Europe“ siehe Kapitel 1.4.4.)

\section{Priorität 2: Die Weiterentwicklung des Europäischen Forschungsraums}

Die zweite Priorität im Rahmen des österreichischen Vorsitzes im Rat der Europäischen Union im Bereich von Wissenschaft, Forschung und Innovation betrifft den Europäischen Forschungsraum (ERA). Dessen Ziel ist es, einen Binnenmarkt des Wissens in Europa

29 Vgl. Council of the European Union (2018). 
zu schaffen, in dem Forscherinnen und Forscher mit ihrem Wissen ein Zuhause ohne Grenzen finden und Technologien für einen gemeinsamen Markt entwickelt werden. Im Mittelpunkt des ERA stehen die von jedem Mitgliedstaat durchzuführenden Reformen. Damit soll jedes Land die Effizienz und Wirksamkeit seines Innovationssystems steigern und die transnationale Zusammenarbeit auf allen Ebenen stärken.

In Bezug auf den Europäischen Forschungsraum hat der Rat am 30.11.2018 auch die Schlussfolgerungen zum Europäischen Forschungsraum angenommen und verabschiedet. ${ }^{30}$ Diese Schlussfolgerungen des Rates, die der österreichische Vorsitz nach dem ERAC-Plenum (Plenum des European Research Area and Innovation Committee) im September 2018 in Salzburg vorgeschlagen hatte, wurden Anfang Oktober 2018 in der Arbeitsgruppe Forschung erfolgreich verhandelt. Die Schlussfolgerungen bestehen aus drei Teilen: (a) Anerkennung der wichtigen Fortschritte, welche die verschiedenen mit dem ERA in Zusammenhang stehenden Gruppen bei der Umsetzung der ERA-Agenda erzielt haben; b) Entscheidung der Forschungsministerinnen und -minister über die „ERABeratungsstruktur" auf der Grundlage der einschlägigen Überprüfung und des ERAC-Berichts; ${ }^{31}$ (c) Ausblick auf die Zukunft des ERA mit der Aufforderung an die Europäische Kommission, bis 2020 eine neue ERA-Mitteilung zu veröffentlichen, sowie die Aufforderung an die zukünftigen Ratsvorsitze zur Veranstaltung regelmäßiger ERA-Ministerinnen- und Ministerkonferenzen.

Der Punkt (b) der Schlussfolgerungen beruht auf einem Auftrag des Rates für Wettbewerbsfähigkeit aus dem Jahr 2015, die bestehende ERA-Beratungsstruktur in Europa zu überprüfen. ${ }^{32}$ Die Überprüfung, die durch den Ratsvorsitz in Zusammenarbeit mit ER$A C$ durchgeführt wurde, umfasste den Inhalt und die
Umsetzung der sechs Prioritäten des Europäischen Forschungsraums für die Reform nationaler Forschungssysteme. Dabei handelt es sich um folgende Prioritäten: ${ }^{33}$

- Steigerung der Effizienz und Effektivität der nationalen Forschungs- und Innovationssysteme,

- Einen Beitrag zur Lösung der großen gesellschaftlichen Herausforderungen (den sogenannten "Grand Challenges") durch die Förderung öffentlicher bzw. öffentlich-privater Partnerschaften - zum Beispiel in Form von JPIs (Joint Programming Initiatives) zu leisten sowie öffentliche Investitionen in Forschungsinfrastrukturen optimal zu nutzen,

- Die Reformierung des Arbeitsmarkts für Forscherinnen und Forscher zur Schaffung eines offenen europäischen Forschungsarbeitsmarkts,

- Die Förderung der Gleichstellung der Geschlechter in der Forschung,

- Die Optimierung der Zirkulation und des Transfers von Wissen durch Öffnung von Wissenschaft und Innovation („open science“ und „open innovation“), sowie

- Die Stärkung der internationalen Zusammenarbeit in Wissenschaft, Forschung und Innovation.

Als Ergebnis dieser von ERAC geführten Überprüfung schlug der Vorsitz die Schlussfolgerungen des Rates zur Steuerung des Europäischen Forschungsraums vor. Die Ministerinnen und Minister haben diese Schlussfolgerungen am 30.11.2018 einstimmig angenommen. ${ }^{34}$ In den Schlussfolgerungen werden die bisher erzielten Fortschritte bei der Schaffung eines Binnenmarktes für Wissen, insbesondere des neuen ESFRI-Fahrplans 2018 für Forschungsinfrastrukturen ${ }^{35}$, und die Governancestruktur der European Open Science Cloud $^{36}$ bestätigt. Die Schlussfolgerungen ebnen schließlich auch den Weg für eine überarbeitete Reformagenda für den Europäi-

\footnotetext{
30 Ebenda.

$31 \mathrm{Vg}$. ERAC (2018).

32 Vgl. Council of the European Union (2015).

33 Vgl. ERAC (2015).

$34 \mathrm{Vgl}$. Council of the European Union (2018).

35 Vgl. ESFRI (2018).

36 Vgl. https://era.gv.at/object/news/4439
} 
schen Forschungsraum, die im Jahr 2020 erarbeitet werden soll.

\section{Priorität 3: Unterstützung sektoraler Politik}

Forschung und Innovation spielen in vielen anderen Politikbereichen eine immer wichtigere Rolle. Aus diesem Grund bestand die dritte Priorität der österreichischen Ratspräsidentschaft im Bereich der Wissenschafts-, Forschungs- und Innovationspolitik darin, Sektorpolitiken durch mehr Zusammenarbeit zu unterstützen. Zur Befriedigung der Nachfrage nach neuem Wissen und Innovation in vielen sektoralen Bereichen ist es notwendig, "Horizon Europe“ und andere EU-Programme immer enger miteinander zu verknüpfen, um intelligente und effiziente Synergien zu erzielen. Der österreichische Vorsitz hat daher einige der sektoralen Politikbereiche aktiv in den Verhandlungsprozess von „Horizon Europe“ einbezogen, wie den Europäischen Fonds für regionale Entwicklung, ERASMUS+, das „Digital Europe“-Programm oder den Europäischen Verteidigungsfonds. Wenn die Verhandlungen über 16 verschiedene EU-Programme genug gediehen sind, können schließlich künftige Vorsitze endgültige Schlussfolgerungen zu den Synergien mit „Horizon Europe“ ziehen.

Neben naheliegenden sektoralen Bereichen, die von Wissenschaft, Forschung und Innovation stark beeinflusst werden, wie z.B. Klimawandel oder Digitalisierung, bilden auch die Ziele der Vereinten Nationen für nachhaltige Entwicklung ${ }^{37}$ einen wichtigen Bezugsrahmen für die Nachfrage nach Forschung und Innovation. Die österreichische Präsidentschaft betrachtete Forschung und Innovation daher als generischen Politikbereich, der für alle sektoralen Politiken wirksam werden muss. Insbesondere zählen dazu:

- Der Beitrag von Forschung zum Umgang mit gesundheitspolitischen Fragen;

- Der Beitrag von Forschung zur Entwicklung wirtschaftlicher und technologischer Wachstumspfade, insbesondere im Zusammenhang mit der Digi- talisierung der Gesellschaft oder der Erforschung des Weltraums;

- Der Beitrag von Forschung zum Wandel der Energiesysteme für die Zukunft Europas;

- Die Interaktion zwischen Forschung und verantwortungsbewussten Bürgerinnen und Bürgern;

- Die Schnittstelle zur Bildungspolitik u.a. zur Ermöglichung einer neuen Generation von Mobilitätsprogrammen in Europa und andere.

\section{Überblick über die österreichischen \\ Ratspräsidentschaftsveranstaltungen}

Insgesamt waren rd. 40 Veranstaltungen im Wissenschafts-, Forschungs- und Innovationspolitikbereich Teil der Aktivitäten der österreichischen Präsidentschaft im zweiten Halbjahr 2018. Von den Treffen der Forschungsministerinnen und Forschungsminister bis zu Expertinnen- und Expertenkonferenzen luden die Veranstaltungen während der österreichischen Ratspräsidentschaft dazu ein, über Fragen zu „Horizon Europe“, zum Europäischen Forschungsraum und über die Schnittstelle von Forschung und Innovation zu Sektorpolitiken zu diskutieren.

Zehn Veranstaltungen, die im Rahmen der österreichischen EU-Ratspräsidentschaft organisiert wurden, haben einen direkten Bezug zum kommenden europäischen Forschungsrahmenprogramm „Horizon Europe“ hergestellt. Diese Veranstaltungen reichten von Marie Skłodowska-Curie über Industrial Technologies, vom Beitrag der Sozial- und Geisteswissenschaften zur missionsorientierten Forschung bis zur Konferenz „Imagine Digital - Connect Europe“" (siehe Tabelle 8-2 in Anhang I). Die Ergebnisse dieser Veranstaltungen wurden auch dokumentiert ${ }^{38}$ und sodann dem Verhandlungsteam in Brüssel zur Verfügung gestellt.

Wie auch in Hinblick auf „Horizon Europe“ ergänzten zahlreiche Veranstaltungen im Rahmen der österreichischen Ratspräsidentschaft die Verhandlungen über die Schlussfolgerungen auf EU-Ebene zum Europäischen Forschungsraum (ERA) bzw. hatten speziel- 
le Bereiche des Europäischen Forschungsraums im Fokus (siehe Tabelle 8-3 in Anhang I). ERAC, ESFRI, Open Science, Joint Programming und viele andere Foren erörterten Themen des ERA. Ein Spezialgebiet, das auch eine wichtige Schnittstelle zum ERA hat, ist die Hochschulbildung. Mit insgesamt fünf Veranstaltungen an der Schnittstelle zwischen Hochschulbildung und Forschung unterstrich der österreichische Ratsvorsitz die Bereitschaft zu einer besseren Zusammenarbeit zwischen diesen Politikbereichen im Rahmen der Reformagenda.

Darüber hinaus unterstützten mehrere Veranstaltungen während der österreichischen Präsidentschaft im zweiten Halbjahr 2018 den einschlägigen Fachdiskurs in sektoralen Politikfeldern (siehe Tabelle 8-4 in Anhang I). Die Expertinnen- und Expertenkonferenzen unterstrichen dabei vor allem den Zusammenhang zwischen Bildung, Forschung, technologischer Entwicklung, Innovation und den jeweils anderen Politikbereichen.

\subsubsection{Die Performance Österreichs in Horizon 2020}

Die von der Europäischen Kommission über eCORDA mit Stichtag 21.01.2019 bereitgestellten Daten, die auch im EU-Performance Monitoring der FFG (03/2019) weiterverarbeitet werden, bestätigen den guten Erfolg österreichischer Institutionen und Forscherinnen und Forscher aus Industrie und Wissenschaft im laufenden achten europäischen Forschungsrahmenprogramm Horizon 2020. Die Gesamtsumme der Bewilligungen für Österreich liegt bei 1,11 Mrd. €. Damit wurde die Milliardeneurogrenze deutlich überschritten. In Österreich ansässige Institutionen bzw. Forscherinnen und Forscher nahmen an 8,8 \% aller 21.472 Projekte, die im Rahmen von Horizon 2020 gefördert wurden, teil.

Der Anteil der österreichischen Beteiligungen an den insgesamt 104.427 Beteiligungen in den geförderten Horizon 2020 Projekten beträgt 2,8 \%. Dabei handelt es sich um 2.919 Beteiligungen in insgesamt 1.894 Projekten. In 1.344 Projekten gibt es nur einen österreichischen Partner. In 550 Projekten nehmen mindestens zwei österreichische Partner teil. Gemessen an der Anzahl der Beteiligungen liegt Österreich mit 2.919 Beteiligungen bzw. 2,8\% im internationalen Vergleich an der neunten Stelle hinter Schweden (insgesamt 3.139 Beteiligungen) und knapp vor Griechenland (2.917) und der Schweiz (2.788). Die meisten Beteiligungen weisen naturgemäß die großen europäischen Länder Deutschland (12.582), UK (11.750) sowie Spanien (10.717) und Frankreich (10.094) auf.

Der von Österreich eingeworbene Anteil der bewilligten Förderungen aus dem Horizon 2020 Budget liegt bei 2,8\% und entspricht damit dem Beteiligungsanteil. Der Anteil österreichischer Koordinatorinnen und Koordinatoren an allen Koordinierenden liegt bei 2,6\%. Im internationalen Vergleich zeigt sich, dass unter allen österreichischen Koordinationen diejenigen, die von Unternehmen koordiniert werden, verhältnismäßig überrepräsentiert sind (Österreich: 32,6\%; alle: 27,3\%), während österreichische Koordinationen aus dem Hochschulsektor vergleichsweise unterrepräsentiert sind (Österreich: 41,0\%; alle: 47,7\%).

Mit einer Erfolgsrate ${ }^{39}$ von $17,9 \%$ auf Ebene der Beteiligungen liegt Österreich deutlich über der durchschnittlichen Horizon 2020 Erfolgsrate von $15,3 \%$ und nach Belgien (18,7 \%) an zweiter Stelle unter den Mitgliedsstaaten der Europäischen Union.

Auffallend ist die unterschiedliche Teilnahme österreichischer Beteiligter an den einzelnen Säulen und deren Untergliederungen. Aus Tabelle 1-2 „Österreichs Abschneiden in Horizon 2020nach Säulen, Projektteilnahmen, Projekten, Koordinationen und Budget" zeigt sich, dass Österreich besonders überdurchschnittlich in der Programmlinie "Science with and for Society" abschneidet. Hier liegt der Anteil österreichischer Koordinationen bei 9,3\%, der eingeworbene Budgetanteil bei $6,8 \%$ und der Anteil an

39 Erfolgsrate = Beteiligungen auf der „mainlist“ / „eligible applications“. 
Projektbeteiligungen bei $5,4 \%$. Allerdings ist diese Programmlinie mit verhältnismäßig bescheidenem Budget dotiert. Auffallend niedrig sind die Beteiligungen aus Österreich hingegen in den ebenfalls mit niedrigem Budget ausgestatteten Programmlinien "Cross-cutting issues" (1,6\% aller Beteiligungen und Koordinationen") sowie EURATOM (1,0\% aller Beteiligungen und $1,9 \%$ aller Koordinationen).

Gesamtgesehen sind hingegen aus budgetärer Sicht die großen Programmbereiche („Säulen“) „Societal Challenges“, "Excellent Science“ und „Industrial Leadership“ am bedeutendsten. Das meiste Budget konnte diesbezüglich in der Säule 3 "Societal Challenges" in Höhe von 430,51 Mio. € für Österreich eingeworben werden. Der österreichische Anteil in Säule 3 entspricht 2,9\% an den in allen Verträgen budgetierten Förderungen dieser Säule. In der Säule 1 „Excellent Science“ wurden 389,33 Mio. € von in Österreich tätigen Forscherinnen und Forschern eingeworben, was einem Anteil von 2,7 \% in dieser Säule entspricht. In der Säule 2 „Industrial Leadership“wurden 257,44 Mio. € von Österreich eingeworben, was einem Budgetanteil von 3,1 \% entspricht. Der relative Anteil in Säule 2 liegt demnach deutlich über dem österreichischen Durchschnitt. Mit Anteilen von 2,3\% an den Beteiligungen sowie 2,3\% bei den Koordinationen liegen die österreichischen Beiträge in der Säule „Excellent Science" deutlich unter dem österreichischen Horizon 2020-Durchschnitt von 2,8\% bzw. 2,6\%. Entsprechend darüber liegen die österreichischen Anteile in den Säulen "Industrial Leadership" (3,07 \% bzw. 3,02 \%) und "Societal Challenges" (3,04\% sowie 3,04\%).

Innerhalb der Säule 3 "Societal Challenges" finden sich die höchsten Beteiligungsanteile österreichischer Institutionen in den thematischen Clustern „Intelligenter, umweltfreundlicher und integrierter Verkehr"mit 4,1\% im Vergleich zu allen Beteiligungen in diesem Cluster (Koordinationen: 3,9\% und Budget: $3,6 \%)$, "Integrative, innovative und reflexive Gesellschaften" mit 4,0\% (K: $3,6 \%$ sowie $B: 4,4 \%$ ), sowie
"Sichere, saubere und effiziente Energie" mit 3,6\% (K: $3,6 \% ; \mathrm{B}: 3,6 \%$ ). Diese thematischen societal challenges können im europäischen Vergleich als österreichische Stärkefelder betrachtet werden. Unterdurchschnittlich niedrigere Beteiligungen finden sich insbesondere in den Clustern „Ernährungs- und Lebensmittelsicherheit, nachhaltige Land- und Forstwirtschaft, marine, maritime und limnologische Forschung und Biowirtschaft" mit 2,1\% (K: 1,9\%; B: $1,8 \%)$ sowie "Gesundheit, demografischer Wandel und Wohlergehen" mit 2,0 \% (K: 2,3 \%; B: 2,0\%). Innerhalb der Säule "Industrial Leadership“ finden sich die höchsten Beteiligungsanteile österreichischer Institutionen insbesondere in den thematischen Clustern „Materialien ${ }^{400}$ mit 3,9\% (Koordinationen: 2,4\%; Budget: $4,5 \%$ ), "IKT" mit 3,4\% (K: 4,1\%; B: 3,4\%) sowie „Biotechnologie“ mit 3,3 \% (K: 1,0 \%; B: 3,8 \%). Diese können als industrierelevante thematische Stärken Österreichs ausgewiesen werden. Die Beteiligungsanteile innerhalb des Bereichs „Risikofinanzierung " sind hingegen mit 2,1\% gering, wenngleich hier der Anteil österreichischer Koordinationen überdurchschnittlich hoch bei 10,0\% liegt und der österreichische Budgetanteil mit $4 \%$ ebenfalls hoch ist. In der Säule „Excellent Science“ finden sich mit 3,2 \% überdurchschnittlich hohe Beteiligungsanteile österreichischer Antragsteller innerhalb der Programmlinie „künftige und neu entstehende Technologien (FET)" (Koordinationen: 4,5\%; Budget: 3,2\%) sowie im „European Research Council (ERC)“ mit 2,7\% (K: 2,7 \%; B: 2,9\%). Vergleichsweise niedrigere Beteiligungsanteile finden sich in den "Marie-SkłodowskaCurie-Maßnahmen (MSCA)" mit 2,1\% (K: 1,7\%; B: $2,4 \%)$ und bei den "Forschungsinfrastrukturen" mit $2 \%$ (K: 3,3\%; B: 1,8 \%).

Die meisten österreichischen Beteiligungen - bezugnehmend auf die Gesamtanzahl - in Horizon 2020 stammen aus dem Unternehmenssektor (38,0\%), davon sind fast zwei Drittel kleine und mittlere Unternehmen (KMU). Danach folgen der Hochschulsektor $(27,9 \%)$ und der außeruniversitäre Forschungsbe-

40 Diese Abkürzung steht für das Programm NMP (Nanotechnologies, Advanced Materials and Production). 
reich $(22,6 \%)$. Insgesamt machen diese drei Sektoren $88,5 \%$ der österreichischen Beteiligungen in Horizon 2020 aus. Der Rest entfällt auf den öffentlichen Bereich (5,3\%) bzw. in die Kategorie „other" (6,3\%).

Auch in Bezug auf diese Sektoren ist die Aufteilung nach den einzelnen Säulen und Programmlinien sehr unterschiedlich. Gemessen an den eingeworbenen Förderungen beträgt der Anteil des österreichischen Hochschulbereichs in der Säule 1 („Excellent Science“) 68,8\%. Das ist - wenig überraschend durch einen hohen Anteil an Beteiligungen im ERC in Höhe von 74,8 \% erklärbar. Aber auch bei den Programmlinien FET und MSCA liegt der Anteil des Hochschulsektors gemessen an den eingeworbenen Förderungen, bei ungefähr zwei Drittel. Der entsprechende Anteil des außeruniversitären Bereichs liegt in der Säule 1 bei 19,5\% und jener des Unternehmenssektors liegt bei 10,6\%. In den Säulen 2 (,Industrial Leadership") und 3 ("Societal Challenges") hingegen zeigt sich - gemessen an den eingeworbenen Förderungen - ein gänzlich anderes Beteiligungsmuster. In diesen beiden Säulen liegen die Beteiligungen aus dem österreichischen Unternehmensbereich vor den Beteiligungen des heimischen außeruniversitären Sektors. Der Anteil des österreichischen Hochschulbereiches liegt hingegen jeweils unter $20 \%$. Gemessen an den eingeworbenen Förderungen liegt der Anteil des österreichischen Unternehmenssektors in Säule 2 bei 54,6\%. Der entsprechende Anteil des österreichischen außeruniversitären Sektors beträgt 23,0 \% und der Anteil des Hochschulsektors liegt bei 17,5\%. In Säule 3 („Societal Challenges“) liegt der Anteil des österreichischen Unternehmenssektors gemessen an den eingeworbenen Förderungen bei 40,4\%. Vergleichsweise hoch ist in Säule 3 auch der entsprechende Anteil des außeruniversitären Sektors mit 29,1\%. Besonders hoch liegt der Anteil des außeruniversitären Sektors in den Themenclustern "Sichere Gesellschaften“ mit 44,5\%, "Integrative, innovative und reflektierende Gesellschaften" $(43,5 \%)$ und „Ernährungs- und Lebensmittelsicherheit, nachhaltige Land- und Forstwirtschaft, marine, maritime und limnologische Forschung und die Biowirtschaft" (32,8\%). Der entsprechende Anteil des Hochschulsektors liegt in Säule 3 hingegen lediglich bei $19,4 \%$. In der horizontalen Programmlinie "Wissenschaft mit der und für die Gesellschaft" teilt sich die österreichische Beteiligung nach Organisationstyp, gemessen an den eingeworbenen Förderungen, folgendermaßen auf: Hochschulsektor: 30,7 \%; Unternehmenssektor: $13,0 \%$; sowie außeruniversitärer Sektor: 40,2\%. In der Programmlinie „Verbreitung von Exzellenz und Ausweitung der Beteiligung "liegen die Anteile bei 51,2\% (Hochschulsektor) und 38,6\% (au-

Tabelle 1-2: Österreichs Performance in Horizon 2020 nach Säulen, Projektteilnahmen, Projekten, Koordinationen und Budget

\begin{tabular}{|c|c|c|c|c|c|c|c|c|c|c|c|c|}
\hline & $\begin{array}{l}\text { Bewilligte } \\
\text { Beteiligungen } \\
\text { (alle Staaten) }\end{array}$ & $\begin{array}{l}\text { Bewilligte } \\
\text { österr. } \\
\text { Beteiligungen }\end{array}$ & $\begin{array}{l}\text { Anteil } \\
\text { Österreich } \\
\text { an allen } \\
\text { Staaten } \\
\text { (in \%) }\end{array}$ & $\begin{array}{l}\text { Bewilligte } \\
\text { Projekte } \\
\text { (alle Staaten) }\end{array}$ & $\begin{array}{l}\text { Bewilligte } \\
\text { Projekte } \\
\text { mit österr. } \\
\text { Beteiligung }\end{array}$ & $\begin{array}{l}\text { Anteil } \\
\text { österr. } \\
\text { Projekte } \\
\text { an allen } \\
\text { Projekten } \\
\text { (in \%) }\end{array}$ & $\begin{array}{l}\text { Bewilligte } \\
\text { Koordinationen } \\
\text { (alle Staaten) }\end{array}$ & $\begin{array}{c}\text { Bewilligte } \\
\text { Koordinationen } \\
\text { (Österreich) }\end{array}$ & $\begin{array}{l}\text { Anteil Projekte } \\
\text { mit österr. } \\
\text { Koordinatoren } \\
\text { an allen } \\
\text { Koordinatoren } \\
\text { (in \%) }\end{array}$ & $\begin{array}{c}\text { EU-Beitrag } \\
\text { (alle } \\
\text { Staaten) } \\
\text { (in Mio. €) }\end{array}$ & $\begin{array}{c}\text { EU-Beitrag } \\
\text { (Österreich) } \\
\text { (in Mio. €) }\end{array}$ & $\begin{array}{l}\text { Anteil } \\
\text { Österreich } \\
\text { am EU- } \\
\text { Beitrag } \\
\text { (in \%) }\end{array}$ \\
\hline $\mathrm{H} 2020$ & 104.427 & 2.919 & $2,80 \%$ & 21.472 & 1.894 & $8,82 \%$ & 21.421 & 558 & $2,60 \%$ & 39.382 & $1.107,52$ & $2,81 \%$ \\
\hline EC Treaty & 103.302 & 2.907 & $2,81 \%$ & 21.421 & 1.889 & $8,82 \%$ & 21.472 & 557 & $2,60 \%$ & 38.742 & $1.105,41$ & $2,85 \%$ \\
\hline Excellent Science & 34.492 & 784 & $2,27 \%$ & 11.887 & 583 & $4,90 \%$ & 11.887 & 267 & $2,25 \%$ & 14.586 & 389,33 & $2,67 \%$ \\
\hline $\begin{array}{l}\text { Societal } \\
\text { Challenges }\end{array}$ & 42.327 & 1.287 & $3,04 \%$ & 5.064 & 803 & $15,86 \%$ & 5.064 & 154 & $3,04 \%$ & 14.828 & 430,51 & $2,89 \%$ \\
\hline $\begin{array}{l}\text { Spreading } \\
\text { Excellence } \\
\text { and Widening } \\
\text { Participation }\end{array}$ & 844 & 25 & $2,96 \%$ & 204 & 22 & $10,78 \%$ & 204 & 1 & $0,49 \%$ & 527 & 4,93 & $0,94 \%$ \\
\hline $\begin{array}{l}\text { Science with and } \\
\text { for Society }\end{array}$ & 1.381 & 75 & $5,43 \%$ & 129 & 56 & $43,41 \%$ & 129 & 12 & $9,30 \%$ & 272 & 18,57 & $6,84 \%$ \\
\hline Cross-cutting & 645 & 10 & $1,55 \%$ & 129 & 9 & $6,98 \%$ & 129 & 2 & $1,55 \%$ & 270 & 4,64 & $1,72 \%$ \\
\hline Euratom & 1.125 & 12 & $1,07 \%$ & 51 & 5 & $9,80 \%$ & 51 & 1 & $1,96 \%$ & 640 & 2,10 & $0,33 \%$ \\
\hline
\end{tabular}

Quelle: EK/FFT per 03/2019; Berechnungen KMU Forschung Austria. 
Beruniversitäre Forschung). 10,2 \% sind dem Sektor "öffentlicher Bereich“ zuordenbar; eine österreichische Unternehmensbeteiligung ist in dieser Programmlinie nicht vorhanden.

In Bezug auf die österreichischen Bundesländer zeigt sich, dass 50,5\% der österreichischen Beteiligungen in Horizon 2020 auf den Standort Wien entfallen. Der Anteil der Steiermark ist mit 20,9\% ebenfalls überdurchschnittlich hoch. Danach folgen Oberösterreich (8,5\%) und Niederösterreich (7,5\%). Die wenigsten Beteiligungen sind aus Vorarlberg $(0,8 \%)$ und dem Burgenland (0,7\%) zu verzeichnen. Die hohe Beteiligung aus Wien ist vor allem der starken Konzentration des Hochschulsektors und des außeruniversitären Forschungsbereichs in Wien geschuldet. Die Verteilung der Beteiligungen des Unternehmenssektors in Horizon 2020 ist hingegen weniger stark konzentriert. Auch hier liegt Wien zwar mit einem Anteil von $39,9 \%$ an erster Stelle, aber die entsprechenden Anteile der anderen Bundesländer, insbesondere der Steiermark (21,6\%), Oberösterreich $(10,9 \%)$, Niederösterreich $(10,6 \%)$ und Kärnten $(6,8 \%)$ sind vergleichsweise höher.

\subsubsection{Die Umsetzung von Horizon 2020 in Österreich}

Die Federführung der Koordination der österreichischen EU-FTI-Politik obliegt gemäß Bundesministeriengesetz dem Bundesministerium für Bildung, Wissenschaft und Forschung. Die Umsetzung von Horizon 2020 in Österreich erfolgt im Wesentlichen im Rahmen von Aktivitäten und Maßnahmen, die in folgenden drei Bereichen gesetzt werden:41

1. Beratung und Betreuung von Horizon 2020 und ERA im Bereich „Europäische und internationale Programme" der FFG (FFG-EIP)
2. EU-Performance Monitoring (FFG)

3. Das ERA-Observatorium Austria als übergeordneter Rahmen für die Integration der einzelnen Aufgabenstellungen im Bereich der Governance der EU-FTI-Politik in Österreich.

\section{Beratung und Betreuung von Horizon 2020 und ERA im Bereich „Europäische und internationale Programme" der FFG}

Die Aufgabe des Bereiches „Europäische und internationale Programme" der FFG (FFG-EIP) ist es, die Beteiligung Österreichs an Programmen, Initiativen und Aktionen der europäischen und internationalen Forschungs- und Technologiekooperation - insbesondere am EU-Forschungsprogramm - nachhaltig zu stärken. ${ }^{42}$ Den Rahmen dafür bildet eine Beauftragung der FFG durch den Bund, vertreten durch mehrere Ministerien und durch die WKO für den Zeitraum 2014-2020. Beauftragt sind insbesondere diverse Informations-, Beratungs- und Vernetzungsleistungen, die allen österreichischen Interessentinnen und Interessenten sowie Teilnehmenden an europäischen Forschungsinitiativen zugutekommen. Der Bereich „Europäische und internationale Programme" der FFG informiert regelmäßig über 30.000 österreichische Interessentinnen und Interessenten und Teilnehmende an europäischen Forschungsinitiativen zu europäischen Programmen und Initiativen. Pro Jahr werden durchschnittlich 6.500 Beratungen zu den europäischen FTI-Programmen durchgeführt.

Neben Horizon 2020 werden im FFG-EIP, teilweise zusammen mit Partnern ${ }^{43}$, auch EUREKA, Eurostars, COSME, das Enterprise Europe Network, COST und eine Vielzahl von europäischen FTI-Partnerschaftsinitiativen ${ }^{44}$ betreut. Das Servicespektrum für Unternehmen, Universitäten, Forschungseinrichtungen etc. reicht von der projektspezifischen Betreuung bis zur

41 Vgl. Biegelbauer et al. (2018).

42 Vgl. https://www.ffg.at/ffg/eip

43 Vgl. z.B. aws, WKO, Wirtschaftskammern der Bundesländer, Bundesländeragenturen.

44 Der europäische Forschungsraum umfasst neben Horizon 2020 weitere Beteiligungsmöglichkeiten, wie z.B. Joint Programming Initiativen (JPI), Future and Emerging Technology (FET) Flagships, Gemeinsame Technologieinitiativen (JTI), Public-Private-Partnerships (PPP-Initiativen) oder auch die Europäische Technologieplattformen (ETP). Vgl. hierzu auch: https://www.ffg.at/Europa/ partnership_initiativen 
Tabelle 1-3: Informations- und Beratungsinstrumente des FFG-EIP nach Aufgabenbereichen

\begin{tabular}{|c|c|}
\hline Aufgabenbereich & Instrumente \\
\hline $\begin{array}{l}\text { Bewusstseinsbildung und } \\
\text { Informationsvermittlung }\end{array}$ & $\begin{array}{l}\text { 1. FFG-EIP Website: beinhaltet allgemeine Informationen über die Gesamtstruktur des europäi- } \\
\text { schen Programmportfolios sowie Förderangebote für alle Zielgruppen. Zusätzlich bieten spezifische } \\
\text { Seiten gezielte Unterstützung bei der Implementierung von H2O2O-Projekten (z.B. rechtliche und } \\
\text { finanzielle Aspekte). } \\
\text { 2. eNewsletter: Zusammenschau über nationale und europäische Nachrichten } \\
\text { 3. „Awareness“ und Informationsveranstaltungen: Informationen zum H2O2O-Programmportfolio } \\
\text { und der zugehörigen „Programmlogik (Veranstaltungen zum Start von Ausschreibungsrunden, } \\
\text { Roadshows, Kooperationsbörsen etc.) }\end{array}$ \\
\hline $\begin{array}{l}\text { Programm- und } \\
\text { Projektberatung }\end{array}$ & $\begin{array}{l}\text { 4. Persönliche Beratung \& Proposal Checks für potenzielle Teilnehmende aus Wissenschaft und } \\
\text { Wirtschaft werden in allen Projektphasen angeboten. An Universitäten und Organisationen mit ei- } \\
\text { genen „Unterstützungsstrukturen“ werden Forschende und Multiplikatoren im Rahmen des Second- } \\
\text { level Supports bei programmspezifischen Fragen beraten. Multiplikatoren/Intermediäre werden } \\
\text { durch den Einsatz unterschiedlicher Instrumente aus der Toolbox befähigt, den First-level Support } \\
\text { sukzessive eigenständig wahrzunehmen. } \\
\text { 5. FFG Akademie: Im Rahmen der FFG Akademie werden Trainingsveranstaltungen und Webinare } \\
\text { des EIP zwecks Informationsvermittlung und Wissensaufbaus gebündelt. Ein besonderer Schwer- } \\
\text { punkt liegt auf Projektentwicklung, Antragstellung sowie dem Management von H2020-Projekten. } \\
\text { Diese werden ergänzt durch das Format EU-Networking zur besseren Vernetzung der österreichi- } \\
\text { schen FTI-Akteure mit europäischen Expertinnen und Experten. Diskussionsrunden im Rahmen des } \\
\text { EU-Networkings beschäftigen sich mit aktuellen Entwicklungen der europäischen Forschungspolitik } \\
\text { und Forschungsförderung. }\end{array}$ \\
\hline $\begin{array}{l}\text { Strategische Beratung und ERA } \\
\text { Orientierungswissen }\end{array}$ & $\begin{array}{l}\text { 6. ERA-Themendossiers/Analytische Programmberichte: Diese analysieren die österreichische } \\
\text { Beteiligung in bestimmten Themen (H2O2O und europäische FTI-Partnerschaftsinitiativen) und } \\
\text { zeigen wichtige Entwicklungen und Handlungsfelder auf. Quantitative Daten liefert das EU-Perfor- } \\
\text { mance-Monitoring. Die Hauptzielgruppe sind Delegierte und Policymaker, angepasst werden diese } \\
\text { Informationen auch öffentlich zur Verfügung gestellt. } \\
\text { 7. Policy Briefs: „In_brief“ sind anlassbezogene Policy Briefs über aktuelle Entwicklungen mit stra- } \\
\text { tegischer und/oder operativer Relevanz für die österreichische FTI-Community. } \\
\text { 8. Analysen: Analysen von Evaluierungsergebnissen sowie Netzwerk- und Beteiligungsanalysen } \\
\text { (ex ante und ex post) gewinnen an Bedeutung. Netzwerk- und Beteiligungsanalysen werden unter } \\
\text { Einbeziehung der Auftraggeberinnen und Auftraggeber, des EU-Performance-Monitorings und den } \\
\text { „themenverantwortlichen Akteuren“ in den Ressorts durchgeführt. }\end{array}$ \\
\hline
\end{tabular}

Quelle: Biegelbauer et al. (2018, 10f.), adaptierte Darstellung.

gezielten Unterstützung in der strategischen Positionierung im europäischen Forschungs- und Innovationsumfeld, wobei ein immer stärkeres Augenmerk auf eine systemorientierte Unterstützung und eine Selbstbefähigung der Akteure gelegt wird.

Tabelle 1-3 gibt einen Überblick über die Informations- und Beratungsinstrumente des FFG-EIP.

Darüber hinaus ist FFG-EIP die offiziell nominierte Nationale Kontaktstelle (NCP) der europäischen Kommission für Horizon 2020 und alle europäischen FTI-Partnerschaftsinitiativen, die in Verbindung mit Horizon 2020 und ERA stehen.

\section{EU-Performance Monitoring (FFG)}

Zentrale Aufgabe des EU-Performance Monitorings (EU-PM) ist die Erfassung, Verarbeitung, Aufbereitung und Kommunikation von Daten über die Beteili- gung österreichischer Organisationen an den EUProgrammen für Forschung und Innovation. ${ }^{45}$ Durch die Beauftragung des EU-PM an die FFG werden nationale und EU-Beteiligungsdaten unter einem Dach gebündelt. Durch die synergetische Nutzung von inhaltlichem und technischem Know-how, basierend auf der fachlichen Expertise der FFG, soll so "strategische Intelligenz" für FTI-Akteure geboten werden.

Neben der Datenbereitstellung, also der Sammlung, Aufbereitung, Auswertung und Kommunikation zuverlässiger Daten über die Teilnahme Österreichs in relevanten Förderprogrammen der EU und der Integration von Informationen aus verfügbaren Datenquellen in eine umfassende Datenbasis, werden auch spezifische Produkte erstellt. Diese umfassen u.a. regelmäßige periodische Auswertungen und adhoc Auswertungen auf Anfrage, qualitative und 
quantitative Analysen und Interpretationen über die EU-FTI-Performance Österreichs, eine inhaltliche Zusammenschau von EU-Daten mit relevanten nationalen Förderdaten der FFG und den Betrieb eines Internetportals.

Ziele dieser Aktivitäten sind im Wesentlichen ein kontinuierliches Monitoring des österreichischen Abschneidens in den EU-Forschungsprogrammen, der Vergleich mit anderen Ländern (internationale Positionierung), die Verteilung innerhalb Österreichs, die Analyse von Stärken und Schwächen in einzelnen Bereichen ( $z$.B. nach Themen, Organisationen und Branchen) sowie die Unterstützung für strategische und politische Weichenstellungen.

\section{Das ERA-Observatorium Austria}

Das ERA Observatorium umfasst eine Reihe von Aktivitäten, die den Informationsaustausch und die bessere Abstimmung zwischen jenen Akteuren ermöglichen sollen, die eine wesentliche Rolle bei der Formulierung und Vermittlung österreichischer Positionen in Bezug auf die europäische FTI-Politik spielen. ${ }^{46}$ Das übergeordnete Ziel der Instrumente des ERA-Observatoriums ist die Unterstützung der eigenverantwortlichen Entscheidungsfindung insbesondere von politiknahen FTI-Akteuren im EU-Kontext. Konkret verfolgt das ERA-Observatorium diese Zielsetzungen:

1. Bereitstellung von Informationen und Austauschmöglichkeiten über relevante EU-Politiken:

a. Über das ERA Portal Austria ${ }^{47}$ stellt das BMBWF Informationen zu Entwicklungen im Europäischen Forschungsraum, gemeinsamen Aktivitäten (z.B. zu den europäischen FTI-Partnerschaftsinitiativen) und zum österreichischen Forschungssystem zur Verfügung.

b. Mit dem Veranstaltungsformat „Europa Forum Forschung" (EFF) wird die FTI-Community in Österreich mehrmals pro Jahr mit aktuellen EU FTI-Themen adressiert.
2. Bereitstellung einer bedarfsgerechten Beratung und Betreuung der verschiedenen ministeriellen Ressorts für H2O2O:

a. EIP-Beauftragung (siehe oben)

b. Lenkungsausschuss FFG-EIP

c. Thematische H2O2O Expertinnen- und Expertengruppen: In mehreren Themengebieten arbeiten österreichische Delegierte mit Delegierten anderer Länder zusammen und bilden Allianzen (Programmausschüsse von H2020; dabei werden sie von nationalen thematischen Gruppen unterstützt).

d. Delegierten-Roundtable: Delegierte treffen sich durchschnittlich einmal im Quartal zu den H2020 Programmkomitees und tauschen Informationen über ihre Arbeit aus.

e. Bereitstellung einer strategischen Beratung der Politik (ERA Council Forum Austria) und von Prozessen (ERA-Roundtable, Arbeitsgruppen zu EIT, JPI etc.)

f. Das ERA Council Forum Austria ist ein Gremium bestehend aus nationalen und internationalen Expertinnen und Experten, das den österreichischen Minister für Bildung, Wissenschaft und Forschung an der Schnittstelle zwischen der europäischen Forschungspolitik und dem nationalen Wissenschafts-, Forschungs- und Innovationssystem berät. Es bietet strategische Orientierung und Beratung im Kontext von „Europa 2020“, „Innovationsunion“, „ERA-Partnerschaft" sowie „H2020“ an. Das Forum von fünf Mitgliedern wird von der ehemaligen Präsidentin des ERC, Helga Nowotny, geleitet und tritt mehrmals pro Jahr zusammen. Im Rahmen der jährlichen Europatagung hat das ERA Council Forum eine zentrale Rolle in der Diskussion aktueller Entwicklungen in Bezug auf die Ziele des Europäischen Forschungsraumes.

g. Bei den ERA-Roundtables werden Entwicklungen im europäischen Forschungsraum disku-

$46 \mathrm{Vgl}$. https://era.gv.at/directory/166

47 Ebenda. 
tiert sowie Beratungen zu nationalen Maßnahmen im Kontext der Realisierung des Europäischen Forschungsraums (ERA) in den sechs Prioritäten vorgenommen: Effektivere nationale Forschungssysteme (ERA-Priorität 1), länderübergreifende Zusammenarbeit (ERA-Priorität 2), ein offener Arbeitsmarkt für Forscherinnen und Forscher (ERA-Priorität 3), Gleichstellungsaspekte (EAR-Priorität 4), Wissenstransfer/Open Access (ERA-Priorität 5), sowie Internationale Zusammenarbeit (ERA-Priorität 6). Am ERARoundtable nehmen die Akteure teil, die für die nationale Umsetzung der ERA-Prioritäten zuständig sind.

h. Die Arbeitsgruppen zum European Institute of Technology (EIT) und JPIs dienen primär der Abstimmung ministerieller Positionen zu diesen europäischen Instrumenten. Die Teilnehmerinnen und Teilnehmer setzen sich dementsprechend in erster Linie aus Ministerien, den Agenturen FFG und FWF, den Interessenvertretungen WKO und teils auch IV, sowie der uniko und den unmittelbar mit EIT und JPIs beauftragten Organisationen zusammen.

3. Bereitstellung eines wirkungsorientierten Monitorings der österreichischen Teilnahme an H2O2O und ERA über das EU-Performance Monitoring und das ERA Reporting Board.

\subsubsection{Das neue Rahmenprogramm gemäß Vorschlag der Europäischen Kommission}

Das neunte europäische Forschungsrahmenprogramm „Horizon Europe" beginnt voraussichtlich am 01.01.2021 und hat eine Laufzeit bis 31.12.2027. Bei der Ausgestaltung des Programms wurden verschiedene Inputs, insbesondere die Ergebnisse der Interimsevaluation von Horizon 2020, Stakeholder-Konsultationen, wie auch externe Expertise ${ }^{48}$ berücksichtigt. Für Forschung und Innovation werden im mehrjährigen Finanzrahmen insgesamt rd. 100 Mrd. € seitens der Europäischen Kommission zur Verfügung gestellt, davon 97,6 Mrd. € für das Rahmenprogramm (inklusive 3,5 Mrd. € für den Fonds InvestEU) und

Tabelle 1-4: Übersicht über den Aufbau von Horizon Europe gemäß dem Vorschlag der Europäischen Kommission

\begin{tabular}{|c|c|c|}
\hline $\begin{array}{c}\text { Säule I } \\
\text { Offene Wissenschaft } \\
(25,8 \text { Mrd. } €)\end{array}$ & $\begin{array}{c}\text { Säule II } \\
\text { Globale Herausforderungen und } \\
\text { industrielle Wettbewerbsfähigkeit } \\
(52,7 \text { Mrd. } €)\end{array}$ & $\begin{array}{c}\text { Säule III } \\
\text { Offene Innovation } \\
(13,5 \text { Mrd. } €)\end{array}$ \\
\hline Europäischer Forschungsrat $-16,6$ Mrd. $€$ & $\begin{array}{l}\text { Cluster: } \\
\text { - Gesundheit } \\
\text { - Kultur, Kreativität und inklusive } \\
\text { Gesellschaft } \\
\text { - Zivile Sicherheit für die Gesellschaft } \\
\text { - Digitalisierung, Industrie und Weltraum } \\
\text { - Klima, Energie und Mobilität } \\
\text { - Lebensmittel, Bioökonomie, natürliche } \\
\text { Ressourcen, Landwirtschaft und Umwelt }\end{array}$ & Europäischer Innovationsrat - 10 Mrd. $€$ \\
\hline $\begin{array}{l}\text { Marie-Skłodowska-Curie-Maßnahmen - } \\
\text { 6,8 Mrd. € }\end{array}$ & $\begin{array}{l}\text { Gemeinsame Forschungsstelle - } \\
2,2 \text { Mrd. } €\end{array}$ & $\begin{array}{l}\text { Europäische Innovationsökosysteme } \\
-0,5 \text { Mrd. } €\end{array}$ \\
\hline Infrastrukturen - 2,4 Mrd. € & & $\begin{array}{l}\text { Europäisches Institut für Innovation und } \\
\text { Technologie- } 3 \text { Mrd. } €\end{array}$ \\
\hline \multicolumn{3}{|c|}{ Stärkung des Europäischen Forschungsraums (2,1 Mrd. €) } \\
\hline \multicolumn{3}{|c|}{ Teilen von Exzellenz - 1,7 Mrd. $€$} \\
\hline \multicolumn{3}{|c|}{ Reformierung und Stärkung des europäischen F\&I-Systems - 0,4 Mrd. $€$} \\
\hline
\end{tabular}

Quelle: Rat der Europäischen Union (2019).

48 Insbesondere berücksichtigt wurden die Empfehlungen der HLG-Berichte „LAB-FAB-APP“ (Europäische Kommission, 2017), „Europe is back: Accelerating breakthrough innovation“ (Europäische Kommission, 2018c), sowie der Bericht von Mariana Mazzucato (2018) „Mission-Oriented Research \& Innovation in the European Union“. 
2,4 Mrd. € für Euratom vorgeschlagen. Diese Budgetbeträge sind allerdings vorläufig, da dem geplanten Finanzrahmen noch alle Mitgliedstaaten (im Rat der EU einstimmig) sowie das EU-Parlament (mehrheitlich) zustimmen müssen. Tabelle 1-4 gibt einen Überblick über die von der Europäischen Kommission vorgeschlagene Struktur von Horizon Europe und der jeweiligen budgetären Ausstattung der Programmteile.

Die erste Säule "Offene Wissenschaft" umfasst die bereits in Horizon 2020 implementierten Programmteile „Europäischer Forschungsrat", „MarieSkłodowska-Curie-Maßnahmen" und "Infrastrukturen" und soll die wissenschaftliche Basis der Union erweitern und stärken. Die Bezeichnung "Offene Wissenschaft" weist zudem auf die stärkere Orientierung des europäischen Rahmenprogramms zu einer "open-science-policy“ hin. Generell soll Forschung unter Horizon Europe für die Gesellschaft zugänglicher gestaltet und die Dissemination sowie die Nutzung von F\&I-Ergebnissen gestärkt werden. Neben den Publikationen aus Forschungsprojekten sollen zunehmend auch Forschungsdaten öffentlich zugänglich gemacht bzw. deren Veröffentlichung angestrebt werden. ${ }^{49}$

Die zweite Säule „Globale Herausforderungen und industrielle Wettbewerbsfähigkeit Europas" umfasst fünf themenspezifische Cluster zur Bewältigung der großen globalen Herausforderungen, die wiederum in Interventionsbereiche („areas of intervention") ${ }^{50}$ unterteilt sind. Säule II soll die Verbreitung von qualitativ hochwertigem neuem Wissen, neuer Technologien und nachhaltiger Lösungen unterstützen. Weiters soll sie die Wettbewerbsfähigkeit der europäischen Industrie und die Wirkung von Forschung und Innovation auf die Entwicklung stärken. Außerdem soll Säule II innovative Lösungen in der Industrie (insbeson- dere in KMU und Start-ups) und der Gesellschaft zur Bewältigung globaler Herausforderungen unterstützen. Als Instrumente eingesetzt werden Calls, EUweite F\&I-Missionen („R\&I Missions“) und EU FTI-Partnerschaften. EU-weite F\&I-Missionen stellen ein neues Instrument im Rahmenprogramm dar. Sie umfassen ein Bündel an Maßnahmen, sollen sich durch hohe Sichtbarkeit auszeichnen und Ziele von hoher gesellschaftlicher Relevanz adressieren. Forschung und Innovation sollen im Rahmen dieser Missionen verstärkt auf die Gesellschaft und die Bedürfnisse der europäischen Bürgerinnen und Bürger Bezug nehmen. Folgende Bereiche für Missionen wurden definiert: (1) Anpassung an den Klimawandel, inkl. gesellschaftliche Transformation, (2) Krebs, (3) gesunde Ozeane, Meere, Küsten- und Binnengewässer, (4) klimaneutrale und „smarte" Städte, (5) Bodengesundheit und Lebensmittel ${ }^{51}$.

Umgesetzt werden diese Aufträge unter Beteiligung von Bürgerinnen und Bürgern, diversen Stakeholdern, dem Europäischen Parlament und den Mitgliedsstaaten. Die Auswirkungen dieser Missionen auf Wissenschaft, Technologie, und Gesellschaft sollen dabei über das Wirkungspotenzial von Einzelmaßnahmen hinausgehen. ${ }^{52}$ Europäische FTI-Partnerschaften sollen künftig nur dann eingerichtet werden, wenn die geplanten Wirkungen nicht durch andere Maßnahmen des europäischen Rahmenprogramms oder durch nationale Aktivitäten alleine erreicht werden können. Sie sollen grundsätzlich zeitlich begrenzt werden. Schließlich ist die Gemeinsame Forschungsstelle (JRC) ebenfalls der zweiten Säule zugeordnet. Die gemeinsame Forschungsstelle ist der wissenschaftliche Dienst der EU-Kommission und unterstützt die europäische Politik mit unabhängigen wissenschaftlichen Erkenntnissen, Expertisen und

49 "Open access to research data shall be ensured in line with the principle 'as open as possible, as closed as necessary" , siehe Artikel 10(1) des „Proposal for a Regulation of the European Parliament and of the Council establishing Horizon Europe - the Framework Programme for Research and Innovation, laying down its rules for participation and dissemination" (Europäische Kommission, 2018e).

50 Beispiele hierfür sind: „Artificial Intelligence and Robotics“, "Clean Transport and Mobility“, "Cultural Heritage“ etc.

51 Vgl. Rat der Europäischen Union (2019).

52 Mariana Mazzucato (2018) nennt mitunter folgende fiktive Beispiele für solche Missionen: „100 carbon neutral cities by 2030 , „A plastic-free ocean“, und „Decreasing the burden of dementia“. 
technischer Unterstützung während des gesamten Politikzyklus. ${ }^{53}$

Eingegliedert in die dritte Säule „Offene Innovation", die sich an innovationstreibende Akteure aus allen Wirtschaftsbereichen richtet, sind der Europäische Innovationsrat, die Europäischen Innovationsökosysteme und das Europäische Institut für Innovation und Technologie. Der Europäische Innovationsrat soll marktorientierte, potenziell disruptive und mit hohem Risiko behaftete Innovationen unterstützen, wozu ihm zwei themenoffene, komplementäre Programme zur Verfügung stehen werden: Das Programm „Pathfinder" unterstützt hochrisikoreiche Projekte zur Entwicklung radikal innovativer Technologien und ist auf die Frühphase des Innovationszyklus ausgerichtet (Ideenphase bis Prototypenentwicklung). Der Fokus des zweiten Programms, "Accelerator", liegt auf der Markteinführungsphase von Innovationen bzw. der Scale-up-Phase von Startups und soll so die wirtschaftliche Verwertung von Innovationsleistungen unterstützen. Die Interventionen richten sich insbesondere an Startups und KMU. Der Europäische Innovationsrat setzt damit im Rahmen von Horizon Europe zwei europaweite bottom-up Innovationsförderprogramme auf. Die Europäischen Innovationsökosysteme dienen hingegen der Vernetzung von Innovationsakteuren auf regionaler und nationaler Ebene, während das Europäische Institut für Innovation und Technologie (EIT) die Kooperation unterschiedlicher Akteure aus Forschung, Bildung und Wirtschaft unterstützt - mit dem Ziel, Innovationen und Entrepreneurship in der EU zu stärken.

Außerhalb der Säulenstruktur von Horizon Europe sollen weitere finanzielle Mittel (rd. 2,1 Mrd. €) zur Stärkung des Europäischen Forschungsraumes zur Verfügung gestellt werden, die sich in die Bereiche
„Teilen von Exzellenz" und „Reformierung und Stärkung des europäischen F\&I-Systems" unterteilen. Bei „Teilen von Exzellenz“ sollen Mitgliedsstaaten und Regionen, die im FTI-Bereich strukturell schwächer sind, gezielt in Richtung des Aufbaus von Exzellenz unterstützt werden (Teaming, Twinning, Fortführung von COST). Im Rahmen der „Reformierung und Stärkung des europäischen F\&I-Systems" sind ForesightAktivitäten, das Monitoring sowie die Evaluierung von Horizon Europe geplant.

Darüber hinaus strebt Horizon Europe Synergien mit einer Reihe weiterer EU-Programme an. ${ }^{54}$ Erfolgreich evaluierte Projekte können ein „Exzellenzsiegel“ („Seal of Excellence Certificate“) erhalten, wodurch diese im Rahmen anderer Programme (unter anderem etwa durch den Europäischen Fonds für regionale Entwicklung - EFRE) gefördert werden sollen. Verknüpfungen zu anderen EU-Programmen ${ }^{55}$ sollen zudem gefördert, Förderrichtlinien zwischen den Programmen vereinheitlicht, flexible Kofinanzierungsregeln erstellt und Ressourcen auf EU-Ebene gebündelt werden. Die Offenheit des Programms für internationale Partnerschaften mit Organisationen aus Drittstaaten (EFTA-Mitgliedstaaten, Beitrittskandidaten, ENP-Staaten) soll weiterhin erhalten bleiben. Darüberhinausgehend sollen die Kooperationsmöglichkeiten mit weiteren Drittstaaten ermöglicht werden, sofern diese bestimmte Kriterien ${ }^{56}$ erfüllen.

Die Verhandlungen über den Vorschlag der Europäischen Kommission wurden zwischen Rat und Europäischem Parlament im April 2019 vorläufig abgeschlossen, es konnte eine vorläufige Einigung über die Inhalte des Programmes erzielt werden. Eine genaue Analyse und Darstellung des endgültigen Rahmenprogramms kann erst im Lichte der noch ausstehenden Budgetverhandlungen über den mehrjähri-

53 Vgl. https://ec.europa.eu/info/departments/joint-research-centre_de

54 Die angestrebten Synergien sind beschrieben im Annex IV des „Proposal for a Regulation of the European Parliament and of the Council establishing Horizon Europe - the Framework Programme for Research and Innovation, laying down its rules for participation and dissemination" (Europäische Kommission, 2018e).

55 Wie beispielsweise: COSME, European Space Programme, Digitale Europe Programme und European Defence Fund.

56 Für diese Kriterien siehe Artikel 12 des „Proposal for a Regulation of the European Parliament and of the Council establishing Horizon Europe - the Framework Programme for Research and Innovation, laying down its rules for participation and dissemination“ (Europäische Kommission, 2018e). 
gen Finanzrahmen der EU erfolgen. Außerdem müssen ergänzende Teile des Rahmenprogramms unter finnischer EU-Präsidentschaft verhandelt werden (z.B. Synergien mit anderen EU-Programmen, Drittstaatenbeteiligung).

\subsubsection{Resümee}

Im Bereich der FTI-Politik konnte Österreich als Vorsitzland des Rats der Europäischen Union trotz schwieriger Rahmenbedingungen und unter groBem zeitlichem Druck einige Erfolge verzeichnen. Überschattet von den laufenden Brexit-Verhandlungen und - damit zusammenhängend - ohne einen vereinbarten mehrjährigen Finanzrahmen der Europäischen Union für den Zeitraum 2021-2027 konnte auf Basis intensiver Abstimmungen und Verhandlungen in einer Rekordzeit von nur fünf Monaten eine Einigung über die wesentlichen Inhalte der Horizon Europe-Verordnung erzielt werden. Diese wurde am 30.11.2018 im Rat für Wettbewerbsfähigkeit durch die Annahme einer sogenannten „partiellen allgemeinen Ausrichtung “ beschlossen. Dem Vorschlag der Europäischen Kommission zufolge soll von 2021-2027 ein Betrag von ca. 100 Mrd. $€$ für Horizon Europe zur Verfügung stehen. Schwerpunkte von Horizon Europe werden wieder die Unterstützung von exzellenter Forschung, Mobilität und Ausbildung von Forscherinnen und Forschern sowie Forschungsinfrastrukturen sein. Die großen gesellschaftlichen und wirtschaftlichen Herausforderungen sollen in Zukunft in themenübergreifenden Clustern angegangen und unter anderem durch die sogenannten "Missionen“ zu spezifischen Problemstellungen umgesetzt werden. Eine weitere Neuerung wird der „Europäische Innovationsrat" sein, der bahnbrechende Innovationen (wie z.B. durch "Blended Finance“ = Kombination von $\mathrm{Zu}$ schuss- und Eigenkapital-Elementen für rasch wachsende Unternehmen mit Breakthrough-Innovationen) fördern soll.

Die zweite Priorität im Rahmen des österreichischen Vorsitzes im europäischen Rat im Bereich von
Wissenschaft, Forschung und Innovation betraf den Europäischen Forschungsraum (ERA). Diesbezüglich hat der Rat am 30.11.2018 einstimmig Schlussfolgerungen zum Europäischen Forschungsraum angenommen und verabschiedet. Diese wurden Anfang Oktober 2018 in der Arbeitsgruppe Forschung erfolgreich verhandelt. Die Schlussfolgerungen anerkennen wichtige Fortschritte bei der Umsetzung der ERAAgenda, beinhalten Konsequenzen aus der Überprüfung der ERA-Beratungsstrukturen und bieten einen Ausblick auf die Zukunft des ERA. Die Schlussfolgerungen sollen vor allem den Weg für eine überarbeitete Reformagenda für den Europäischen Forschungsraum im Jahr 2020 ebnen.

Das Europäische Forschungsrahmenprogramm war und ist für Österreich eine wichtige Quelle für die kompetitive Forschungsförderung auf höchstem Niveau. Die aktuellen Daten der Europäischen Kommission bestätigen den guten Erfolg österreichischer Institutionen und von Forscherinnen und Forschern aus Industrie und Wissenschaft im laufenden achten europäischen Forschungsrahmenprogramm Horizon 2020. Bislang konnten aus Horizon 2020 mehr als eine Milliarde Euro erfolgreich eingeworben werden. In Österreich ansässige Institutionen bzw. Forscherinnen und Forscher nahmen dabei durchschnittlich an $8,82 \%$ aller 21.472 Projekte, die im Rahmen von Horizon 2020 gefördert wurden, teil. Gemessen an der Anzahl der Beteiligungen liegt Österreich im internationalen Vergleich an der neunten Stelle. Mit einer Erfolgsrate von $17,87 \%$ liegt Österreich deutlich über der durchschnittlichen Horizon 2020-Erfolgsrate von $15,32 \%$ und nach Belgien an zweiter Stelle unter den Mitgliedstaaten der Europäischen Union.

Aus budgetärer Sicht sind die großen Programmbereiche („Säulen“) "Gesellschaftliche Herausforderungen", "Wissenschaftsexzellenz" und "Führende Rolle der Industrie" am bedeutendsten. Im Unterschied zu anderen EU-Mitgliedsländern konnte Österreich das meiste Budget aus der Säule 3 „Gesellschaftliche Herausforderungen" einwerben. Als österreichische Stärkefelder in dieser Säule können im europäischen Vergleich die thematischen Cluster 
„intelligenter, umweltfreundlicher und integrierter Verkehr", "integrative, innovative und reflexive Gesellschaften" und "sichere, saubere und effiziente Energie" angesehen werden. Innerhalb der Säule "Führende Rolle der Industrie" sind die thematischen Cluster "Materialien", "IKT" sowie "Biotechnologie“ ausgewiesene österreichische Stärkefelder.

Insgesamt stellt der in Österreich ansässige Unternehmenssektor die meisten Beteiligungen in Horizon 2020 (37,99\%). Davon sind fast zwei Drittel KMU. Danach folgen der Hochschulsektor (27,85\%) und der außeruniversitäre Forschungsbereich (22,61\%).

Die professionell agierenden Beratungs- und Betreuungsstrukturen tragen durch ihre Arbeit zum erfolgreichen Abschneiden in Horizon 2020 bei. Hierbei ist insbesondere der Bereich „Europäische und internationale Programme" der FFG (FFG-EIP) zu nennen. Den Rahmen dafür bildet eine Beauftragung der FFG durch den Bund, vertreten durch mehrere Ministerien und durch die WKO für den Zeitraum 2014-2020. Pro Jahr werden durchschnittlich 6.500 Beratungen zu europäischen FTI-Programmen durchgeführt.

\subsection{Strategische Maßnahmen, Initiativen und Weiterentwicklungen}

Zur Erreichung der Ziele der FTI-Strategie aus dem Jahr 2011 wurde sowohl auf Ebene der Bundesregierung als auch auf Ressortebene eine Vielzahl an strategischen Maßnahmen und Initiativen gestaltet und weiterentwickelt. Die zur Konkretisierung und Koordination der Umsetzung eingerichtete Task Force FTI, die aus Vertreterinnen und Vertretern der relevanten Ressorts besteht (BMF, BMBWF, BMVIT, BMDW, Vorsitz: BKA), führte auch 2018 ihre Tätigkeiten fort. Im Folgenden werden die aktuellsten Entwicklungen bei strategischen Prozessen, FTI-relevanten Maßnahmen sowie bei der Umsetzung neuer Projekte und Programme überblicksmäßig dargestellt.

\section{Zukunftsoffensive für Forschung, Technologie} und Innovation

Basierend auf dem Regierungsprogramm hat die Bundesregierung mit dem Ministerratsvortrag 25/63 „Zukunftsoffensive für Forschung, Technologie und Innovation" vom August 2018 die Umsetzung von folgenden Maßnahmen im Bereich Forschung, Technologie und Innovation beschlossen:

- Aktualisierung der Forschungsstrategie (FTI-Strategie)

- Initiative zur Stärkung und Weiterentwicklung der kompetitiven Grundlagenforschung in Österreich zur Förderung der Spitzenforschung (Exzellenz)

- Forschungsfinanzierungsgesetz - Pakt für Forschung

- Zusammenlegung des Rates für Forschung und Technologieentwicklung (RFTE), des Wissenschaftsrates und des ERA Council Forums als Beratungsgremium der Bundesregierung ergänzt um volkswirtschaftliche Kompetenz

- Umsetzung einer Forschungsförderungsdatenbank

Erste Ergebnisse werden im Rahmen des FTI-Gipfels 2019 präsentiert.

\section{Umsetzung von ausgewählten Teilstrategien}

\section{Umsetzung Open Innovation Strategie für Österreich}

Österreich hat im Juli 2016 als erster und bisher einziger EU-Mitgliedsstaat eine umfassende nationale "Open Innovation Strategie" (Ol-Strategie) beschlossen. ${ }^{57}$ Seitdem wurden seitens der mit der Umsetzung betrauten Ministerien BMVIT und BMBWF sowie Stakeholdern auf Bundes-, Landes- und Gemeindeebene bereits eine Vielzahl an Aktivitäten und Maßnahmen gesetzt. Im Folgenden werden exemplarisch aktuelle Umsetzungsbeispiele im Berichtsjahr 2018 aufgezeigt.

Das BMVIT setzt auf thematisch unterschiedlich ausgerichtete Innovationslabore und vor allem Test-

57 Vgl. http://openinnovation.gv.at/wp-content/uploads/2016/08/Open-Innovation-barrierefrei.pdf 
umgebungen, die eine breite Basis zur Wissensgenerierung unter Einbeziehung von Stakeholdern ermöglichen. So werden Testumgebungen für die Funktion und den Gebrauch von Drohnen und zum automatisierten Fahren forciert. Um die wichtigen Bereiche der Infrastruktur bestmöglich zu koordinieren und zu schützen, wird seit 2018 die Nutzung von Ol-Methoden auch im Bereich Cyber Security forciert. Gemeinsam mit der Digitalisierungsagentur plant das BMVIT weiters eine "Bundesländer-Road Show" mit Erklärungsmethoden zu 3D-Druck, Breitbandausbau und Drohnen. Außerdem hält das BMVIT weiterhin am überaus erfolgreichen Programm „Massiv Open Online Courses", an dem monatlich durchschnittlich 20.000 User teilnehmen, fest.

Mit der öffentlichen Forschungsinfrastruktur-Datenbank trägt das BMBWF zum Aufbau und Betrieb einer Innovationslandkarte maßgeblich bei (siehe ausführlicher Abschnitt 3.3.1, 3.3.5 und 4.4.6)

Weiters wurden seitens des BMBWF mit dem Future Learning Lab der PH Wien gemeinsam mit erfahrenen Pädagoginnen und Pädagogen im Sommersemester 2018 Lernsequenzen entwickelt, die das MINT-Interesse und den Gestaltungswillen junger Menschen in allen Schulformen der Sekundarstufe fördern sowie Verständnis für Industrie 4.0-Prozesse schaffen sollen. Anschließend wurden Lehrpersonen von $\mathrm{rd}$. 20 Schulen mit den erstellten Lernsequenzen vertraut gemacht und im Einsatz der bereitgestellten 3D-Drucker geschult. Die bereitgehaltenen Unterrichtssequenzen werden darüber hinaus als Open Content unter CC BY Lizenz in einem entsprechend gestalteten Wiki zur Verfügung gestellt.

Auch andere Ressorts tragen maßgeblich zur erfolgreichen Implementierung der Maßnahmen der OIStrategie bei: BMDW und BMVIT steuern in bewährter Form über die gemeinsam getragene Initiative „Innovationsfördernde Öffentliche Beschaffung“ (IÖB) mittels Matchmaking-Plattform, Crowdsourcing-Challenges und Community Management einen wesentlichen Beitrag zur Anwendung von Ol-Methoden in der öffentlichen Verwaltung bei. ${ }^{58}$

Das BMEIA unterstützt das Monitoring der OlStrategie mit jährlichen internationalen Statusberichten zur Entwicklung von Ol in anderen Staaten. Diese weisen auch für das Berichtsjahr 2018 auf ein tendenziell gestiegenes Interesse an OI in verschiedenen Staaten hin (z.B. Estland, Norwegen, Schweden, Slowakei, Südafrika, USA); allerdings hat bis jetzt neben Österreich kein zweiter Staat eine explizite Open Innovation Strategie entwickelt.

Besonders erfreulich ist auch die verstärkte Einbindung der Bundesländer, die sich mitunter durch die erstmalige Abhaltung eines Ol-Workshops in Salzburg manifestierte: Im Juni 2018 wurde erstmals gemeinsam von der ITG Salzburg, den beiden Ressorts BMVIT und BMBWF sowie dem Land Salzburg ein regionaler Open Innovation Stakeholderworkshop mit dem Titel "Open Innovation Praxistag Salzburg“ durchgeführt, der vor allem regionale Good PracticeBeispiele aus dem Open Innovation-Bereich adressierte.

Über ihre Programme und Förderleistungen sind die nationalen Förderagenturen wichtige Intermediäre zur Umsetzung der Open Innovation Strategie. Folglich verankerte die FFG OI in bestehenden Programmlinien und fördert die Umsetzung der OI-Strategie durch gezielte Maßnahmen, wie etwa durch das von der Nationalstiftung geförderte „Impact Innovation-Programm". Der FWF hat sich als Teil des internationalen Konsortiums "cOAlition $S^{4}$ dazu verpflichtet, Forschungsergebnisse nach dem 01.01.2020 aus öffentlichen Fördermitteln in konformen OpenAccess-Zeitschriften oder auf konformen Open-Access-Plattformen zu veröffentlichen. Die aws unterstützt die Umsetzung der Ol-Strategie, indem sie sich der Entwicklung von fairen Sharing- und Abgeltungsmodellen für Crowdwork widmet; die aws koordiniert damit auch die Arbeitsgruppe zu „Faire(r) Vergütung in Open Innovation" im Rahmen des NCP-IP. Die Ludwig Boltzmann Gesellschaft (LBG) hat sich

58 Vgl. www.ioeb-innovationsplattform.at/ 
zum Ziel gesetzt, Open Innovation in Science (OIS) über die Umsetzung von konkreten Projekten systematisch in der Praxis zu verankern.

Es gibt zahlreiche Good Practice-Beispiele, die einen offenen Wissensfluss zwischen Forschungstätigkeit und Anwendung demonstrieren. So werden z.B. im Rahmen des EU-Forschungsprojekts „RiConfigure" (Reconfiguring Research and Innovation Constellations) ${ }^{59}$ die Vervielfältigung von AkteursKonstellationen in Innovationsprozessen untersucht. In sogenannten Social Labs wird die Innovation unter Beteiligung von Akteurinnen und Akteuren aus Industrie, Forschung, öffentlichen Einrichtungen und Zivilgesellschaft weiterentwickelt. In Österreich gibt es hierzu das Social Lab zu Quadruple Helix Innovation, an welchem das Institut für Höhere Studien gemeinsam mit dem ÖBB Open Innovation Lab in Abstimmung mit den federführenden Ol-Ressorts BMVIT und BMBWF arbeitet.

Weiters hat das Österreichische Patentamt (ÖPA) Daten zu Schutzrechten wie Patenten, Gebrauchsmustern und Marken im Sinne einer Open Data Initiative aufbereitet und umfangreich öffentlich zugänglich gemacht. Universitäten und Fachhochschulen setzen in ihrem Wirkungsbereich entsprechende Projekte mit Ol-Bezug um; ein Beispiel hierfür sind themenspezifische Hackathons.

Die gelisteten Beispiele geben lediglich einen groben Überblick über laufende OI-Initiativen ${ }^{60}$, veranschaulichen jedoch die zunehmende Umsetzungsbereitschaft quer über alle Stakeholder-Bereiche, die sich über die volle inhaltliche Breite der in der OIStrategie für Österreich definierten Maßnahmen erstreckt.

\section{Umsetzung Zukunftsstrategie Life Sciences und Pharmastandort Österreich}

Ziel der im November 2016 präsentierten „Zukunftsstrategie Life Sciences und Pharmastandort Österreich“61 ist es, die wirtschaftliche und wissenschaftliche Wettbewerbsfähigkeit des für den Standort Österreich bedeutenden Sektors zu erhalten und auszubauen. Auch im zweiten Umsetzungsjahr konnten in den neun Handlungsfeldern (namentlich Grundlagenforschung, Forschungsinfrastrukturen, Big Data, Personalisierte Medizin, Klinische Forschung, Wissenschafts-Wirtschaftskooperationen, Unternehmen, Produktion \& Markt, Dialog Wissenschaft-Gesellschaft) festgelegte Maßnahmen initiiert, weiterverfolgt und umgesetzt werden. Folgende Maßnahmen und Aktivitäten im Zuständigkeitsbereich des BMBWF wurden dabei realisiert:

Im Handlungsfeld „Forschungsinfrastrukturen“ wurden 2018 entscheidende Schritte zum Beitritt Österreichs zur ESFRI-Forschungsinfrastruktur „EuroBiolmaging "62 im Bereich bildgebender Verfahren gesetzt, die in ein offizielles Beitrittsschreiben Österreichs Anfang 2019 gemündet haben. Nach der sehr positiven Evaluierung des nationalen BiobankenNetzwerks „BBMRI.at“63 (Partner der europäischen Forschungsinfrastruktur BBMRI-ERIC ${ }^{64}$ ) durch eine internationale Expertinnen- und Experten-Jury konnte die zweite Förderphase des Netzwerks erfolgreich gestartet werden. Außerdem wurde BBMRI.at auch in den Leistungsvereinbarungen der relevanten Universitäten verankert, um die Weiterentwicklung des Betriebs der Biobanken bzw. Reinvestitionen in Infrastruktur an den Standorten zu sichern. Bezüglich "Core Facilities" wurden weiters vorbereitende

59 Vgl. www.riconfigure.eu

60 Eine tabellarische Übersicht über laufende OI-Initiativen findet sich im Anhang I.

61 Vgl. https://bmbwf.gv.at/fileadmin/user_upload/forschung/Life_Science_Strategie_barrierefrei.pdf

62 Vgl. http://www.eurobioimaging.eu/

63 Vgl. http://bbmri.at/

64 Vgl. http://www.bbmri-eric.eu/ 
Schritte zur „Vienna Biocenter Vision 2030“ gesetzt, die den Betrieb der Vienna Biocenter Core Facilities $\mathrm{GmbH}\left(\mathrm{VBCF} \mathrm{GmbH}^{65}\right.$ ) für weitere zehn Jahre gewährleisten soll.

In Überschneidung mit dem Handlungsfeld „Big Data" wurden ebenso wichtige Aktivitäten zur Etablierung von e-Infrastrukturen in den Leistungsvereinbarungen der relevanten Universitäten berücksichtigt und damit notwendige Voraussetzungen zur Vorbereitung der Beteiligung an europäischen Forschungsinfrastrukturen (wie z.B. ELIXIR) geschaffen. Zur nachhaltigen Koordination von Forschungsaktivitäten im Bereich „Personalisierte Medizin“ wurde die Beteiligung an der im Oktober 2017 gegründeten Österreichischen Plattform für Personalisierte Medizin (ÖPPM) ${ }^{66}$ nunmehr auch in den Leistungsvereinbarungen der relevanten Universitäten aufgenommen.

Im Bereich „Klinische Forschung" ist die interministerielle und interinstitutionelle Arbeitsgruppe unter Koordination des Bundesamts für Sicherheit im Gesundheitswesen (BASG/AGES) weiterhin aktiv, um die Umsetzung der „EU Clinical Trials Regulation“, die voraussichtlich Ende 2020 in Geltung treten wird, vorzubereiten. Für die Entwicklung der IT-Schnittstellen der Ethikkommissionen der Medizinischen Universitäten zum zentralen EU Portal, über das alle Anträge für klinische Studien zukünftig administriert werden, hat das BMBWF 1,3 Mio. $€$ im Rahmen der Leistungsvereinbarungen zur Verfügung gestellt.

Im Zuständigkeitsbereich des BMDW wurden folgende Maßnahmen und Aktivitäten umgesetzt:

Für das Translational Research Center (TRC), das eine Finanzierungs- und Kompetenzlücke zwischen Grundlagenforschung und anwendungsorientierter Forschung schließen soll, sind Verhandlungen zwischen dem BMDW und der aws mit einer internationalen Fonds-Konstruktion am Laufen.

Im Rahmen der geförderten Christian Doppler Labors und Josef Ressel Zentren kommt bereits rd. ein
Drittel aller im Jahr 2018 aktiven Labors (97) aus den thematischen Clustern "Life Sciences und Umwelt" bzw. „Medizin“. Im Berichtszeitraum wurden in diesen Themenclustern fünf Christian Doppler Labors und ein Josef Ressel Zentrum eröffnet. Ebenso kommt eines der drei 2018 neu bewilligten COMET-Zentren aus dem Bereich Life Sciences. Der Diskussionsprozess zur Errichtung eines Wissenstransferzentrums (WTZ) im Bereich Medizinprodukte wurde fortgesetzt.

Die Initiative "LISA“ (Life Science Austria) stattete im Jahr 2018 acht Life Science-Gründungsvorhaben mit insgesamt 1,5 Mio. $€$ PreSeed-Mitteln und neun Unternehmen mit 6,1 Mio. $€$ Seedfinancing aus. Während des LISAvienna Business Treffs 2018 wurden die Finanzierungsinstrumente des BMDW für Life Science Unternehmen vorgestellt. Darüber hinaus betreut LISA auch das Standortmarketing im Bereich Life Sciences, bei sechs Messeauftritten im Ausland wurden österreichische Biotech Start-ups und Unternehmen repräsentiert; die größte saisonale europäische Life-Sciences-Messe (BIO-Europe Spring 2019) in Wien bot vielfältige Möglichkeiten der Vernetzung und der Präsentation des Österreichischen Life Science Standortes.

Weiters wurde der Life-Sciences-Preis "Best of Biotech 2019" (BoB, Preis für den besten Businessplan) neu gestaltet, und die "Life Science Research Awards Austria 2018“ der Österreichischen Gesellschaft für Molekulare Biowissenschaften und Biotechnologie (ÖGMBT) wurden für die beiden Kategorien Grundlagenforschung und anwendungsorientierte Forschung vergeben; erstmals wurde ein Sonderpreis für herausragende Forschung mit gesellschaftlicher Relevanz ausgeschrieben. Mehrere Konferenzen wurden unterstützt, darunter die „BioNanoMed 2018" in Graz zum Thema "Nanotechnology Enables Personalized Medicine" und die "Life Science Success 2018“" mit dem Rahmenthema "Alles digital" in Wien.

65 Vgl. https://www.viennabiocenter.org/facilities/

66 Vgl. https://www.personalized-medicine.at/ 
Der "Life Science Report Austria 2018“ gemeinsam mit einem Firmenverzeichnis für den österreichischen Forschungs- und Wirtschaftsstandort im Bereich Life Sciences wurde erstellt und publiziert. Der Report belegt ein durchgängiges Wachstum des Life Sciences-Sektors bezüglich der Anzahl an Unternehmen (Steigerung um 11\% im Vergleich zum Life Science Report 2015), in der Anzahl der Beschäftigten des Sektors $(+7,4 \%)$ bzw. auch in der Höhe des Umsatzes (+ $17,2 \%)$, sowie eine kontinuierlich positive Entwicklung des Wissenschaftsstandortes in den Life Sciences durch die hierin aktiven Universitäten und außeruniversitären Forschungseinrichtungen.

\section{Umsetzung IP-Strategie}

Zwei Jahre nach Inkrafttreten der IP-Strategie ${ }^{67}$ durch den Beschluss der Bundesregierung im Februar 2017 konnten fast 90 \% der vorgeschlagenen Maßnahmen umgesetzt werden. Die rasche Umsetzung und die gute Aufnahme der vorgeschlagenen Maßnahmen legen nahe, dass bestehende Bedürfnisse der Community passend adressiert werden konnten. So wurden zahlreiche neue Angebote bereits in den ersten Monaten sehr stark in Anspruch genommen:

Die Onlineplattform IP Hub, deren Einrichtung beim Österreichischen Patentamt eine wesentliche Maßnahme der IP-Strategie darstellt, etablierte sich als zentrale Anlaufstelle für alle, die sich für Beratungs- und Förderangebote rund um das Thema Schutz von Geistigem Eigentum informieren möchten. Es werden laufend neue Angebote und Partner in die Serviceplattform aufgenommen; derzeit umfasst die Plattform insgesamt 76 Angebote von 20 Partnern.

Bei der Implementierung eines verbesserten Dienstleistungsangebots im Patent- und Markenbereich wurden seitens des Österreichischen Patentamts für jedes einzelne Dienstleistungsservice interne Evaluierungsdesigns erarbeitet, mit deren Unterstützung die Wirkung überprüft und die Angebote gegebenenfalls weiterentwickelt werden. Die im Markenbereich neuen Dienstleistungen des Österrei- chischen Patentamtes, die Markenähnlichkeitsprüfung und der zusätzlich mit einer rechtlichen Einschätzung zur Schutzfähigkeit ausgestattete Pre Check, werden von den Kundinnen und Kunden gut angenommen. Ebenso die Möglichkeit der raschen Markenanmeldung Fast Track wird stark in Anspruch genommen - im Jahr 2018 wurden bereits $45 \%$ aller Online-Anmeldungen über Fast Track abgewickelt, was eine nationale Markenregistrierung in nur zehn Tagen ermöglicht.

Der Patent Scheck, ein gemeinsam mit der FFG speziell zur Stärkung der IP-Kompetenz und IP-Nutzung von KMU und Start-ups konzipiertes Förderinstrument, konnte 2018 eine weitere Steigerung der Nachfrage gegenüber dem Vorjahr verzeichnen. Insgesamt sind über 800 Anträge bei der FFG eingelangt.

Auch die seitens der aws ab 2017 ausgerollten neuen Unterstützungsmaßnahmen im IP-Bereich werden sehr gut angenommen. Die IPR-Förderungs- und Beratungsdienstleistungen der aws für Unternehmen sind gut etabliert und zielen insgesamt auf die „Hebelwirkung" von IP bei der Realisierung von Innovationen in Unternehmen ab. Mit dem Förderungsprogramm IP. Coaching unterstützt die aws KMU bei der Entwicklung und Implementierung einer maßgeschneiderten Strategie zur Nutzung des geistigen Eigentums (IP-Strategie). Diese nachhaltige IP-Strategie wird im Zuge des aws Coachings mit dem Geschäftsmodell und den Innovationen des Unternehmens erarbeitet und abgestimmt. Bislang haben sich 65 Unternehmen zum IP.Coaching gemeldet.

IP.Market unterstützt KMU sowie technologieentwickelnde Forschungseinrichtungen kunden- und marktorientiert bei der Verwertung ihres geistigen Eigentums (langfristiger strategischer wichtiger Zukunftstechnologien) außerhalb des Unternehmens (Lizenzierung) bzw. außerhalb der Forschungseinrichtung (Fremdverwertung). Das Programm umfasst Beratungs- und Vermarktungsleistungen wie auch $\mathrm{Zu}$ schüsse.

67 Vgl. BMWFW und BMVIT (2016). 
Für die „Nationale Kontaktstelle für Wissenstransfer und Geistiges Eigentum (NCP-IP)“ wurde im Herbst 2018 eine neue Website ${ }^{68}$ erfolgreich gelauncht. Damit wurde sowohl die Sichtbarkeit des NCP-IP erhöht, um die für den Wissens- und Technologietransfer essentiellen IP-Informationen noch besser zu verbreiten als auch die Vertragsmusterbank IPAG69, die seit 2014 rd. 25.000 Zugriffe auf aktuelle sowie kostenlos zur Verfügung stehende Vertragsmuster im Technologietransfer verzeichnet, integriert. Zusätzlich werden vom NCP-IP laufend neue (internationale) Themen im Zusammenhang mit Wissenstransfer aufgegriffen und im Rahmen von IP-relevanten Veranstaltungen mit Vertreterinnen und Vertretern aus Wissenschaft und Wirtschaft diskutiert. Im Zuge der Umsetzung der Open Innovation Strategie wurde z.B. die Arbeitsgruppe „Motivation und fairer Austausch in offenen Innovationsprozessen" eingerichtet. Ziel dieser AG ist es, bis Ende 2019 die Vertragsmusterbank IPAG um ein neues Open Innovation Toolkit zu erweitern.

Im Bildungsbereich wurden Maßnahmen gesetzt, um speziell Lehrpersonen und Lernende auf den Umgang mit IP vorzubereiten, vor allem im Hinblick auf die vorwissenschaftlichen Arbeiten und die Diplomarbeiten in der Berufsbildung. So werden beispielsweise an den Pädagogischen Hochschulen Seminare wie "Schutzrechtswissen für Lehrende" mit Fokus auf Urheberrecht, Patent, Marke, Design, Verwertung von IP etc. angeboten.

Die Weiterentwicklung bzw. Nachschärfung der Schutzrechts- und Verwertungsstrategien der österreichischen Universitäten wurde auch in den aktuellen Leistungsvereinbarungen des BMBWF mit den Universitäten (2019-2021) sowie mit der Österreichischen Akademie der Wissenschaften und dem IST Austria (2018-2020) vorangetrieben. Die regionalen Wissenstransferzentren setzen ihre Arbeit auf Basis der Leistungsvereinbarungen mit den Universitäten sowie mit Unterstützung aus Mitteln des Österreich- fonds fort und sollen damit u.a. auch IP-relevantes Wissen weiter ausbauen.

\section{Umsetzung Strategische Weiterentwicklung der Rahmenbedingungen für Geistes-, Sozial- und Kulturwissenschaften}

Im Strategiedokument zur strategischen Weiterentwicklung der Rahmenbedingungen der Geistes-, Sozial- und Kulturwissenschaften $(G S K)^{70}$, das Ende 2017 veröffentlicht wurde, wurden insgesamt 41 Maßnahmen in fünf Themenfeldern gebündelt, nämlich in: 1) Freiräume für Forschung, 2) Qualitäts- und Leistungsmessung, 3) Internationalisierung, 4) Alternative Vernetzungsräume, sowie 5) Nachwuchsförderung. 2018 wurden sie maßgeblich zur Umsetzung gebracht: Von den 41 Maßnahmen wurden 17 umgesetzt und bei über der Hälfte liegen Teilergebnisse vor. Ziel ist es, alle Maßnahmen bis Ende 2021 umzusetzen und diese von einer Monitoring-Gruppe begleiten zu lassen. Als zentrales Ergebnis zahlreicher Gespräche mit Forschungsförderungsinstitutionen zeigt sich, dass die GSK als Teil eines breiten Innovationsbegriffs gesehen werden. Diese Perspektive fließt auch verstärkt in die Umsetzung von Forschungsförderungsprogrammen ein.

In den Geisteswissenschaften wurde im Bereich der Museen und Archive Awareness für die spezifischen Anforderungen der Forschung geschaffen, insbesondere wenn es um die Digitalisierung von historischen Büchern und Archivalien geht.

60 neue GSK-Forschungsinfrastrukturen wurden 2018 in der Forschungsinfrastrukturdatenbank des BMBWF abgebildet. Über die Eintragung in der Datenbank wird u.a. Archiven, Museen und Sammlungen, aber auch sozialwissenschaftlichen Datenerhebungen die Möglichkeit geboten, sich zu präsentieren und so nationale und internationale Kooperationen anzubahnen. Auf nationaler Ebene gibt es eine Vielzahl kleinerer geisteswissenschaftlicher Forschungsinfrastrukturen, wie regionale und lokale Sammlun- 
gen und Archive, die für die Forschung einen hohen Wert aufweisen, aber aufgrund ihrer vergleichsweisen geringen Sichtbarkeit noch nicht so intensiv von der Forschung genutzt werden, wie es möglich wäre. Das Potenzial, dass die Daten aus Forschungsinfrastrukturen genutzt werden, ist groß. $70 \%$ der Forschenden in den Geisteswissenschaften gaben in einer österreichweiten Befragung an, Fremddaten, also Daten, die nicht selbst erhoben wurden, für Forschungszwecke zu verwenden. ${ }^{71}$

Das Austrian Social Science Data Archive (AUSSDA) wurde im Rahmen der Leistungsvereinbarungsverhandlungen mit den Universitäten Wien, Graz und Linz institutionell langfristig verankert; die Universität Innsbruck wird im Laufe der nächsten LV-Periode einen Beitritt zum Konsortium prüfen.

\section{Umsetzung Kreativwirtschaftsstrategie}

Um das BMDW bei der Steuerung und Überprüfung der Umsetzung der konkreten Maßnahmen der Kreativwirtschaftsstrategie $^{72}$ zu unterstützen, wurde 2018 ein Kreativwirtschaftsbeirat aus nationalen und internationalen Expertinnen und Experten ins Leben gerufen. Dieser soll ein jährliches Monitoring der Maßnahmen durchführen sowie entsprechende Empfehlungen aussprechen.

Am 04.10.2018 fand der achte European Creative Industries Summit im Zuge der österreichischen EURatspräsidentschaft erstmals in Wien statt. Rund 150 Teilnehmerinnen und Teilnehmer diskutierten mit den Playern der europäischen Kreativwirtschaft sowie den Verantwortlichen auf europäischer Ebene über Ausrichtung und Schwerpunkte der europäischen Innovationspolitik. Gerade an der Schnittstelle von Kreativwirtschaft und Digitalisierung entstehen bahnbrechende Innovationen, beispielsweise in den Bereichen Gesundheit, Mobilität, grüne Energie oder Klimawandel. Ziel des European Creative Industries Summit 2018 war es zu zeigen, wie die Kreativwirtschaft die Gesamtwirtschaft sowie die Entwicklung von Regionen und der Gesellschaft als Ganzes durch Cross-Innovationen vorantreibt.

Im Rahmen der monetären Förderung kreativwirtschaftsbasierter, nicht-technologischer Innovationen wurde 2018 ein spezieller Fokus auf Digitalisierungsprojekte gelegt, um der Schlüsselrolle der Kreativwirtschaft als Digitalisierungstreiber Rechnung zu tragen. Bei jeweils zwei halbjährlichen Calls der beiden Zuschussprogramme „aws impulse XL“ und „aws impulse XS" konnten in Summe über 50 innovative Projektideen mit mehr als vier Mio. $€$ unterstützt und in die Tat umgesetzt werden.

Zur Stärkung der unternehmerischen Kompetenzen Kreativschaffender in Hinblick auf Internationalisierung wurde in Zusammenarbeit mit der Außenwirtschaft Austria der WKO der Pilot „Kreativwirtschaftswebinare" gestartet. Dabei werden innovative Geschäftsmodelle weltweit gescoutet und im Rahmen digitaler Webinare der österreichischen Kreativwirtschaft vorgestellt. So soll ein Know-how-Transfer von internationalen Märkten nach Österreich als Anregung für neue Geschäftsmodelle in Österreich und zur Förderung von innovativen Ideen stattfinden.

Um wirtschaftliche Kompetenz, Kreativität und Kooperationen unter Kreativschaffenden weiter zu stärken, wurden die Kreativwirtschaftscoachings "C

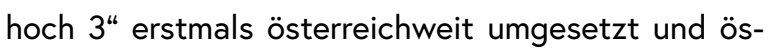
terreichweite Netzwerktreffen für alle Teilnehmerinnen und Teilnehmer initiiert. Unter dem Motto „Innovationen Raum geben“ wurden eintägige Innovationscamps für Kreativunternehmen mit der Bezeichnung „Kreativwirtschaftswerkstätten“ angeboten, um gemeinsam Fragestellungen zu erarbeiten, Kooperationen einzugehen und Innovationen anzustoßen. Im Rahmen der "impulse lectures" Reihe wurden aktuelle Fragestellungen aus dem Bereich soft \& creative innovation aufgegriffen, u.a. die Themen social design \& social impact oder Biomimicry - Designprinzipien aus der Natur \& Lernwerkzeuge für naturinspiriertes Design.

71 Vgl. Bauer et al. $(2015,112)$.

72 Vgl. https://www.kreativwirtschaft.at/wp-content/uploads/2016/06/Kreativwirtschaftsstrategie_f\%C3\%BCr_\%C3\%96sterreich. pdf 


\section{Bioökonomie - eine Strategie für Österreich}

Im März 2019 wurde die erste Bioökonomie-Strategie $^{73}$ für Österreich im Ministerrat beschlossen. Sie adressiert die Frage, wie die Gesellschaft nachhaltig und verantwortungsvoll mit natürlichen Ressourcen umgehen und dabei gleichzeitig erfolgreich wirtschaften kann. Bioökonomie steht für ein Wirtschaftskonzept, das fossile Ressourcen (Rohstoffe und Energieträger) durch nachwachsende Rohstoffe in möglichst allen Bereichen und Anwendungen ersetzen soll. Sie umfasst dabei alle industriellen und wirtschaftlichen Sektoren, die biologische Ressourcen produzieren, ver- und bearbeiten oder nutzen. Bioökonomie soll damit die große Chance bieten, globalen Herausforderungen wie dem fortschreitenden Klimawandel, der Lebensmittel- und Wasserknappheit oder den zunehmenden Umweltbelastungen zu begegnen und gleichzeitig die ökonomische Entwicklung zu stärken.

Das Arbeitspapier "Bioökonomie-FTI-Strategie" ${ }^{\text {774 }}$ der AG 2 zu „Klimawandel und Ressourcenknappheit“ der Task Force FTI, die zur besseren Koordination der zahlreichen Aktivitäten und Politikfelder im Bereich der Bioökonomie ins Leben gerufen wurde, identifziert wesentliche Forschungsaufgaben und wichtige Zielkonflikte, die einer Bearbeitung bedürfen. In die Erstellung der Bioökonomie FTI-Strategie wurden frühzeitig zahlreiche Expertinnen und Experten aus Wissenschaft und Forschung eingebunden.

Da die wirtschaftliche Transformation hin zur Bioökonomie ein komplexer Prozess ist, stellen adäquate FTI-Instrumente, die alle Innovationsphasen abdecken, eine wesentliche Voraussetzung dar. Neben der technologischen Entwicklung ist die systemische Verbindung von technischen mit wirtschaftlichen, politischen, gesellschaftlichen und ethischen Aspekten essentiell für die erfolgreiche Umsetzung einer wis- sensbasierten Bioökonomie. Bioökonomie-Forschung muss daher - gestützt auf Grundlagenforschung - inter- und transdisziplinär ausgerichtet sein, sich an Prinzipien wie „Open Innovation"75 und „Responsible Science ${ }^{\text {"76 }}$ orientieren und Kooperationen fördern. Die Bioökonomiestrategie nennt daher folgende Handlungsfelder im Bereich Wissenschaft und Forschung ${ }^{77}$ :

- Grundlagenforschung zu Ressourcen-Verfügbarkeit, ökologischen Funktionen (Boden, Biodiversität etc.), Standortbedingungen und sozialen Rahmenbedingungen biobasierter Wirtschaft;

- (Physikalische, chemische, biologische) Analytik von Materialeigenschaften;

- Abschätzung der institutionellen und rechtlichen Rahmenbedingungen sowie regionaler und lokaler Effekte der Bioökonomie;

- Themenspezifische, interdisziplinäre (Grundlagenund angewandte Forschung verbindende) Analysen und Bewertungen unter Berücksichtigung geistes-, sozial- und kulturwissenschaftlicher Forschung;

- Angewandte Forschung: Erhöhung der Anstrengungen im Bereich Produkt- und Prozessentwicklungen bei stofflicher und energetischer Verwertung biogener Materialien;

- Erhebung des Nutzungspotenzials von biobasierten Materialien unter Berücksichtigung ökologischer, ökonomischer und sozialer Effekte;

- Systemische Bewertung der Wechselwirkungen zwischen dem Klimawandel und der verstärkten Biomasseproduktion sowie der Biodiversität;

- Entwicklung neuer Produkte aus biogenen Rohstoffen bzw. Abfällen und Nebenprodukten;

- Themenspezifische Initiativen der Universitäten und Hochschulen zu Bildung sowie Aus- und Fortbildung (im Sinne des Gesamtösterreichischen Universitätsentwicklungsplans);

73 Vgl. BMNT et al. (2019).

74 Vgl. https://nachhaltigwirtschaften.at/resources/nw_pdf/biooekonomie-fti-strategie-ag2-2018.pdf

75 Vgl. http://openinnovation.gv.at/

76 Vgl. https://www.responsiblescience.at/

77 Vgl. BMNT et al. (2019). 
- Schaffung von rechtlichen und organisatorischen Rahmenbedingungen für die Bioökonomie und private Investoren.

Österreich konnte sich bereits in den vergangenen Jahren als Kompetenzträger für Bioökonomie positionieren. So ist beispielsweise ein Drittel der österreichischen Universitäten im Bereich Bioökonomie aktiv. Hervorzuheben ist dabei speziell die Universität für Bodenkultur Wien, die bereits frühzeitig den internationalen Entwicklungstrend hin zu Bioökonomie gestaltet hat und 2019 die Gründung eines Zentrums für Bioökonomie vorsieht, das als Anlaufstelle für Stakeholder aus Wirtschaft und Politik dienen soll. Neben institutionellen Einrichtungen widmen sich insbesondere Kooperationsplattformen den Prozessketten "Landwirtschaft - Lebensmittel - Biotechnologie" und "Forstwirtschaft - Holzwirtschaft", sowie den Verbindungen zwischen den Ressourcenströmen. So wird im Rahmen von 16 Christian-Doppler-Laboren und vier Josef-Ressel-Zentren unter Beteiligung von Universitäten, Fachhochschulen und Unternehmen an bioökonomierelevanten Forschungsfragen gearbeitet.

Auf Basis der Bioökonomiestrategie wurde Anfang 2019 mit der Erstellung eines Aktionsplans für Bioökonomie begonnen, der sich an alle relevanten Akteure in diesem Bereich richtet. Den Rahmen für die Auswahl der Maßnahmen des Aktionsplans bilden die in der Strategie identifizierten Zielfelder:

1. Erreichung der Klimaziele

2. Reduktion der Abhängigkeit von nicht erneuerbaren Rohstoffen

3. Förderung von Innovation

4. Förderung wirtschaftlicher Entwicklung

5. Sicherung und Schaffung von Arbeitsplätzen

6. Förderung nachhaltiger gesellschaftlicher Transformation
Artificial Intelligence Mission Austria 2030 AIM AT 2030

Die Regierung arbeitet aktuell ressortübergreifend an der „Artificial Intelligence Mission 2030“, der Bundesstrategie für Künstliche Intelligenz, kurz: AIM AT $2030^{78}$. Bis Sommer 2019 werden hierfür von Arbeitsgruppen, welche bereits im Jänner dieses Jahres ihre Arbeit aufgenommen haben, auf Basis des "White Papers" des Österreichischen Rates für Robotik und Künstliche Intelligenz, Vorschläge erarbeitet. Im Herbst wird dann das Strategiedokument vorliegen, welches das Handlungspotenzial wie auch den Rahmen für Österreich als Schlüsselfigur im internationalen Kontext aufzeigt. Ein primäres Ziel ist die Reduktion der Abhängigkeit von internationalen Marktführern. Zu diesem Zweck soll vor allem in Grundlagenforschung investiert werden, um Österreich künftig auch als internationalen Forschungsstandort für $\mathrm{Al}$ zu positionieren. ${ }^{79}$

\section{Aktuelle Entwicklungen im Hochschulbereich}

\section{Umsetzung Sustainable Development Goals (SDGs) an den Universitäten}

Die Resolution "Transformation unserer Welt, die Agenda 2030 für nachhaltige Entwicklung“ wurde bei der Generalversammlung der Vereinten Nationen beschlossen. Sie besteht aus einem Katalog von 17 Zielen und 169 Unterzielen für nachhaltige Entwicklung in der Periode von 2016 bis 2030. Ziel ist es, unter Mitwirkung aller Staaten der Welt, globalen Herausforderungen wie Armut, Hunger, Ungleichheiten, Krisen und Konflikten wie auch dem Klimawandel zu begegnen. Die kohärente Umsetzung der SDGs in Österreich wurde mit einem Ministerratsbeschluss vom 12.01.2016 vereinbart und war auch Thema des Beschlusses der Bologna-Ministerkonferenz am 25.05.2018. ${ }^{80}$

78 Vgl. https://www.bmvit.gv.at/innovation/publikationen/ikt/downloads/aimat_ua.pdf

79 Vgl. BMVIT und BMDW (2019).

80 Vgl. EHEA Paris 2018 Ministerial Conference (2018). 
Die Umsetzung der SDGs im Hochschulbereich erfolgt auf vielfältige Weise, wie durch Verankerung in den Leistungsvereinbarungen der Universitäten, im Gesamtösterreichischen Universitätsentwicklungsplan und einem systemorientierten Forschungsansatz zu den gesellschaftlichen Herausforderung in der FTIStrategie des Bundes. Durch ihren Bildungs- und Forschungsauftrag sind die österreichischen Universitäten prädestiniert, in der Umsetzung der SDGs durch fächerübergreifende Kooperation, visionäres Denken und innovative Lösungsvorschläge eine für die Gesellschaft wichtige Rolle zu übernehmen. In diesem Kontext agiert auch das Netzwerk „UniNEtZ - Universitäten und Nachhaltige EntwicklungsZiele". Es ist bestrebt, die SDGs an den Universitäten in Forschung, Lehre und Hochschulmanagement zu verankern. Entsprechende verbindliche Patenschaften bzw. Mitgliedschaften von Universitäten zur Umsetzung einzelner SDGs wurden in den Leistungsverhandlungen 2019-2021 zwischen den Universitäten und dem BMBWF festgeschrieben. Mitglieder in UniNEtZ sind derzeit 15 Universitäten sowie die Zentralanstalt für Meteorologie und Geodynamik (ZAMG), die Geologische Bundesversuchsanstalt (GBA) und das Climate Change Center Austria (CCCA). Zu den Zielen zählen die Erstellung eines universitäts- und fächerübergreifenden Optionenpapiers, das die Bundesregierung bei der Umsetzung der Nachhaltigkeitsziele unterstützen soll, die inter- und transdisziplinäre universitätsübergreifende Vernetzung, die Interaktion mit Stakeholdern aus Politik, Verwaltung, Wirtschaft und Zivilgesellschaft, sowie der Aufbau von Wissenskompetenz zu die SDGs betreffenden Themen.

\section{Ausbau der strukturierten Doktoratsausbildung und kooperatives Modell}

Die Förderung des wissenschaftlichen Nachwuchses ist das zentrale Element, um den Wissenschaftsstandort Österreich im internationalen Wettbewerb nachhaltig zu stärken.

Österreich hat sich im Hinblick auf die Entwicklung der Anzahl erfolgreicher Doktorandinnen und Doktoranden keine absolute Zahl als Ziel gesetzt.
Strategie ist vielmehr, eine Verbesserung des Verhältnisses Studierende zu Absolventinnen und Absolventen herbeizuführen, Drop-outs nach Möglichkeit zu vermeiden und die Anzahl der Abschlüsse zu erhöhen. Zur Zielerreichung wird ein qualitativer Ansatz verfolgt. Das österreichische Modell des strukturierten Doktorats stützt sich dabei auf qualitative Eckpfeiler (siehe Abschnitt 3.2.5). Auf dessen qualitative Weiterentwicklung wird auch in den Leistungsvereinbarungen 2019-2021 besonderes Augenmerk gelegt.

Die Förderung eines Doktoratsprogramms zwecks Kooperation zwischen Universitäten und Fachhochschulen, wie 2015 von der Hochschulkonferenz empfohlen, ist - vorbehaltlich budgetärer Bedeckbarkeit - in Vorbereitung.

Unter "kooperativem Doktorat" wird verstanden, dass eine Universität und eine Fachhochschule gemeinsam Doktoratsprogramme anbieten. Von Relevanz ist, dass das Promotionsrecht weiterhin ausschließlich von einer Universität verliehen wird, die Betreuung der Doktorandinnen und Doktoranden hingegen gemeinsam erfolgt. Ergänzt werden diese Bestrebungen durch weitere Maßnahmen zur Unterstützung der Karriereentwicklung von Forschenden, etwa durch Förderung der internationalen Mobilität, des intersektoralen und interdisziplinären Wissenstransfers via z.B. Marie-Sklodowska-Curie-Maßnahmen der Europäischen Union sowie durch eine ganz grundsätzliche qualitative Verbesserung der Doktoratsausbildung.

Im Wintersemester 2018/19 startete das erste kooperative Doktoratskolleg der TU Wien gemeinsam mit der FH Technikum Wien zum Themenbereich „Resilient Embedded Systems".

\section{Umsetzungsstand zur Hochschulplanung}

Ab 2019 werden folgende strategische Dokumente weitergeführt bzw. neu eingeführt:

- Neu: Österreichischer Hochschulentwicklungsplan (HoP)

- Rollierung It. UG: Gesamtösterreichischer Universitätsentwicklungsplan (GUEP)

- Fortführung: Zukunft Hochschule (ZH) 
Einführung: „Österreichischer

Hochschulentwicklungsplan" zur

Hochschulbildung

Der Hochschulentwicklungsplan (HoP) wird jenes strategische Dokument darstellen, das auf den gesamten österreichischen Hochschulbildungsbereich fokussiert. Das heißt, der HoP wird als "strategisches Dach" für die bestehenden Strategiepapiere GUEP, Fachhochschulentwicklungs- und -finanzierungsplan sowie zum Pädagogischen Hochschulen Entwicklungsplan (PH-EP) etabliert. Aktuell befindet sich der HoP in Ausarbeitung und soll 2020 fertiggestellt sein.

Fokussierte der Hochschulplan 2011 auf Prozesse, so wird der Hochschulentwicklungsplan Aussagen zu Entwicklungsgrößen machen, z.B. wieviel Prozent eines Altersjahrgangs sollten im tertiären Bereich ausgebildet werden? Welcher Hochschulsektor soll sich quantitativ und qualitativ wie entwickeln?

Mit der Erstellung des Hochschulentwicklungsplans kommt das BMBWF den wiederkehrenden Aufforderungen des Rechnungshofs aber auch des Parlamentes nach, ein Planungsinstrument zu erstellen, das den gesamten österreichischen Hochschulraum umfasst.

\section{Rollierung des GUEP: Überarbeitung des GUEP in 2019 lt. § 12b UG}

Der Gesamtösterreichische Universitätsentwicklungsplan (GUEP) ist das strategische Planungsdokument des BMBWF, in dem jene Ziele priorisiert sind, welche die Weiterentwicklung der Universitäten prägen. Die Erstversion des GUEP bezog sich auf den Planungshorizont 2016-2021 und wurde 2015 nach einem Konsultationsprozess mit 42 hochschulischen Institutionen erstellt. In Vorbereitung auf die Leistungsvereinbarungsverhandlungen im Jahr 2018 und den Abschluss der Leistungsvereinbarungen 20192021 wurde der GUEP bereits 2017 für den Planungshorizont 2019-2024 rollierend überarbeitet. Bis Ende
2019 wird dieser nun erneut inhaltlich weiterentwickelt, um als leitendes Strategiepapier für die Universitäten bei Erstellung ihrer jeweiligen Universitätsentwicklungspläne zu dienen. Diese wiederum sind wesentliche Grundlage für den Erstellungsprozess der kommenden Leistungsvereinbarungen 20222024.

\section{Fortsetzung von Zukunft Hochschule}

Das im Frühjahr 2016 durch das BMBWF gestartete Projekt "Zukunft Hochschule“, das 2017 erfolgreich abgeschlossen wurde, soll in überarbeiteter Form fortgesetzt werden. Das Projekt hatte die strategische Weiterentwicklung des österreichischen Hochschulsystems - insbesondere eine bessere Abstimmung zwischen öffentlichen Universitäten und Fachhochschulen - zum Ziel. Ergebnisse aus Zukunft Hochschule 2016/17 sind in den FH-Plan und in die Leistungsvereinbarungen 2019-21 eingeflossen.

Die Fortführung des Erfolgsprojekts dient der weiteren Vertiefung der Abstimmung zwischen den Hochschulsektoren sowie der inhaltlichen Vorbereitung der Schwerpunktthemen für die Entwicklungen ab 2022. Neu wird insbesondere die Einbindung der Pädagogischen Hochschulen sowie der Privatuniversitäten in den gemeinsamen Abstimmungsprozess sein.

Die Themen, die zur Diskussion stehen, nehmen eine Spannbreite von neuen Lernwelten und ihren virtuellen und realen Infrastrukturen (wie z.B. ShortCycle-Programmen) bis hin zu Fragen zu innovativen Studienformaten ein. Schwerpunktthemen werden auch künftig die Abgrenzung der Hochschulsektoren zueinander und zu anderen Bildungsanbietern, sowie generell die künftigen inhaltlichen Entwicklungen in den Portfolios der Hochschulsektoren, die Abstimmung im Studienbereich (Durchlässigkeit/Anrechnung) sowie die Kooperationen zwischen den Hochschulsektoren sein. 
2. Die großen

Förderagenturen des Bundes 
In Österreich befinden sich zahlreiche Förderungseinrichtungen für Forschung, Technologie und Innovation auf Ebene des Bundes und der Bundesländer. Der größte Teil der bundesseitigen Förderungen wird von drei großen Agenturen, dem Wissenschaftsfonds FWF, der österreichischen Forschungsförderungsgesellschaft FFG und der Austria Wirtschaftsservice Gesellschaft aws vergeben bzw. im Auftrag des Bundes abgewickelt. Während der FWF gemeinsam mit dem FFF - einer zentralen Vorgängerorganisation der FFG - bereits im Jahr 1968 gegründet wurde, entstanden FFG und aws aus der Zusammenlegung verschiedener Agenturen in den Jahren 2004 bzw. 2002. ${ }^{1}$

In diesem Kapitel werden diese drei großen Förderagenturen des Bundes, ihre gesetzlichen Grundlagen, aktuelle Kennzahlen und Schwerpunkte sowie neue strategische Initiativen und Förderungsprogramme dargestellt.

Der Wissenschaftsfonds FWF (Fonds zur Förderung der wissenschaftlichen Forschung) ist Österreichs zentrale Einrichtung zur Förderung der Grundlagenforschung. Zum Aufgabenbereich zählen die Stärkung und der Ausbau des Wissenschaftssystems und der Standortattraktivität des Landes, wobei die Unterstützung von Forscherinnen und Forschern durch Einzelprojekte das traditionell wichtigste Förderungsprogramm darstellt. Die Projektförderung erfolgt hauptsächlich bottom-up auf Basis von Anträgen, die ein internationales Peer-review Verfahren durchlaufen. Im Jahr 2018 wurden vom FWF 684 Projekte (2017: 642) mit einem Budget von insgesamt 230,8 Mio. $€$ (2017: 217,3 Mio. $€$ ) neu bewilligt. Aufgrund eines deutlichen Anstiegs des Antragsvolumens auf rd. 950 Mio. $€$ (2017: 879,4 Mio. $€$ ) sank die gesamte Bewilligungsquote (nach Summe) dennoch leicht von $22,4 \%$ auf $22,1 \%$, während die Bewilligungsquote der Einzelprojekte mit rd. 28 \% gehalten werden konnte.
Die Österreichische Forschungsförderungsgesellschaft FFG ist die nationale Förderinstitution für unternehmensnahe Forschung und Entwicklung in Österreich. Sie bietet vor allem Unternehmen, aber auch Forschungseinrichtungen und Hochschulen ein breites Angebot verschiedener Instrumente: Das Portfolio reicht von niederschwelligen Programmen, die den Einstieg in eine kontinuierliche Forschungsund Innovationstätigkeit erleichtern bis hin zur Förderung und Finanzierung von thematischen Verbünden und Kompetenzzentren. Über die finanzielle Unterstützung hinaus bietet die FFG auch Dienstleistungen und Beratungen an - so agiert sie z.B. als Nationale Kontaktstelle für die Forschungsprogramme der Europäischen Union und als Schnittstelle zur Europäischen Weltraumagentur. Im Jahr 2018 betrugen die vertraglich zugesicherten Förderungen (inklusive Haftungen und Darlehen) 617,6 Mio. $€$, dies entspricht einem Barwert von 500,8 Mio. $€$.

Die Austria Wirtschaftsservice Gesellschaft aws ist die Förderbank des Bundes. Durch die Vergabe von zinsengünstigen Krediten, Zuschüssen und Garantien werden Unternehmen bei der Umsetzung ihrer innovativen Projekte unterstützt, insbesondere dann, wenn die erforderlichen Mittel durch sonstige Finanzierungen nicht ausreichend aufgebracht werden können. Ergänzend werden spezifische Informations-, Beratungs-, Service- und Dienstleistungen für angehende, bestehende und expandierende Unternehmen angeboten. Im Jahr 2018 lag die gesamte Finanzierungsleistung mit 2.189,5 Mio. $€$ rd. 91,2 \% über dem Vergleichswert des Vorjahres, wobei temporär angebotene und breitenwirksame Zuschussprogramme (vor allem der Beschäftigungsbonus) für den hohen Anstieg des Zuschussvolumens (von 223,6 Mio. $€$ im Jahr 2017 auf 1.241,2 Mio. € im Jahr 2018) verantwortlich zeichneten. Die Bewilligungsquote betrug $54 \%$.

1 Für einen historischen Überblick vgl. Pichler et al. (2007). 


\subsection{Wissenschaftsfonds (FWF)}

\section{Gesetzliche Grundlagen und Förderzweck}

Der Wissenschaftsfonds FWF (Fonds zur Förderung der wissenschaftlichen Forschung) ist Österreichs zentrale Einrichtung zur Förderung der Grundlagenforschung, sowie der künstlerisch-wissenschaftlichen Forschung. Der FWF wurde 1968 gegründet und ist eine durch ein Bundesgesetz (Forschungs- und Technologieförderungsgesetz, FTFG) ${ }^{2}$ eingerichtete juristische Person öffentlichen Rechts. Lt. § 2 des FTFG wurde der FWF zum Zweck der Förderung der Forschung, die dem Erkenntnisgewinn und der Erweiterung sowie Vertiefung der wissenschaftlichen Kenntnisse dient und nicht auf Gewinn gerichtet ist, eingerichtet. Das Bundesministerium für Bildung, Wissenschaft und Forschung (BMBWF) agiert als Aufsichtsbehörde des FWF.

\section{Instrumente, Kennzahlen und Schwerpunkte}

Die Programme des FWF gliedern sich in drei große Programmgruppen:

Förderung von Spitzenforschung - Neues entdecken, die volumenmäßig größte Gruppe, die unter anderem auch das wichtigste FWF Programm, die Einzelprojektförderung, umfasst.

\section{Ausbau der Humanressourcen - Talente fördern} mit Programmen zur Nachwuchs- und Mobilitätsförderung, sowie

2. Wechselwirkung Wissenschaft - Gesellschaft Ideen umsetzen, die eine Vielzahl eher kleinerer Programme und Initiativen an der Nahtstelle zu Wirtschaft und Gesellschaft enthält.

Für die Jahre 2018 bis 2021 wurden dem FWF ein Budget in der Höhe von 184 Mio. $€$ pro Jahr und zusätzliche Mittel für den gleichen Zeitraum in der Höhe von 110 Mio. $€$ genehmigt. In der Mehrjahresplanung wurde entsprechend eine schrittweise Anhebung der Budgetmittel bis auf ein Niveau von 224 Mio. $€$ bis 2021 eingeplant. Weitere Budgetausweitungen erfolgten über die Höherdotierung der Natio- nalstiftung für Forschung, Technologie und Entwicklung (NFTE), die zusätzlichen Mittel der Bundesländer sowie Stiftungsmittel.

Aufgrund der Budgetausweitung stiegen im Jahr 2018 die Neubewilligungen um rd. 6 \%. Die gesamte Bewilligungsquote (berechnet als Quotient von Antragsvolumen und Neubewilligungsvolumen) sank aufgrund des gestiegenen Antragsvolumens dennoch leicht von $22,4 \%$ auf $22,1 \%$, während die Bewilligungsquote der Einzelprojekte mit rd. $28 \%$ auf hohem Niveau gehalten werden konnte (siehe Tabelle 2-3).

Das Kuratorium des FWF hatte im Jahr 2018 über 2.500 Projektanträge mit einem Gesamtvolumen von rd. 950 Mio. $€$ zu entscheiden. Dieser deutliche Anstieg des Antragsvolumens gegenüber dem Vorjahr (2017: 879,4 Mio. €) zeigt einen stetig steigenden Bedarf der Wissenschaftsgemeinschaft in Österreich, der aufgrund des anhaltenden Wachstums des Wissenschaftsstandortes auch künftig nicht abflachen wird. Dieser Anstieg mag - ebenso wie der Rückgang im Vorjahr - auch teilweise auf die im August 2018 erfolgte Aufhebung der Antragsbeschränkungen zurückzuführen sein: So konnte die Anzahl der beantragbaren Projekte von zwei auf drei angehoben und die Limitierung der maximal beantragbaren Fördersumme aufgehoben werden.

Dabei erhöhte sich die Anzahl der Projektanträge (nach einem leichten Rückgang im Jahr 2017) von 2.493 auf 2.501 Anträge nur unwesentlich um 0,3 \% (vgl. Tabelle 2-1), bei den Neubewilligungen beträgt der Zuwachs 6,5\%.

Ähnlich wie bei der Anzahl fallen auch die Veränderungen in den Volumina aus: bei den Antragssummen $7,9 \%$, bei den Neubewilligungen 6,2\% (vgl. Tabelle 2-3). Dies deutet auf eine stetige Zunahme der Projektgröße wie auch auf längere Projektzeiten hin.

Der FWF fördert vor allem Forscherinnen und Forscher, insgesamt konnte die Anzahl der vom FWF finanzierten Personen auf 4.155 erhöht werden, in Vollzeitäquivalenten macht dies 2.843 aus. Es handelt

2 Vgl. https://www.ris.bka.gv.at/GeltendeFassung.wxe?Abfrage=Bundesnormen\&Gesetzesnummer=10009523 
Tabelle 2-1: Anzahl der Förderungen 2017-2018

\begin{tabular}{|c|c|c|c|c|c|c|c|c|}
\hline \multirow{2}{*}{ Programm } & \multicolumn{2}{|c|}{ Projektanträge } & \multirow{2}{*}{$\begin{array}{c}\begin{array}{c}\text { Frauen als } \\
\text { PL in \% }\end{array} \\
2018\end{array}$} & \multirow{2}{*}{$\begin{array}{r}\begin{array}{l}\text { Projekt- } \\
\text { MA }^{1}\end{array} \\
2018\end{array}$} & \multicolumn{2}{|c|}{ Neubewilligungen } & \multicolumn{2}{|c|}{$\begin{array}{l}\text { Bewilligungsquote } \\
\text { in \% }\end{array}$} \\
\hline & 2017 & 2018 & & & 2017 & 2018 & 2017 & 2018 \\
\hline Einzelprojekte & 1.025 & 1.052 & 28,2 & 733 & 295 & 298 & 28,8 & 28,3 \\
\hline Internationale Programme & 466 & 456 & 26,5 & 242 & 106 & 121 & 22,7 & 26,5 \\
\hline Spezialforschungsbereiche (SFB) - Neuanträge $e^{2,3}$ & 33 & 62 & 22,6 & 55 & 7 & 29 & 5,3 & 10,3 \\
\hline Spezialforschungsbereiche (SFB) - Verlängerungen ${ }^{3}$ & 24 & - & - & - & 20 & - & 83,3 & - \\
\hline Zukunftskollegs & - & 61 & 47,5 & 46 & - & 7 & - & 11,5 \\
\hline START-Programm & 88 & 84 & 20,2 & 25 & 6 & 6 & 6,8 & 7,1 \\
\hline Wittgenstein-Preis & 20 & 21 & 19,0 & k.A. & 1 & 2 & 5,0 & 9,5 \\
\hline Doktoratskollegs - Neuanträge ${ }^{2}$ & 5 & - & - & - & 4 & - & 25,0 & - \\
\hline Doktoratskollegs - Verlängerungen & 8 & 8 & 0,0 & $126^{4}$ & 7 & 6 & 87,5 & 75,0 \\
\hline doc.funds & 45 & - & - & - & 7 & - & 15,6 & - \\
\hline Erwin-Schrödinger-Auslandsstipendien & 146 & 132 & 32,6 & 53 & 53 & 53 & 36,3 & 40,2 \\
\hline Lise-Meitner-Programm & 209 & 238 & 35,3 & 70 & 50 & 70 & 23,9 & 29,4 \\
\hline Hertha-Firnberg-Programm & 83 & 91 & 100 & 22 & 21 & 22 & 25,3 & 24,2 \\
\hline Elise-Richter-Programm & 74 & 83 & 100 & 44 & 17 & 29 & 23,0 & 34,9 \\
\hline Klinische Forschung & 81 & 79 & 41,8 & 43 & 13 & 17 & 16,0 & 21,5 \\
\hline $\begin{array}{l}\text { Programm zur Entwicklung und Erschließung der } \\
\text { Künste }\end{array}$ & 67 & 68 & 47,1 & 44 & 9 & 11 & 13,4 & 16,2 \\
\hline Top Citizen Science & 18 & 10 & 10,0 & 9 & 7 & 5 & 38,9 & 50,0 \\
\hline $\begin{array}{l}\text { Interregionales Projektnetzwerk „Südtirol-Tirol- } \\
\text { Trentino“ }\end{array}$ & 38 & 56 & 16,1 & 11 & 2 & 8 & 5,3 & 14,3 \\
\hline Open Research Data & 40 & - & - & - & 12 & - & 30,0 & - \\
\hline Wissenschaftskommunikationsprogramm & 23 & - & - & - & 5 & - & 21,7 & - \\
\hline Gesamt & 2.493 & 2.501 & 34,3 & 1.523 & 642 & 684 & 25,5 & 26,7 \\
\hline
\end{tabular}

1 Die Angaben entsprechen dem beantragten Projektpersonal in bewilligten Projekten. Diese Angaben müssen nicht identisch sein mit den später in den Projekten tatsächlich finanzierten Personen.

2 Die Bewilligungsquote errechnet sich aus bewilligten Voll- zu Konzeptanträgen. Konzeptanträge sind in der vorliegenden Tabelle nicht abgebildet. Die Gesamtbewilligungsquote berücksichtigt diesen Zusammenhang ebenfalls.

3 Gewertet sind SFB-Teilprojekte.

4 Die Angabe inkludiert beantragtes Projektpersonal und beantragte "fully funded by FWF“-PhD-Stellen. Weitere teil-finanzierte PhD-Stellen sind nicht berücksichtigt.

Quelle: FWF

Tabelle 2-2: Durch den FWF finanziertes Forschungspersonal 2017-2018

\begin{tabular}{|c|c|c|c|c|c|}
\hline Forschungspersonal & $\begin{array}{r}\text { VZÄ } 2017 \\
\text { (Stichtag 31.12.) } \\
\end{array}$ & $\begin{array}{r}\text { davon Frauen } \\
\text { in } \% \\
\end{array}$ & $\begin{array}{r}\text { VZÄ } 2018 \\
\text { (Stichtag 31.12.) } \\
\end{array}$ & $\begin{array}{r}\text { davon Frauen } \\
\text { in } \% \\
\end{array}$ & $\begin{array}{r}\text { Veränderung } \\
\text { Frauenanteil in \% }\end{array}$ \\
\hline Wissenschaftliches Personal & 2819,3 & 42,15 & 2843,1 & 44,39 & $+2,24$ \\
\hline PostDoc & 1114,9 & 37,46 & 1134,7 & 41,11 & $+3,65$ \\
\hline PraeDoc & 1373,6 & 42,49 & 1381,0 & 44,02 & $+1,53$ \\
\hline Anderes Personal & 330,9 & 56,51 & 327,4 & 57,29 & $+0,78$ \\
\hline
\end{tabular}

Quelle: FWF.

sich hier in erster Linie um Promovierende, die in FWF-Projekten arbeiten. Der Frauenanteil unter den FWF-finanzierten Forschenden ist im Jahr 2018 nach einem Rückgang im Jahr 2017 - in allen Personalkategorien wieder gestiegen, und zwar über das Niveau des Jahres 2016 hinaus (vgl. Tabelle 2-2). Auch bei den Projektleitungen sind Frauen stärker präsent: Waren dies 2017 noch $28 \%$, so stieg der Anteil im Jahr 2018 auf über 34,5\%. Dies kann sowohl auf die gestiegenen Bewilligungswahrscheinlichkeiten zurückzuführen sein, als auch als Erfolg der vielfachen Bemühungen des FWF gesehen werden, die Teilhabe von Frauen in FWF-Projekten auf allen Ebenen zu unterstützen. 
Tabelle 2-3: Förderungssummen in Mio. €, 2017-2018

\begin{tabular}{|c|c|c|c|c|c|c|c|c|}
\hline \multirow[t]{2}{*}{ Programm } & \multicolumn{2}{|c|}{$\begin{array}{l}\text { Beantragte } \\
\text { Förderungssummen }\end{array}$} & \multicolumn{2}{|c|}{$\begin{array}{c}\text { Neu- } \\
\text { bewilligungen }\end{array}$} & \multicolumn{2}{|c|}{$\begin{array}{l}\text { Bewilligungsquote } \\
\text { in } \%\end{array}$} & \multicolumn{2}{|c|}{$\begin{array}{l}\text { Gesamtbewilligungen } \\
\text { (inkl. Ergänzende } \\
\text { Bewilligungen)' }\end{array}$} \\
\hline & 2017 & 2018 & 2017 & 2018 & 2017 & 2018 & 2017 & 2018 \\
\hline Einzelprojekte & 337,4 & 360,2 & 97,8 & 102,7 & 29,0 & 28,5 & 98,7 & 104,7 \\
\hline Internationale Programme & 131,6 & 126,9 & 27,5 & 30,0 & 20,9 & 23,6 & 27,6 & 30,6 \\
\hline Spezialforschungsbereiche (SFB) - Neuanträge ${ }^{2}$ & 13,3 & 26,4 & 3,3 & 13,3 & 4,3 & 11,0 & 3,3 & 13,6 \\
\hline Spezialforschungsbereiche (SFB) - Verlängerungen & 11,0 & - & 8,4 & - & 76,8 & - & 9,1 & - \\
\hline Zukunftskolleg & - & 118,9 & - & 13,0 & - & 10,9 & - & 13,0 \\
\hline START-Programm & 101,1 & 98,4 & 6,8 & 7,1 & 6,7 & 7,2 & 6,8 & 7,2 \\
\hline Wittgenstein-Preis & 30,0 & 31,5 & 1,5 & 2,8 & 5,0 & 8,9 & 1,5 & 2,8 \\
\hline Doktoratskollegs - Neuanträge ${ }^{2}$ & 11,6 & - & 7,7 & - & 19,0 & - & 7,7 & - \\
\hline Doktoratskollegs - Verlängerungen & 23,5 & 26,0 & 17,9 & 19,0 & 76,0 & 73,2 & 19,5 & 22,2 \\
\hline doc.funds & 65,3 & - & 11,3 & - & 17,3 & - & 11,3 & - \\
\hline Erwin-Schrödinger-Auslandsstipendien & 18,7 & 17,5 & 7,2 & 7,1 & 38,4 & 40,5 & 8,0 & 8,0 \\
\hline Lise-Meitner-Programm & 32,6 & 38,8 & 7,9 & 11,4 & 24,2 & 29,4 & 8,2 & 11,9 \\
\hline Hertha-Firnberg-Programm & 19,1 & 21,3 & 4,8 & 5,2 & 25,3 & 24,2 & 5,0 & 5,5 \\
\hline Elise-Richter-Programm & 21,4 & 24,0 & 4,8 & 8,3 & 22,2 & 34,5 & 5,1 & 8,6 \\
\hline Klinische Forschung & 22,7 & 24,2 & 4,0 & 5,4 & 17,5 & 22,2 & 4,0 & 5,4 \\
\hline $\begin{array}{l}\text { Programm zur Entwicklung und } \\
\text { Erschließung der Künste }\end{array}$ & 25,1 & 25,2 & 3,4 & 4,1 & 13,5 & 16,5 & 3,4 & 4,2 \\
\hline Top Citizen Science & 0,8 & 0,5 & 0,3 & 0,2 & 41,5 & 47,1 & 0,3 & 0,2 \\
\hline $\begin{array}{l}\text { Interregionales Projektnetzwerk } \\
\text { „Südtirol-Tirol-Trentino“ }\end{array}$ & 4,9 & 8,8 & 0,3 & 1,3 & 5,8 & 14,3 & 0,3 & 1,3 \\
\hline Open Research Data & 8,2 & - & 2,2 & - & 27,0 & - & 2,2 & - \\
\hline Wissenschaftskommunikationsprogramm & 1,1 & - & 0,2 & - & 23,2 & - & 0,2 & - \\
\hline Gesamt $^{3}$ & 879,4 & 948,7 & 217,3 & 230,8 & 22,4 & 22,1 & 222,6 & 239,5 \\
\hline
\end{tabular}

1 Die Gesamtkosten enthalten zusätzlich zu Neubewilligungen ergänzende Bewilligungen in bereits laufenden Projekten. Diese ergänzenden Bewilligungen umfassen u.a. Inflationsabgleichungen und Buchhaltungsausgleichungen.

2 Die Bewilligungsquote errechnet sich aus bewilligten Voll- zu Konzeptanträgen. Konzeptanträge sind in der vorliegenden Tabelle nicht abgebildet. Die Gesamtbewilligungsquote berücksichtigt diesen Zusammenhang ebenfalls.

3 Enthält bei Gesamtbewilligungen zusätzliche Bewilligungen in Programmen, in denen in 2017 oder 2018 keine Neubewilligungen erfolgten.

Quelle: FWF.

\section{Strategische Entwicklungen}

Im Jahr 2018 fanden zahlreiche wichtige Ereignisse statt, die für den FWF auch künftig von zentraler strategischer Bedeutung sein werden:

- Im Jahr 2018 feierte der FWF sein 50-jähriges Bestehen mit dem „BE OPEN - Science \& Society Festival" während der EU-Ratspräsidentschaft Österreichs. Mit dem Festival zielte der FWF darauf ab, das Vertrauen in die Wissenschaft zu stärken und die Bedeutung der Grundlagenforschung hervorzuheben. Aus zahlreichen Gesprächen ist die Stakeholder-Initiative PEARL entstanden, die das Netzwerk zwischen dem FWF und Organisationen aus Wirtschaft und Gesellschaft weiter ausbauen soll. Im Jahr 2018 wurden erste Pilotprojek- te gestartet, die 2019 nach einer finalen Evaluierung die Grundlage für eine Verstetigung dieser Initiative sein sollen.

- Von strategischer Bedeutung ist weiterhin die auf Basis des Regierungsprogramms formulierte Zukunftsoffensive der für Forschung zuständigen Ministerien (siehe Ministerratsvortrag vom August 2018 „Zukunftsoffensive für Forschung, Technologie und Innovation") sowie die Beauftragung der Ausarbeitung einer Exzellenzinitiative für Österreich.

- Auch hat der FWF gemeinsam mit Partnerorganisationen in Zentraleuropa begonnen, seine internationalen Kooperationen in dieser Region zu intensivieren. Diese Central European Science 
Partnership (CEUS) wird 2019 noch weiter ausgebaut werden.

- In Fortführung seiner Politik für Open Access und Open Science war der FWF einer der starken Promotoren der "cOAlition S", einer Koalition europäischer Förderungsorganisationen, die den freien Zugang zu wissenschaftlichen Publikationen mit dem „Plan S“ entscheidend vorantreiben will. Ziel ist die Formulierung von gemeinsamen Prinzipien und eines Umsetzungsplanes bis 2020. ${ }^{3}$ Auch wurde mit der Einführung einer adaptierten Open Research Data Policy und eines Data Management Plans im FWF ein weiterer Schritt im Bereich Open Science gesetzt. ${ }^{4}$

- Einen weiteren Schwerpunkt stellt der Ausbau von Maßnahmen im Bereich wissenschaftlicher Ethik und Integrität dar ${ }^{5}$ : Neben einer jährlichen Veröffentlichung der behandelten Verdachtsfälle wissenschaftlichen Fehlverhaltens sowie der aktiven Beteiligung am Netzwerk SOPs4RI (Standard Operating Procedures for Research Integrity) befindet sich ein "Code of Conduct" im Rahmen einer Arbeitsgruppe der Hochschulkonferenz in Ausarbeitung.

- Umstellung auf PROFI: Im Jahr 2018 wurde der umfassende Konsultationsprozess mit den Forschenden, Forschungsinstitutionen und FWF-Gremien zur Umsetzung der Projektförderung über Institutionen (PROFI) abgeschlossen. Beginnend mit 2018 werden in einer ersten Phase die Förderungsprogramme doc.funds, Zukunftskollegs und Forschungsgruppen nach PROFI bereits abgewickelt. In den Jahren 2019/20 werden in Folge bestehende Programme auf das neue Förderungsformat umgestellt.

- Strategie 2019-2021: Ende 2018 legte der FWF seine Strategie 2019-2021 (Mehrjahresprogramm 2019-2021) vor. Drei Schwerpunkte stehen dabei im Vordergrund: Die Qualitätssicherung, die Konsolidierung des Förderungsportfolios und der Dialog mit der Gesellschaft. ${ }^{6}$

Tabelle 2-4: Neue Initiativen und Förderungsprogramme 2018

\begin{tabular}{|c|c|c|}
\hline Förderungsprogramm/ Initiative & Zielgruppe & Zielsetzung \\
\hline $\begin{array}{l}\text { Zukunftskollegs (in Kooperation } \\
\text { mit ÖAW): Postdoc-Programm für } \\
\text { innovative, interdisziplinäre Teams }\end{array}$ & $\begin{array}{l}\text { Nachwuchswissenschaftlerinnen und } \\
\text {-wissenschaftler (Postdocs aus dem In- und } \\
\text { Ausland von } 1 \text { bis max. } 5 \text { Jahre nach der } \\
\text { Promotion) aller Wissenschaftsdisziplinen }\end{array}$ & $\begin{array}{l}\text { Förderung junger Postdocs; } \\
\text { Mittelfristige Zusammenarbeit (bis zu } 4 \text { Jahren) zu } \\
\text { einem interdisziplinären, komplexen, aktuellen Thema } \\
\text { in gemischten Teams von mind. } 3 \text { bis max. } 5 \text { Forschen- } \\
\text { den; fachgrenzenübergreifende Zusammenarbeit zu } \\
\text { innovativen Themen }\end{array}$ \\
\hline $\begin{array}{l}\text { Forschungsgruppen: Standortunab- } \\
\text { hängige Vernetzung von inter- oder } \\
\text { multidisziplinären Teams aus drei bis } \\
\text { fünf Forschenden }\end{array}$ & $\begin{array}{l}\text { Ein Team aus 3-5 international herausra- } \\
\text { genden Forscherinnen und Forschern aller } \\
\text { Wissenschaftsdisziplinen }\end{array}$ & $\begin{array}{l}\text { Förderung von Kooperationsprojekten zwischen } 3 \text { bis } \\
\text { max. } 5 \text { Forschenden an kleineren Standorten bzw. in } \\
\text { bestimmten Wissenschaftsdisziplinen, die in kleineren } \\
\text { Formaten kooperieren, um in inter- oder multidiszip- } \\
\text { linären Forschungsprojekten ein Thema zu vertiefen } \\
\text { oder neu zu konsolidieren }\end{array}$ \\
\hline $\begin{array}{l}\text { Quantenforschung und -technologie } \\
\text { (QFTE) (in Kooperation mit FFG) }\end{array}$ & $\begin{array}{l}\text { Wissenschaftlerinnen und Wissenschaftler } \\
\text { aus der Quantenforschung nach der } \\
\text { Promotion, deren wissenschaftliche } \\
\text { Qualifikation durch referierte, internationa- } \\
\text { le Publikationen belegt ist }\end{array}$ & $\begin{array}{l}\text { Wissenstransfer aus der Grundlagenforschung der } \\
\text { Quantenphysik in die Entwicklung und Anwendung } \\
\text { von Quantentechnologien und umgekehrt. Forsche- } \\
\text { rinnen und Forscher erhalten damit eine Karriereper- } \\
\text { spektive im Unternehmenssektor als Alternative zu } \\
\text { einer rein akademischen Laufbahn }\end{array}$ \\
\hline
\end{tabular}

Quelle: FWF.

3 Vgl. die Unterstützung von Plan S von cOAlition S: https://www.coalition-s.org/

4 Vgl. https://www.fwf.ac.at/en/research-funding/open-access-policy/open-access-to-research-data/

5 Vgl. https://www.fwf.ac.at/de/forschungsfoerderung/research-integrity-research-ethics/

6 Vgl. https://www.fwf.ac.at/fileadmin/files/Dokumente/Ueber_den_FWF/Publikationen/FWF-relevante_Publikationen/fwfmehrjahresprogramm-2019-2021.pdf 


\section{Veränderungen im Instrumentenportfolio}

Der FWF arbeitet seit einiger Zeit intensiv und in enger Abstimmung mit den Universitäten, der ÖAW wie auch der FFG an neuen Formaten, die dem Bedarf der Wissenschaftsgemeinschaft Rechnung tragen, Lücken im bestehenden Portfolio schließen oder neue Partnerschaften ermöglichen. Tabelle 2-4 beschreibt neue Programme oder Instrumente, die im Jahr 2018 initiiert wurden.

\section{2 Österreichische Forschungsförderungs- gesellschaft (FFG)}

\section{Gesetzliche Grundlagen und Förderzweck}

Die Österreichische Forschungsförderungsgesellschaft ist die nationale Förderungsagentur für die angewandte Forschung und Innovation in Österreich. Sie wurde durch das Bundesgesetzblatt I Nr. 73/2004 „Forschungsförderungsgesellschaft Errichtungsgesetz" (FFG-Gesetz) mit 01.09.2004 gegründet und steht zu 100 \% im Eigentum der Republik Österreich. Träger der Agentur sind das Bundesministerium für Verkehr, Innovation und Technologie (BMVIT) und das Bundesministerium für Digitalisierung und Wirtschaftsstandort (BMDW). Als Anbieter von Förderungsdienstleistungen ist die FFG aber auch im Auftrag anderer nationaler, regionaler und internationaler Institutionen tätig, unterstützt die Abwicklung der Programme des Klima- und Energiefonds, bietet Förderungen im Rahmen von Bundesländerkooperationen an und begutachtet die Anträge für die Forschungsprämie.

Die FFG unterstützt die FTI-Politik in 1) der Verbreiterung der Innovationsbasis, 2) dem Strukturwandel (wie z.B. durch die Förderung von Start-ups und besonders riskanter und strategisch zentraler F\&E-Vorhaben) und 3) der Stärkung der österreichischen Forschungs- und Innovationsbasis in strategischen Themenfeldern (wie z.B. Energie, Produktion, Mobilität, IKT etc.). Die Verbesserung des Zusammenspiels zwischen Wissenschaft und Wirtschaft, Nachwuchsförderung und Förderung von Forscher- karrieren in der angewandten Forschung in Wissenschaft und Wirtschaft sowie die Förderung der Chancengleichheit sind weitere Zielsetzungen der FFG, die über ein ausdifferenziertes Portfolio unterschiedlichster Instrumente umgesetzt werden.

\section{Instrumente, Kennzahlen und Schwerpunkte}

Die Bandbreite der von der FFG eingesetzten Instrumente reicht von niederschwelligen Einstiegsformaten bis hin zur Förderung von Spitzenforschung. Dabei werden jeweils spezifische Förderziele mit unterschiedlichen Projektarten verknüpft. Die Förderinstrumente der FFG bilden diese verschiedenen Förderansätze wie folgt ab:

- Einstieg: Ausloten von Forschungs- und Entwicklungsthemen bzw. Innovationsoptionen und erste projektvorbereitende Schritte

- Forschungs-, Entwicklungs- und Innovationsprojekt (FEI-Projekt): Konkrete F\&E-Projekte von der orientierten Grundlagenforschung bis zu marktnahen Entwicklungsprojekten

- Markteinführung: Ergebnisse aus der F\&E-Phase werden im Markt eingeführt.

- Struktur: Aufbau und Verbesserung von Strukturen und Infrastrukturen für Forschung und Innovation

- Personen: Förderung von Nachwuchs, Qualifizierung von F\&E-Mitarbeiterinnen und -Mitarbeitern, Verbesserung von Chancengleichheit

- Aufwendungen: Durchführung von beauftragten F\&E-Leistungen zu spezifischen Fragestellungen Die Zahlen und Fakten zu den FTI-Förderungen der FFG werden im Folgenden dargestellt. Zu beachten ist, dass in den präsentierten Eckdaten zu den Förderungsaktivitäten der FFG auf die FTI-Agenden fokussiert und die Förderung im Rahmen der für das BMVIT abgewickelten Breitbandinitiative nicht berücksichtigt ist. In Tabelle 2-5 und Tabelle 2-6 werden für das Jahr 2018 die Zahl der Projekte, die damit verbundenen Beteiligungen und die Anzahl der unterschiedlichen Akteure (Unternehmen, Forschungseinrichtungen etc.) sowie die vertraglich zugesicherten Fördermittel aufgelistet. Letztere betrugen insgesamt 
Tabelle 2-5: FFG-Förderungsaufkommen, ohne Breitband, 2018

\begin{tabular}{l|c|c|c|c|c|c|c|}
\multirow{2}{*}{ Programmgliederung } & \multicolumn{2}{|c|}{ Projekte } & \multicolumn{2}{c|}{ Beteiligungen } & \multicolumn{2}{c}{ Akteure } \\
\cline { 2 - 7 } & $\mathbf{2 0 1 7}$ & $\mathbf{2 0 1 8}$ & $\mathbf{2 0 1 7}$ & $\mathbf{2 0 1 8}$ & $\mathbf{2 0 1 7}$ & $\mathbf{2 0 1 8}$ \\
\hline FFG (gesamt) & $\mathbf{3 . 6 0 2}$ & $\mathbf{3 . 8 5 4}$ & $\mathbf{5 . 8 7 0}$ & $\mathbf{6 . 6 2 2}$ & $\mathbf{3 . 4 0 7}$ & $\mathbf{3 . 8 9 7}$ \\
\hline Bereich Basisprogramme & 1.664 & 1.794 & 2.192 & 2.355 & 1.608 & 1.709 \\
\hline Bereich Thematische Programme & 1.475 & 1.502 & 2.318 & 2.526 & 1.485 & 1.646 \\
\hline Agentur für Luft- und Raumfahrt & 421 & 490 & 1.271 & 1.617 & 991 & 1.027 \\
\hline Europäische und internationale Programme & 33 & 32 & 77 & 87 & 57 & 61
\end{tabular}

Quelle: FFG.

Tabelle 2-6: FFG-Fördersummen nach Bereich, ohne Breitband, 2017 und 2018 (in Mio. €)

\begin{tabular}{l|c|c|c|c|c|c|c}
\multirow{2}{*}{ Programmgliederung } & \multicolumn{2}{c}{ Gesamtkosten } & \multicolumn{2}{c}{ Gesamtförderung } & \multicolumn{2}{c}{ Barwert } \\
\cline { 2 - 8 } & $\mathbf{2 0 1 7}$ & $\mathbf{2 0 1 8}$ & $\mathbf{2 0 1 7}$ & $\mathbf{2 0 1 8}$ & $\mathbf{2 0 1 7}$ & $\mathbf{2 0 1 8}$ \\
\hline FFG (gesamt) & $\mathbf{1 . 1 0 2 . 5}$ & $\mathbf{1 . 2 4 4 . 6}$ & $\mathbf{5 6 2 . 5}$ & $\mathbf{6 1 7 . 6}$ & $\mathbf{4 3 4 . 3}$ & $\mathbf{5 0 0 . 8}$ \\
\hline Bereich Basisprogramme & 606.8 & 491.1 & 307.7 & 294.4 & 179.5 & 177.7 \\
\hline Bereich Strukturprogramme & 217.4 & 317.7 & 90.6 & 118.8 & 90.6 & 118.8 \\
\hline Bereich Thematische Programme & 267.7 & 348.1 & 155.5 & 196.6 & 155.5 & 196.6 \\
\hline Agentur für Luft- und Raumfahrt & 9.1 & 9.2 & 7.3 & 7.0 & & 7.3 & 7.0 \\
\hline Europäische und internationale Programme & 1.5 & 0.9 & 1.4 & 0.8 & & 1.4 & 0.8
\end{tabular}

Quelle: FFG.

(inklusive Haftungen und Darlehen) 617,6 Mio. $€$, dies entspricht einem Barwert von 500,8 Mio. $€$. Im Vergleich zum Jahr 2017 ergibt sich damit eine Zunahme des Barwerts der Förderungen in der Höhe von 15,3\% (2017: 434,3 Mio. $€$ Barwert). Durch diese Förderung wurden Forschungsvorhaben im Gesamtausmaß von mehr als 1,2 Mrd. $€$ (plus 12,9\% gegenüber 2017) initiiert. Insgesamt beläuft sich die Zahl der geförderten Projekte auf 3.854, die 6.622 Beteiligungen und 3.897 unterschiedliche Akteure umfassten. Das entspricht einer Zunahme der geförderten Akteure gegenüber dem Vorjahr (3.407 Akteure) um 14,4\%, während die Zahl der Projekte um 7,0 \% zunahm. Diese Steigerungen machen deutlich, dass die FFG erfolgreich zur Stärkung des Forschungsstandortes und zur laufenden Verbreiterung der Forschungsbasis in Österreich beiträgt.

Die größte Bedeutung innerhalb dieses Programmportfolios weist der Programmbereich der Basisprogramme auf, auf den der größte Anteil des Fördervolumens sowohl gemessen am Barwert, an der Anzahl der Projekte wie auch an der Höhe der induzierten Gesamtkosten entfällt. Knapp 1.800 Projekte mit ei- nem Barwert von 177,7 Mio. $€$ finden sich innerhalb dieses Bereichs, womit ihr Anteil am gesamten Förderaufkommen der FFG 46,5 \% (Projekte) bzw. 35,5\% (Barwert) beträgt.

Auf die thematischen Programme entfallen 196,6 Mio. $€$ an zugesagten Fördermitteln, gemessen am Barwert. Mit Hilfe dieser Programme werden in ausgewählten Technologiefeldern Schwerpunkte gesetzt. Ziel ist es, in diesen Feldern kritische Massen an Forschungsaktivitäten zu induzieren und damit einen Anschluss Österreichs an die internationale Technologiegrenze (technological frontier) zu gewährleisten. Die Bandbreite der ausgewählten Technologiefelder ist dabei sehr weit. Die quantitativ bedeutsamsten umfassen Energie, IKT, Mobilität und Transport, Produktionstechnologien, Sicherheitsforschung oder den Luftfahrtsektor. Auch der Programmbereich ALR (Agentur für Luft- und Raumfahrt) kann zu diesen thematischen Spezialisierungen gezählt werden, wird aber als eigener Bereich geführt und ist auch so aufgelistet.

Den dritten großen Programmbereich der FFG stellen die Strukturprogramme dar. Auf sie entfallen 
118,8 Mio. € Fördermittel (Barwert). Dieser Bereich beinhaltet u.a. das Programm COMET (knapp 85 Mio. $€$ Barwert an Förderungen im Jahr 2018), das österreichische "Leuchtturmprogramm“ zur Förderung der Zusammenarbeit zwischen Wissenschaft und Wirtschaft durch den Aufbau gemeinsamer Forschungskompetenzen. Das Programm orientiert sich insbesondere an Exzellenz, der Einbindung von internationalem Forschungs-Know-how sowie dem Aufbau und der Sicherung der Technologieführerschaft von Unternehmen zur Stärkung des österreichischen Forschungsstandorts.

Das Förderangebot der FFG steht grundsätzlich allen Akteuren bzw. Organisationstypen offen, der Schwerpunkt liegt bei der Förderung der angewandten Forschung von Unternehmen, was sich an ihrem Anteil von 61 \% (bzw. 376,8 Mio. $€$ in absoluten Zahlen) an den gesamten Förderungen der FFG niederschlägt (siehe Tabelle 2-7). An zweiter Stelle folgen die (außeruniversitären) Forschungseinrichtungen, die eine Förderung von insgesamt 135,9 Mio. $€$ zugesagt bekommen haben. Ihr Anteil an der gesamten FFG-Förderung beträgt damit $22 \%$. Die Universitäten erhielten im Jahr 2018 Förderungszusagen in der Höhe von 88,2 Mio. € bzw. 14,3\% der FFG-Förderungen.

Die FFG klassifiziert die geförderten Projekte nach Technologiefeldern, wobei diese Klassifikation auf Basis der "CORDIS Subject Index Classification Codes" (SIC) erfolgt. Damit ist es auch möglich, die thematische (bzw. technologische) Struktur der FFGForschungsförderung darzustellen. Das entsprechende Ergebnis dieser Zuordnung findet sich in Abbildung 2-1.

$\mathrm{Zu}$ beachten ist, dass sich die unterschiedlichen SIC-Technologiefelder in ihrer "Bandbreite" sehr unterscheiden können. Breiten, ja „generischen“ Technologiefeldern wie "Industrielle Fertigung“ oder "IKTAnwendungen" stehen sehr eng definierte Technologiefelder (z.B. landwirtschaftliche Biotechnologie, Luftverkehr und -technologien, Quantentechnologien) gegenüber. Dennoch ergibt sich aus dieser Klassifikation ein interessantes Bild hinsichtlich der thematischen Ausrichtung der geförderten Projekte.

Mit einem zugesagten Barwert von 64,9 Mio. $€$ erhält das Technologiefeld "Industrielle Fertigung" am meisten Fördermittel von Seiten der FFG, gefolgt von „Oberflächenverkehr und -technologien“7 (56,5 Mio. €), „Elektronik/Mikroelektronik“ (48,7 Mio. €) und Werkstofftechnik (48,0 Mio. €). Zählt man die beiden expliziten IKT-Themenfelder "IKT-Anwendungen" sowie "Informationsverarbeitung, Informationssysteme" zusammen (die im CORDIS-SIC System als getrennte Felder definiert sind) zeigt sich, dass die IKT mit einem Barwert von insgesamt knapp 73,8 Mio. $€$ sogar an erster Stelle stünde. Berücksichtigt man, dass IKT-Themen als generische Technologie auch in vielen anderen Themenfeldern behandelt werden sowie einige Technologiefelder engen Kon-

Tabelle 2-7: FFG-Förderungen nach Organisationstyp (Kosten, Förderungen und Barwert in Mio. $€$ ), ohne Breitband, 2018

\begin{tabular}{l|r|r|r|r|r} 
Organisationstyp & Akteure & Beteiligungen & Gesamtkosten & $\begin{array}{c}\text { Gesamt- } \\
\text { förderung }\end{array}$ & Barwert \\
\hline Unternehmen & 2.542 & 3.770 & 769,6 & 376,8 & 260,4 \\
\hline Forschungseinrichtungen & 203 & 976 & 324,7 & 135,9 & 135,8 \\
\hline Hochschulen & 582 & 1.199 & 122.9 & 88,2 & 88,2 \\
\hline Intermediäre & 34 & 46 & 5,8 & 3,4 & 13,0 \\
\hline Sonstige & 536 & 631 & 21,7 & 13,2 & $\mathbf{6 1 7 , 6}$ \\
\hline Gesamt & $\mathbf{3 . 8 9 7}$ & $\mathbf{6 . 6 2 2}$ & $\mathbf{1 . 2 3 6 , 1}$ & $\mathbf{5 0 0 , 8}$
\end{tabular}

Quelle: FFG.

7 Darunter wird in der „CORDIS Subject Index Classification“ der Landverkehr (also Straße und Schiene) subsumiert. 
Abbildung 2-1: FFG-Förderung nach Technologiefeldern, 2018 (Barwerte in Mio. €)

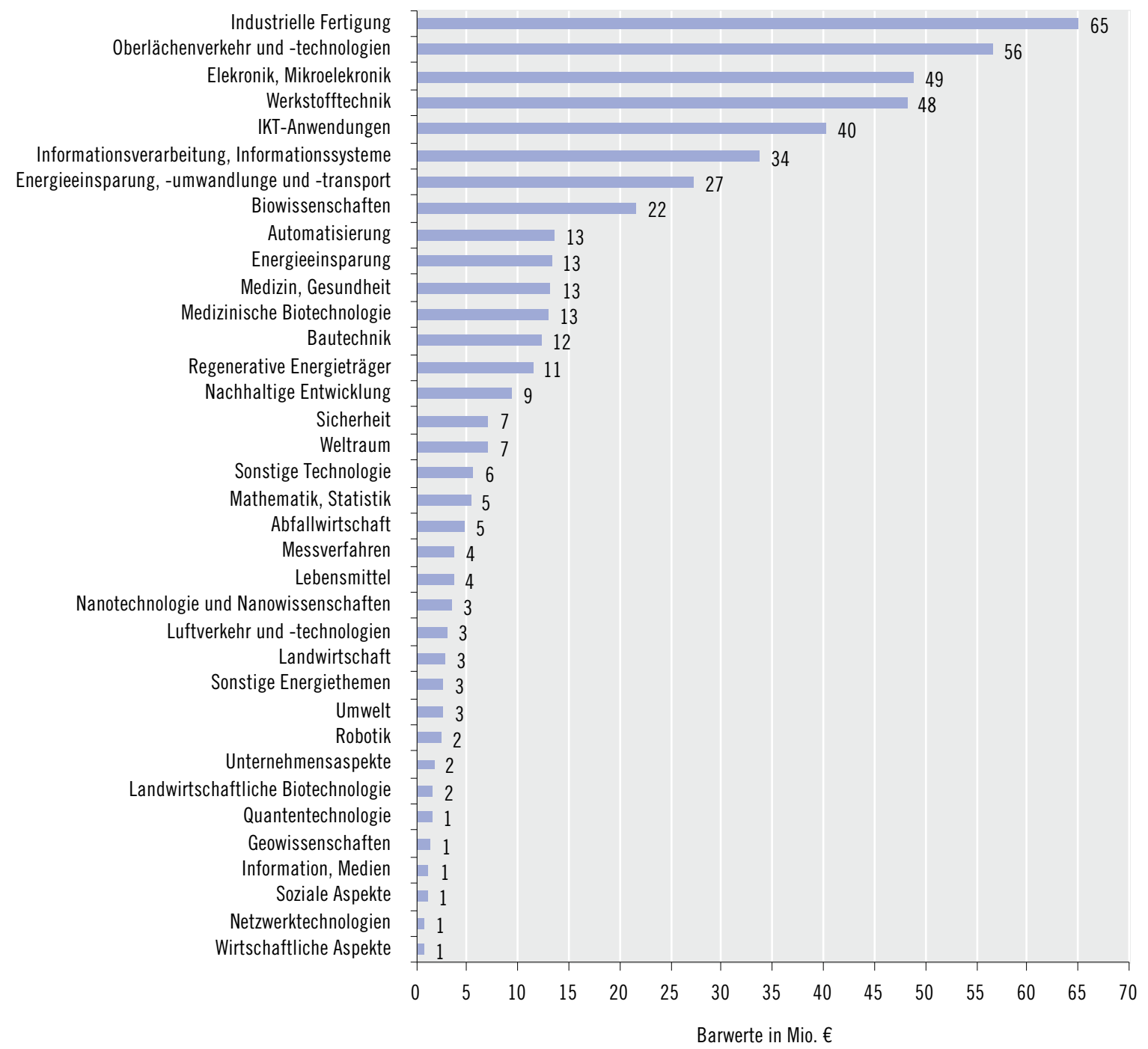

Anm.: Technologiefelder unter 1 Mio. € Förderbarwerte sind nicht dargestellt.

Quelle: FFG.

nex zu IKT aufweisen (z.B. Elektronik/Mikroelektronik, Automatisierung, Robotik) wird deutlich, dass dieses Technologiefeld eines der wesentlichen Schwerpunktthemen der angewandten Forschungsförderung in Österreich darstellt. Tatsächlich zeigt eine Sonderauswertung ${ }^{8}$ der FFG, dass der Anteil der Förderungsmittel, die in Projekte mit starken Digitalisierungsaspekten fließen in den letzten Jahren deutlich gestiegen ist und zwar von $40 \%$ im Jahr 2015 auf $61 \%$ im Jahr 2018. Innerhalb dieses Schwerpunkts der Digitalisierung spielen die Themenfelder „Künstliche Intelligenz" und "Robotik“ eine wichtige Rolle. Im Durchschnitt der Jahre 2017 und 2018 flossen jeweils annähernd 100 Mio. $€$ an Förderungsmitteln der FFG in diese Schlüsseltechnologien der Digitalisierung. Betrachtet man die jeweiligen Programme, in denen diese Mittel abgerufen werden, zeigt sich, dass die Basisprogramme mit einem Anteil von $51 \%$ voran liegen, d.h. dass die Forschung zu KI und Robotik auch wesentlich bottom-up getrieben ist. An

8 Vgl. Egerth und Pseiner (2019). 
zweiter Stelle liegt das Programm „Produktion der Zukunft", 10 \% der gesamten Fördermittel für $\mathrm{KI}$ und Robotik werden über dieses einschlägige Programm verteilt. Es folgen COMET und "IKT der Zukunft" mit 9 \% bzw. 8 \%. Diese Zahlen zeigen, dass die Forschung zu diesen neuen Schlüsseltechnologien mit einer ausgeglichenen Kombination von bottom-up und top-down Instrumenten vorangetrieben wird.

\section{Strategische Entwicklungen}

Ein wichtiger strategischer Meilenstein im Jahr 2018 war die Einrichtung der Digitalisierungsagentur (kurz DIA). Die DIA, die selbst keine Förderprogramme umsetzt, wird vom BMDW sowie vom BMVIT finanziert und ist als eigener Bereich in der Österreichischen Forschungsförderungsgesellschaft eingerichtet. Damit unterstützt die Österreichische Bundesregierung die digitale Transformation Österreichs. Zielsetzung ist, Österreich zu einem international führenden Standort für digitale Exzellenz und Innovation auszubauen. Die DIA begleitet Gesellschaft, Verwaltung und insbesondere Österreichs Wirtschaft in Digitalisierungsfragen. Sie unterstützt sowohl mit Know-how und Expertise als auch mit eigenen Projekten speziell für Unternehmen und versteht sich als Vernetzer der Digitalisierungscommunity. Die DIA begleitet auch in ihrer Funktion als Beraterin der Bundesregierung die Digitalisierungsoffensive Digital Austria. Erstmals finden damit Projekte, Kompetenzen und Akteure aus Gesellschaft, Wirtschaft und Verwaltung unter einem Dach zusammen.

Die folgenden Projekte skizzieren die praktische Umsetzung der Aufgabenstellung:

- Community Map: Der Zugang zu digitalen Initiativen, das Wissen über mögliche Kooperationspartner bei der Digitalisierung ist in Österreich derzeit nur dezentral und unstrukturiert vorhanden. Hier setzt die DIA an: Sie vernetzt die verschiedenen Akteure und unterstützt bei der Skalierung von konkreten Projekten. In einer stetig wachsenden Community-Map auf https://www.digitalaustria. gv.at/ werden die zentralen Akteure der Digitalisierung sichtbar gemacht. Die Community-Map zeigt Player, Projekte und Initiativen zur Digitalisierung in Österreich. Die visualisierte Datenbank schafft eine Basis für den Aufbau von Beziehungen der wichtigsten Stakeholder, Initiativen und Organisationen. Damit können sich interessierte Unternehmen ganz einfach informieren zu Fragen wie: Wo in meiner Umgebung findet Digitalisierung statt? Mit wem kann mein KMU kooperieren?

- DIAloge - Lernen von den Besten. In jeder Branche zeigen erfolgreiche KMU, wie sie die Chancen der Digitalisierung bereits perfekt nutzen. Mit der neuen Veranstaltungsreihe DIAloge schafft die Digitalisierungsagentur ein Forum, bei dem die digitalen Vorreiter ihr Wissen weitergeben. Ein Branchentalk vernetzt die innovativen Erfolgsbetriebe mit etablierten Unternehmen, KMU lernen von den Besten. Damit wird das Wissen direkt in den Betrieben aufgebaut. Bereits im März starten die österreichweiten DIAlog-Veranstaltungen für die Betriebe, insgesamt sind im ersten Jahr im Rahmen einer Roadshow Veranstaltungen in jedem österreichischen Bundesland geplant.

- Team KMU: Vor allem Leitbetriebe sind in der Digitalisierung gefordert und verfügen über die Ressourcen, die digitale Transformation ihrer Unternehmen voranzutreiben. Damit sie wettbewerbsfähig bleiben, braucht es die digitale Kompetenz der Zulieferbetriebe. Genau hier setzt das Team KMU an: Leitbetriebe entwickeln mit ihren Partnern und der Unterstützung der DIA anschlussfähige Digitalisierungsprozesse. Damit werden Ecosysteme geschaffen, die besser und effizienter gemeinsam agieren.

- Digital Austria Day: Erfolgreiche Digitalisierungsprojekte brauchen Talente, die das Berufsfeld erobern. Nach dem Vorbild des etablierten Töchtertags/Girls Day ist der Digital Austria Day ein integrativer Aktionstag, bei dem sich Unternehmen mit ihren Digitalisierungsschwerpunkten öffnen und präsentieren. Sie geben einen erlebnisorientierten und damit emotionalen Zugang zu Digitalisierungsprojekten und lenken als österreichweite Initiative den Fokus auf die Digitalisierungs- 
Tabelle 2-8: Neue Entwicklungen im Instrumentenportfolio

\begin{tabular}{l|l|l}
\hline Förderinstrumente & Zielgruppe & Zielsetzung \\
\hline $\begin{array}{l}\text { Digital Pro Bootcamps } \\
\text { https://www.ffg.at/ausschreibung/digital- }\end{array}$ & KMU & $\begin{array}{l}\text { Qualifizierung für die Nutzung digitaler } \\
\text { Technologien }\end{array}$ \\
\hline $\begin{array}{ll}\text { pro-bootcamps-1-ausschreibung } \\
\text { Entwicklung jeweils maßgeschneiderter } \\
\text { Curricula für die Ausbildung zu Digital } \\
\text { Professionals }\end{array}$ \\
\hline $\begin{array}{l}\text { Digital Innovation Hubs } \\
\text { https://www.ffg.at/dih/1. }\end{array}$ & KMU & $\begin{array}{l}\text { Verbesserung der innovationsunterstützenden } \\
\text { Infrastruktur }\end{array}$ \\
\hline Ausschreibung2018 & $\begin{array}{l}\text { Zugang zu Expertise, Infrastruktur und } \\
\text { Coaching für Innovationsvorhaben, } \\
\text { inhaltlicher Schwerpunkt liegt auf dem } \\
\text { Einsatz neuer Technologien }\end{array}$
\end{tabular}

Quelle: FFG.

power der KMU in Österreich. Im Herbst 2019 werden in Österreich erstmals Unternehmen unter dem Schwerpunkt „Insight Digitalization“ ihre Türen für Schülerinnen und Schüler in ganz Österreich öffnen. Ein ergänzender „Insight Digitalization" Wettbewerb für Schulen vertieft das Thema auch bereits auf Schulebene.

- Exemplarisch für die strategische Weiterentwicklung ist auch der neue Zugang im Auswahlverfahren für die Vorzeigeregionen Energie, eine Initiative des Klima- und Energiefonds (KLIEN). Im Zentrum stehen eine großfächige Validierung und Demonstration innovativer Energietechnologien in realen Einsatzumgebungen. Dies verlangt langfristige Planbarkeit, um auch die notwendige Unternehmensbeteiligung und Einbindung der Nutzerinnen und Nutzer sicherzustellen.

- Zur Abwicklung der Vorzeigeregion Energie wurde 2017/18 ein neues zweistufiges Auswahlverfahren eingesetzt. Ziel war es, sowohl die notwendige Konzentration der verfügbaren Mittel (kritische Größen) als auch die längerfristige Perspektive (2018-2025) der Initiative operativ handhabbar zu machen. Die Reduktion des Antragsaufwands war zudem ein relevanter Zusatznutzen. Im Rahmen dieses Auswahlverfahrens traten in einer ersten Stufe langfristig ausgerichtete strategische Konzepte miteinander in den Wettbewerb. In der Folge können drei durch eine internationale Jury aus- gewählte Vorzeigeregionen ${ }^{9}$ zu den beurteilten Gesamtkonzepten passende Einzelprojekte einreichen (zweite Stufe).

\section{Veränderungen im Instrumentenportfolio}

Im Sinne der Entwicklung neuer Förderformate wurde das FFG-Portfolio 2018 in mehrere Richtungen erweitert. Zum einen haben die beiden Piloten „Impact Innovation" und "Ideen Lab" ihren Praxistest bestanden und stehen vor der Überführung ins FFG-Portfolio. Zum anderen wurden im Schwerpunkt Digitalisierung neue Akzente Richtung Qualifikation und Diffusion gesetzt (siehe Tabelle 2-8). Im Folgenden werden diese Neuerungen im Detail dargestellt:

- Impact Innovation: Nach zwei Ausschreibungen in der Pilotphase - finanziert aus Eigenmitteln der FFG (1. Ausschreibung) und Mitteln der Nationalstiftung (2. Ausschreibung) - wurde das Format insbesondere in Bezug auf die Zielgruppenansprache und die Bewertungskriterien weiterentwickelt. Derzeit (Stand März 2019) läuft die Evaluierung der Pilotphase, die Zwischenergebnisse sind durchwegs positiv. Eine Überführung in den Regelbetrieb ist somit für das Jahr 2019 geplant.

- Ideen Lab: Die Resonanz auf die erste Ausschreibung, finanziert mit Mitteln der Nationalstiftung, war sehr positiv. Aus 112 Bewerbungen wurden 14 Teilnehmerinnen und 16 Teilnehmer zum Ideen Lab eingeladen. Thema war: „Mensch 4.0? - die Zu-

9 Siehe https://www.vorzeigeregion-energie.at/ 
kunft der Zusammenarbeit Mensch-Maschine“. Die „mitgebrachten“ Ideen wurden am Ende zu fünf Projektskizzen verdichtet, von denen drei eingeladen wurden, einen Vollantrag einzubringen. Inzwischen haben alle drei Projekte eine positive Förderentscheidung erhalten.

- Digital Pro Bootcamps: Adressiert wird der Qualifizierungsbedarf in der Nutzung digitaler Technologien. In den 9-wöchigen Bootcamps sollen Mitarbeiterinnen und Mitarbeiter österreichischer Unternehmen (KMU-Fokus) entlang eines maßgeschneiderten Curriculums zu Digital Professionals qualifiziert werden. Die erste Ausschreibung wurde Mitte November 2018 geöffnet und schließt Ende Mai 2019. Das Format wird vom BMDW finanziert.

- Digital Innovation Hubs (DIH). Dahinter steht eine strukturelle Maßnahme, mit der die innovationsunterstützende Infrastruktur für kleine und mittlere Unternehmen vor Ort verbessert wird. Der inhaltliche Fokus liegt auf dem Einsatz digitaler Technologien. Den KMU sollen mit den DIH Spezialisten zur Seite gestellt werden, die Zugang zu state-of-the-art Expertise, Infrastruktur und Coaching für konkrete Innovationsvorhaben bieten. Die erste Ausschreibung hatte Ende Februar 2019 Einreichschluss, die Hearings finden Ende April statt.

\subsection{Austria Wirtschaftsservice (aws)}

\section{Gesetzliche Grundlagen und Förderzweck}

Die Austria Wirtschaftsservice GmbH (aws) ist die im Eigentum der Republik Österreich befindliche Förderungsbank für die österreichische Wirtschaft. Sie wurde durch das Austria Wirtschaftsservice-Errichtungsgesetz (BGBI. 130/2002) mit Wirksamkeit zum 31.12.2001 am 01.10.2002 mit sondergesetzlicher Re- gelung errichtet. Die Eigentümerinteressen werden vom Bundesministerium für Verkehr, Innovation und Technologie (BMVIT) sowie vom Bundesministerium für Digitalisierung und Wirtschaftsstandort (BMDW) vertreten, welche sowohl die Geschäftsführung als auch den Aufsichtsrat der aws bestellen. Auftraggeber sind die beiden Eigentümer (BMDW und BMVIT) sowie weitere Bundesministerien, Länder und öffentliche Stellen. ${ }^{10}$

Die aws ist gemäß ihrem gesetzlichen Auftrag die zentrale Anlaufstelle zur Förderung von Wachstum und Innovation ( $\S 2$ des aws-Gesetzes). Die dort festgeschriebenen, wesentlichen Aufgaben der aws umfassen die Sicherung und Schaffung von Arbeitsplätzen, die Stärkung der Wettbewerbsfähigkeit unter Berücksichtigung der besonderen Bedeutung der Technologie- und Innovationsförderung sowie Standortsicherung durch die Vergabe und Abwicklung der unternehmensbezogenen Wirtschaftsförderung des Bundes und die Erbringung von Finanzierungs- und Beratungsleistungen zur Unterstützung der Wirtschaft. ${ }^{11}$

\section{Instrumente, Kennzahlen und Schwerpunkte}

Das Förderungsinstrumentarium der aws ist auf eine Verbesserung der Ressourcenbasis von Innovationsund Wachstumsprojekten des Unternehmenssektors mit den beiden Schwerpunkten "Gründung" sowie "Wachstum und Industrie“ ausgerichtet. Die zentralen monetären Instrumente sind:

- aws-Garantien zur Schaffung des Zugangs zu privaten Finanzierungsmitteln für Gründungen, Innovationsprojekte oder Wachstumssprünge, um fehlende oder unzureichende bankmäßige Sicherheiten auszugleichen. ${ }^{12}$

- erp-Kredite zur Ausfinanzierung von Investitionsvorhaben unter Nutzung günstiger Konditionen hinsichtlich Laufzeit und Verzinsung. ${ }^{13}$

- aws-Zuschüsse als Stärkung der Eigenkapital-

10 Vgl. https://www.aws.at/rechtsgrundlage-eigentuemer/

11 Vgl. BMDW und BMVIT (2018).

12 Vgl. https://www.aws.at/foerderungen/aws-garantien-fuer-investitionen-in-oesterreich/

$13 \mathrm{Vgl}$. https://www.aws.at/professionals/zinssaetze-konditionen/kreditkonditionen/ 
Tabelle 2-9: Anzahl der Förderungen und Fördersummen in Mio. €, 2018

\begin{tabular}{|c|c|c|c|c|c|c|c|c|c|}
\hline \multirow{2}{*}{$\begin{array}{l}\text { Programm/ } \\
\text { Instrument }\end{array}$} & \multicolumn{2}{|c|}{ Anzahl Anträge } & \multicolumn{2}{|c|}{$\begin{array}{c}\text { Anzahl } \\
\text { Neubewilligungen }\end{array}$} & \multirow{2}{*}{$\begin{array}{c}\begin{array}{c}\text { Bewilligungsquote } \\
\text { (in \%) }\end{array} \\
2018\end{array}$} & \multicolumn{2}{|c|}{$\begin{array}{l}\text { Neubewilligungen in } \\
\text { Mio. } €\end{array}$} & \multirow{2}{*}{$\begin{array}{r}\text { Barwert Neu- } \\
\text { bewilligungen } \\
\text { in Mio. } €\end{array}$} & \multirow{2}{*}{$\begin{array}{r}\begin{array}{c}\text { Gesamt- } \\
\text { projektkosten } \\
\text { in Mio. } €\end{array} \\
2018\end{array}$} \\
\hline & 2018 & 2017 & 2018 & 2017 & & 2018 & 2017 & & \\
\hline Garantie & 1.755 & 1.666 & 1.165 & 1.114 & 67 & 335,4 & 306,4 & 22,3 & 485,9 \\
\hline Kredit & 1.761 & 1.782 & 1.296 & 1.367 & 77 & 600,0 & 600,0 & 14,5 & 734,6 \\
\hline Zuschuss & 3.681 & 18.917 & 13.800 & 2.932 & $\left.33^{*}\right)$ & $1.241,2$ & 223,6 & $1.240,6$ & $5.559,8$ \\
\hline Beteiligung & 669 & 623 & 44 & 69 & 9 & 12,9 & 15,4 & 0,0 & 36,3 \\
\hline Gesamt & 7.866 & 22.988 & 16.305 & 5.482 & 54 & $2.189,6$ & $1.145,4$ & $1.277,4$ & $6.816,6$ \\
\hline
\end{tabular}

*) Bewilligungsquote bei den Zuschüssen ohne das Programm Beschäftigungsbonus.

Quelle: aws.

basis von Unternehmen bei der Finanzierung von Investitionsprojekten. ${ }^{14}$

- Ergänzend zu Garantien, Krediten und Zuschüssen setzt die aws Instrumente zur Verbesserung der Eigenkapitalausstattung von Unternehmen ein. Dazu werden einerseits öffentliche Mittel als Hebel für die Einbringung privaten Kapitals genutzt, andererseits reduzieren entsprechende Vermittlungsleistungen die Suchkosten zwischen Unternehmen und Investorinnen und Investoren. Förderungsdienstleistungen der aws beschränken sich allerdings nicht auf monetäre Leistungen, sondern beinhalten auch wirkungsverstärkende Awareness-, Beratungs-, Informations- und CoachingLeistungen.

Die Leistungsentwicklung der aws weist für 2018 substanzielle Steigerungen auf, die bei den OutputIndikatoren der Anzahl von Neubewilligungen sowie der erbrachten Finanzierungsleistungen für monetäre Förderungen einen außerordentlichen Anstieg erkennen lassen.

Verbesserungen an bestehenden Dienstleistungen sowie die hohe Investitionsdynamik der österreichischen Unternehmen haben die Vollausschöpfung der Kreditprogramme mit einem Volumen von 600 Mio. $€$ sowie Steigerungen bei den Garantieprogrammen ermöglicht; gerade im Garantiebereich wird aber auch deutlich, dass österreichische Unternehmen bei der Finanzierung von Gründungs-, Innovations- und Wachstumsprojekten häufig auf Unterstützung beim
Zugang zu Kredit- und Kapitalmärkten angewiesen sind. Demgegenüber machen sich beim Zuschussinstrumentarium Anreicherungen des aws-Portfolios um überwiegend temporär angebotene Programme wie insbesondere Beschäftigungsbonus, Investitionszuwachsprämie, Lohnnebenkostenförderung und Risikokapitalprämie in außerordentlichen Steigerungen bei Zusagen, Finanzierungsleistungen und Förderungsbarwerten bemerkbar.

Die gesamte Finanzierungsleistung liegt mit 2.189,5 Mio. € rd. 91,2\% über dem Vergleichswert des Vorjahres, wobei der Kreditbereich ein gleichbleibendes Volumen, die Beteiligungen einen Rückgang um $16,2 \%$ und die Garantien eine Steigerung um 9,5\% erreichten. Gleichzeitig ermöglichten temporär angebotene und breitenwirksame Zuschussprogramme (vor allem der Beschäftigungsbonus) eine beinahe Versechsfachung des Zuschussvolumens, d.h. einen Anstieg von 223,7 Mio. € im Jahr 2017 auf 1.241,2 Mio. $€$ im Jahr 2018. Dementsprechend zeigt auch der Barwert vergebener Förderungen einen Anstieg um 395,3\% auf 1.277,4 Mio. $€$ (siehe Tabelle 2-9).

In Hinblick auf die sektorale Verteilung der Förderungsbegünstigten entfällt der größte Anteil der neu bewilligten Mittel auf die Sachgüterproduktion; bereinigt um nicht einer Branche direkt zugeordnete Fälle kommt sie 2018 auf einen Anteil von $45 \%$, was auch die konjunkturelle Entwicklung mit außerordentlich starker Investitionsdynamik der Industrie 
widerspiegelt. Leichte Steigerungen zeigt auch der Handel, der einen Anteil von $16 \%$ aufweist, während im Gegenzug der Anteil neu bewilligter Mittel für den Dienstleistungssektor auf $20 \%$ zurückgegangen ist.

Gemessen an Förderungszusagen sind kleine Unternehmen mit einem Anteil von $80 \%$ die mit Abstand stärkste Gruppe unter den Geförderten. Trotz kleinerer Projekte entfallen auf EPU, Kleinstunternehmen und andere Kleinunternehmen mit Anteilen von $10 \%, 16 \%$ und $23 \%$ auch in Hinblick auf den Mitteleinsatz fast die Hälfte der eingesetzten Förderungsmittel, während Mittelunternehmen und Großunternehmen hier Anteile von 20 \% bzw. $32 \%$ erreichen.

Im Bundesländervergleich zeigt sich eine traditionell starke Stellung der beiden Bundesländer Oberösterreich und Niederösterreich, auf die mit $27 \%$ bzw. $19 \%$ insgesamt fast die Hälfte der Finanzierungsleistung der aws des Jahres 2018 entfällt. Weitere $15 \%$ entfallen auf Wien und immerhin $12 \%$ auf die Steiermark, zwei Bundesländer, die 2018 eine besonders starke Dynamik aufweisen. Die Bundesländer Kärnten, Salzburg und Tirol verzeichnen Anteile von jeweils rd. $7 \%$, Vorarlberg kommt - wie im Vorjahr - auf einen Anteil von $3 \%$, während das Burgenland im Vorjahresvergleich die höchste Steigerung aufweist, um nunmehr rd. $2 \%$ der in Österreich erbrachten Gesamtfinanzierungsleistung zu erreichen. Insgesamt zeigt die regionale Förderungsverteilung 2018 eine ausgewogenere Struktur als in den Vorjahren, was auf die Breitenwirksamkeit temporär eingesetzter Zuschussprogramme zurückzuführen ist.

\section{Strategische Entwicklungen}

Die strategische Ausrichtung des Instrumentenportfolios der aws stand während der letzten Jahre im Zeichen kontinuierlicher Weiterentwicklungen, die sowohl auf eine Erhöhung der Wirksamkeit bei geförderten Unternehmen als auch auf Vereinfachungen des Zugangs zu Förderungen sowie die Reduktion des administrativen Aufwands abzielen. Abseits der 2018 expansiv wirkenden Sondereffekte temporärer Maßnahmen beruht die Weiterentwicklung des Portfolios nur bedingt auf neu eingeführ- ten Programmen. Vielmehr stützt sie sich auf zahlreiche Adaptierungen von Bedingungen und Konditionen der Förderungen, die insgesamt eine Erhöhung der Attraktivität von aws-Förderungen zur Finanzierung von Innovations- und Wachstumsprojekten zur Folge haben und gleichzeitig heterogenen Finanzierungserfordernissen der Unternehmen durch einen abgestimmten Instrumenteneinsatz gerecht werden. Das gilt insbesondere für die Zielgruppe der innovativen Start-ups, für die eine projektbezogene öffentliche Unterstützung von F\&E-Aktivitäten allein oft nur bedingt zur Schaffung einer den Wachstumsbestrebungen des Unternehmens angemessenen Finanzierungsbasis beitragen kann. Zudem gilt es gerade im Gründungsbereich, oft auch die Informations- und Wissensbasis mit geeigneten Förderungsmaßnahmen zu adressieren.

Dementsprechend setzt die aws im Gründungsbereich einen mittlerweile gut ausgereiften und abgestimmten Mix an Instrumenten ein, der nicht nur den Zugang zu Fremdkapital über Garantien und erp-Kredite erleichtert, sondern dem Verlauf der Unternehmensentwicklung folgende typische Mängel in der Eigenkapitalausstattung bzw. hinsichtlich der Zugänglichkeit von privatem Eigenkapital adressiert:

Den Eckpfeiler einer Pipeline öffentlicher Unterstützung für hochinnovative Gründungen in sehr frühen Phasen - die durch ein entsprechend hohes Risiko und hohe Hürden beim Zugang zu privatem Eigenkapital charakterisiert sind - bildet das als bedingt rückzahlbarer Zuschuss ausgestaltete Seed-financing (Seed). Die für die Jahre 2017-2019 budgetär verankerte Seed-Erweiterung erlaubte 2018 eine Ausweitung von PreSeed und Seed auf 54 Zusagen mit einer Finanzierungsleistung von 18,7 Mio. $€$; Impulse für die Frühphasenfinanzierung in dieser GröBenordnung sind auch für 2019 vorgesehen.

Die Seed-Familie bildet einen zentralen Ausgangspunkt öffentlicher Unterstützung, indem innovative Gründungsvorhaben nach erfolgreicher Qualifizierung in einem kompetitiven Auswahlverfahren mittels Zuschüsse und Beratungsleistungen sowohl in ihrer Finanzierungsbasis als auch hinsichtlich ihrer Wis- 
sensbasis gestärkt werden. Im Rahmen der Beratungsleistungen wird u.a. auch der Innovationsprozess dieser Startups durch eine individuelle Strategie für geistige Eigentumsrechte unterstützt. ${ }^{15}$ In Ergänzung zum Hochtechnologiesegment stehen für innovative Vorhaben der Kreativwirtschaft mit impulse XS und impulse $X^{16}$ weitere Zuschussinstrumente zur Verfügung, wobei ebenfalls ein selektives Jurysystem zur Anwendung kommt. Im Jahr 2018 wurden dabei 53 Projekte mit einer Finanzierungsleistung von 4,1 Mio. $€$ unterstützt. Für innovative neuartige Konzepte von Inkubatoren und deren inkubierten Startups stehen 2018/19 ebenfalls noch 1,5 Mio. $€$ zur Verfügung.

Wesentliches Ziel der aws-Förderungsstrategie für das Segment innovativer Gründungen ist aber auch die sukzessive Erweiterung ihrer Kapitalbasis um privates Eigenkapital. Dazu dienen sowohl monetäre Förderungen, die eine Risikoteilung zwischen öffentlicher Hand und privaten Investorinnen und Investoren ermöglichen bzw. Anreize für die Ausweitung privaten Kapitaleinsatzes im Gründungsbereich setzen, als auch Instrumente zur Reduktion der Suchkosten. Die aws setzt auf unterschiedliche Förderungsansätze zur Lösung spezifischer Problemlagen der Finanzierungsbasis oder der Wissensbasis, wobei eine Teilnahme in zeitlicher Abfolge oder kombiniert in Frage kommt:

- Der mit 68,5 Mio. $€$ dotierte aws-Gründerfonds ${ }^{17}$ stellt seiner Zielgruppe Eigenkapital zu marktkonformen Konditionen zur Verfügung, wobei die Bandbreite der Investments von 0,1 Mio. $€$ bis zu 3 Mio. $€$ reicht. Der Fonds ermöglicht durch CoInvestments eine Risikoteilung mit bzw. eine Hebelung von privatem Kapital. Bisher konnten bereits 30 Portfoliounternehmen mit einem Gesamt- kapital von 153,7 Mio. $€$ - davon 124,7 Mio. $€$ private Co-Investments - finanziert werden.

- Das von der aws betriebene Matching-Service i2 Business Angels ist eine auf die Reduktion von Suchkosten zielende Vermittlungsdienstleistung für junge Unternehmen und private Investorinnen und Investoren. Ein kontinuierlicher Ausbau dieser Plattform auf derzeit rd. 350 teilnehmende Business Angels und etwa 800 Unternehmensprojekte ermöglicht jährlich rd. 20 erfolgreiche Vermittlungen. Damit wird innovativen Gründungen aber nicht nur privates Kapital leichter zugänglich gemacht, sondern auch ein Zugang zu Netzwerken und zu Expertise erfahrener Angels ermöglicht.

- Als wichtige Ergänzung der Business-Angel-Aktivitäten hat die aws gemeinsam mit dem Europäischen Investitionsfonds (EIF) den aws-Business Angel Fonds ${ }^{18}$ eingerichtet. Der mit 32,5 Mio. $€$ dotierte Fonds verstärkt nach dem Prinzip von CoInvestments die Bereitstellung von Eigenkapital durch Business-Angels in österreichischen Startups und bewirkt damit eine weitere Belebung der heimischen Business-Angel-Szene.

- Die 2011 erstmals eingeführte Venture Capital Initiative $(\mathrm{VCl})^{19}$ ist als fund-of-fund-Programm zur Mobilisierung privaten Kapitals konzipiert und stimulierte mit einer aws-Beteiligung von rd. 37 Mio. $€$ bereits über 100 Mio. $€$ an Kapital für Gründungsprojekte und erste Wachstumsphasen innovativer Start-ups; erst im Oktober 2018 wurde ein weiterer Call mit 10 Mio. $€$ dotiert.

\section{Veränderungen im Instrumentenportfolio}

Bereits im Jahr 2017 vorgenommene Weichenstellungen zur Einführung des Beschäftigungsbonus haben für 2018 annähernd eine Verdopplung der aws-Finan-

15 Nutzungsmuster von Patenten werden beispielsweise heute über das ursprüngliche Verwertungsverbot hinaus verwendet, um Investoren anzuziehen, Einkommen aus Auslizenzierung zu generieren, - sei es aus strategischen Gründen (Sperrpatente, also nicht vom eigenen Unternehmen genutzte Patente, die aber dem Wettbewerb Handlungsspielraum nehmen), oder sei es aus marketingmäßigen Gründen - um diese zur Erstellung von Normen/Standards zu nutzen oder um (F\&E-)Kooperationen zu ermöglichen. 
zierungsleistung auf rd. 2,2 Mrd. € ermöglicht. Für das Jahr 2019 sieht die aws als Förderungsbank der Republik vor, der heimischen Wirtschaft eine Finanzierungsleistung von mehr als $1 \mathrm{Mrd}$. $€$ in Form von Garantien, Krediten, Zuschüssen, Beteiligungen sowie Service und Beratung zu erbringen. Während bei breitenwirksamen Zuschussprogrammen angesichts zeitlicher Befristungen und budgetärer Beschränkungen in Programmen wie Beschäftigungsbonus, Risikokapitalprämie und KMU-Investitionszuwachsprämie eine insgesamt rückläufige Förderungstätigkeit im Zuschussbereich abzusehen ist, lässt sich für Garantien und Kredite ein weiterhin hohes Förderungspotenzial realisieren.

Substanzielle Beiträge dazu liefern insbesondere die Anfang 2017 gestarteten und 2018 fortgeführten Anpassungen der Förderungsbedingungen bei Garantien und Krediten. Dazu gehören beispielsweise erhöhte betragliche Obergrenzen, gesteigerte Risikobereitschaft sowie reduzierte Bearbeitungs- und Garantieentgelte oder auch die Vereinheitlichung der erp-Kreditrichtlinien in einem neuen Wachstums- und Innovationsprogramm bei gleichzeitiger Beibehaltung des Kreditzinssatzes auf einem historischen Tiefststand. Im Fokus stehen dabei gleichermaßen innovative Investitionsprojekte bereits etablierter Unternehmen wie auch jene von Neugründungen und jungen Unternehmen; für letztere gelten weiterhin die Sonderkonditionen des Gründungskleinkredits mit einem Fixzinssatz von $0,5 \%$ über die gesamte Laufzeit.

Im Bereich der Netzwerk- und Matchmaking-Programme erfolgte 2018 ein weiterer Ausbau des in Kooperation mit der FFG angebotenen internationalen Netzwerkprogramms "Global Incubator Network" (GIN). Mit der im September von der Nationalstiftung für Forschung und technologische Entwicklung zugesagten Erhöhung des Budgets soll - neben einer Intensivierung der Kontakte mit den bereits bestehenden Kooperationspartnern - mit China (Region Shanghai) auch ein neuer Zielmarkt erschlossen wer- den. Ein weiteres Netzwerkprogramm, das 2016 gestartete Industry-Startup.Net der aws, bietet Startups eine Vernetzungsmöglichkeit mit etablierten Unternehmen (Corporates) - mit dem Ziel, durch die Vermittlung von Kooperationen u.a. besseren Marktzugang für die Start-ups und die raschere Nutzung innovativer Entwicklungen für die Corporates zu ermöglichen. Mit mehr als 250 Mitgliedern ist IndustryStartup.Net bereits jetzt die größte Plattform für die Vermittlung von Kooperationen in Österreich in dieser Art. Über 20 bereits vermittelte Kooperationen zeigen das große Potential dieses Programms, das 2019 durch neue Services weiter ausgebaut wird.

Sowohl 2018 als auch 2019 spielen Produktneueinführungen bei aws-Förderungsmaßnahmen eine eher geringe Rolle; erwähnenswert ist hierbei der mit 3 Mio. $€$ von der Nationalstiftung dotierte Digital Innovation Call, der sich an Start-ups mit digitalen Produkten und Dienstleistungen im Bezugsrahmen der 17 Sustainable Development Goals der Vereinten Nationen wendet. Im Vordergrund steht die Fokussierung auf bewährte Instrumente unter Berücksichtigung der Erfahrungen, die in Pilotphasen oder anlässlich erster Erprobung jüngst eingeführter Instrumente gemacht wurden. Letzteres gilt beispielsweise für IP.Market (das Hilfestellungen zur externen kommerziellen Verwertung und Marküberleitung von Innovationen und Technologien anbietet) und License. IP (das KMU bzw. Neugründungen bei der Suche und Lizensierung technologischer Lösungen unterstützt), zwei Service- und Beratungsleistungen der IPR-Programmfamilie, zu der auch IP.Coaching (ein Programm zur Entwicklung und Implementierung einer IP-Strategie technologieorientierter KMU) gehört, das 2018 in adaptierter Form weitergeführt wurde.

Um sich im großen, hier nur auszugsweise dargestellten Instrumentenportfolio der aws zurechtzufinden und sich schnell und unbürokratisch über passende Fördermöglichkeiten informieren zu können, hat die aws im Jahr 2018 den DigiCoach, eine Online-Beratungsplattform, ins Leben gerufen. ${ }^{20}$ Mit Hil-

20 Vgl. https://www.aws.at/foerderbaum/aws-digicoach/ 
fe des DigiCoach erhalten Start-ups, KMU, Investorinnen und Investoren nach Beantwortung weniger Fragen (z.B. zur Branche und dem Tätigkeitsbereich des Unternehmens) maßgeschneiderte Informationen über die zur Verfügung stehenden aws-Förderoptionen. Darüber hinaus können mit dem Fördermanager ${ }^{21}$ nach erfolgter Registrierung die jeweiligen Förderprodukte ausgewählt und online beantragt werden. Der Fördermanager assistiert und begleitet das Unternehmen durch die einzelnen Abschnitte des Antrags und gibt einen Überblick über erforderliche Unterlagen und anstehende Termine. Mit den
Pitch-your-idea-Beratungsevents ${ }^{22}$ hat die aws kürzlich ein weiteres Format implementiert, bei dem Start-ups um die passende Förderung pitchen können. Nach einer fünfminütigen Präsentation der Geschäftsidee und einer folgenden Fragenrunde zum Vorhaben und zur Finanzierung bekommen die Unternehmen ein unmittelbares Feedback, welches Förderungsprogramm am besten zur Geschäftsidee passt. Förderprogramme und Beratungsleistungen sind einem ständigen Wandel ausgesetzt - die digitale Transformation kann dabei zweifellos als wesentlicher Treiber der Veränderung identifiziert werden.

21 Vgl. https://www.aws.at/service/web-services/aws-foerdermanager/

22 Vgl. https://www.aws.at/foerderbaum/pitch-your-idea/ 
3. Review
FTI-Strategie 2020

3. Review
FTI-Strategie 2020

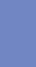


Die österreichische Bundesregierung hat am 08.03.2011 erstmals eine Strategie für Forschung, Technologie und Innovation (FTI-Strategie) ${ }^{1}$ beschlossen, die vom Bundeskanzleramt und dem Bundesministerium für Finanzen, dem Bundesministerium für Unterricht, Kunst und Kultur, dem Bundesministerium für Verkehr, Innovation und Technologie, dem Bundesministerium für Wissenschaft und Forschung sowie dem Bundesministerium für Wirtschaft, Familie und Jugend unter Einbeziehung sämtlicher Konsultationen im Vorfeld entwickelt wurde. So fand im Zuge dessen in den Jahren 2007-2008 ein österreichweiter Forschungsdialog ${ }^{2}$ statt, welcher das Ziel hatte, diverse Erfahrungen, Ideen und Visionen der gesamten Forschungscommunity für die Entwicklung des österreichischen Forschungsstandorts bis $2020 \mathrm{zu}$ sammeln. Des Weiteren wurde internationale Expertise in Form eines CREST-Reviews $2008^{3}$ eingeholt, es folgte eine Systemevaluierung mit einem Ergebnisbericht in $2009^{4}$ und der Rat für Forschung und Technologieentwicklung selbst erstellte eine Strategie $2020^{5}$ ebenfalls in 2009. Zehn Jahre später, 2017/18, führte die OECD einen Review of Innovation Policy in Österreich durch, dessen Ergebnisse erst jüngst im Rahmen der Europatagung 2018 präsentiert wurden.

Zur Umsetzung der FTI-Strategie wurde laufend berichtet. So finden sich in den jährlich erscheinenden Forschungs- und Technologieberichten Darstellungen zu den Zielen und Maßnahmen der FTI-Strategie, sowie zur Arbeit der Task Force FTI und der Arbeitsgruppen - aufbereitet für das Parlament wie auch für die Bürgerinnen und Bürger. Ergänzend wurde auch immer auf die Umsetzung besonderer Ressortvorhaben eingegangen. Ebenso folgten jährlich Umsetzungsberichte über Teilstrategien, wozu sich

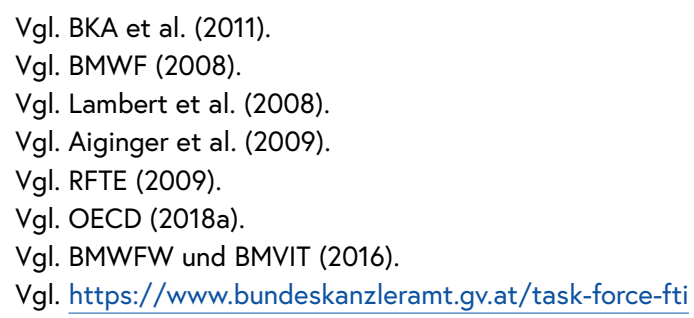

die Bundesregierung - wie z.B. bei der Open Innovation Strategie - gegenüber dem Parlament verpflichtet hatte. Schließlich wurde im Rahmen des Forschungs- und Technologieberichts 2016 auch ein Mid-Term Review ${ }^{7}$ der FTI-Strategie durchgeführt.

Mit diesem Bericht liegt erstmals ein Gesamt-Review der FTI-Strategie - alle Jahre der FTI-Strategie umfassend und aus externer Expertinnen- und Expertensicht erstellt - vor. Basierend auf quantitativen und qualitativen Analyseverfahren werden die Umsetzung und die Erreichung der Ziele der FTI-Strategie jeweils untersucht. Als Grundlage dient hierfür auch eine seitens aller an der FTI-Strategie beteiligten Ressorts erstellte Maßnahmenliste, welche wiedergab, welche Maßnahmen seitens der Ressorts bereits umgesetzt wurden bzw. welche Maßnahmen bislang noch nicht umgesetzt wurden oder (angesichts z.B. veränderter Rahmenbedingungen) nicht mehr relevant waren bzw. sind. Darüber hinaus wurden Gespräche mit den Mitgliedern der Task Force $F T{ }^{8}$ geführt, um auch explizit ihre Sicht hinsichtlich der Entwicklung des österreichischen Innovationsystems in das Review und in diesem Kontext auch den Stellenwert der FTI-Strategie reflektierend aufnehmen zu können.

Die folgenden Abschnitte dienen somit dazu, die Ziele und Maßnahmen der FTI-Strategie hinsichtlich ihrer Umsetzung darzulegen und zu analysieren. Zum Schluss hin erfolgt zudem eine Kurzzusammenfassung der wesentlichsten Befunde des OECD Review of Innovation Policy (2018), bevor ein Gesamtresümee über die großen Entwicklungslinien des österreichischen Innovationssystems gezogen wird. Die Befunde sollen mitunter als Input für die nächste Strategie, die FTI-Strategie 2030, dienen. 


\subsection{Einbettung der FTI-Strategie im Kontext nationaler und internationaler Entwicklungen}

Die Entwicklung der FTI-Strategie von 2011 und damit verbunden die Formulierung ambitionierter Ziele begann demnach, als nach einem Ende der Finanz- und Wirtschaftskrise allgemein noch von wachsenden, dynamischen Entwicklungspfaden ausgegangen wurde. ${ }^{9}$ Allerdings blieben in den darauf folgenden Jahren die budgetären Mittel hinter den ursprünglichen Erwartungen zurück. Zusätzlich zu dieser bereits im Midterm Report zur Umsetzung der FTI-Strategie in 2016 wahrgenommenen Änderung, hat sich insbesondere seit 2015 eine Krise der Europäischen Union zugespitzt, deren Entwicklung bzw. längerfristigen Aus- wirkungen auch heute noch nicht abschätzbar sind. Nichtsdestotrotz oder gerade deswegen waren die Bestrebungen auf europäischer Ebene ambitioniert, den Europäischen Forschungsraum weiterzuentwickeln. Hierzu zählen die Kommunikation der Europäischen Kommission 2012, die fünf Schwerpunktbereiche und ein Konzept zur Vollendung des EFR im Jahr 2014 definiert $^{10}$, der Start von Horizon 2020 im Jahr 2014 und die damit verbundene Orientierung der Forschungspolitik an gesellschaftlichen Herausforderungen, sowie die Verabschiedung der Europäischen ERA Roadmap 2015. Etwa ein Jahr später, im April 2016, beschloss dann der Ministerrat die österreichische ERA-Roadmap mit einem verbindlichen Aktionsplan. All diese genannten Entwicklungen waren nicht vorherzusehen und waren daher auch nicht Teil der FTI-

\section{Abbildung 3-1: Die FTI-Strategie 2020 sowie weitere nationale Teilstrategien im Kontext europäischer}

Entwicklungen

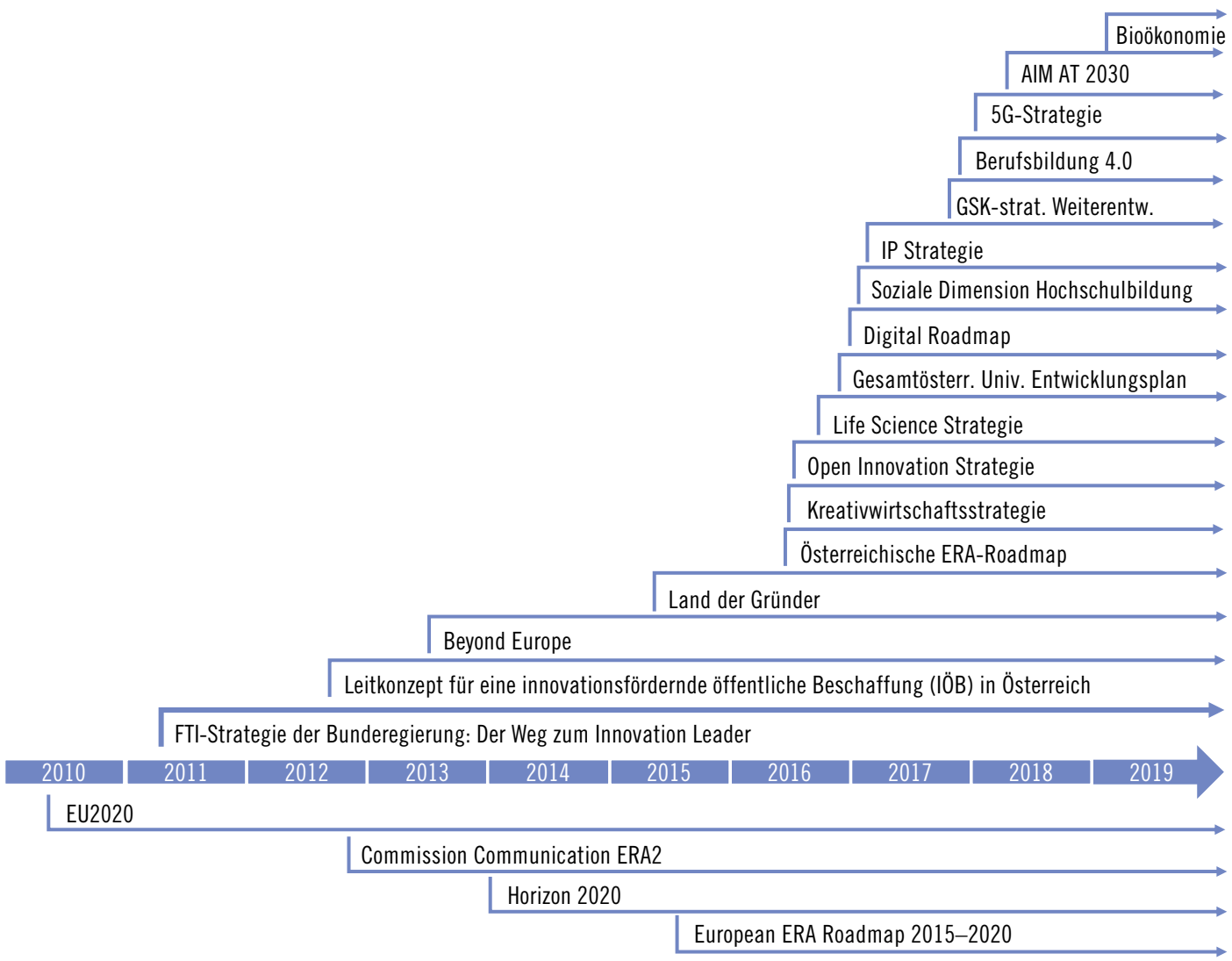

Quelle: Darstellung Technopolis.

9 Ebenda.

10 Vgl. Europäische Kommission (2012). 
Strategie von 2011, wurden im Laufe der Zeit aber aufgegriffen und mittels jeweiliger Teilstrategie seitens der österreichischen FTI-Politik adressiert.

\section{Die Rolle der nationalen Teilstrategien}

Tatsächlich wurden von 2011 an bis heute insgesamt 17 Teilstrategien vor dem Hintergrund der FTI-Strategie 2020 - Abbildung 3-1 illustriert dies - verabschiedet. Aktuell ist eine nationale Strategie zu Artificial Intelligence als Teil einer breiteren Digitalisierungsstrategie in Ausarbeitung.

Über all die Jahre galt die FTI-Strategie 2020 dabei als Orientierungsrahmen, waren Ziele und Maßnahmen hier formuliert, und wurden diese dann durch weitere, neue, aktuelle Themen via Teilstrategien ergänzt. Zu den Themen, die hierbei aufgegriffen wurden, zählten z.B. Open Innovation, Life Sciences, geistiges Eigentum (IP), oder die Geistes-, Sozialund Kulturwissenschaften. Die Teilstrategien füllten damit für die österreichische FTI-Politik strategisch wichtige Lücken und sie ergänzten die FTI-Strategie damit auch aus gesamtsystemischer Sicht. Auf nationaler Ebene waren die Teilstrategien von der Orientierung an gesellschaftlichen Herausforderungen, einer vergleichsweise hohen Bereitschaft für offene Beteiligungsverfahren, wohl aber auch von der breiten politischen Anerkennung der Relevanz dieses Politikbereichs, die sich erstmals in der FTI-Strategie 2020 manifestierte, geprägt.

Die ambitionierte und sektorübergreifende Vision, Innovation Leader zu werden, diente über all die Jahre als kohärenter Rahmen, welcher maßgebend für die damit einhergehenden Ziele und Maßnahmen war. Insgesamt wurde so die Überzeugung gelebt, dass der Bereich Forschung, Technologie und Innovation sich nach den Besten (im internationalen Kontext) zu orientieren hat und sich daher weiterentwickeln muss. Damit hat die Vision, welche zugleich das Hauptziel der FTI-Strategie 2020 war, ihr Ziel erreicht - nämlich als klares, beständiges Signal über alle anderen Teilziele der österreichischen FTI-Politik zu wirken - auch wenn gemäß des European Innovation Scoreboard es nicht gelang, in die Gruppe der Innovation Leader vorzustoßen.

\section{Die Rolle der Task Force FTI und das Monitoring}

Zwecks besserer Koordination der zahlreichen Politikfelder, welche die FTI-Strategie 2020 berühren, wurde eine interministerielle Task Force FTI unter dem Vorsitz des Bundeskanzleramts (BKA) auf hoher Beamtinnen- und Beamtenebene ins Leben gerufen. Die Aufgabe der Task Force FTI ist die Begleitung der Umsetzung der FTI-Strategie wie auch teils deren Konkretisierung, sowie die Abstimmung der Aktivitäten der mit der Umsetzung der FTI-Strategie befassten einzelnen Ressorts in strategischer wie auch systemorientierter Hinsicht. ${ }^{11}$ Damit hatte die Task Force FTI auch positive Effekte auf die interministerielle Kooperation und stärkte die Vertrauensbildung auf Governance-Ebene. Außerdem wurden in speziellen Themenbereichen Arbeitsgruppen eingerichtet, um die interministerielle Koordination auch auf operativer Ebene noch zusätzlich zu unterstützen. Manche der in Abbildung 3-1 angeführten Strategien sind sogar auf dieser Ebene entstanden, d.h. aus den Arbeitsgruppen hervorgegangen.

Folgende Arbeitsgruppen (AG) unterstützten die Umsetzung der FTI-Strategie auf Governance-Ebene:

- AG1: Humanpotenziale

- AG2: FTI-Schwerpunkte: Klimawandel/ Knappe Ressourcen

- AG3: FTI-Schwerpunkt: Lebensqualität und demographischer Wandel

- AG 4: Forschungsinfrastruktur

- AG 5: Wissenstransfer und Gründungen

- AG 6: Unternehmensforschung

- AG 7: Europa und Internationales

- 7a: Internationalisierung und FTI-Außenpolitik

- 7b: Aktionsplan Österreich und der Europäische

Wissensraum 2020

- AG 8: Internationale Rankings

Zusätzlich zu diesen neun von Beginn an eingerichte-

11 Vgl. https://www.bundeskanzleramt.gv.at/task-force-fti 
ten Arbeitsgruppen wurden im Laufe der Zeit noch weitere Arbeitsgruppen gebildet, wie z.B. eine begleitende Arbeitsgruppe zum OECD-Review. Einige dieser kurzfristig, nach Bedarf etablierten Arbeitsgruppen wurden mittlerweile auch wieder aufgelöst bzw. sind heute nicht mehr aktiv.

Die Umsetzung der Maßnahmen zur Erreichung der in der FTI-Strategie von 2011 postulierten Ziele obliegt den zuständigen Ressorts. Funktional in die Umsetzung eingebunden sind natürlich auch die zahlreichen Akteure des österreichischen FTI-Systems, insbesondere die Förderagenturen und die Forschungs- und Hochschuleinrichtungen. In diesem Sinne ist die Umsetzung der Strategie von den zuständigen Ministerien getragen, in ihrer nachhaltigen Wirkung lebt diese jedoch, wenn sie - ausgehend von einem auf der Governance-Ebene gesetzten Impuls - von den im nationalen Innovationssystem agierenden Stakeholdern aufgegriffen wird und dann die Ziele auch von diesen umgesetzt werden. So haben z.B. die Universitäten oder die ÖAW Ziele der FTIStrategie in ihren jeweiligen Leistungsvereinbarungen verankert. Wie die Berichte über die Teilbereiche der Strategie infolge zeigen, ist gerade dieser nachhaltige, systemverändernde Effekt in wesentlichen Punkten erzielt worden.

Die FTI-Strategie wurde auch einem Monitoring unterzogen. So wurde der Rat für Forschung und Technologieentwicklung damit betraut, alljährlich einen Bericht zur wissenschaftlichen und technologischen Leistungsfähigkeit Österreichs zu erstellen. Im Fokus stand dabei eine Bewertung der Performance Österreichs in Bezug auf die Zielsetzungen und Maßnahmen der FTI-Strategie per se sowie die Leistungen des österreichischen Wissenschafts- und Innovationssystems im internationalen Vergleich, vorrangig gemessen an den Leistungen der Innovation Leader-Ländern. ${ }^{12}$

\subsection{Neuerungen im Bildungssystem}

FTI-Strategie 2020 mit der Vision:

"Talente entfalten, Leidenschaft wecken - Das Bildungssystem nachhaltig umgestalten“"

Ein gutes Bildungssystem über alle Stufen (inklusive Weiterbildung) ist die Basis eines aufstrebenden Innovationsystems, insbesondere wenn es darum geht, als Hochlohnland mit kleiner Größe wie Österreich im internationalen Wettbewerb erfolgreich zu sein. Gut ausgebildete Menschen bestimmten das Potential, nicht nur neues Wissen und neue Technologien zu generieren, sondern dieses auch umzusetzen. Die FTI-Strategie hat sich damit - wie folgt im Einzelnen dargestellt - eine Reihe von Zielen und Maßnahmen gesetzt, um das österreichische Bildungssystem nachhaltig zu verbessern.

\subsubsection{Strukturreformen des Bildungssystems und verbesserte Bildungsübergänge}

Bei der Verabschiedung der FTI-Strategie in 2011 wurden die frühe Selektion nach Ausbildungs- und Bildungssträngen und deren Auswirkungen auf Studien- und Berufswahl problematisiert. Im Gegensatz zum OECD-Durchschnitt von $61 \%$ haben damals in Österreich nur $39 \%$ eines Altersjahrgangs eine Hochschulzugangsberechtigung erworben. Des Weiteren wurde auf das ausgeprägte Gender-Ungleichgewicht in den technisch-naturwissenschaftlichen Ausbildungen einerseits und im sprachlich-pädagogischen Bereich andererseits hingewiesen, das zu einer „Vermännlichung“ bzw. „Verweiblichung“ ganzer Berufsfelder führe.

Zur Behebung der dargestellten Mängel wurden daher Reformen vorgeschlagen, die auf „die Entschärfung der sozialen Selektivität, die bessere Durchlässigkeit zwischen den Bildungsgängen bzw. -wegen, eine durchgängige Qualitätssteigerung im Unterricht und in der Hochschullehre, die verbesser-

12 Vgl. https://www.rat-fte.at/leistungsberichte.html 
te Integration von Zuwandernden und einen Ausgleich der Gender-Ungleichgewichte in der Forschung" abzielen. „Die Quote der Schulabbrecherinnen und -abbrecher soll bis 2020 auf 9,5\% reduziert werden. Die Maturantenquote soll bis 2020 auf $55 \%$ einer Alterskohorte angehoben werden." 13

Als Maßnahmen ${ }^{14}$ wurden daher in der FTI-Strategie 2020 vorgeschlagen:

- Verbesserte Frühförderung durch verpflichtendes, kostenfreies Kindergartenjahr (halbtags) für Fünfjährige

- Erhöhung der Anzahl an Ganztagesschulen und Ausweitung der bedarfsorientierten Ganztagesbetreuung

- Ausbau der Berufsmatura für Lehrlinge und der Berufsreifeprüfung für Erwachsene als Studienzugang

- Einführung von österreichweiten Bildungsstandards und teilstandardisierten Abschlussprüfungen

- Weiterentwicklung des Schulsystems im Hinblick auf bessere individuelle Förderung und eine Erhöhung der Durchlässigkeit insbesondere im Bereich der Sekundarstufe 1

- Stärkung der Humanpotenziale im Bereich Mathematik, Informationstechnologie, Naturwissenschaft, Technik durch gezielte Förderung im (vor-) schulischen Unterricht und an universitären Einrichtungen

- Ausbau der Berufsorientierung und der Studienberatung (z.B. Studienchecker, Studieren probieren) bereits in der Schule

\section{Verbesserte Frühförderung}

Die Expertinnen und Experten der Länder, des „Charlotte-Bühler-Instituts für praxisorientierte Kleinkindforschung" und des Bundesministeriums für Unterricht, Kunst und Kultur haben 2009 gemeinsam einen bundesländerübergreifenden Bildungsrahmenplan für Kinder im Alter von null bis sechs Jahren entwickelt. ${ }^{15}$ Alle Bundesländer haben dem Einsatz des Bildungsplans zugestimmt. Er gibt sämtlichen Einrichtungen in allen Bundesländern eine Anleitung zur bestmöglichen Förderung der Kinder durch die Kindergartenpädagoginnen und -pädagogen. Im Rahmen von 15a-Vereinbarungen zwischen Bund und Ländern wurden Kennwerte festgelegt und ein Controllingsystem aufgebaut.

Konkrete Maßnahmen beziehen sich auf:

- Sprachstandsfeststellung im Kindergarten

- Eine halbtägige kostenlose und verpflichtende Förderung im letzten Kindergartenjahr im Ausmaß von 20 Stunden an mindestens vier Tagen in der Woche

- Die Gestaltung des Übergangs vom Kindergarten in die Schule

- Den Ausbau des institutionellen Kinderbetreuungsangebots für unter 3-jährige Kinder

- Die Flexibilisierung und Erweiterung der Öffnungszeiten im Sinne einer besseren Vereinbarkeit von Familie und Beruf

- Die Einführung neuer kompetenzorientierter Lehrpläne an der Bundesbildungsanstalt für Elementarpädagogik, Entwicklung eines Kollegs mit Zusatzqualifikation für Hortpädagogik

- Die verbindliche Vermittlung von grundlegenden Werten und Prinzipien im Kindergarten, wie z.B. Gleichberechtigung, Toleranz und Partizipation

Gemäß Kindertagesheimstatistik der Statistik Austria wurden die Krippen und Kleinkindbetreuungseinrichtungen seit $2010 / 11$ von 1.200 auf ca. $2.100 \mathrm{im}$ Jahr 2017/18 massiv ausgebaut. Mit dem Ausbau der Krippen und Kleinkindbetreuungseinrichtungen ging auch eine deutliche Steigerung der Betreuungsquote einher. Lag der Anteil der 0- bis 2-jährigen Kinder in institutionellen Kinderbetreuungseinrichtungen 2010 österreichweit bei $17,1 \%$, so liegt er 2017 bei $26,1 \%$ und damit um 9 Prozentpunkte höher. Die Anzahl der

\footnotetext{
13 Vgl. BKA et al. $(2011,16)$.

14 Vgl. BKA et al. $(2011,17)$.

15 Vgl. Charlotte Bühler Institut (2009).
} 
Kindergärten blieb im selben Zeitraum nahezu unverändert (2010/11: 4.690, 2017/18: 4.570). Die Betreuungsquote der 3- bis 5-jährigen Kinder stieg dennoch seit 2010 bis 2017 von 90,7\% auf 93,4\% an. Dennoch gab es österreichweit durchschnittlich 15,9 Schließtage in Krippen und Kleinkindbetreuungseinrichtungen und 26,5 Schließtage in Kindergärten im Jahr 2017, was durchaus eine Herausforderung für die Vereinbarkeit von Beruf und Familie darstellen kann. ${ }^{16}$

\section{Erhöhung der Anzahl an Ganztagesschulen und}

Ausweitung der bedarfsorientierten Ganztagesbetreuung

Von 2011 bis 2018/19 bestanden Fördermöglichkeiten aus 15a-Vereinbarungen zum Ausbau der ganztägigen Schulformen. 2018 lag die ganztägige Betreuungsquote bei rd. $26 \%$, rd. 177.600 Schülerinnen und Schüler besuchten eine ganztägige Schulform. Im Anschluss daran erfolgt ein bedarfsorientierter Ausbau mit BIG-Mitteln; bis 2032/33 wird eine Betreuungsquote von $40 \%$ angestrebt.

\section{Ausbau der Berufsmatura für Lehrlinge und der Berufsreifeprüfung für Erwachsene als Studienzugang}

In 2008 wurde die Initiative "Lehre mit Matura“ gestartet. Mit der Novelle zum Berufsausbildungsgesetz 2015 wurde die Möglichkeit geschaffen, die Lehrzeit bei gleichzeitiger Vorbereitung auf die Berufsreifeprüfung aliquot zu verlängern. Die Curricula der Berufsreifeprüfung wurden den Anforderungen der standardisierten Reifeprüfung angepasst. Das Programm "Coaching und Beratung von Lehrlingen und Lehrbetrieben" (seit Herbst 2015) unterstützt Lehrlinge zudem bei allen ausbildungsbezogenen Fragen sowie bei ihrer individuellen Karriereplanung. 2018 bereiteten sich rd. 9.500 Lehrlinge auf die Berufsreifeprüfung vor. In Summe gibt es derzeit über 6.700 Absolventinnen und Absolventen der Berufsmatura für Lehrlinge.

\section{Einführung von österreichweiten}

\section{Bildungsstandards und teilstandardisierten} Abschlussprüfungen

2009 wurden die Bildungsstandards ${ }^{17}$ für Schülerinnen und Schüler der vierten und achten Schulstufe festgelegt und im Mai 2012 erstmals überprüft. Die teilstandardisierte Abschlussprüfung ist an den allgemein bildenden höheren Schulen (AHS) seit 2014/15 umgesetzt.

Des Weiteren wurde die standardisierte, kompetenzorientierte Reife- und Diplomprüfung im Regierungsübereinkommen 2008 festgelegt und als Gesetz im Nationalrat 2010 beschlossen. Im Schuljahr 2015/16 haben erstmals alle AHS und BHS (teil-) standardisierte kompetenzorientierte neue Reifeprüfungen bzw. Reife- und Diplomprüfungen durchgeführt.

\section{Weiterentwicklung des Schulsystems im} Hinblick auf bessere individuelle Förderung Im Rahmen des bundesweiten Qualitätsschwerpunkts "Individualisierung des Unterrichts“ wurden die Qualitätsinitiative Schulqualität Allgemeinbildung (SQA) und Qualitätsinitiative Berufsbildung (QIBB) entwickelt. Beide Qualitätsinitiativen bilden die Konkretisierung des Nationalen Qualitätsrahmens für das österreichische Schulwesen in Richtung Individualisierung, Kompetenzorientierung und inklusiver Settings („neue Oberstufe“). Die beiden Qualitätssysteme werden sukzessive zusammengeführt. Der Ausbau des Angebots an Diagnose- und Förderinstrumenten dient der individuellen Lernbegleitung im Rahmen der neuen Oberstufe. In dieser sollen „Semestrierte Lehrpläne" (Lehrstoffe des Lehrplans sind einzelnen Semestern zugeordnet) und semesterweise Beurteilung zu einer Reduktion von Klassenwiederholungen führen. Bei drohender negativer Beurteilung erfolgt eine „Frühwarnung“ sowie eine Förderung durch "individuelle Lernbegleitung“. 
Die flächendeckende Umsetzung der neuen Oberstufe war ursprünglich für das Schuljahr 2017/18 geplant. Aufgrund von Rückmeldungen aus der Praxis wurde den Schulleitungen die Möglichkeit eröffnet, den Start zu verschieben, um geplante Reformmaßnahmen an ihrer Schule im Schulalltag besser zu verankern. Bis Ende 2019 wird die neue Oberstufe unter Einbindung aller betroffenen Zielgruppen evaluiert, bevor sie 2021/22 gegebenenfalls in adaptierter Form flächendeckend umgesetzt wird.

\section{Erhöhung der Durchlässigkeit insbesondere im Bereich der Sekundarstufe 1}

Die Umsetzung der Neuen Mittelschule wurde mit dem Schuljahr 2017/18 abgeschlossen. 2018 wurden im „pädagogischen Paket“ neue Regelungen zur Einführung von zwei Leistungsniveaus beschlossen. Diese werden gemeinsam mit neuen Lehrplänen 2020 umgesetzt. Ob das „pädagogische Paket“ die intendierten Ziele erfüllen kann wird sich voraussichtlich erst nach vier Jahren, im Sommer 2023 zeigen.

\section{Stärkung der Humanpotentiale im Bereich \\ Mathematik, Informationstechnologie, Naturwissenschaft, Technik durch gezielte}

Förderung im schulischen Unterricht und an universitären Einrichtungen

Im Rahmen dieser Maßnahme wurde im Laufe der Jahre eine Reihe von Projekten und Initiativen wie folgt durchgeführt:

- Seit 2001 existieren im Rahmen des Projekts IMST (Innovationen machen Schulen Top) ${ }^{18}$ neun Ländernetzwerke zum Austausch von Modellen guter Praxis. Ca. 3.000 Lehrkräfte beteiligen sich jährlich. Insgesamt wurden bislang rd. 60 Unterrichtsprojekte zur Förderung des mathematischnaturwissenschaftlichen Unterrichts durchgeführt.
- Seit 2017 wird ein MINT-Gütesiegel für Schulen vergeben. Bis Jänner 2019 wurden insgesamt 216 Bildungseinrichtungen in Österreich ${ }^{19}$ mit dem MINT-Gütesiegel ausgezeichnet.

- Die Initiative Yo!Tech ${ }^{20}$ (seit 2005 durch das BMBWF unterstützt) organisiert Veranstaltungen, die Schülerinnen und Schüler der Ober- und Unterstufe Einblicke in technisch- naturwissenschaftliche Ausbildungen ermöglicht.

- Für das Projekt „Jugend innovativ“21 gibt es jährlich rd. 600 Einreichungen aus vorwiegend maturaführenden Schularten, die in einzelnen Fällen auch Schutzrechte anmelden und auf internationale Erfolge verweisen können. Der Wettbewerb umfasst die Kategorien Design, Engineering, Science, Sustainability und Young Entrepeneurs.

- Die Initiative „Science Experts ${ }^{.22}$ hat das Ziel, an sogenannten "Brennpunktschulen“ das Interesse für Wissenschaft und Forschung zu wecken bzw. zu fördern. Schülerinnen und Schüler der Neuen Mittelschulen (NMS) können damit auch erste Kontakte mit selbsttätiger Vorwissenschaft knüpfen.

- Der Girls' Day ${ }^{23}$ ist ein einmal im Jahr stattfindender Aktionstag, der Mädchen und Frauen motiviert, technische und naturwissenschaftliche Berufe zu ergreifen. Damit strebt der Girls' Day an, den Anteil der weiblichen Beschäftigten in sogenannten "Männerberufen" zu erhöhen und dem Fachkräftemangel in der Wirtschaft entgegenzuwirken.

\section{Sparkling Science}

Ein weiteres wichtiges Programm zur Förderung des Humanpotentials ist das Sparkling Science Programm des BMBWF, das die forschungsorientierte Zusammenarbeit zwischen Schulen und Wissenschaft in den Fokus rückt. Weitere Details zum Programm finden sich in Abschnitt 3.5.5.

18 Vgl. https://www.imst.ac.at/

19 Vgl. https://www.mintschule.at/mint-landkarte/

$20 \mathrm{Vgl}$. http://www.yo-tech.at/home.html

21 Vgl. https://www.jugendinnovativ.at

22 Vgl. https://bmbwf.gv.at/das-ministerium/veranstaltungen/fotos-und-nachlese/science-experts-2018/

23 Vgl. https://www.frauen-familien-jugend.bka.gv.at/frauen/gleichstellung-arbeitsmarkt/girls-day-girls-day-mini.html 


\section{Ausbau der Berufsorientierung und der}

\section{Studienberatung (z.B. Studienchecker, Studieren} probieren) bereits in der Schule

Seit dem Schuljahr 2008/09 findet eine Bildungswahl- und Berufsorientierung in der 7./8. Schulstufe AHS bzw. im 8./9. Jahrgang BHS statt. Der Unterstützung bei Berufs- und Studienwahl dienen die Programme "Studienchecker" und "Maturantinnen- und Maturantenberatung“ sowie "Studieren probieren“ der ÖH. Seit 2013 stellen zudem bei der größten Bildungsmesse Österreichs, BeSt in Wien, „Role Models" die Möglichkeit untypischer Bildungswege vor - mitunter mit dem Ziel, Mädchen und Frauen für MINT-Fächer zu begeistern.

In diesem Kontext hat auch die Zukunftsoffensive MINT-Fachkräfte das Ziel, bis 20233.000 neue Ausbildungsplätze im MINT-Bereich zu schaffen. Die Initiative umfasst Maßnahmen auf vier Ebenen: 1) Gezielter Ausbau von IT-Ausbildungen an höheren technischen Schulen; 2) Gezielter Ausbau der Fachrichtung „Digital Business" an den Handelsakademien; 3) Gezielter Ausbau neuer Kollegs für neue Zielgruppen (wie z.B. die Etablierung eigener, neuer IT-Kollegs); sowie 4) Gezielter Ausbau der FH-Studiengänge im MINT-/IT-Bereich. ${ }^{24}$

\subsubsection{Qualitätsverbesserung der}

\section{Hochschullehre}

Die FTI-Strategie 2020 hielt fest, dass sowohl die Beteiligungsquote als auch die Absolventinnenund Absolventenquote im Hochschulbereich unter dem OECD-Durchschnitt liegen. „Mit einer Akademikerquote von 34,6\% - definiert als Anteil von Personen mit tertiärem sowie gleichwertig postsekundärem Bildungsabschluss an der 30-34-jähri- gen Wohnbevölkerung - liegt Österreich unter dem EU-Durchschnitt von 38 \%. In Österreich nehmen (insgesamt) $43 \%$ eines Altersjahrgangs ein Studium an einer Hochschule oder Fachhochschule auf, im OECD-Durchschnitt sind es $56 \%$ " ${ }^{25}$ Als weiteres Problem wurde "ein ausgeprägtes Gender-Ungleichgewicht in den technisch-naturwissenschaftlichen Ausbildungen einerseits und im sprachlichpädagogischen Bereich andererseits " ${ }^{\prime 26}$ festgestellt. Außerdem wurde auf die im internationalen Vergleich ungünstigen „Rahmenbedingungen für die universitäre Lehre und insbesondere die Betreuungsverhältnisse" hingewiesen, "was sich negativ auf das Abschneiden österreichischer Hochschulen in den Universitätsrankings auswirkt. Der sehr unterschiedliche Andrang zu den Studien bringt nicht nur entsprechend unterschiedliche Studienbedingungen, sondern auch unterschiedliche Chancen am Arbeitsmarkt mit sich." ${ }^{\text {"27 }}$

Als Ziel definierte folglich die FTI-Strategie, dass der „Anteil der 30-34-Jährigen, die ein Hochschulstudium abgeschlossen haben oder über einen gleichwertigen Abschluss verfügen, bis 2020 auf 38 \% erhöht werden“28 soll. Um dies zu erreichen, sollen „die Studienbedingungen an den Hochschulen wesentlich verbessert werden, wozu auch neue Finanzierungsmodelle für die Hochschullehre etabliert

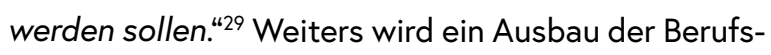
orientierung und der Studienberatung sowie die Einrichtung flexibler Studieneingangsphasen in allen Diplom- und Bachelorstudien angestrebt.

Die hierzu erforderlichen Maßnahmen ${ }^{30}$ lauteten:

- Entwicklung eines "österreichischen Modells" für eine künftige Teilung der Finanzierung der Universitäten nach studierendenbezogenen Mitteln (Lehre) und Forschung

24 Vgl. https://bmbwf.gv.at/fileadmin/user_upload/Aussendung/MINT-Fachkräfte__Internationaler_Frauentag/190306_PKUnterlage_OÖ_FH_MINT-Fachkräfte_final_AW_korrAP.pdf

25 Vgl. BKA et al. $(2011,14)$.

26 Vgl. BKA et al. $(2011,16)$.

27 Vgl. BKA et al. $(2011,15)$.

28 Vgl. BKA et al. $(2011,16)$.

29 Ebenda.

30 Vgl. BKA et al. $(2011,17)$. 
- Verbesserung der Betreuungsrelationen von Lehrenden zu Studierenden

- Entwicklung von Qualitätsindikatoren für den Lehrbetrieb im Hochschulbereich

\section{Verbesserung der Betreuungsrelationen von Studierenden zu Lehrenden}

Die Maßnahmen zur kapazitätsorientierten und studierendenbezogenen Universitätsfinanzierung werden in Abschnitt 3.3.2 dargestellt. Diese neue Universitätsfinanzierung ist eine wichtige Voraussetzung für eine Verbesserung der Betreuungsrelationen von Lehrenden zu Studierenden. Eine weitere Voraussetzung, um dieses Ziel zu erreichen, ist die Schaffung neuer Professorinnen- und Professorenstellen in besonders stark nachgefragten Studienfeldern. Seit der Beschlussfassung der FTI-Strategie wurden sukzessive Maßnahmen in diese Richtung gesetzt. Im Zeitraum 2011/12 wurden Investitionsmittel zur Verbesserung der hochschulischen Lehre an Universitäten in der Höhe von 40 Mio. $€$ bereitgestellt. Insgesamt wurden damit 78 Projekte an 18 Universitäten bzw. Hochschulen finanziert.

In den Leistungsvereinbarungen 2013-2015 wurden im Rahmen des Qualitätspakets "Lehre“ in den besonders stark nachgefragten Studienfeldern Architektur, Biologie, Informatik, Pharmazie und Wirtschaft 95 neue Professorinnen- und Professorenstellen eingerichtet. Zur Erhöhung der Transparenz werden zudem im Gesamtösterreichischen Universitätsentwicklungsplan die faktischen und angestrebten Betreuungsverhältnisse ausgewiesen.

Aktuell strebt der Gesamtösterreichische Universitätsentwicklungsplan ${ }^{31}$ für die Leistungsvereinbarungsperiode 2019-2021 eine Verbesserung der kalkulatorischen Betreuungsrelation in Richtung 1:40 an. Die Universitäten sind daher in der aktuellen Leistungsvereinbarungsperiode 2019 - 2021 auch angehalten, insgesamt mindestens 358,1 zusätzliche Professuren und Vollzeitäquivalente auszuschreiben, von
Tabelle 3-1: Betreuungsrelation auf StudienfeldEbene, Studienjahr 2015/16

\begin{tabular}{l|r}
\hline Studienfeld & Betreuungsrelation \\
\hline Erziehungswissenschaft und & $1: 37,4$ \\
Ausbildung von Lehrkräften & $1: 20$ \\
\hline Künste & $1: 52,7$ \\
\hline Geisteswissenschaften & $1: 79,6$ \\
\hline Sozial- und Verhaltenswissenschaften & $1: 124,5$ \\
\hline Journalismus und Informationswesen & $1: 73,4$ \\
\hline Wirtschaft und Verwaltung & $1: 77,2$ \\
\hline Recht & $1: 37,2$ \\
\hline Biowissenschaften & $1: 29,4$ \\
\hline Exakte Naturwissenschaften & $1: 23,4$ \\
\hline Mathematik und Statistik & $1: 37,9$ \\
\hline Informatik & $1: 33,8$ \\
\hline Ingenieurwesen und technische Berufe & $1: 40,3$ \\
\hline Herstellung und Verarbeitung & $1: 58,8$ \\
\hline Architektur und Baugewerbe & $1: 38,1$ \\
\hline Landwirtschaft, Forstwirtschaft und & $1: 21,5$ \\
\hline Fischereiwirtschaft & $1: 19,2$ \\
\hline Veterinärmedizin & $1: 23,2$ \\
\hline Gesundheit & $1: 81,8$ \\
\hline Persönliche Dienstleistungen & $1: 53,2$ \\
\hline Umweltschutz & \\
\hline Nicht bekannt/keine näheren Angaben & \\
\hline Quelle: BMBWF (2017, 110). & \\
\hline & \\
\hline
\end{tabular}

denen jedenfalls 287 sogenannte Laufbahnstellen sein müssen, die Jungwissenschaftlerinnen und Jungwissenschaftlern vorbehalten sind.

\section{Entwicklung von Qualitätsindikatoren für den Lehrbetrieb im Hochschulbereich}

Im Hochschulentwicklungsplan wird der Stellenwert guter Lehre insofern definiert bzw. hervorgehoben, als sie „im globalen Wettbewerb der Standorte internationalen Standards entsprechen und neben der Bewertung von Forschungsaspekten auch Karriererelevanz für die Lehrenden (...) erhalten (muss). Als Zeichen von Qualität ist außerdem zu werten, inwieweit forschungsgeleitete Lehre, über den fachlichen Kontext hinaus, auch der Heterogenität bzw. Diversität

31 Vgl. BMWFW (2017a). 
der Studierenden und gesellschaftlicher Herausforderungen gerecht wird." ${ }^{32}$

Angesichts dessen wurde eigens eine Arbeitsgruppe der Hochschulkonferenz zur "Stärkung der Qualität der hochschulischen Lehre" mit der Erarbeitung von Vorschlägen zur Weiterentwicklung der Qualität in der Lehre und Stärkung ihres Ansehens beauftragt. Diese liegen seit Ende 2014 vor. ${ }^{33}$ Die Empfehlungen wurden in der Hochschulkonferenz am 11.12.2014 beschlossen und im März 2015 der Öffentlichkeit präsentiert, wobei zwischen vier lehrrelevanten Dimensionen unterschieden wird:

- Lehrtätigkeit (im Hinblick auf die individuelle Kompetenz der einzelnen Lehrenden);

- Lehrangebot (im Hinblick auf Fach, Profil, Standort, Curriculum und Ressourcen);

- Lehrbetrieb (im Hinblick auf die organisatorische Durchführung, Betreuung und den Stellenwert der Lehre);

- Lehrinput und Lehroutput (im Hinblick auf Systemeffizienz).

Alle diese Dimensionen wurden und werden in den Leistungsvereinbarungen mit den Universitäten berücksichtigt. So umfasste ein Schwerpunkt in den Leistungsvereinbarungen 2016-2018 Maßnahmen im Bereich der Studiengestaltung und der Lehr- und Lernorganisation ebenso wie die Weiterentwicklung der Lehrkompetenz im Rahmen der Personalentwicklung (Teaching Competence-Programme, Angebote zur Basisqualifizierung oder Weiterqualifizierung für Lehrende sowie spezifische Angebote zur Erweiterung der Lehrkompetenzen im Hinblick auf E-Didaktik und den Einsatz digitaler Medien in der Lehre). Für die Leistungsvereinbarungsperiode 2019-2021 werden neben der Stabilisierung bzw. Verbesserung von Betreuungsrelationen (siehe Abschnitt 3.2.1) aktuell folgende thematische Schwerpunkte gesetzt:

- Hinwirken auf Erhöhung der Anteile des hoch qualifizierten Universitätspersonals;
- Nutzung von Open Educational Resources und Learning Analytics;

- Verbesserung der Lehrvermittlung (Didaktik) und Studienorganisation mit Fokus auf:

- Digitalisierung, Studierendenzentriertheit und Prüfungswesen;

- Entwicklung neuer (studierendenzentrierter und barrierefreier) digitaler Lehrmethoden;

- Angemessene Berücksichtigung didaktischer Kompetenzen in Qualifizierungs- und Berufungsverfahren sowie in der Aus- und Weiterbildung des Universitätspersonals;

- (Strukturelle) Studierbarkeit und Ermöglichung von Studienabschlüssen innerhalb angemessener Studiendauer;

- Beurteilung der Lehre durch Studierende, Monitoring und Befragung von Absolventinnen und Absolventen, externe Evaluierung der Studierbarkeit;

- Soziale Dimension in der Hochschulbildung.

\section{Flankierende Maßnahmen und Auszeichnungen}

Die Website „Atlas der guten Lehre“34 stellt Good Practice-Beispiele im Bereich der Lehre öffentlich dar. Durch ihre Funktion als Überblicksinstrument und zum gegenseitigen Informationsaustausch soll sie die stetige Verbesserung der Qualität in der Lehre unterstützen und entsprechende Aktivitäten sichtbar machen. Zielgruppe ist die interessierte Öffentlichkeit und jene Personen, die an Hochschulen mit der Weiterentwicklung der Lehre befasst sind.

Um einen intensiveren Austausch zur Lehre zu unterstützen, wurde neben der Website auch die Veranstaltungsreihe "Dialog zur hochschulischen Lehre“ ins Leben gerufen. Die Veranstaltungsreihe umfasste 2017 und 2018 vier Veranstaltungen, die vom BMDW in Kooperation mit dem Österreichischen Austauschdienst (OeAD) organisiert wurden. Die Veranstaltungen bieten eine Plattform, damit sich Lehrende und 
Personen, die mit der Weiterentwicklung der Qualität der Lehre beschäftigt sind, austauschen und aktuelle Herausforderungen kritisch diskutieren können. Die Themenschwerpunkte der vier Veranstaltungen 2017 waren: Stellenwert der Lehre-Anreizsetzung für hochschuldidaktische Weiterbildung, Kompetenzorientiertes Prüfen, Digitalisierung und digitale Kompetenzen in der hochschulischen Lehre, sowie forschungsgeleitete Lehre.

An vielen Universitäten wird die Bedeutung von guter Lehre durch die Auszeichnung von Lehrenden demonstriert. Entsprechende Preise bringen die Anerkennung durch materielle und ideelle Auszeichnungen zum Ausdruck und werden oft im Rahmen von sogenanntem „Tag der Lehre“ vergeben. Die Würdigung besonders engagierter Lehrender schafft auch einen Anreiz für Wissenschaftlerinnen und Wissenschaftler, in guter Lehre einen wichtigen Bestandteil ihrer Karriere zu sehen. Bei den Preisen kann es sich um allgemeine Auszeichnungen für „exzellente Lehre" oder "innovative" Lehre handeln, wie z.B. bei den zwei gleichnamigen Preisen der Wirtschaftsuniversität Wien, dem „Lehre Plus!Preis“ der Universität Innsbruck oder dem „Preis für exzellente Lehre“ der Technischen Universität Graz. Einige Universitäten vergeben Preise mit jährlich wechselnden thematischen Schwerpunkten, wie z.B. die Universität Wien mit dem „UNIVIE Teaching Award“ oder die Universität Graz mit dem Preis „Lehre: Ausgezeichnet!“. Die Universität Klagenfurt hat, wie in der Leistungsvereinbarung 2016-2018 vereinbart, 2018 erstmals wieder einen Lehrpreis (ApplAAUs! - Preis für herausragende Lehre vergeben) im Rahmen der Lehr- und Lerntage vergeben. ${ }^{35}$ Auch wird die zunehmende Bedeutung digitaler Medien in der universitären Lehre durch die Vergabe spezieller Preise deutlich. Sonderpreise in der Kategorie „E-Learning“ werden z.B. von der Universität Graz („Digitale Lehre: Ausgezeichnet!“), der Veterinärmedizinischen Universität Wien (Vetucation ${ }^{\circledast}$ Award), der Universität Innsbruck (E-Learning-
Preis) und seit 2017 auch von der Wirtschaftsuniversität Wien (E-Teaching Award) vergeben.

Schließlich hat das BMBWF gemeinsam mit der Universitätenkonferenz, der Fachhochschul-Konferenz, der Österreichischen Privatuniversitäten Konferenz und der Österreichischen Hochschülerlnnenschaft den „Ars Docendi - Staatspreis für exzellente Lehre" neugestaltet. Seit 2015 wird der „Ars Docendi“ jährlich für Lehrende öffentlicher Universitäten, Fachhochschulen und Privatuniversitäten vergeben. Der Preis wurde 2018 in den folgenden fünf thematischen Kategorien vergeben und war mit jeweils $7.000 €$ dotiert:

- Digitale Lehr- und Lernelemente in Verbindung mit traditionellen Vermittlungsformen;

- Konzepte und Beispiele im Bereich kooperativer Lern- und Arbeitsformen über Hochschulen und Hochschulsektoren hinweg;

- Forschungs- und kunstgeleitete Lehre, insbesondere die Förderung von kritischem Denken, Dialogorientierung, Methodenkompetenz;

- Umsetzung hochschulischer Internationalisierungskonzepte in der Lehre;

- Herausragende Betreuung von wissenschaftlichen und künstlerischen Abschlussarbeiten (MA, Dipl., Diss.).

2019 können erstmals auch Lehrende Pädagogischer Hochschulen ausgezeichnet werden.

\section{Soziale Dimension in der Hochschulbildung}

Im Jahr 2016 erarbeitete das BMBWF (damals BMWFW) in einem diskursiven Prozess mit Hochschulen, Sozialpartnern und Beratungseinrichtungen die "Nationale Strategie zur sozialen Dimension in der Hochschulbildung. Für einen integrativeren Zugang und eine breitere Teilhabe" (BMWFW, 2017). Dieser Prozess wurde einerseits durch die Bologna-Ministerinnen- und Ministerkonferenz in Jerewan 2015 angestoßen, bei der sich die Mitgliedstaaten darauf verständigten, nationale Strategien zur Verbesserung

35 Vgl. https://www.aau.at/blog/applaaus-preis-fuer-herausragende-lehre-vergeben/ 
der sozialen Dimension im Hochschulbereich zu entwickeln (d.h. Hochschulstudierende sollen bei Zugang, Teilhabe und Abschluss die Zusammensetzung der Bevölkerung widerspiegeln). Andererseits enthielt das Regierungsprogramm 2013-2018 entsprechende Zielsetzungen zur sozialen Dimension, die auch in Strategiedokumenten des Ressorts (wie im Gesamtösterreichischen Universitätsentwicklungsplan) verfolgt werden.

Die Umsetzung dieses ersten gesamthaften Strategiedokuments zu diesem Thema ist bis 2025 vorgesehen, schließt alle Hochschulsektoren mit ein und fordert Hochschulen in ihrem gesamten Leistungsspektrum, vor allem in den Bereichen Lehre, Studium und gesellschaftliche Verantwortung („Dritte Mission"). Die soziale Dimension in der Hochschulbildung ist somit auch als Reformanstrengung im hochschulpolitischen Mainstream angelangt.

Die "Nationale Strategie zur sozialen Dimension in der Hochschulbildung" legt drei Zieldimensionen mit jeweils drei Aktionslinien (Handlungsfeldern) und konkreten Maßnahmen fest.

- Zieldimension I: „Integrativerer Zugang“ adressiert vor allem die Qualität und Zugänglichkeit von Informations- und Beratungsangeboten und Outreach-Aktivitäten sowie die Anerkennung und Validierung nicht-formaler und informeller Kompetenzen, um den Zugang vereinfacht und effektiver zu gestalten und mehr Heterogenität im Hochschulzugang zu gewährleisten.

- Zieldimension II: „Abbruch verhindern, Studienerfolg verbessern" bezieht sich auf die Studienorganisation (u.a. bessere Vereinbarkeit des Studiums mit anderen Lebensbereichen), die Phase des Studieneinstiegs (u.a. „Willkommenskultur“) sowie die Qualität der Lehre zur Steigerung der Heterogenitäts- und Diversitätssensibilität.

- Zieldimension III: „Rahmenbedingungen schaffen und hochschulpolitische Steuerung optimal einsetzen" spricht einerseits Hochschulsystemfragen an (z.B. Weiterentwicklung des Studienrechts, Monitoring der „Studierbarkeit“, Erhöhung der sozialen Treffsicherheit im Hochschulzugang, Überprü- fung der Hochschulfinanzierung in ihrer Wirkung auf die soziale Dimension), andererseits die Schaffung geeigneter Governance-Strukturen an den Hochschulen (u.a. durch Integration der sozialen Dimension in hochschulische Strategieüberlegungen), und ebenso die Studienförderung, bezüglich der es mit der Novelle zum Studienförderungsgesetz 2017 bereits eine wesentliche Verbesserung gab.

Die Zielsetzungen bilden sich darüber hinaus in neun quantitativen Zielen ab, wie z.B. im Abbau der Unterrepräsentanz von Studierenden mit Eltern ohne Matura (insgesamt und in Human- und Zahnmedizin), in der Steigerung der Studienanfängerinnen und -anfänger mit nicht-traditionellem Hochschulzugang oder Migrationshintergrund, oder auch in der Verbesserung des Geschlechterverhältnisses in allen Studienfeldern.

Im Kontext der kapazitätsorientierten Universitätsfinanzierung ( $\$ 12 a$ (4) UG 2002) wurde ermöglicht, dass zur Sicherstellung der Umsetzung der Maßnahmen zur sozialen Dimension bis zu 0,5\% des Globalbudgets (insgesamt rd. 45 Mio. €) einbehalten werden können. Daher wurden in allen Leistungsvereinbarungen 2019-2021 mit den Universitäten entsprechende Maßnahmen vereinbart, an deren Nachweis der Umsetzung bis spätestens zum LV-Begleitgespräch im Herbst 2020 die Mittelfreigabe gebunden ist. Über ein Drittel der Universitäten hat sich dafür entschieden, ein gesamthaftes institutionelles Strategievorhaben zu erarbeiten (hierzu zählen die Wirtschaftsuniversität Wien, die Medizinische Universität Graz, die Veterinärmedizinische Universität Wien, die Universität für angewandte Kunst Wien, die Universität Salzburg, die Universität Klagenfurt, das Mozarteum Salzburg und die Kunstuniversität Graz). Die übrigen Universitäten haben maßgebliche Vorhaben in die Leistungsvereinbarung integriert, die sich auf Outreach-Maßnahmen, das Monitoring des Studienzugangs, die Unterstützung beim Studieneinstieg oder in der Studieneinstiegsphase, sowie auf die Studierfähigkeit von nicht-traditionellen Studierendengruppen beziehen. 


\subsubsection{Verbesserte Integrationsangebote}

Die FTI-Strategie 2020 bemängelte damals, dass „verfügbare Potenziale und Qualifikationen von Zuwandernden in zu geringem Ausmaß entwickelt und in Wissenschaft und Wirtschaft zu wenig genutzt ${ }^{\text {*36 }}$ werden. "Migrantinnen und Migranten weisen auch in der zweiten und dritten Generation meist signifikant schlechte Bildungsniveaus auf. Sprachbarrieren erschweren den Bildungszugang. ${ }^{\text {" }} 7$

Um eine verbesserte Integration von Zuwandernden zu erreichen, soll „der Anteil der Schülerinnen und Schüler mit einer anderen Erstsprache als Deutsch, die die zweite Sekundarstufe abschließen, von derzeit 40 auf $60 \%$ steigen." ${ }^{38}$

Als Maßnahmen ${ }^{39}$ wurden hierfür vorgeschlagen:

- Vermehrter Einsatz von Lehrenden nichtdeutscher Muttersprache und interkulturellen Mitarbeiterinnen und Mitarbeitern

- Verstärkte Sprachförderung

- Flexibel gestaltete Anerkennung und Nostrifikation von Diplomen und anderen Abschlüssen
Vermehrter Einsatz von Lehrenden nichtdeutscher Muttersprache und interkulturellen Mitarbeiterinnen und Mitarbeitern

Im Rahmen des Projekts "Diversität und Mehrsprachigkeit in pädagogischen Berufen" erstellte eine interdisziplinäre Expertinnen- und Expertengruppe aus acht Pädagogischen Hochschulen Empfehlungen zur Verankerung migrationsbedingter Diversität und Mehrsprachigkeit im österreichischen Bildungssystem. Diese Empfehlungen beziehen sich auf die Bereiche Organisation, Personal, Curricula, Forschung und Lehre. ${ }^{40}$ Ein Team der Universität Wien entwickelte zudem ein Förderdiagnoseinstrument für Lehramtsstudierende zur Begleitung der (wissenschaftlichen und professionsbezogenen) Sprachentwicklung in deutscher Sprache. ${ }^{41}$

\section{Verstärkte Sprachförderung mit Fokus auf Elementar- und Primarpädagogik sowie Aus- und Fortbildung von Pädagoginnen und Pädagogen Um den Übergang vom Kindergarten in die Volks-}

Abbildung 3-2: Entscheidungsmatrix für die Aufnahme in die erste Schulstufe

\begin{tabular}{|c|c|c|}
\hline & $\begin{array}{l}\text { Schulreife aufgrund »körper- } \\
\text { licher und geistiger Reife» } \\
\text { JA }\end{array}$ & $\begin{array}{l}\text { Schulreife aufgrund »körper- } \\
\text { licher und geistiger Reife» } \\
\text { NEIN }\end{array}$ \\
\hline $\begin{array}{l}\text { Schulreife aufgrund } \\
\text { Beherrschung U-Sprache }\end{array}$ & Ordentlicher Status & Ordentlicher Status \\
\hline JA & 1. Schulstufe & Vorschulstufe \\
\hline \multirow{3}{*}{$\begin{array}{l}\text { Schulreife aufgrund } \\
\text { Beherrschung U-Sprache } \\
\text { NEIN }\end{array}$} & Außerordentlicher Status & Außerordentlicher Status \\
\hline & $\begin{array}{l}\text { Deuschförderklasse } \\
\text { (1. Schulstufe) }\end{array}$ & $\begin{array}{l}\text { Deuschförderklasse } \\
\text { (Vorschulstufe) }\end{array}$ \\
\hline & $\begin{array}{l}\text { 1. Schulstufe } \\
\text { mit Deutschförderkurs }\end{array}$ & $\begin{array}{l}\text { Vorschulstufe } \\
\text { mit Deutschförderkurs }\end{array}$ \\
\hline
\end{tabular}

Quelle: BMBWF (2018a).

36 Vgl. BKA et al. $(2011,14)$.

37 Ebenda.

38 Vgl. BKA et al. $(2011,16)$.

39 Vgl. BKA et al. $(2011,17)$.

40 Vgl. BMUKK (2013).

41 Vgl. Knappik (2013). 
schule zu optimieren, wurden in allen Bundesländern Kooperationsformen zwischen Kindergärten und Volksschulen entwickelt. Dabei kommen individualisierte und differenzierende Unterrichtsformen, Formen der alternativen Leistungsbeurteilung und Konzepte einer umfassenden Sprachförderung zum Einsatz. Spezielle Maßnahmen gibt es für Kinder mit Deutsch als Zweitsprache, für Seiteneinsteigerinnen und -einsteiger sowie für Kinder mit Sprachentwicklungsstörungen. Die Entscheidungsmatrix zur Aufnahme in die erste Schulstufe ist in Abbildung 3-2 dargestellt.

Nationale und internationale wissenschaftliche Befunde weisen darauf hin, dass der Leistungsabstand zwischen Kindern mit Erstsprache Deutsch und Kindern mit einer anderen Erstsprache in Österreich besonders hoch ist und sich in den letzten Jahren sogar noch vergrößert hat. 2015 haben die Ergebnisse der Bildungsstandards Deutsch in der 4. Schulstufe gezeigt, dass $67 \%$ der Kinder mit Deutsch als Erstsprache die vorgegebenen Standards für das Leseverständnis erreichen oder übertreffen, jedoch nur $39 \%$ der Kinder mit nicht-deutscher Erstsprache. Knapp ein Drittel dieser Kinder (27\%) hat Mühe mit den einfachsten Leseaufgaben und erreicht die Bildungsstandards nicht; weitere $35 \%$ erreichen die Bildungsstandards nur teilweise.

Mit dem Schuljahr 2018/19 traten daher neue Lehrpläne für Deutschförderklassen in Kraft. Auf Basis österreichweit einheitlicher und standardisierter Testverfahren werden jene Kinder und Jugendliche ermittelt, die unzureichende Kenntnisse der Unterrichtssprache aufweisen und dem Unterricht nicht folgen können. Sie erhalten den Status „außerordentliche Schüler/in" und werden einem Deutschförderkurs zugeteilt. Für die Sprachstartgruppe gibt es eine Erhöhung auf 15 (bisher elf) Förderstunden in der Volksschule bzw. auf 20 Förderstunden in der Sekundarstufe. $A b$ acht Schülerinnen und Schülern pro Schule wird eine Deutschförderklasse eingerichtet. Bei einer geringeren Anzahl werden die Schülerinnen und Schüler in der Regelklasse nach dem Lehrplan für die Deutschförderklassen unterrichtet.

Nach jedem Semester findet eine einheitliche Sprachstandüberprüfung statt, die eine semesterweise Übertrittmöglichkeit in den Regelunterricht ermöglicht. Schülerinnen und Schüler, die einen Deutschförderkurs besucht haben, können im nächsten Schuljahr in die nächsthöhere Schulstufe übertreten, wenn sie in allen Pflichtgegenständen positiv beurteilt wurden.

In Deutschförderklassen und Deutschförderkursen werden verbindlich Diagnoseinstrumente eingesetzt, um die Kompetenzen zu erfassen und Kinder bzw. Jugendliche diagnosebasiert zu fördern. Hierzu wird vom BMBWF das Instrument „Unterrichtsbegleitende Sprachstandsbeobachtung Deutsch als Zweitsprache" (USB-DaZ) empfohlen.

Um die Kompetenzen auch seitens des Lehrpersonals auf die Anforderungen der Integration besser vorzubereiten, wird seit 2009 an den Pädagogischen Hochschulen ein Lehrgang zur Qualifizierung von Pädagoginnen und Pädagogen und des Sprachförderpersonals (im Ausmaß von 6 ECTS) angeboten. Seit 2013 gibt es auch das Bundeszentrum für Interkulturalität, Migration und Mehrsprachigkeit (BIMM). ${ }^{42}$ Es handelt sich hierbei um ein Netzwerk der 13 Pädagogischen Hochschulen mit dem Ziel, das Themenfeld sprachliche und kulturelle Bildung in der Migrationsgesellschaft in der Pädagoginnen- und Pädagogenbildung zu verankern.

2013 wurde das Bundesrahmengesetz zur Einführung einer neuen Ausbildung für Pädagoginnen und Pädagogen verabschiedet. Dessen Ziel ist die Professionalisierung und Attraktivierung pädagogischer Berufe. Trägerinnen der neuen Ausbildungen sind die Pädagogischen Hochschulen und Universitäten, die in enger Kooperation Lehramtsausbildungen für die Sekundarstufe Allgemeinbildung auf tertiärem Niveau anbieten. Zur Umsetzung der neuen Pädagoginnen- und Pädagogenbildung wurden vier regionale Hochschulverbünde gebildet. Die gemeinsam einge-

42 Vgl. https://bimm.at/ 
richteten Lehramtsstudien werden österreichweit seit dem Studienjahr 2016/17 angeboten.

\section{Flexibel gestaltete Anerkennung und Nostrifizierung von Diplomen und anderen}

\section{Abschlüssen}

Ein Fünf-Punkte-Programm zur verbesserten Berufsanerkennung von Akademikerinnen und Akademikern aus Drittstaaten zielt auf eine zeitgemäße Berufsanerkennung durch rasche und effiziente Verfahren ab. Die Punkte umfassen: 1) Zur besseren und umfassenderen Information der Drittstaatsangehörigen wurde die bestehende Infostelle NARIC (National Academic Recognition Information Centre) im Wissenschaftsministerium in der Öffentlichkeit stärker als Infoservicestelle positioniert und eine Informationsoffensive gestartet. Die eigens eingerichtete Homepage ${ }^{43}$ wird stark nachgefragt. 2) Da es für die Antragstellenden oftmals schwierig war, herauszufinden, wo sie ihre Ansuchen einreichen können, wurde mit dem NARIC eine direkte Informations- und Einreichstelle geschaffen. Diese leitet die Antragstellenden dann auch an die jeweils zuständige Universität weiter. 3) Die maximale Wartezeit für Bearbeitung der Anträge wurde von sechs auf drei Monate halbiert. 4) Da fast die Hälfte der jährlichen Anträge für Nostrifizierungen in Österreich den Medizinbereich und damit die drei Medizinischen Universitäten Wien, Graz und Innsbruck sowie die Universität Linz (Medizinische Fakultät) betreffen, soll eine gemeinsame Geschäftsstelle der drei Medizinischen Universitäten und der Universität Linz für eine effizientere Abwicklung sorgen. 5) Das Service der NARIC steht nun auch verstärkt als Bewerbungshilfe zur Verfügung. Arbeitgeberinnen und Arbeitgeber können mit dem Service der NARIC die Ausbildung von Drittstaatsangehörigen kostenlos vergleichen und bewerten. Diese Bewertung ist unbürokratisch meist innerhalb weniger
Tage möglich. Dabei handelt es sich um ein Gutachten von offizieller Stelle, nicht aber um eine Anerkennung im akademischen Sinn (Nostrifizierung).

\subsubsection{Steigerung der Mobilität}

Der Austausch der Universitäten mit anderen Forschungseinrichtungen sowie mit forschungs- und technologieintensiven Wirtschaftsbranchen soll den Wissensaustausch begünstigen und so die Leistungsfähigkeit aller Beteiligten erhöhen. Studien zeigen, dass Arbeitnehmerinnen und Arbeitnehmer, die als Studierende Aufenthalte an ausländischen Universitäten absolviert haben, später höhere Einkommen erzielen. ${ }^{44}$ Mobilität wird in der wissenschaftlichen Literatur allgemein als Kanal der Wissensübertragung gesehen und trägt so zu wirtschaftlicher Prosperität bei. ${ }^{45}$ Es wird davon ausgegangen, dass Arbeitnehmerinnen und Arbeitnehmer in wissensintensiven Branchen und unter diesen speziell Wissenschaftlerinnen und Wissenschaftler ihr Wissen durch Mobilität erweitern können, indem sie neue Verfahren und neues Wissen kennenlernen und nach Hause oder an einen anderen Ort transferieren. Eine solche geografische Ausbreitung des Wissens wird auch Wissensspillover (knowledge spillovers) genannt. Auch in der FTI-Strategie 2020 wird explizit das Ziel, "Mobilität steigern zu wollen“46, genannt.

Folgende Maßnahmen ${ }^{47}$ wurden hierzu festgehalten:

- Gezielte Steigerung der Mobilität von Studierenden und Graduierten in ausgewählte Länder

- Verbreiterung des Austausches für Schülerinnen und Schüler, Studierende und Lehrpersonen auf allen Ebenen mit der forschungs-, technologieund innovationsintensiven Wirtschaft und dem Ausland

Die Entwicklung der Mobilität ordentlicher Studie-

43 Vgl. http://www.nostrifizierung.at

44 Vgl. Kratz und Netz (2018).

$45 \mathrm{Vgl}$. Fornahl et. al (2005).

46 Vgl. BKA et al. $(2011,17)$.

47 Ebenda. 
Abbildung 3-3: Anteil der Incomings und Outgoings an den ordentlichen Studierenden an Universitäten, 2001-2017

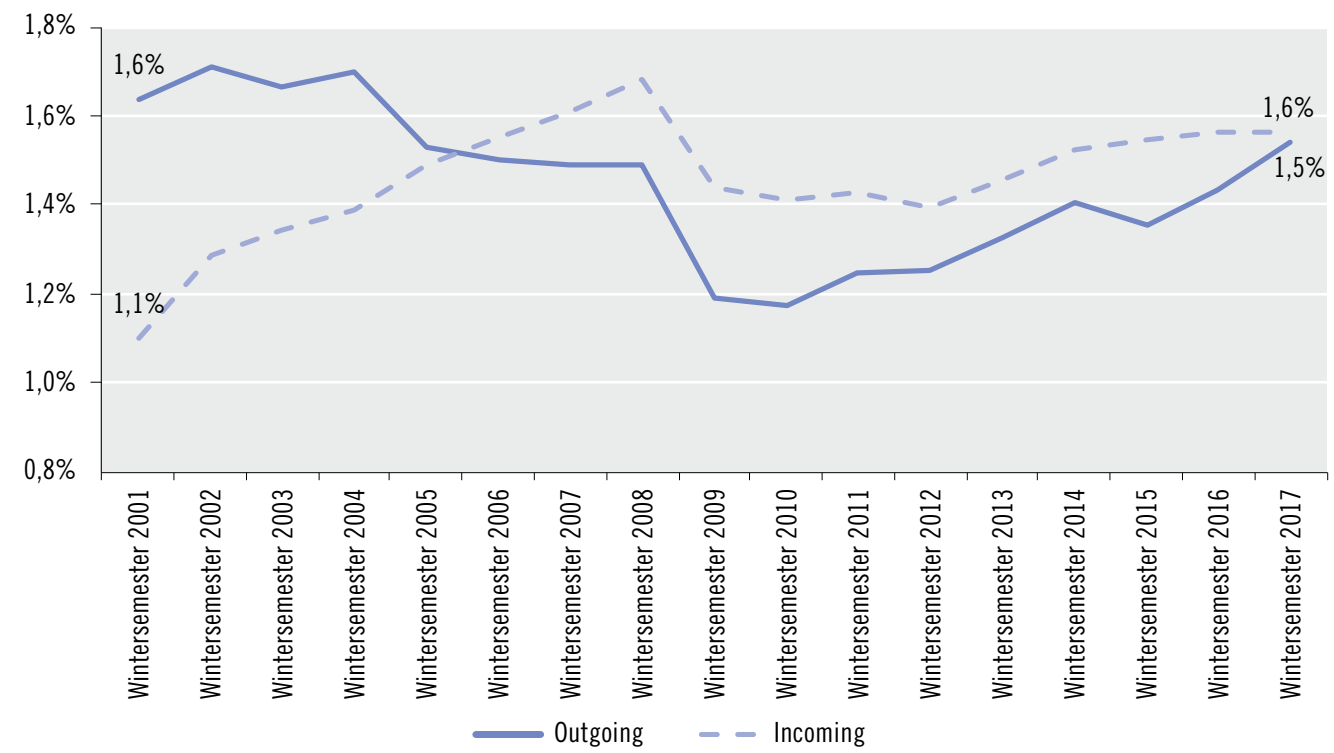

Quelle: BMBWF, uni:data (2019), Datenmeldungen der Universiäten auf Basis UniStEV zum jeweiligen Stichtag.

render an Österreichs Universitäten betreffend, zeigt sich, wie auch Abbildung 3-3 veranschaulicht, dass in den vergangenen Jahren sowohl der Anteil der Incomings als auch der Anteil der Outgoings leicht angestiegen ist, sodass im Wintersemester 2017 insgesamt rd. 4.300 Studierende einen Auslandsaufenthalt im Rahmen eines geförderten Mobilitätsprogramms absolvierten. Etwa gleich viele Incomings kamen im Wintersemester 2017 an eine österreichische Universität.

Die Europäische Union betreibt eine Reihe von Programmen, um die internationale Mobilität von Studierenden zu erhöhen. Das prominenteste Programm ist dabei Erasmust, welches Studienaufenthalte an ausländischen Hochschulen sowie studienrelevante Praktika, u.a. für kürzlich Graduierte fördert. Mit dem Erasmus+ Stipendium, haben Studierende (wie auch Schülerinnen und Schüler, Lehrlinge und Lehrende) die Möglichkeit, einen geförderten Auslandaufenthalt, an einer Erasmus-Partnerhochschule zu absolvieren. Erbrachte Studienleistungen werden an der Heimathochschule anerkannt, sofern sie dem in Voraus vereinbarten Studienprogramm, dem Learning Agreement entsprechen. Des Weiteren werden Lehraufenthalte und Fort- und Weiterbildungsmaßnahmen von Hochschul- und allgemeinem Personal gefördert. Das seit 1987 bestehende Erasmus-Programm wird zwar von der Europäischen Union verwaltet, steht seit 2014 aber auch anderen Ländern außerhalb der EU und Europas offen; Österreich nimmt seit 1992 teil und ist somit länger dabei, als es EU-Mitglied ist. Wie in Abbildung 3-4 zu sehen, stieg die Zahl der an Erasmus teilnehmenden Studierenden, die an einer österreichischen Hochschule eingeschrieben sind, kontinuierlich an. Mit dem Studienjahr 2017/18 haben bereits mehr als 107.000 aus Österreich hinausgehende Studierende einen Erasmus-Auslandsaufenthalt absolviert.

Weitere Programme zur Steigerung der Mobilität und der Verbreiterung des Austausches sind: das Central European Exchange Program for University Studies (CEEPUS), das die Mobilität von Studierenden und Lehrenden mit dem Ziel unterstützt, die Zusammenarbeit und Vernetzung mit den Staaten Mit- 


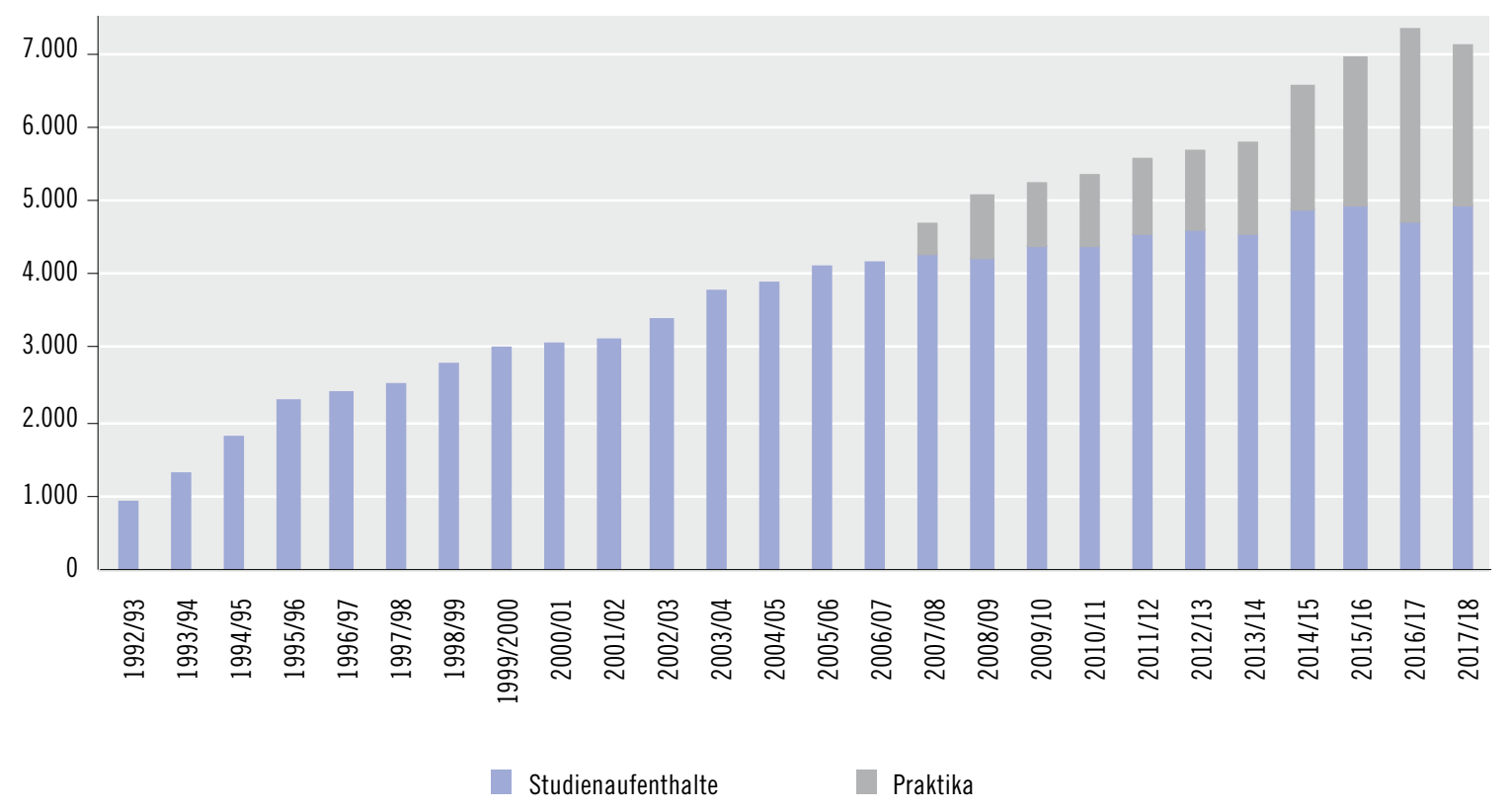

Quelle: OeAD.

tel-, Ost- und Südosteuropas weiter auszubauen; ${ }^{48}$ AKTIONEN, das Programm zur Förderung der bilateralen Zusammenarbeit im Hochschulbereich mit dem Ziel der Vernetzung des wissenschaftlichen Nachwuchses mit der Slowakei, Tschechien und Ungarn; Marietta-Blau-Stipendien, für welche sich Doktoratsstudierende aller Fachbereiche bewerben können, um zwölf Monate im Ausland forschen zu können; Fulbright, ein Programm zur Förderung der bilateralen Zusammenarbeit zwischen Österreich und den USA im Bereich der Bildung, Wissenschaft und Forschung durch Mobilität von Studierenden, Lehrenden und Forschenden zwischen den beiden Ländern, kofinanziert durch österreichische und US-amerikanische Regierungsbeiträge sowie Drittmitteln.

Zu weiteren Stipendien, die Mobilität fördern, zählen: etwa aus dem Bereich der Geistes-, Sozial- und Kulturwissenschaften die Doctoral Research Fellowships, die in Zusammenarbeit mit den ÖsterreichZentren in New Orleans, Minnesota, Alberta und Jerusalem vom BMBWF vergeben werden; Andrássy-
Stipendien zur Absolvierung eines zweijährigen Masterstudiums in Budapest, Doktoratsstipendien für das Europäische Hochschulinstitut (EHI), ErwinSchrödinger-Auslandsstipendien des FWF für Postdocs, um Forschungserfahrungen an führenden Forschungseinrichtungen weltweit zu sammeln, sowie das Lise-Meitner-Programm des FWF, das hochqualifizierte ausländische Wissenschaftlerinnen und Wissenschaftler aller Fachdisziplinen nach Österreich bringen soll.

Studierendenmobilität findet in Form von Credit-Mobility oder Degree-Mobility statt. Neben der Teilnahme an Programmen steht es Studierenden offen, Auslandsaufenthalte auch selbst zu organisieren. Bei der Credit-Mobility wird der studienbezogene Auslandsaufenthalt über Credits für das Studium zu Hause angerechnet. Lt. Universitätsbericht 2017 haben rd. 7.900 Studierende im Studienjahr 2016/17 einen Auslandsaufenthalt im Rahmen eines internationalen Mobilitätsprogramms absolviert. Im selben Studienjahr haben 8.500 an ausländischen Universi-

48 Partnerländer sind Albanien, Bosnien-Herzegowina, Bulgarien, Kroatien, Mazedonien, Montenegro, Moldau, Polen, Rumänien, Serbien, Slowakei, Slowenien, die Tschechische Republik und Ungarn; zusätzlich Universitäten im Kosovo. 
täten eingeschriebene Studierende in Österreich einen Aufenthalt absolviert. Bei der Degree-Mobilität wird ein ganzes Studium oder ein Studienzyklus im Ausland absolviert. Lt. Universitätsbericht 2017 betrieben 2015 rd. 16.000 Österreicherinnen und Österreicher ein Studium an Hochschulen im OECDAusland. Umgekehrt studierten im Wintersemester 2016 rd. 59.300 ausländische Studierende im Rahmen einer Degree-Mobilität an einer österreichischen Universität (wovon rd. 40 \% ausländischer DegreeMobilitätsstudierender aus Deutschland kamen); insgesamt sind dies $78 \%$ aller ordentlichen ausländischen Studierenden. Einer Auswertung des BMBWF zufolge haben $27 \%$ der inländischen Absolventinnen und Absolventen einen studienbezogenen Auslandsaufenthalt zu dieser Zeit absolviert.

Um die Mobilität nicht nur in quantitativer, sondern vor allem auch in qualitativer Hinsicht zu verbessern, hat das BMBWF 2016 eine „Hochschulmobilitätsstrategie“ zur Förderung transnationaler physischer Hochschulmobilität von Studierenden, Lehrenden und jungen Forschenden im Sinne von Early Stage Researchers und dem allgemeinen Personal im Hochschulbereich veröffentlicht. Aktuell wird diese gemeinsam mit den Hochschulen weiterentwickelt und im Sinne eines gesamthaften Zugangs um die Internationalisierung der Lehre erweitert.

Die in der FTI-Strategie angestrebte Mobilität in ausgewählte Länder wird durch eine Reihe von Programmen gefördert, wobei die ausgewählten Länder v.a. in Nordamerika, Europa (neben der Europäischen Union insbesondere in Mittelosteuropa und Südosteuropa) sowie in Teilen Asiens liegen. Zur Intensivierung der internationalen Kooperation im Bereich Forschung und Technologie hat Österreich bereits in den frühen 1990er-Jahren begonnen, ein Netz an Wissenschaftsaußenstellen aufzubauen. Der Schwerpunkt dieses Netzes lag anfangs in den südosteuropäischen Ländern, die sogenannten Austrian Science and Research Liaison Offices (ASO) wurden vor allem in den östlichen Nachbarländern betrieben. Angesichts der erfolgreichen Intensivierung der bilateralen Beziehungen und des Beitritts der Sitz- länder zur EU bzw. deren Assoziierung zu den EUForschungsrahmenprogrammen wurde die ASO dann durch außereuropäische Offices of Science and Technology Austria (OSTA) abgelöst. Aktuell werden zur Vertiefung der Forschungs- und Technologiekooperation mit wichtigen außereuropäischen Forschungsnationen jeweils ein OSTA in Washington (gegründet 2001, zuständig für Kanada, Mexiko und die USA) und in China (gegründet 2012, zuständig für China und die Mongolei) betrieben. Beide Büros stellen ministerienübergreifende Initiativen dar. Zu ihren Aufgaben zählen die Unterstützung der Universitäten und Forschungseinrichtungen beim Aufund Ausbau bilateraler Beziehungen, die Anbahnung und Durchführung von Kooperationen mit Einrichtungen in den Sitzländern sowie, die Ziele der FTI-Strategie betreffend, die Unterstützung der Vernetzung der österreichischen Wissenschaftlerinnen und Wissenschaftler. Zwecks Stärkung der Vernetzung betreibt das OSTA in Washington auch das Research and Innovation Network Austria (RINA), das derzeit über 2.700 in Nordamerika tätige österreichische Wissenschaftlerinnen und Wissenschaftler umfasst, und veranstaltet jährlich den Austrian Research and Innovation Talk (ARIT). Das OSTA in Peking arbeitet zurzeit am Aufbau eines Alumni-Netzwerks und veranstaltete hierzu 2018 die „Austrian Alumni Session“ in Peking.

Neben dem RINA des OSTA stellt das Austrian Scientists and Scholars in North America (ASCINA) das zweite große wissenschaftliche österreichische Netzwerk in Nordamerika dar. Ziel ist die Förderung des privaten und beruflichen Austauschs und die gegenseitige Unterstützung im Rahmen von Nordamerika-Aufenthalten. Eine andere Form von Netzwerken stellen das Eurasia-Pacific Uninet, das seit 2010 besteht und dem rd. 40 Bildungseinrichtungen aus 14 Ländern angehören; veranstaltet werden u.a. Sommerakademien, sowie das ASEAN-European Academic University Network (ASEA-UNINET) aus rd. 75 Universitäten aus 19 Ländern in Europa und Asien für die Kooperationen in Lehre und Forschung mit den Ländern Südostasiens, dar. 
Insgesamt setzt Österreich damit eine Vielzahl von Maßnahmen zwecks Erhöhung der Mobilität von Studierenden und Wissenschaftlerinnen und Wissenschaftlern um. Die Teilnahme am Erasmus-Programm ist dabei sicherlich von zentraler Bedeutung, wie die steigenden Teilnahmezahlen in den vergangenen Jahren zeigen.

\subsubsection{Verbesserte Rahmenbedingungen für Forscherinnen und Forscher an Hochschulen}

Die FTI-Strategie hat das Ziel gesetzt, "die Rahmenbedingungen für Forscherinnen und Forscher an Österreichs Hochschulen zu verbessern". ${ }^{49}$ Der Aktionsplan für einen wettbewerbsfähigen Forschungsraum fasst die Ausgangslage 2015 dabei folgendermaßen zusammen: „Die Karriereperspektiven für Forschende an österreichischen Universitäten sind von Rahmenbedingungen geprägt, die durchgängige Karrieren erschweren, nur enge und späte Karriereperspektiven eröffnen und eingeschlagene Karrierewege zu Einbahnstraßen werden lassen. Die daraus resultierende berufliche Perspektivenlosigkeit und Unsicherheit, der viele Nachwuchswissenschaftlerinnen und -wissenschaftler in Österreich ausgesetzt sind, hat zur Folge, dass viele Talente dem Land verloren gehen". ${ }^{0}$

Im Gesamtösterreichischen Universitätsentwicklungsplan (GUEP 2019-2024) zählt die Förderung des wissenschaftlichen Nachwuchses und die Entwicklung geeigneter Karrieremodelle und -pfade damit zu den priorisierten Systemzielen. Dies korrespondiert mit den in der FTI-Strategie angesetzten Maßnahmen ${ }^{51}$ :

- Verstärkte Förderung von Doktorandinnen und Doktoranden und Post-Docs durch Ausbau strukturierter Programmangebote

- Weiterentwicklung des Kollektivvertrags und des Universitätsgesetzes zur Umsetzung des TenureTrack-Systems (Umsetzung eines Karrieremodells mit Optionen zur unbefristeten Anstellung in Abhängigkeit von Leistungsevaluationen)

- Transparente und leistungsbezogene Vergabe von Laufbahnstellen an Hochschulen

\section{Einführung und Ausbau strukturierter Doktoratsprogramme}

Das Doktorat an österreichischen Universitäten befand sich im Berichtszeitraum in einem Umstellungsprozess, der mit den Bologna-Reformen und der Einführung des 3-jährigen PhD 2009 begonnen hat, und der 2019 weitestgehend abgeschlossen ist. Spätestens 2018 ist das letzte „alte“ Doktoratsprogramm ausgelaufen, es ist daher davon auszugehen, dass derzeit - von möglichen Einzelfällen abgesehen - keine Doktoratsstudierenden an Österreichs Universitäten ihr Doktorat mehr im Rahmen "alter“ Studiengänge absolvieren. Das Auslaufen alter Studiengänge 2017 führte auch zu einem leichten Knick der Studierendenzahlen: Die Zahl der ohnehin bereits seit 2011 stetig rückläufigen ordentlichen Doktoratsstudien ging in einem Jahr von 25.503 (WS 2016) auf 22.374 (WS 2017), also um rd. $14 \%$, zurück. ${ }^{52}$ Die Zahl der erfolgreichen Doktoratsabschlüsse hingegen entwickelte sich bis zum Studienjahr 2015/16 relativ konstant, bei jährlichen Abschlüssen um 2.200. Im Studienjahr 2016/17 verzeichneten die Doktoratsabschlüsse einen ersten deutlichen Anstieg auf 2.586 Abschlüsse. Im Studienjahr 2017/18 stiegen die Doktoratsabschlüsse erneut an und erreichten einen Höchststand von 2.755 Abschlüssen; dies waren $24 \%$ mehr als im Studienjahr 2015/16.

Ein Kernpunkt der Reformen des Doktoratsstudiums war, das Verständnis, das Doktorat als ersten Schritt einer wissenschaftlichen Karriere anzusehen, zu etablieren. Damit lag auch die Weiterentwicklung der qualitätsgesicherten Doktoratsausbildung im weiteren Ausbau „strukturierter

49 Vgl. BKA et al. $(2011,17)$.

50 Vgl. BMWFW (2015a, 10).

51 Vgl. BKA et al. $(2011,17)$.

52 Vgl. BMBWF uni:data (2019). 
Doktoratsprogramme" ${ }^{453}$. Strukturierte Programme haben das Ziel, Doktorandinnen und Doktoranden als "early stage researchers" möglichst früh in die wissenschaftliche Gemeinschaft einzubinden sowie klar geregelte, transparente und objektive(re) Verfahren und Abläufe in der Doktoratsausbildung sicherzustellen. Die Struktur orientiert sich dabei an internationalen Vorbildern, etwa an sogenannten Graduate Schools im englischen oder skandinavischen Raum bzw. am deutschen Modell der Graduiertenkollegs.

Obgleich die Regelungen an den einzelnen Universitäten variieren, orientiert sich der konkrete Ablauf eines strukturierten Doktoratsstudiums in Österreich in der Regel an folgendem Muster: Nach Einreichung eines Exposés erfolgt eine (fakultäts-)öffentliche Präsentation des Forschungsvorhabens. Im Fall der positiven Evaluierung des Vorhabens durch einen Beirat oder ein ähnlich gelagertes Gremium wird eine „Dissertationsvereinbarung" abgeschlossen, die auch einen Zeitplan beinhaltet, im Rahmen dessen die Dissertation fertiggestellt werden soll, sowie einen Ar- beitsplan. An einigen Universitäten werden zudem jährliche Fortschrittsberichte, teils auch Belege wissenschaftlicher Tätigkeit, etwa Konferenzteilnahmen oder Publikationen, gefordert. Auch können Schulungen (wie z.B. zu transferable skills) vorgesehen sein.

Beispielhaft lässt sich das Verfahren anhand der graphisch-schematischen Darstellung der Universität Wien in Abbildung 3-5 nachvollziehen.

Insbesondere bei der abschließenden Leistungsüberprüfung haben sich zunehmend Verschiebungen ergeben, wobei ein Trend zur Trennung der Rolle der Betreuerinnen und Betreuer sowie der Gutachterinnen und Gutachter zu beobachten ist. ${ }^{54}$ Analog zu peer-review Verfahren auf höheren Karriere-Ebenen wird auch in der abschließenden Beurteilung von Dissertationen, die in früheren Modellen der Betreuerin/dem Betreuer oblag, nunmehr auf externe Gutachten gesetzt. In einigen neuen Doktoratsprogrammen ist die Betreuerin bzw. der Betreuer nicht länger in die Benotung bzw. die abschließende Evaluierung miteinbezogen. Dadurch wandelt sich auch die Betreuerrolle, die in diesem Modell Rechenschaft über die Betreuungsleistung

\section{Abbildung 3-5: Ablauf eines strukturierten Doktoratsstudiums an der Universität Wien}

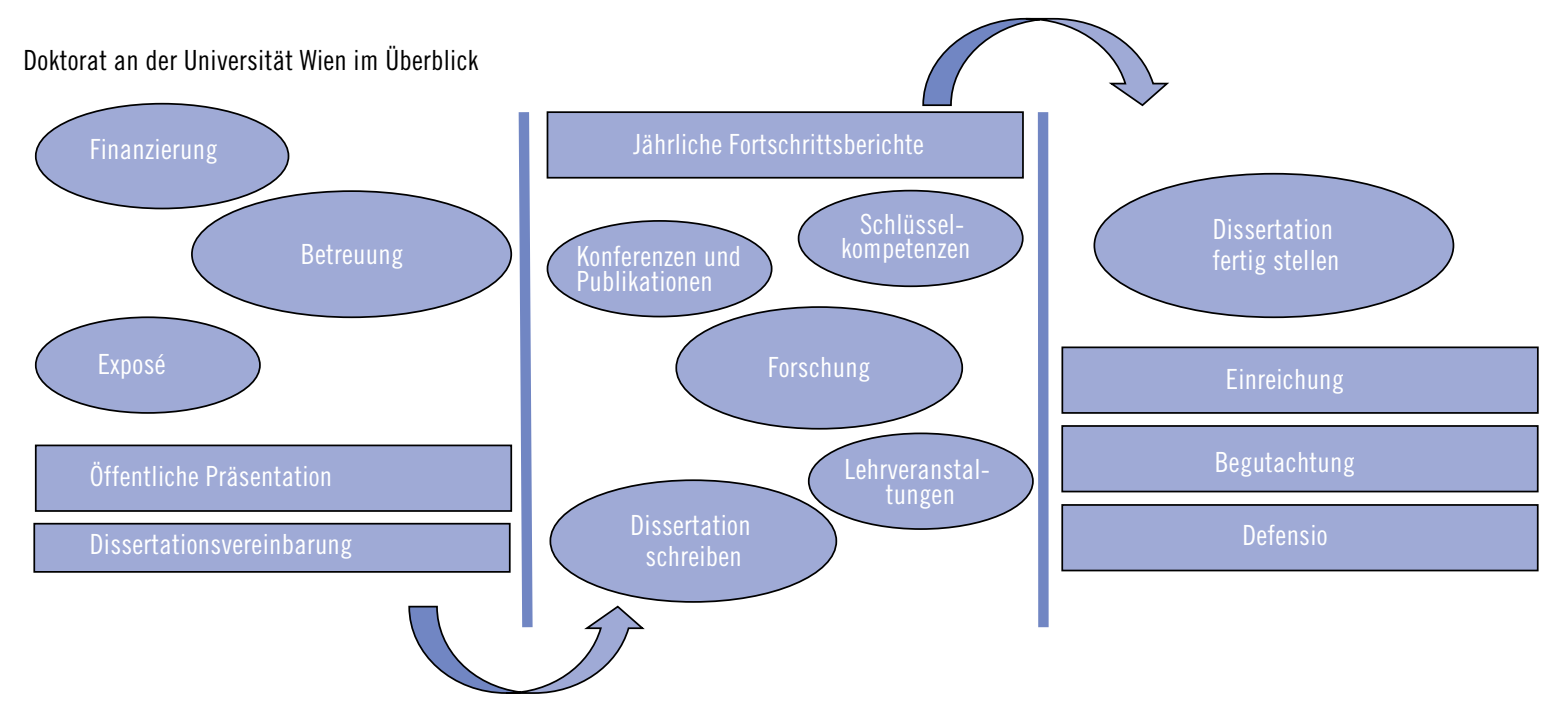

Quelle: Universität Wien: https://doktorat.univie.ac.at/doktoratsablauf/

53 Vgl. Österreichische Hochschulkonferenz (2015).

54 Vgl. Österreichische Universitätenkonferenz $(2015,9)$. 
ablegen muss. Oftmals umfassen strukturierte Doktoratsprogramme daher mittlerweile auch Weiterbildungen für Betreuerinnen und Betreuer, um sie auf diese (neue) Rolle adäquat vorzubereiten.

Im neuen Doktorats-Förderprogramm des FWF (https://www.fwf.ac.at/de/forschungsfoerderung/fwfprogramme/docfunds/ ) ist diese Trennung bereits als grundlegende Anforderung festgeschrieben. Nur jene Doktoratsprogramme, in denen die „personelle Trennung von Betreuung und Beurteilung der Dissertation (wo studienrechtlich konform) ${ }^{455}$ umgesetzt wird, kommen für eine Förderung seitens des FWF in Frage. Auch geht die Entwicklung (v.a. ausgehend von den MINT-Fächern) zunehmend weg von der Einzelbetreuung hin zu einer Betreuung durch ein Team von Wissenschaftlerinnen und Wissenschaftlern. Die Einbettung in Kohorten und Teams von Studierenden ist ebenfalls ein Leitmerkmal von Graduate Schools.

In diesem Sinne wurden an zahlreichen Universitäten sogenannte "Doctoral Schools" bzw. "Doctoral Academies" in ausgewählten Themenfeldern ins Leben gerufen. Nach einem selektiven Auswahlverfahren stehen den aufgenommenen Doktoratsstudierenden weitreichende Unterstützungsleistungen (auch finanzieller Natur, wie z.B. für Konferenzteilnahmen) zu. Es ist jedoch darauf hinzuweisen, dass die Aufnahme in eine Doctoral School bzw. in ein strukturiertes Doktoratsprogramm nicht notwendigerweise gleichbedeutend mit einer Anstellung an der Universität ist. Dies gilt derzeit letztlich für alle strukturierten Doktoratsprogramme in Österreich, die zunächst vor allem eine intensivere Betreuung und die Einbindung in das wissenschaftliche Umfeld ermöglichen sowie einen höheren Grad von Verbindlichkeit seitens der Studierenden herstellen sollen.

Vor diesem Hintergrund stellt die von Seiten des Ministeriums wiederholt geforderte Erhöhung des Anteils von Doktoratsstudierenden mit Anstellungsverhältnis (wie im GUEP 2019-2024 in den Systemzielen 2 und 5 formuliert) eine Herausforderung für die Universitäten dar. Lt. Universitätsbericht 2017 verfügten im WS 2016 rd. 8.000 Doktoratsstudierende an österreichischen Universitäten über ein Anstellungsverhältnis zur Universität, das entspricht etwa einem Drittel aller Doktoratsstudierenden (35\%, 7.869 Beschäftigungsverhältnisse bei 22.374 ordentlichen Doktoratsstudien). ${ }^{56}$ Davon sind jeweils etwa die Hälfte als Projektmitarbeiterinnen und -mitarbeiter in drittmittelfinanzierten Forschungsprojekten bzw. als global finanzierte Universitätsassistentinnen und -assistenten tätig. Ca. zwei Drittel (65 \%, 5.147) aller angestellten Doktoratsstudierenden waren im WS 2017 mit mindestens 30 Wochenstunden beschäftigt. Der Rückgang bei ordentlichen Doktoratsstudien, der mit dem Auslaufen „alter“ Studiengänge 2017 einherging, führte auch zu einem Anstieg des Anteils der Doktoratsstudierenden mit einem Anstellungsverhältnis zur Universität. Ihr Anteil stieg von $31 \%$ im WS 2016 auf 35\% im WS 2017.

Auch die FTI-Strategie ist dahingehend zu interpretieren, dass sie nicht nur die Einbindung in einen strukturierteren Studienablauf bzw. eine verbesserte Betreuungsleistung avisiert, sondern vor allem auch die vermehrte Finanzierung und Absicherung von Nachwuchswissenschaftlerinnen und Nachwuchswissenschaftlern im Rahmen eines Doktoratsprogramms anzielt. Ein Weg, dieses Ziel zu erreichen, führt über höhere Zugangshürden. Die Universitäten haben in der Vergangenheit wiederholt strengere Selektionskriterien auch für den Bereich des Doktorats gefordert. Dies ist mit der UG Novelle 2017 möglich geworden, weshalb davon auszugehen ist, dass sich der Anteil von Doktoratsstudierenden mit Anstellungsverhältnis auf diese Weise künftig erhöhen wird. Auch im Bereich des Doktorats geht damit der Trend zu einer frühe(re)n Entscheidung mit Signalwirkung, einer Vorverschiebung von Karriereentscheidungen und zu einer Überleitung in eine Karriereschiene, um karrieretechnischen „Einbahnstraßen“"57 in späteren Lebensphasen vorzubeugen bzw. diese

55 Vgl. https://www.fwf.ac.at/de/forschungsfoerderung/fwf-programme/docfunds/

56 Vgl. BMBWF uni:data (2019).

57 Vgl. BMWFW (2015a, 9). 
zu vermeiden. Die Entscheidung für oder wider eine akademische Karriere erfolgt in diesem Modell bereits nach Abschluss des Erststudiums (d.h. vor Aufnahme in ein PhD-Programm).

\section{Förderung der Doktoratsausbildung durch den FWF}

Der Wissenschaftsfonds FWF trägt maßgeblich zur Ausbildung von Doktorandinnen und Doktoranden an Österreichs Universitäten bei. Dies betrifft rd. 2.000 Prädoc-Positionen sowohl in geförderten Forschungsprojekten, als auch die im Rahmen der hochselektiven strukturierten Doktoratsprogramme, den sogenannten „Doktoratskollegs (DK)“. Das Programm wurde 2015 letztmalig neu ausgeschrieben und zugleich die Doktorats-Förderschiene auf eine neue Grundlage gestellt: das sogenannte doc.funds-Schema. Die noch laufenden 40 Doktoratskollegs blieben davon unberührt. So wurden im Jahr 2018 sechs DKs für weitere vier Jahre verlängert.

Das neue Förderinstrument strukturierter Doktoratsprogramme setzt dabei nicht länger auf gänzlich neu aufgesetzte thematische Kollegs, wie dies zuvor bei den Doktoratskollegs der Fall war, sondern fördert stattdessen bereits länger als zwei Jahre an den Universitäten erfolgreich bestehende strukturierte Doktoratsprogramme. Diese müssen funktionierende Strukturen bereits etabliert haben (u.a. in Bezug auf Auswahlverfahren, Betreuung - darunter die explizite Anforderung von Teambetreuungen, Monitoring, sowie Unterstützungs- und Weiterbildungsmaßnahmen auch für Betreuerinnen und Betreuer) und in einen Forschungsrahmen eingebettet sein. Laufende FWFDKs können sich erst nach Abschluss eines laufenden DKs um ein doc.funds Programm bewerben.

Diese Weiterentwicklung des Programms trägt den wesentlichen Entwicklungen Rechnung, die sich seit dessen Einführung an den Universitäten gezeigt haben. Das Programm doc.funds setzt auf bereits etablierten Ausbildungsformaten an den einzelnen Institutionen auf und fördert deren thematische Weiterentwicklung, anstatt sich auf den Auf- und Ausbau von Strukturen zu beschränken. Letztlich zielt die neue Förderschiene darauf ab, bereits bestehende qualitätsvolle Doktoratsprogramme in Kombination mit exzellenten Forschungsschwerpunkten zu unterstützen.

\section{Die strukturierte Doktoratsausbildung als Wettbewerbsindikator in der Universitätsfinanzierung}

Die Empfehlungen der Hochschulkonferenz zur Erarbeitung von Kriterien zur Förderung der qualitativen Weiterentwicklung der Doktoratsausbildung (2015) mündeten in eine Umsetzung durch Hochschulraumstrukturmittel und flossen in das System der Universitätsfinanzierung NEU bzw. die Leistungsvereinbarungen 2019-2021 ein. Im Rahmen der Universitätsfinanzierung NEU wurde ein Anreizsystem für die Schaffung bzw. angemessene Ausstattung strukturierter Doktoratsprogramme geschaffen. „Strukturierte Doktoratsprogramme" werden in diesem Modell als ein "Wettbewerbsindikator" berücksichtigt; d.h. je erfolgreicher eine Universität in der Bereitstellung strukturierter Doktoratsprogramme ist, desto höher fällt auch die budgetäre Ausstattung durch die Universitätsfinanzierung aus. ${ }^{58}$ Allerdings werden dabei nur jene Programme über diesen Mechanismus gefördert, die u.a. Teambegleitung sowie die personelle Trennung von Betreuung und Beurteilung der Dissertation vorsehen. ${ }^{59}$

Die - insbesondere von Fachhochschulvertreterin-

58 Zur Definition strukturierter Doktoratsprogramme in der Wissensbilanzverordnung, die u.a. Teambegleitung sowie die personelle Trennung von Betreuung und Beurteilung als kennzeichnendes Merkmal beinhalten müssen, vgl. BMWFW (2017c, 93).

59 Die exakte Definition zu strukturierten Doktoraten It. BMBWF lautet wie folgt (siehe auch Erläuterungen zur Erstellung der Wissensbilanz, gemäß der Verordnung über die Wissensbilanz, BGBI.II Nr. 202/2018):

- Einreichen eines Exposés innerhalb des ersten Jahres nach Zulassung zum Studium

- Öffentliche Präsentation des Dissertationsvorhabens

- Abschluss einer Dissertationsvereinbarung inklusive Zeit- und Arbeitsplans

- Betreuung bzw. Begleitung durch ein Team

- Personelle Trennung von Betreuung bzw. Begleitung der Dissertation und deren Beurteilung 
nen und -vertretern angeregte - Diskussion um das Promotionsrecht an Fachhochschulen, die sich auch im Rahmen der Differenzierungsdebatte und der Anregung einer verstärkten Durchlässigkeit zwischen den Sektoren im Berichtszeitraum verstärkt hat (u.a. auch im Rahmen des Projekts „Zukunft Hochschule“), ist derzeit von geringer politischer Relevanz. In der Diskussion hat sich die Universitätenkonferenz deutlich für die Beibehaltung des „Alleinstellungsmerkmals der Universitäten“ ausgesprochen. ${ }^{60}$ Vielmehr sollen in Zukunft Kooperationsprojekte zwischen forschungsintensiveren Fachhochschulen mit Universitäten angeregt und ausgebaut werden, wie sie etwa bereits z.B. in Oberösterreich (Johannes Kepler Universität Linz und Fachhochschule Oberösterreich) erfolgreich bestehen.

\section{PostDoc Phase und neue Wege zur Professur}

Die Postdoc Phase, also die oft schwierige, entscheidende Übergangszeit zwischen dem Abschluss des Doktorats und einer unbefristeten Festanstellung, kann als Bottleneck einer akademischen Karriere bezeichnet werden. ${ }^{61}$ Auch im internationalen Umfeld stehen (zu) viele PhDs einer (zu) geringen Anzahl möglicher Festanstellungen, insbesondere Professuren, an Universitäten gegenüber. Vor allem in Disziplinen, in denen außeruniversitäre Anstellungsmöglichkeiten vergleichsweise gering ausgeprägt sind (etwa in den GSK), kann dies prekäre Lebens- und Arbeitssituationen für die Betroffenen zur Folge haben, darüber hinaus in einer vergleichsweise späten Lebensphase.

Daher sind während der Berichtsperiode zahlreiche Ansätze und Regelungen getroffen worden, um die Postdoc-Phase besser zu strukturieren und zu regeln bzw. dem wissenschaftlichen Nachwuchs Perspektiven und Wege zur Festanstellung zu eröffnen und die Planbarkeit akademischer Karrieren zu erhöhen. Dies betrifft insbesondere auch die Umsetzung des österreichischen "Laufbahnmodells“, das im Rahmen des Kollektivvertrags für die Arbeitnehmerinnen und Arbeitnehmer der Universitäten (Uni-KV) entwickelt wurde, und welcher 2009 in Kraft getreten ist. Es handelt sich dabei um ein durchgängiges Karrieremodell mit Optionen zur unbefristeten Anstellung in Abhängigkeit von Leistungsevaluationen, im Rahmen derer zu einem frühe(re)n Zeitpunkt Klarheit darüber geschaffen wird, ob eine Entfristung für die Betreffende bzw. den Betreffenden absehbar ist.

Das Schema, das sich an internationalen Vorbildern, vor allem am US-amerikanischen tenure-track orientiert, sieht im Wesentlichen folgenden Ablauf vor: Nach einer internationalen Ausschreibung und einem kompetitiven Besetzungsverfahren, das professoralen Berufungsverfahren durchaus vergleichbar ist, muss mit der erfolgreichen Bewerberin bzw. dem erfolgreichen Bewerber innerhalb von zwei Jahren eine sogenannte "Qualifizierungsvereinbarung" geschlossen werden. In der Folge hat die nunmehrige „Assistenzprofessorin“ bzw. der „Assistenzprofessor“ vier Jahre Zeit, die vereinbarten Kriterien, etwa eine bestimmte Anzahl hochrangiger Publikationen oder Betreuungsleistungen in der Lehre, zu erfüllen. Die zum Teil höchst umfassenden und anspruchsvollen Anforderungskataloge orientieren sich dabei nicht länger nur an wissenschaftlichen, sondern zunehmend auch an gesellschaftlichen Exzellenzkriterien (Stichwort „Responsible University"). Ob die Qualifizierungsvereinbarung als erfüllt gelten kann, wird nach Ablauf der Frist in einem - an den einzelnen Universitäten unterschiedlich gestalteten - Evaluierungsverfahren festgestellt. Ist der Ausgang des Verfahrens positiv, erfolgt die Überleitung in ein unbefristetes Anstellungsverhältnis als sogenannte „Assoziierte Professorin“ bzw. „Assoziierter Professor“.

Im Berichtszeitraum der FTI-Strategie stieg die Zahl der Laufbahnstelleninhaber von 633 im WS 2011 auf $1.452 \mathrm{im}$ WS 2018 an, sie hat sich also mehr als

Das Exposé und die öffentliche Präsentation des Dissertationsvorhabens vorzuweisen, sind die Voraussetzung für das AbschlieBen einer Dissertationsvereinbarung. Vgl. BMWFW $(2017 c, 93)$.

60 Vgl. Österreichische Universitätenkonferenz $(2015,7)$.

61 Vgl. Brechelmacher et al. $(2015,17)$. 
verdoppelt. Dem stehen im WS 2018 rd. 2.600 ordentliche Professorinnen und Professoren (Universitätsprofessorinnen und -professoren nach $\$ 98$ und $\S 99$ Abs. 1-4) gegenüber. Der Frauenanteil an Laufbahnstellen betrug im WS 2018 36\%, dagegen waren lediglich rd. $25 \%$ der Universitätsprofessuren von Frauen besetzt. ${ }^{62}$ Der Anteil von Laufbahnstellen am wissenschaftlichen Stammpersonal hat sich von rd. 4 \% im WS 2011 auf rd. 9 \% im WS 2018 erhöht. ${ }^{63}$

Organisationsrechtlich war die „Assoziierte Professur" bis 2015 dem akademischen Mittelbau, nicht der Professorinnen- und Professorenkurie, zugeordnet; es handelte sich in Österreich bis zu diesem Zeitpunkt daher nicht um einen „echten" tenure-track im Sinne eines Karrierewegs, der bis zur Professur führt. Traditionell war das österreichische akademische Karrieresystem durch eine sogenannte „unbridged disjunction" ${ }^{44}$ - eine unüberbrückbare hierarchische Trennung zwischen Universitätsprofessorinnen und -professoren und dem Rest des akademischen Personals - gekennzeichnet. Die Professur bleibt allein über einen besonderen Bestellungsvorgang, das „Berufungsverfahren“, erreichbar.

Trotz der weiterhin bestehenden Sonderstellung der Professor/inn/enschaft wurden im Berichtszeitraum - auch im Hinblick auf die von der FTI-Strategie angeregte Orientierung an internationalen Vorbildern - erste Anstrengungen unternommen, diese "Kluft“ zu überbrücken bzw. alternative Wege in die Professur einzubeziehen. Mit der UG Novelle 2015 wurde es erstmals ermöglicht, dass "Assoziierte Professorinnen und Professoren", die ein den "internationalen kompetitiven Standards entsprechendes Auswahlverfahren" durchlaufen haben, mit Erfüllung der Qualifizierungsvereinbarung in die Kurie der Universitätsprofessorinnen und Universitätsprofessoren aufrücken können. Damit sind assoziierte Professorinnen und Professoren den berufenen Professorinnen und Professoren organisationsrechtlich gleichgestellt (wenn auch nicht arbeitsrechtlich). Der 2015 eingeführte neue §99 Abs. 5 UG (die hochselektive, kompetitive Auswahl und internationale Ausschreibung) und der ebenfalls neue §99 Abs. 6 UG (das Aufrücken in die Professorinnen- und Professorenkurie) bedingen einander dabei wechselseitig.

Darüber hinaus ermöglichte der neu geschaffene §99 Abs. 4 UG einer begrenzten Anzahl von habilitierten Universitätsdozentinnen und -dozenten (diese entsprechen den „Außerordentlichen Professorinnen und Professoren" nach altem Dienstrecht) sowie „Assoziierten Professorinnen und Professoren“ (also Laufbahnstelleninhabern) über ein vereinfachtes Berufungsverfahren eine Universitätsprofessur zu erlangen, womit sie sowohl arbeitsrechtlich und organisationsrechtlich gleichgestellt sind.

Bezüglich der Akzeptanz dieser "Neuzugänge“ in der Professorinnen- und Professorenkurie fehlen bislang empirische Evidenzen, zumal es sich derzeit noch um eine überschaubare Fallzahl handelt: Im WS 2018 gehörten erst 15 „Assoziierte Professorinnen und Professoren“ über $§ 99$ Abs. 6 der Professorinnen- und Professorenkurie an; 54 Universitätsprofessorinnen und -professoren sind auf Grundlage eines vereinfachten Berufungsverfahrens ( $\$ 99$ Abs. 4) aufgerückt. Letztlich sind es auch die Bestellungsmodalitäten der aufgerückten Professorinnen und Professoren, die ausschlaggebend dafür sein werden, welche Akzeptanz die Stelleninhaber innerhalb der Professorinnen- und Professorenkurie finden werden.

In Erfüllung der Vorgaben der FTI-Strategie wurde im Berichtszeitraum ein durchlässigeres Karrieresystem in Richtung eines "echten" tenure-track, eines Karrierewegs in die Professur, geschaffen. Dennoch wird das Erreichen der Professur letztlich nur einer Minderheit des akademischen Personals vorbehalten bleiben. Die überwiegende Mehrheit der an österreichischen Universitäten angestellten wissenschaft-

62 Siehe hierzu auch Abschnitt 3.2.6.

63 Wissenschaftlich-künstlerisches Stammpersonal umfasst - statistisch gesehen - die Verwendungen 11, 12, 14, 16, 21, 23, 26, 27, $28,81,82,83,84,85,86$ und 87 gemäß BidokVUni. Vgl. BMBWF uni:data (2019).

$64 \mathrm{Vgl}$. Ben-David (1991, 198). 
lich-künstlerischen Mitarbeiterinnen und Mitarbeitern steht in „befristeten Dienstverhältnissen mit wenigen Jahren Laufzeit und geringen Aussichten, in den festen Personalstand der Universität aufgenommen zu werden ${ }^{465}$. Es wurden jedoch weitere Anstrengungen unternommen, um Forscherinnen und Forschern an Hochschulen auch auf sub-professoraler Ebene bessere Karriereperspektiven zu ermöglichen. Dazu zählen die Einführung der unbefristet angelegten Positionen „Senior Scientist“ und „Senior Lecturer" durch den Kollektivvertrag sowie die Neuregelung der sogenannten "Kettenvertragsregelung“. Der § 109 UG war ursprünglich zum Schutz der Arbeitnehmerinnen und Arbeitnehmer vor der Aneinanderreihung mehrerer befristeter Beschäftigungsverhältnisse erlassen worden und sah vor, dass befristete Anstellungsverhältnisse nach Ablauf von sechs bzw. acht Jahren (bei Teilzeittätigkeit) in unbefristete umgewandelt werden müssen. Dies hatte an den Universitäten teils abrupte Abbrüche von Forscherkarrieren zur Folge. Die Betroffenen mussten bei Überschreitung der Frist ihre Stellen verlassen - auch aufgrund der Weigerung der Universitäten, befristet angelegte Stellen in unbefristete umzuwandeln. Der Gesetzgeber trug diesem Umstand mit der UG Novelle 2015 Rechnung und flexibilisierte die Regelungen. Abgesehen davon, dass Tätigkeiten als studentische Mitarbeiterinnen und Mitarbeiter nicht länger angerechnet werden, beginnt nunmehr die Zählung bei einem Wechsel in eine andere Verwendung von Neuem, was z.B. den Sprung von einer auf sechs Jahre befristeten Universitätsassistenz (Postdoc) auf eine ebenfalls auf sechs Jahre befristete Stelle als Projektmitarbeiterin bzw. Projektmitarbeiter ermöglicht. Dies stellt sicherlich eine Erleichterung für zahlreiche wissenschaftliche Mitarbeiterinnen und Mitarbeiter dar.

\subsubsection{Forcierung von Gleichstellung in der Forschung}

Frauen stellen in Österreich bereits seit dem Jahr 2000 über 50 \% der Universitätsabsolventinnen und -absolventen, dennoch sind sie in vielen Bereichen der Forschung noch immer unterrepräsentiert, vor allem auf den höheren Hierarchieebenen, in der industriellen Forschung sowie in vielen Naturwissenschaften und in den meisten Ingenieurwissenschaften. In der FTI-Strategie wurde daher das Ziel verankert, ausgeglichene Geschlechterverhältnisse in der Forschung zu schaffen.

Dazu wurden Maßnahmen ${ }^{66}$ in mehreren Bereichen vorgesehen:

- Gender Budgeting in allen Forschungsförderungsmaßnahmen

- Individuelle Förderungsmaßnahmen für Frauen im wissenschaftlichen Nachwuchs

- Maßnahmen zur besseren Vereinbarkeit von Beruf und Familie

Diese Maßnahmen setzen auf verschiedenen Ebenen an, nämlich (i) auf der Ebene des Bundeshaushaltes, (ii) in den Förderungsagenturen des Bundes, (iii) in den Forschungseinrichtungen und deren Governance, sowie (iv) auf der individuellen Ebene einzelner Forscherinnen. Sowohl Ansätze des Gender Mainstreamings als auch der Frauenförderung werden dabei in einer ganzen Reihe von konkreten Maßnahmen verwirklicht.

\section{Legistische Maßnahmen, Gender Budgeting und Governance}

Mit dem Inkrafttreten der Haushaltsrechtsreform 2013 wurde Gender Budgeting gesetzlich verankert. Dabei sind auf allen Budget-Ebenen Ziele, Maßnahmen und Indikatoren vorgesehen, die auf die tatsächliche Gleichstellung von Frauen und Männern abzie-

65 Vgl. BMWFW (2015a, 10).

66 Vgl. BKA et al. $(2011,17)$. 
len. Gender Budgeting wird im Rahmen der wirkungsorientierten Budgetierung, der wirkungsorientierten Folgenabschätzung sowie im Wirkungscontrolling umgesetzt. Als Gender- bzw. Gleichstellungsziele haben alle drei mit Forschung befassten Ministerien im Bundesvoranschlag $2018^{67}$ eine Erhöhung der Frauenanteile in ihren jeweiligen Wirkungsbereichen festgelegt: Das Wirkungsziel des BMBWF ist "ein ausgeglichenes Geschlechterverhältnis in Führungspositionen und Gremien sowie beim wissenschaftlichen/künstlerischen Nachwuchs" ${ }^{\text {"B8. }}$. Das BMVIT verfolgt das Ziel, die Zahl der Beschäftigten in Technologie und Innovation zu steigern und zwar "mit besonderem Augenmerk auf Erhöhung des Anteils von Frauen "69, und das Gleichstellungsziel des BMDW ist die bessere Nutzung des in Österreich vorhandenen Potentials an Fachkräften, „insbesondere durch Erhöhung des Anteils von Frauen in Forschung, Technologie und Innovation “70.

\section{Grundlagen für die Gleichstellung an Universitäten, Fachhochschulen und Privatuniversitäten}

Wesentliche Grundlagen für eine tatsächliche Gleichstellung von Frauen und Männern an Universitäten, Fachhochschulen und Privatuniversitäten werden kontinuierlich durch Novellen der einschlägigen Materien geschaffen bzw. weiterentwickelt:

Für die öffentlichen Universitäten wurden im Zuge zweier Novellen des Universitätsgesetzes $2002^{71} \mathrm{im}$ Jahr 2015 weitere Schritte gesetzt, um die Gleichstellung voranzubringen. Akzente wurden dabei im Bereich der Vereinbarkeit von Beruf und Familie (siehe unten), der Zusammensetzung von Gremien und der Förderung von wissenschaftlichen Laufbahnen gesetzt. Fragen der Gleichstellung sind jeweils Gegenstand der Leistungsvereinbarungen (LV) zwischen dem BMBWF und den Universitäten. Dementsprechend wurden auch für die Leistungsvereinbarungsperiode 2019-2021 zahlreiche Vorhaben und Ziele vereinbart, die die Erhöhung der Repräsentanz von Frauen (potenzialorientierte Zielvorgaben für wissenschaftliche/künstlerische Führungspositionen), die Einbeziehung der Dimension Geschlecht in universitäre Strukturen bzw. Prozesse und die Einbeziehung der Dimension Geschlecht in Forschung und Lehre stärken. In der zurückliegenden LV-Periode 20162018 setzten die Universitäten vor allem in den beiden erstgenannten Schwerpunktbereichen Ziele und Vorhaben um.

Das QSRG $2011^{72}$ verpflichtet auch die Fachhochschulen und Privatuniversitäten explizit zur Gleichstellung von Männern und Frauen sowie zur Frauenförderung - bis dahin hatte es solche Bestimmungen ausschließlich für öffentliche Universitäten gegeben. Die Akkreditierung dieser Einrichtungen ist seither an entsprechende Regelungen in Entwicklungsplänen und Satzungen geknüpft. Auch bei der Zusammensetzung von Gremien und Organen sind ausgewogene Geschlechterverhältnisse anzustreben.

\section{Gleichstellung in der Forschungsförderung: FWF, FFG}

Im Bereich der Forschungsförderung hat der FWF neben den Grundsätzen der Exzellenz und des Wettbewerbs, sowie der Unabhängigkeit und Internationalität in seinem Leitbild Transparenz und Fairness, Gender Mainstreaming und Chancengleichheit als

67 Vgl. BMF (2018).

$68 \mathrm{Vgl}$. BMF $(2018,364)$.

$69 \mathrm{Vgl}$. BMF $(2018,408)$.

$70 \mathrm{Vgl}$. BMF $(2018,399)$.

71 Vgl. Bundesgesetz über die Organisation der Universitäten und ihre Studien (Universitätsgesetz 2002 - UG), BGBI. I Nr. 21/2015 bzw. BGBI. I Nr. 131/2015.

72 Vgl. Bundesgesetz, mit dem ein Gesetz über die externe Qualitätssicherung im Hochschulwesen und die Agentur für Qualitätssicherung und Akkreditierung Austria (Hochschul-Qualitätssicherungsgesetz - HS-QSG) und ein Bundesgesetz über Privatuniversitäten (Privatuniversitätengesetz - PUG) erlassen werden sowie das Fachhochschul-Studiengesetz (FHStG), das Bildungsdokumentationsgesetz, das Gesundheits- und Krankenpflegegesetz, das Hebammengesetz und das MTD-Gesetz geändert werden (Qualitätssicherungsrahmengesetz - QSRG), Juli 2011. 
wesentliche Grundprinzipien festgelegt. ${ }^{73}$ Das dem BMBWF zur Genehmigung vorgelegte Mehrjahresprogramm 2019-2021 des FWF stellt zudem hinsichtlich der strukturellen Verankerung der Gleichstellung eine deutliche Verbesserung dar. In der neuen FWFStrategie 2019-2020 werden Gleichstellung und Chancengleichheit als zentrale Anliegen des FWF und als Querschnittsmaterie deutlich hervorgehoben, deren Umsetzung als wichtige Führungsaufgabe definiert ist. Zudem bekennt sich der FWF uneingeschränkt zu Gleichstellung und Diversität bei der Gremien-Zusammensetzung, zu fairen und transparenten Verfahren und zu konkreten Zielsetzungen im Bereich Gleichstellung inklusive deren Überprüfung (z.B. 50 \% Frauenanteil im FWF-Kuratorium). Der Aktionsplan sieht u.a. den Ausbau des Monitorings zur Chancengleichheit vor, die entsprechende Weiterentwicklung der jeweiligen Programme, spezielle Maßnahmen zur Erhöhung der Anzahl von Antragstellerinnen sowie eine Kostenstelle Gender-Forschung in den FWF-Programmen selbst. ${ }^{74}$ Der FWF hat zudem mit Anfang 2019 seine Antragsrichtlinien für Einzelprojekte überarbeitet. Die Projektbeschreibung im Antrag hat nunmehr potenzielle geschlechtsund genderrelevante Aspekte im Projekt sowie deren Umsetzung zu beinhalten. Auch wenn keine genderrelevanten Forschungsfragen aufkommen, ist dieser Punkt kurz zu thematisieren. Die entsprechende Adaptierung der Antragsrichtlinien für alle anderen Programme erfolgt im Laufe dieses Jahres.

Auch die FFG hat im Sinne des Gender Mainstreamings die Berücksichtigung von Gender- und Gleichstellungsaspekten in die Bewertung von Anträgen sowie in die Berichtslegung integriert, ähnlich, wie dies in Horizon 2020 praktiziert wird. Für die FFG gilt außerdem im Rahmen der wirkungsorientierten Budgetierung das Ziel, den Anteil von Frauen in Entscheidungsgremien der FTI-Förderungsprogramme zu steigern.

Eine ganze Reihe von Maßnahmen fördert Frauen im wissenschaftlichen Nachwuchs individuell. Dazu zählen die vom BMBWF finanzierten Stipendienprogramme des FWF, das Postdoc-Programm "Herta Firnberg" und das Senior Postdoc-Programm "Elise Richter" sowie das L'ÓREAL-Stipendium für junge Grundlagenforscherinnen in MINT-Fächern. Dazu kommen Maßnahmen an den einzelnen wissenschaftlichen Einrichtungen, welche auch im Rahmen der aktuellen Leistungsvereinbarungen mit dem BMBWF verankert sind.

Hervorzuheben sind hier auch die Gabriele-Possanner-Preise des BMBWF, die alle zwei Jahre für "wissenschaftliche Leistungen, die der Geschlechterforschung in Österreich förderlich sind" vergeben werden, und die auch einen Impuls zur Integration der Geschlechterperspektive in die Forschung setzen.

\section{Laura Bassi - Centres of Expertise}

Das Förderprogramm "Laura Bassi Centres of Expertise" des BMDW, das neben der Förderung von Forscherinnen in der Leitung eines Forschungszentrums auch die Verankerung einer neuen Forschungskultur zum Ziel hatte, findet mit dem Programm Laura Bassi 4.0 eine Fortsetzung und Weiterentwicklung. Der Fokus liegt auf der Digitalisierung, da der Gender-Gap im Bereich IKT virulent ist und die Gefahr besteht, dass sich implizite Mechanismen der Ungleichheit im Zuge des digitalen Wandels verhärten. Das Programm zielt damit auf die Gestaltung einer chancengerechten Digitalisierung ab, indem inter- und transdisziplinäre Forschungs- und Innovationsvorhaben mit hoher gesellschaftlicher Relevanz von Konsortien, in denen Frauen stark und in führenden Rollen repräsentiert sind, gefördert werden. Zur Bekämpfung der strukturellen Ungleichheit in Wissenschaft und Forschung müssen sich die beteiligten Organisationen der geförderten Konsortien zur Durchführung einer Genderanalyse und anschließend zur Setzung von Maßnahmen zur Verbesserung der Chancen-

73 Vgl. https://fwf.ac.at/de/ueber-den-fwf/leitbild/

74 Vgl. FWF (2018). 
gleichheit in der Organisation verpflichten. Als Begleitmaßnahme wird zudem ein Netzwerk „Digitalisierung und Chancengleichheit" aufgebaut. In den Netzwerktreffen werden die impliziten Mechanismen der Ungleichheit thematisiert und bearbeitet. ${ }^{75}$

\section{w-fFORTE}

Im Rahmen des Programms w-fFORTE (Wirtschaftsimpulse von Frauen in Forschung und Technologie) $)^{76}$ setzt das BMDW Maßnahmen für Frauen in Wissenschaft und Forschung. In den Veranstaltungsreihen „Im Fokus: Karriere" für Forscherinnen, Personalverantwortliche und Führungskräfte aus Unternehmen und Forschungseinrichtungen sowie in ManagementWorkshops für Top-Forscherinnen werden Karrierewege und Führungsfragen thematisiert sowie Soft Skills für Führungsaufgaben vermittelt.

\section{Talente}

Im Rahmen des Humanressourcenförderprogramms des BMVIT (Förderschwerpunkt Talente) wird mit der Programmlinie "Talente nützen: Chancengleichheit" ein Schwerpunkt auf Frauen gesetzt. ${ }^{77}$ FEMtech Praktika für Studentinnen dienen dem Einstieg von Nachwuchswissenschaftlerinnen in die angewandte naturwissenschaftlich-technische Forschung. Zur Verbesserung der Rahmenbedingungen und der Bewusstseinsbildung über strukturelle Hemmnisse von Frauen in der Forschung unterstützt „FEMtech Karriere" Organisationen beim Aufbau von Genderkompetenz zur Verbesserung des Personalmanagements, der Öffentlichkeitsarbeit und der Work-LifeBalance in der Organisation, um Frauen in die Forschung zu bringen und deren Karrieren vorantreiben zu können. Des Weiteren werden Forschungsprojekte mit Genderrelevanz im Rahmen von FEMtech Forschungsprojekten gefördert, um die Integration der Genderperspektive in der Forschung voranzutreiben. Für Schülerinnen und Schüler werden im Rahmen von
"Talente entdecken: Nachwuchs" Praktika gefördert, wobei mit der Vorgabe, 50 \% der Praktika an Personen aus AHS und nicht-technischen BHS/BMS zu vergeben, der geschlechtsspezifischen Segregation in den Schulen entgegengewirkt wird.

\section{Vereinbarkeit von Beruf und Familie}

Einen weiteren Schwerpunkt bilden Maßnahmen zur besseren Vereinbarkeit von Beruf und Familie. Die vom BMBWF umgesetzte Erhöhung der Anzahl von Ganztagesschulen und die Ausweitung der Ganztagesbetreuung an sich kommt auch Wissenschaftlerinnen und Wissenschaftlern sowie Forscherinnen und Forschern mit schulpflichtigen Kindern zugute. Angesichts der speziellen Anforderungen für diese Personengruppen sind jedoch auch einschlägige Maßnahmen, die an den Forschungseinrichtungen gesetzt werden, von großer Bedeutung. Mit der UG-Novelle 2015 wurden daher Normen geschaffen, die die Vereinbarkeit an den Universitäten befördern sollen: So wurde die Vereinbarkeit als leitender Grundsatz, der bei der Erfüllung aller universitären Aufgaben zu berücksichtigen ist, festgelegt. Zudem wurden die Universitäten verpflichtet, Gleichstellungspläne, in denen u.a. die Vereinbarkeit zu regeln ist, als Teil der Satzung zu verankern. Ferner wurden in den Leistungsvereinbarungen für 2019-2021 zahlreiche Vorhaben und Ziele zu Fragen der Vereinbarkeit verankert, etwa solche zur (Weiter-)Entwicklung von speziellen Kinderbetreuungsangeboten.

Ähnliches gilt für die Leistungsvereinbarungen mit dem Institute of Science and Technology Austria (IST Austria) sowie mit der ÖAW: Das IST Austria wurde 2014 mit dem Audit „berufundfamilie“ zertifiziert, hat die dreijährige Umsetzungsphase 20152017 erfolgreich durchlaufen und strebt in der laufenden Leistungsvereinbarungsperiode eine Rezertifizierung an. Ein Ausbau der Maßnahmen zur besseren Vereinbarkeit von Beruf und Familie wur-

75 Vgl. FFG (2018).

76 Vgl. http://www.w-fforte.at/

77 Vgl. https://www.femtech.at/ 
den auch in der Leistungsvereinbarung 2018-2020 mit der ÖAW vereinbart.

\section{Zielerreichung}

Betrachtet man die Entwicklungen der Präsenz von Frauen in F\&E in den letzten Jahren quantitativ, so ergibt sich ein gemischtes Bild:

Eine positive Entwicklung lässt sich an den öffentlichen Universitäten feststellen: Bei den Studierenden insgesamt liegt der Anteil der Frauen konstant über $50 \%$ und bei den Erstabschlüssen noch höher (2017: 57,7\%), Frauen sind also nach den Zahlen die erfolgreicheren Studierenden und sie stellen inzwischen auch die Mehrzahl der Zweitabschlüsse (2017: 52,5\%). Der Anteil der Frauen unter den wissenschaftlichen und künstlerischen Assistentinnen und Assistenten ist seit 2010 ebenfalls deutlich gestiegen und zeigt mit $46,2 \%$ ein schon beinahe ausgewogenes Verhältnis. Besonders stark war das Wachstum der Frauenanteile unter den Professorinnen und Professoren, von $15,7 \%$ (2010) auf 24,4 \% (2017), womit das Wirkungsziel für 2017 von 23,5\% sogar übertroffen wurde. Auch bezüglich Erreichung einer Geschlechterparität in Gremien konnten klare Fortschritte erzielt werden.

In den Unternehmen sowie im kooperativen Sektor hingegen stagnieren die Anteile der Frauen in F\&E auf niedrigem Niveau. Das Ziel einer geschlechtergerechten FTI-Landschaft darf daher auch in einer künftigen FTI-Strategie nicht aus den Augen verloren werden. Zum Erreichen eines Gender-Gleichgewichts in Wissenschaft und Forschung sind weiterhin Anstrengungen auf verschiedenen Ebenen erforderlich. Dabei ist die einschlägige österreichische Gleichstellungspolitik im internationalen und europäischen Kontext eingebettet, insbesondere die Vorgaben durch die UN-Konvention zur Beseitigung jeder Form der Diskriminierung von Frauen sowie die Ziele zur Gleichstellung der Geschlechter in der Europäischen ERA Roadmap berücksichtigend. ${ }^{78}$

\subsection{Stärkung der Basis für die Wissensgesellschaft}

FTI-Strategie 2020 mit der Vision:

„Erkenntnis schaffen, Exzellenz forcieren - Die Basis der Wissensgesellschaft festigen"

Die Bedeutung der Grundlagenforschung im Innovationssystem steigt, je mehr sich ein Land der Technologiegrenze annähert und zu einem führenden Innovationsland aufsteigt. Grundlagenforschung ist per se von Neugier getrieben, erweitert die Grenze der wissenschaftlichen Erkenntnis und ist die Basis neuen Wissens. Die Stärkung der Grundlagenforschung stellt damit ein prioritäres Ziel der österreichischen FTI-Politik dar.

\subsubsection{Stärkung der Grundlagenforschung}

Um die Grundlagenforschung zu stärken wurde in der FTI-Strategie 2020 eine Reihe von Zielen definiert, welche „die Steigerung der Investitionen in die Grundlagenforschung bis 2020 auf das Niveau führender Forschungsnationen", "Strukturreformen des Hochschulsystems“, „eine Reformierung des Modells der Universitätsfinanzierung“, „eine Erhöhung der im Wettbewerb einzuwerbenden Drittmittel des Wissenschaftsfonds FWF“, „die Unterstützung der Profilbildung der Universitäten durch Exzellenzcluster", sowie "eine bessere Abstimmung der Lehr- und Forschungsthemen an den Universitäten und eine bessere Zusammenarbeit mit den außeruniversitären Forschungseinrichtungen " umfassen. ${ }^{79}$

Die Stärkung der Grundlagenforschung soll anhand folgender Maßnahmen ${ }^{80}$ erfolgen:

- Entwicklung eines "österreichischen Modells" für eine künftige Teilung der Finanzierung der Universitäten nach studierendenbezogenen Mitteln (Lehre) und Forschung

- Ausbau der Drittmittelfinanzierung der Hochschul-

78 Vgl. European Research Area and Innovation Committee (2015).

79 Vgl. BKA et al. (2011, 21).

80 Ebenda. 
forschung über im Wettbewerb evaluierte Projekte des Wissenschaftsfonds FWF mit pauschalierter Abdeckung der Overheads in der Höhe von $20 \%$

- Hebung des Grundlagenforschungsanteiles an Universitäten und an forschenden Unternehmen durch moderate und kontinuierliche Förderung des Christian-Doppler-Modells (CD-Labors und JR-Zentren) oder nachhaltige Unterstützung bzw. Dotierung des Christian-Doppler-Fördermodells an Universitäten

- Implementierung einer österreichischen Exzellenzinitiative mit Einrichtung von bis zu zehn Exzellenzclustern bis zum Jahr 2020

- Weiterentwicklung der Leistungsvereinbarungen zu einem Instrument für die bessere Abstimmung der Forschungsthemen unter den Universitäten und zur Forcierung der Zusammenarbeit mit anderen Forschungseinrichtungen

- Refinanzierung der vor 2004 beschafften Infrastrukturen auf Basis einer Bestandserhebung und Teilfinanzierung neuer Infrastrukturen für Kooperationen mit universitären und außeruniversitären Forschungseinrichtungen

- Strukturelle Reform der ÖAW durch Erstellung eines Entwicklungsplans, Abschluss von Leistungsvereinbarungen sowie Einführung eines modernen Finanz- und Liquiditätsmanagements

\section{Entwicklung eines „österreichischen Modells“ für eine künftige Teilung der Finanzierung der Universitäten nach studierendenbezogenen Mitteln (Lehre) und Forschung.}

Diese Maßnahme wird in Abschnitt 3.3.2 ausführlich beschrieben.

\section{Ausbau der Drittmittelfinanzierung der Hochschulforschung über im Wettbewerb evaluierte Projekte des Wissenschaftsfonds FWF mit pauschalierter Abdeckung der Overheads in der Höhe von $20 \%$}

Die Summe der Drittmittelerlöse aus F\&E-Projekten der Universitäten lag im Jahr 2010 bei 525 Mio. $€$, davon stammen $23 \%$ beziehungswiese rd. 120,8 Mio. $€$ der Erlöse vom FWF. ${ }^{81}$ Bis zum Jahr 2017 stiegen die Drittmittelerlöse um $28 \%$ an und liegen aktuell bei 673,2 Mio. $€$. Davon stammten 23,5\% beziehungsweise 158,3 Mio. € vom FWF. Auch wenn in den vergangenen sieben Jahren der Anteil des FWF in der Finanzierung nicht mehr gesteigert werden konnte, so wuchsen die finanziellen Mittel des FWF in absoluten Zahlen dennoch an und zeigen insgesamt die große Bedeutung des FWF bei der Finanzierung von Spitzenforschung an den Universitäten.

Die Bewilligungssumme von FWF-Fördermitteln ist erwartungsgemäß für die einzelnen Universitäten sehr unterschiedlich (vgl. Abbildung 3-6), was neben der Größe der jeweiligen Universität auch von den jeweiligen fachlichen Schwerpunkten abhängig ist. Die Medizinische Universität Wien konnte 2017 95,3 Mio. $€$ an Drittmitteln einwerben, davon knapp $19 \%$ (17,9 Mio. €) vom FWF. Im Vergleich: Die Universität Wien warb 2017 79,3 Mio. $€$ an Drittmitteln ein, davon kam ein beträchtlicher Teil von 47,7\% (38,1 Mio. €) vom FWF.

Im Gesamtösterreichischen Universitätsentwicklungsplan wurde außerdem mit der „Sicherstellung einer ausgewogenen und zum Profil der Universität passenden Drittmittelzusammensetzung durch Erstellung/Weiterentwicklung von Drittmittelstrategien seitens der Universitäten" 82 ein Ziel bezüglich 
Abbildung 3-6: Alle Drittmittelerlöse aus F\&E-Projekten und Erlöse aus Projekten des FWF, 2017

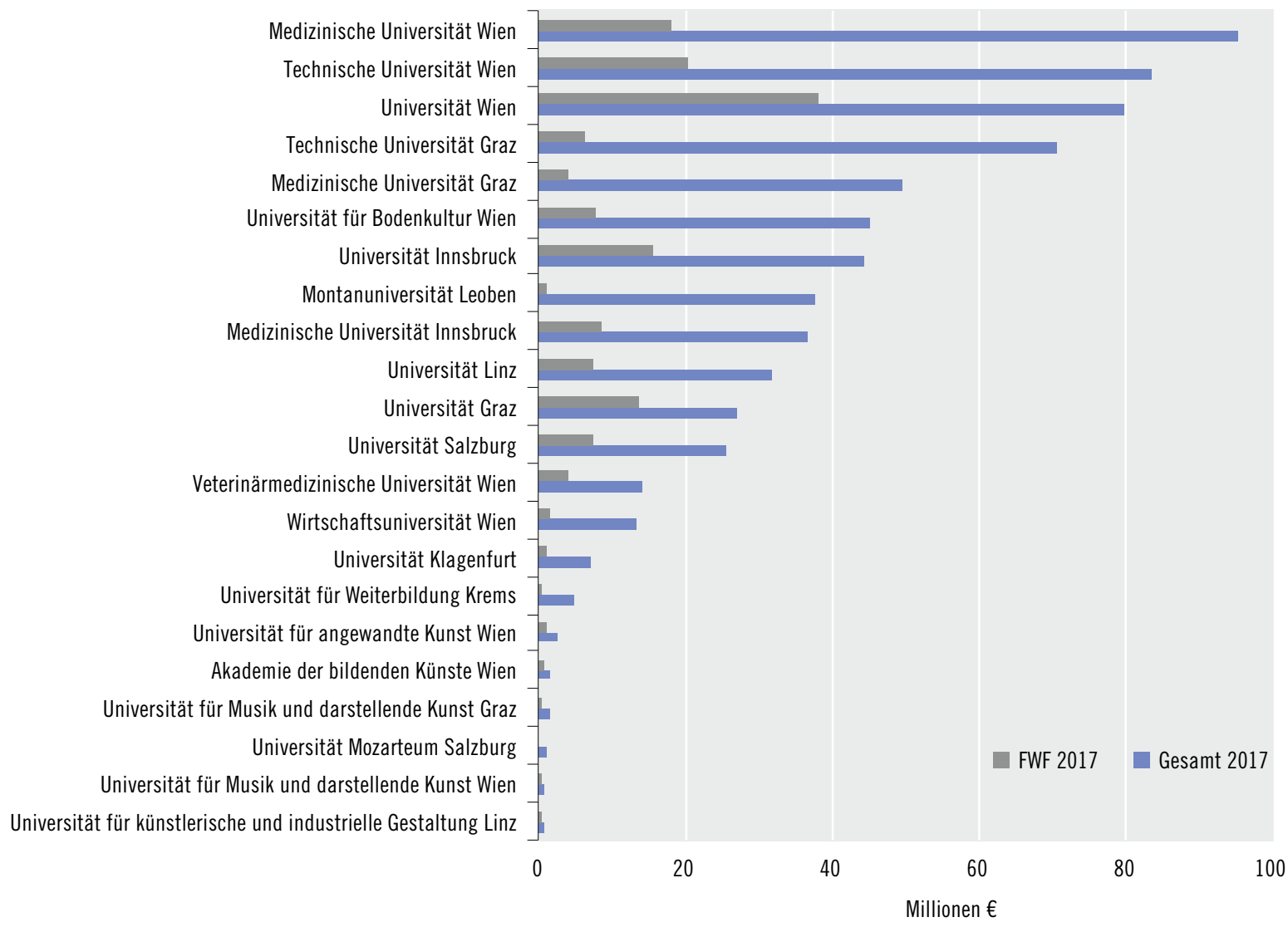

Quelle: BMBWF uni:data

der Drittmittelfinanzierung der Hochschulen festgelegt. Dieses Ziel soll über Verankerung in den universitären Entwicklungsplänen bis 2024 umgesetzt werden.

\section{Implementierung einer österreichischen}

Exzellenzinitiative mit Einrichtung von bis zu zehn Exzellenzclustern bis zum Jahr 2020

Wie im Regierungsprogramm 2017-2022 angekündigt, wurde im August 2018 im Ministerrat eine „Zukunftsoffensive für Forschung, Technologie und Innovation" ${ }^{\text {"83 }}$ beschlossen. Neben einem Forschungsfinanzierungsgesetz soll auch eine Exzellenzinitiative erarbeitet und dann auch umgesetzt werden. „Ziel ist es, disziplinären Engführungen durch inter- und transdisziplinäre Zugangsweisen entgegenzutreten, durch Vernetzung und Kooperation Exzellenzpotenzial der nationalen Forschungseinrichtungen zu erschließen und Exzellenzfelder an der Schnittstelle zwischen etablierten und neu entstehenden Forschungsfeldern zu entwickeln. ${ }^{\text {84 }}$

Für die Ausarbeitung von strategischen Überlegungen, grundlegenden Strategieelementen, Modulen und geeigneten Maßnahmen unter Einbindung der Scientific Community wurde vom BMBWF ein Team aus Expertinnen und Experten bestehend aus dem RFTE-Vorsitzenden, dem Vorsitzenden des ÖWR, der Vorsitzenden des ERA Council Forum Austria und

83 Vgl. BMBWF et al. (2018).

84 Vgl. BMBWF et al. $(2018,2)$. 
dem FWF Präsidenten beauftragt, ein Konzept wurde beauftragt und ausgearbeitet.

Alle Beratungsgremien und die OECD empfehlen die Umsetzung einer Exzellenzinitiative, da diese auch einen wichtigen Impuls für bereits laufende positive Entwicklungen darstellt (Erhöhung der FWF-Finanzierung und der ÖAW-Finanzierung, Universitätsfinanzierung-Neu, Auf- und Ausbau des IST Austria).

\section{Weiterentwicklung der Leistungsvereinbarungen} zu einem Instrument für die bessere

Abstimmung der Forschungsthemen unter den Universitäten und zur Forcierung

der Zusammenarbeit mit anderen

\section{Forschungseinrichtungen}

Das BMBWF schließt mit allen 22 öffentlichen Universitäten für eine Periode von drei Jahren Leistungsvereinbarungen ab. Das Instrument der Leistungsvereinbarung wird stetig weiterentwickelt. Um jedoch darüber hinaus die Planungssicherheit und Transparenz zu erhöhen, wird seit 2016 der Gesamtösterreichische Universitätsentwicklungsplan (GUEP) ${ }^{85}$ angewandt. Dieser umfasst zwei Leistungsvereinbarungsperioden und priorisiert jene Ziele, welche die Weiterentwicklung der Universitäten prägen. Der aktuelle GUEP 2019-2024 sieht damit auch zahlreiche Maßnahmen zur besseren Abstimmung unter den Universitäten vor. So soll etwa der Dialog und die Kooperation zwischen den Hochschulsektoren (z.B. durch gemeinsame Studien- und Lehrangebote) gefördert und ausgebaut, interuniversitäre Kooperation in kleinen Fächern systematisch gestärkt, gemeinsame Aktivitäten im Zuge von Studieninformation forciert und ein aktiveres Durchlässigkeitsmanagement etabliert werden. Zudem soll die Grundlagenforschung an Österreichs Universitäten gestärkt werden, indem die Schaffung von Freiräumen für neue, innovative und unkonventionelle Forschung wie auch die Weiterentwicklung der kompetitiven und projektbezoge- nen Komponenten der Forschungsfinanzierung weiters unterstützt werden.

Wie auch in Abschnitt 3.3.4 dargestellt, wurde mit der Österreichischen Akademie der Wissenschaften (ÖAW) 2012 erstmals eine Leistungsvereinbarung abgeschlossen. Seither werden Leistungsvereinbarungen jeweils für drei Jahre abgeschlossen, die zur Profilbildung und Schwerpunktsetzung im österreichischen Forschungsraum beitragen. Die im November 2017 abgeschlossene Leistungsvereinbarung für die Jahre 2018-2020 sieht eine substantielle Budgetsteigerung um 8 \% bzw. 30 Mio. $€$ auf 363 Mio. $€$ vor. Dies bedeutet eine klare Stärkung der Grundlagenforschung in Österreich. Schwerpunkt der aktuellen Leistungsvereinbarungsperiode ist insbesondere auch die Förderung des wissenschaftlichen Nachwuchses. Im Rahmen der Vereinbarungen liegt ein besonderer Fokus auf Profilbildung, gemeinsamen Berufungen mit dem Universitätssektor, gemeinsamer Infrastrukturnutzung und Stimulierung von Forschungskooperationen.

Auch mit dem IST Austria, das im Jahr 2006 gemeinsam von der Österreichischen Bundesregierung und dem Land Niederösterreich gegründet wurde, wurde 2015 erstmals eine Leistungsvereinbarung abgeschlossen. Darin wurden Zielsetzungen und Maßnahmen für die Periode 2015-2017 festgelegt. Das Monitoring erfolgt jährlich im Rahmen von Begleitgesprächen sowie auf Basis der vereinbarten Berichtslegung. Ebenfalls ist die Mission und Ausrichtung des IST Austria als ausschließlich der Hervorbringung wissenschaftlicher Exzellenz verpflichtete Institution mit Hauptaugenmerk auf die internationale Wettbewerbsfähigkeit in ausgewählten Forschungsbereichen (Life Sciences, Physical Sciences und Formal Sciences) festgeschrieben. Mit dem IST Austria wurde im Jänner 2018 eine neue Leistungsvereinbarung für die Periode 2018-2020 abgeschlossen. In diesem Zeitraum erhält es max. 219 Mio. $€$ an Bundesmitteln, wobei 90 Mio. $€$ davon leistungsabhängig vergeben werden und an die Einwerbung von Drittmitteln und

85 Vgl. BMWFW (2017a). 
die Erfüllung definierter forschungsimmanenter Qualitätskriterien gebunden sind. Gegenüber der ersten Vereinbarung (157 Mio. $€$ ) ist das Budget damit, entsprechend der aktuellen Größe und des geplanten Ausbaus des Instituts, deutlich gewachsen. Der budgetäre Rahmen für die gemeinsame Finanzierung durch Bund und Land Niederösterreich ist für eine zehnjährige Finanzierungsperiode, aktuell von 2017 bis 2026, definiert. ${ }^{86}$ Die Forcierung von Kooperationen im österreichischen Forschungsraum sind vielfältig. IST Austria forscht und publiziert im regen Austausch mit dem gesamten österreichischen Forschungssystem. Die Forschungsinfrastruktur ist "open for collaboration“.

Die wissenschaftliche Evaluierung 2015 hält fest, dass das IST Austria einen hervorragenden Start hatte, die Herausforderungen, die mit einer Gründung einhergehen, gut gemeistert und damit eine gute Basis für die zukünftige Entwicklung gelegt hat. ${ }^{87}$

\section{Refinanzierung der vor 2004 beschafften Infrastrukturen auf Basis einer \\ Bestandserhebung und Teilfinanzierung neuer Infrastrukturen für Kooperationen mit universitären und außeruniversitären Forschungseinrichtungen}

Im Jahr 2016 wurde im Rahmen des „Aktionsplans für einen wettbewerbsfähigen Forschungsraum" die öffentliche Forschungsinfrastruktur-Datenbank ${ }^{88}$ als Informationsplattform umgesetzt. Neben Informationen über Forschungsinfrastruktur-Kooperationen bietet die Datenbank auch eine jährliche nationale Bestandsliste an Forschungsinfrastrukturen, die bei den Leistungsvereinbarungen mit den Universitäten, bei Infrastruktur-Ausschreibungen in Österreich sowie bei verschiedenen Monitoring-Prozessen unterstützend eingesetzt wird.
2014 wurde seitens des BMBWF (damals BMWFW) ein Sonderbauprogramm für Universitätsbauten in der Höhe von 200 Mio. $€$ initiiert. Ziel war die Umsetzung von Sanierungsmaßnahmen in sicherheitsrelevanten Bereichen (z.B. Brandschutz) oder für thermische Sanierungen. Die Umsetzung von Neubauten war nicht Teil dieses Sonderbauprogramms. Die Projekte wurden aus den Gewinnen der Bundesimmobiliengesellschaft im Zeitraum 2013-2017 finanziert. ${ }^{89}$ In Anlehnung an das Sonderbauprogramm für Universitätsbauten aus dem Jahr 2014 wurde auch im Februar 2017 ein neues Sonderbauprogramm durchgeführt. Neben Sanierungen wurden diesmal auch Neuund Erweiterungsbauten finanziert, um die Infrastruktur an den Universitäten zu optimieren. Insgesamt wurden 150 Mio. $€$ in zwölf Bauprojekte investiert, die wie auch 2014 aus den Gewinnen der Bundesimmobiliengesellschaft finanziert wurden. ${ }^{90}$

Weitere Maßnahmen zum Ausbau und zur Weiterentwicklung der Forschungsinfrastruktur werden in Abschnitt 3.3.5 dargestellt. Trotz der umfassenden Anstrengungen wurden It. ForschungsinfrastrukturDatenbank rd. 20 \% des Forschungsinfrastrukturbestands vor 2004 beschafft. Es bedarf daher auch zukünftig Bemühungen, den Bereich der Forschungsinfrastrukturen auf aktuellen Stand zu bringen.

\section{Strukturelle Reform der ÖAW durch Erstellung eines Entwicklungsplans, Abschluss von \\ Leistungsvereinbarungen sowie Einführung eines modernen Finanz- und Liquiditätsmanagements}

Wie auch in Abschnitt 3.3.4 dargestellt, wurde 2012 erstmals eine Leistungsvereinbarung zwischen der ÖAW und dem BMBWF (damals BMWF) abgeschlossen. Darin wurden organisatorische und strukturelle Reformen, wie die Übertragung von ÖAW-Instituten an Universitäten festgelegt. ${ }^{91}$ Seitdem wird in einem

86 Vgl. BMWFW und BMVIT (2016).

87 Vgl. Kornberg et al. (2016).

$88 \mathrm{Vgl}$. https://forschungsinfrastruktur.bmbwf.gv.at/de

89 Vgl. BMWFW (2014).

90 Vgl. BMBWF (2017).

91 Vgl. BMWFW und BMVIT (2016) 
Dreijahresrhythmus eine Leistungsvereinbarung abgeschlossen und darin werden wesentliche Kennzahlen zur Weiterentwicklung der ÖAW definiert.

\subsubsection{Finanzierung der Universitäten nach dem neuen Finanzierungsmodell}

Lt. FTI-Strategie stellen sich "die Rahmenbedingungen für die universitäre Lehre und insbesondere die Betreuungsverhältnisse (...) im internationalen Vergleich ungünstig dar, was sich negativ auf das $A b$ schneiden österreichischer Hochschulen in den Universitätsrankings auswirkt. Der sehr unterschiedliche Andrang zu den Studien bringt nicht nur entsprechend unterschiedliche Studienbedingungen, sondern auch unterschiedliche Chancen am Arbeitsmarkt mit sich. ${ }^{\text {992 }}$ Zur Behebung dieser Mängel wird die „Entwicklung eines ,österreichischen Modells‘ für eine künftige Teilung der Finanzierung der Universitäten nach studierendenbezogenen Mitteln (Lehre)

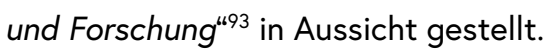

Im ersten Halbjahr 2017 wurde im Rahmen der Reform-Steuerungsgruppe „Studienplatzfinanzierung" vom BMBWF (damals BMWFW) in Abstimmung mit der österreichischen Universitätenkonferenz und unter Einbeziehung des BMF ein Modell zur Festlegung universitätsbezogener bzw. bundesweiter Studienplatzzahlen entwickelt. Im August 2017 wurde sodann ein entsprechender Gesetzesentwurf für eine kapazitätsorientierte, studierendenbezogene Universitätsfinanzierung zur Begutachtung ausgesandt, und im Jänner 2018 wurde das neue Budgetmodell vom Nationalrat beschlossen. Wichtigstes Ziel dabei ist die Verbesserung der Betreuungsrelationen in stark nachgefragten Fächern.

\section{Budgetsäulen und Fächergewichtungen}

Für den Gesamtbetrag der den Universitäten aus Bundesmitteln zur Verfügung gestellten Mittel werden im Rahmen der neuen Universitätsfinanzierung die zu erwartenden Studierendenzahlen und die Betreuungsverhältnisse berücksichtigt. Für besonders stark nachgefragte Studienfelder bzw. Studien wird die österreichweit anzubietende Mindestanzahl an Studienplätzen für Studienanfängerinnen und -anfänger pro Studienjahr und Studienfeld bzw. Studium festgelegt. Das Gesamtbudget teilt sich auf drei Budgetsäulen für die universitären Leistungsbereiche auf in: (a) Lehre, (b) Forschung bzw. Entwicklung und Erschließung der Künste (EEK), sowie (c) Infrastruktur und strategische Entwicklung.

Die Budgetsäule Lehre setzt sich gemäß UG $\S 12$ aus folgenden Beträgen zusammen:

- Einen „Betrag für alle österreichweit in den einzelnen Fächergruppen mindestens anzubietenden Studienplätze." ${ }^{94}$ Die Anzahl dieser Studienplätze in den einzelnen Fächergruppen wird auf Basis der prüfungsaktiven Studien in ordentliche Bachelor-, Master- und Diplomstudien festgelegt; prüfungsaktive Studien sind Studien von Studierenden, die im Studienjahr mindestens 16 ECTS oder 8 Semesterwochenstunden an positiv beurteilten Prüfungsleistungen erbracht haben; anders formuliert: Prüfungsaktiv ist ein Studium dann, wenn die bzw. der Studierende im betreffenden Studium mind. 16 ECTS Punkte oder positiv beurteilte Studienleistungen im Umfang von 8 Semesterstunden erbracht hat.

- Einen „Betrag, welcher anhand von mindestens einem wettbewerbsorientierten Indikator berechnet wird und höchstens $20 \mathrm{vH}$ der Budgetsäule Lehre betragen darf. ${ }^{495}$ 
Die Budgetsäule Forschung bzw. EEK setzt sich gemäß UG § 12 aus folgenden Beträgen zusammen:

- Einen „Betrag für die österreichweit in den einzelnen Fächergruppen mindestens zu beschäftigenden Personen (in Vollzeitäquivalenten) in ausgewählten Verwendungsgruppen." ${ }^{46}$ Konkret handelt es sich bei diesen Personen um wissenschaftliches/künstlerisches Personal.

- Einen „Betrag, welcher anhand von mindestens einem wettbewerbsorientierten Indikator berechnet wird und höchstens $20 \mathrm{vH}$ der Budgetsäule Forschung/EEK betragen darf. ${ }^{497}$

Die Budgetsäule Infrastruktur und strategische

Entwicklung umfasst insbesondere Mittel für:

- Bestehende vertragliche Verpflichtungen, die nicht über die Budgetsäulen Lehre oder Forschung bzw. EEK abgedeckt werden (z.B. Gebäudeinfrastruktur, klinischer Mehraufwand),

- Incentives in Forschung/EEK und Lehre, die direkt finanziert werden sollen, wie z.B. digitale Offensive oder die soziale Dimension,

- Notwendige Durchfinanzierungen und die wirtschaftliche Absicherung des bestehenden Leistungsangebotes der Universitäten.

Die einzelnen Fächergruppen werden gewichtet, wobei insbesondere die unterschiedlichen Ausstattungsnotwendigkeiten und die tatsächlichen Kostenstrukturen berücksichtigt werden. Für die Verteilung der Mittel werden Finanzierungssätze ermittelt. Die Finanzierungssätze für die Lehre ergeben sich aus dem für diese Budgetsäule verfügbaren Gesamtbetrag und der Anzahl der österreichweit in den einzelnen Fächergruppen mindestens anzubietenden Studienplätze unter Berücksichtigung der entsprechenden Fächergruppengewichtungen. Analog werden die Finanzierungssätze für die Forschung/EEK aus dem für diese Budgetsäule verfügbaren Gesamtbetrag und der Anzahl der österreichweit in den einzel- nen Fächergruppen mindestens zu beschäftigenden Personen unter Berücksichtigung der entsprechenden Fächergruppengewichtungen ermittelt.

\section{Dreiteiliger Ablaufprozess}

Die neue Universitätsfinanzierung folgt einem dreiteiligen Ablaufprozess, bestehend aus (a) Planungs-, (b) Umsetzungs- und (c) Analyseprozess.

\section{Planungsprozess}

Das für den Planungsprozess maßgebliche Dokument ist der Gesamtösterreichische Universitätsentwicklungsplan (GUEP), in dem jene Ziele und Rahmenparameter definiert sind, welche die Weiterentwicklung der Universitäten prägen. ${ }^{98}$ Der GUEP umfasst zwei Leistungsvereinbarungsperioden und stellt Planungssicherheit und Transparenz sicher. Auf Basis des GUEP und unter Berücksichtigung der budgetären Rahmenbedingungen des Bundes wird in Abstimmung mit dem BMF alle drei Jahre das Universitätsbudget für die kommende Leistungsvereinbarungsperiode festgelegt. Der Gesamtbetrag wird gemäß dem Drei-Säulen-Modell auf die Teilbereiche Lehre, Forschung/EEK sowie Infrastruktur und strategische Entwicklung aufgeteilt. Außerdem werden die Anteile der nach Wettbewerbsindikatoren zu vergebenden Mittel und die Gewichtungen für die sieben Fächergruppen in Lehre und Forschung/EEK in der Universitätsfinanzierungsverordnung festgelegt. Die Entwicklungspläne der Universitäten haben sich inhaltlich an den Zielsetzungen des GUEP zu orientieren. Die Entwicklungspläne enthalten Angaben über die geplanten und weiterzuführenden Forschungsprojekte/EEK, zum Studienbetrieb, sowie zu den Weiterbildungsaktivitäten und gesellschaftlichen Zielsetzungen. Die gesellschaftlichen Ziele beziehen sich unter anderem auf die Wirkungen der Universitäten auf Wirtschaft und Gesellschaft, Nachhaltigkeit, die dritte Mission sowie Maßnahmen zur besseren sozialen

96 Ebenda

97 Ebenda.

98 Vgl. BMWFW (2017a). 
Durchlässigkeit, zur sozialen Dimension in der Lehre sowie zur Einbeziehung von unterrepräsentierten Gruppen in die Hochschulbildung.

\section{Umsetzungsprozess}

Im Zuge des Umsetzungsprozesses werden die gesamtösterreichischen Ziele und Leistungsbeiträge auf die einzelnen Universitäten umgelegt sowie die Leistungsvereinbarungen unter Berücksichtigung der universitätsspezifischen Schwerpunkte mit den Universitäten verhandelt und abgeschlossen. Für die Umsetzung erhalten die Universitäten ein Globalbudget, dessen Höhe sich primär über spezifische Indikatoren für Lehre und Forschung/EEK (wie zuvor dargestellt) berechnet.

\section{Analyseprozess}

Das Monitoring der in den Leistungsvereinbarungen festgelegten Ziele, Vorhaben und Maßnahmen erfolgt laufend im Rahmen der zweimal jährlich stattfindenden Begleitgespräche zwischen Universität und BMBWF. In diesem Zusammenhang werden auch die Entwicklungen im Bereich der Basisindikatoren analysiert. Sämtliche Erkenntnisse und Analyseergebnisse dienen zudem als Input für die Optimierung des Planungsprozesses für die nächste Leistungsvereinbarungsperiode.

\section{Zugangsregelungen und Festlegung von Studienplatzzahlen}

Parallel mit der kapazitätsorientierten Universitätsfinanzierung sollen den Universitäten bei Überschreitung von studienspezifischen Betreuungsrichtwerten sowohl bundesweit als auch universitätsbezogen Zugangsregelungen ermöglicht werden. Dies erfolgt über das Instrument der Universitätszugangsverordnung. Diese legt fest, welche Studienfelder/Studien aufgrund gesetzlich definierter Kriterien besonders stark nachgefragt sind, und welche Anzahl an Studienplätzen für Studienanfängerinnen und -anfänger mindestens zur Verfügung zu stellen ist. Eine öster- reichweite Festlegung von Studienplatzzahlen für Anfängerinnen und Anfänger erfolgt, wenn:

- der bundesweite Durchschnittswert der Betreuungsrelation der letzten fünf Jahre in einem Studienfeld das 1,75-fache des Betreuungsrichtwertes übersteigt,

- in diesem Studienfeld im Durchschnitt der letzten fünf Jahre österreichweit mehr als 1.000 Prüfungsaktive zu verzeichnen waren und

- das Kapazitätsproblem zumindest an zwei Universitäten besteht.

Lt. UG 2002 § 71b wird gegenwärtig in folgenden Studien eine Mindestanzahl an Studienplätzen festgelegt ${ }^{99}$ :

$\begin{array}{lr}\text { Architektur und Städteplanung } & 2.020 \\ \text { Biologie und Biochemie } & 3.700 \\ \text { Erziehungswissenschaft } & 1.460 \\ \text { Fremdsprachen } & 3.020 \\ \text { Informatik } & 2.800 \\ \text { Management und Verwaltung/Wirtschaft und } & \\ \text { Verwaltung, allgemein/Wirtschaftswissenschaft } & 10.630 \\ \text { Pharmazie } & 1.370 \\ \text { Publizistik und Kommunikationswissenschaft } & 1.530 \\ \text { Recht } & 4.300\end{array}$

Die Aufteilung der festgelegten Anzahl an Studienplätzen auf die einzelnen Universitäten erfolgt im Rahmen der Leistungsvereinbarungen unter Berücksichtigung universitätsspezifischer Faktoren (Kapazitäten, Nachfrage am Arbeitsmarkt, Forschungsstärke sowie bisherige Zahl der Studienplätze). In den betroffenen Studienfeldern bzw. Studien ist das Rektorat berechtigt, die Zulassung zu diesem Studium entweder durch ein Aufnahmeverfahren vor der Zulassung oder durch die Auswahl der Studierenden bis längstens ein Semester nach der Zulassung zu regeln. Im Rahmen des Aufnahme- oder Auswahlverfahrens ist innerhalb einer vom Rektorat festzulegen-

99 Vgl. Bundesgesetz über die Organisation der Universitäten und ihre Studien (Universitätsgesetz 2002 - UG). 
den Frist eine verpflichtende Registrierung der Studienwerberinnen und -werber vorzusehen. Das Verfahren darf nur dann durchgeführt werden, wenn die Anzahl der registrierten Studienwerberinnen und -werber die festgelegte Anzahl an Studienplätzen übersteigt.

Für das Aufnahme- oder Auswahlverfahren sind folgende Vorgaben maßgebend:

- Eine Überprüfung der für das den Ausbildungserfordernissen des jeweiligen Studiums entsprechenden leistungsbezogenen Kriterien;

- Das Aufnahme- oder Auswahlverfahren darf zu keiner Diskriminierung aufgrund des Geschlechts sowie der sozialen Herkunft führen;

- Der Prüfungsstoff muss auf der Homepage der Universität rechtzeitig und kostenlos zur Verfügung gestellt werden;

- Das Aufnahme- bzw. Auswahlverfahren muss mehrstufig gestaltet sein; allfällige mündliche Komponenten dürfen nicht das alleinige Kriterium für das Bestehen des Aufnahme- oder Auswahlverfahrens sein.

\subsubsection{Ausbau der Drittmittelfinanzierung}

Um die Grundlagenforschung in Österreich zu stärken, formulierte die FTI-Strategie die Ziele „die Drittmittelfinanzierung der Hochschulforschung über im Wettbewerb evaluierte Projekte des Wissenschaftsfonds FWF mit pauschalierter Abdeckung der Overheads in der Höhe von $20 \%$ auszubauen" sowie "die Profilbildung der Universitäten durch die Errichtung von Exzellenzclustern zu unterstützen“. 100

\section{Verstärkte Einwerbung von kompetitiven Mitteln}

Das Ziel der FTI-Strategie, in der Forschungsförderung an Hochschulen verstärkt auf kompetitive Mittelvergabe, und damit auf die Akquise von Forschungsprojekten zu setzen, wurde in den Gesamt- österreichischen Universitätsentwicklungsplänen (2015-2017 und 2019-2024) weiter präzisiert: Im Rahmen des GUEP-Systemziels 2c („Weiterentwicklung kompetitiver und projektbezogener Komponenten der Forschungsfinanzierung ") werden die Universitäten konkret dazu aufgefordert, Drittmittelstrategien zu entwickeln sowie innovative Drittmittelkonzepte zu schaffen, die u.a. auch universitätsinterne kompetitive Elemente berücksichtigen. Darüber hinaus werden die Universitäten angehalten, „die Teilnahme der Forschenden an internationalen und nationalen Exzellenzprogrammen zu fordern, zu fördern und die dafür notwendigen Rahmenbedingungen zur Verfügung zu stellen". ${ }^{101}$ Diese Vorgaben wurden in der Folge in den Leistungsvereinbarungen verankert und werden laufend umgesetzt.

Auch von Seiten des BMBWF soll die Finanzierung der Forschung österreichweit zukünftig noch stärker über einen nach internationalen Standards geleiteten Wettbewerb gestaltet werden, was letztlich v.a. auch auf eine Stärkung der über den Wissenschaftsfonds FWF vergeben Mittel hinausläuft, wie sie im Rahmen der FTI-Strategie gefordert wird. Folglich ist es im Rahmen des Bundesfinanzrahmengesetzes 2018-2022 zu einer weiteren Aufstockung des FWFBudgets gekommen, wodurch zu einer weiteren Qualitätsorientierung der Forschungsförderung beigetragen wird.

Tatsächlich hat sich die Drittmittelquote, also der Anteil am gesamten Forschungsetat der Universitäten aus extern finanzierten Forschungsprojekten, seit 2011 von rd. 28,3\% auf 29,1\% erhöht. Die Erlöse aus extern finanzierten Forschungsprojekten an österreichischen Universitäten sind seit 2011 um 22,6 \% gestiegen. Sie betrugen 2011 rd. 549 Mio. $€$, im Jahr 2017 waren es bereits rd. 673 Mio. $€{ }^{102}$

Das Forschungsbudget der Universitäten, d.h. die forschungswirksamen Hochschulausgaben des Bun-

$100 \mathrm{Vgl.} \mathrm{BKA} \mathrm{et} \mathrm{al.}(2011,21)$.

101 Vgl. BMBWF $(2017,17)$.

102 Vgl. BMBWF uni:data, Tabelle 7.6. 
Abbildung 3-7: Herkunft der universitären Einnahmen aus F\&E-Projekten sowie Projekten der Entwicklung und Erschließung der Künste 2017
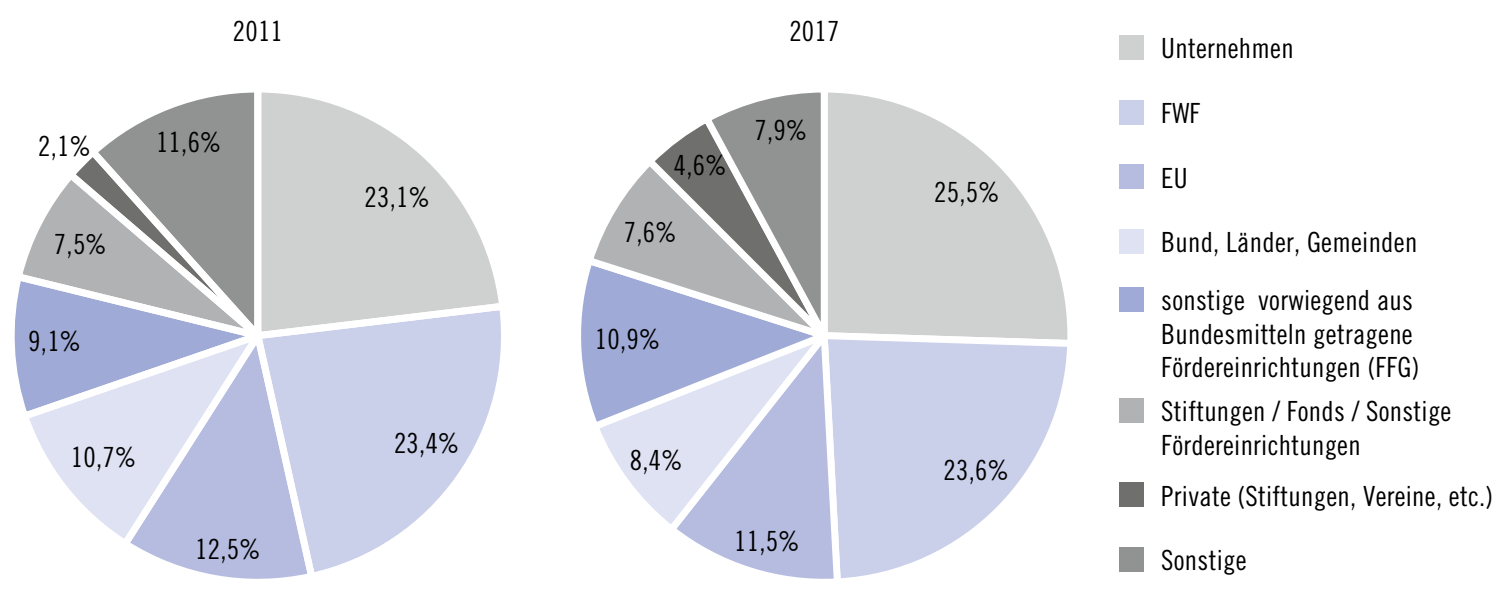

Quelle: BMBWF uni:data, Tabelle 7.6, Darstellung WPZ Research.

des, hat sich im selben Zeitraum um rd. 18 \% erhöht, von rd. $1.388 \mathrm{im}$ Jahr 2011 auf rd. 1.638 Mio. Euro im Jahr 2017. ${ }^{103}$ Die über Drittmittel finanzierte universitäre Forschung ist somit im Berichtszeitraum etwas stärker gestiegen als das Globalbudget für Forschung und Entwicklung an den Universitäten. Das strategische Ziel der FTI-Strategie, stärker auf kompetitive Mittelvergabe zu setzen, bleibt dennoch weiterhin gültig.

Betrachtet man die Finanzierungsquellen, d.h. die Herkunft der universitären Einnahmen aus F\&E-Projekten genauer, wird rd. ein Drittel der Forschungsprojekte aus privaten Mitteln finanziert, rd. zwei Drittel seitens der öffentlichen Hand (inkl. EU). Eine Aufschlüsselung über die Herkunft der universitären Einnahmen aus F\&E-Projekten im Jahr 2017 gibt Abbildung 3-7 wieder. Dabei haben sich im Berichtszeitraum (2011-2017) vor allem die Anteile der von Unternehmen und Privaten finanzierten Forschungsprojekte erhöht. Auch der FFG-Anteil ist von rd. $9 \%$ auf $11 \%$ gestiegen. Ebenso sind die Förderungen aus Mitteln des FWF anteilsmäßig leicht gestiegen, wo- hingegen Förderungen von „Bund, Ländern und Gemeinden" sowie EU-Förderungen anteilsmäßig seit 2011 leicht zurückgegangen sind.

In absoluten Zahlen haben sich die von den Universitäten aus EU-Programmen lukrierten Projektförderungen im selben Zeitraum jedoch von 68,7 Mio. $€$ (2011) auf 76,6 Mio. $€$ (2017) erhöht. Forscherinnen und Forscher aus Österreich sind bei der Einwerbung von Geldern im Rahmen des EU-Forschungsrahmenprogramms „Horizon 2020“ bzw. dem Vorgängerprogramm FP7 (2009-2013) überdurchschnittlich erfolgreich. Abbildung 3-8 gibt einen Überblick über den Anteil Österreichs an im Rahmen von Horizon 2020 in verschiedenen Programmzweigen ausgeschütteten Mitteln wieder (insgesamt liegt der Anteil bis 2018 bei rd. 2,9\%. Zum Vergleich: Der österreichische Anteil am EU-Budget lag 2017 bei rd. 2,5\%; Österreich hat 1,7\% der Bevölkerung der EU-28). ${ }^{104}$

Insbesondere im Themenbereich "Science with and for Society" schneidet Österreich mit rd. $7 \%$ aller von der EU in diesem Themenfeld ausgeschütteten Gelder sehr gut ab. Insgesamt ist die Rückfluss-

\footnotetext{
103 Quelle: Statistik Austria: Allgemeine forschungswirksame Hochschulausgaben des Bundes 2000 - 2019: „General University Funds" auf Basis der Beilagen T der Arbeitsbehelfe und "Detailübersichten Forschungswirksame Mittelverwendungen des Bundes" zu den Bundesfinanzgesetzen.

104 Quelle: Europäische Kommission - Eurostat, BMF.
} 


\section{Abbildung 3-8: Anteil Österreichs an EU-Forschungsgeldern (Horizon 2020), Stand 06/2018}

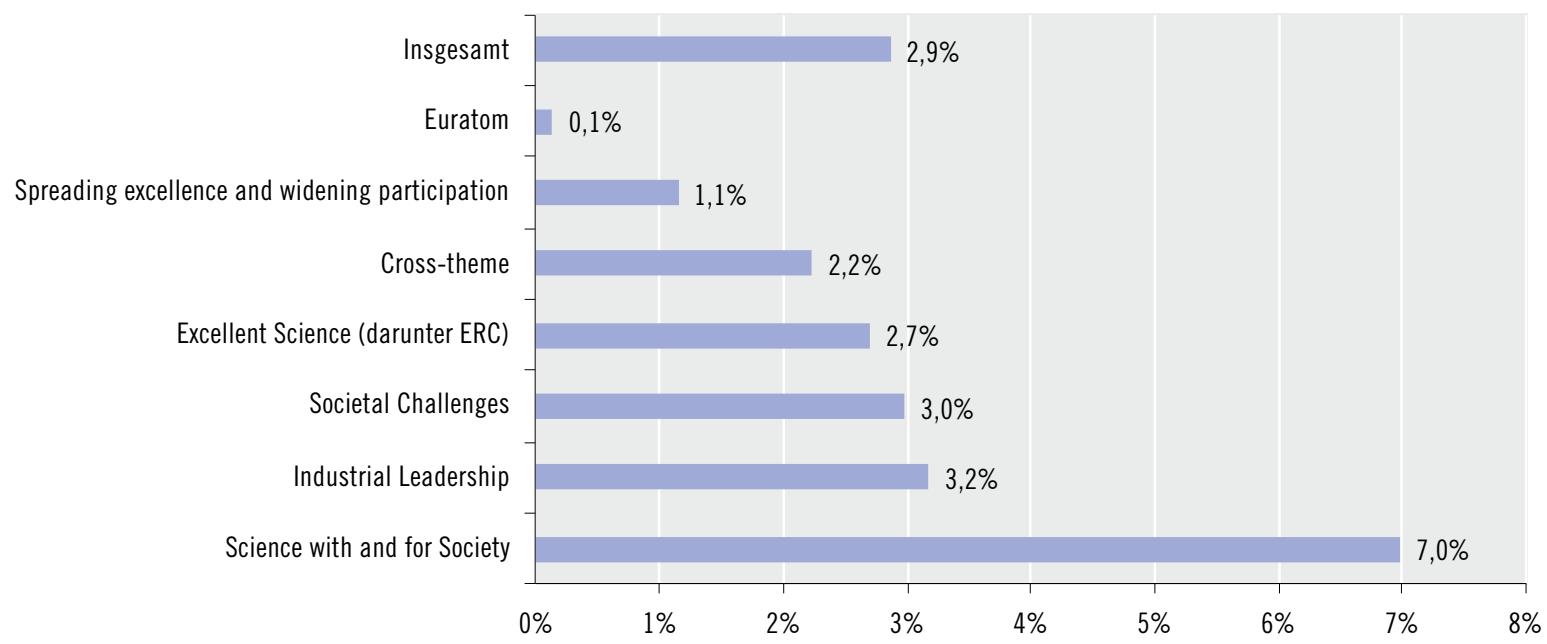

Quelle: BMBWF uni:data, Tabelle 7.5

quote im Bereich Forschung positiv. Österreich erhält mehr aus dem - kompetitiv verteilten, projektbasierten - EU-Forschungsbudget zurück, als es einzahlt, das Land zählt also im Forschungsbereich zu den Nettoprofiteuren: Während Österreich im Jahr 2017 rd. 2,5\% des EU-Haushaltes beigesteuert hat, sind 2,8 \% der EU-Forschungsgelder nach Österreich geflossen. ${ }^{105} \mathrm{Im}$ europäischen Vergleich belegt Österreich in absoluten Zahlen der eingeworbenen EUMittel den respektablen neunten Platz, in Relation zu den eingezahlten Mitteln die Zehnte Stelle. ${ }^{106}$ Bei der Erfolgsrate, d.h. der Bewilligung eingereichter Forschungsvorhaben, liegt Österreich sogar an zweiter Stelle.

Ebenso im Bereich der europäischen Spitzenforschung waren österreichische Forscherinnen und Forscher im Berichtszeitraum höchst erfolgreich. Die erfolgreiche Einwerbung sogenannter ERC Grants, d.h. Förderungen des European Research Council, ist als "Qualitätssiegel" für Forschungsleistungen zu interpretieren, handelt es sich dabei doch um die renommierteste, begehrteste und international sichtbarste
EU-Spitzenforschungsförderung ${ }^{107}$ (vgl. Abbildung 3-9). Mit insgesamt 243 Grants für Pionierforschung bis 2018 hält Österreich im internationalen Vergleich innerhalb der EU eine Position im guten Mittelfeld an neunter Stelle, in Relation zur Bevölkerungsgröße innerhalb der EU belegt Österreich sogar den sechsten Platz (rd. 27,5 ERC Grants pro Mio. Einwohnerinnen bzw. Einwohner). ${ }^{108}$

In den vergangenen Jahren wurden zahlreiche Maßnahmen gesetzt, um vor allem europäische Programme und Strukturen bestmöglich zu nutzen und so ein Maximum an Rückfluss zu sichern. Dazu zählt u.a. auch die Weiterentwicklung des Beratungssystems für Horizon 2020 durch die Österreichische Forschungsförderungsgesellschaft. Des Weiteren wurden insbesondere über das Instrument der Leistungsvereinbarungen exzellenzbezogene Maßnahmen an den Universitäten forciert, oder mit der Einrichtung von zahlreichen Christian-Doppler-Labors, bzw. diesbezügliche Anreizsysteme geschaffen und die Universitäten dazu angehalten, entsprechende Drittmittelstrategien zu entwickeln. Darüber hinaus

105 BMBWF, uni:data, Tabelle 8.9; BMF.

106 Im Jahr 2016, vgl. BMBWF (2018b, 103ff).

$107 \mathrm{Vgl}$. BMBWF (2018b, 101f).

108 Einschließlich ERC-Ausschreibungen 2018, exkl. Advanced Grant 2018. Quelle: Eurostat: ERC: https://erc.europa.eu/projectsfigures/statistics (abgerufen am 13. Februar 2019). 
Abbildung 3-9: Österreich: Anzahl der eingeworbenen ERC Grants für Pionierforschung (kumuliert seit 2007)

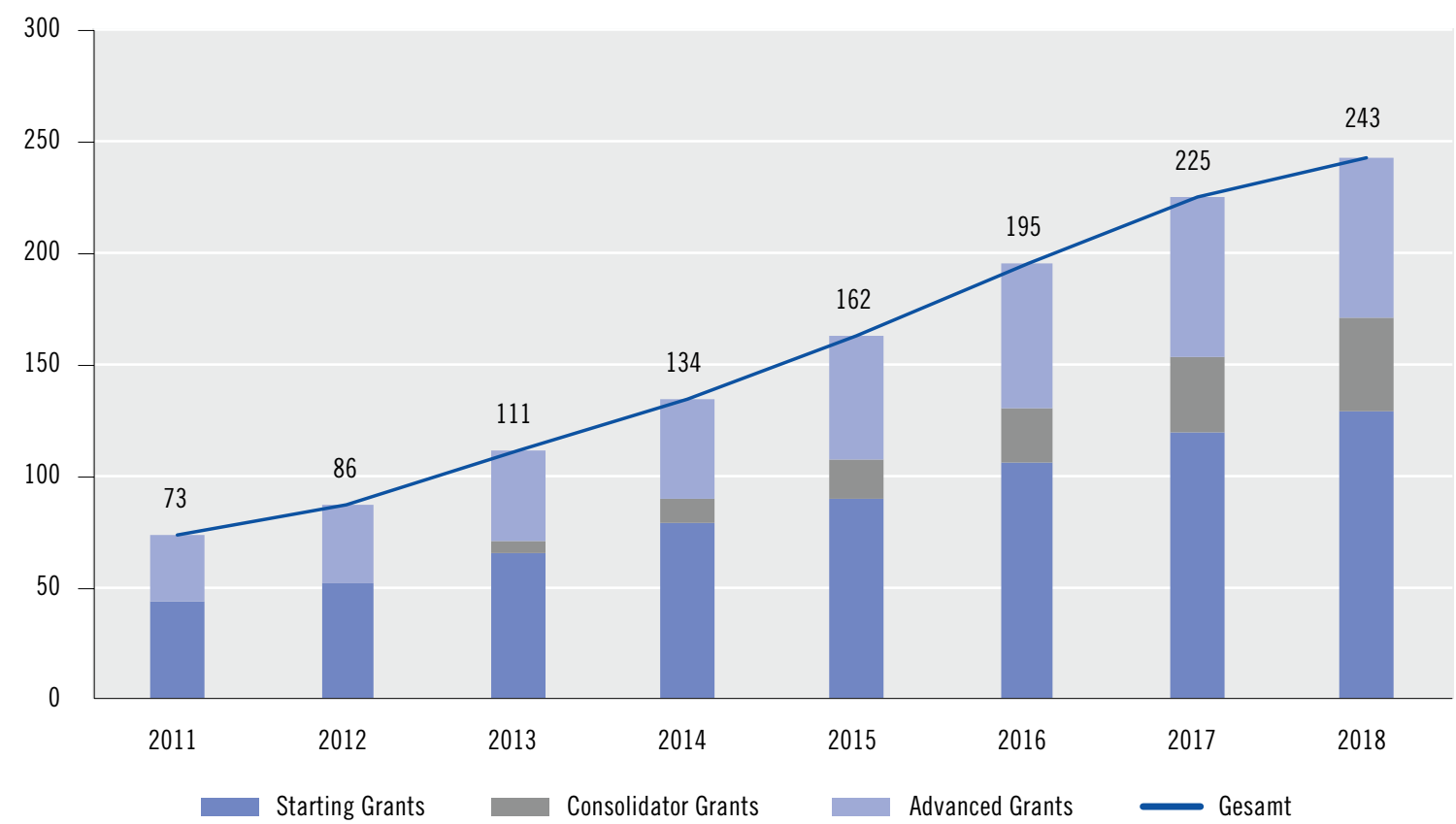

Quelle: ERC, https://erc.europa.eu/projects-figures/statistics, Kumulative Darstellung WPZ Research; Anmerkung: Die im Jahr 2018 neu eingeworbenen Advanced Grants sind noch nicht inkludiert.

basiert der Erfolg auf europäischer Ebene auch auf einer starken nationalen Forschungsförderung im kompetitiven Bereich. So erhielten bspw. 80 \% der Forschenden mit einem ERC Advanced Grant zuvor eine FWF-Förderung. Die nationalen und europäischen Forschungsförderungen ergänzen und bedingen sich gegenseitig. Eine starke nationale Förderung und eine entsprechend starke Forschungslandschaft sind Grundvoraussetzung, um auch auf europäischer Ebene erfolgreich zu sein. Somit werden nationale Investitionen durch den erhöhten Erfolg auf europäischer Ebene zum Teil auch gegenfinanziert.

In der Leistungsvereinbarung der Universität Wien 2019-2021 ist etwa festgehalten, dass die Rahmenbedingungen, die für eine projektorientierte Forschung dienlich sind, weiter verbessert werden sollen. Dazu zählt etwa die „Optimierung der internen Servicestrukturen im Drittmittelbereich". Ebenso plant die Universität die Umsetzung einer „Drittmittelstrukturstrategie", die sowohl Maßnahmen auf institutioneller Ebene als auch Maßnahmen auf der Ebe- ne der Wissenschaftlerinnen und Wissenschaftler umfasst und mitunter befristete Entlastungen im Lehrbereich für die Dauer der Antragstellung hochkarätiger Drittmittelprojekte (u.a. ERC, SFB) vorsehen kann.

Als beispielhaft für geplante Aktivitäten und Schwerpunktsetzungen im Bereich der Drittmitteleinwerbung kann auch die Universität Innsbruck gelten, die in ihrer Leistungsvereinbarung weitreichende Fortbildungs- und Unterstützungsangebote festlegt, um die Beteiligung an EU-Forschungsrahmenprogrammen zu steigern: so etwa ein jährliches Schulungsprogramm zu Horizon 2020, sowie die Umsetzung und laufende Weiterentwicklung eines umfassenden ERC Mentoring-Konzepts mit anschlieBender Wirkungsgradmessung.

Die Universität Wien strebt darüber hinaus an, sich verstärkt auch in der anwendungsorientierten Forschung zu positionieren. So ist eine „Diversifikation der Förderungen", mithin die Stimulierung von Einwerbungen nicht-grundlagenorientierter Drittmittelprojekte, beabsichtigt. Dies soll mitunter 
durch eine bessere Verschränkung von Technologietransfer- und Antragsaktivitäten sowie durch Beteiligungen im Rahmen des European Innovation Council (EIC) gelingen.

\section{Erhöhung der Mittel, die durch den FWF vergeben werden}

Die FTI-Strategie peilte, wie bereits zuvor erwähnt, eine Reform der Hochschulfinanzierung im Sinne einer verstärkten wettbewerbsorientierten Mittelvergabe an. Dies betrifft in Österreich insbesondere die Rolle und Ausstattung des Wissenschaftsfonds FWF als das wichtigste Förderinstrument für Grundlagenforschung. Die Aufstockung der durch den FWF verteilten Mittel wird auch durch die OECD im Review of Innovation Policy 2018 für Österreich empfohlen. In dem Bericht wird auf die im internationalen Vergleich geringe Dotation der im Wettbewerb vergebenen Mittel für die Grundlagenforschung in Österreich hingewiesen: "The comparatively low funding of the FWF [...] is an impediment to excellent research [in Austria]" ${ }^{\text {"109. }}$.

Im Einklang mit den Strategievorgaben erhöhte sich das FWF-Budget von 490 Mio. $€$ in der Periode 2013-2015 auf rd. 552 Mio. $€$ für die Jahre 20162018, was einer Budgetsteigerung von rd. $12,5 \%$ entspricht. Im Jahr 2011 wurden Drittmittelprojekte an den Universitäten in der Höhe von 128 Mio. $€$ vom FWF gefördert, 2017 waren es 158 Mio. $€$; dies entspricht einer Steigerung des Fördervolumens von rd. $23 \%{ }^{110}$

Was die Abdeckung von Overheadkosten anbelangt, so konnte diese derweilen nicht über den FWF umgesetzt werden, stattdessen wird die Finanzierung von Overheads für Forschung im Rahmen der Universitätsfinanzierung NEU berücksichtigt. Das neue Modell sieht die Einführung von Wettbewerbsindikatoren in der Forschungsfinanzierung vor, ein solcher Indikator sind „Erlöse aus F\&E-
Projekten/Projekten der Entwicklung und ErschlieBung der Künste". Nach derzeitiger Schätzung werden die Universitäten je eingenommenem Euro vom FWF 25-27 Cent pro Jahr zusätzlich aus diesem "Wettbewerbstopf “"11 erhalten. Diese Mittel gehen ins Globalbudget und können von Seiten der Universitäten natürlich auch als Kompensation für Overheads zur Abdeckung von direkten administrativen und Infrastrukturkosten der FWF-Forschungsprojekte genutzt werden. Vor dem neuen Finanzierungsmodell gab es eine solche Berücksichtigung von indirekten Kosten/Aufwendungen für Forschungsprojekte auch bereits, und zwar im Rahmen der Hochschulraumstrukturmittel (unter dem Indikator „Wissenstransfer") für die Leistungsvereinbarungsperioden 2013-2015 und 2016-2018.

\section{Errichtung von Exzellenzclustern}

Die Implementierung einer österreichischen Exzellenzinitiative mit der Einrichtung von bis zu zehn Exzellenzclustern bis zum Jahr 2020 stellte eine weitere, von der FTI-Strategie avisierte Maßnahme dar. Auch die OECD unterstrich in ihrem Review 2018 die Bedeutung eines solchen Vorhabens für den Forschungsstandort Österreich. Für nähere Ausführungen siehe hierzu Abschnitt 3.3.1.

\subsubsection{Strukturelle Reformen der außeruniversitären Forschung}

Neben dem Hochschulsektor hat sich in Österreich eine vielfältige und ausdifferenzierte Szene von auBeruniversitären Forschungseinrichtungen etabliert, in die rd. ein Drittel der öffentlichen Ausgaben für Forschung und Entwicklung fließt. Die F\&E-Erhebung der Statistik Austria unterscheidet vier Sektoren: Unternehmen (kooperativer und firmeneigener Bereich), Hochschulen, Staat und privater gemeinnütziger Sektor. Der kooperative Bereich des

109 Vgl. OECD (2018a, 29).

110 Vgl. BMBWF, uni:data, Tabelle 7.6.

111 Nach BMBWF-internen Angaben. 
Unternehmenssektors beinhaltet demnach Forschungsdienstleistungseinrichtungen, die regelmäBig F\&E für Unternehmen betreiben. Er umfasst vorrangig die Mitglieder der Vereinigung der Kooperativen Forschungseinrichtungen der österreichischen Wirtschaft (ACR), des Austrian Institute of Technology (AIT), Joanneum Research und die Kompetenzzentren der COMET-Programmlinien. Gemäß Statistik Austria sind 201761 Erhebungseinheiten dem kooperativen Bereich des Unternehmenssektors zuzurechnen. Diese investierten über 825 Mio. $€$ in Forschung und Entwicklung und beschäftigten mehr als 5.300 VZÄ. ${ }^{112}$

Im Bereich der strukturellen Reformen der außeruniversitären Forschung wurden im Rahmen der FTIStrategie 2020 drei Ziele gesetzt, nämlich „die Entwicklung klarer Rollenbilder entlang von definierten Leistungszielen für die verschiedenen Einrichtungen des außeruniversitären Forschungssektors", „die Stärkung der internen Strukturen der Forschungseinrichtungen durch Reformen und durch Anpassung an neue Anforderungen", sowie "die Optimierung der Gesamtstruktur des außeruniversitären Forschungssektors durch eine bessere Abstimmung “ 113

Zu diesem Zweck wurden in der FTI-Strategie folgende Maßnahmen ${ }^{114}$ festgehalten:

- Strukturelle Reformen einzelner Institutionen sowie Fortführung der internationalen, strategischen Positionierung des Austrian Institute of Technology (AIT)

- Finanzierung der außeruniversitären Einrichtungen in Form von befristeten Leistungs- und Fördervereinbarungen, die als Kriterien etwa Publikationen oder Patente aufweisen

- Flexible Gestaltung der Forschungsstrukturen mit Anreizen für die (Re-)Integration von außeruniversitären Einrichtungen in die Universitäten oder andere, größere Forschungsstrukturen
- Einrichtung außeruniversitärer Strukturen vorwiegend in Form zeitlich befristeter Institutionen

- Erneuerung und Vereinheitlichung der Rechtsgrundlagen durch Neufassung des Forschungsorganisationsgesetzes (FOG)

\section{Positionierung der Austrian Cooperative Research (ACR)}

Die Wettbewerbsfähigkeit und Innovationskraft der $\mathrm{KMU}$ zu stärken und ins Blickfeld zu rücken, ist angesichts der Kleinstrukturiertheit der österreichischen Wirtschaft ein vorrangiges Ziel seit den ersten technologiepolitischen Ansätzen. Heute, in Zeiten zunehmender Internationalisierung und unter globalisierten Produktionsbedingungen, gilt dies mehr denn je. Eine wichtige Rolle spielt dabei die Austrian Cooperative Research (ACR), eine Vereinigung von derzeit 18 kooperativen, außeruniversitären sowie gemeinnützigen Forschungseinrichtungen ${ }^{115}$.

Die ACR ist eine Einrichtung zur Bündelung von Interessen im Bereich der außeruniversitären, branchen- und wirtschaftsnahen F\&E. Sie zielt damit auf die Bearbeitung eines sehr spezifischen und fokussierten Teils des österreichischen Innovationssystems ab, den ihre Mitglieder, die ACR-Institute, teils in Kooperation mit anderen Institutionen sowie der Wirtschaft, abdecken. Als Netzwerk von privaten Forschungsinstituten, die F\&E für Unternehmen betreiben, richtet sich das Angebot speziell an KMU, die in ihrer Innovationstätigkeit unterstützt werden sollen. ${ }^{116}$ Derzeit verfügen die ACR-Institute über rd. 800 hochqualifizierte Mitarbeiterinnen und Mitarbeiter (davon sind rd. 30 \% Frauen im Bereich FTI tätig und beträgt der Anteil an Akademikerinnen und Akademikern mehr als $70 \%$ ). Zwei Drittel der Leistungen erbringen die ACR-Institute für KMU. Der Gesamtumsatz der ACR-Forschungsinstitute stieg 2017 gegenüber dem Vorjahr um 4,7\% auf ins-

112 Vgl. Statistik Austria (2015).

$113 \mathrm{Vgl}$. BKA et al. $(2011,22)$.

$114 \mathrm{Vgl}$. BKA et al. $(2011,23)$.

115 Vgl. https://www.acr.ac.at/acr-institute/

116 Vgl. ACR (2018). 
gesamt 64,4 Mio. €, davon sind 56,7 Mio. $€$ des Umsatzes leistungsbezogen. ${ }^{117}$

Eine Evaluierung aus dem Jahr 2015 zeigt, dass die ACR-Institute in Nischen nicht nur eine Brücke von der grundlagennahen Forschung hin zu KMU-Innovationen bilden, sondern auch zwischen größeren Unternehmen und KMU wichtige Player sind. Die gröBeren Unternehmen fungieren dabei als Schrittmacher, KMU können aus den Kooperationen lernen. Durch die Zusammenarbeit (auch) mit größeren Unternehmen können die ACR-Institute letztlich wiederum einen Nutzen für die österreichische KMU-Landschaft stiften. ${ }^{118}$

Die aktuelle Forschungsstrategie hat die ACR 2015 für den Zeitraum bis 2020 formuliert. Das Fundament der Strategie bilden die FEI-Kernkompetenzen der ACR-Institute. Darüber hinaus sind schwerpunktübergreifende Zukunftsfelder definiert, die wirtschaftliche wie auch gesellschaftliche Herausforderungen adressieren. Neben thematisch definierten Leuchttürmen (wie z.B. Innovative Charakterisierungsverfahren, Digitalisierung \& Strukturwandel etc.) wurde auch ein Förderprogramm für „Strategische Projekte" ins Leben gerufen. Das Förderprogramm soll die Institute dabei unterstützen, gemeinsames Know-how aufzubauen und ihre Infrastruktur laufend zu verbessern. Die Förderung wird im Wettbewerb vergeben. Das langfristige Ziel des Programms ist es, Unternehmen mit der bestmöglichen Infrastruktur und Expertise bei der Entwicklung und Einführung marktfähiger Produkte und Dienstleistungen zu unterstützen. ${ }^{119}$

\section{Restrukturierung des Austrian Institute of Technology (AIT)}

Mit 01.01.2017 erfolgte eine organisatorische Neugliederung des AIT. Die ehemaligen fünf Departments wurden in die neue Center-Struktur mit nunmehr acht Centers (namentlich Digital Safety \& Security; Energy; Health \& Bioresources; Innovation Systems \& Policy; Low-Emission Transport; Mobility Systems; Technology Experience; sowie Vision, Automation \& Control) überführt. Die Neuordnung wurde im Rahmen eines Strategieprozesses erarbeitet, um entsprechend auch einer Themenbündelung Rechnung zu tragen. Außerdem wurde die departmentübergreifende Zusammenarbeit evaluiert und für die Strategieperiode 2018-2021 neu aufgestellt. Das System sieht nun die Zusammenarbeit in Business Cases vor, die unter der Leitung eines Centers stehen. ${ }^{120}$ Durch die Organisationsreformen soll sichergestellt werden, dass die internen Strukturen des AIT gestärkt und an neue Anforderungen, wie die "Grand Challenges", angepasst werden.

Bereits in der Rahmenvereinbarung für die Jahre 2014-2017 wurden „Key Performance Indicators“ für ein kontinuierliches Benchmarking im Sinne der Sicherstellung eines möglichst effizienten Einsatzes öffentlicher Mittel durch kontinuierliche Erhöhung der Leistungsfähigkeit des AIT festgelegt. Unter diesen Leistungsindikatoren sind bspw. die Anzahl der Patente, der Publikationen oder auch die Zahl an Dissertantinnen und Dissertanten. Die Indikatoren entwickeln sich seit 2010 sehr positiv: Wurden im Jahr 2010 noch 16 Patente erteilt, waren es 2017 mit 37 bereits mehr als doppelt so viele. Auch die Anzahl der Publikationen in wissenschaftlich referierten Zeitschriften mit Impact Faktor hat sich von 138 auf 243 nahezu verdoppelt. Die Zahl an Dissertantinnen und Dissertanten ist ebenso angestiegen, von 103 in 2010 auf 229 in 2017; mit 34\% kommen 2017 auch um sechs Prozentpunkte mehr Dissertantinnen und Dissertanten aus dem internationalen Raum. ${ }^{121}$ Die Entwicklung der Kennzahlen deutet damit darauf hin, dass die Fortführung der internationalen, strategischen Positionierung des AIT gelungen ist.

117 Vgl. https://www.acr.ac.at/ueber-uns/

$118 \mathrm{Vgl}$. Convelop (2015).

119 Vgl. ACR (2018).

$120 \mathrm{Vgl.} \mathrm{AIT} \mathrm{(2018).}$

$121 \mathrm{Vgl}$. AlT (2018) und AIT (2011). 
Finanzierung der außeruniversitären

Einrichtungen in Form von befristeten Leistungsund Fördervereinbarungen

Seitens des BMVIT werden laufend befristete Rahmen- bzw. Förderungsvereinbarungen mit AIT, Joanneum Research und Salzburg Research abgeschlossen, in denen klare Zielvorgaben definiert sind. In Zukunft sollen manche dieser Verträge über das Forschungsfinanzierungsgesetz ${ }^{122}$ geregelt werden.

\section{Flexible Gestaltung der Forschungsstrukturen mit Anreizen für die (Re-)Integration von außeruniversitären Einrichtungen in die Universitäten oder andere, größere Forschungsstrukturen}

Für die Periode 2012-2014 wurde erstmals eine Leistungsvereinbarung zwischen der ÖAW und dem BMBWF abgeschlossen. Ein Schwerpunkt dieser ersten Periode bildeten insbesondere strukturelle und organisatorische Anpassungen: Die Zahl der Forschungsinstitute wurde von damals 63 auf aktuell 28 - um mehr als die Hälfe - reduziert. Zahlreiche ÖAWInstitute wurden an Universitäten übertragen, um die Profilbildung zu fördern und um optimale Rahmenbedingungen für exzellente Grundlagenforschung sicherzustellen. ${ }^{123}$ Prioritäres Ziel der Leistungsvereinbarung mit der ÖAW war vor allem, die Stärkefelder der ÖAW selbst auszubauen. Vor diesem Hintergrund wurde auch das Basisbudget der ÖAW erhöht und konnte diese ihre Drittmittel in fünf Jahren um zwei Drittel steigern. ${ }^{124}$ Darüber hinaus wurde ein Schwerpunkt auf die Digitalisierung in den Wissenschaften gelegt (wie z.B. durch die Weiterführung und den Ausbau des Austrian Centre for Digital Humanities).

Der Pfad der institutionellen Erneuerung wurde auch in den nachfolgenden Leistungsvereinbarungsperioden verfolgt. In der jüngst abgeschlossenen Leistungsvereinbarung für die Jahre 2018-2020 wur- de das Budget um $8 \%$ auf 363 Mio.€ erhöht und der thematische Schwerpunkt vor allem auch auf die Förderung des wissenschaftlichen Nachwuchses gelegt. Im Rahmen eines Monitorings werden wichtige Leistungskennzahlen dokumentiert und geprüft. Es kann daher geschlossen werden, dass Maßnahmen zur flexiblen Gestaltung der Forschungsstrukturen mit Anreizen für die $(\mathrm{Re})$ Integration von außeruniversitären Einrichtungen in die Universitäten oder andere gröBere Forschungsstrukturen erfolgreich umgesetzt wurden.

\section{Einrichtung außeruniversitärer Strukturen vorwiegend in Form zeitlich befristeter Institutionen}

Bei zahlreichen Programmen im Bereich Wissenschafts-Wirtschaftskooperationen herrscht eine klare zeitliche Befristung. Aus Tabelle 3-2, welche die Laufzeit sämtlicher Programme dargestellt, wird deutlich, dass die Maßnahme, außeruniversitäre Strukturen vorwiegend in Form zeitlich befristeter Institutionen einzurichten, somit vollumfänglich umgesetzt wurde. Details zu den Programmen finden sich in Abschnitt 3.4.3.

Erst im Dezember 2018 wurde mit Silicon Austria Labs $\mathrm{GmbH}(\mathrm{SAL})^{125}$ eine weitere außeruniversitäre Forschungseinrichtung gegründet. Als Spitzenforschungszentrum für Electronic Based Systems mit Standorten in Graz, Villach und Linz liegt der Fokus auf vier Schlüsseltechnologien: 1) Sensor Systeme, 2) Leistungselektronik, 3) Hochfrequenztechnologien und 4) Systemintegration. Als Forschungspartner für Wissenschaft und Wirtschaft soll entlang der gesamten Wertschöpfungskette geforscht werden. Hauptgesellschafter der Silicon Austria Labs ist die Republik Österreich über das BMVIT (50,1\%). Bis Ende 2023 sollen an den drei Standorten rd. 400 Mitarbeiterinnen und Mitarbeiter arbeiten und die

122 Vgl. Kapitel 1.

123 Vgl. BMWFW und BMVIT (2016).

$124 \mathrm{Vgl}$. https://www.oeaw.ac.at/oesterreichische-akademie-der-wissenschaften/die-oeaw/article/mahrerzeilinger-spitzenpositionen-staerken-innovation-foerdern/

$125 \mathrm{Vgl}$. https://silicon-austria-labs.com 
Tabelle 3-2: Laufzeit Wissenschafts-Wirtschaftskooperationen

\begin{tabular}{llc}
\hline \multirow{2}{*}{ Programm } & & \multicolumn{1}{c}{ Laufzeit (in Jahren) } \\
\hline COMET Competence Centre Programme & COMET Module & max. 8 \\
\cline { 2 - 3 } & K1 Zentrum & max. 8 \\
\cline { 2 - 3 } K2 Zentrum & 7 \\
\cline { 2 - 3 } CDG & COMET Projekt & 5 \\
\hline BRIDGE & CD-Labor & max. 3 \\
\hline JR-Zentrum & $1-3$ \\
\hline COIN Cooperation \& Innovation & COIN Netzwerk & $2-5$ \\
\hline Research Studios Austria & COIN Aufbau & 4 \\
\hline AplusB-Zentren & & 10 \\
\hline Laura Bassi Centres of Expertise & AplusB-Programm & 5 \\
\hline Innovationsscheck & aws AplusB Scale-up & 7 \\
\hline Spin-off Fellowships & & max. 1 \\
\hline
\end{tabular}

Quelle: aws, CDG und FFG.

Silicon Austria Labs damit zum drittgrößten Forschungszentrum Österreichs machen. Dafür stehen 140 Mio. $€$ seitens des Bundes und der Bundesländer Steiermark, Kärnten und Oberösterreich, sowie weitere 140 Mio. $€$ seitens der Elektroindustrie zur Verfügung. Nach Ablauf der Pilotphase im Jahr 2023 ist eine Zwischenevaluierung der Aktivitäten geplant.

\section{Erneuerung und Vereinheitlichung der Rechtsgrundlagen im Kontext des FOFINAG}

Die Vereinheitlichung und Erneuerung der Rechtsgrundlagen wird soweit relevant in das Forschungsfinanzierungsgesetz, welches im Regierungsprogramm 2017-2022 vorgesehen ist und im Ministerrat im Sommer 2018 angekündigt wurde, einfließen (siehe hierzu auch Kapitel 1).

\subsubsection{Ausbau und Weiterentwicklung von Forschungsinfrastrukturen}

Auch was Forschungsinfrastrukturen betrifft, setzte die FTI-Strategie Ziele, konkret dass „die Forschungsinfrastrukturen in Österreich als Basis für

126 Vgl. BKA et al. $(2011,23)$.

127 Ebenda. exzellente Forschung und zur internationalen Positionierung der österreichischen Forschung koordiniert ausgebaut werden sollen" und "die Nutzung der Forschungsinfrastrukturen der Profilbildung der Universitäten und außeruniversitären Forschungseinrichtungen [als Träger der Forschungsinfrastrukturen] eine optimale Abdeckung hinsichtlich Stärken und Synergieeffekten garantieren soll“ ${ }^{\star 126}$.

Eine konkurrenzfähige Infrastrukturausstattung der Forschungsinstitutionen und der Zugang zu internationalen Infrastrukturen ist eine wesentliche Voraussetzung für einen attraktiven Forschungsstandort. Daher wurden folgende Maßnahmen ${ }^{127}$ zur Zielerreichung definiert:

- Erarbeitung einer verbindlichen „Nationalen Roadmap für Forschungsinfrastruktur"

- Anreize zur Vernetzung von Infrastrukturen zur Erreichung kritischer Massen, wie etwa die Finanzierung von Großinfrastrukturen in Abhängigkeit von Konzepten koordinierter Nutzung (wie im Fall von Hochleistungsrechnern)

- Ausbau der Kooperation von Forschungseinrichtungen und Unternehmen auf der Basis gemeinsamer Infrastrukturnutzung 
- Beteiligung Österreichs an europäischen und internationalen Infrastrukturen im Rahmen der ESFRI-Roadmap

- Entwicklung der rechtlichen Rahmenbedingungen für die Nutzung von Infrastrukturen, wie Biobanken und statistischer Datenbestände

\section{Erarbeitung einer verbindlichen „Nationalen Roadmap für Forschungsinfrastruktur"}

Wettbewerbsfähige Forschungsinfrastrukturen sind ein Schlüsselfaktor, um anspruchsvolle Forschung zu betreiben, wichtige technologische Fortschritte zu erzielen und damit den Herausforderungen der Zukunft gewachsen zu sein. Basierend auf der FTIStrategie wurde daher der "Österreichische Forschungsinfrastruktur-Aktionsplan" erarbeitet, der die Herausforderungen für den Bereich der grundlagenforschungsgetriebenen und anwendungsorientierten Forschungsinfrastrukturen thematisiert. ${ }^{128}$ In den Bereichen Grundlagenforschung wie auch anwendungsorientierte Forschungsinfrastrukturen werden jeweils Status-quo erhoben und Ziele zur Weiterentwicklung definiert. Die Forschungsinfrastrukturen in Österreich als Basis für exzellente Forschung sollen koordiniert ausgebaut werden. AuBerdem soll die Profilbildung der Universitäten und außeruniversitären Forschungseinrichtungen als Träger der Forschungsinfrastrukturen für eine optimale Abdeckung von Stärken und Synergieeffekten in der Nutzung von Forschungsinfrastrukturen sorgen. Der "Österreichische ForschungsinfrastrukturAktionsplan" liefert bis ins Jahr 2020 die Grundlage für forschungsinfrastrukturpolitische Ausrichtungen und berücksichtigt dabei auch europäische Initiativen und Strategien. Der Aktionsplan wurde seitens der Europäischen Kommission als ex-ante Konditionalität (Vorliegen einer Roadmap) für die potenzielle Nutzung von finanziellen Mitteln europäischer Fonds für regionale Entwicklung (EFRE) ab dem Jahr 2014 anerkannt.

\section{Anreize zur Vernetzung von Infrastrukturen zur Erreichung kritischer Massen, wie etwa die Finanzierung von Großinfrastrukturen in} Abhängigkeit von Konzepten koordinierter Nutzung (wie im Fall von Hochleistungsrechnern) Im Rahmen der Hochschulraum-Strukturmittel wurde im Jahr 2013 erstmals ein Teilbetrag von 63 Mio. € für die Leistungsvereinbarungsperiode 2013-2015 als Anschubfinanzierung von Kooperationsprojekten zur Verfügung gestellt. Die Mittel wurden kompetitiv vergeben, Voraussetzung war die Beteiligung mindestens einer weiteren Institution aus dem Wissenschafts-, Hochschul-, Kunst- oder Kulturbereich oder der Wirtschaft. Damit sollte einerseits ein Beitrag zu einem in Lehre und Forschung abgestimmten Hochschul- und Forschungsraum geleistet und andererseits die Durchlässigkeit und Kooperation zwischen den Bildungseinrichtungen sowie zwischen Wissenschaft und Wirtschaft gestärkt werden. So wurden damals beispielsweise kooperative Forschungsinfrastrukturen, wie der Hochleistungsrechner im Vienna Scientific Cluster an der TU Wien oder der Supercomputer MACH der JKU gefördert.

In der Leistungsvereinbarungsperiode 2016-2018 wurden die Hochschulraum-Strukturmittel für Kooperationen auf 97,5 Mio. $€$ aufgestockt. Die Ausschreibungsverfahren für die Bereiche Lehre, Forschung/ EEK und Verwaltungsinnovation wurden gesondert durchgeführt, um zielgerichteter auf die spezifischen Anforderungen einzugehen. Insgesamt wurden 56 Projekte ausgewählt, davon 43 Kooperationsvorhaben zur Verbesserung der F\&E-Infrastrukturausstattung der Universitäten und 13 Kooperationsvorhaben im Bereich "unkonventioneller" Forschung bzw. innovativer Arts-based Research. So flossen 42 Mio. $€$ in die Verbesserung der F\&E-Infrastruktur und acht Mio. $€$ in die Unterstützung exzellenzfördernder und zugleich strukturentwickelnder Forschungskooperationsprojekte. Themenfelder der Projekte waren z.B. neue Therapieansätze zur Erforschung von Fettstoff-

$128 \mathrm{Vgl}$. BKA et al. (2014) 
wechselerkrankungen oder die Vorbereitung Österreichs auf die digitale Revolution. ${ }^{129}$ Darüber hinaus wurden koordinierte Nutzungen und Konzepte von zukünftigen Forschungsinfrastrukturen in den Leistungsvereinbarungen 2016-2018 und 2019-2021 der Universitäten festgeschrieben.

\section{Ausbau der Kooperation von}

Forschungseinrichtungen und Unternehmen auf der Basis gemeinsamer Infrastrukturnutzung Im Rahmen des „Aktionsplans für einen wettbewerbsfähigen Forschungsraum" wurde im Jahr 2016 die öffentliche Forschungsinfrastruktur-Datenbank $k^{130}$ eingerichtet. Die öffentliche ForschungsinfrastrukturDatenbank ermöglicht es, Forschungsinfrastrukturen für neue Kooperationsprojekte zu finden oder auch anzubieten. Mit Stand Februar 2019 sind über 1.300 Forschungsinfrastrukturen von fast 100 Forschungseinrichtungen und Unternehmen aus Österreich in der öffentlichen Datenbank erfasst. Da hohe Anschaffungs-, Betriebs- und Folgekosten von Forschungsinfrastruktur häufig die Nutzung durch mehrere Institutionen notwendig machen, kann die Forschungsinfrastruktur-Datenbank als wichtiger Impuls für Forschungs- und Entwicklungskooperationen gesehen werden. Außerdem ermöglicht sie Unternehmen und Forschungseinrichtungen wesentliche Einblicke in die Infrastruktur der österreichischen Forschungslandschaft und erleichtert damit auch Wissenschafts-Wirtschaftskooperationen. ${ }^{131}$ Seitens der OECD wird die Forschungsinfrastruktur-Datenbank in Österreich ein als Best Practice-Modell einer digitalen Plattform für Forschungsinfrastruktur-Kooperationen geführt und dient zahlreichen anderen Staaten als Vorbild. ${ }^{132}$

Auch im Rahmen von COMET erfolgt der Ausbau der Kooperation von Forschungseinrichtungen und
Unternehmen auf der Basis gemeinsamer Infrastrukturnutzung (siehe Abschnitt 3.4.3). Das BMDW fördert seit 2012 zudem gemeinsam angeschaffte FTIInfrastruktur an ACR-Instituten und damit verbunden die Erschließung neuer Kooperationspartner, insbesondere KMU (siehe Abschnitt 3.3.4). Ebenso sind die Silicon Austria Labs mit Standorten in Graz, Villach und Linz ein weiteres Beispiel für eine gelungene Kooperation zwischen Forschungseinrichtungen und Unternehmen auf Basis gemeinsamer Infrastruktur (siehe Abschnitt 3.3.4). Des Weiteren wurden seitens des BMBWF und des BMVIT einige Großforschungsinfrastrukturen, wie das "Zentrum am Berg“ an der Montanuniversität Leoben, oder das Wasserbaulabor und die Pilotfabrik zu Industrie 4.0 an der TU Wien finanziert. ${ }^{133}$

Angesichts dieser Entwicklungen kann daher geschlossen werden, dass der von der FTI-Strategie angestrebte Ausbau der Forschungsinfrastruktur systematisch implementiert und damit die Förderung von Kooperationen zwischen den Forschungsakteuren erfolgreich realisiert wurden, sowie diese auch in $\mathrm{Zu}$ kunft fortgesetzt werden.

\section{Beteiligung Österreichs an europäischen und internationalen Infrastrukturen im Rahmen der ESFRI-Roadmap}

Durch die steigende Komplexität und die sehr hohen Investitionssummen werden Forschungsinfrastrukturen zunehmend durch mehrere Staaten gemeinsam finanziert und betrieben. Daher wurde bereits im Jahr 2002 das „European Strategy Forum on Research Infrastructures" (ESFRI) ins Leben gerufen, um eine eigenständige Forschungsinfrastrukturstrategie für Europa zu entwickeln. ${ }^{134}$ ESFRI soll neue Forschungsinfrastrukturen von europäischem Interesse identifizieren, um den Forschungsstandort Europa at-

129 Vgl. BMBWF (2017).

$130 \mathrm{Vgl}$. https://forschungsinfrastruktur.bmbwf.gv.at/de

131 Vgl. BMWFW (2015a).

132 Vgl. OECD (2017).

133 Vgl. BMWFW und BMVIT (2016).

$134 \mathrm{Vgl}$. http://ec.europa.eu/research/infrastructures/index.cfm?pg=esfri 
traktiv und konkurrenzfähig zu halten. Aus diesem Grund wurde erstmals 2006 eine Roadmap für die wichtigsten Vorhaben erstellt, die weltweit erstmalig alle Wissenschaftsbereiche abdecken sollte. 2008, 2010, 2016 und zuletzt 2018 unter österreichischer EU-Ratspräsidentschaft veröffentlichte ESFRI jeweils eine überarbeitete und aktualisierte Version dieser Roadmap. Derzeit befinden sich 18 Projekte und 37 sog. „ESFRI Landmarks“ (bereits umgesetzte bzw. finanzierte Forschungsinfrastrukturen) auf dieser Roadmap.

ESFRI dient den EU-Staaten und anderen im EUForschungsrahmenprogramm assoziierten Staaten als Plattform für Diskussion und Abstimmung der Forschungsinfrastrukturen. Zwar verfügt ESFRI über keine eigenen Fördermittel, dennoch spielt es im europäischen Entscheidungsprozess eine große Rolle für die Errichtung der nächsten Generation von Großforschungseinrichtungen. Kein Land, auch kein groBes Land, beteiligt sich an allen ESFRI-Infrastrukturen. Die Verschiedenheit der Fachgebiete, die unterschiedlichen wissenschaftlichen Gemeinschaften und deren Bedürfnisse machen es notwendig, über die Teilnahme an jedem einzelnen Vorhaben individuell zu entscheiden. Österreich ist 2019 an insgesamt 13 ESFRI-Infrastrukturen beteiligt:

Die biomedizinische Forschungsinfrastruktur BBMRI ERIC (Biobanking and Biomolecular Resources Research Infrastructure) ist bisher die einzige ESFRIInfrastruktur in Österreich mit Sitz in Graz. Seit Februar 2019 beteiligt sich Österreich auch an Euro-Biolmaging, eine Forschungsinfrastruktur im Bereich bildgebender Verfahren („Imaging“) für die Life Sciences und die medizinische Forschung. Die Universität Linz ist der österreichische Koordinator von SHARE ERIC (Survey on Health, Ageing and Retirement in Europe), einer Forschungsinfrastruktur, die Panel-Daten zu den Themen Gesundheit, Erwerbsleben und Altern sammelt. Die ÖAW beteiligt sich über das Austrian Center for Digital Humanities (ACDH) in Kooperation mit österreichischen Universitäten an CLARIN ERIC (Common Language Resources and Technology Infrastructure) und DARIAH ERIC (Digital
Research Infrastructure for the Arts and Humanities), zwei Infrastrukturkonsortien in den Bereichen digitale Sprachressourcen und Geisteswissenschaften. Weiters nimmt Österreich koordiniert durch das IHS an der europäischen Bevölkerungsbefragung European Social Survey (ESS ERIC) und über das Austrian Social Science Data Archive (AUSSDA) am Konsortium der sozialwissenschaftlichen Datenarchive in Europa (CESSDA ERIC) teil. Auf dem Gebiet der Werkstoffwissenschaften im Rahmen bereits bestehender wissenschaftlicher Mitgliedschaften ist Österreich an den Upgrades der Synchrotronstrahlenquelle ESRF sowie der Neutronenquelle ILL beteiligt. Im Bereich der Astronomie finanziert Österreich im Rahmen der ESO-Mitgliedschaft das E-ELT und über die Universität Innsbruck wird die Vorbereitung der Forschungsinfrastruktur Cherenkov Telescope Array (CTA) organsiert. Im Rahmen der ältesten Großforschungsbeteiligung Österreichs im Bereich der Physik fließen bedeutende österreichische Beiträge auch an den HL-LHC am CERN. An der ESFRI-Infrastruktur zu High Performance Computing - PRACE - ist Österreich über das Austrian Academic Computer Network beteiligt.

Im Rahmen der FTI-Strategie 2011 der Bundesregierung wurde ein Österreichischer Forschungsinfrastruktur-Aktionsplan 2014-2020 erarbeitet, der die Grundlage für die Beteiligung an weiteren ESFRI-Infrastrukturen bildet, wobei zukünftige Mitgliedschaften jedenfalls strikt bedarfsgeleitet geprüft werden müssen und unter Budgetvorbehalt stehen. Neue Mitgliedschaften werden bei konstanten Budgets in Zukunft auch Fragen der Schließung bzw. der Beendigung von bestehenden Mitgliedschaften aufwerfen.

Neben den ESFRI-Initiativen ist Österreich außerdem noch an acht weiteren Großforschungsinfrastrukturen von pan-europäischem Interesse beteiligt; zu diesen zählen CERN, EMBL, ESO, ESRF etc. Insgesamt beläuft sich die Zahl der internationalen forschungsbezogenen Mitgliedschaften im Bereich des BMBWF auf 38. Gerade mit diesen Beteiligungen soll auch die internationale Einbettung und Wettbe- 
werbsfähigkeit der Forschung sichergestellt und der Zugang zu modernsten Forschungseinrichtungen und aktuellsten Daten(-sammlungen) für österreichische Forscherinnen und Forscher gewährleistet werden.

\section{Entwicklung der rechtlichen}

Rahmenbedingungen für die Nutzung von Infrastrukturen wie Biobanken und statistischer Datenbestände

Im Jahr 2018 wurde das Datenschutz-Anpassungsgesetz 2018 - Wissenschaft und Forschung (WFDSAG 2018) im Nationalrat beschlossen. Damit wurde u.a. die Voraussetzung wie folgt geschaffen:

- Schaffung der Voraussetzungen für Registerforschung

- Sicherstellung des Betriebs von Biobanken und anderen wissenschaftlichen Archiven

- Entbürokratisierung von Projektgenehmigungen und Datenschutz-Folgenabschätzungen

- Abbau von Hindernissen für innovative Technologien und Partnerschaften

- Klarstellungen zur Verarbeitung personenbezogener Daten auf internationaler Ebene ${ }^{135}$

Weitere Entwicklungen zum verbesserten Zugang zu Informationen über öffentlich finanzierte Forschung sind in Abschnitt 3.5.5 dargestellt.

\subsection{Steigerung der Wissensverwertung und Wertschöpfung}

FTI-Strategie 2020 mit der Vision:

„Wissen verwerten, Wertschöpfung steigern Die Potentiale der Innovation aktivieren“

Unter der Vision „Wissen verwerten, Wertschöpfung steigern" wurde in der FTI-Strategie die Leistungsfähigkeit des Innovationssystems auf breiter Ebene angesprochen. Dabei wird von einem breiten Innovationsansatz ausgegangen, der technologische, forschungsgetriebene und nicht-technologische In- novationen sowohl in der Sachgüterproduktion als auch im Dienstleistungssektor ebenso einschließt wie ökologische und soziale Innovationen oder Innovationen im öffentlichen Bereich.

Die FTI-Strategie verweist in diesem Zusammenhang darauf, dass Österreich in der Phase des Aufholprozesses vor der Strategieerstellung vorwiegend einen angebotsseitigen Ansatz verfolgte. Die nachfrageseitige Stimulierung von Innovation ist seither zunehmend ein Thema. Hiermit sind die Gestaltung der öffentlichen Beschaffung, Normensetzung, Definition von Standards und der regulative Rahmen für die Wirtschaftstreibenden angesprochen, die einen wesentlichen Einfluss auf die Nachfrage nach innovativen Lösungen und die Größe der Märkte für innovative Produkte aufweisen können.

Die Strategie macht, trotz des beachtlichen Aufholprozesses der 2000er Jahre, noch Defizite aus sowohl auf der Inputseite (Forschungsquote insgesamt, Finanzierungsanteil der Wirtschaft an der Forschungsquote) wie auch auf der Outputseite (insbesondere der Anteil der Wertschöpfung in den forschungs-, technologie-, ausbildungs- und wissensintensiven Branchen im Unternehmenssektor, der Umsatzanteil von Marktneuheiten, der Anteil von wissensintensiven Dienstleistungen, der Technologiegehalt von Exportprodukten und Dienstleistungen, sowie der Anteil der Beschäftigung im Medium-Tech- und High-Tech-Bereich der Sachgüterproduktion).

Vor diesem Hintergrund wurden in der FTI-Strategie die Ziele formuliert, "die Wertschöpfung im Inland [zu] steigern, indem wir forschungsintensive Wirtschaft und wissensintensive Dienstleistungen forcieren und dabei verstärkt nachfrageseitige Instrumente in der Beschaffung, der Regulierung oder der Standardisierung zur Stimulierung von Innovationen einsetzen“. Dazu sollte „die Anzahl der systematisch Forschung und Entwicklung betreibenden Unternehmen [...] bis 2013 insgesamt um etwa 10\% und bis 2020 insgesamt um etwa 25\% erhöht werden“, 
"die international erfolgreichen österreichischen Leitbetriebe [...] in ihrer tragenden Rolle für das Innovationssystem gestärkt und die KMU in ihrer Forschungs- und Innovationsleistung aktiviert werden" und „Attraktivität des Standorts Österreich für die Ansiedlung forschungs- und technologieintensiver Unternehmen" weiter verbessert werden. Außerdem sollte "das Innovationsniveau in den Unternehmen [...] durch Steigerung der Anteile der radikalen Innovationen" nachhaltig angehoben werden und die „Produkt- und Dienstleistungsstruktur durch Erhöhung der Wissens- und Innovationsintensität der Unternehmen "verbessert werden. ${ }^{136}$

\subsubsection{Aktivierung und Steigerung von Unternehmensforschung}

Um die Unternehmensforschung in Österreich zu forcieren, wurden in der FTI-Strategie sowohl allgemeine als auch konkrete Maßnahmen definiert. Die konkreten Maßnahmen wurden jeweils durch eine nicht geplante, de facto Entwicklung ergänzt:

Allgemeine Maßnahmen lt. FTI-Strategie
Ausbau der direkten Förderung und deren optimale
Abstimmung mit der indirekten Förderung zur Aktivierung und
Steigerung der Unternehmensforschung und der innovativen
Leistung von Unternehmen

Verbesserung der Rahmenbedingungen für und Intensivierung der Bemühungen um die Ansiedlung weiterer forschungsintensiver Unternehmen und den Aufbau von Headquarter-Funktionen

\section{Ausbau der direkten Förderung und deren optimale Abstimmung mit der indirekten} Förderung zur Aktivierung und Steigerung der Unternehmensforschung und der innovativen Leistung von Unternehmen

Die Input-Seite der öffentlichen Hand, d.h. die budgetäre Entwicklung zur „Förderung zur Aktivierung und Steigerung der Unternehmensforschung und der innovativen Leistung von Unternehmen" wurde bereits in Abschnitt 1.2.2 dargestellt. Hier sind insbesondere die bereitgestellten Budgets des BMVIT sowie des BMDW relevant, die Grosso Modo auf eine positive Entwicklung verweisen können. Bei der Verteilung der direkten Förderung von angewandter Unternehmensforschung ist insbesondere auf das Portfolio der Forschungsförderungsgesellschaft (FFG; siehe Kapitel 2.2) zu verweisen. Die FFG hat mittels eines bottom-up Ansatzes im Bereich Basisprogramme insbesondere die Verbreiterung der Innovationsbasis bei österreichischen Unternehmen im Fokus. Aber auch die Strukturprogramme mit dem Netzwerkcharakter ihrer Förderansätze sind auch dazu geeignet, Unternehmen mit wenig Forschungserfahrung anzusprechen. Der Bereich „Thematische Programme" nimmt mit seinen Schwerpunkten eine spezielle Stellung ein, die eine Sogwirkung für Unternehmensforschung durch die konkrete thematische Ausrichtung entfalten kann.

Das Ziel der Aktivierung der Unternehmensforschung, d.h. vormals nicht oder kaum forschungsakti-

\section{Konkrete Maßnahmen}

Stärkung der bottom-up Förderung (z.B. themenoffenes Christian-Doppler-Modell) zur Verbreiterung der Innovationsbasis durch Innovationsschecks etc. Weiterentwicklung der indirekten (steuerlichen) F\&E-Förderung

Internationale Marketinginitiative „Forschungsplatz Österreich" durch die Betriebsansiedlungsgesellschaft ABAInvest in Austria

Zusammenführung der Programme Competence Headquarters und Frontrunner im Jahr 2017 ve Unternehmen zum Einstieg in (eigene) F\&E-Tätigkeiten zu bewegen, kann nicht allein mit Forschungsförderung erreicht werden. Wie auch bei anderen Zielen ist für dessen Erreichung ein Maßnahmenbündel nötig, das auch potenziell mittelfristig wirksame Aktivitäten im Bereich der Bildung und Infrastruktur anspricht, wie auch rechtliche und organisatorische Rahmenbedingungen (z.B. im Bereich der Gründungs- 
Tabelle 3-3: Anzahl von Unternehmen mit eigener Forschung in Österreich, 2009-2015

\begin{tabular}{|c|c|c|c|c|c|c|}
\hline & 2009 & 2011 & 2013 & 2015 & $\begin{array}{r}\text { Veränderung } \\
2009-13\end{array}$ & $\begin{array}{r}\text { Veränderung } \\
2009-15\end{array}$ \\
\hline Weniger als 10 Beschäftigte & 908 & 1.191 & 1.135 & 1.283 & $25,0 \%$ & $41,3 \%$ \\
\hline $10<50$ Beschäftigte & 831 & 941 & 930 & 1.038 & $11,9 \%$ & $24,9 \%$ \\
\hline $50<250$ Beschäftigte & 780 & 818 & 805 & 833 & $3,2 \%$ & $6,8 \%$ \\
\hline $250<1.000$ Beschäftigte & 357 & 361 & 384 & 379 & $7,6 \%$ & $6,2 \%$ \\
\hline 1.000 und mehr Beschäftigte & 70 & 73 & 72 & 78 & $2,9 \%$ & $11,4 \%$ \\
\hline Gesamt & 2.946 & 3.384 & 3.326 & 3.611 & $12,9 \%$ & $22,6 \%$ \\
\hline
\end{tabular}

Quelle: STATISTIK AUSTRIA, F\&E-Erhebungen 2009-2015, firmeneigener und kooperativer Bereich.

Darstellung: KMU Forschung Austria.

Tabelle 3-4: Anzahl der Erstfördernehmer (Unternehmen) und deren Anteil an den Fördernehmern

\begin{tabular}{|c|c|c|c|c|c|c|c|c|}
\hline FFG Bereiche & 2011 & 2012 & 2013 & 2014 & 2015 & 2016 & 2017 & 2018 \\
\hline BP ohne Innovationsschecks & $\begin{array}{r}174 \\
(25 \%)\end{array}$ & $\begin{array}{r}165 \\
(25 \%)\end{array}$ & $\begin{array}{r}157 \\
(23 \%)\end{array}$ & $\begin{array}{r}130 \\
(20 \%)\end{array}$ & $\begin{array}{r}178 \\
(26 \%)\end{array}$ & $\begin{array}{r}281 \\
(37 \%)\end{array}$ & $\begin{array}{r}410 \\
(41 \%)\end{array}$ & $\begin{array}{r}361 \\
(36 \%)\end{array}$ \\
\hline Innovationsschecks & $\begin{array}{r}367 \\
(66 \%)\end{array}$ & $\begin{array}{r}299 \\
(64 \%)\end{array}$ & $\begin{array}{r}255 \\
(62 \%)\end{array}$ & $\begin{array}{r}235 \\
(57 \%)\end{array}$ & $\begin{array}{r}192 \\
(58 \%)\end{array}$ & $\begin{array}{r}163 \\
(56 \%)\end{array}$ & $\begin{array}{r}154 \\
(50 \%)\end{array}$ & $\begin{array}{r}186 \\
(53 \%)\end{array}$ \\
\hline SP ohne Talente & $\begin{array}{r}84 \\
(29 \%)\end{array}$ & $\begin{array}{r}168 \\
(32 \%)\end{array}$ & $\begin{array}{r}125 \\
(32 \%)\end{array}$ & $\begin{array}{r}287 \\
(33 \%)\end{array}$ & $\begin{array}{r}33 \\
(29 \%)\end{array}$ & $\begin{array}{r}144 \\
(29 \%)\end{array}$ & $\begin{array}{r}159 \\
(32 \%)\end{array}$ & $\begin{array}{r}189 \\
(33 \%)\end{array}$ \\
\hline Talente & $\begin{array}{r}38 \\
(15 \%)\end{array}$ & $\begin{array}{r}43 \\
(12 \%)\end{array}$ & $\begin{array}{r}72 \\
(20 \%)\end{array}$ & $\begin{array}{r}64 \\
(17 \%)\end{array}$ & $\begin{array}{r}38 \\
(13 \%)\end{array}$ & $\begin{array}{r}56 \\
(17 \%)\end{array}$ & $\begin{array}{r}52 \\
(15 \%)\end{array}$ & $\begin{array}{r}30 \\
(11 \%)\end{array}$ \\
\hline $\begin{array}{l}\text { TP inkl. ALR } \\
\text { (ohne Breitband) }\end{array}$ & $\begin{array}{r}144 \\
(29 \%)\end{array}$ & $\begin{array}{r}121 \\
(29 \%)\end{array}$ & $\begin{array}{r}118 \\
(27 \%)\end{array}$ & $\begin{array}{r}175 \\
(31 \%)\end{array}$ & $\begin{array}{r}128 \\
(24 \%)\end{array}$ & $\begin{array}{r}181 \\
(30 \%)\end{array}$ & $\begin{array}{r}137 \\
(27 \%)\end{array}$ & $\begin{array}{r}214 \\
(31 \%)\end{array}$ \\
\hline
\end{tabular}

Quelle: FFG, Datenstand 21.01.2019; Definition Erstfördernehmer: erstmalige Förderung eines Unternehmens durch die FFG insgesamt seit deren Gründung im Jahr 2004. EIP ist nicht darstellbar. Bereich BP ohne Innovationsschecks ab 2016 mit neu aufgelegtem Patentscheck, der zu hohen Erstfördernehmerzahlen führte. TP inkl. ALR: Thematische Programme inkl. Agentur für Luft- und Raumfahrt. Darstellung: KMU Forschung Austria.

unterstützung). Aufbauend auf dieser Grundlage können dann unterschiedliche Formate der Forschungsförderung Wirkung entfalten, die den Einstieg in die Forschung erleichtern, und damit die Forschungsbasis in Österreich verbreitern sollen (FFG, teilweise auch in der aws sowie den Bundesländern).

Der Fortschritt zur Erreichung dieses Zieles kann primär anhand der F\&E-Statistik der Statistik Austria nachgezeichnet werden. Auch wenn gewisse Unschärfen bei der Erhebung nicht vermeidbar sind ${ }^{137}$, lässt sich aus der F\&E Statistik ablesen, dass die Zahl der forschungsaktiven Unternehmen tendenziell steigt. Diese Entwicklung ist bereits seit Beginn der F\&E-Erhebung zu beobachten (2002: 1.942; 2007: 2.521).

Die Anzahl der Unternehmen mit internen F\&EAusgaben in den jeweiligen Jahren hat sich von 2009 bis 2013 um knapp 13 \% erhöht, und von 2009 bis 2015 um über $22 \%$ (vgl. Tabelle 3-3). Dies beinhaltet kontinuierlich wie auch fallweise F\&E-treibende Unternehmen, die im Sinne der FTI-Strategie beide als "systematisch“ F\&E-treibende Unternehmen zu verstehen sind. Damit scheint die Erreichung des Zieles einer Steigerung von $25 \%$ bis 2020 sehr realistisch.

Der Beitrag der direkten Forschungsförderung zur Verbreiterung der Forschungsbasis bei Unternehmen kann näherungsweise aufgrund der Antragsstatistik der FFG, als wichtigster Akteur in der angewandten Forschungsförderung, abgebildet werden. Tabelle 3-4 zeigt die Anzahl an Erstfördernehmenden seit 2011 auf Ebene der FFG-Bereiche bzw. eine Ebene darunter. Innerhalb des Bereichs "Basisprogramme“ sind die Innovationsschecks aufgrund ihres expliziten Ziels der Verbreiterung der Forschungsbasis se- 
parat ausgewiesen. Im Bereich „Strukturprogramme“ (inkl. der COMET Zentren) ist das Programm „Talente“ aufgrund seines spezifischen, für die Strukturprogramme nicht repräsentativen, Fokus auf personenbezogene Nachwuchsförderung separat dargestellt.

Die Innovationsschecks nehmen eine wesentliche Funktion für den Einstieg von Unternehmen in das FFG-Portfolio ein. Die letzte Evaluierung der Innovationsschecks ${ }^{138}$ zeigte, dass im Zeitraum 2007-2016 sogar $68 \%$ der Begünstigten Erstfördernehmer bei der FFG waren; 25 \% dieser Erstfördernehmer haben daraufhin F\&E-Folgeprojekte gestartet. Diese mögliche Verbreiterung der Innovationsbasis spiegelt sich teilweise auch in der F\&E-Erhebung der Statistik Austria wider. Die Evaluierung schlussfolgerte, dass ca. 21 \% der im Zeitraum 2007-2013 neu in den F\&EErhebungen erfassten Unternehmen einen der Innovationsschecks erhielten.

Seit 2016 sorgt der neu aufgelegte Patentscheck für einen weiteren Schub in den Erstförderzahlen im Bereich Basisprogramme. Ansonsten lägen die Bereiche "Strukturprogramme (ohne Talente)" und "Thematische Programme" bei den Erstfördernehmerraten höher (diese fluktuieren rd. 14 Jahre nach Gründung der FFG nach wie vor um $30 \%$ ).

Das Ziel der Steigerung von Unternehmensforschung, d.h. bereits mehr oder weniger forschungsaktive Unternehmen verstetigen bzw. vertiefen ihre F\&E-Tätigkeiten, wird von der FTI-Strategie ebenso festgehalten. Dem liegt die Annahme zugrunde, dass mit einer Verstetigung der F\&E-Aktivitäten in zumindest fallweise forschenden Unternehmen das Ziel einer Erhöhung der F\&E-Quote leichter erreicht wird. Zu diesem Befund kommt auch eine aktuelle Studie für Deutschland, und konkretisiert dies folgendermaBen: Frietsch et al. identifizierten zur Erreichung des 3,5 \% F\&E-Quotenziels für Deutschland das größte Potential bei mittelgroßen Unternehmen, die entweder in den forschungs- und wissensintensiven Wirtschaftszweigen beheimatet sind oder bereits länger in $F \& E$ investieren, wenn mit einer Förderung eine Verstetigung der F\&E-Aktivitäten in gelegentlich oder mit unterschiedlicher Intensität forschenden Unternehmen erreicht wird. ${ }^{139}$

Der Fortschritt zur Erreichung des Ziels der Steigerung der Unternehmensforschung in Österreich kann

Abbildung 3-10: Anzahl F\&E-aktiver Unternehmen und deren F\&E-Umsatzintensität nach der Technologie- und Wissensintensität der Wirtschaftszweige (OECD-Definition) im Zeitverlauf

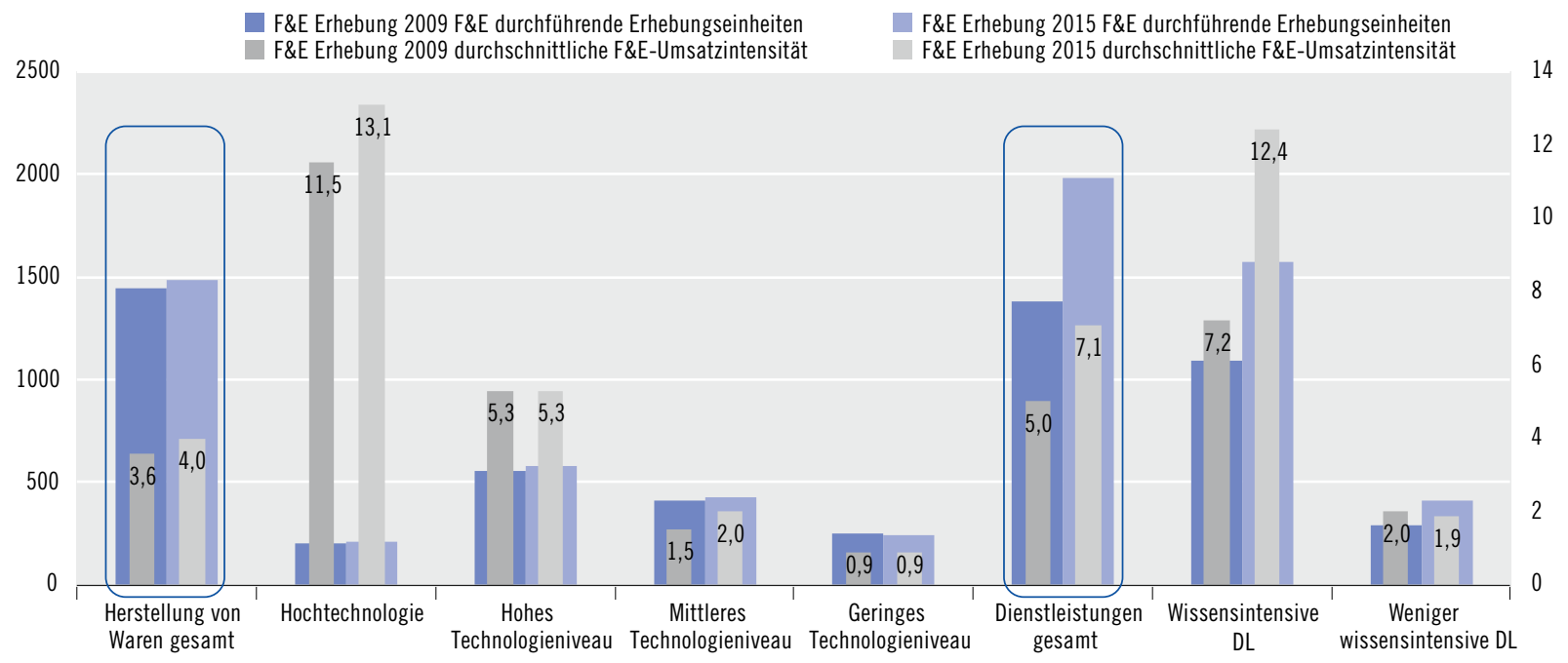

Quelle: STATISTIK AUSTRIA, F\&E-Erhebung 2009 und 2015, firmeneigener Bereich, Darstellung: KMU Forschung Austria.

$138 \mathrm{Vgl}$. Jud et al. (2017).

$139 \mathrm{Vgl}$. Frietsch et al. (2019). 
wiederum im Kontext der F\&E-Statistik der Statistik Austria diskutiert werden. Wie in Abschnitt 1.2 bereits dargestellt, stieg die Finanzierung der in Österreich durchgeführten Forschung und experimentellen Entwicklung durch den Unternehmenssektor im Zeitraum 1981-2017 nominell kontinuierlich an. Dies gibt jedoch noch keine Informationen zur Verteilung der F\&E-Aktivitäten. Einen Hinweis dazu gibt ein Überblick zur Entwicklung der Anzahl sowie der F\&E-Umsatzintensität von Unternehmen gemäß der folgenden Auswertung der F\&E-Erhebung (dargestellt in Abbildung 3-10). Zur besseren Übersicht werden nur die Ergebnisse aus den F\&E-Erhebungen 2009 und 2015 gegenübergestellt. Die umrahmten Balken zeigen die aggregierten Ergebnisse für die Sektoren Herstellung von Waren sowie Dienstleistungen, wobei die steigende Anzahl an F\&E-aktiven Unternehmen überwiegend auf den Dienstleistungssektor, und hier schwerpunktmäßig auf die wissensintensiven Dienstleistungen, zurückzuführen ist. Diese zeigen auch einen starken Anstieg am durchschnittlichen Anteil von F\&E-Ausgaben am Umsatz (F\&E-Umsatzintensität). Im produzierenden Gewerbe ist die höchste Dynamik beim Anteil der F\&E-Ausgaben in den Unternehmen der Hochtechnologiebranchen zu beobachten.
Die Innovationsleistung der österreichischen Wirtschaft stützt sich inzwischen vermehrt auf Forschung und Entwicklung, auch wenn der Zwischenbefund lautet, dass die höheren F\&E-Inputs noch nicht ausreichend am Markt realisiert werden (siehe Abschnitt 3.6). Einen Einblick dazu gibt Abbildung 3-11, die anhand der Ergebnisse der letzten vier Innovationserhebungen die Entwicklung des Anteils innovationsaktiver Unternehmen an den österreichischen Unternehmen darstellt. Die umrahmten Bereiche in der Abbildung zeigen ein insgesamt höheres Niveau von innovationsaktiven Unternehmen im Sektor Herstellung von Waren im Vergleich zu den Dienstleistungen. Die detaillierte Auswertung nach der OECD-Klassifizierung der Technologie- und Wissensintensität zeigt, dass die Innovationsneigung umso höher ist, je technologie- und wissensintensiver die jeweiligen Branchen eingestuft werden. Insgesamt hat sich die Innovationsneigung nach der Wirtschaftskrise wieder erholt, bzw. insbesondere bei den Unternehmen mit niedrigeren Technologieniveaus erhöht. Die Untergliederung zeigt ebenso, dass die Dynamik bei den Dienstleistungen besonders auf den wissensintensiven Bereich zurückgeht.

\section{Abbildung 3-11: Anteil innovationsaktiver Unternehmen nach der Technologie- und Wissensintensität der Wirtschaftszweige (OECD-Definition) im Zeitverlauf}

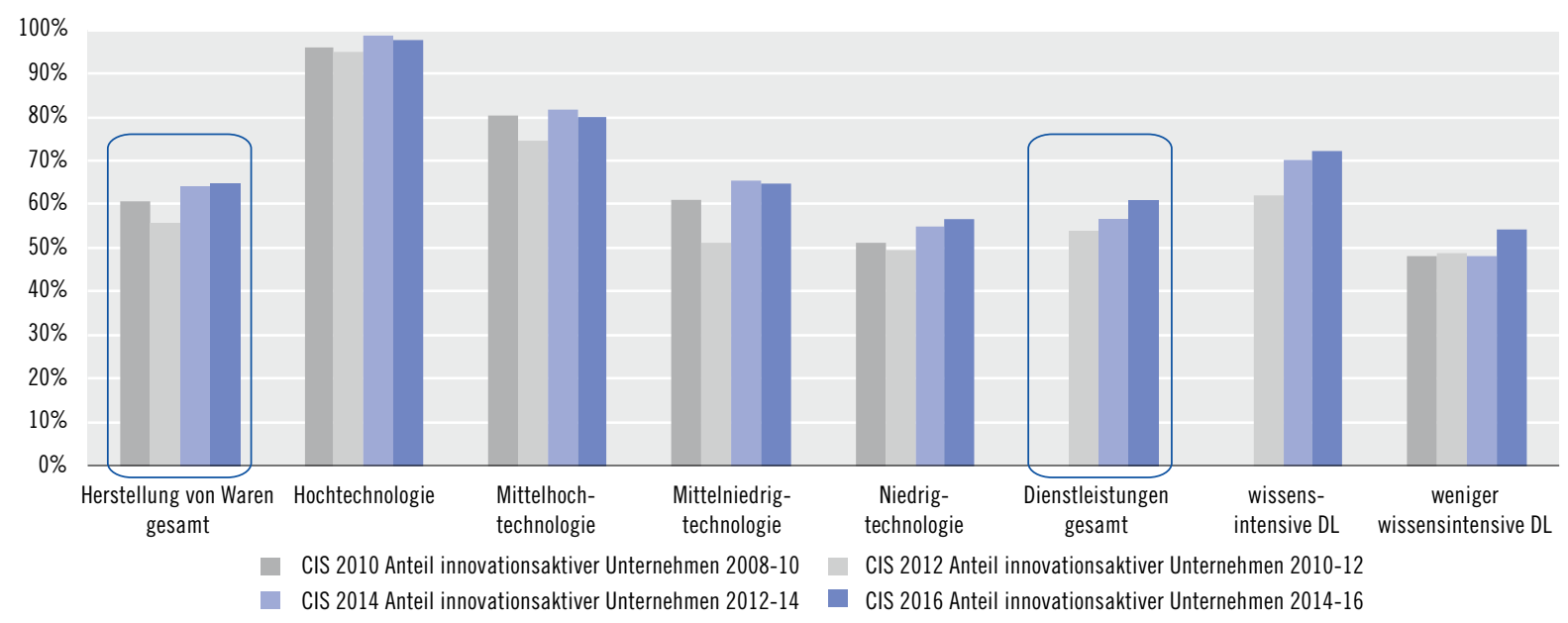

Quelle: STATISTIK AUSTRIA, Europäische Innovationserhebung 2010-2016. Anm.: Wissensintensive Dienstleistungen sind für 2010 nicht dargestellt, da diese anders definiert waren. Die OECD-Klassifikation der Wirtschaftszweige ist in der F\&E-Statistik weiter gefasst als in der Innovationserhebung. Darstellung: KMU Forschung Austria. 
Abbildung 3-12: Unternehmen mit Produktinnovationen und Marktneuheiten

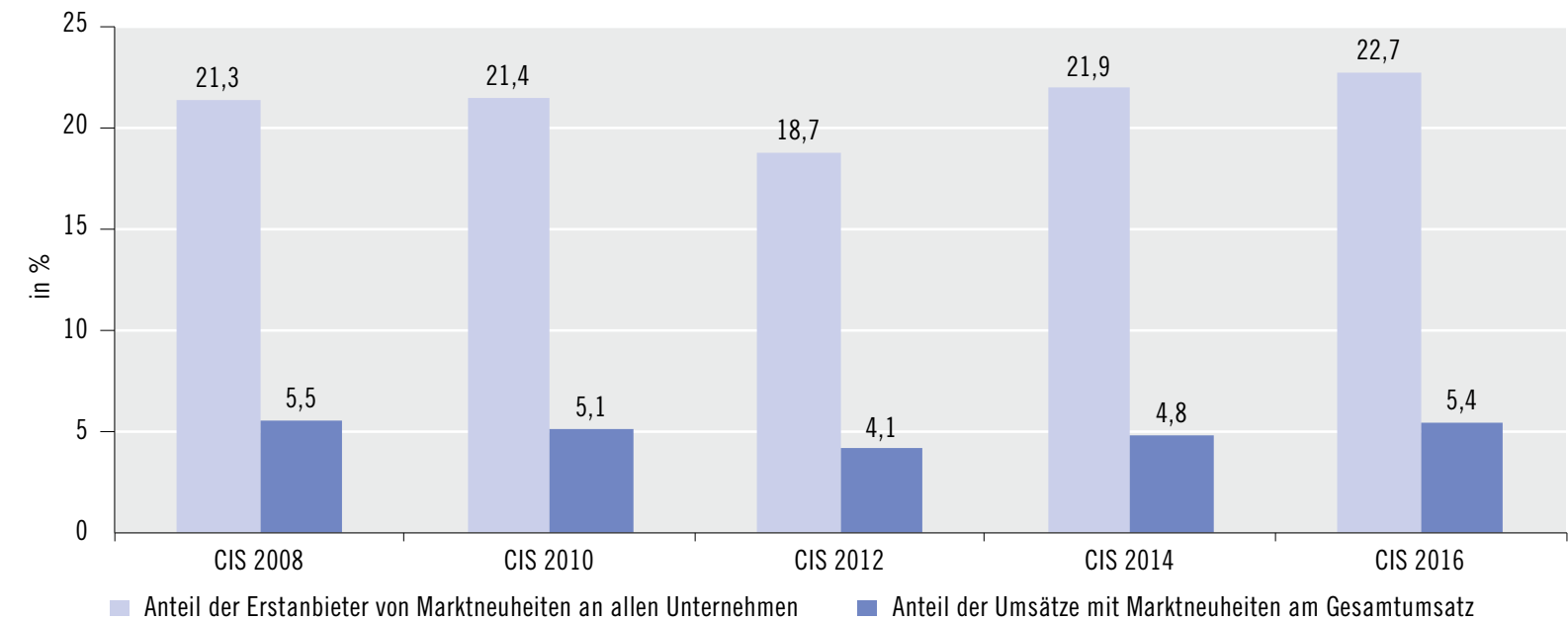

Quelle: STATISTIK AUSTRIA, Europäische Innovationserhebung 2008-2016. Darstellung: KMU Forschung Austria.

Die FTI-Strategie zielt ebenso auf eine nachhaltige Steigerung der Anteile von radikalen Innovationen, die neu für den Markt sind, allerdings ohne konkrete Maßnahme. ${ }^{140}$ Wie bereits an anderer Stelle festgestellt ${ }^{141}$, ist der im Oslo Manual verwendete Begriff der radikalen Innovation auf der Output-Ebene angesiedelt und definiert den Neuheitsgrad unterschiedlicher Innovationstypen. Radikale Innovationen können, müssen aber nicht, zu wirtschaftlichem Erfolg führen. Unterschiedliche Surveys (darunter das europaweite $\mathrm{CIS}$, das Wirkungsmonitoring der FFG-Förderungen etc.) unterstützen diese Annäherung insofern, als Produktinnovationen, die als neu für den eigenen Markt bzw. für den internationalen Markt angesehen werden, deutlich öfter zu konkreten Umsätzen führen

Abbildung 3-12 zeigt anhand der Innovationserhebungen der letzten zehn Jahre die Entwicklung von Marktneuheiten. Hier wird auch der Einfluss der Wirtschaftskrise sichtbar, was sich insbesondere in der Erhebung 2012 niederschlägt. Insgesamt aber deuten die Zahlen auf eine strukturelle Herausforderung hin, da sich die Umsatzanteile von Markt- neuheiten nach der Krise nur mit einer zeitlichen Verzögerung zur Lancierung von Marktneuheiten erholten und erst 2016 das Vorkrisenniveau erreichten. Damit scheint der Hebel hin zu einer nachhaltigen Steigerung der Anteile von radikalen Innovationen noch ausbaufähig zu sein.

Die indirekte (steuerliche) F\&E-Förderung wurde in den vergangenen Jahren mehrmals angehoben: im Jahr 2011 von $8 \%$ auf $10 \%, 2016$ auf $12 \%$ und 2018 auf $14 \%$. Kennzeichnend für die Forschungsprämie ist, dass sie seit 2011 das einzige steuerliche Instrument in Österreich zur Förderung von F\&E ist, das allen Unternehmen gleichermaßen zugänglich ist. Die Forschungsprämie wirkt dabei vor allem bei kontinuierlich F\&E-betreibenden Unternehmen unterstützend und ist bei international tätigen, forschungsintensiven Unternehmen für die Standortsicherung relevant. ${ }^{142}$

„Die geplante Steuerreform sieht zudem zusätzliche Ausweitungen und Vereinfachungen vor (siehe: Ministerratsvortrag 55/15 aus 2019). So sollen zukünftig insbesondere Start-ups und kleine Unterneh-

140 Da kein (international vergleichbares) objektives Messverfahren verfügbar ist, wird als Annäherung an das Thema oftmals der europaweit standardisierte Community Innovation Survey (CIS) als Diskussionsgrundlage herangezogen, etwas seltener auch die Patentstatistik.

141 Vgl. BMWFW und BMVIT (2017).

142 Vgl. Ecker et al. (2017). 
men besser unterstützt werden, indem in der Bemessungsgrundlage ein fiktiver Unternehmerlohn berücksichtigt wird. Ebenso soll die derzeit bestehende Verbindung der Prämienbeantragung mit der Einkommen- oder Körperschaftsteuererklärung beseitigt sowie eine Teilauszahlung der Prämie ermöglicht werden, damit Unternehmen rascher über die Prämie verfügen können.“

Eine umfassende Abstimmung von direkter und indirekter Forschungsförderung, wie in der FTI-Strategie angesprochen, ist anvisiert. Die geplanten Entwicklungen hinsichtlich eines verbesserten Zugangs zu Registerdaten (Änderungen im Bundesstatistikgesetz, Zusammenführung von Registerdaten) würden die bessere Datenverfügbarkeit in diesem Zusammenhang unterstützen.

\section{Verbesserung der Rahmenbedingungen für und Intensivierung der Bemühungen um die} Ansiedlung weiterer forschungsintensiver Unternehmen und den Aufbau von Headquarter-

\section{Funktionen}

Zur Verbesserung der Rahmenbedingungen zur Ansiedlung weiterer forschungsintensiver Unternehmen hat das BMDW im Zeitraum 2008 bis 2018 Mittel in Höhe von ca. 7,8 Mio. $€$ zur Verfügung gestellt. Die Austrian Business Agency (ABA) hat bislang 188 F\&Erelevante Ansiedlungsprojekte realisiert, die 3.414 geschaffene Arbeitsplätze sowie eine Investitionssumme von 978,2 Mio. $€$ nach sich zogen (Quelle: ABA). ${ }^{143}$ Das Aktivitätsportfolio der ABA besteht aus einem Mix von PR-Arbeit, Vernetzung, konkreten Akquisitionsmaßnahmen und Investorenveranstaltungen unter Einbindung des BMDW.

Die Zusammenführung der Programme Competence Headquarters und Frontrunner im Jahr 2017 hat zu einer Vereinfachung der Förderlandschaft geführt, die positiv zu bewerten ist. Die kürzlich fertiggestellte Evaluierung der Frontrunner Initiative bescheinigt dem Programm eine positive Genese und empfiehlt in Zukunft verstärkt Diversifizierungsstrategien von Unternehmen zur Unterstützung des Strukturwandels zu fördern. ${ }^{144}$

Einen quantitativen Einblick in die F\&E-Ausgabenstruktur gibt die Entwicklung der F\&E-Finanzierung. In Kapitel 1.2 wurde bereits diskutiert, dass die Dynamik der F\&E-Ausgaben in den letzten Jahren stark vom Unternehmenssektor getragen wurde. Abbil-

\section{Abbildung 3-13: Entwicklung der F\&E-Finanzierung aus dem Ausland}

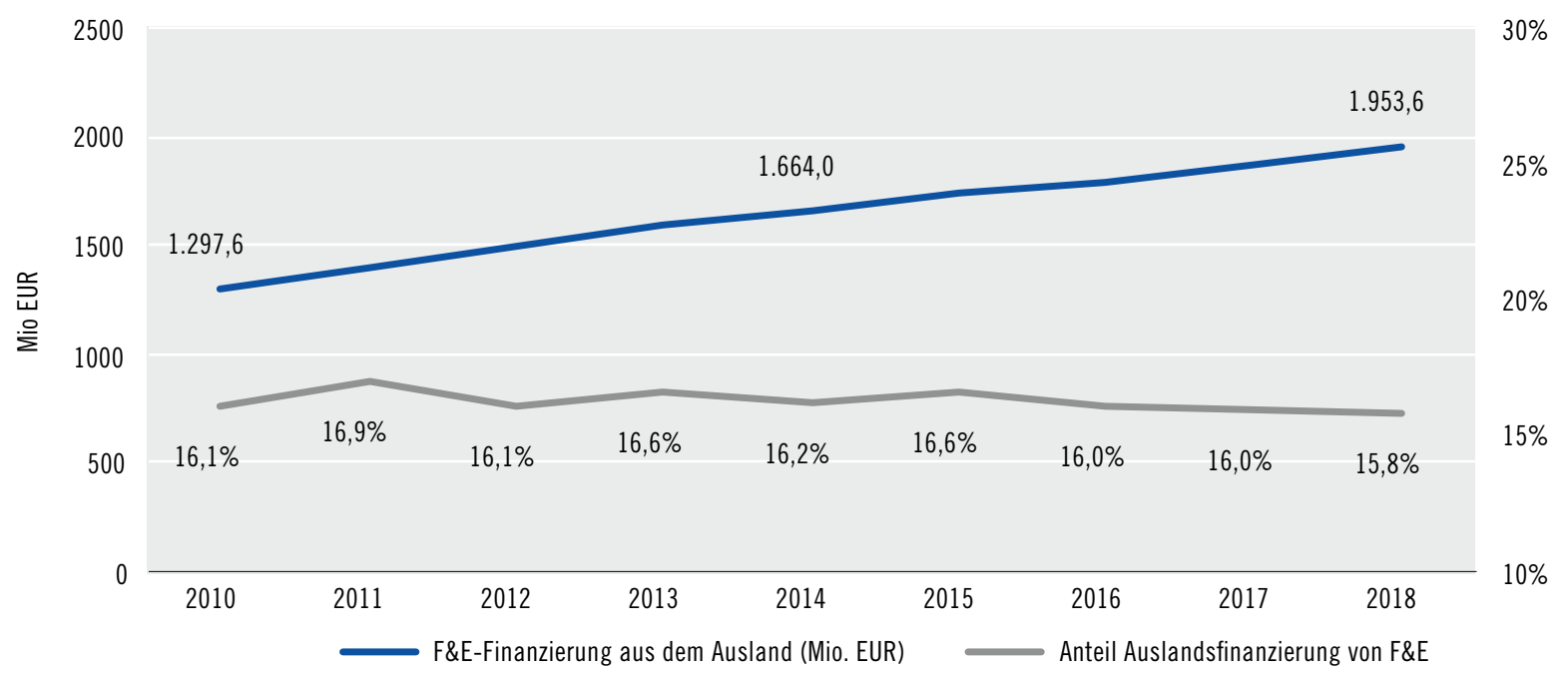

Quelle: STATISTIK AUSTRIA, Globalschätzung 2018, 2016-2018: Schätzung Statistik Austria. Darstellung: KMU Forschung Austria. 
dung 3-13 verdeutlicht, dass trotz kontinuierlich steigender F\&E-Ausgaben aus dem Ausland der Anteil der Auslandsfinanzierung leicht zurückgeht, da die heimischen Unternehmen eine noch höhere Dynamik aufweisen.

Eine aktuelle Studie zu den Motiven „für die Ansiedlung von F\&E-Einheiten in Wien“ im Vergleich zu anderen internationalen Standorten betont einmal mehr die Wichtigkeit eines breiten Mix an Faktoren. ${ }^{145}$ Diese sind das Vorhandensein von innovativen Talenten, ausgebildet von exzellenten Einrichtungen, sowie eine effiziente, international ausgerichteten Verwaltung (Stichwort: Arbeitserlaubnis). Während die Verfügbarkeit hochqualifizierter Humanressourcen, das Forschungsumfeld und die Zusammenarbeit mit Forschungseinrichtungen, Universitäten und Fachhochschulen die wichtigsten Determinanten für F\&E-affine Ansiedlungsentscheidungen darstellen, werden der vorhandene Kostenmix und Förderungen, sowie die Lebensqualität als Stärkefelder derzeit noch zu wenig in Form einer kohärenten Ansiedlungspolitik kommuniziert. Entwicklungsbedarfe sehen die Studienautorinnen und -autoren speziell darin, die Ausbildung am Hochschulstandort (insbesondere IKT und ihrer interdisziplinären Vernetzung) attraktiver zu gestalten, sowie das Profil am Forschungsstandort und darauf aufbauen das Marketing zu schärfen.

\subsubsection{Nachfrageseitige Stimulierung von Innovationen durch die öffentliche Hand}

Nachfrageorientierte Innovationspolitik wird verstanden als nachfrageseitiges, politisches Handeln, das Ineffizienzen in Innovationsprozessen bzw. Innovationssystemen adressiert. Grundsätzlich lassen sich drei Typen nachfrageorientierter Innovationspolitik unterscheiden: Regulierung (Standardisierung, Gesetzgebung, Normen), Förderung der privaten Nachfrage und öffentliche Beschaffung von innovativen Gütern und Dienstleistungen. Der öffentlichen Be- schaffung, also dem Einkauf von Gütern und Dienstleistungen durch staatliche Organisationen oder Unternehmen der öffentlichen Hand, kommt in diesem Zusammenhang schon durch das tatsächliche Nachfragevolumen eine zentrale Bedeutung zu (im Durchschnitt aller EU-Staaten ca. 14 \% des BIP). Über strategische Einkäufe hinaus kann der Staat auch aktiv Einfluss auf die Nachfrage nach neuen, innovativen Produkten und Dienstleistungen nehmen, etwa über die Definition von Produktanforderungen und die Schaffung direkter Kaufanreize bzw. Subventionen.

\section{Umsetzung IÖB-Leitkonzept}

Auf Basis der FTI-Strategie der Bundesregierung (bzw. insbesondere des darin erwähnten verstärkten Einsatzes nachfrageseitiger Instrumente in der Beschaffung, der Regulierung oder der Standardisierung zur Stimulierung von Innovationen) wurde 2012 in Österreich unter der Federführung des BMWFJ (jetzt BMDW) und BMVIT sowie unter Einbeziehung relevanter Stakeholder und in Kooperation mit der Bundesbeschaffung $\mathrm{GmbH}$ (BBG) das Leitkonzept für eine innovationsfördernde öffentliche Beschaffung (IÖBLeitkonzept) ${ }^{146}$ entwickelt. Insbesondere die darin entwickelten Maßnahmen auf operativer und rechtlicher Ebene können als vollumfänglich, jene auf strategischer Ebene als zumindest weitgehend umgesetzt angesehen werden. Der dafür notwendige politische Rückhalt existiert, wenn auch in unterschiedlicher Intensität und Breite, und wird nicht zuletzt in der Berücksichtigung von IÖB in strategischen Dokumenten und insbesondere im Regierungsprogramm der aktuellen Regierungskoalition (u.a. mit dem Ziel, 2 \% des Beschaffungsvolumens zentraler Beschaffer für IÖB einzusetzen) deutlich. Die Umsetzung des IÖB-Leitkonzepts erfolgt durch die koordinierenden Ressorts. Darüber hinaus gibt es Kooperationen, Kommunikations- und Diskussionskanäle mit dem Bundesministerium für öffentlichen Dienst und Sport (BMÖDS) und dem Bundesministerium für Nachhaltigkeit und Tou-

145 Vgl. Lasinger et al. (2019).

146 Vgl. BMWFJ, et al. (2012). 
rismus (BMNT). Außerdem sind in den Bundesministerien Koordinatoren für IÖB-Aktivitäten eingesetzt worden, deren Zusammenarbeit ein wesentliches Element der Koordinierungsaufgaben des Bundes darstellt und die als Beschaffungskoordinatoren bereits im ursprünglichen Leitkonzept Erwähnung fanden. Jüngst wurde das IÖB-Leitkonzept einer umfassenden Evaluierung unterzogen. ${ }^{147}$

Lt. dem IÖB-Leitkonzept von 2012 sollten zudem Innovationsstrategien und Beschaffungs- bzw. Umsetzungspläne öffentlicher Bedarfsträger erarbeitet werden. Diese IÖB-Pläne existieren derzeit noch nicht. Einer der Gründe hierfür liegt in der mangelhaften Informations- und Datenbasis bezüglich des Beschaffungsvolumens und der Gegenstände von Beschaffungsvorhaben. Dennoch sind verschiedene Vorarbeiten bzw. Vorbereitungen bereits getroffen worden. Aufseiten des IÖB-Dienstleistungsangebots (der IÖB-Servicestelle) wird unterstützende strategische Beratung angeboten. Allerdings wurde dieses Instrument bislang noch wenig durch die Ressorts in Anspruch genommen.

Das IÖB-Leitkonzept sah auf operativer Ebene die Einrichtung einer IÖB-Servicestelle vor. Diese nahm den Betrieb mit 01.09.2013 auf. Neben internen Schulungen in der BBG fanden auch Seminare an der Verwaltungsakademie des Bundes in Form von Grundlagen- und Vertiefungsseminaren statt. Es wurden zudem spezielle Schulungen für neue Mitarbeiterinnen und Mitarbeiter der BBG zu den Themen Innovation, KMU sowie soziale und ökologische Nachhaltigkeit konzipiert.

Das IÖB-Servicenetzwerk umfasst derzeit folgende Organisationen: die IÖB-Servicestelle, die IÖBKompetenz- stellen (aws, FFG, Bundesimmobiliengesellschaft, die Österreichische Energieagentur und die Österreichische Gesellschaft für Straßen- und Verkehrswesen), die IÖB-Kontaktstellen (Bundesländervertretung, Wirtschaftskammer Österreich, Industriellenvereinigung, die Magistratsabteilung 23 der Stadt Wien und die ITG Salzburg). Das Austrian Institute of Technology (AIT) begleitet als wissenschaftliche Institution den Prozess der Umsetzung des IÖB-Leitkonzepts in Österreich. Derzeit läuft ein Prozess der strategischen Neuausrichtung des Servicenetzwerks bzw. des IÖB-Beirats als dessen Koordinationsorgan.

\section{Maßnahmen und Instrumente}

Als umgesetzte Maßnahmen im Zusammenhang mit der IÖB-Initiative sind der Projektwettbewerb sowie die Initiierung und Durchführung von Pilotprojekten (unabhängig von einer Involvierung der federführenden Ministerien oder der IÖB-Servicestelle) zentral. Der Projektwettbewerb bietet einen finanziellen Anreiz, innovationsfördernde Beschaffungsvorhaben trotz des erhöhten Aufwands umzusetzen und verstärkt die Signalwirkung innerhalb des öffentlichen Sektors. IÖB-Projekte können von beschaffenden Stellen schon nach Abschluss der Konzeptionsphase (d.h. vor einer Ausschreibung) für den Projektwettbewerb eingereicht werden. Im Zeitraum 2013-2018 wurden 28 Projekte im Rahmen von Teilnahmen am Projektwettbewerb ausgewählt und zum Teil bereits abgeschlossen bzw. befinden sich noch in der Umsetzungsphase. Zwischen 2013 bis Februar 2019 wurden insgesamt 35 Pilotprojekte mit einem bisher erreichten Gesamtvolumen von 19,8 Mio. $€$ gestartet bzw. umgesetzt. Da es keine allgemeingültige Definition eines Pilotprojekts gibt, kann in Summe eher von mindestens 40 bis 50 Pilotprojekten in unterschiedlichen Stadien der Umsetzung ausgegangen werden.

Im Zuge der Etablierung der Online-Plattform „innovationspartnerschaft.at" ${ }^{\text {"148 }}$ wurden die Instrumente "Challenges" und "Marktplatz Innovation" implementiert, die der direkten Initiierung von IÖBProjekten dienen. "Challenges" werden von der IÖBServicestelle ausgerufen, um den Bedarf einer beschaffenden Stelle nach einer innovativen Lösung, 
die noch nicht bekannt ist, zu decken. Auf der Onlineplattform ist es für Beschaffer möglich, solche "Challenges" einzugeben, die dann von der IÖB-Servicestelle geprüft werden. Wird eine Challenge ausgerufen, erhalten Unternehmen die Möglichkeit, Lösungen darzustellen, die sie direkt über die Plattform hochladen können. Beschaffer können sich so im Vorfeld einer Ausschreibung ohne viel Aufwand und vor allem unverbindlich über mögliche Lösungsangebote informieren („Markterkundung“). Über die eingelangten Lösungsvorschläge bekommen Beschaffer zudem konkrete Anhaltspunkte zu möglichen Lösungswegen und den Kosten. Die eingelangten Lösungsvorschläge werden von Vertretern der beschaffenden Stelle (Projektleiterinnen und -leiter, Abteilungsleiterinnen und -leiter etc.) im Rahmen einer Jurysitzung bewertet, wobei im Anschluss daran Gewinner unter den eingebrachten Lösungsvorschlägen gekürt werden. Diese können dann im weiteren Verlauf stärker in den Beschaffungsprozess eingebunden werden und im Idealfall auch ein konkretes Angebot legen. Zwischen September 2013 und Jänner 2019 wurden insgesamt 19 "Challenges“ mit einem nachfolgenden Auftragsvolumen von derzeit insgesamt $311.000 €$ ausgerufen. Aufgrund der langen Vorlaufzeit der "Challenges" vor konkreten Beschaffungsvorhaben bzw. des fehlenden Mechanismus' der Rückmeldung der (Budgets der) über "Challenges" konkret ausgelösten Projekte ist eine exakte Abschätzung der Beschaffungsvolumina derzeit nicht möglich.

Am „Marktplatz Innovation“ können sich beschaffende Stellen und Bedarfsträger über innovative Lösungen von Unternehmen erkundigen. Die dort angebotenen Lösungen werden von der IÖB-Servicestelle und Vertretern aus Kompetenz- und Kontaktstellen sowie der Wirtschaft im Rahmen eines Review-Prozesses auf ihren innovativen Charakter in Jurysitzungen überprüft. Per Februar 2019 befinden sich 132 IÖB-taugliche Produkte und Dienstleistungen auf dem Marktplatz. Prinzipiell kann jede und jeder auf den Marktplatz zugreifen und über die dort angegebenen Kontaktdaten die Anbieter von Innovationen direkt kontaktieren. Die BBG greift systematisch auf den „Marktplatz Innovation“ zu, d.h. einerseits wird der Marktplatz im Zuge der Markterkundung eingesetzt, andererseits wurden insgesamt sechs IÖBtaugliche Lösungen des Marktplatzes bereits in das Standardsortiment der BBG aufgenommen. Allerdings gibt es derzeit keine Möglichkeit für die IÖBServicestelle, die Anzahl erfolgter Kontaktaufnahmen und daraus eventuell entstandener Beschaffungsvorhaben direkt zu erfassen.

Die Berücksichtigung von IÖB in Förderprogrammen vor allem der FFG und aws ist aus derzeitiger Sicht als nur teilweise gegeben anzusehen. Unternehmen, die im Rahmen geförderter Projekte innovative Lösungen entwickeln, werden von den beiden Förderagenturen auf die Möglichkeiten der Teilnahme an öffentlicher Beschaffung allgemein sowie die IÖB und die entsprechenden Online-Angebote der Servicestelle (d.h. vor allem den „Marktplatz Innovation") hingewiesen. Allerdings gibt es diesbezüglich keine Informationen, inwiefern dies tatsächlich systematisch und umfassend geschieht. Die Förderagenturen fungieren in diesem Zusammenhang vor allem als Know-how-Träger und Ansprechpartner für Beschaffer. Derzeit fehlt den Agenturen allerdings die Möglichkeit, auf Nachfrage seitens der Beschaffer und Bedarfsträger geförderte Projekte nach relevanten Lösungen zu durchsuchen.

Ein zentrales Element einer systematische(re)n Nutzung von IÖB war die Schaffung einer entsprechenden Grundlage durch die Aufnahme von Innovation als sekundäres Beschaffungsziel in das Bundesvergabegesetz (BVergG). Dies wurde bereits 2013 an der im Leitkonzept vorgeschlagenen Stelle umgesetzt (nunmehr BVergG 2018 §20 (7)).

Zur Umsetzung des Beschaffungsinstrumentes Innovationspartnerschaft (EU-Richtlinie 2014/24/EU) ist 2018 eine entsprechende Novelle des Vergaberechts vorgenommen worden. Damit wird die Beschaffung von nicht am Markt verfügbaren innovativen Lösungen erleichtert. Das neue Vergabeverfahren ermöglicht, die Entwicklung und den anschließenden Erwerb einer innovativen Leistung in einem Beschaf- 
fungsvorgang abzuwickeln. Derzeit läuft eine entsprechende Ausschreibung. ${ }^{149}$

Die IÖB-Servicestelle unterstützt in Kooperation mit der aws Beschafferinnen und Beschaffer im Rahmen des neuen Förderungsprogramms „aws IÖBToolbox" (finanziert aus Mitteln des ÖsterreichFonds). Darin werden öffentliche Auftraggeber bei der Planung und Umsetzung von IÖB-Challenges sowie innovativen Beschaffungsprojekten unterstützt. Ab April 2019 wird eine Einreichung für eines der beiden Module möglich: IÖB-Transfer (Investitionsförderung für innovative Beschaffungsprojekte mit einer Förderungshöhe von max. $100.000 €$ bzw. max. 50 \% der förderbaren Projektkosten) oder IÖB-Prepare (Förderung von Beratungskosten für die Gestaltung und Durchführung von IÖB-Challenges mit einer Förderungshöhe von max. $15.000 €$ bzw. max. $100 \%$ der förderbaren Projektkosten).

\section{Monitoring}

Das Monitoring und Benchmarking umfasst neben der Teilnahme an internationalen Benchmarkings und der Mutual Learning Exercise (MLE) auch die Fortschrittsberichte der Servicestelle sowie Aufbau und Führung einer Datenbank mit Good Practice-Projekten. Im aktuellen „Benchmarking of national innovation procurement policy frameworks“"150 der EU-Kommission rangiert Österreich unter 30 Staaten an der zweiten Stelle. Besonders hervorgehoben wird dabei, dass Österreich über einen der detailliertesten und klarsten Aktionspläne für öffentliche Beschaffung verfügt und gut strukturierte Monitoring- und Anreizsysteme etabliert hat.

Das Monitoring der entsprechend beobachtbaren Beschaffungsvolumina ist im Rahmen einer Piloterhebung der Statistik Austria getestet worden, woraus wesentliche Impulse für weitergehende Entwicklungen der Wirkungsmessung abgeleitet wurden. Es besteht derzeit jedoch kein System der Messung der
IÖB-Anteile an öffentlicher Beschaffung. Derzeit wird jedoch an der Implementierung eines IÖB-Monitorings über bestehende e-Vergabeplattformen gearbeitet.

\subsubsection{Stärkung und Ausbau von Wissenschaft- Wirtschaftskooperationen}

Kooperationen zwischen Wissenschaft und Wirtschaft sind eine wichtige Determinante unternehmerischer Innovationsfähigkeit. Auf der einen Seite wird neues Wissen aus der Grundlagenforschung und angewandten Forschung Unternehmen verfügbar gemacht und damit die Möglichkeit zur wirtschaftlichen Verwertbarkeit geschaffen. Auf der anderen Seite erhält auch die Wissenschaft Impulse aus der Wirtschaft, die wiederum zu neuen Forschungsaktivitäten Anlass geben können.

Die Zusammenarbeit zwischen Unternehmen und Forschungseinrichtungen kann auf vielfältige Weise stattfinden und bedarf aufgrund von Kooperationsbarrieren der politischen Unterstützung durch unterschiedliche Instrumente. Österreich verfügt mittlerweile über ein umfassendes Portfolio an Instrumenten zur Förderung von WissenschaftWirtschaftskooperationen, das beständig angepasst wird. Die Entwicklung der Kooperationsintensität zeigt seit den 1990er Jahren einen positiven Trend und mittlerweile gehört Österreich zu den führenden EU-Ländern bei Wissenschaft-Wirtschaftskooperationen. Dieser Prozess wurde maßgeblich durch politische Maßnahmen angestoßen und gefördert. ${ }^{151}$ Als Meilensteine auf diesem Weg können die Neuorganisation der Christian Doppler Gesellschaft im Jahr 1995 und die Einführung des heutigen COMET-Programms im Jahr 2008 gelten. Das COMET-Programm bietet verschiedene Formate an, organisiert die Bündelung von wissenschaftlichen und wirtschaftlichen Kompetenzen in Zent-

149 Vgl. https://www.ffg.at/fue-Innovationspartnerschaft_asfinag2018

$150 \mathrm{Vgl}$. https://ec.europa.eu/newsroom/dae/document.cfm?doc_id=55186

151 Für eine umfassendere Darstellung des Politikmix zur Förderung von Wissenschaft-Wirtschaftskooperationen vgl. Ecker et al. 2018. 
Tabelle 3-5: Maßnahmen zur Förderung von Wissenschaft-Wirtschaftskooperationen in der FTI-Strategie und Förderinstrumente von BMVIT, BMDW und BMBWF

\begin{tabular}{|c|c|}
\hline Allgemeine Maßnahmen It. FTI-Strategie & Konkrete Maßnahmen (Auswahl) \\
\hline $\begin{array}{l}\text { (1) Weiterentwicklung der Unterstützungsmaßnahmen für } \\
\text { Forschungskooperationen, Netzwerke und strategische } \\
\text { Allianzen mit Fokus auf Exzellenz und Nachhaltigkeit } \\
\text { (wie COMET, Bridge, COIN) und von Modellen der } \\
\text { thematisch orientierten Grundlagenforschung (wie CDG) }\end{array}$ & $\begin{array}{l}\text { - } \text { BRIDGE } \\
\text { - Christian Doppler Labors } \\
\text { - COIN-Netzwerke } \\
\text { - COMET - Competence Centers for Excellent Technologies } \\
\text { - Laura Bassi Centres of Expertise } \\
\text { - Stiftungsprofessuren } \\
\text { - Josef Ressel-Zentren } \\
\text { - Netzwerke der Wissenstransferzentren }\end{array}$ \\
\hline $\begin{array}{l}\text { (2) Stärkung der Hebel- und Transferfunktion von Clustern } \\
\text { und Intermediären }\end{array}$ & $\begin{array}{l}\text { - AplusB-Programm } \\
\text { - Jumpstart } \\
\text { - Nationale österreichische Clusterplattform } \\
\text { - COIN-Aufbau }\end{array}$ \\
\hline \multicolumn{2}{|l|}{$\begin{array}{l}\text { (3) Identifikation von Stärkefeldern zur Bündelung von } \\
\text { Ressourcen und zur Ausschöpfung von Synergien sowie } \\
\text { Unterstützung der Entwicklung von Forschungs- und } \\
\text { Entwicklungs-(Leit-)Themen (zwischen Industrie und } \\
\text { Wissenschaft/Forschung) }\end{array}$} \\
\hline $\begin{array}{l}\text { (4) Unterstützung der „Anbindung“ von österreichischen } \\
\text { Unternehmen und wissenschaftlichen und } \\
\text { Forschungseinrichtungen an EU und internationale } \\
\text { Programme }\end{array}$ & $\begin{array}{l}\text { - ERA-NET } \\
\text { - Europäische und Internationale Programme der FFG } \\
\text { - Joint Programming }\end{array}$ \\
\hline $\begin{array}{l}\text { (5) Unterstützung der Unternehmen in der Sicherung und } \\
\text { Durchsetzung von geistigem Eigentum und dessen } \\
\text { Verwertung }\end{array}$ & $\begin{array}{l}\text { - NCP-IP Nationale Kontaktelle für geistiges Eigentum } \\
\text { (inklusive Intellectual Property Agreement Guide) } \\
\text { - EU Gemeinschaftspatent } \\
\text { - EUREKA } \\
\text { - Finanzielle Unterstützung von internationalen EU-ERA und } \\
\text { INCO-Net Projekten } \\
\text { - aws IP Programme (IP Coaching, License IP und IP market) } \\
\text { - Patentscheck } \\
\text { - Umsetzung Nationale IP-Strategie (2017) } \\
\text { - Wissenstransferzentren und IPR Verwertung } \\
\end{array}$ \\
\hline $\begin{array}{l}\text { (6) Ausbau von Initiativen zur Stärkung der } \\
\text { Humanpotenziale im Bereich der angewandten } \\
\text { Forschung und Stärkung der intersektoralen und } \\
\text { internationalen Mobilität }\end{array}$ & $\begin{array}{l}\text { - AIT-Kolleg } \\
\text { - Talente-Programm } \\
\text { - Forschungskompetenzen für die Wirtschaft } \\
\text { - Jugend Innovativ und aws first }\end{array}$ \\
\hline
\end{tabular}

ren bzw. Großprojekten und hat damit zur Institutionalisierungeinerinternational wettbewerbsfähigen kooperativen Forschung auf hohem wissenschaftlichen Niveau im österreichischen Innovationssystem beigetragen.

Die FTI-Strategie hat bereits 2011 die Entwicklung der Wissenschaft-Wirtschaftskooperationen als sehr positiv in Österreich eingeschätzt. Um die Kooperationsbeziehungen in Zukunft noch weiter zu stärken, wurden Ziele für den Ausbau von Kooperationsbeziehungen gesetzt. Demnach sollen „die Kooperationsintensität österreichischer Unternehmen weiters er- höht und die strategisch orientierte Zusammenarbeit zwischen Wissenschaft und Wirtschaft gestärkt werden - mit besonderem Fokus auf Exzellenz und Nachhaltigkeit"; in diesem Zusammenhang sind daher auch „die Barrieren und Schwellenängste von Unternehmen, insbesondere von KMU, für Kooperationen mit Wissenschaft/Forschung abzubauen und der Zugang von innovativen Unternehmen zu externen Ressourcen zu erleichtern" sowie "die Unternehmen zu unterstützen, dass diese vermehrt ihre Technologieführerschaft ausbauen und in Innovationsspitzenpositionen vorstoßen.“152 
Tabelle 3-6: Indikatoren zu Wissenschaft-Wirtschaftskooperationen des European Innovation Scoreboard (letztverfügbare Zahlen nach EIS 2018), Österreich, EU und die Innovation Leader

\begin{tabular}{|c|c|c|c|c|c|c|}
\hline & \multicolumn{3}{|c|}{$\begin{array}{l}\text { Kofinanzierung von öffentlichen F\&E-Ausgaben } \\
\text { durch Unternehmenssektor (\% des BIP) }\end{array}$} & \multicolumn{3}{|c|}{$\begin{array}{l}\text { Ko-Publikationen von öffentlichen } \\
\text { Forschungseinrichtungen und Unternehmen pro } \\
\text { Mio. Einwohnerinnen bzw. Einwohner }\end{array}$} \\
\hline & 2008 & 2011 & 2015 & 2010 & 2011 & 2017 \\
\hline AT & 0,04 & 0,04 & 0,05 & 72,68 & 71,88 & 82,30 \\
\hline DK & 0,03 & 0,03 & 0,03 & 156,83 & 162,21 & 162,82 \\
\hline EU-28 & 0,05 & 0,05 & 0,05 & 40,16 & 42,61 & 40,93 \\
\hline $\mathrm{FI}$ & 0,08 & 0,08 & 0,05 & 89,32 & 89,48 & 85,40 \\
\hline Innovation Leader* & 0,05 & 0,05 & 0,04 & 94,10 & 97,85 & 94,77 \\
\hline LU & 0,01 & 0,02 & 0,01 & 35,85 & 33,21 & 25,40 \\
\hline $\mathrm{NL}$ & 0,13 & 0,09 & 0,08 & 102,81 & 110,77 & 99,35 \\
\hline SE & 0,05 & 0,04 & 0,04 & 118,94 & 127,34 & 130,56 \\
\hline UK & 0,03 & 0,03 & 0,02 & 60,82 & 64,10 & 65,11 \\
\hline
\end{tabular}

*Innovation Leader bezeichnet das ungewichtete arithmetische Mittel der Innovation Leader.

Die sechs in der FTI-Strategie allgemein formulierten Maßnahmen sind in Tabelle 3-5 gemeinsam mit einer Auswahl an zugeordneten konkreten Maßnahmen des Bundes (Stand Jänner 2019) dargestellt. Die konkreten Maßnahmen umfassen sehr unterschiedliche Instrumente und Förderintensitäten. Monetär betrachtet werden für die Programme zur Umsetzung von Maßnahme (1) die meisten Mittel ausgegeben, wobei hierbei wiederum das COMET-Programm die höchste finanzielle Dotierung aufweist. ${ }^{153}$

Die Vielzahl an Maßnahmen hat beigetragen, Wissenschaft-Wirtschaftskooperationen in Österreich weiter auszubauen bzw. zu bestärken. Die Zielerreichung soll im Folgenden anhand einiger Indikatoren sowie mittels internationalen Vergleichs beantwortet werden. Eine ursächliche Erklärung der Entwicklung der Indikatoren aufgrund der Wirkung einzelner Maßnahmen ist nicht möglich.

Tabelle 3-6 gibt einen Überblick über die zwei Indikatoren zu Wissenschaft-Wirtschaftskooperationen, die im European Innovation Scoreboard (EIS) enthalten sind. Diese beiden Maßzahlen können vor allem zur Messung des Ziels, inwieweit sich die Kooperati- onsintensität österreichischer Unternehmen weiters erhöht bzw. verfestigt hat, herangezogen werden. Zusammen mit dem Anteil an KMU mit Innovationskooperationen bilden diese zwei Indikatoren die Subdimension "Linkages“ im EIS. Österreich konnte in dieser Subdimension seine Position im Zeitraum 2011-2017 durch einen Sprung vom neunten Rang auf den dritten Rang deutlich verbessern und liegt nunmehr lediglich hinter Belgien und den Niederlanden. Im Detail zeigt sich auch bei den Indikatoren in Tabelle 3-6 eine positive Entwicklung. Bei der Kofinanzierung öffentlicher F\&E-Ausgaben in \% des BIP durch Unternehmen stieg der Anteil von 0,04\% auf 0,05\%; dieser Anteil liegt damit auch über dem Durchschnittswert der Innovation Leader, die im gleichen Zeitraum einen abnehmenden Finanzierungsanteil der Unternehmen aufweisen. Bei den Ko-Publikationen weist Österreich ebenfalls einen positiven Trend auf; der entsprechende Wert stieg um ca. $15 \%$ von 71,88 im Jahr 2011 auf 82,30 im Jahr 2017. Demgegenüber stagnierte die Entwicklung für die EU-28 sowie die der Innovation Leader. Obwohl Österreich hier noch unter dem durchschnittlichen Niveau der Innovation Leader liegt, lässt sich damit als Zwischenergebnis

153 So betrug etwa das gesamte Fördervolumen seitens des Bundes für COMET für den Zeitraum 2008-2018 etwa 575 Mio. $€$ (ohne Bundesländer). Vgl. Ecker et al., 2018. 
Abbildung 3-14: Anteil der Produkt- und Prozessinnovatoren mit Innovationskooperation mit Universitäten, Fachhochschulen oder anderen höheren Bildungseinrichtungen, in \%

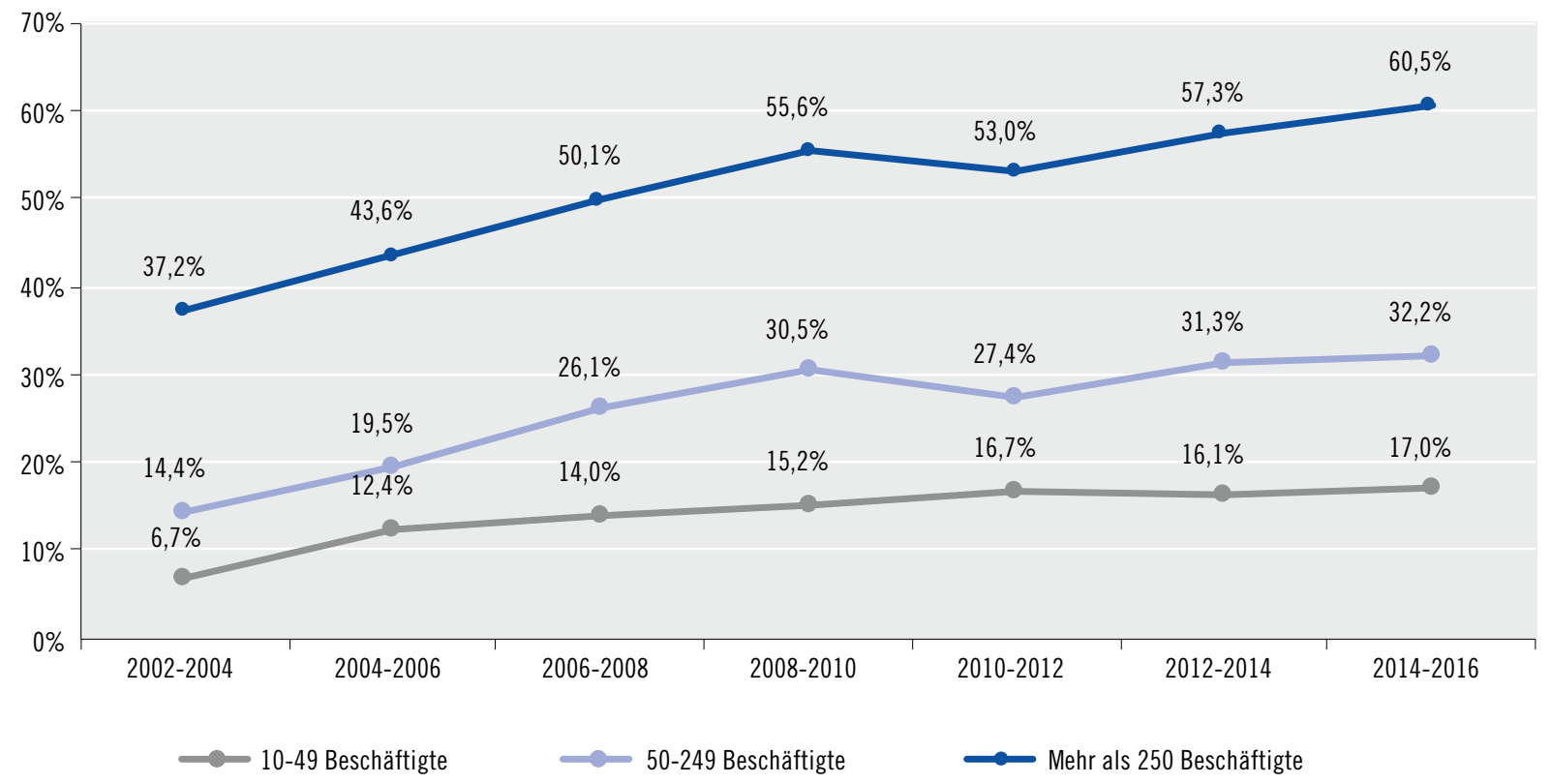

Quelle: Statistik Austria, CIS 2004, CIS 2006, CIS 2008, CIS 2012, CIS 2014, CIS 2016.

und auf Basis der im EIS erfassten Indikatoren festhalten, dass das Ziel der FTI-Strategie, WissenschaftWirtschaftskooperationen in Österreich weiter auszubauen, erreicht wurde: Es konnte sowohl die Kooperationsintensität erhöht als auch der Abstand zu den Innovation Leadern reduziert werden.

Adressiert die erste Analyse den Unternehmenssektor insgesamt, so gilt es im nächsten Schritt zu untersuchen, ob sich die Kooperationsintensität zwischen Wissenschaft und Wirtschaft insbesondere auch bei den KMU verbessert hat. Diese Überprüfung kann durch die Analyse der Entwicklung der Kooperationsaktivitäten von Unternehmen mit Forschungseinrichtungen erfolgen. Die Unternehmen werden hierzu in verschiedene Größenklassen eingeteilt.

Abbildung 3-14 stellt diese Entwicklung für die wissenschaftlichen Kooperationspartner Universitäten, Fachhochschulen und andere höhere Bildungseinrichtungen von 2002-2016 dar. Es zeigt sich für alle Unternehmensgrößengruppen eine Zunahme des Anteils an Unternehmen mit Wissenschaft-Wirt- schaftskooperationen. Diese Feststellung gilt auch für die Periode nach Einsetzung der FTI-Strategie im Jahr 2011. Großunternehmen und mittelgroße Unternehmen konnten deutlich stärker zulegen als Unternehmen mit weniger als 50 Beschäftigten. Über den gesamten Zeitraum 2002-2016 wuchs die Kooperationsneigung bei Kleinunternehmen am höchsten und bei Großunternehmen am geringsten. Dieser Befund bestätigt sich grundsätzlich auch, wenn anstatt tertiärer Bildungseinrichtungen "Sonstige staatliche oder öffentliche Forschungseinrichtungen" als Kooperationspartner betrachtet werden. Hierunter fallen etwa das AIT oder Joanneum Research. Einschränkend ist anzumerken, dass KMU (Unternehmen mit weniger als 250 Beschäftigte) seit 2010-2012 geringfügige Abnahmen ihrer Kooperationsintensität aufweisen. Im internationalen Vergleich für die Periode 2012-2014 liegt Österreich bei einer Auswertung für öffentliche und private Forschungseinrichtungen als Kooperationspartner sogar vor allen Innovation Leadern auf Platz eins. ${ }^{154}$ 
Schließlich wurde von der FTI-Strategie auch das Ziel gesetzt, dass mehr Unternehmen Technologieführerschaft erreichen und in Innovationsspitzen vorstoßen. Um diesbezüglich die Zielerreichung zu überprüfen, werden in den Leistungsberichten des RFTE die Patentanmeldungen nach PCT relativ zum BIP analysiert. ${ }^{155}$ Demnach konnte Österreich im Zeitraum 2008 bis 2015 die Patentanmeldungen nach PCT pro Mrd. des BIP von 4,4 auf 4,7 steigern. Allerdings zeigt sich seit 2011 eine Abnahme von 5,13 auf 4,70. Der österreichische Wert lag 2015 bei etwa $85 \%$ des Durchschnittswerts der Innovation Leader. Für den Zeitraum 2008-2015 ist damit ein Aufholprozess Österreichs zu verzeichnen, allerdings hat die Dynamik seit 2011 nachgelassen.

Neben der Patentanalyse werden im Folgenden noch die Daten aus dem European Industrial R\&D Investment Scoreboard, in dem jährlich die 1.000 Unternehmen der EU mit den höchsten F\&E-Ausgaben erfasst werden, zur weiteren Betrachtung herangezogen. Die Auswahl an Unternehmen - erfasst durch den European Industrial R\&D Investment Scoreboard - kann als europäische Innovationsspitze aufgefasst werden und die Anzahl an österreichischen Unternehmen unter diesen 1.000 Unternehmen als Indikator für das Erreichen von Spitzenpositionen. Im Zeitraum 2011-2017 nahm die Anzahl der im European Industrial R\&D Investment Scoreboard vertretenen österreichischen Unternehmen von 27 auf 32 zu. Beispielhaft und als Vergleich hierzu sei Schweden, das im Jahr 2017 mit 77 Unternehmen nach wie vor deutlich mehr Unternehmen in Spitzenpositionen aufweist als Österreich.

Insgesamt scheint damit das Ziel einer Ausweitung der Unternehmen mit Technologieführerschaft nur teilweise erreicht worden zu sein. Einschränkend ist jedoch anzumerken, dass gerade der hochinnovative und auf Nischen spezialisierte Mittelstand der österreichischen Industrie nur unzureichend durch die beiden verwendeten Maßzahlen abgebildet wird.
Zusammenfassend ergibt sich für die Erreichung der Ziele - und somit auch für die Umsetzung der Maßnahmen im Gesamten - für Wissenschaft-Wirtschaftskooperationen ein positiver Befund. Eine Zunahme der Kooperationsintensitäten ist zu verzeichnen und Österreich kann als ein führendes Land in diesem Bereich klassifiziert werden.

\subsubsection{Stärkung von Entrepreneurship an den Universitäten}

Um die Interaktion zwischen Wissenschaft und Wirtschaft, den Wissens- und Technologietransfer zwischen diesen beiden Sektoren und um schließlich auch den Unternehmergeist und die Zahl der innovativen Unternehmensgründungen aus den Hochschulen heraus zu erhöhen, hielt die FTI-Strategie 2011 hierfür insbesondere zwei Maßnahmen ${ }^{156}$ fest:

- Stärkung von Finanzkompetenz und Entrepreneurship an den Universitäten, u.a. durch die Einrichtung von Wissenstransferzentren

- Erarbeitung von neuen Finanzierungsmodellen mit Venture-Capital-Beteiligung zur Verwertung von universitären Intellectual Property Rights (IPR) bis hin zur Einrichtung von universitätsbezogenen Venture-Beteiligungsgesellschaften

Um die Abstimmung und Entwicklung von Unterstützungsmaßnahmen für die Förderung von Wissensund Technologietransfer, Unternehmensgründungen etc. zu verbessern, wurde im Rahmen der Task Force FTI die Arbeitsgruppe "Wissenstransfer und Gründungen" eingerichtet.

\section{IP National Contact Point (NCP-IP)}

Die Nationale Kontaktstelle für Geistiges Eigentum (NCP-IP) wurde per Beschluss der Bundesregierung am 02.03.2010 auf Basis einer Empfehlung der Europäischen Kommission („IP-Recommendation”) gemeinsam von BMBWF, BMDW und BMVIT eingerichtet. Die operative Umsetzung erfolgt von aws und FFG über

155 Dieser Indikator ist auch im EIS enthalten und dient als eine von drei Maßzahlen für die Subdimension „Intellectual assets“. $156 \mathrm{Vgl}$. BKA et al. $(2011,30)$. 
abgestimmte jährliche Arbeitsprogramme. Zielsetzung des NCP-IP ist es, die Zusammenarbeit von Wissenschaft und Wirtschaft interministeriell abgestimmter Maßnahmen weiter zu stärken, öffentliche Forschungseinrichtungen. Universitäten und Unternehmen beim Umgang mit Geistigem Eigentum zu unterstützen (wie z.B. durch Workshops, Schulungen, Vertragsmuster) und Österreich in den einschlägigen europäischen Gremien zu vertreten.

Umgesetzt wurde bisher das Vorreiterprojekt Intellectual Property Agreement Guide (IPAG), welches kostenlos online Vertragsmuster im Technologietransfer in deutscher und englischer Sprache für Forschungseinrichtungen und Unternehmen zur Verfügung stellt. Das Projekt IPAG bestätigt den hohen Bedarf an fundierten rechtlichen Grundlagen im Wissenstransfer (rd. 25.000 Zugriffe seit Beginn). In Umsetzung der Open Innovation Strategie der Bundesregierung werden ab Ende 2019 Unternehmen und Forschungseinrichtungen bei der Schaffung von fairen Open Innovation Prozessen durch ein weiteres neues interaktives Toolkit unterstützen. Zur Vernetzung der Akteure des österreichischen Innovationsystems wurden seit Errichtung des NCP 2010 über 50 Veranstaltungen organisiert, um den Austausch zwischen Vertreterinnen und Vertretern aus Wissenschaft und Wirtschaft weiter zu intensivieren und die Vertrauensbildung zu stärken. Zusätzlich erfolgte der Launch der neuen NCP-IP Homepage ${ }^{157}$ mit umfassenden Informationen zum Thema Wissens- und Technologietransfer.

\section{Wissenstransferzentren und IPR Verwertung}

Das Programm „Wissenstransferzentren und IPR Verwertung" (2013-2018) schuf Anreize für eine verstärkte regionale Kooperation der Wissens- und Technologietransferstellen der österreichischen Universitäten im Rahmen der Wissenstransferzentren Ost, Süd und West sowie für die Etablierung des thematischen Wissenstransferzentrums Life Sciences. Dafür stand ein Investitionsvolumen von 11,25 Mio. $€$ zur Verfügung. An den regionalen Zentren nahmen alle österreichischen Universitäten mit 16 Kooperationsprojekten teil. Durch das Programm sollen insbesondere Synergiepotentiale der Wissens- und Technologietransferstellen der Universitäten gestärkt werden, insbesondere wenn es darum geht, Kompetenzen im Wissens- und Technologietransfer ausund aufzubauen, Netzwerkbeziehungen zwischen Hochschulen, Forschungseinrichtungen und Wirtschaftsunternehmen zu intensivieren und Verwertungspotentiale zu erweitern und besser auszuschöpfen. Dabei wurden sowohl die technischen und medizinischen Studienrichtungen als auch jene der Geistes-, Sozial- und Kulturwissenschaften (GSK) und der Kunst österreichweit miteingeschlossen.

Im Rahmen der Förderung von Patentkosten in Höhe von insgesamt 5 Mio. $€$ bis Ende 2018 langten bis zum November 2018 (=Ende der Einreichfrist) insgesamt 738 Anträge der Universitäten ein, wobei bislang 99,4\% der Fördermittel zugesagt wurden.

Im Zuge der Prototypenförderung PRIZE wurden 2014, 2015, 2016 und 2017 von einer internationalen Fachjury erfolgsversprechende Projekte zur Förderung ausgewählt. Insgesamt wurden damit vom BMDW rd. 4 Mio. $€$ für die Entwicklung patentfähiger Prototypen zur Verfügung gestellt.

Eine externe Zwischenevaluierung sowie der Monitoringbericht von BMBWF und BMDW im Rahmen der WFA waren positiv und zeigen, dass die strategischen und operativen Vorgaben des Programms weitgehend umgesetzt werden konnten. Darauf aufbauend sollen im Rahmen der Leistungsvereinbarungen 2019-2021 das Community Building der Wissenstransferzentren bzw. deren erfolgreiche Projekte fortgeführt werden. Aus Mitteln des ÖsterreichFonds in der Höhe von 6 Mio. € wurde im April 2019 das „Impulsprogramm für den österreichischen Wissens- und Technologietransfer" von der aws ausgeschrieben, welches unter Berücksichtigung neuer Schwerpunkte wie die verstärkte Einbeziehung von Fachhochschulen zusätzliche Anreize für die Erwei-

157 Vgl. https://www.ncp-ip.at/ 
terung der bisherigen universitären Netzwerke durch Fachhochschulen, Schulen, Kindergärten und Unternehmen speziell im MINT-Bereich bieten soll. Zusätzlich erfolgen eine Patentförderung für Universitäten und Fachhochschulen sowie eine Prototypenförderung für aussichtsreiche Projekte aus der wissenschaftlichen Forschung. ${ }^{158}$

\section{Spin-off Fellowships}

Im Zuge der Gründerland-Initiative der Bundesregierung wurde auch das "Spin-off Fellowships“-Programm ${ }^{159}$ entwickelt. Die sogenannten Spin-off Fellowships sollen einen zusätzlichen Anreiz bieten, Forschungsergebnisse aus dem Hochschulbereich in Geschäftsideen umzusetzen und diese bis zur Gründung eines Unternehmens voranzutreiben. Adressaten sind Studierende und Forschende an Universitäten und Forschungseinrichtungen, die bereits marktrelevante Forschungsergebnisse erzielt haben. Mit einem Spin-off Fellowship erhalten diese die Möglichkeit, über einen Zeitraum von max. 1,5 Jahren (freigestellt von Verpflichtungen wie Lehre und Forschung) an der jeweiligen Hochschule oder Forschungseinrichtung an ihrer Geschäftsidee zu arbeiten, um anschließend ein akademisches Spin-off zu gründen. Seit Programmbeginn sind bisher 15 Projekte gestartet worden. Das „Spin-off Fellowship“-Programm läuft bis 31.12.2021. Im Falle einer Unternehmensgründung können Förderprogramme wie AplusB, Seed oder PreSeed anschließende Unterstützung bieten.

\section{AplusB-Programm}

AplusB-Zentren unterstützen Firmengründungen aus Universitäten, Fachhochschulen und außeruniversitären Forschungseinrichtungen. Von 2002 bis 2017 wurde das AplusB-Programm im Auftrag des BMVIT von der FFG abgewickelt. In diesem Zeitraum wurden österreichweit in sieben (zeitweise neun) AplusBZentren akademische Unternehmensgründungen und Spin-offs unterstützt und gefördert. Bis April 2018 wurden damit 829 Projekte betreut, wovon 710 ein Unternehmen gegründet haben. Insgesamt wurden über 3.000 (vorwiegend für hochqualifizierte) Arbeitsplätze geschaffen. ${ }^{160}$ Seit 2017 wird das Programm unter "AplusB Scale-up“ fortgeführt und wechselte die Abwicklung von der FFG zur aws. ${ }^{161}$ Der Fokus der Förderung hat sich ebenfalls geändert. Mit dem neuen Programm „AplusB Scale-up“ sollen nun insbesondere $\mathrm{FTI}-G$ ründungen mit hohem Wachstumspotential bzw. hoher Wachstumsneigung unterstützt werden. ${ }^{162}$

\section{Verankerung von Entrepreneurship an den Universitäten}

Österreichs Universitäten sind nicht nur Hauptträger der Grundlagenforschung, sie nehmen auch vor dem Hintergrund der Profilentwicklung eine zentrale Rolle in der Standortentwicklung ein. ${ }^{163}$ Ähnlich wie bei Unternehmen wurde daher auch für die österreichischen Universitäten eine "Leitinstitutionen-Initiative“ entwickelt und diese in drei aufeinanderfolgenden Leistungsvereinbarungen und im Gesamtösterreichischen Universitätsentwicklungsplan verankert. Ebenso festgehalten ist hier die Motivation zu universitären Aktivitäten, die entrepreneurship-orientiertes Denken in Lehre und Forschung fördern bzw. einbeziehen (wie z.B. durch spezielle Lehrveranstaltungen). In der Leistungsvereinbarungsperiode 20162018 wurden daher auch gezielt Vorhaben gefördert, die Maßnahmen zur Personalentwicklung und Weiterqualifizierung des Universitätspersonals - u.a. zur

$158 \mathrm{Vgl}$. www.aws.at/foerderungen/wissenstransferzentren/, www.aws.at/patentfoerderung und www.aws.at/foerderungen/prototypenfoerderung/

159 Vgl. https://www.ffg.at/spin-off-fellowships

$160 \mathrm{Vgl}$. https://www.ffg.at/aplusb-academia-plus-business

$161 \mathrm{Vgl}$. https://www.aws.at/foerderungen/aws-aplusb-scale-up/

$162 \mathrm{Vgl}$. https://www.bmvit.gv.at/innovation/zentren/aplusb/index.html

$163 \mathrm{Vgl}$. Janger et al. (2017a). 
Vermittlung von Entrepreneurship-Kompetenzen beinhalteten. ${ }^{164}$

Projekte der regionalen Wissenstransferzentren wie „Ideen Garten“, „Skinnovation“, die "Gründungsgarage“ oder Prämierungsevents wie „Von der Innovation zur Wissenschaft" haben darüber hinaus merklich zur Steigerung der Gründungsmotivation und des Entrepreneurial Spirit an Österreichs Universitäten beigetragen. ${ }^{165}$ Es wurden auch Gründungslehrstühle an den Universitäten Klagenfurt, Linz und Wien eingerichtet. Außerdem nimmt Österreich aktuell an einer von Europäischer Kommission und OECD gemeinsam durchgeführten HElnnovate-Länderstudie teil - nicht zuletzt, um die Hochschulen zu motivieren, Entrepreneurship und Innovation in sämtlichen Dimensionen ihrer Aufgabenbereiche mitzudenken bzw. strategisch zu verankern.

\subsubsection{Unterstützung von}

\section{Unternehmensgründungen und Stärkung der Risikokapitalfinanzierung}

In der FTI-Strategie wird der im internationalen Vergleich deutlich unterdurchschnittliche Anteil an jungen, schnell wachsenden Unternehmen als Defizit von Österreichs Innovationssystem hervorgehoben. ${ }^{166}$ Tatsächlich zeigt der jüngste European Innovation Scoreboard, dass Österreich 2017 im Vergleich zu 2010 in der Kategorie „Beschäftigung in schnell wachsenden Unternehmen“ („employment in fast-growing enterprises") weiter zurückgefallen ist. ${ }^{167}$ Zwar hat sich die Performance der EU als Ganzes ebenfalls verschlechtert, Österreich verlor jedoch noch mehr Anteile, was bedeutet, dass Ös- terreich sowohl relativ zur EU wie zu sich selbst zurückgefallen ist. Diese Entwicklung ist auch dafür verantwortlich, dass die Innovationsdimension "Beschäftigung" (,employment impacts“) neben „Verkauf“ („sales impacts“) im aktuellen European Innovation Scoreboard als größte Schwäche Österreichs identifiziert wird. ${ }^{168}$ Ein Indikator der Statistik Austria, nämlich jener zu „Wissens- und forschungsintensive Neugründungen [von Unternehmen]", zeigt nach Jahren der Stagnation für das letzte verfügbare Jahr (2016) immerhin eine kräftige Steigerung. ${ }^{169}$

Um sich im Bereich der Beschäftigten in schnell wachsenden Unternehmen zu verbessern, braucht Österreich zunächst Unternehmen, die das Potential haben, in der Zukunft zu wachsen. Unternehmen, die schnell wachsen, sind üblicherweise solche, die innovativ sind. Zur Finanzierung ihrer Projekte benötigen sie Investorinnen und Investoren, da die Unternehmensgründerinnen und -gründer nur selten über entsprechende Eigenmittel verfügen. Daher betont die FTI-Strategie auch die Notwendigkeit der Verfügbarkeit von Risikokapital für die Finanzierung wachstumsorientierter Unternehmen. Die in Österreich vorherrschende Form der Unternehmensfinanzierung durch Bankkredite erweist sich als Schwäche, da Banken aufgrund ihres Geschäftsmodells nur selten bereit sind, das hohe Risiko einzugehen, das mit unternehmerischer Innovationstätigkeit, insbesondere in der Frühphase, zwangsläufig verbunden ist. Die FTI-Strategie sieht daher die Stärkung des Risikokapitalmarkts als spezifische Herausforderung Österreichs, d.h. das Ziel der FTI-Strategie adressiert eine „Vergröße-

164 Vgl. BMBWF (2017).

165 Ebenda.

$166 \mathrm{Vgl}$. BKA et al. $(2011,29)$.

167 Dieser Indikator ist definiert als Anteil der Beschäftigten in der oberen Hälfte der innovativsten Branchen („number of employees in highgrowth enterprises in 50 \% 'most innovative' industries"). In den Ausgaben des European Innovation Scoreboard werden aufgrund der Datenverfügbarkeit jeweils die Daten des Vor-Vorjahres erfasst. Im jüngsten Innovation Scoreboard von 2018 stammen die meisten Daten aus dem Jahr 2017, jene für den Indikator „Beschäftigung in schnell wachsenden Unternehmen“ daher aus dem Jahr 2015.

$168 \mathrm{Vgl}$. Europäische Kommission (2018b, 69).

169 Vgl. Unternehmensdemografie der Statistik Austria; die Zahlen für 2016 sind bei Drucklegung „vorläufig“. 
rung des Volumens an Wagniskapital“ 170, das österreichischen Start-ups zur Finanzierung zur Verfügung steht. ${ }^{171}$

Vor diesem Hintergrund hat die FTI-Strategie folgende Maßnahmen ${ }^{172}$ zur Unterstützung von innovativen und technologiebasierten Unternehmen sowie zwecks Stimulierung der Risikokapitalfinanzierung definiert:

- Entwicklung von Angebot und Nachfrage an Risikokapital

- Schaffung eines rechtlichen Rahmens zur Eigenkapitalstärkung von jungen, technologie- und wachstumsorientierten Unternehmen

- Ausbau der Risikokapitalinitiative für die Stimulie- rung von Frühphaseninvestitionen unter Berücksichtigung der bisherigen Entwicklungen

- Optimierung und Vervollständigung der bereits bestehenden Unterstützungsmaßnahmen für technologiebasierte und innovative Unternehmensgründungen, vor allem Maßnahmen für die Startphase (vgl. PreSeed, Seedfinancing, BusinessAngels, Technologiemarketing etc.)

\section{Entwicklung von Angebot und Nachfrage an Risikokapital}

Abbildung 3-15 zeigt das Wagniskapital-Investitionsvolumen anteilig am BIP für Österreich sowie für die im Innovation Scoreboard 2018 ausgezeichneten In-

\section{Abbildung 3-15: Wagniskapital-Investitionen in ausgewählten Ländern nach Standort des Portfolio-}

Unternehmens, 2007-2017, in Promille des BIP

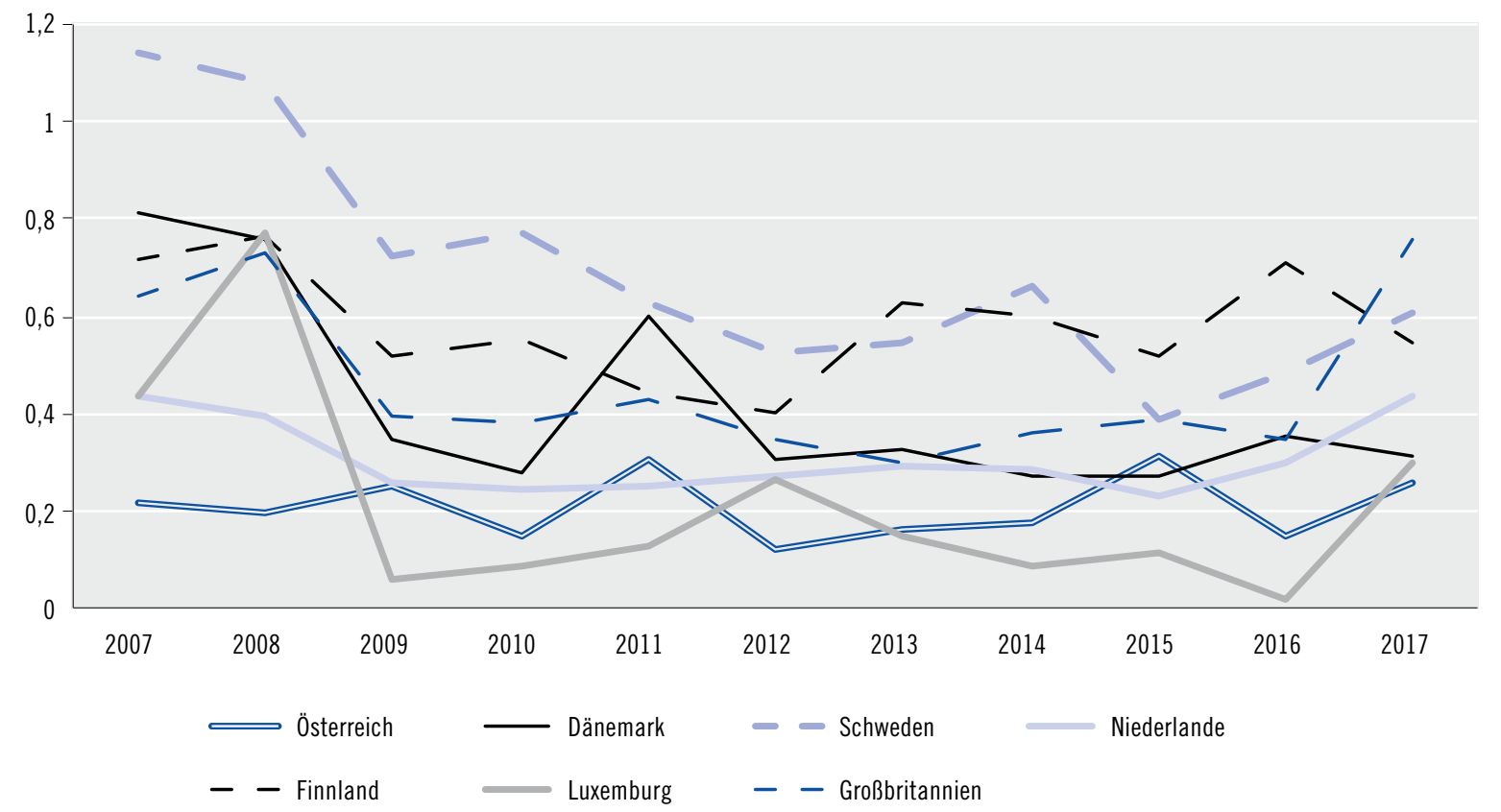

Quelle: INVEST Europe (Investitionen) und Eurostat (BIP), Berechnungen und Darstellung WPZ Research

170 Vgl. BKA et al. $(2011,29)$ und BMWFW, BMVIT $(2016,71)$.

171 Ein Start-up bezeichnet ein junges Unternehmen, das forschungsintensiv produziert und durch ein hohes Risiko gekennzeichnet ist. Wagniskapital (Venture Capital) bezeichnet privates oder staatliches Beteiligungskapital, das in Start-ups investiert wird, um das Unternehmen nach einem gewissen Zeitraum möglichst mit Gewinn wieder zu veräußern. Wagniskapital stellt somit eine Sonderform von Private Equity dar, das gezielt in Start-ups investiert wird. Mit dem Begriff Risikokapital ist manchmal Private Equity inkl. Wagniskapital gemeint, manchmal auch nur Wagniskapital. Da in der FTI-Strategie mit Risikokapital nur Wagniskapital gemeint ist, werden diese Begriffe auch hier synonym verwendet.

172 Vgl. BKA et al. $(2011,30)$ 
Abbildung 3-16: Wagniskapital-Investitionen in ausgewählten Ländern nach Standort der Beteiligungsfirma, 2007-2017, in Promille des BIP

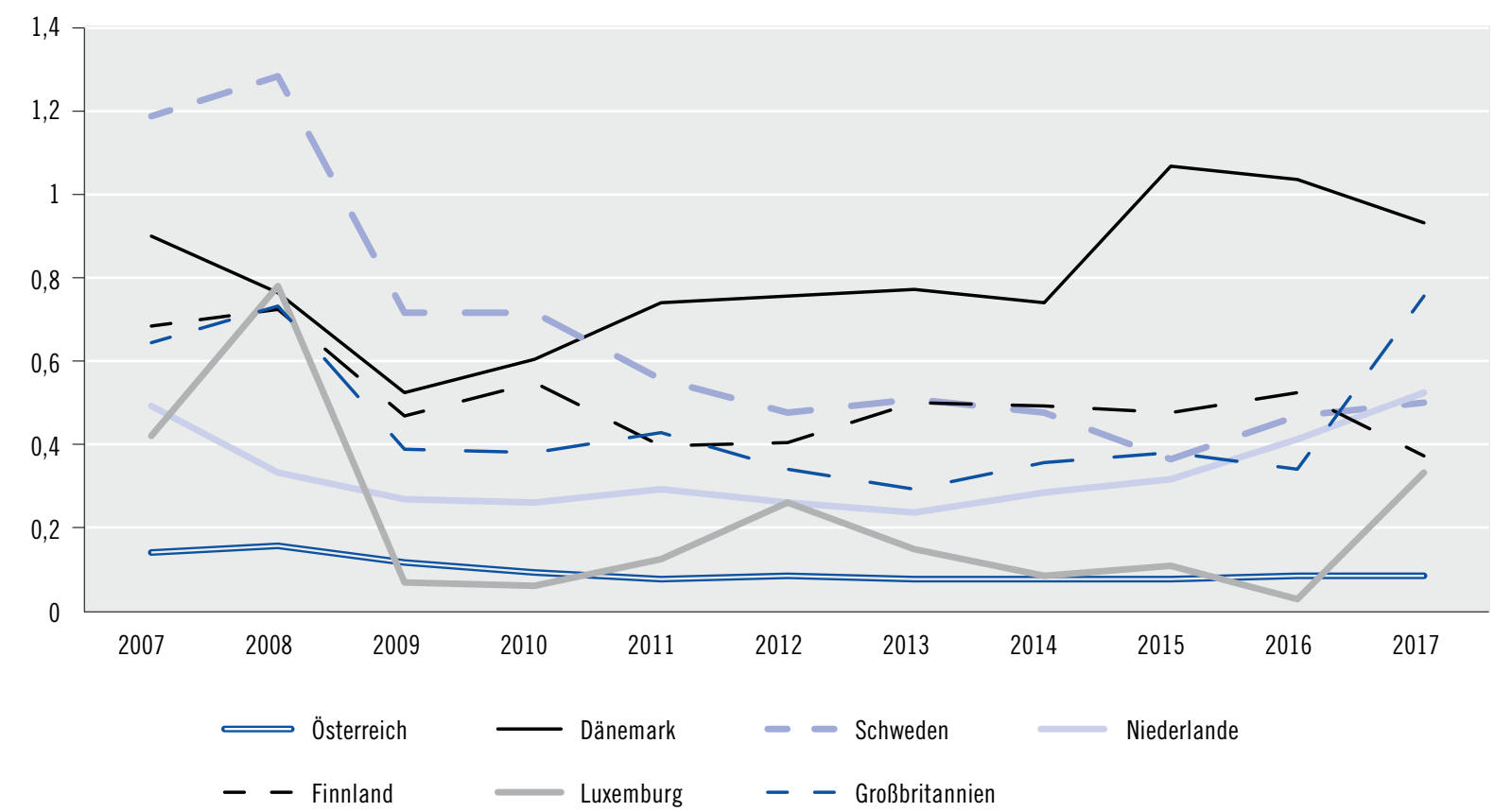

Quelle: INVEST Europe (Investitionen) und Eurostat (BIP), Berechnungen und Darstellung WPZ Research.

novation Leader Dänemark, Schweden, Niederlande, Finnland, Luxemburg und Großbritannien für den Beobachtungszeitraum 2007-2017.173 Österreich liegt zwar in den meisten Jahren an letzter oder vorletzter Stelle, holt gegenüber den Innovation Leadern jedoch kontinuierlich auf: Lag das Verhältnis von Österreichs Anteil zum Mittelwert der Anteile der Innovation Leader 2007 und 2008 noch bei deutlich unter einem Drittel, so liegt er seit 2013 durchgehend bei über zwei Fünftel, im Jahr 2015 lag Österreich sogar annähernd gleichauf, 2017 lag das Verhältnis bei etwas mehr als der Hälfte. ${ }^{174}$

Noch bedeutender ist, dass Österreich als einziges der sieben Länder im Beobachtungszeitraum eine, wenn auch nur leichte, steigende Tendenz aufweist. ${ }^{175}$ Während das investierte Wagniskapital-Volumen in Österreich von der Finanz- und Wirtschaftskrise nicht merklich beeinflusst wurde, haben sich alle anderen sechs untersuchten Länder von diesem Schock immer noch nicht erholt und zeigen über den gesamten Zeitraum eine fallende Tendenz.

Abbildung 3-16 zeigt das Wagniskapital-Investitionsvolumen, das durch heimische Beteiligungsfirmen angelegt wird, wiederum im Verhältnis BIP für dieselben Länder. Das Kapital muss nicht notwendigerweise in Start-ups im eigenen Land investiert werden, aller-

173 Man beachte, dass der Abstand Österreichs wesentlich kleiner ist als in der FTI-Strategie angegeben. In Letzterer (S. 29) wird für das Jahr 2010 für Österreich ein Wert von 0,03\%, für Schweden hingegen ein Wert von 0,23\% angegeben. Diese Diskrepanz ist vermutlich darauf zurückzuführen, dass in der FTI-Strategie die Werte aus dem damals aktuellen Innovation Scoreboard übernommen wurden. Diese enthalten jedoch, erstens, zusätzlich zu Wagniskapital im engeren Sinn (Seed-Phase, Start-up-Phase, „later stage venture") auch Wachstumskapital, Rescue- und Turnaround-Kapital sowie Replacement-Kapital, welche üblicherweise zwar als Private Equity, nicht aber als Wagniskapital klassifiziert werden. Zweitens handelt es sich in der FTI-Strategie vermutlich, wie im Innovation Scoreboard, um Mittelwerte der vorangegangenen drei Jahre. In diesem Bericht ist mit Wagniskapital (und somit auch Risikokapital) stets Wagniskapital im engeren Sinn gemeint, und Jahresangaben beziehen sich auf das tatsächlich angegebene Jahr.

174 Die Werte sind im Einzelnen: 2007: 0,31; 2008: 0,26; 2009: 0,66; 2010: 0,38; 2011: 0,74; 2012: 0,34; 2013: 0,44; 2014: 0,47; 2015: 0,99; 2016: 0,40; 2017: 0,53.

175 Berechnet nach dem Kleinste-Quadrate-Schätzer; für weitere Details siehe Keuschnigg und Sardadvar (2019). 
dings zeigt die empirische Evidenz sehr deutlich, dass die geografische Distanz eine entscheidende Determinante darstellt: Üblicherweise investieren Beteiligungsfirmen in Start-ups im eigenen Land, oft sogar in der eigenen Region, allenfalls in benachbarten Ländern. Vor diesem Hintergrund ist umso bemerkenswerter, dass das Volumen, dass durch österreichische Wagniskapitalfonds investiert wird, in jedem Jahr wesentlich kleiner ist als das Volumen, das in österreichische Start-ups investiert wird. Daraus folgt, dass die Nachfrage nach Wagniskapital in Österreich größer ist als das zur Verfügung stehende Angebot.

\section{Schaffung eines rechtlichen Rahmens zur Eigenkapitalstärkung von jungen, technologie- und wachstumsorientierten Unternehmen}

Eine mögliche Ursache für den in Abbildung 3-16 deutlich sichtbaren Abstand Österreichs hinsichtlich der Etablierung von Beteiligungsfirmen ist die fehlende Rechtssicherheit. Diese Unsicherheit betrifft dabei jedoch weniger die Rechtsform der Beteiligungsfirmen, denn die für Risikokapitalgesellschaften international übliche Rechtsform einer "Limited Partnership" ist mit der in Österreich möglichen Kommanditgesellschaft vergleichbar. ${ }^{176}$ So sieht z.B. das Mittelstandsfinanzierungsgesellschaften-Gesetz (MiFiGG) Kommanditgesellschaften als Rechtsform vor; das Alternative Investmentfonds Manager-Gesetz (AIFMG) bestimmt, wer einen Wagniskapitalfonds mit Publikumsgeldern betreiben darf. Dies alles ist jedoch unabhängig von der Frage, ob es einen geeigneten Rechtsrahmen für Risikokapitalfonds in Österreich gibt, zu sehen. Das eigentliche Problem ist vielmehr, dass es in Österreich an steuerlicher Rechtssicherheit fehlt. Tatsächlich existiert keine Rechtsgrundlage, die definiert, welche Kriterien von einer Risikokapitalgesellschaft zu erfüllen sind.

Hinsichtlich Crowdfunding wurde 2015 in Zusammenarbeit mit dem BMWFW (heute: BMDW) und dem BMASK (heute: BMASGK) ein Rechtsrahmen geschaffen, der auch Eigenkapitalinstrumente umfasst und sich insbesondere an junge und innovative Unternehmen richtet. ${ }^{177}$ Darauf aufbauend werden internationale Modelle für rechtliche Rahmenbedingungen für eigenkapitalstärkende Maßnahmen vor dem Hintergrund europäischer Rahmenbedingungen analysiert und auf Umsetzbarkeit in Österreich geprüft.

\section{Ausbau der Risikokapitalinitiative für die Stimulierung von Frühphaseninvestitionen unter Berücksichtigung der bisherigen Entwicklungen}

Die Durchführung einer Risikokapitalinitiative wurde im Regierungsprogramm 2008-2013 formuliert. ${ }^{178}$ Die Umsetzung obliegt in erster Linie der aws. Die Eigenkapitalinitiativen der aws erweitern die verfügbaren Finanzinstrumente, mobilisieren privates Risikokapital und verbessern insgesamt die mittel- und langfristige Finanzierungsstruktur von KMU. Hierzu zählt die „Venture-Capital-Initiative“, die mit Mitteln der Nationalstiftung für Forschung, Technologie und Entwicklung finanziert ist, ${ }^{179}$ und über die sich die aws zurzeit an zehn Fonds beteiligt, von denen sich (mit Stand Dezember 2018) drei in der Investitionsphase befinden: 1) Speedinvest II EuVECA GmbH \& Co KG, ein Fonds mit Sitz in Wien und im Silicon Valley, der sich an Unternehmen in der Frühphase im Bereich In-

176 Eine „Limited Partnership“ besteht aus einem „General Partner", der auch die Geschäfte führt und die Gesellschaft nach außen vertritt. Investorinnen und Investoren stellen die Limited Partner dar, indem sie sich wie Aktionäre (oder Kommanditisten) beteiligen. Wie bei einer Kommanditgesellschaft haftet also für Verbindlichkeiten der Gesellschaft mind. ein Gesellschafter unbeschränkt (Generalpartner, Komplementär) und mind. ein weiterer Gesellschafter nur beschränkt (Limited Partner, Kommanditist). Mitsprache- und Kontrollrechte der Limited Partner sind tendenziell eher eingeschränkt als jene der Kommanditisten, obwohl es hier sowohl im angelsächsischen wie im deutschsprachigen Raum Unterschiede gibt.

177 Vgl. https://www.bmdw.gv.at/EUundInternationaleMarktstrategien/Wirtschaftsrecht/Seiten/Alternativfinanzierungsgesetz-.aspx 178 Vgl. BKA (2017, 42).

179 Die Nationalstiftung für Forschung, Technologie und Entwicklung dient folgenden Zielen: (i) der nachhaltigen Finanzierung von Forschungsvorhaben; (ii) der Ausrichtung auf langfristige Zielsetzungen der Forschungs- und Technologiepolitik; sowie (iii) der exzellenten, internationalen Positionierung österreichischer Forschung und Technologieentwicklung. Sie dient dabei als Finanzierungsinstrument, ohne neue Verwaltungs- und Abwicklungsstrukturen zu schaffen. Die Fördermittel der Stiftung werden nicht direkt an Förderungswerber, sondern an vom Bund getragene Fördereinrichtungen ausgeschüttet, welche die Mittel im Rahmen ihrer Förderrichtlinien an einzelne Förderungswerber weitergeben. 
formationstechnologien (Internet, e-Commerce, mobile Anwendungen, Finanztechnologie) beteiligt; 2) APEX Ventures EuVECA GmbH \& Co KG, die in technologiebasierte Unternehmen aller Branchen investiert; sowie 3) SHS IV Medtech Investments GmbH \& Co. KG ("SHS IV"), die in wachstumsstarke Unternehmen aus den Bereichen Medizintechnik, Diagnostik, angewandte Technologien und industrielle Biotechnologie im deutschsprachigen Raum investiert. Als Vorbild kann der 2011 gegründete Fonds Speedinvest I gesehen werden, der als sehr erfolgreich gilt und an dem die öffentliche Hand mit 28 Prozent als Leitinvestor beteiligt ist; darüber hinaus sind private Investorinnen und Investoren sowie die Mitarbeiterinnen und Mitarbeiter an diesem Fonds beteiligt. Dieser Fonds hatte einige sehr erfolgreiche „Exits" (d.h. gewinnbringende Verkäufe von Beteiligungen an Portfoliounternehmen) und konnte daher in seiner zweiten Auflage ein Mehrfaches an privatem Risikokapital mobilisieren.

Zu den Eigenkapitalinitiativen der aws zählt auBerdem der „aws Business Angel Fonds”, der als KoInvestitionsprogramm Direktbeteiligungen von Business Angels verdoppelt und der in Österreich seit 2013 als Teil der Initiative „European Angels Fund“ des Europäischen Investitionsfonds (EIF) besteht. Hier war Österreich eines der ersten Länder, die eine derartige Kooperation mit dem EIF eingegangen sind. Ein weiteres Programm der Eigenkapitalinitiative ist der „aws Gründerfonds“, der sich als Risikokapitalfonds an österreichischen Unternehmen in ihrer Gründungs- und Wachstumsphase beteiligt. ${ }^{180}$ Der "aws Mittelstandsfonds" schließt zwar Investitionen in Start-ups explizit aus, finanziert allerdings Wachstumsprojekte (z.B. zur Entwicklung neuer Produkte oder Verfahren, Vertriebsaufbau und MarkterschlieBung u.a.), Anschlussfinanzierungen und Ko-Investments mit in- und ausländischen Partnern. Im Rahmen der "Cleantech-Initiative" ist die aws bei einer
Schweizer Beteiligungsgesellschaft beteiligt, die sich ihrerseits an Cleantech-Unternehmen in Österreich (im Bereich erneuerbare Energien, Energieeffizienz, Energiespeicherung etc.) beteiligt, mit dem Ziel, Wachstum und Expansion solcher Unternehmen zu fördern. Zusätzlich werden bereits seit 1997 über das Programm „i2 Business Angels" Start-ups an interessierte Investorinnen und Investoren vermittelt.

\section{Optimierung und Vervollständigung der bereits bestehenden Unterstützungsmaßnahmen}

für technologiebasierte und innovative Unternehmensgründungen, vor allem Maßnahmen für die Startphase (vgl. PreSeed, Seedfinancing, Business-Angels, Technologiemarketing etc.)

Die im vorangegangenen Abschnitt genannten Programme der aws sind auch in diesem Bereich relevant, zusätzlich fördert das Programm „aws JumpStart“ Inkubatoren und Akzeleratoren sowie von ihnen betreute Start-ups. Unterstützung zur Gründung von innovativen Unternehmen leistet auch die FFG mittels des Programms "Spin-off Fellowship“, das zum Ziel hat, an Hochschulen und Forschungseinrichtungen Impulse zu setzen, um die Zahl an Spin-offs zu erhöhen. Angestrebt wird die Verwertung geistigen Eigentums im Rahmen einer Unternehmensgründung zu einem sehr frühen Zeitpunkt. Sehr früh setzt auch das Förderprogramm „aws First" an. Ziel von „aws First“ ist es, den Unternehmergeist in Österreich zu fördern und junge Menschen bei der Entwicklung und Umsetzung innovativer Ideen zu unterstützen. ${ }^{181}$

Weitere Maßnahmen betreffen die empirische Erfassung der Start-ups. So wurde im Rahmen eines Workshops die Definition von innovativen, technologieorientierten und forschungsintensiven Gründungen 2014 festgelegt und diese mit der Novelle der Unternehmensdemografie-Statistikverordnung 2015 dann umgesetzt. Daran anschließend wurde 2018 der

180 Der aws Gründerfonds ist mit einem Fondsvolumen von 68,5 Mio. $€$ und einem Beteiligungsvolumen von $100.000 €$ bis 3 Mio. $€$ der vom Volumen her wichtigste Fonds. Er wurde im Forschungs- und Technologiebericht 2016 beschrieben und ist nicht zu verwechseln mit dem aws Mittelstandsfonds, der Investitionen in Start-ups explizit ausschließt.

181 Im Jahr 2018 wurden zwölf Teams mit insgesamt 42 Teilnehmenden im Alter von 18 bis 26 Jahren im aws First Programm gefördert. Davon haben bereits drei Teams mit ihrer Projektidee ein Unternehmen gegründet. 
erste „Austrian Startup Monitor" veröffentlicht, der die empirischen Fakten zusammenfasst. ${ }^{182}$ Der Gründerpreis "Phönix“ zeichnet die besten Start-ups, Spin-offs und Prototypen in fünf Kategorien aus. Besonderes Augenmerk wird dabei auch auf Frauen in Start-ups und als Gründerinnen gelegt.

So wurde im Jahre 2018 über JumpStart auch ein von einem weiblichen Team geführter Inkubator namens „Female Founders“ gefördert. Dieser Inkubator richtet sich speziell an Gründerinnen und hat das Ziel, Frauen in der Gründerszene verstärkt zu verankern. Tatsächlich ist der Anteil der Frauen unter den Gründern sehr gering, und es lässt sich empirisch zeigen, dass ein höherer weiblicher Anteil im Management die Chance eines Unternehmens reduziert, risikofinanziert zu sein. ${ }^{183}$

Insgesamt zeichnet sich somit für Österreich ein Bild, in dem eine lebendige und über mannigfaltige Initiativen geförderte Start-up-Szene mit einer Finanzierungslücke konfrontiert ist. Das bedeutet, dass aufgrund von Kapitalmangel eine unbekannte Zahl potenziell ertragreicher Projekte nicht realisiert wird. Der „aws Business Angel Fonds” und der „aws Gründerfonds" sind zwei Beispiele, wie durch staatliche Initiative ein Mix aus öffentlichen und privaten Mitteln diese Lücke zumindest teilweise füllt. Der aws Business Angel Fonds hat zur Professionalisierung der österreichischen Business-Angels-Szene beigetragen, indem er sich gezielt an Business Angels richtet, welche Erfahrung und Expertise im entsprechenden Investitionsgebiet vorweisen können. Private Mittel werden vom Staat verdoppelt, was in der Praxis bedeutet, dass sich der Staat bei seinen eigenen Investitionen auf das Urteil Privater verlässt. Der aws Gründerfonds hingegen stellt ein Beispiel eines professionell verwalteten Risikokapitalfonds dar, der ganz überwiegend durch staatliche Mittel finanziert wird, jedoch nach den gängigen, marktorientierten
Prinzipien vergleichbarer privater Fonds betrieben wird. ${ }^{184}$ Und dies mit Erfolg: Der aws Gründerfonds weist eine hohe Mobilisierung von privatem Risikokapital auf Unternehmensebene auf, so können 1 Mio. $€$ aus dem aws Gründerfonds rd. 4,4 Mio. € private Mittel mobilisieren.

Zusammenfassend kann konstatiert werden, dass das Angebot an Wagniskapital, das in Österreich zur Verfügung steht, noch nicht ausreicht, um die entsprechende Nachfrage zu decken. Immerhin zeigt Österreich aber im Gegensatz zu den Innovation Leader-Ländern beim Wagniskapitalvolumen, das in heimische Unternehmen investiert wird, für den Beobachtungszeitraum 2007-2017 keine fallende Tendenz. Österreich liegt in diesem Bereich allerdings immer noch zurück. Wie Erfahrungen in Ländern, die im Bereich der Wagnisfinanzierung führend sind, zeigen, stehen gezielte staatliche Investitionen zumeist am Beginn eines lebendigen Start-up-Umfelds. Durch die öffentlichen Fonds (aws Gründerfonds, aws Business Angels Fonds) sowie durch die Unterstützung privater (nationaler und internationaler) Fonds im Rahmen der Venture-Capital-Initiative der aws gelingt es, den Risikokapitalmarkt zumindest zu mobilisieren, wobei es in Zukunft gilt, hier noch mehr Potentiale aufzubauen bzw. auszuschöpfen.

\subsubsection{Verbesserung von wettbewerblichen \\ Rahmenbedingungen}

Es ist allgemein unbestritten, dass Wettbewerb positiv auf Innovation wirkt. ${ }^{185}$ Wie Untersuchungen zeigen, wirkt Wettbewerbspolitik hierbei vor allem auf die Phase der Kommerzialisierung von Innovationen und deren Verbreitung. Dabei kommt der Wettbewerbspolitik auch die Rolle als wichtiges Korrektiv und die Funktion eines Gegengewichtes im Verhältnis zu anderen Instrumenten der FTI-Politik zu. Insbe-

$182 \mathrm{Vgl}$. Leitner et al. (2018).

$183 \mathrm{Vgl}$. Sardadvar (2018).

184 Der aws Gründerfonds ist zu 65 Mio. $€$ aus Bundesmitteln und zu 3,5 Mio. $€$ aus privaten Mitteln (Erste Bank) dotiert. Der aws Gründerfonds investiert zumeist gemeinsam mit anderen (privaten) Investorinnen und Investoren in Start-ups.

$185 \mathrm{Vgl}$. Shapiro (2002). 
sondere betrifft dies das System geistigen Eigentums, das über temporäre Monopole und damit über eine Wettbewerbsminderung, Innovationsanreize setzt. Aber auch der Themenkomplex Normen und Normungsprozesse muss hier angeführt werden. Dieser wirkt grundsätzlich ebenfalls innovationsstimulierend, kann aber auch indirekt dazu missbraucht werden, Kartelle zu schaffen und damit Marktzugänge (und Innovation) zu blockieren. Schließlich sind direkte Wirkungen auf Innovation zu erwarten, da erfolgreiche Wettbewerbspolitik neben der Sicherstellung angemessener Preise auch auf die Verbesserung der Qualität der Güter und damit die Forcierung von Innovation abstellt. Deshalb ist eine funktionierende Wettbewerbspolitik mit entsprechend vorhandenen schlagkräftigen Institutionen, die Wettbewerb sicherstellen, auch notwendig. ${ }^{186}$

Unter dem Ziel "Verbesserung von wettbewerbli-

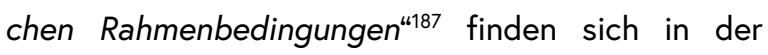
FTI-Strategie in der Folge vier Maßnahmen ${ }^{188}$, welche die Wettbewerbspolitik betreffen:

- Die Maßnahme „Abbau administrativer Hürden in den Bereichen der Unternehmensgründung und Dienstleistungsregulierung" stellt vor allem auf wettbewerbserhöhende Veränderungen der Rahmenbedingungen $a b$, insbesondere hinsichtlich erleichterter Möglichkeiten, neue Unternehmen zu gründen.

- Die weiteren drei Maßnahmen befassen sich mit institutionellen Aspekten, konkret mit der Organisation und Ausgestaltung der Bundeswettbewerbsbehörde (,Reform der Bundeswettbewerbsbehörde (Aufgaben, Befugnisse, Ressourcen)", „Durchführung von sektorspezifischen Analysen
(z.B. Treibstoffmarkt, Lebensmittel)" sowie „Überprüfung der wettbewerbspolitischen Regeln im Hinblick auf Innovationshemmnisse").

\section{Abbau administrativer Hürden in den \\ Bereichen der Unternehmensgründung und Dienstleistungsregulierung}

Den Zielen der FTI-Strategie entsprechend sollen durch unterschiedliche Maßnahmen u.a. die Gründung von Unternehmen wesentlich erleichtert und die entsprechenden finanziellen Belastungen verringert werden. Zentrale Elemente hierbei sind die Anforderungen des Gründungsprozesses selbst sowie die Frage nach den Erfordernissen der Gewerbeanmeldung.

\section{Unternehmensserviceportal (USP)}

In 2017 und 2018 erfolgte bereits die Umsetzung von mehreren Erleichterungen für Gründerinnen und Gründer im Sinne des Bürokratieabbaus. Die Unternehmensgründung für Einzelunternehmerinnen und -unternehmer über das Unternehmensserviceportal (USP) ist bereits seit 31.07.2017 möglich, seit 01.01.2018 existiert diese Möglichkeit auch für EinPersonen-GmbHs. Von der Gewerbeanmeldung über die Finanzamtsmeldung bis hin zur Sozialversicherungsmeldung kann damit der gesamte Gründungsprozess elektronisch durchgeführt werden. Für $\mathrm{GmbHs}$ mit einem einzigen Gesellschafter-Geschäftsführer ist auch vorgesehen, dass die Erklärung über die Errichtung der Gesellschaft und die Anmeldung zur Eintragung in das Firmenbuch ebenfalls über das USP abgegeben werden können. ${ }^{189}$ Die Mehrheit aller Unternehmensgründungen, die in Ös-

186 Gleichwohl ist festzuhalten, dass zentrale innovationsrelevante Agenden der Wettbewerbspolitik auf EU-Ebene bzw. bei der EU-Kommission verankert sind. So kann die EU-Kommission gemäß Art. 102 TFEU das Verhalten von Unternehmen regulieren, die ihre Marktmacht missbrauchen, oder bei marktverzerrenden Strukturen und Konzentrationen, die sich z.B. aus bestimmten Unternehmenszusammenschlüssen ergeben und eine EU-übergreifende Dimension haben (Regulierung EC 139/2004), direkt eingreifen. Insgesamt besteht in diesem Themenkomplex eine weiterreichende Arbeitsteilung zwischen der Europäischen Kommission und nationalen Wettbewerbsbehörden im Rahmen des Netzwerks der europäischen Wettbewerbsbehörden (European Competition Network, ECN).

$187 \mathrm{Vgl}$. BKA et al. (2011, 31).

188 Ebenda.

189 Vgl. https://www.usp.gv.at/Portal.Node/usp/public/content/gruendung/egruendung/269403.html 
terreich als Ein-Personen-Unternehmen gegründet werden, dürfte davon profitieren. Teamgründungen (als besonders wachstumsstarke Unternehmen und Gründungen, die für Investorinnen und Investoren deutlich attraktiver sind) sind derzeit davon ausgenommen. Die USP-Gründung für Ein-Personen-GmbHs ist derzeit aber auch nur möglich, wenn diese mit der Mindestsatzung auskommen. Für innovative Unternehmensgründungen, selbst Ein-Personen-Unternehmen, existieren hingegen zu viele Bedarfe an Anpassungen der Mindestsatzung, um davon profitieren zu können. Weiterhin führen Anpassungen an einer ursprünglich genutzten Mindestsatzung, etwa aufgrund von Intellectual Property Rights (IPR) oder der Aufnahme weiterer Gesellschafter dazu, dass die Gründerin bzw. der Gründer nochmals den gesamten ursprünglichen Weg durchlaufen muss.

\section{Änderungen gesetzlicher Rahmenbedingungen}

Auch die Gewerbeordnung ist in den letzten Jahren in mehrfacher Hinsicht überarbeitet worden. Nicht zuletzt auf der Basis des entsprechenden Ministerratsvortrags vom Juli 2016 betreffend die „Modernisierung der Gewerbeordnung und Erleichterungen im gewerblichen Betriebsanlagenrecht" und in Folge durch den parlamentarischen Beschluss zur Änderung der Gewerbeordnung am 05.07.2017 hat es zahlreiche Veränderungen diesbezüglich ${ }^{190}$ gegeben, die auf Entlastungen der Unternehmen abzielten. Dazu zählen u.a. die Freistellung des Gewerbeverfahrens von Gebühren und Verwaltungsabgaben des Bundes, insbesondere für Gewerbeanmeldungen und sämtliche derzeit noch gebühren- und abgabepflichtigen Verfahren im Berufsrecht und die Etablierung des Grundsatzes „Beraten statt Strafen“ im Betriebsanlagenrecht.

Für die Modernisierung der Gewerbeordnung zentral war die Einführung der Gewerbelizenz mit
01.05.2018. Zusätzlich zur Gewerbeberechtigung (Recht, ein Gewerbe auszuüben) wurde damit eine personenbezogene Gewerbelizenz (Recht, eine gewerbliche Tätigkeit auszuüben) geschaffen. ${ }^{191} \mathrm{Die} \mathrm{Ge}-$ werbelizenz umfasst sämtliche Gewerbe einschließlich der Nebenrechte. Die erste Gewerbeberechtigung ist immer anzumelden (unabhängig davon, ob reglementiertes oder freies Gewerbe). Liegt bereits eine Gewerbelizenz vor, müssen weitere reglementierte Gewerbe wie bisher angemeldet, weitere freie Gewerbe lediglich angezeigt werden. Die Erweiterung der Nebenrechte hat ebenfalls dazu geführt, dass unter bestimmten Bedingungen keine gesonderte Anmeldung oder Anzeige nötig ist.

Ein derzeit noch ungelöstes Problem besteht in der unklaren Datenbasis für eine Einschätzung der Dauer entsprechender Verfahren. Mittlerweile wurde hierfür ein Verfahrensmonitoring geschaffen, das zwischen städtischen Agglomerationen und ländlichen Gebieten unterscheidet.

Unter bestimmten Bedingungen können Gründerinnen und Gründer Abgabenbefreiungen auf Basis des Neugründungs-Förderungsgesetzes (NeuFöG ${ }^{192}$ ) in Anspruch nehmen, das sind im Wesentlichen der Wegfall von Stempelgebühren und Bundesverwaltungsabgaben, der Grunderwerbsteuer für die Einbringung von Grundstücken auf gesellschaftsvertraglicher Basis, der Gerichtsgebühren für die Eintragungen in das Firmenbuch usw.. Außerdem ist die Mindeststeuer im Rahmen der Körperschaftsteuer bei neu gegründeten Gesellschaften mit beschränkter Haftung $(\mathrm{GmbH})$ in den ersten zehn Jahren reduziert.

Eine der Voraussetzungen der Eintragung einer Gesellschaft mit beschränkter Haftung in das Firmenbuch ist der Abschluss eines schriftlichen Gesellschaftsvertrags ${ }^{193}$, was der Form des Notariatsakts bedarf. Im Gründungsprozess wird der zeitliche

190 Vgl. https://www.help.gv.at/Portal.Node/hlpd/public/content/171/Seite.1710834.html

191 Vgl. Gewerbeordnung 1994 - GewO 1994 § 38.

192 Vgl. Bundesgesetz, mit dem die Neugründung von Betrieben und die Übertragung von Klein- und Mittelbetrieben gefördert wird (Neugründungs-Förderungsgesetz - NeuFöG).

193 Vgl. Gesetz vom 6. März 1906, über Gesellschaften mit beschränkter Haftung (GmbH-Gesetz - GmbHG) § 4 Abs. 3. 
und finanzielle Aufwand für die gesetzlich vorgeschriebene Involvierung von Notaren vielfach als unverhältnismäßig hoch eingeschätzt. Um den zeitlichen Aufwand und die finanziellen Kosten zu senken, wird von Expertinnen und Experten gefordert, die Erfordernisse des zwingenden Notariatsakts sowie der notariellen Beurkundung aus dem Gesellschaftsrecht zu entfernen oder zumindest stark einzuschränken. ${ }^{194}$ Zumindest die Art der Abwicklung wird mit dem ab 01.01.2019 geltenden Elektronischen Notariatsform-Gründungsgesetz ${ }^{195}$ ergänzt bzw. dahingehend erweitert, dass elektronische Kommunikationsmöglichkeiten persönliche Termine ersetzen können.

\section{Wettbewerbsrecht und}

\section{Bundeswettbewerbsbehörde}

In Bezug auf die Bundeswettbewerbsbehörde (BWB) ist festzuhalten, dass eine zentrale Zielsetzung der FTI-Strategie die Reform der BWB war. Diese Maßnahme wurde mit der Reform des Wettbewerbsrechtes vom 01.03.2013 umgesetzt. Die BWB - die erst 2002 im Rahmen einer umfassenden institutionellen Reform aus der Taufe gehoben wurde - erhielt in der Folge weitere Ermittlungsbefugnisse, die auch in Bezug zu Änderungen im materiellen Recht zu stellen sind (Stichwort: kollektive Marktbeherrschung oder Bestimmungen für Private Enforcement). Gleichwohl wurden auch Änderungen in der Organisationsstruktur angedacht. Seit August 2017 bestehen zwei Abteilungen - eine Rechtsabteilung und eine Prozessabteilung -, womit eine entsprechende langjährige Forderung hinsichtlich der Schaffung moderner Organisationsstrukturen und Abteilungen aufgegriffen wurde. ${ }^{196}$

Seit Bestehen der BWB wurden (Stand Ende 2017) mehr als 140 Hausdurchsuchungen durchgeführt, mehr als 4.600 nationale Unternehmenszusammen- schlüsse geprüft, 536 Kartellfälle bearbeitet sowie 196 Mio. $€$ an Geldbußen verhängt. ${ }^{197}$ Diesen Einnahmen sind Kosten von akkumuliert 33 Mio. $€$ gegenüberzustellen. Die BWB ist von zunächst 17 auf 40 Mitarbeiterinnen und Mitarbeiter gewachsen (Stand Ende 2017).

Die in der FTI-Strategie postulierte Maßnahme zu sektorspezifischen Analysen wurde ebenfalls (und wird laufend nach Bedarf) umgesetzt. Hier sieht das Wettbewerbsgesetz die Möglichkeit einer allgemeinen Untersuchung eines Wirtschaftszweiges durch die BWB vor, sofern die Umstände vermuten lassen, dass der Wettbewerb in dem betreffenden Wirtschaftszweig eingeschränkt oder verfälscht ist. Bislang wurden sieben derartige Analysen durchgeführt. ${ }^{198}$

Hinsichtlich der in der FTI-Strategie angeführten Maßnahme bezüglich Überprüfung der wettbewerbspolitischen Regeln in Hinblick auf Innovationshindernisse ist zunächst festzuhalten, dass eine derartige Überprüfung laufend im Rahmen von Begutachtungsverfahren erfolgt, auch vor dem zuvor erwähnten allgemeinen Ziel qualitativ hochwertig(er)e Güter zu erhalten (was vielfach vorgelagerte Innovationsaktivitäten erfordert). Weitere Verbesserungen beim materiellen Recht, z.B. hinsichtlich kollektiver Marktbeherrschung, also der Situation, wo mehrere Unternehmen marktbeherrschend sind, selbst wenn jedes für sich nicht der Definition der Einzelmarktbeherrschung genügt, haben auch für Start-Ups und „Newcomer" Relevanz, da damit der Markteintritt erleichtert wird.

Somit können in Summe jene Punkte, die sich mit der BWB im Rahmen der FTI-Strategie beschäftigen, als abgeschlossen bzw. als laufend umgesetzt betrachtet werden. Gleichwohl besteht z.B. hinsichtlich der Frage weiterer Reformen - z.B. hinsichtlich der Zusammenlegung der Kompetenzen von Bundes-

194 Vgl. Ruhland et al. (2017).

195 Bundesgesetz, mit dem das GmbH-Gesetz und die Notariatsordnung geändert werden (Elektronische Notariatsform-Gründungsgesetz - ENG).

$196 \mathrm{Vgl}$. Bundeswettbewerbsbehörde (2018).

197 Ebenda.

198 Bankomatgebühren, Strom und Gas, Treibstoff, Lebensmittel, Zement und Beton, Mobilfunk, Apothekenmarkt (Quelle: https:// www.bwb.gv.at/branchenuntersuchungen/). 
wettbewerbsbehörde und des Kartellanwalts ${ }^{199}$ Handlungsbedarf, was auch im aktuellen Regierungsprogramm aufgegriffen wird.

\subsection{Verbesserungen in der Governance und strategische Maßnahmen}

FTI-Strategie 2020 mit der Vision:

"Lenkung geben, Rahmen setzen - Die politische Steuerung effizient organisieren"

Effektivität und Intelligenz eines Systems sind eng mit der Governance desselbigen verknüpft. Das Thema Bildung, Wissenschaft, Technologie und Innovation ist in den vergangenen Jahren immer breiter und komplexer geworden. Immer mehr verantwortliche Stellen sind zwecks Koordination eingerichtet bzw. immer mehr Stakeholder und Akteure zu diesem Thema hinzugezogen worden. Gute Abstimmung und das Vermeiden von Doppelgleisigkeiten bei klaren Zielvorgaben - unter Wahrung von Unabhängigkeit - sind damit die großen strategischen Ziele.

\subsubsection{Verbesserung von Governance-Strukturen} Im Bereich der Governance-Strukturen setzte sich die FTI-Strategie 2020 vier zentrale Zielvorgaben, nämlich eine klarere Abstimmung der Kompetenzen der verantwortlichen Ministerien durch effiziente Koordinationsmechanismen, Optimierung der „Aufgabenverteilung zwischen Ressorts und Förderungsagenturen [...] durch höhere operative Unabhängigkeit der Agenturen bei gleichzeitig verstärkter strategischer Steuerung", Bereinigung von Doppelgleisigkeiten auf Ebene der Förderagenturen und Steigerung der „Systemeffektivität und Systemintel- ligenz $[\ldots]$ durch vermehrte Ziel- und Outputsteuerung" ${ }^{* 200}$.

Das österreichische FTI-System weist aufgrund seiner historischen Entwicklung eine beträchtliche Anzahl von Akteuren auf, die in vielfältigen Wechselwirkungen miteinander stehen. Zugleich erfordern die aktuellen wirtschaftlichen und gesellschaftlichen Entwicklungen zunehmend Handlungsfelder übergreifende und abgestimmte Konzepte zur Erreichung der Ziele der FTI-Strategie. Die zugrundeliegenden Analysen ${ }^{201}$ der FTI-Strategie haben im Governance System einige Schwächen identifiziert, deren Ursachen in den "versäulten" und "fragmentierten Strukturen" gesehen werden.

Derzeit sind für die Bereiche der FTI-Politik verschiedene Bundesministerien zuständig. Es sind dies die drei Fachressorts Bundesministerium für Bildung, Wissenschaft und Forschung (BMBWF), Bundesministerium für Verkehr, Innovation und Technologie (BMVIT) und Bundesministerium für Digitalisierung und Wirtschaftsstandort (BMDW) ${ }^{202}$, sowie das Bundesministerium für Finanzen (BMF). Dem Bundeskanzleramt (BKA) kommt eine koordinierende Funktion in diesem Bereich zu.

Der Rat für Forschung und Technologieentwicklung (RFTE) ist das zentrale Beratungsgremium der Bundesregierung im Bereich der FTI-Politik. Der RFTE unterbreitet Vorschläge für die mittel- bis langfristige strategische Ausrichtung der FTI-Politik und gibt Einzelempfehlung und Stellungnahmen zu (laufenden) Initiativen und Maßnahmen ab. Der Österreichische Wissenschaftsrat berät den/die Bundesminister/in für Bildung, Wissenschaft und Forschung, Parlament und Universitäten in Fragen der Wissenschaftspolitik und Kunst. Das ERA Council Forum berät den/die zuständige/n Bundesminister/in in Fragen des Europäischen Forschungsraums und zu Horizon 2020. Ge-

199 Vgl. Böheim (2008).

200 Vgl. BKA et al. $(2011,34)$.

$201 \mathrm{Vgl}$. BKA et al. (2011, 32).

202 In der abgelaufenen Legislaturperiode vereinte das BM für Wissenschaft, Forschung und Wirtschaft (BMWFW) die Kompetenzen für Wissenschaft und angewandte Wirtschaftsforschung. Allerdings waren die beiden Politikbereiche in getrennten Verwaltungsbereichen innerhalb des Ressorts angesiedelt. 
mäß Regierungsprogramm 2017-2022 wird eine Zusammenführung dieser drei Räte angestrebt. ${ }^{203}$

Die Umsetzung der verschiedenen FTI-Politiken seitens der Ressorts erfolgt durch eine Reihe von Agenturen (wie z.B. aws, FFG, OeAD), Vereinen (z.B. $C D G$ ) und Förderungsfonds (Wissenschaftsfonds, Klima und Energiefonds, usw.) sowie durch Finanzierungsinstrumente wie Nationalstiftung und Österreich Fonds. Die im Laufe der Zeit geschaffenen Organisationen haben die Effektivität der FTI-Politik zweifellos erhöht, stellen gleichzeitig aber auch erhöhte Anforderungen an die strategische und operationale Steuerungsfähigkeit des Systems. In diesem Zusammenhang kommt den Bundesministerien einerseits die Rolle des Eigentümers bzw. der Aufsichtsbehörde dieser Institutionen zu, andererseits nehmen die Ministerien aber auch die Rolle als Auftraggeber der jeweiligen Forschungs- und Technologieprogramme wahr; bei der Nationalstiftung sind die Bundesministerien im Stiftungsrat vertreten.

Vor diesem Hintergrund wird in der FTI-Strategie daher auch explizit auf das Ziel einer besseren Arbeitsteilung zwischen Ressorts und Agenturen verwiesen. Als problematisch wird gesehen, dass die Agenturen die Aufgaben der Strategieentwicklung und des Agenda Settings in zu großem Umfang wahrnehmen, die zuständigen Ressorts hier zu wenig präsent sind; im Gegensatz dazu die Ressorts in einem zu hohen Ausmaß in der Detailsteuerung der Maßnahmenumsetzung involviert sind. Letzteres wirkt sich insbesondere auf den administrativen Aufwand negativ aus; alles in allem schmälert eine solche Governance die Effizienz des Systems.

Vor diesem Hintergrund wurden in der FTI-Strategie folgende Maßnahmen ${ }^{204}$ gesetzt:

- Einrichtung einer Task Force Forschung, Technologie und Innovation auf hoher Verantwortungsebene mit folgenden Aufgaben: Begleitung, Konkreti- sierung und Koordination der Umsetzung der FTIStrategie; strategische und systemorientierte Abstimmung und Koordination der Aktivitäten der einzelnen Ressorts; Behandlung der Empfehlungen des Rates für Forschung und Technologieentwicklung

- Der Rat für Forschung und Technologieentwicklung ist das strategische Beratungsorgan der Bundesregierung in Fragen der FTI-Politik. Er erarbeitet dazu im engen Dialog mit den Ressorts Empfehlungen für die mittel- und langfristige Ausrichtung dieses Politikfeldes. Dazu gibt sich der Rat für Forschung und Technologieentwicklung ein jährliches Arbeitsprogramm.

- Strategische Steuerung der Förderungsagenturen durch Leistungsvereinbarungen auf Basis von Output- und Impact-Zielvorgaben

- Die Erhöhung der Systemeffizienz beim Klima- und Energiefonds (KLIEN) durch verstärkte Nutzung von Synergien und Weiterentwicklung des vorhandenen Instrumentenportfolios

\section{Einrichtung einer Task Force Forschung, Technologie und Innovation}

Diese Maßnahme hat sich mittlerweile erfolgreich etabliert. Die Task Force tagt großteils auf Ebene der Sektionsleitungen mehrmals jährlich und gewährleistet einen transparenten und reibungslosen Austausch der Information über die Aktivitäten der jeweiligen Ressorts. Die Task Force hat sich zudem als geeignetes Instrument der strategischen Steuerung erwiesen. So werden über dieses Gremium aktuell die konzeptiven Arbeiten für die Erstellung und Umsetzung der FTI Strategie 2030 durchgeführt. Die FTI Task Force erfüllt somit die Aufgabe der Koordinierung der FTI-Politik auf Ebene des Bundes sehr gut. 
Jährliches Arbeitsprogramm des Rates für

Forschung und Technologieentwicklung

als strategisches Beratungsorgan der

Bundesregierung in Fragen der FTI-Politik

Auch diese Maßnahme wurde im Rahmen der Implementierung der FTI-Strategie erfolgreich umgesetzt.

Es erfolgen derzeit laufende Diskussionen sowie ein Austausch sowohl in den Ratssitzungen als auch anlassbezogen bei den gemeinsamen Besprechungen der Task Force mit Mitgliedern des RFTE. Mit Hilfe dieser Aktivitäten wird dem hohen Koordinierungsbedarf und der Notwendigkeit einer konsistenten strategischen Planung dieses Politikbereichs Rechnung getragen. Ein zentrales Element stellt in diesem Zusammenhang der Bericht zur wissenschaftlichen und technologischen Leistungsfähigkeit Österreichs dar. Dieser beschreibt jährlich anhand von Indikatoren den Fortschritt Österreichs in Bezug auf die FTIStrategie der Bundesregierung und bildet damit eine wesentliche Grundlage für die laufende Beurteilung gesetzter Maßnahmen bzw. gegebenenfalls für erforderliche Adaptionen.

Von der Beschleunigung des technologischen Wandels in der jüngeren Vergangenheit besonders betroffen sind die Bereiche Digitalisierung, Künstliche Intelligenz und Robotik. Als Reaktion auf diese rasante Entwicklung wurde vom BMVIT der österreichische Rat für Robotik und Künstliche Intelligenz (KI) im Jahr 2017 eingerichtet. Der Rat erstellt laufend Stellungnahmen zu Fragen von Robotik und Künstlicher Intelligenz durch ein interdisziplinäres Gremium von Expertinnen und Experten, welche eine technologische, wirtschaftliche, gesellschaftliche und rechtliche Betrachtung einnehmen können. Damit gehen auch laufend Empfehlungen und Vorschläge für geeignete Implementierungsmaßnahmen diesen Themenkomplex betreffend einher.

Die Einrichtung des Rates für Robotik und KI kann auch als ein Beispiel für die gelungene zeitnahe Anpassung der österreichischen FTI-Strategie an aktu- elle technologische und gesellschaftliche Trends angesehen werden.

\section{Strategische Steuerung der \\ Förderungsagenturen durch}

Leistungsvereinbarungen auf Basis von Outputund Impact-Zielvorgaben

Im Bereich der Governance der Förderungsagenturen besteht trotz substantieller Fortschritte jedoch noch weiterer Handlungsbedarf. Mit der Forschungsförderungsgesellschaft mbH (FFG) und der Austria Wirtschaftsservice $\mathrm{GmbH}$ (aws) verfügt die österreichische Bundesregierung über zwei Agenturen zur Implementierung und laufenden Abwicklung von Forschungs- und Wirtschaftsförderungsprogrammen öffentlicher Auftraggeber. FFG und aws entstanden durch Zusammenführung von vier bzw. drei Organisationseinheiten im Forschungs- und Wirtschaftsförderungsbereich im Jahr 2002. Mit der Konsolidierung der institutionellen Strukturen in diesem Bereich hat die FTI-Politik wesentlich an Schlagkraft gewonnen. Es sind durch diese Organisationsstruktur aber in der Folge auch spezifische Anforderungen an die Rollenverteilung und -wahrnehmung von politischer Führung, und der Arbeitsteilung zwischen Bundesministerien und den Agenturen entstanden. ${ }^{205}$

Im Zuge der Umsetzung der FTI-Strategie wurde von den zuständigen Eigentümerressorts von aws und FFG, dem BMVIT und dem BMWFW (nunmehr BMDW), die Steuerungsvorgaben an die FFG vereinheitlicht. Dies erfolgte durch ein Themenmanagement, das einheitliche Instrumente, Ausschreibungen und Verfahren definiert.

Die strategischen Vorgaben für FFG und aws wurden aus der FTI-Strategie abgeleitet und werden im jeweiligen Mehrjahres- und Arbeitsprogramm der Agenturen konkret ausformuliert. Sowohl für FFG und aws bestehen mittlerweile mehrjährige Rahmenverträge, die zur verbesserten strategischen Steuerung beitragen. 
Ein weiterer Aspekt der verbesserten strategischen Steuerung stellt deren Einbettung in die im Haushaltsrecht verankerte Wirkungsorientierte Folgenabschätzung (WFA) dar. Mittlerweile sind die Indikatoren auf Ebene der Programmdokumente für aws und FFG von den Eigentümer- und Auftraggeberressorts mit dem Bundesministerium für Finanzen akkordiert.

Eine entsprechende mehrjährige Planung über ein Mehrjahresprogramm und die Umsetzung über ein jährliches Arbeitsprogramm, das mit der WFA abgestimmt ist, wurde auch bei der Österreichischen Austauschdienst $\mathrm{GmbH}(\mathrm{OeAD})$ vom zuständigen BMBWF verwirklicht. Ebenso erfolgt die Steuerung des FWF durch das BMBWF über ein Mehrjahresprogramm und über die Genehmigung der jährlichen Arbeitsprogramme.

Zwecks Identifikation weiterer Verbesserungspotentiale in der Steuerung der Agenturen aws und FFG haben die Eigentümerresorts erst jüngst eine Evaluierung bei Fraunhofer und KMU Forschung in Auftrag gegeben. Die Ergebnisse der Studie 2017 bestätigen, dass die Agenturen aws und FFG hochprofessionell und effektiv agieren. Demnach wurden in den mittlerweile relativ großen Organisationseinheiten „zur Gewährleistung einer hohen Professionalität, Serviceorientierung, Effektivität und Effizienz zahlreiche Prozesse und Strukturen entwickelt und implementiert, die sich im Großen und Ganzen bewährt haben."206 Es wurden allerdings auch strukturelle Defizite im Bereich der Steuerung der Agenturen identifiziert. So halten die Studienautorinnen und -autoren fest, dass das GovernanceSystem der Agenturen von anhaltend hoher Komplexität geprägt ist. Als kritisch werden die hohe Anzahl von Ausführungsverträgen und das Fehlen eines einheitlichen, koordinierten Steuerungsverständnisses seitens der Eigentümer angesehen. Demnach haben die Agenturen auf die veränderten Rahmenbedingungen reagiert und an strategischer Intelligenz, Einfluss und Autonomie gewonnen; aus- geblieben sei aber die Entwicklung eines geeigneten Steuerungsverständnisses auf Ebene der Ressorts. In der Studie werden demnach drei Reformoptionen unterbreitet, wobei in der ambitionierten Variante der Übergang zu einer ergebnisorientierten Steuerung mittels Globalbudgets empfohlen wird. Damit soll mehr Entscheidungskompetenz und Verantwortung an die Agenturen übertragen werden, während sich die Ressorts verstärkt auf politisch strategische Aspekte, die Minimierung von Informationsasymmetrien sowie die Controllingfunktion fokussieren sollen.

Die Ergebnisse der Evaluierung stellen die Grundlage für einen Reformprozess der strategischen Steuerung der Agenturen durch die Ressorts dar. Dieser Reformprozess ist mit der Erstellung des Forschungsfinanzierungsgesetzes abgestimmt.

\section{Erhöhung der Systemeffizienz beim Klima- und Energiefonds (KLIEN) durch verstärkte Nutzung von Synergien und Weiterentwicklung des vorhandenen Instrumentenportfolios}

Die FTI-Förderungen des Klima- und Energiefonds wurden in das Themenmanagement des BMVIT integriert und werden von der FFG entsprechend umgesetzt. Auf Ebene der Instrumente werden nunmehr standardisierte Förderinstrumente und Prozesse angewendet, sodass der KLIEN im Rahmen von Abstimmungsgesprächen mit den Fachabteilungen des BMVIT und im Arbeitsprogramm der FFG integriert ist.

Insgesamt wurden im Bereich der Governance die Zielvorgaben der FTI-Strategie weitgehend erfüllt. Die Aufgabe der verbesserten Koordinierung der komplexen Strukturen der FTI-Landschaft in Österreich wurde mit getätigten Maßnahmen erfüllt. Im Bereich der Steuerung der Agenturen wurden die bestehenden Defizite adressiert, allerdings zeigt die aktuelle Evaluierung von aws und FFG, dass noch weiterer Handlungsbedarf besteht, um die Effektivität und Effizienz der Prozesse zu erhöhen. Die aktuellen Maßnahmen der Ressorts zielen auf Basis der 
vorliegenden Befunde darauf ab, diese bestehende Lücke zu schließen.

\subsubsection{Strategische, thematische Schwerpunktsetzungen}

Innovationssysteme unterscheiden sich unter anderem dadurch, inwieweit der Staat mit seinen Instrumenten auf die inhaltliche Ausrichtung der Innovationsaktivitäten Einfluss nimmt. Die beiden Idealtypen sind dabei einerseits ein liberaler, bottom-up-Ansatz, der Vorgaben inhaltlicher Natur ablehnt und alleine Marktsignale sowie wissenschaftliche Neugier als steuernde Instanz akzeptiert. Zum anderen ist ein interventionistischer top-down-Ansatz denkbar, der nicht nur thematische Vorgaben macht, sondern etwa auch konkrete Technologien als förderwürdig benennt. Inhaltlich eng definierte thematische Förderprogramme stellen hohe Anforderungen an das Wissen der Entscheidungsträger; dieses kann primär über einen breiten Dialog mit Gesellschaft, Wirtschaft und Wissenschaft erarbeitet werden.

In der innovationspolitischen Realität finden sich zahlreiche Mischformen dieser beiden Positionen, die darüber hinaus auch zwischen Technologiefeldern und Entwicklungsstand von Technologien variieren können. In letzter Zeit wird die thematische Orientierung der Innovationspolitik zunehmend bedeutsamer und unter den Stichwörtern "Grand Challenges" oder "Social Challenges" diskutiert. Eine Politik, die explizit die damit angesprochenen thematischen Herausforderungen aufgreift und mit entsprechenden Instrumenteneinsatz adressiert, wird als missionsorientierte Innovationspolitik bezeichnet. ${ }^{207}$ Letztlich soll diese dazu beitragen, dass innovationsgetriebene Volkswirtschaften nicht nur quantitativ expandieren, sondern sich auch qualitativ im Einklang mit gesellschaftlichen Zielen (wie sie etwa in den Sustainable Development Goals oder der Europa-2020-Strategie zum Ausdruck kommen) entwickeln.
Die thematische Orientierung von FTI-Politik in Österreich wird seit einigen Jahren auch durch die Smart Specialisation Strategie als Teil der EU 2020-Strategie mitbestimmt. Diese zielt wesentlich auf eine thematisch fokussierte und innovationsbasierte Regionalentwicklung entlang von regionalen Potentialen ab. Die Bundesländer haben hierzu unter Berücksichtigung der FTI-Strategie sowie in Abstimmung mit dem Bund regionale thematische Schwerpunkte definiert. ${ }^{208}$

Vor diesem Hintergrund definierte die FTI-Strategie des Bundes 2011 auch fünf Ziele hinsichtlich thematischer Schwerpunktsetzungen. Dabei wird zwischen "generischen Querschnittsfeldern" und "Grand Challenges" unterschieden; beispielhaft erwähnt die FTI-Strategie auch vier Querschnittsfelder, nämlich die Informations- und Kommunikationstechnologien (IKT), die Materialwissenschaften, die Life Sciences und die Geistes-, Sozial- und Kulturwissenschaften (GSK). Im Zuge der Arbeit am Konzept der Smart Specialisation wurde das Thema Bioökonomie und Nachhaltigkeit als weitere thematische Priorität festgelegt. 209 Unter "Global Challenges" subsummiert die Strategie die drei Herausforderungen Klimawandel, Umgang mit knappen Ressourcen und Sicherung der Lebensqualität angesichts des demographischen Wandels. Während die Ziele (2), (3) und (5) den Prozess zur Definition von thematischen Schwerpunkten betreffen, sind die Ziele (1) und (2) einer stärkeren thematischen Fokussierung unter Berücksichtigung ökonomischer Spezialisierungsmuster gewidmet. Die fünf Ziele demnach sind:

1. „...Österreichs Wettbewerbsfähigkeit in generischen Querschnittsfeldern der Wissenschaft und Technologie durch Fokussierung der Aktivitäten in international wettbewerbsfähigen Größeneinheiten stärken. Dabei ist auf die Stärkefelder der heimischen Wissenschaft und Wirtschaft Bezug zu nehmen. Kompetenzen und Potenziale österreichischer Unternehmen, die in der Umsetzung der For-

207 Vgl. Europäische Kommission (2017).

$208 \mathrm{Vgl}$. Schuch und Testa (2018)

209 Vgl. BMWFW und BMVIT (2016). 
schungsergebnisse zur Bewältigung der Grand Challenges beitragen können, sind besonders zu berücksichtigen.

2. Schwerpunktsetzungen in Forschung und Technologieentwicklung sollen auf der Basis von systematischen Auswahl- und Entscheidungsprozessen stattfinden. Dabei gilt es, auf ausreichende Begründung staatlicher Schwerpunktsetzung zu achten, um Markt- und Systemversagen zu verhindern.

3. Eine neue Schwerpunktdefinition für spezifische Herausforderungen soll zu einer konzertierten Abstimmung der Aktivitäten in einem systemumfassenden Einsatz aller betroffenen Ressorts im Rahmen der Task Force Forschung, Technologie und Innovation führen.

4. Systemumfassende Schwerpunkte sind insbesondere zur Adressierung großer gesellschaftlicher Herausforderungen der Zukunft (Grand Challenges) zu etablieren.

5. Die Definition von Schwerpunkten soll auf Basis vorlaufender Analysen erfolgen, befristete Wir- kung haben und einer begleitenden Überprüfung unterworfen werden. ${ }^{210}$

Die den Zielen zugeordneten Maßnahmen beziehen sich unmittelbar stärker auf jene Ziele, die sich mit dem Prozess der Erarbeitung von Schwerpunkten befassen (betrifft die Ziele (2), (3) und (5)). Die in Tabelle 3-7 dargestellte Übersicht ordnet den in der Strategie genannten allgemeinen Maßnahmen eine Auswahl konkreter Maßnahmen zu, die seit Erstellung der Strategie im Jahr 2011 von den zuständigen Ministerien gesetzt wurden. ${ }^{211}$

Darüber hinaus ist zu berücksichtigen, dass die Innovationspolitik des Bundes eine Reihe von thematischen Förderprogrammen betreibt, welche insbesondere die Ziele (1) und (4) adressieren. Von besonderer Bedeutung sind dabei die Thematischen Programme der FFG. Diese umfassen fünf Schwerpunkte (Energie, Stadt und Umwelt; IKT; Produktions-, Nanound Quantentechnologien; Mobilität; Sicherheit) und ein Modul zu transnationalen Initiativen und Calls. ${ }^{212}$ Für den Bereich Umwelt und Nachhaltigkeit spielt

Tabelle 3-7: Maßnahmen der FTI-Strategie zur Setzung strategischer, thematischer Schwerpunkte

\begin{tabular}{|c|c|}
\hline Allgemeine Maßnahmen It. FTI-Strategie & Konkrete Maßnahmen (Auswahl) \\
\hline $\begin{array}{l}\text { (1) Entwicklung nationaler Strategien für generische } \\
\text { Wissenschafts- und Technologiefelder }\end{array}$ & $\begin{array}{l}\text { - Neuausrichtung des IKT-F\&E-Portfolios entlang „IKT der Zukunft“: } \\
\text { Definition von vier IKT-Themenfeldern, die in gesellschaftlichen } \\
\text { Herausforderungen Lösungen bereitstellen können } \\
\text { - Schwerpunkt im Bereich IKT: Efit21, Dachmarke für ein Bündel von } \\
\text { IKT-Initiativen und Projekten im Bildungsbereich } \\
\text { - Erstellung einer Zukunftsstrategie Life Sciences und } \\
\text { Medizinstandort Österreich } \\
\text { - Erstellung einer Strategie zu Künstlicher Intelligenz }\end{array}$ \\
\hline $\begin{array}{l}\text { (2) Etablierung der Kategorien „Ressortübergreifende } \\
\text { Forschungs-, Technologie- und Innovationsschwerpunkte“ } \\
\text { und „Ressortschwerpunkte“ sowie Festlegung von } \\
\text { Mechanismen und Strukturen zu ihrer Implementierung und } \\
\text { Umsetzung }\end{array}$ & $\begin{array}{l}\text { - Ressortschwerpunkte BMDW: Smart and Digital Services, } \\
\text { Biotechnologie, Industrie 4.0, Digitale Wirtschaft/IKT, } \\
\text { Digitalisierung } \\
\text { - Ressortschwerpunkte BMVIT: Energie- und Umwelttechnologien, } \\
\text { Raumfahrttechnologie, Sicherheitstechnologie, Mobilität, } \\
\text { Nanotechnologie }\end{array}$ \\
\hline
\end{tabular}

210 Vgl. BKA et al. $(2011,37)$

211 Ebenda.

212 Vgl. https://www.ffg.at/thematische-programme 
der Klima- und Energiefonds, finanziert durch das Bundesministeriums für Nachhaltigkeit und Tourismus und das Bundesministerium für Verkehr, Innovation und Technologie eine zentrale Rolle. ${ }^{213}$

\section{Zur Zielerreichung der Steigerung der Wettbewerbsfähigkeit in ausgewählten}

Technologiesegmenten bzw. der Etablierung systemumfassender Schwerpunkte

Ziel (1) und Ziel (4) über die Steigerung der Wettbewerbsfähigkeit in ausgewählten Technologiesegmenten bzw. die Etablierung systemumfassender Schwerpunkte sollen im Folgenden anhand ausgewählter Bereiche mittels quantitativer Kennzahlen zur relativen Spezialisierung überprüft werden. Liegt der auf Basis von Patentdaten berechnete Wert der Kennzahl „Revealed Technological Advantage“ (RTA) unter eins, so weist das Land keine Spezialisierung auf; bei einem Wert über eins gilt das Land als im betreffenden Bereich spezialisiert. Tabelle 3-8 zeigt den RTA für vier verschiedene Technologiefelder, die mit Ausnahme der hier fehlenden Geistes-, Sozial- und Kulturwissenschaften (GSK) in etwa den "Generischen Querschnittsfeldern" der FTI-Strategie entsprechen.

Österreich verfügt im Sinne des RTA nur bei Umwelttechnologien über eine Spezialisierung. Darüber hinaus lässt sich feststellen, dass nur bei IKT durch eine Steigerung des RTA-Wertes von 0,47 (2002-
2005) auf 0,54 (2012-2015) eine Zunahme der Spezialisierung erzielt werden konnte. Vergleicht man die Werte Österreichs für die Jahre 2012-2015 mit jenen der Vergleichsländer, so ergibt sich ein relativ klares Bild: Österreich ist in Summe deutlich weniger spezialisiert in den vier in der FTI-Strategie genannten Technologiefeldern. Bei Umwelttechnologien liegen etwa nur die Niederlande hinter Österreich, bei Nanotechnologie liegt Österreich an letzter Stelle. Zusammengenommen ergeben diese Daten daher keine starke Evidenz für eine positive Zielerreichung von Ziel (1) und Ziel (5) und deren gesetzten Maßnahmen.

Der Befund eines relativ niedrigen Spezialisierungsgrades wird auch durch Abbildung 3-17 bestätigt. Demnach gibt Österreich mit 70\% einen relativ hohen Anteil an staatlichen Mitteln ohne direkte sozioökonomische Zweckwidmung aus. Dies entspricht der Dominanz eines Fördersystems nach dem bottom-up Prinzip. ${ }^{214}$

Für die Beurteilung der Erreichung der Ziele (2) und (5), welche dem Prozess der Selektion von strategischen Schwerpunkten gewidmet sind, existiert keine quantitative Datenbasis. Auf Basis qualitativer Evidenz weist die OECD jedoch darauf hin, dass in Österreich im Vergleich zu anderen Staaten (wie etwa Deutschland oder Großbritannien) nach wie vor relativ selten von systematischen Prozessen zur Identifikation von Schwerpunkten Gebrauch gemacht

Tabelle 3-8: Revealed Technological Advantage nach ausgewählten Technologiefeldern, 2002-2005 und 2012-2015

\begin{tabular}{|c|c|c|c|c|c|c|c|c|}
\hline & Biotec & ologie & & & Nanote & ologie & Umweltt & inologie \\
\hline & 2002-05 & 2012-15 & 2002-05 & 2012-15 & 2000-03 & $2010-13$ & 2002-05 & 2012-15 \\
\hline Dänemark & 3,53 & 3,04 & 0,45 & 0,52 & 0,64 & 0,27 & 1,45 & 2,24 \\
\hline Deutschland & 0,72 & 0,74 & 0,49 & 0,42 & 0,56 & 0,39 & 1,27 & 1,23 \\
\hline EU-28 & 0,99 & 1,07 & 0,62 & 0,57 & 0,61 & 0,59 & 1,13 & 1,19 \\
\hline Finnland & 0,63 & 0,65 & 1,49 & 1,17 & 0,23 & 0,52 & 1,02 & 1,15 \\
\hline Großbritannien & 1,54 & 1,58 & 0,82 & 0,86 & 0,79 & 0,85 & 0,97 & 1,15 \\
\hline Niederlande & 1,12 & 1,67 & 1,23 & 0,65 & 0,78 & 1,11 & 0,70 & 0,83 \\
\hline Österreich & 0,82 & 0,67 & 0,47 & 0,54 & 0,44 & 0,25 & 1,21 & 1,04 \\
\hline Schweden & 1,28 & 1,04 & 0,76 & 1,16 & 0,64 & 0,51 & 1,13 & 1,16 \\
\hline
\end{tabular}

Quelle: OECD Science, Technology and Industry Scoreboard 2017; OECD Key Nanotech Indicators; OECD Key Biotech Indicators.

213 Vgl. https://www.klimafonds.gv.at/ 214 Vgl. Ecker et al. (2018). 
Abbildung 3-17: Staatliche Forschungsausgaben für F\&E nach sozioökonomischen Zielsetzungen, 2016

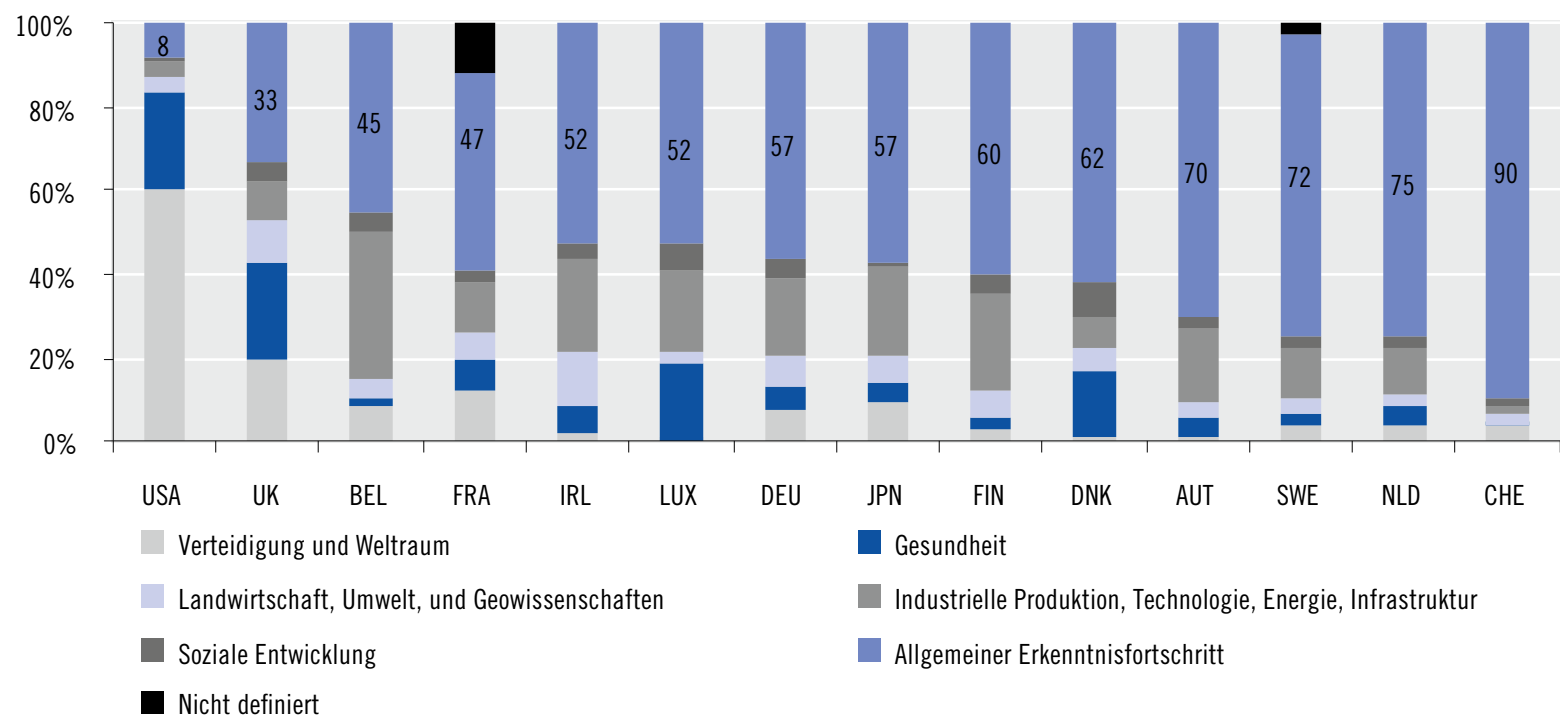

Quelle: OECD (2018b).

wird. ${ }^{215}$ Dies deutet darauf hin, dass die Ziele (2) und (5) noch nicht zur Gänze erreicht worden sind.

Die Einrichtung der interministeriellen Arbeitsgruppen sowie die Erarbeitung von Strategien stellen wichtige Elemente zur Verbesserung der Koordination von ressortübergreifenden Aktivitäten zur Bewältigung der "Grand Challenges" dar. Ziel (3) über die konzertierte Abstimmung der Aktivitäten der Ministerien kann damit jedenfalls von den Voraussetzungen her als erfüllt angesehen werden.

Insgesamt forderte die FTI-Strategie damit (zumindest teilweise) eine verstärkte Setzung von strategischen Themen sowie eine Professionalisierung der Selektion von thematischen Schwerpunkten. Die verbundenen Maßnahmen umfassen neben thematisch orientierten Förderprogrammen vor allem auch die Einrichtung von Arbeitsgruppen zur Abstimmung ressortübergreifender Themen im Sinne der "Grand Challenges“. Eine Überprüfung der Zielerreichung zeigt auf, dass die diesbezüglich gesetzten Maßnahmen allerdings noch nicht zu einem höheren Spezialisierungsgrad der Wirtschaft geführt haben. Dies dürfte auch die Folge eines Fördersystems sein, das bislang durchaus erfolgreich themenoffene Programme gegenüber einer top-down Förderphilosophie favorisierte.

\subsubsection{Optimierungen im Förderungssystem}

Fördersysteme für Innovationsprozesse umfassen eine Vielzahl von Programmen, Instrumenten und Akteuren. Neben direkten und indirekten monetären Förderinstrumenten, sind auch die regulatorischen Rahmenbedingungen, wie etwa Produktmarktregulierungen oder Patentrecht, wichtige Ansätze einer umfassend konzipierten Innovationspolitik.

Die Komplexität der Organisation des Fördersystems steigt weiterhin durch die Tatsache, dass unterschiedliche Akteure auf verschiedenen Ebenen des Innovationssystems mit der Planung, Durchführung sowie der Nachfrage nach diesen Förderungen befasst sind. Fördersysteme sind dabei einerseits historisch gewachsen und werden andererseits ständig an neue Bedingungen angepasst. Dies führt zum einen dazu, dass nationale Förderregime Spezifika aufweisen, die sie von anderen Ländern unterscheiden.

215 Vgl. OECD (2018b). 
Zum anderen hat sich wohl auch deshalb bislang kein Konsens darüber herausgebildet, wie ein optimales Fördersystem ausgestaltet sein sollte. Trotzdem haben sich einige Best Practice-Ansätze durchgesetzt, die beim Design von Instrumenten, Programmen und deren Zusammenwirken im Rahmen des Policy-Mix berücksichtigt werden sollten. ${ }^{216}$

Das österreichische Fördersystem ist stark differenziert und geht so auf die unterschiedlichsten Bedarfslagen der Fördernehmer ein. Die anfänglich stärker entwickelte direkte Förderung wurde zunehmend durch eine steuerbasierte, indirekte F\&E-Förderung ergänzt; mittlerweile sind beide Förderschienen monetär betrachtet von annähernd ähnlicher Bedeutung. Themenoffenen Programmen stehen thematische Programme gegenüber, wobei erstere dominieren. Einzelprojekte werden ebenso wie kooperative Forschungsprojekte gefördert, wobei sowohl kurzfristigere Kooperationsprojekte als auch strukturbildende Institutionen im kooperativen Sektor unterstützt werden.

Neben einer hohen Programmdichte und Instrumentenvielfalt zeichnet sich das österreichische Fördersystem auch durch relativ hohe Förderintensitä- ten aus. Bezieht man die direkten und indirekten F\&E-Förderungen für den Unternehmenssektor auf das BIP, so lag Österreich 2015 mit 0,27 \% vor allen Innovation Leaders; Frankreich und Belgien wiesen mit 0,39\% noch höhere Werte auf.

Vor dem Hintergrund nationaler und internationaler Entwicklungen formulierte die FTI-Strategie vier Ziele zur Verbesserung des nationalen Förderungssystems ${ }^{217}$ :

1. „Etablierung eines gesamthaften Politikansatzes im Förderungssystem, der das im jeweiligen Kontext effizienteste Bündel an Maßnahmen koordiniert zum Einsatz bringt;

2. Weiterentwicklung der direkten Forschungsförderung in Ausrichtung auf den Einsatz eines adäquaten Instrumentenmix;

3. Vereinheitlichung der Rechtsgrundlagen für die Forschungsförderung;

4. Verstärkung des Prinzips der Allokation durch Wettbewerb."

Die Ziele (1) und (2) beziehen sich dabei auf das Zusammenwirken der Instrumente und der sich daraus ergebenden Notwendigkeit zur Abstimmung derselben. Ziel (3) ist den förderrechtlichen Grundlagen

\section{Tabelle 3-9: Maßnahmen der FTI-Strategie zur Optimierung des Fördersystems}

\begin{tabular}{|c|c|}
\hline Allgemeine Maßnahmen It. FTI-Strategie & Konkrete Maßnahmen (Auswahl) \\
\hline $\begin{array}{l}\text { (1) Abschaffung der Forschungsfreibeträge gem. } \\
\S 4 \text { Abs. } 4 \text { EStG; Anhebung der Forschungs- } \\
\text { prämie gem. § 108c EStG von } 8 \% \text { auf } 10 \%\end{array}$ & - Umgesetzt \\
\hline $\begin{array}{l}\text { (2) Optimierung der direkten Forschungs- } \\
\text { förderung }\end{array}$ & $\begin{array}{l}\text { - Verringerung der Vielfalt thematischer Programme } \\
\text { - Verschiedene Ausschreibungstermine von F\&E-Programmen wurden/werden } \\
\text { zusammengelegt. } \\
\text { - Die Instrumente der direkten Förderung wurden standardisiert. }\end{array}$ \\
\hline $\begin{array}{l}\text { (3) Etablierung eines modernen homogenen } \\
\text { Forschungsförderungsrechts als Basis für alle } \\
\text { Förderungen des Bundes }\end{array}$ & $\begin{array}{l}\text { - Neue FFG- und FTI-Richtlinien sind mit } 01.01 .2015 \text { in Kraft getreten, } \\
\text { sämtliche Programmdokumente wurden an die neuen Regelungen angepasst. } \\
\text { - Forschungsfinanzierungsgesetz ist im Regierungsprogramm 2017-2022 } \\
\text { vorgesehen (siehe Kapitel 1). }\end{array}$ \\
\hline $\begin{array}{l}\text { (4) Steigerung des Anteils der kompetitiven } \\
\text { Förderung in der Grundlagenforschung }\end{array}$ & $\begin{array}{l}\text { - Budget des Wissenschaftsfonds (FWF) wird in den Jahren } 2018 \text { bis } 2021 \text { um } \\
\text { insgesamt } 110 \text { Mio. } € \text { erhöht. }\end{array}$ \\
\hline $\begin{array}{l}\text { (5) Verstärkung der Leistungsorientierung in der } \\
\text { Institutionenförderung durch Leistungs- und } \\
\text { Zielvereinbarungen für Basisfinanzierungen }\end{array}$ & $\begin{array}{l}\text { - Leistungsvereinbarungen des BMBWF mit ÖAW und IST Austria } \\
\text { - Rahmen- bzw. Förderungsvereinbarungen von BMVIT mit AIT, JR und SRFG } \\
\text { abgeschlossen } \\
\text { - ACR Strategie+ } \\
\text { - Forschungsfinanzierungsgesetz ist im Regierungsprogramm 2017-2022 } \\
\text { vorgesehen (siehe Kapitel 1). }\end{array}$ \\
\hline
\end{tabular}


gewidmet und Ziel (4) bezieht sich primär auf den Vergabemodus von Förderungen an Institutionen im Allgemeinen und Universitäten im Speziellen (siehe auch Maßnahmen (4) und (5) in Tabelle 3-9).

Die fünf allgemeinen Maßnahmen zur Optimierung des Fördersystems It. FTI-Strategie sind in der linken Spalte von Tabelle 3-9 dargestellt; die rechte Spalte zeigt eine Auswahl der konkreten Maßnahmen der zuständigen Ministerien. Die Maßnahmen (1) und (2) beziehen sich wesentlich auf die Ziele (1) und (2); Maßnahme (3) ist Ziel (3) zugeordnet und die Maßnahmen (4) und (5) adressieren Ziel (4) ${ }^{218}$.

\section{Zur Zielerreichung und Umsetzung der}

\section{Maßnahmen zur Optimierung des Fördersystems}

Eine Überprüfung der Ziele (1) und (2) über die Optimierung des Instrumenten- bzw. Programmix ist nur qualitativ möglich. Durch die Bündelung der steuerlichen Forschungsförderung im Rahmen der Forschungsprämie ${ }^{219}$ wurde eine wichtige Maßnahme für eine Vereinfachung des Fördersystems gesetzt. Trotz der gesetzten Maßnahmen zur Optimierung der direkten Forschungsförderung wird das jetzige System nach wie vor von einigen Institutionen als verbesserungswürdig eingestuft. So wird beispielsweise vom RFTE darauf hingewiesen, dass nach wie vor überlappende Mehrfachstrukturen, Überregulierungen, Zersplitterungen oder unklare Zuständigkeiten bestünden. ${ }^{220}$ Der Rechnungshof kommt auf Basis einer Prüfung ausgewählter Forschungsprogramme des Bundes in den Jahren 2012-2016 zum Schluss, dass die Anzahl der Forschungsprogramme in den Jahren 2012 bis 2016 um rd. 8 \% von 52 auf 56 anstieg, und dass die Zersplitterung der F\&E-bezogenen Agenden beim Bund $\mathrm{zu}$ thematischen Überschneidungen in den Forschungsprogrammen führe. ${ }^{221}$ Auch die OECD sieht in ihrem Länderbericht über das österreichische Innovationssystem weiteren Bedarf zur Koordination der individuellen Programme. ${ }^{222}$ Zusammenfassend ergibt sich damit für die Erreichung der Ziele (1) und (2) kein eindeutiges Bild. Während die indirekte Förderung vereinfacht wurde, scheint es bei der direkten Förderung weiterhin Bedarf zur Koordination und Konsolidierung zu geben.

Das Ziel (3) über die Etablierung eines einheitlichen Forschungsförderungsrechts wurde durch neue Richtlinien (FFG- und FTI-Richtlinie), die im Jahr 2015 in Kraft getreten sind, ein Stück weit realisiert. Durch das geplante Forschungsfinanzierungsgesetz sind weitere signifikante Verbesserungen der rechtlichen Grundlagen zu erwarten. In diesem Sinne kann Ziel (3) als in nächster Zukunft weitgehend realisiert betrachtet werden.

Die vermehrte Allokation von Fördermitteln mittels Wettbewerbsmechanismen ist Gegenstand von Ziel (4). Die Beurteilung der Zielerreichung ist dadurch eingeschränkt, dass bislang keine standardisierte Metrik zur Erfassung des kompetitiv vergebenen Anteils an öffentlichen F\&E-Mitteln existiert. ${ }^{223}$ Es müssen daher plausible Näherungswerte benützt werden. Eine Überprüfung der kompetitiven Mittelvergabe an Universitäten kann auf einem Zeitvergleich der Entwicklung des Globalbudgets mit den Mitteln von FWF, FFG und EU durchgeführt werden. Die Mittel von FWF, FFG und EU werden als kompetitiv vergebene Mittel gesehen. Hierbei zeigt sich zunächst eine Zunahme des Globalbudgets der Universitäten von der LV-Periode 2010-2012 zur LV-Periode 2016-2018 um ca. 17,3 \%.224 Demgegenüber kam es bei der Summe der kompetitiv eingeworbenen Mittel von FWF, FFG und EU zwischen 2010 und 2017 zu einem Anstieg um $29 \% .{ }^{225}$ Folglich kann der Schluss

218 Ebenda.

219 Erhöhung der Forschungsprämie für F\&E-Aufwendungen ab dem 1.1.2016 von 10 \% auf 12 \%, ab dem 1.1 .2018 von $12 \%$ auf 14 \%. 220 Vgl. RFTE (2018a).

$221 \mathrm{Vgl}$. Rechnungshof (2018).

222 Vgl. OECD (2018a).

223 Ebenda.

224 Vgl. BMBWF (2017), Tabelle 2.1.2-1.

225 Vgl. BMWFW (2014) und BMWFW (2017) Tabelle 7.6. 
gezogen werden, dass im betrachteten Zeitraum die wettbewerbliche Mittelallokation im Universitätssektor an Bedeutung zunahm. Diese würde jedenfalls die Erreichung von Ziel (4) bedeuten.

Im internationalen Vergleich erweist sich das gesamte österreichische Forschungsfördersystem allerdings nach wie vor als relativ wenig vom Wettbewerbsmechanismus bestimmt. Eine Pilotstudie der EU-Kommission hat für 14 Länder Indikatoren entwickelt, die einen Vergleich der Fördersysteme ermöglichen sollen. Dabei werden die Mittel, die auf Basis von ex-ante oder ex-post Leistungskriterien vergeben werden, als Anteil an den gesamten öffentlichen F\&E-Mitteln betrachtet. Für das Jahr 2013 liegt Österreich hier mit etwa $35 \%$ zusammen mit Dänemark, Frankreich und Italien am unteren Ende der Vergleichsländer. Deutschland, Finnland, die Niederlande, Schweden, Großbritannien und die Schweiz weisen bis zu doppelt so hohe Anteile auf. Im Zeitvergleich ergibt sich für Österreich eine Steigerung des kompetitiven Mittelanteils von ca. 27 \% im Jahr 2004 auf etwa 35 \% im Jahr 2013.

Dieser generelle Befund wird auch durch eine OECD-Studie bestätigt. 226 Darin wird der Anteil an Projektförderungen an den gesamten Forschungsausgaben des Bundes als Indikator für den Einsatz von wettbewerblichen Allokationsmechanismen herangezogen. Der aktuellste Datenpunkt für den internationalen Vergleich liegt hier bei 2011; der Wert Österreichs betrug im Jahr 2011 25,5\%, und Österreich lag demnach zu diesem Zeitpunkt an letzter Stelle unter den elf Vergleichsländern. So wies beispielsweise Dänemark einen Anteil von 35,3\% und Deutschland einen Anteil von $38,6 \%$ auf. Im Zeitverlauf ist für Österreich seit 2005 keine nachhaltige Zunahme des Anteils an Projektförderungen zu konstatieren.

Die Beurteilung der Zielerreichung von Ziel (4) fällt damit ambivalent aus. Für die Universitäten lässt sich eine Zunahme der kompetitiven Mittelvergabe konstatieren, während das gesamte Fördersystem nach wie vor eine relativ geringe Orientierung am Wettbewerbsprinzip aufzuweisen scheint.

Zusammengefasst verfügt Österreich über ein gut ausgebautes Fördersystem für Forschung- und Innovationsaktivitäten. Die Ziele zur Optimierung des Fördersystems It. FTI-Strategie wurden in unterschiedlichem Maße erreicht. Die Verbesserung der Abstimmung des Förderportfolios konnten nur teilweise realisiert werden. Einige der in der FTI-Strategie genannten Probleme (wie etwa Programmüberfrachtung oder mangelnde Fokussierung des Förderinstrumentariums) scheinen nach wie vor nur teilweise gelöst worden zu sein. Die rechtlichen Grundlagen werden insbesondere durch das neue Forschungsfinanzierungsgesetz modernisiert. Die Vergabe nach dem Wettbewerbsprinzip hat im Universitätssektor zugenommen; das gesamte Fördersystem erweist sich im internationalen Vergleich aber als ein System mit relativ geringer Wettbewerbsorientierung in der Allokation von Fördermitteln für Forschung und Innovation.

\subsubsection{Stärkung der internationalen Positionierung Österreichs}

Seit jeher war es ein prioritäres Ziel Österreichs, internationale Anbindungen zu nutzen und sich damit auch international strategisch zu positionieren. Folglich formulierte auch die FTI-Strategie vier Ziele zur Stärkung der internationalen Positionierung Österreichs: Ein übergeordnetes Ziel fordert eine „abgestimmte Wissenschafts- und Forschungsaußenpolitik mit entsprechenden institutionellen Strukturen". Zwei Ziele adressieren die Zusammenarbeit in der EU: Eines betrifft die „aktive Mitgestaltung an der ,European Knowledge Area' und eine entsprechende Positionierung Österreichs", das andere die „stärkere österreichische Beteiligung an europäischen Förderungsprogrammen und eine weitere Erhöhung der Rückflussquote". Zwei weitere Ziele betreffen die "globale Zusammenarbeit mit außereuro- 
Tabelle 3-10: Performance in den Rahmenprogrammen FP6 bis $\mathrm{H} 2020$

\begin{tabular}{l|r|r|r} 
& FP6: 2002-2006 & FP7: 2007-2013 & H2020: 2014-2020 \\
\hline Beteiligungen insgesamt & $\mathbf{7 4 . 5 8 4}$ & 136.388 & 93.716 \\
\hline Beteiligungen Österreich & 1.957 & 3.595 & 2.587 \\
\hline Österreich in \% & $2,62 \%$ & $2,64 \%$ & $2,76 \%$ \\
\hline Förderung insgesamt in Mio. $€$ & 16.697 & 45.236 & \\
\hline Förderung Österreich in Mio. $€$ & 426 & 1.185 & 35.245 \\
\hline Österreich in \% & $2,55 \%$ & $2,62 \%$ & 984 \\
\hline
\end{tabular}

Quelle: FFG EU PM Portal, (Datenstand 29.9.2018).

päischen sowie mit mittel- ost- und südeuropäischen Ländern" ${ }^{\text {227. }}$.

Bezugnehmend auf die Europäische Union konstatiert die FTI-Strategie 2020 mit Verweis auf die erfolgreiche Beteiligung an den Forschungsrahmenprogrammen FP4 bis FP7 eine sehr gute Einbindung Österreichs in den Europäischen Forschungsraum als Ausgangslage. Verfolgt man diese Entwicklung weiter bis zum heutigen Stand der Teilnahme bei Horizon 2020, so lässt sich - gemessen an den Beteiligungen und den Förderungen - sogar noch eine Steigerung der bereits damals guten Performance feststellen: Österreich konnte den Anteil der Förderungen von 2,55\% in FP6 auf 2,79\% in Horizon 2020 steigern, den Anteil der Beteiligungen von 2,62\% auf 2,76 \% (vgl. Tabelle 3-10). Das Ziel der weiteren Erhöhung der Rückflussquote konnte demnach erreicht werden.

Anschließend an die damalige Kritik vonseiten der Systemevaluierung ${ }^{228}$ an einer Mentalität, die zu sehr an Programmrückflüssen orientiert und zu wenig an strategischer Mitgestaltung getragen sei, forderte die FTI-Strategie auch ein kohärentes Maßnahmenbündel zur optimalen Nutzung der Chancen des europäischen Forschungsraumes sowie zur Platzierung österreichischer Interessen. Die Erreichung dieses Ziels ist weit schwieriger zu bewerten, wiewohl die Vielfalt an Aktivitäten und Maßnahmen eine aktive Rolle zeigt, die Österreich bei der Gestaltung des europäischen Forschungsraumes einnimmt. Anderer- seits macht die Evaluierung der Umsetzung von H2020, EUREKA, COSME, EEN und ERA in Österreich ${ }^{229}$ deutlich, dass zur Erreichung des umfassend postulierten Strategiezieles weitere Anstrengungen in der strategischen Positionierung, der Governance und den Unterstützungsstrukturen notwendig sind. Dabei ist das Ausmaß dieser Veränderungen von der angestrebten Positionierung Österreichs in Bezug auf die europäische FTI-Politik abhängig. Diese Positionierung und die damit verbundenen Veränderungen werden in drei Szenarien reflektiert.

Im Szenario 1 geht es um eine inkrementelle Weiterentwicklung des gut funktionierenden Status-quo ohne wesentliche Veränderungen vorzunehmen. Im Einklang mit diesem Bild verbleibt die Positionierung Österreichs in EU-Initiativen weitgehend bei den FTIInstitutionen selbst und im Aufgabenbereich der Fachministerien, die diese Positionierung sowie die Platzierung österreichischer Interessen nach jeweils ressortspezifischen Prioritätensetzungen vornehmen. Hingegen wird eine deutlich proaktivere Mitgestaltung europäischer FTI-Politik, um die sich daraus ergebenden Chancen der EU FTI-Politik gezielter und effektiver für Österreich zu nutzen, im „smartalignment"-Szenario (Szenario 2) vorgestellt. Zentrale Ansatzpunkte dafür wären die Entwicklung einer europäischen Beteiligungsstrategie, um in prioritären Feldern eine prägende Rolle spielen zu können. Erforderlich hierfür wäre eine engere Verschränkung nationaler und europäischer Programme, die sich bei- 
spielsweise in einer selektiven Beteiligung an strategischen EU-Initiativen oder in einer verbesserten inhaltlichen Komplementarität/Abstimmung zwischen nationalen und europäischen Förderausschreibungen manifestieren könnte. Zur Realisierung dieses Szenarios bedürfte es jedoch neben einer akzeptierten politischen Leadership Rolle auch der Unterstützung durch eine starke FFG mit ihren nationalen und europäischen Aktivitäten, sowie verstärkten interministeriellen Abstimmungsmechanismen. Beim „distributed empowerment"-Szenario (Szenario 3) schließlich würde die Ausweitung der Mitgestaltung und Beteiligung an der europäischen FTI-Politik vor allem über die Stärkung und bessere Vernetzung der FTI-Institutionen selbst erfolgen. Dieser Prozess der Selbstermächtigung erfordert jedoch Unterstützung seitens der Politik, um die unterkritische Ressourcenausstattung (z.B. zur wettbewerblichen Kofinanzierung) zu überwinden, die ein wesentlicher Grund dafür ist, dass österreichische FTI-Institutionen nur eine Nebenrolle in den größer dimensionierten europäischen Initiativen spielen. Dieses Szenario setzt eine eigenständige Entwicklungsstrategie der Organisationen voraus, sowie die Fähigkeit zur Selbstorganisation in schlagkräftigen nationalen Netzwerken. Die Beteiligung der FTI-Akteure an größeren Initiativen wäre durch eine entsprechende Schwerpunktverschiebung in der FFG zu unterstützen. Ähnlich wie im zweiten Szenario ist auch hier eine enge Verschränkung der nationalen Programme mit den europäischen Initiativen sinnvoll.

Hinsichtlich der Zusammenarbeit mit Ländern auBerhalb der EU ortet die FTI-Strategie Ausbaupotential und die Notwendigkeit eines koordinierten Vorgehens. Genannt werden die USA, die BRIC-Länder, die mittel-, ost- und südosteuropäischen Nachbarländer sowie Asien. Auch hier kann das Ausmaß der Zielerreichung nicht eindeutig definiert oder gemessen, sondern nur der Umsetzungsstand der Aktivitäten und Maßnahmen berichtet werden.
In der FTI-Strategie wurden hierzu drei Maßnahmen ${ }^{230}$ formuliert:

- Einrichtung einer ständigen Arbeitsgruppe zur Koordination und Implementierung einer österreichischen Außenwissenschafts- und Technologiepolitik, bestehend aus den Fachressorts

- Entwicklung eines Aktionsplanes „Österreich und der Europäische Wissensraum 2020“, ausgearbeitet von BMWFW und BMVIT unter Einbindung der relevanten Ressorts und Stakeholder

- Entwicklung einer kohärenten Kooperationsstrategie für verschiedene Schwerpunkträume: Mittel,Ost- und Südosteuropa, Nordamerika, Asien und BRIC-Staaten

\section{Einrichtung einer ständigen Arbeitsgruppe zur Koordination und Implementierung einer österreichischen Außenwissenschafts- und Technologiepolitik, bestehend aus den \\ Fachressorts}

Eine Arbeitsgruppe "Internationalisierung und FTIAußenpolitik" der Task Force FTI (AG 7a) wurde eingerichtet und hat Mitte 2013 das Strategiepapier „Beyond Europe - Die Internationalisierung Österreichs in Forschung, Technologie und Innovation über Europa hinaus“231 erarbeitet. Ausgangspunkt der formulierten Empfehlungen war der Befund, dass Österreich im Vergleich mit den im Innovationsbereich führenden Ländern Europas (den Innovation Leader) im Bereich der Internationalisierungsaktivitäten einen erheblichen Handlungsbedarf aufweist. Im Kontext der Empfehlungen wurden drei Prioritätsklassen für Länder und Regionen formuliert sowie Themen und Maßnahmen skizziert (gezielte top-down Maßnahmen in bilateraler und thematischer Kooperation, allgemeine bottom-up Maßnahmen in multilateraler und themenoffener Kooperation, Ausbau der WTZAbkommen, Unterstützung der universitären Kooperationen). Ebenfalls geplant waren Verbesserungen der Außenvertretung Österreichs durch den Aus- und 
Aufbau von OSTA (Offices of Science and Technology Austria) und die Einrichtung von FTI-Attachés an österreichischen Botschaften in ausgewählten Schwerpunktländern der Priorität 1 und 2. Aus dieser Strategie wurden in den Folgejahren folgende Maßnahmen umgesetzt:

- Start des Programms „Beyond Europe“: Im Jahr 2015 erfolgte eine erste Ausschreibung des themen- und regionenoffenen Programms "Beyond Europe" des BMDW. Das Programm wurde fortgesetzt (die dritte Ausschreibung erfolgte im Dezember 2018) und im Jahr 2018 erfolgreich evaluiert. "Beyond Europe“ richtet sich an österreichische Unternehmen, die mit Partnern in außereuropäischen Ländern innovative Entwicklungsprojekte durchführen;

- Installierung des „Office of Science and Technology Austria - OSTA“ an der österreichischen Botschaft in Peking im Jahr 2012;

- Erweiterung der WTZ-Abkommen (Wissenschaftlich-Technische Zusammenarbeit) mit außereuropäischen Ländern sowie mit Mittel,- Ost- und Südosteuropa ${ }^{232}$;

- Ausbau der Zusammenarbeit mit außereuropäischen Forschungspartnern und Förderungsorganisationen vonseiten des FWF sowie vonseiten BMVIT und FFG vor allem im asiatischen Raum (z.B. thematische Ausschreibungen mit der Chinese Academy of Science und der Universität Shanghai), Implementierung der Initiative „Global Incubator Network (GIN)";

- Unterstützung österreichischer Forschender und Unternehmen, die im Rahmen von Horizon 2020 mit Drittstaaten zusammenarbeiten durch spezifische Information und Beratung seitens der FFG ${ }^{233}$, Mitwirkung an der strategischen Weiterentwicklung von EUREKA mit Südkorea, Kanada, Südafrika und Chile als assoziierte Länder und aktive Teil- nahme an Globalstars sowie am Eurostars-Programm.

\section{Entwicklung eines Aktionsplanes „Österreich und der Europäische Wissensraum 2020“ ausgearbeitet von BMWFW und BMVIT unter Einbindung der relevanten Ressorts und \\ Stakeholder}

Eine zweite Arbeitsgruppe der Task Force FTI wurde unter dem Titel „Aktionsplan Österreich und der Europäische Wissensraum 2020" als Arbeitsgruppe 7b eingerichtet. Diese hat Mitte 2013 einen Strategieplan („Österreichischer EU-Aktionsplan“234) unter dem Leitszenario "Stärkung österreichischer Akteure ermöglicht eine erfolgreiche Europäisierung" mit sechs Prioritäten und insgesamt 72 Maßnahmen vorgelegt und treibt nunmehr die Umsetzung der formulierten Maßnahmen für eine optimale Positionierung Österreichs in den Rahmenprogrammen und im ERA voran. 2016 wurde die österreichische „ERA Roadmap“235 auf Basis der „European Research Area Roadmap 2015-2020" beschlossen. Sie benennt sieben Handlungsfelder in sechs Prioritäten, die für den weiteren Ausbau des Europäischen Forschungsraums als wesentlich erachtet werden. Allfällige Anpassungen aufgrund der ERA-Ratsschlussfolgerungen vom 30.11.2018 werden 2019 diskutiert. Weiters werden die Verhandlungen auf europäischer Ebene betreffend "Horizon Europe", dem Nachfolgeprogramm von „Horizon 2020", im Jahr 2019 fortgeführt. Dabei geht es auch um die Frage, in welchem Maße die definierten Themenfelder für „Missionen“ sowie „Partnerschaften“ im Rahmen nationaler Schwerpunktsetzungen Berücksichtigung finden sollen.

Die FFG hat ihre Unterstützungs- und Betreuungsaktivitäten sowohl für die europäische als auch für die globale Zusammenarbeit erweitert und intensiviert. Es erfolgte eine schrittweise Umgestaltung von

232 Für eine Liste aller WTZ-Abkommen sowie aktueller Fördermöglichkeiten von Auslandsaufenthalten siehe https://oead.at/de/ projekte/internationale-kooperationen/wissenschaftlich-technische-zusammenarbeit/

233 Vgl. z.B. https://www.ffg.at/ausschreibungen/H2020_internationale-kooperation

234 Vgl. https://era.gv.at/directory/159

235 Vgl. ERA Observatorium Austria und BMWFW (2016). 
reinen Maßnahmen der Informationsbereitstellung zu Beratungsleistungen, die vom „empowerment-Ansatz“ geprägt sind. Die kürzlich abgeschlossene Evaluierung der Umsetzung europäischer Programme in Österreich bestätigt diesen erfolgreichen Weg und schlägt weitere Maßnahmen zur Stärkung der strategischen Orientierung vor. ${ }^{236}$ Mit der Einführung des "EU-Performance Monitoring“ (EU-PM) verarbeitet die FFG Daten zu den EU-Rahmenprogrammen und wertet diese unter besonderer Berücksichtigung der österreichischen Beteiligungen aus. Es handelt sich um ein nutzerfreundliches interaktives Tool, in welches nun auch die nationalen Förderungsprogramme integriert werden sollen.

\section{Entwicklung einer kohärenten}

Kooperationsstrategie für verschiedene

Schwerpunkträume: Mittel,- Ost- und

Südosteuropa, Nordamerika, Asien und BRIC-

\section{Staaten}

Über die Internationalisierungsbemühungen der letzten Jahre hinaus, die sich in Umsetzung der FTIStrategie und des zur Intensivierung der Kooperation mit den wichtigsten Schwerpunkträumen entwickelten Strategiepapiers „Beyond Europe“ auf Nordamerika, Asien und die BRIC-Staaten konzentrierten, wurde im Jahr 2018 auch ein Ausbau der Kooperation mit Afrika begonnen. Dieser wurde während des High Level Forum Africa-Europe, das im Rahmen der österreichischen EU-Ratspräsidentschaft am 18.12.2018 veranstaltet wurde, vorgestellt. Hintergrund dieser Initiative ist die große wirtschaftliche Dynamik Afrikas in den letzten Jahren, die internationale Verantwortung für die Sustainable Development Goals (SDGs), die damit verbundene Notwendigkeit der Festigung produktiver, wirtschaftlicher und sozialer Strukturen vor Ort und das gestiegene Bewusstsein in Entwicklungs- und Schwellenländern über die Bedeutung endogener F\&E-Potentiale. Der Ausbau der Kooperation mit Afrika basiert im Wesentlichen auf drei Säulen:
- Erstens wurde ein Memorandum of Understanding zur wissenschaftlich-technischen Zusammenarbeit (WTZ) mit dem Wissenschaftsministerium in Ägypten unterzeichnet. Eine weitere WTZ-Vereinbarung mit dem äthiopischen Wissenschafts- und Technologieministerium ist in Vorbereitung. Diese bilden zusammen mit der bereits vorhandenen WTZ-Kooperationsvereinbarung mit dem Wissenschaftsministerium in Südafrika die ersten bilateralen Kooperationsvereinbarungen im Bereich Wissenschaft und Forschung zwischen Österreich und ausgesuchten afrikanischen Ländern.

- Die zweite Säule bietet das neue BMBWF-Programm für Entwicklungsforschung. Dieses löst die bis Ende 2018 existierende Kommission für Entwicklungsforschung (KEF) bei der OeAD GmbH ab. Das neue Programm, das von der OeAD GmbH abgewickelt wird, hat ein Budget von bis zu $315.000 €$ pro Jahr. Mit dem Programm möchte das BMBWF die Entwicklungsforschung verstärken, die Forschungskooperation mit Entwicklungsländern, insbesondere - aber nicht ausschließlich - mit Afrika, Schritt für Schritt weiter ausbauen und die österreichischen Hochschulen und Forschungseinrichtungen in ihrem Engagement bei der Umsetzung der Sustainable Development Goals (SDGs), die 2015 von den Vereinten Nationen beschlossen wurden, unterstützen. Finanziert werden Mobilitäts- und Sachkosten in Österreich sowie im Partnerland.

- Ergänzend wird in einer dritten Säule das Forschungsnetzwerk österreichischer und afrikanischer Hochschul- und Forschungseinrichtungen aufgebaut, das in der Aufbauphase von der Universität für Bodenkultur Wien koordiniert und von der OeAD administrativ implementiert wird. Ziel des Forschungsnetzwerkes ist der Ausbau der Forschungskooperation mit Ländern in Afrika durch Förderung von Netzwerkaktivitäten und Projektkooperationen zwischen österreichischen und afrikanischen Hochschul- und Forschungsein-

236 Vgl. Dinges et al. (2018). 
richtungen. Das BMBWF finanziert das Netzwerk mit $250.000 €$ pro Jahr für die Supportstruktur bei der OeAD sowie die Vernetzungsaktivitäten. Die Netzwerkaktivitäten umfassen Alumni-Aktivitäten, Trainings, Workshops, Seminare, Konferenzen, bi- bzw. multilaterale Forschungsprojekte mit mindestens einer afrikanischen Mitgliedsinstitution und Projektanbahnungen für gemeinsame Anträge in Horizon Europe, Erasmus+, FWF- und FFG-Projekte. Eine thematische Einschränkung ist nicht vorhanden.

Ergänzt werden diese Maßnahmen durch die bereits bestehenden themen- wie auch länderoffenen Programme wie jene des FWF oder des „Beyond Europe“Programms des BMDW.

\subsubsection{Ausbau der Verbindung Forschung und Gesellschaft}

Auch für das Verhältnis zwischen Forschung und Gesellschaft hat die Bundesregierung In der FTI-Strategie konkrete Ziele formuliert. Angestrebt werden „eine Kultur der Wertschätzung von Forschung, Technologie und Innovation" und eine Förderung des Verständnisses dafür, „dass diese einen wesentlichen Beitrag zur Steigerung von Lebensqualität und gesellschaftlichem Wohlstand leisten “237. Um dies zu erreichen, soll ein stabiles, auch infrastrukturelles Umfeld für vielfältige Formen des Dialogs von Wissenschaft und Gesellschaft im Sinn einer "Scientific Citizenship" aufgebaut werden. Innerhalb der Wissenschaft sollen Verantwortung und Integrität der Wissenschaft durch institutionalisierte Prozesse gestärkt werden.

Definiert wurde eine Fülle von Maßnahmen ${ }^{238}$ :

- Einrichtung eines zentralen Veranstaltungsortes für den Dialog Wissenschaft/Forschung und Gesellschaft

- Förderung von Dialogaktivitäten für Forschung, Technologie und Innovation
- Durchführung einer regelmäßigen nationalen Leistungsschau zur Darstellung von Forschung als zukunftgestaltende gesellschaftliche Leistung

- Ausbau der unabhängigen Technologiefolgenabschätzung

- Etablierung hoher Standards der wissenschaftlichen Integrität:

- strenge Richtlinien im Umgang mit Interessenskonflikten bei der Auftragsforschung

- Offenlegung von Wertesystemen in der Forschung

- Die Ergebnisse von öffentlich finanzierten bzw. geförderten Forschungsprojekten sind in geeigneter Art und Weise der Öffentlichkeit zugänglich zu machen

- Stärkung der dafür vorgesehenen Organisationen

- Schaffung einer klaren gesetzlichen Regelung von Forschungsethikkommissionen in Bezug auf Prüfauftrag, Rechtsqualität der Stellungnahmen und Verfahrensregeln

Zusätzlich finden sich entlang der Maßnahmen auch Aktivitäten, die nicht direkt in der FTI Strategie angesprochen wurden, jedoch die Vielfältigkeit und die Dynamik in der österreichischen Forschungslandschaft dokumentieren.

\section{Dialogaktivitäten für Forschung, Technologie und Innovation}

Im Anliegen der Förderung des Dialogs zwischen FTI und Gesellschaft spiegelt sich die internationale Diskussion zum Thema Wissenschaft und Gesellschaft. Deren Anfang war, dass davon ausgegangen wurde, dass eine passive, in Fragen der Wissenschaft ungebildete Öffentlichkeit über die Leistungen und den Nutzen der Forschung lediglich informiert werden müsse, um Akzeptanz zu schaffen. ${ }^{239}$ Mittlerweile ist hier das Bild eines Dialogs zwischen den verschiedenen Akteuren in den Vordergrund getreten und Ansätze zum „public engagement", also zur Einbezie-

237 Vgl. BKA et al. $(2011,43)$.

238 Ebenda.

239 Vgl. z.B. das international wegweisende Dokument der Royal Society (1985). 
hung der Öffentlichkeit in Forschungsprozesse, haben neben "klassischen" Formaten der Leistungsschau auch in Österreich an Bedeutung gewonnen, wie am Beispiel der unten dargestellten Aktivitäten etwa zu Citizen Science und Responsible Science deutlich wird. Neben der vorwiegend monodirektionalen Information und Kommunikation über Wissenschaft und Forschung werden also zunehmend Maßnahmen zur bidirektionalen Kooperation zwischen Wissenschaft und Gesellschaft gesetzt. Dadurch können das Verständnis für die Rolle und Bedeutung von FTI in der Öffentlichkeit verbessert und zudem die sozialen Dimensionen wissenschaftlicher Tätigkeit in der Forschungspraxis besser gehandhabt werden. AuBerdem eröffnen sich neue Erkenntniswege für die Forschung, etwa über Citizen Science. Der Begriff Citizen Science umfasst unterschiedliche Arten der Beteiligung von Bürgerinnen und Bürgern an wissenschaftlichen Aktivitäten. Vom Datensammeln bis zur Initiierung von Forschungsprojekten finden innovative Kombinationen von lokalem, praktischem Wissen der Gemeinschaft mit dem systematisierten Wissen der Forscherinnen und Forschern statt.

Die Bundesregierung hat verschiedene Dialogformate entwickelt und umgesetzt und im Licht der Erfahrungen auch immer wieder erneuert. So unterstützte das BMVIT im „forum bmvit" bis 2016 die Vernetzung zwischen den Akteuren aus Politik, Forschung, Industrie und Wirtschaft im Rahmen von Veranstaltungen zu aktuellen Innovationsthemen. Mit "fti remixed"240 wurde eine Wissenschaftskommunikationsplattform speziell für Jugendliche eingerichtet, mit der diese über FTI und die damit verbundenen beruflichen Perspektiven informiert werden. Primär an ein jugendliches Publikum richtet sich auch die im Rahmen von Horizon 2020 finanzierte „European Researchers 'Night" ${ }^{\text {"241 }}$, eine europaweite Aktion, bei der parallel an vielen Orten eine Fülle von Veran- staltungen stattfinden, wobei interaktive Formate im Vordergrund stehen.

Das BMBWF spricht auch mit seiner Veranstaltungsreihe "Science Talks"242 in Form von Podiumsdiskussionen mit Wissenschaftlerinnen und Wissenschaftlern zu aktuellen Themen die interessierte Öffentlichkeit an. Diese Veranstaltungen finden in der „Aula der Wissenschaften“ statt, die ebenfalls als Teil der FTI-Strategie als zentraler Veranstaltungsort für den Dialog Wissenschaft/ Forschung und Gesellschaft eingerichtet wurde. Das BMBWF fasst im Jahr 2015 seine Maßnahmen zur Umsetzung der FTI-Strategie im „Aktionsplan für einen wettbewerbsfähigen Forschungsraum" zusammen. Die Aktionslinie "Den Dialog zwischen Wissenschaft und Gesellschaft vertiefen" setzt dabei besonders auf die Konzepte von Responsible Science, Citizen Science und Crowdsourcing sowie Open Innovation.

Eine der größten österreichischen Maßnahmen in diesem Kontext ist das Programm "Sparkling Science ${ }^{243}$ des BMBWF, das Elemente des CitizenScience-Diskurses beinhaltet und diesen in Österreich deutlich prägt. Sparkling Science wird vom Österreichischen Austauschdienst (OeAD) abgewickelt. Das Programm hat den Anspruch, "hochwertige Forschung mit voruniversitärer Nachwuchsförderung“244 zu verbinden. Dies geschieht durch die Förderung anspruchsvoller wissenschaftlicher Projekte, in denen Schülerinnen und Schüler aktiv am Forschungsprozess teilnehmen. Die Forschung soll von besonderen wissenschaftlichen Erkenntnissen profitieren, die Bildung davon, dass Schülerinnen und Schüler (und Lehrerinnen und Lehrer) im Zuge des Schulunterrichts direkt mit dem neuesten Stand des Wissens als auch mit wissenschaftlichen Herangehensweisen in Berührung kommen.

Das Programm Sparkling Science war während seiner Laufzeit Gegenstand von zwei Evaluierungen

240 Vgl. https://www.fti-remixed.at/

$241 \mathrm{Vgl}$. http://sci4all.eu/de/

$242 \mathrm{Vgl}$. https://bmbwf.gv.at/das-ministerium/veranstaltungen/science-talk/

243 Vgl. https://www.sparklingscience.at/

$244 \mathrm{Vgl}$. BMWFW und OeAD $(2016,2)$. 
(2009 und 2013) $)^{245}$ sowie je einer Analyse der wissenschaftlichen und der institutionellen Wirkun$\operatorname{gen}^{246}$. Das Programm hat seine Zielgruppen erfolgreich mobilisiert und ist ausgesprochen gut angenommen worden, vor allem von Schulen, Universitäten und Pädagogischen Hochschulen. Die Erfahrungen mit den geförderten Projekten in Sparkling Science sind insgesamt ausgesprochen positiv, und sowohl die beteiligten Forscherinnen und Forscher als auch die Schülerinnen und Schüler berichten von hoher Motivation und einer auch den Inhalten zuträglichen positiven Dynamik in den Projekten. Die Kontakte und Aktivitäten aus den Sparkling Science-Projekten werden oft über das finanzierte Projekt hinaus, etwa in Form von weiteren gemeinsamen Forschungsarbeiten oder Lehrtätigkeiten, weitergeführt. Das Programm hat damit klar zur Verbesserung der Schnittstelle zwischen Schulen und Hochschulen beigetragen. Zudem hat es einen wesentlichen Impuls von „klassischen“, einseitigen Pfaden der Wissenschaftskommunikation weg hin zur tatsächlichen Zusammenarbeit in gemeinsamer Forschung gegeben. Die dadurch aufgebauten Partnerschaften und Kompetenzen bei den teilnehmenden Institutionen bilden eine Grundlage für künftige Aktivitäten, die jedoch weiterhin der Förderung bedürfen, da die Forschung in hohem Maß auf Drittmittel angewiesen ist.

Seit 2015 werden im Rahmen von Sparkling Science verstärkt Elemente von Citizen Science unterstützt. Dazu zählen vor allem die „Citizen Science Awards", welche eine größere Sichtbarkeit bewirken sollen, sowie die Erweiterung von Sparkling-ScienceProjekten um Elemente des Crowdsourcings. Darunter wird die Einbindung von Bürgerinnen und Bürgern über die im Konsortium direkt involvierten Schülerinnen und Schüler hinaus im Rahmen der Young-Citizen-Science-Pilotprojekte verstanden. In begleitender Analyse ${ }^{247}$ wurden dabei auch die optimale Ge- staltung von Förderungsmaßnahmen für Citizen Science erforscht.

Auch an Universitäten, Forschungseinrichtungen und Agenturen des Bundes wurden in den vergangenen Jahren strukturierte Citizen Science-Aktivitäten gesetzt, darunter die folgenden:

- Der FWF und der Österreichische Austauschdienst (OeAD) haben in Abstimmung mit dem BMBWF dreimal „Top Citizen Science (TCS)“248 Erweiterungsprojekte (bis max. $50.000 €$ ) zu laufenden FWF-Projekten und Sparkling-Science-Projekten finanziert. Diese Erweiterungsmodule finanzieren Projekterweiterungen im Sinne von Citizen Science, d.h. es sollen Bürgerinnen und Bürger sowie Personen mit spezialisierter Expertise - sogenannte "Knowledge-Communities" - aktiv in laufende Forschungsarbeiten eingebunden und damit die Möglichkeiten für exzellente Forschung erweitert werden. Bisher wurden 31 TCS-Erweiterungsprojekte gefördert.

- Die Ludwig Boltzmann Gesellschaft (LBG) richtete 2016 mit Mitteln der Nationalstiftung das „LBG Open Innovation in Science Center" ein ${ }^{249}$. Das Konzept des Zentrums basiert auf erfolgreichen Pilotprojekten in den Bereichen Crowdsourcing und Training für Open Innovation in der Wissenschaft. Open Innovation in Science (OIS) bedeutet, dass wissenschaftliche Methoden gezielt im Rahmen offener, kollaborativer und partizipativer Prozesse eingesetzt werden. Das Ziel dabei ist es, Neues zu schaffen und einen Mehrwert für die Gesellschaft zu erzielen. Im Rahmen des jüngsten Crowdsourcing-Projekts „Reden Sie mit bei Unfallverletzungen!" haben Patientinnen und Patienten sowie Expertinnen und Experten über die OnlinePlattform „Tell us!" mehr als 800 Forschungsfragen eingereicht.

- Citizen Science war auch ein Thema der von 2014

$245 \mathrm{Vgl}$. Mitterauer und Birke (2009); Birke und Fettelschoß (2013).

$246 \mathrm{Vgl}$. Manahl et al. (2016); Tiefenthaler (2018).

$247 \mathrm{Vgl}$. Tiefenthaler und Warta (2016).

248 Vgl. https://www.fwf.ac.at/de/forschungsfoerderung/fwf-programme/foerderinitiative-top-citizen-science/ und https://www. zentrumfuercitizenscience.at/de/top-citizen-science

249 Vgl. https://ois.lbg.ac.at/ 
bis 2018 bestehenden Wissenstransferzentren ${ }^{250}$. Das BMBWF und das BMDW finanzierten die Aktivitäten im Rahmen des Förderprogramms „Wissenstransferzentren und IPR Verwertung", um den Transfer von Wissen zwischen Wissenschaft in Wirtschaft und Gesellschaft zu intensivieren. Die Initiative wurde von der Austria Wirtschaftsservice $\mathrm{GmbH}$ koordiniert.

- An der Universität für Bodenkultur wurde 2016 die Citizen Science-Plattform „Österreich forscht" gegründet. Mit Ende 2018 finden sich dort 58 laufende und zehn abgeschlossene Projekte von über 30 Institutionen, die zusammen ungefähr 100.000 Citizen Scientists versammeln. ${ }^{251}$ Diese Projekte wurden nicht nur von Universitäten initiiert und koordiniert, sondern auch von außeruniversitären Forschungseinrichtungen, NGOs, Museen, Vereinen, privaten Forschungsinstitutionen und Stiftungen. Neben den Natur-, Geistes- und Sozialwissenschaften sind seit 2018 auch Projekte aus den Kunst- und Kulturwissenschaften vertreten. Eine inter-institutionelle Arbeitsgruppe hat Qualitätskriterien für Citizen Science-Projekte ${ }^{252}$ erarbeitet, die auch international positiv wahrgenommen worden sind.

\section{Leistungsschau: Forschung als}

\section{zukunftsgestaltende gesellschaftliche Leistung}

Im Juni 2014 wurden im Rahmen der Eurobarometer Studie „Öffentliche Wahrnehmung von Wissenschaft, Forschung und Innovation" rd. 28.000 Personen in den 28 EU-Mitgliedstaaten zu den Auswirkungen von Wissenschaft und Technologie auf wesentliche Aspekte des Lebens in den kommenden 15 Jahren befragt. ${ }^{253}$ Die Ergebnisse weisen für Österreich weiter- hin eine vergleichsweise große Skepsis gegenüber Wissenschaft und Technologie aus. Demgegenüber stoßen jedoch diverse Maßnahmen auf reges Interesse der Öffentlichkeit. Zentrale Aktivität in dieser Maßnahmenkategorie ist die "Lange Nacht der Forschung“. ${ }^{254}$ Außerdem dienen auch verschiedene Staatspreise sowohl der Auszeichnung herausragender Leistungen in F\&E als auch deren Präsentation in der Öffentlichkeit. Dies sind insbesondere die Staatspreise "Mobilität" und „Patent“, die zweijährig verliehen werden, sowie die jährlich verliehenen Staatspreise "Innovation“ und "Design“ sowie der Gründerpreis „Phönix“. 2019 wird erstmals ein Staatspreis für „Digitalisierung “ verliehen. ${ }^{255}$ Für besondere Leistungen im Diversitätsmanagement an Hochschulen und Forschungseinrichtungen wird der "Diversitas Award“" verliehen ${ }^{256}$, und die Gabriele-Posanner-Preise zeichnen alle zwei Jahre Leistungen in der Geschlechterforschung aus ${ }^{257}$.

Die „Lange Nacht der Forschung“ (LNF) ist Österreichs größter Forschungs-Event für ein breites $\mathrm{Pu}$ blikum. Seit 2005 fand die LNF in Österreichs groBen Städten, seit 2012 biennal, und seit 2014 bundesweit statt und macht die Leistungen und Herausforderungen der österreichischen Wissenschaft - von Schulprojekten bis Spitzenforschung zugänglich und interaktiv erfahrbar. ${ }^{258}$ Der Besuch ist kostenlos. Universitäten, außeruniversitäre Forschungseinrichtungen, Fachhochschulen, Industrie, Infrastrukturen und Schulen sind aufgerufen, ihre Aktivitäten niederschwellig zu vermitteln, um so ein besseres Verständnis für Forschung und Technologie zu erzielen und die Begeisterung für Forschung und für den offenen Dialog von Wissenschaft und Gesellschaft zu wecken. Zudem wird die vielfältige

250 Vgl. WTZ Ost, WTZ Süd, WTZ West: http://www.wtz.ac.at/, und das thematische WTZ: www.wings4innovation.at

251 Vgl. Citizen Science Network Austria (2018, 22).

252 Vgl. Heigl et al. (2018).

253 Vgl. Europäische Kommission (2014).

$254 \mathrm{Vgl}$. https://www.langenachtderforschung.at

$255 \mathrm{Vgl}$. https://www.bmdw.gv.at/Ministerium/Staatspreise/Seiten/default.aspx

256 Vgl. https://bmbwf.gv.at/wissenschaft-hochschulen/gleichstellung-und-diversitaet/programme-und-initiativen/diversitas/ 257 Vgl. https://bmbwf.gv.at/das-ministerium/staatspreise-und-auszeichnungen/gabriele-possanner-staats-und-foerderpreis/ 258 Details zur Geschichte der LNF siehe RFTE (2015). 
Forschungslandschaft als attraktiver Arbeitsplatz für Nachwuchskräfte vorgestellt. In interaktiven Präsentationen, Mitmachstationen und bei Führungen treten Interessierte mit den Forschenden in Kontakt, legen selbst Hand an Experimente und diskutieren aktuelle Herausforderungen. 2018 fand die achte und bisher größte LNF statt: An 265 Standorten mit 2.601 Stationen wurden über 228.000 Besucherinnen und Besucher aller Altersschichten angesprochen. ${ }^{259}$ Die bundesweiten Maßnahmen der LNF werden vom BMBWF, dem BMDW und dem BMVIT finanziert. Die Österreichische Forschungsförderungsgesellschaft (FFG) ist mit der rechtlichen und finanziellen Abwicklung betraut. Der Rat für Forschung und Technologieentwicklung (RFTE) koordiniert zusammen mit der Koordinationsstelle der LNF die Inhalte und die Kommunikation zwischen den Ressorts und den Bundesländern. Für die operative Abwicklung in den Regionen sind Vertreterinnen und Vertreter der Bundesländer zuständig. Die nächste "Lange Nacht der Forschung" wird 2020 stattfinden.

Des Weiteren werden im Zusammenhang mit diesem Maßnahmenpaket auch die Beurteilung und Erforschung der gesellschaftlichen Wirkung von Wissenschaft betrieben. Die vom RFTE in Auftrag gegebene explorative Studie „Der gesellschaftliche Impact sozialwissenschaftlichen Wissens in Österreich: Wirkungswege, Messung, Potentiale" widmet sich den universitär verankerten Sozialwissenschaften in Österreich und beleuchtet die Möglichkeiten, den gesellschaftlichen Impact zu erfassen und zu messen. ${ }^{260}$

\section{Ausbau der unabhängigen Technologiefolgenabschätzung}

Seit 2017 unterstützen das Institut für Technikfolgenabschätzung (ITA) der Österreichischen Akade- mie der Wissenschaften und das Austrian Institut of Technology (AIT) für drei Jahre das Parlament mit Beratungsleistungen in den Bereichen Technikfolgenabschätzung und Foresight. ${ }^{261}$ Dazu zählen jährliche Monitorings, in denen laufend über aktuelle Trends und die technischen Auswirkungen auf unseren Alltag berichtet wird. Gesondert werden Studien zu Schwerpunktthemen beauftragt. Das Gesamtbudget des von der Parlamentsdirektion ausgeschriebenen Auftrags beläuft sich auf $200.000 €$ pro Jahr.

Für Umgang mit den Risiken der Nanotechnologien für Umwelt, Gesundheit und Sicherheit wurde, basierend auf dem österreichischen Aktionsplan Nanotechnologie, eine ressortübergreifende Herangehensweise gewählt: BMVIT, BMNT und BMASK haben das ITA bis zum Jahr 2020 mit dem Projekt "Nano-Trust" beauftragt. ${ }^{262}$ Das Nano-Trust-Team identifiziert und thematisiert die dringendsten Fragen zu Sicherheit und Gefahren von Nanotechnologien und bietet eine öffentlich zugängliche Informationsplattform. Im Programm Nano-EHS ${ }^{263}$ werden Projekte zur Erforschung der umwelt- und gesundheitsbezogenen Risiken von synthetischen Nanomaterialien gefördert. An diesem von der FFG abgewickelten Programm wirken neben den genannten Ressorts auch das BMDW und die WKÖ an der strategischen Ausrichtung mit. Das Programm läuft bis 2020 .

\section{Etablierung hoher Standards der wissenschaftlichen Integrität}

In der FTI-Strategie wurden für das Ziel der Etablierung hoher Standards der wissenschaftlichen Integrität vier Maßnahmen formuliert: die Entwicklung strenger Richtlinien im Umgang mit Interessenskonflikten bei der Auftragsforschung, die Offenlegung von Wertesystemen in der Forschung, der verbesserte Zugang zu Informationen über öffentlich finanzier-

259 Vgl. https://www.langenachtderforschung.at/2018/index.html $260 \mathrm{Vgl}$. Felt und Fochler (2018).

261 Vgl. https://www.parlament.gv.at/PAKT/AKT/SCHLTHEM/SCHLAG/J2017/132Technikfolgen.shtml

262 Vgl. https://www.oeaw.ac.at/ita/projekte/nanotrust/ueberblick

$263 \mathrm{Vgl}$. https://www.ffg.at/programme/nano-environment-health-and-safety 
te Forschung und die Stärkung der dafür vorgesehenen Organisationen ${ }^{264}$. In diesem Zusammenhang sei auch das Ziel der Schaffung einer klaren gesetzlichen Regelung von Forschungsethikkommissionen genannt. Dazu werden derzeit Positionen eingeholt; so wurden etwa im Rahmen der Begleitgespräche zu den Leistungsvereinbarungen die Standpunkte der Universitäten erhoben.

\section{Entwicklung strenger Richtlinien im Umgang mit Interessenskonflikten bei der Auftragsforschung und die Offenlegung von Wertesystemen in der Forschung}

Im April 2015 wurden die „Richtlinien zur Guten Wissenschaftlichen Praxis (GPW) “265 von der Kommission für wissenschaftliche Integrität überarbeitet und von der Generalversammlung der Österreichischen Agentur für Wissenschaftliche Integrität (ÖAWI) verabschiedet. Deren Implementierung ist zurzeit in Diskussion. Mit Beschluss der Hochschulkonferenz wurde die Arbeitsgruppe Research Integrity/Research Ethics mit der Erarbeitung von Vorschlägen für die Umsetzung der Richtlinien in Hinblick auf den im April 2017 veröffentlichten European Code of Conduct for Research Integrity ${ }^{266}$ betraut. Ergebnisse werden für Anfang 2020 erwartet.

Österreich hat 2015 „Responsible Science” im „Aktionsplan für einen wettbewerbsfähigen Forschungsraum “267 verankert. Eine darin begründete Maßnahme ist die Gründung der Allianz für Responsible Science ${ }^{268}$, der sich bereits zahlreiche Institutionen aus Wissenschaft, Forschung, Bildung und Praxis angeschlossen haben. Im selben Jahr wurde von überwiegend außeruniversitären Forschungseinrichtungen die „Plattform RR/“269 gegründet. Als Kompetenznetzwerk sammelt sie Erfahrun- gen und Best Practices aus RRI-Projekten, um eine kritische Reflexion und Öffnung der Forschung gegenüber gesellschaftlichen Herausforderungen zu fördern. Über diese Plattform werden auch Studien durchgeführt. So hat das BMBWF eine Use Case Study beauftragt, welche die Prinzipien für verantwortungsvolles Forschen im Forschungsfeld Pflege und Betreuung im Kontext der Aktivitäten des „Netzwerks Altern“ erproben soll. 270 Erste Ergebnisse werden Mitte 2019 vorliegen. Europäische und österreichische Responsible Science-Debatten werden in einem konkreten Forschungs- und Anwendungskontext auf ihre praktische Brauchbarkeit hin geprüft und Probleme und Lösungsansätze der Altersforschung in einen öffentlichen Diskurs über verantwortungsbewusste Forschung übersetzt. Die Mitglieder der „RRI-Plattform“ und des „Netzwerks Altern" bringen ihre jeweiligen Forschungshintergründe ein, um neue Fragestellungen zu generieren und zu erörtern. Im Kontext der neuen österreichischen und europäischen Datenschutzrichtlinien sollen Datengenerierung und -nutzung in einem besonders sensiblen Bereich - ältere Menschen - mit gleichzeitig hohem Markt- bzw. Innovationspotential diskutiert und Vorschläge zur Modifizierung bestehender Praktiken erarbeitet werden.

Seitens der österreichischen Bildungs- und Forschungsinstitutionen wird das Thema Integrität bereits vermehrt in der Praxis implementiert. Die Klagenfurter Erklärung von $2016^{271}$ begründet das informelle österreichische Netzwerk der hochschulischen Ombudsstellen, die in den Bereichen Beratungs-, Beschwerde-, Diversitäts-, Informations-, Konflikt-, Krisen-, Qualitäts-, und Verbesserungsmanagement tätig sind. Durch regelmäßigen Informationsaustausch soll eine Kultur der Fairness weiter etabliert,

264 Vgl. BKA et al. (2011, 43).

$265 \mathrm{Vgl}$. https://oeawi.at/richtlinien/

$266 \mathrm{Vgl}$. All European Academies (2017).

267 Vgl. BMWFW (2015a).

$268 \mathrm{Vgl}$. https://www.responsiblescience.at/

269 Vgl. https://www.rri-plattform.at

270 Vgl. BMWFW (2017b).

271 http://www.hochschulombudsmann.at/wp-content/uploads/2016/06/Klagenfurter-Erkl\%C3\%A4rungfinal_bf.pdf 
die Services professionalisiert und Kompetenzen erweitert werden.

\section{Verbesserter Zugang zu Informationen über öffentlich finanzierte Forschung und die Stärkung der dafür vorgesehenen Organisationen}

Neben der Öffnung der Forschung für gesellschaftliche Herausforderungen ist ein zentrales Anliegen der Europäischen Forschungspolitik die allgemeine Verfügbarmachung von Ergebnissen öffentlich finanzierter Forschung, so sie nicht sensible oder schützenswerte Informationen preisgibt. Mit der „Open Innovation - Open Science - Open to the World"272 Initiative setzte die Europäische Kommission im internationalen Vergleich einen Meilenstein für die Verbesserung der Forschungsqualität sowie der Verwertung wissenschaftlicher Ergebnisse. Transparenz, Integrität, Zusammenarbeit, Verwertbarkeit und soziale Innovationsfähigkeit stehen dabei im Zentrum der Debatte (siehe Abbildung 3-18).

Der derzeit wohl meistdiskutierte Bereich ist Open Access (OA). Damit ist der unbeschränkte und kostenlose Zugang zu wissenschaftlicher Information im Internet gemeint. Dazu zählen in erster Linie wissen- schaftliche Publikationen, aber auch Primär- und Metadaten, Quellentexte und digitale Reproduktionen. Mit Open Access ist der Anspruch verbunden, dass die Ergebnisse öffentlich finanzierter Forschung auch für alle Interessierten öffentlich zugänglich sein sollten. Freie Lizenzen regeln die zusätzlichen Nach- und Weiternutzungsrechte. Die OA-Bewegung reagiert auf die Kritik der unnötigen öffentlichen Mehrfachfinanzierung von Forschung: Wenn öffentlich finanzierte Forscherinnen und Forscher publizieren, dann müssen ebensolche begutachten, damit die Publikationen in Formaten erscheinen, welche wiederum durch öffentlich finanzierte Bibliotheken von Verlagen erworben werden. OA bringt auch den Forschenden selbst viele Vorteile, u.a. die erhöhte Sichtbarkeit und Zitation.

Österreich kommt im Bereich von Open Access und Open Data eine Vorreiterrolle zu und Vertreterinnen und Vertreter diverser Institutionen und Initiativen sind international anerkannte Expertinnen und Experten. Bereits seit 2004 verfolgt der FWF eine Open Access-Strategie und ist Kernmitglied der Taskforce zu „Plan S“, einer Verpflichtungserklärung nationaler Forschungsförderorganisationen und internationaler Charities, sowie der Europäischen Kom-

\section{Abbildung 3-18: Die Bereiche offener Wissenschaft}

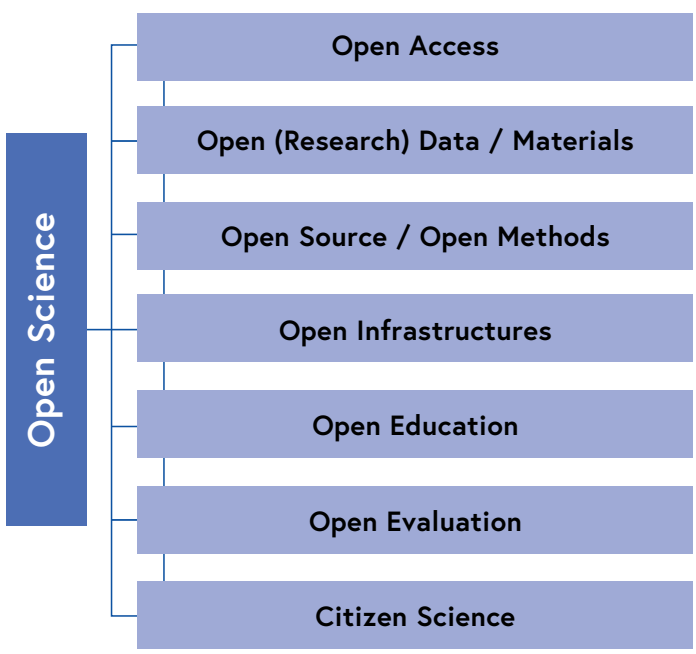

Quelle: Katja Mayer CC BY. Für eine Erklärung der Teilbereiche siehe www.oana.at. 
mission, welche das Ziel anstreben, ab 2020 alle mit ihren Geldern geförderten Publikationen in Open Access-Zeitschriften oder auf Open Access Plattformen zu veröffentlichen. „Plan $S$ “ beschreibt zehn Prinzipien, deren Implementierung zurzeit verhandelt wird und zu der sich die Mitglieder der Koalition verpflichten. Folgend werden die wichtigsten Aktivitäten in Österreich im Bereich OA erläutert.

- Seit 2013 werden vom FWF OA Kosten dokumentiert, seit 2015 wird auch die OA Compliance erhoben. 2017 konnte der FWF eine OA Compliance Rate von $90 \%$ aufweisen. Weiters betreibt der FWF eine Open Access-Journal Initiative als Anschubfinanzierung für hochqualifizierte $O A$ Zeitschriften. Seit 01.01.2019 ist die neue FWF Open Access-Policy um eine verpflichtende Open Research Data Policy und ein verpflichtendes Forschungsdatenmanagement ergänzt. ${ }^{273}$ Die verpflichtende Daten Policy wurde basierend auf den Erfahrungen aus dem Open Research Data Pilot Programm (ORD) ${ }^{274}$ und dem Austausch mit Science Europe entwickelt. ${ }^{275}$

- Das Open Science Network Austria ${ }^{276}$ (vormals Open Access Network Austria - OANA) wurde 2012 als Joint Activity unter dem organisatorischen Dach des Fonds zur Förderung der wissenschaftlichen Forschung (FWF) und der Österreichischen Universitätenkonferenz (UNIKO) gegründet. OANA veröffentlicht regelmäßig Berichte, Empfehlungen und Checklisten. Im jährlichen Rhythmus informiert das Netzwerk in einer öffentlichen Veranstaltung über seine Aktivitäten. 2016 wurden im Ministerrat 16 Open Access-Empfehlungen angenommen. In den Leistungsvereinbarungen der 22 Universitäten findet sich das Bekenntnis zu den Aktivitäten des OANA, verknüpft an die Bereitschaft, Arbeitsgruppen zu beschi- cken. Des Weiteren wird in einer OANA Arbeitsgruppe ein Set an Vorschlägen für eine Open Science-Strategie gemäß den Empfehlungen der EU Kommission und der nationalen ERA Roadmap entwickelt. $^{277}$

- Neben OANA gibt es in Österreich eine Vielzahl an Projekten und Initiativen, die sich mit Open Science beschäftigen. Das österreichische Bibliotheken-Konsortium „Kooperation E-Medien Österreich (KEMÖ)" widmet sich u.a. den koordinierten Erwerb von E-Medien und Nutzungsrechten an EMedien im Rahmen von Konsortien. KEMÖ verhandelt laufend Open Access-Verträge mit Verlagen und kann maßgebliche Erfolge aufweisen. So ist zwar Kostenneutralität noch nicht ganz erreicht, doch 80 \% Rücklauf der Kosten bei den großen Deals bedeuten eine wesentliche Kostenminimierung. Ein weiteres wichtiges Ziel der KEMÖ ist die Erhaltung der Diversität der Wissenschaftslandschaft und die Einbeziehung kleinerer Verlage sowie wissenschaftlicher Dachverbände in Konsortialverträge. So werden österreichweit auch eine Vielzahl an Open Science-Infrastrukturen und Plattformen (mit)finanziert. Aufgrund der Aktivitäten von KEMÖ und den anderen Open Access Initiativen ist Österreich bestens für die Implementation des „Plan S“ vorbereitet.

- Während der Österreichischen EU-Präsidentschaft wurde im Oktober 2018 die European Open Science Cloud (EOSC) feierlich gelauncht. ${ }^{278}$ Mit der EOSC verfolgt Europa das Ziel, eine führende Rolle in der Wissenschaftsdaten-Infrastruktur einzunehmen. Sie ermöglicht europäischen Forscherinnen und Forschern sowie Fachleuten aus Wissenschaft und Technologie ein virtuelles Umfeld mit freiem Zugang, kostenlosen und nahtlosen Services für Speicherung, Management, Analyse und

273 Vgl. https://www.fwf.ac.at/de/forschungsfoerderung/open-access-policy/

274 Vgl. https://zenodo.org/record/803234

275 Vgl. Science Europe (2018).

276 Vgl. https://www.oana.at/

277 Vgl. https://ec.europa.eu/info/news/new-commission-guidance-supports-eu-member-states-transition-open-science2018-apr-25_en

278 Vgl. https://ec.europa.eu/research/openscience/index.cfm?pg=open-science-cloud 
Wiederverwendung von Forschungsdaten, über Grenzen und Forschungsdisziplinen hinweg. Im Rahmen des Launches hat Österreich ein Referenzmodell für die Etablierung von Verwaltung, Services und einer Daten-Architektur innerhalb der European Open Science Cloud präsentiert.

- Im Jänner 2014 wurde das dreijährige, vom BMWFW (heute: BMBWF) geförderte Partnerprojekt eInfrastructures Austria initiiert. Das Projekt verfolgte den koordinierten Aufbau von digitalen Archiv-Infrastrukturen und die Weiterentwicklung von forschungsunterstützenden Services. Durch Vernetzung und Bündelung von Know-how und Ressourcen entstand ein Kompetenznetzwerk, das Hilfestellung bei der Implementierung von Repositorien sowie bei der Erweiterung technischer Systeme, Services und begleitenden Fragestellungen leistet. Das Nachfolgeprojekt „e-Infrastructures Austria Plus" ist ein Projekt von neun österreichischen Universitäten das von 2017-2019 Infrastruktur für eScience in Österreich aufbauen wird. ${ }^{279}$ In sieben Arbeitspaketen werden so unterschiedliche Dinge wie RDM-Policies, "machine-actionable Datenmanagementpläne“, der Aufbau von institutionellen Repositorien für Forschungsdaten, Standards für Metadaten nach dem FAIR Prinzip, Aufbau von DOI (Digital Object Identifier) Infrastruktur und erste Schritte zur Implementierung von Electronic Lab Notebooks behandelt.

- Das vom Bundesministerium für Bildung, Wissenschaft und Forschung geförderte Hochschulraumstrukturmittel-Projekt „Austrian Transition to Open Access (AT2OA)"280 hat das Ziel, die Transformation von Closed zu Open Access bei wissenschaftlichen Publikationen mitzutragen und unterstützende Maßnahmen zu setzen. Im Rahmen der Projektlaufzeit von 2017-2020 sollen durch Neugestaltung der Lizenzverträge mit den Anbietern und durch gezielte Publikationsunterstützung der Forschenden eine Steigerung des österreichischen
Open Access Publikationsoutputs generiert und neue Wege für das Open Access Publizieren eröffnet werden.

- Die disziplinären Repositorien „Austrian Social Science Data Archive (AUSSDA)“ für sozialwissenschaftliche Forschungsdaten und das "Austrian Center for Digital Humanities (ACDH)“ mit seinen Repositorien ARCHE und GAMS für geisteswissenschaftliche Forschungsdaten zeigen exemplarisch, wie nationale Forschungsdatenarchive vermittelt über europäische ESFRI-Forschungsinfrastrukturen zum Aufbau der European Open Science Cloud (EOSC) beitragen. AUSSDA trägt über CESSDA und das ACDH über CLARIN und DARIAH zur EO$S C$ bei. Aktuell arbeitet ein europäisches Konsortium im Rahmen des Horizon2020-Projekts SSHOC am Aufbau von Services für die Geistes-, Sozialund Kulturwissenschaften im Rahmen der EOSC. Neben der Archivierung von Forschungsdaten stellt AUSSDA der sozialwissenschaftlichen Forschungscommunity auch umfangreiche Services, etwa bei der Erstellung von Data Management Plänen (DMPs), zur Verfügung.

Der offene Zugang zu qualitativ hochwertigen Daten ist für die Forschung essentiell. Das Forschungs-Organisationsgesetz (FOG) in der novellierten Fassung von 2018 eröffnet neue Möglichkeiten für wissenschaftliche Forschung an der Schnittstelle von Politik und Gesellschaft. Nach dem Beschluss des Datenschutz-Anpassungsgesetzes 2018 - Wissenschaft und Forschung (WFDSAG 2018) am 20.4.2018 im Nationalrat und der damit verbundenen Novellierung des FOG wurde im BMBWF ein Pilotprojekt gestartet, dessen Zielsetzung darin besteht, anhand eigener Registerdaten die prozessualen, infrastrukturellen sowie legistischen Notwendigkeiten für die transparente und forschungsfreundliche Umsetzung der Registerforschung in Österreich aufzuzeigen. In diesem Zusammenhang wurden sowohl mit der Forschungscommunity als auch mit der Statistik Austria

279 Vgl. https://www.e-infrastructures.at/de/ $280 \mathrm{Vgl}$. https://at2oa.at/home.html 
Abstimmungsgespräche geführt und die Möglichkeiten eines Mikrodatenzentrums für Registerdaten bei der Statistik Austria erörtert. Ebenso wurde eine Informationsveranstaltung für alle Bundesministerien organisiert, um mit ihnen die Erkenntnisse des Pilotprojekts zu teilen und auf Fragen rund um das FOG und die Registerforschung einzugehen. Die österreichische Forschungsgemeinschaft begleitet die Initiative des Gesetzgebers mit der Gründung der Plattform Registerforschung ${ }^{281}$ in 2018 (vgl. Abschnitt 5.1.1).

Ein weiterer Beitrag zur Zugänglichkeit von öffentlich finanzierten Aufträgen im Kontext der FTI-Politik ist das Repositorium der Österreichischen Plattform für Forschungs- und Technologiepolitikevaluierung $(f t e v a l)^{282}$. Dem gemeinsamen Wunsch der fteval-Mitglieder nach einer transparenten Veröffentlichungspolitik folgend, sammelt das fteval-Repositorium Evaluierungsberichte und Studien aus den Bereichen Wissenschafts-, Forschungs-, Technologie und Innovationspolitik in Österreich und macht diese einer interessierten Öffentlichkeit zugänglich.

Forschungsinformationssysteme sind das Rückgrat einer robusten Forschungs- und Innovationspolitik. Der Rechnungshof regte 2016 die Einrichtung einer einheitlichen Forschungsförderungsdatenbank an. Im Auftrag des BMVIT und BMBWF wurde vom RFTE eine Machbarkeitsstudie und eine Kosten-Nutzen-Analyse durchgeführt, welche zu einer Empfehlung für die Einrichtung einer nationalen Datenbank für die umfassende und transparent nachvollziehbare Dokumentation der gesamten Forschungsförderung des Bundes und der Länder führte. ${ }^{283}$ Die Bundesregierung hat das BMF im Einvernehmen mit den anderen in der Task Force FTI-Strategie vertretenen Ressorts beauftragt, ein Projektteam mit der Planung und den vorbereitenden Arbeiten zur Umsetzung einer gebietskörperschaftsübergreifenden
Forschungsförderungsdatenbank einzusetzen und bis zum FTI-Gipfel 2019 ein Umsetzungskonzept vorzulegen.

Erste Schritte in diese Richtung wurden bereits gesetzt: Die seit dem Jahr 2008 webbasierte, öffentlich zugängliche Bundesforschungsdatenbank (BFDAT) wird vom BMBWF betrieben und erfasst die mit Bundesmitteln geförderten Forschungsvorhaben. Das BMVIT schaltete 2018 das „Open4Innovation Portal" frei, auf welchem vor allem Forschungsergebnisse vermittelt und Akteure vernetzt werden.

\subsection{Ergebnisse des OECD Reviews of Innovation Policy in Austria}

Das OECD Review ${ }^{284}$ wurde vom Bundesministerium für Bildung, Wissenschaft und Forschung (BMBWF) sowie vom Bundesministerium für Verkehr, Innovation und Technologie (BMVIT) beauftragt und in der Folge vom OECD Direktorat für Wissenschaft, Technologie und Innovation erarbeitet. Die Präsentation des Endberichtes erfolgte im Rahmen der Europatagung 285 im Dezember 2018.

Das Review zielt darauf ab, ein umfassendes Verständnis der zentralen Elemente, der Beziehungen und Dynamiken im österreichischen Innovationssystem zu erreichen sowie die Möglichkeiten der Politik zur Optimierung des Systems aufzuzeigen. Schließlich soll das Review auch eine Grundlage für die Formulierung der neuen FTI-Strategie 2030 darstellen. Neben einer Zusammenfassung gliedert sich das Review in fünf Kapitel:

- Kapitel 1 („Overall assessment and recommendations") stellt die Charakteristika des österreichischen Innovationssystems sowie die zentralen Befunde und Empfehlungen des Reviews kompakt dar.

$281 \mathrm{Vgl}$. www.registerforschung.at

$282 \mathrm{Vgl}$. https://repository.fteval.at/information.html

283 Vgl. RFTE (2018b).

$284 \mathrm{Vgl}$. OECD (2018a).

$285 \mathrm{Vgl}$. https://era.gv.at/europatagung2018 
- Kapitel 2 („The Austrian innovation system: Evolution and current challenges") skizziert aktuelle Entwicklungen des Innovationssystems anhand zentraler Indikatoren im internationalen Vergleich und diskutiert die damit einhergehenden Herausforderungen.

- Kapitel 3 („Business innovation and Industry 4.0 in Austria") analysiert die zentralen Innovationsakteure und deren Kapazitäten, die öffentliche Unterstützung für Innovation und Unternehmensgründungen und diskutiert das aktuelle Portfolio an politischen Maßnahmen. Besonderes Augenmerk wird auf Industrie 4.0 (inklusive Cloud Computing, Datengenerierung und -verfügbarkeit sowie 5G-Netzwerke) gelegt.

- Kapitel 4 („Improving the performance and attractiveness of higher education institutions and public research institutes in Austria") untersucht die Performance und Attraktivität der Hochschulen und öffentlichen Forschungseinrichtungen. Es zeichnet die Entwicklungen im Hochschulsektor nach und bewertet seine Leistungsfähigkeit bei der Bildung von Humankapital, in der Forschung und hinsichtlich der Verfolgung von "Third Mission"-Aktivitäten. Steuerung und Finanzierung der öffentlichen Universitäten werden ebenso wie die Rollen der verschiedenen öffentlichen Forschungseinrichtungen diskutiert.

- Kapitel 5 („Reconfiguration of science, technology and innovation governance in Austria: Structures for innovation leadership") schließlich widmet sich der Steuerung des Systems. Es bietet einen Überblick über die zentralen Akteure (Ministerien, Förderungsorganisationen, Beratungsorgane) und analysiert Aufgaben, Koordination und Evaluierung einschließlich der Rolle gesellschaftlicher Herausforderungen sowie internationaler Kooperationen.

\section{Ausgangssituation}

Zu Beginn des Reviews steht ein zentraler Befund, aus dem heraus dann die verschiedenen Handlungsfelder ("Main policy challenges") und Maßnahmen („Priority actions“) abgeleitet werden:

Österreich gehört zu den Ländern mit den höchsten F\&E-Intensitäten weltweit. Mit einem Wert von 3,19\% im Jahr 2018 wird Österreich lediglich von Schweden als einzigem EU-Mitglied übertroffen. Die (der Industriestruktur angepasste) F\&E-Intensität des Unternehmenssektors ist OECD-weit führend. Das Wachstum der F\&E-Intensität in den Jahren 1998-2016 nimmt mit fast 1,4 \%-Punkten den zweitgrößten Wert aller OECD-Länder nach Korea ein. Dieser starken Steigerung der F\&E-Ausgaben in den letzten Jahren folgte (bislang) allerdings keine wesentliche Verbesserung der Platzierung Österreichs in den zentralen Innovations-Rankings und kein Aufschließen zu den führenden Ländern in Bezug auf zentrale Output-Indikatoren (einschließlich der Indikatoren zu Digitalisierung und Industrie 4.0). Österreichs Position im internationalen Vergleich blieb damit im Wesentlichen über die Jahre gleich. Das ist u.a. auch darauf rückzuführen, dass die Länder, mit denen sich Österreich unmittelbar vergleicht, sich selbst fortschrittlich entwickelten.

Trotz Weiterentwicklung konnte Österreich in den letzten Jahren jedoch nicht an die Gruppe der Innovation Leader anschließen. Nach dem European Innovation Scoreboard (EIS) $2018^{286}$ findet sich Österreich - wie bereits die Jahre zuvor - im mittleren Feld der "Strong Innovators" auf Rang 10 wieder. Dies lässt sich zum Teil damit begründen, dass das EIS Ökonomien mit hohen Anteilen an High-Tech Sektoren innovativer einstuft als solche mit Innovationsaktivitäten in Sektoren mit mittlerer oder geringer Technologieintensität wie Österreich. ${ }^{287}$ Darüber hinaus verzeichnet Österreich unterdurchschnittli- 
che Bewertungen bei den Kriterien Forschungsexzellenz, Venture Capital Investitionen und intellektuelle Eigentumsrechte.

Ausgehend von diesem Befund formuliert das OECD Review daher die These, dass Österreich eine Reorientierung seiner Forschungs- und Innovationsanstrengungen weg von der Konzentration auf Inputs hin zu einer Konzentration auf Outputs und Wirkungen benötigt: "To join the leading countries in research and innovation, Austria needs a long-term perspective, continued reform efforts and sustained investment that is likely to require adaptation in the mix of policy instruments. In addition, a broader policy approach is required that goes beyond an increase of R\&D intensity."288

Diese Reorientierung wird in der Folge mit Empfehlungen in fünf zentralen Handlungsfeldern („Main policy challenges") konkretisiert:

\section{Handlungsfeld 1: Exzellenz in der Forschung}

Eingedenk der zahlreichen Neuerungen im Hochschulbereich (Unifinanzierung NEU basierend auf Indikatoren, Einführung und Weiterentwicklung des Gesamtösterreichischen Universitätsentwicklungsplans, Schaffung zusätzlicher Karriereperspektiven für Nachwuchswissenschaftlerinnen und Nachwuchswissenschaftler o.ä.) wird empfohlen, das neue System der Universitätsfinanzierung und -steuerung anhand von Indikatoren und Leistungsvereinbarungen so weiterzuentwickeln, dass Anreize für herausragende Forschungsleitungen gesetzt werden. Dazu gehören auch eine Weiterentwicklung der Indikatoren und insbesondere Überlegungen zur Einführung von Outputindikatoren für die Forschung.

Eine weitere zentrale Empfehlung betrifft die Einführung einer Exzellenzinitiative und die Stärkung des kompetitiven Anteils der Finanzierung von Grundlagenforschung. Dies kann durch Steigerungen des FWF-Budgets erreicht werden, wobei die zusätz- lichen Mittel sowohl für existierende Formate als auch für Innovationen im Programmportfolio verwendet werden sollten.

Einen dritten Akzent möchte das OECD Review bei der Rekrutierung arrivierter Forschender wie auch bei der Karriereentwicklung für junge Forschende setzen: Aktuelle Reformen wie die - durch die Adaption des UG im Jahr 2015 ermöglichte - Einführung des neuen "Tenure-track" Modells und die vereinfachte Rekrutierung erfahren eine kritische Würdigung. ${ }^{289}$ Die Implementierung und Handhabung dieser Reformen sollten einem Monitoring unterzogen und weiterentwickelt werden, um die internationale Attraktivität der österreichischen Universitäten weiter zu steigern.

\section{Handlungsfeld 2: Bessere industrielle F\&E-Basis und schnellere Übernahme von Industrie 4.0}

Dieses Handlungsfeld adressiert zwei zentrale Themen: Erstens die Notwendigkeit, innovative Unternehmensgründungen in forschungs- und technologieintensiven Sektoren zu forcieren, die in Österreich traditionell unterrepräsentiert sind. Zweitens den Strukturwandel zu unterstützen, um den Unternehmenssektor stärker und schneller für aktuelle Herausforderungen im Kontext einer zunehmenden Digitalisierung und Industrie 4.0 zu rüsten (Stichwort: Datengenerierung und -nutzung, Robotik, Cloud Computing, Künstliche Intelligenz, Digitale Infrastrukturen etc.).

Bei "Industrie 4.0" handelt es sich um kein genau definiertes technisches Konzept, sondern vielmehr um eine Vielzahl an Phänomenen der Verbindung von industrieller Produktion mit moderner Informationsund Kommunikationstechnik auf Basis intelligenter und vernetzter Systeme. Die Bedeutung einzelner Elemente wie beispielsweise die Verwendung von Industrierobotern, der Einsatz von künstlicher Intelligenz oder von Glasfasertechnologien variiert stark 
von Branche zu Branche. Indikatoren zur Digitalisierung des Unternehmenssektors zeigen, dass sich Österreich auch hier im Mittelfeld befindet. ${ }^{290}$

Entsprechend empfiehlt das Review drei Maßnahmenbündel:

1. Die Stärkung der Unterstützung für innovative Unternehmen mit Wachstumspotential sowie Unternehmensgründungen, um die unternehmerische Forschungsbasis zu erweitern und den Strukturwandel zu beschleunigen;

2. Die Orientierung der öffentlichen Forschungsförderung auf neue technologische Lösungen, neue Kombinationen von Technologien oder die Implementierung neuer wissenschaftlicher Erkenntnisse;

3. Die Ausweitung der F\&E-Kapazitäten in den zentralen Themen von Industrie 4.0 und in strategisch wichtigen Forschungsbereichen (Künstliche Intelligenz, Datenanalytik) sowie die Beschleunigung der Diffusion von Industrie 4.0 Technologien.

\section{Handlungsfeld 3: Humanressourcenbasis von Weltrang}

Trotz der deutlichen Steigerung von Absolventinnen und Absolventen in Naturwissenschaft und Technik und trotz des erfolgreichen Aufbaus des Fachhochschulsektors in den letzten Jahrzehnten existieren immer noch deutliche Schwächen beim Anteil von Frauen in F\&E, bei der Durchlässigkeit zwischen tertiärer und beruflicher Ausbildung, der Doktoratsausbildung, der beruflichen Weiterbildung sowie beim Angebot an Qualifizierungen im Bereich Innovation und Unternehmertum.

Das Review formuliert demnach folgende Empfehlungen:

1. Den weiteren Abbau von Ungleichheiten und Barrieren für Forscherinnen;

2. Die Flexibilisierung und Modularisierung tertiärer und beruflicher Ausbildungen sowie den weiteren Ausbau des Fachhochschulsektors;
3. Den Ausbau der strukturierten Doktoratsausbildung und die bessere Finanzierung der Doktoratsstudierenden.

\section{Handlungsfeld 4: Wissenschaft stärkt Innovation}

Die Zusammenarbeit von Wissenschaft und Wirtschaft ist in Österreich gut etabliert und wird vielfach öffentlich unterstützt - sowohl durch entsprechende Förderungsprogramme als auch durch Infrastrukturen und außeruniversitäre Forschungseinrichtungen. Dennoch ortet das Review hier weiteren Handlungsbedarf hinsichtlich einer strategischeren Ausrichtung: „A key challenge will be to develop new institutional arrangements that provide powerful incentives for path-breaking innovation that links application oriented basic research with industrial innovation across disciplinary boundaries."291

Um dies zu erreichen, schlägt die OECD die folgenden Maßnahmen vor:

1. Die Fokussierung auf weltweit führende und radikale Innovationen in Feldern von hoher strategischer Bedeutung unter aktivem Einbezug von Unternehmen;

2. Die Stärkung der Fähigkeit, themengetriebene Programme zu nutzen. Dies können Programme zur Unterstützung von Forschung und Innovation in neuen Märkten, zur Adressierung gesellschaftlicher Herausforderungen oder zur Verfolgung von Missionen sein;

3. Die Weiterentwicklung des existierenden Netzwerkes außeruniversitärer, technologieorientierter Forschungseinrichtungen (RTOs): Ausweitung ihrer Kapazitäten für herausragende Forschung, Verbesserung ihrer Leistungsbewertung durch ein vergleichbares Set an Indikatoren, Entwicklung einer strategischen und leistungsbasierten Steuerung und Finanzierung.

290 Für eine ausführliche Diskussion von Indikatoren zur Digitalisierung und zu Österreichs Position im internationalen Vergleich siehe auch Abschnitt 1.3.2.

$291 \mathrm{Vgl}$. OECD $(2018 \mathrm{a}, 19)$. 
Handlungsfeld 5: Angemessene Mischung von Maßnahmen und starke politische Steuerung

Österreichs Förderungen für Unternehmensforschung haben sich in den letzten Jahren mit einer zunehmenden Ausrichtung auf die steuerliche Förderung (Forschungsprämie) massiv verändert. Etwa drei Viertel der zusätzlichen F\&E-Ausgaben in den Jahren 2006-2015 sind der Forschungsprämie zuzurechnen. Die Förderungen der FFG wuchsen im gleichen Zeitraum deutlich geringer. Ein weiteres Merkmal des österreichischen Forschungsförderungssystems ist die Dominanz thematisch offener Programme: Mehr als zwei Drittel der öffentlichen Forschungsförderung wird auf themenoffene Formate alloziert (inklusive der Förderungen, die Hochschulen und Forschungseinrichtungen adressieren).

Vor diesem Hintergrund empfiehlt das Review folgende Punkte:

1. Den Instrumentenmix auf künftige Bedarfe und Möglichkeiten anpassen:

- Mehr direkte, kompetitive Förderung für exzellente Forschung und ambitionierte Innovationen (FWF, FFG, andere Forschungsförderungsorganisationen);

- Konzentration auf Unternehmensforschung, die neue technologische Lösungen erforscht oder neue wissenschaftliche Erkenntnisse aufgreift;

- Einen ausgewogenen Mix an Programmen und Instrumenten anstreben, der von niederschwelligen Formaten für KMUs und junge Unternehmen bis zu komplexen F\&E-Kooperationsprogrammen reicht;

2. Gesellschaftliche Herausforderungen adressieren:

- Entwicklung der Kapazitäten, gesellschaftliche Herausforderungen zu adressieren und Missionen zu verfolgen (durch längerfristige Zusammenarbeit zwischen den relevanten Akteuren sowohl in der Grundlagenforschung als auch in der angewandten Forschung);

- Komplementaritäten zwischen nationalen und europäischen Prioritäten ausschöpfen (durch den Abgleich mit den thematischen Feldern von
Horizon Europe und durch die Nutzung des missionsorientierten Ansatzes);

3. Stärkung der FTI-Governance;

- Orientierung des gesamten Innovationssystems auf international sichtbare Exzellenz und große Wirkung; eine neue FTI-Strategie 2020+ kann hier eine Schlüsselrolle spielen und den Rahmen für eine solche Reorientierung bieten;

- Stärkung der operativen Autonomie der Forschungsförderungsgesellschaften (vor allem FFG und aws) bei gleichzeitigem Aufbau strategischer Steuerungskapazitäten in den verantwortlichen Ministerien;

4. Einen starken Rat für Wissenschaft, Forschung und Innovation schaffen;

Dieser eine Rat ist an oberster politischer Ebene verankert (z.B. im Bundeskanzleramt) und hat entweder beratende Funktion oder nimmt Agenden der Politikkoordination und vorausschauenden Entscheidungsfindung wahr;

5. Portfolio Evaluierungen durchführen; Regelmäßige Evaluierungen der Instrumentenportfolios sollen mit einem angemessenen Methodenmix durchgeführt werden und Zugriff auf die dahinter liegenden Daten erlauben.

\subsection{Resümee und Ausblick}

Österreich hat in den vergangenen Jahren einen enormen Aufholprozess im Bereich Forschung, Technologie und Innovation verzeichnet - das ist nicht zuletzt darauf zurückzuführen, dass der Bereich FTI zunehmend im politischen Interesse stand. Als Ausdruck dieses Interesses ist auch die FTI-Strategie, verabschiedet von der Bundesregierung in 2011, zu sehen. Diese Strategie war und ist ein unverkennbares und deutliches Commitment der österreichischen Bundesregierung, Forschung, Technologie und Innovation in allen relevanten Sektoren stärken zu wollen.

Die FTI-Strategie fungierte dabei als Dach für sämtliche Ziele und Maßnahmen, welcher einer För- 
derung, Stärkung, Unterstützung etc. bedurften. Angesichts aktueller Entwicklungen haben sich dann noch Teil-Strategien entwickelt, die zusätzlich bestimmte ausgewählte Themen - sei es z.B. Gründungen, oder sei es Open Innovation - unterstützen und besonders forcieren sollen. Rückblickend lässt sich konstatieren, dass die FTI-Strategie - das akkordierte Vorgehen von Politik und sämtlichen Akteuren im Innovationsystem - gelungen ist. Der Großteil der Ziele wurde erreicht, eine hohe Anzahl von Maßnahmen wurde umgesetzt. Einzig die ganz große Vision der FTI-Strategie 2020, nämlich Österreich (im Europäischen Innovation Scoreboard) als Innovation Leader zu positionieren, konnte nicht erreicht werden.

Österreich hat sich dabei sowohl im Bereich der Wissenschaft als auch im Wirtschafts- und öffentlichen Sektor fortschrittlich entwickelt. Eine zentrale Rolle im Bündeln von Kräften spielte dabei auch die Task Force FTI, das interministerielle Gremium zur Koordination der FTI-Strategie, dessen Ziel es war, die Koordination der mit Forschung, Technologie und Innovation betrauten Ministerien (BMBWF, BMDW und BMVIT) unter Einbindung von BKA und BMF zu verbessern. Die Aufgabe der Task Force FTI, die Begleitung, Konkretisierung und Koordination der Umsetzung der FTI-Strategie sowie die strategische und systemorientierte Abstimmung der Aktivitäten der einzelnen Ressorts, ist nach Einschätzung der Mitglieder außerordentlich gut erfüllt worden. Auch dass die Task Force an oberster Stelle, am Bundeskanzleramt, angesiedelt ist und das BKA eine koordinierende Rolle einnimmt, wird als großer Mehrwert gesehen. Demnach ist die Task Force FTI von der Bundesregierung bereits auch wieder mit der Erarbeitung der neuen FTI Strategie 2030 betraut worden.

Für die Zukunft wird es vor allem darum gehen, bisherige Bemühungen - unter dem Blickwinkel einer gesamtsystemischen Sicht - kontinuierlich weiterzuverfolgen bzw. weiterzuentwickeln und geänderten (bzw. neuen) Rahmenbedingungen entsprechend anzupassen. Als Input dienen - neben dem zuvor dargestellten Review der FTI-Strategie - die Ergebnisse des OECD Reviews und die Leistungsberichte des RFTE, auch bezugnehmend auf die Stärken und Schwächen des österreichischen Innovationssystems.

Als zentral gilt die Forderung, dass auch in Zukunft der Policy Mix in Österreich gut abgestimmt sein muss - zwischen bottom-up und top-down, wie auch zwischen direkter und indirekter Forschungsförderung. Ebenfalls ist - wie von der OECD und dem RFTE festgehalten - eine Verbesserung des Outputs anzustreben (im Sinne einer Verbesserung der Relation von Input zu Output). Tatsächlich weist Österreich heute die zweithöchste Forschungsquote Europas aus, bei so manchen Output-Indikatoren liegt Österreich allerdings nach wie vor im Mittelfeld. Verbunden mit der Frage nach dem gesellschaftlichen Impact gewinnt die Diskussion um den Output damit nochmals eine neue Dimension; das bedeutet aber auch, dass diese Debatte in Zukunft noch viel differenzierter stattfinden muss als bisher. Quantitative Indikatoren werden immer nur ein Teil der Debatte sein.

Ein weiterer wichtiger Aspekt in der österreichischen FTI-Politik ist das Ziel, die Governance laufend zu verbessern bzw. anzupassen. Auch hierzu hat die OECD Empfehlungen gemacht und entsprechend soll auch das Beratungsgremium der Bundesregierung in Zukunft neugestaltet werden. Wie im Regierungsprogramm angekündigt, soll eine Zusammenlegung des Rates für Forschung und Technologieentwicklung, des Wissenschaftsrates und des ERA Council Forums erfolgen. Aus der Synthese dieser drei Gremien soll nach internationalem Vorbild ein neues Beratungsgremium der Bundesregierung, ergänzt um volkswirtschaftliche Kompetenz, entstehen. Zusätzlich soll die Governance des österreichischen Forschungssystems durch das Forschungsfinanzierungsgesetz neu geregelt bzw. optimiert werden. Das Forschungsfinanzierungsgesetz soll dabei vor allem eine längerfristige Finanzierung der zentralen Forschungs- und Forschungsförderungseinrichtungen ermöglichen. Ebenso soll - den Empfehlungen der rezenten aws- 
FFG-Evaluierung folgend - die Steuerung der Förderagenturen durch die Ministerien strategischer werden; gleichzeitig soll den Agenturen mehr operative Autonomie zuerkannt werden. Dieser Schritt ist notwendig, um rasch und effektiv auf neue Herausforderungen reagieren und gleichzeitig auch Schwerpunkte setzen zu können.

Diese Vorhaben sind auch Teil der Zukunftsoffensive $F T I$, welche das BMBWF, das BMVIT und das BMDW gemeinsam auf Basis des Regierungsprogramms als ambitioniertes Paket für die Zukunft von Forschung, Technologie und Innovation in Österreich geschnürt haben. Die Zukunftsoffensive dient vor allem der Effizienzsteigerung mit dem Bestreben, ein hochdynamisches, zukunftsfähiges und mit entsprechenden Strukturen versehenes Innovationssystem in Österreich zu etablieren, und umfasst folgende Punkte:

- Eine neue FTI-Strategie 2030, basierend auf den Prinzipien Exzellenz und Wettbewerb sowie Impact und Offenheit;

- Eine Exzellenzinitiative zur Stärkung und Weiterentwicklung der kompetitiven Grundlagenforschung;

- Zusammenlegung des Rates für Forschung und
Technologieentwicklung, des Wissenschaftsrates und des ERA Council Forums als Beratungsgremium der Bundesregierung ergänzt um volkswirtschaftliche Kompetenz;

- Die Einrichtung einer einheitlichen gesamtösterreichischen Forschungsförderungsdatenbank für eine umfassende und transparent nachvollziehbare Dokumentation der gesamten Forschungsförderung des Bundes und der Länder; ${ }^{292}$

- Den Pakt für Forschung, Technologie und Innovation (FTI-Pakt), welcher basierend auf dem Forschungsfinanzierungsgesetz insbesondere die Finanzierung und die strategische Schwerpunktsetzung der dreijährigen Leistungs- und Finanzierungsvereinbarungen mit den umfassten Einrichtungen festlegt.

Langfristig wird auch die neue FTI-Strategie 2030 von essentiellem Stellenwert sein. So wird es an der Ausgestaltung dieser Strategie liegen, als Rahmen für Politik und FTI-Akteure zu gelten wie auch zur Orientierung der Ausrichtung nationaler Förderungen zu dienen - mit der Vision, in die Gruppe der Innovation Leader vorzurücken.

292 Wobei hier der Schutz von Geschäftsgeheimnissen sichergestellt sein muss. 


.

4
\end{abstract}

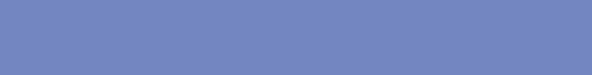

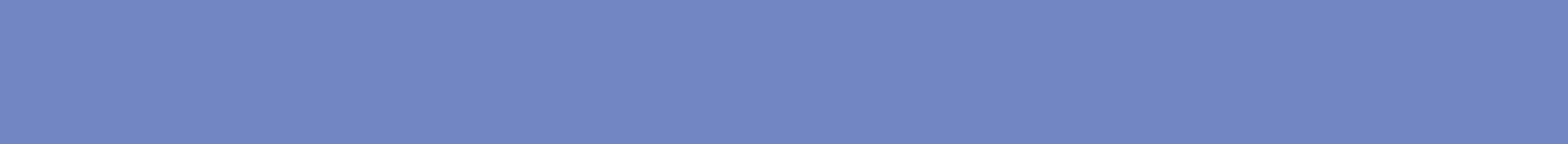

.

.

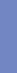

\title{
.
}

(1)

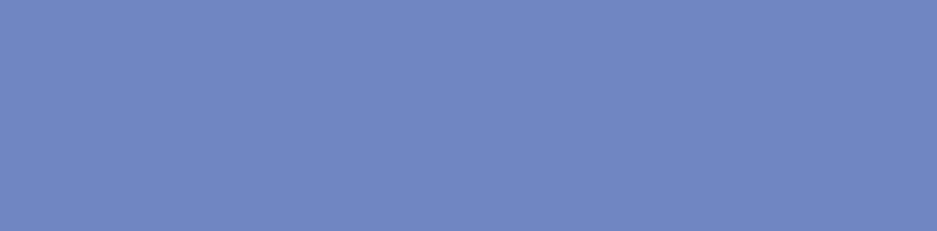
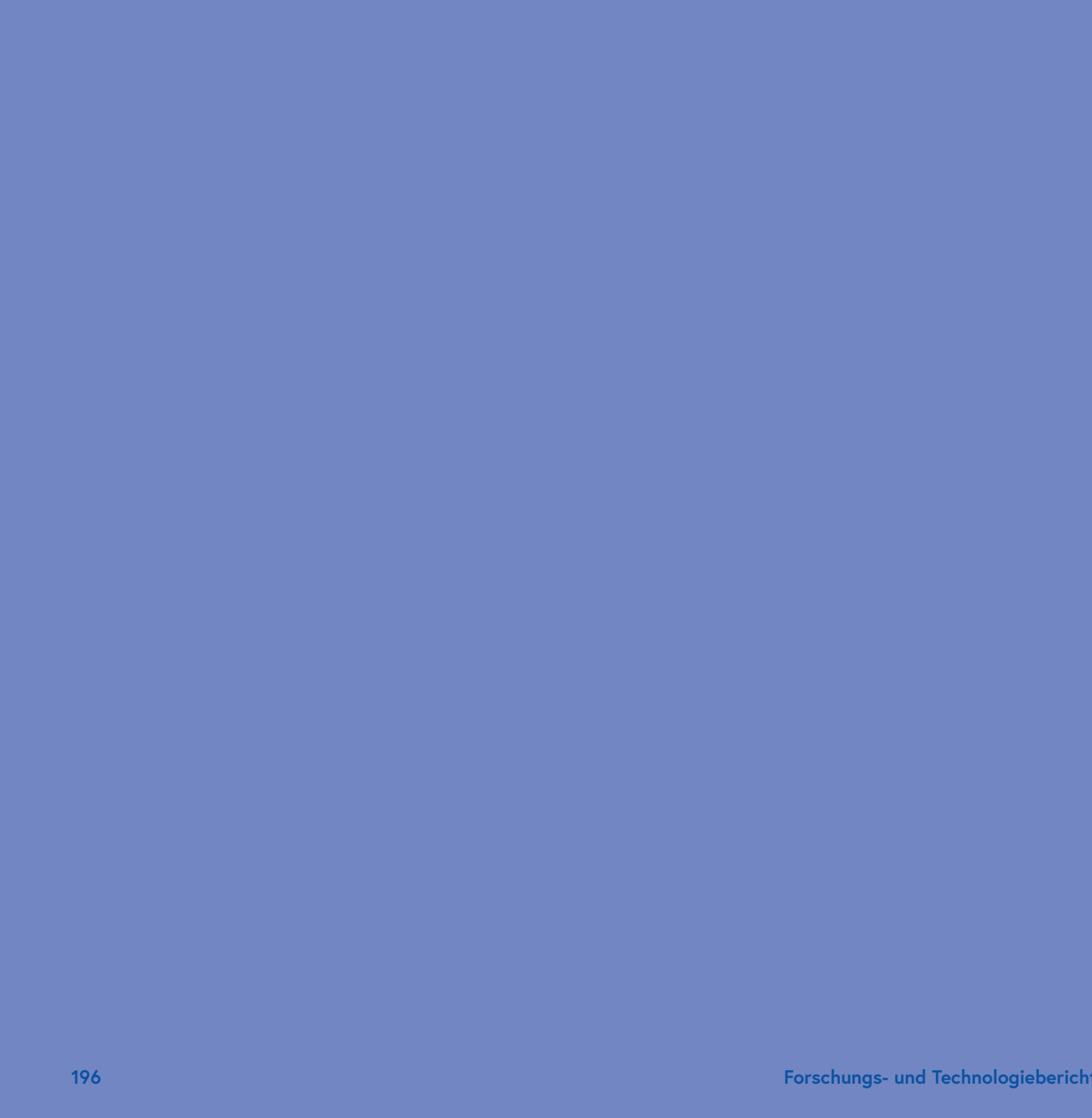
Digitale Infrastrukturen, Produkte und Dienstleistungen führen zu grundlegenden Veränderungen in Wirtschaft, Wissenschaft, Gesellschaft und Politik. Insbesondere der technologische Wandel und Innovationsprozesse werden durch die Digitalisierung substanziell beschleunigt. Aufgrund ihrer umfassenden Bedeutung für die gesellschaftliche und wirtschaftliche Entwicklung kommt der Digitalisierung gegenwärtig höchste Aufmerksamkeit von Seiten der nationalen und internationalen Politik zu. Dies betrifft sowohl die Unterstützung des Bildungssektors als auch die Förderung des Unternehmenssektors und den Ausbau der öffentlichen Infrastruktur hinsichtlich verstärkter Nutzung digitaler Technologien und moderner Kommunikationsdienste; letzteres wird mit der "Digital Roadmap Austria“ umfassend unterstützt.

\subsection{Status-quo der Umsetzung der Digital Roadmap Austria}

Wie in Abschnitt 1.3 dargestellt führt die Europäische Kommission ein laufendes Monitoring der Fortschritte bei der Verbreitung digitaler Technologien in Wirtschaft und Gesellschaft durch; mithilfe des „Digital Economy and Society Index" (DESI) wird ein quantitativer Vergleich unter den Mitgliedstaaten vorgenommen. Im Jahr 2018 erzielte Österreich in diesem Ranking lediglich ein durchschnittliches Ergebnis; d.h. im Gesamtindex lag Österreich auf dem 11. Rang unter allen EU-Mitgliedstaaten. Dabei war gerade die Internetnutzung im Vergleich zu anderen Ländern unterdurchschnittlich. Zwar sind leichte Verbesserungen im Vergleich zum Vorjahr zu erkennen, im Ranking der EU-Mitgliedsstaaten für 2017 konnte Österreich seine Position allerdings nicht verbessern. ${ }^{1}$ Während Österreich bei den digitalen öffentlichen Diensten besonders gut abschneidet und zu den besten fünf innerhalb der EU zählt, ist die Nut- zung von Internetdiensten weniger fortgeschritten. Auch im Jahr 2016 rangierte Österreich im Mittelfeld und belegte lediglich den 10. Platz. ${ }^{2}$

Die mäßige Ausgangsposition in diesem zentralen Politikbereich stellte den Anstoß für die Entwicklung der "Digital Roadmap“ dar. Mit der Digital Roadmap Austria $^{3}$ wurde im Dezember 2016 der Startschuss zu einer gemeinsamen Digitalisierungsstrategie der damaligen Bundesregierung gelegt und erstmals die Aktivitäten aller Ressorts in einem gemeinsamen Strategiepapier der Bundesregierung gebündelt. An der Erstellung der Digital Roadmap haben mehr als 100 Expertinnen und Experten aus allen Ministerien, den Bundesländern, dem Städte- und Gemeindebund sowie Sozialpartner und andere Organisationen mitgewirkt. Im Anschluss beteiligten sich zahlreiche Bürgerinnen und Bürger an einem Online-Konsultationsprozess. Das daraus entstandene Konsultationspapier war die inhaltliche Basis für die Digital Roadmap Austria. Die Roadmap gibt einen Überblick über die aktuellen Herausforderungen und orientiert sich an zwölf Leitprinzipien für die Gestaltung bzw. Umsetzung der Digitalisierung in Österreich. In Summe enthält die Digital Roadmap zwölf Handlungsfelder mit rd. 150 Maßnahmen. Die Digital Roadmap wird als wichtiger Wegweiser in die digitale Zukunft gesehen und stellt damit zugleich die Grundlage für die in Ausarbeitung befindliche neue „Digitalisierungsstrategie" dar.

Vom digitalen Wandel werden alle Bereiche der Wirtschaft direkt oder indirekt beeinflusst. Digitale Technologien ermöglichen neue Geschäftsmodelle, Produkte und Dienstleistungen. Für die Industrie relevant ist insbesondere die Frage, wie bestehende Fertigungs- und Geschäftsprozesse entlang der Wertschöpfungskette mit der Informations- und Kommunikationstechnologie verknüpfbar sind; die Entwicklungen im Bereich „Industrie 4.0“ sind nur ein Ausdruck hiervon.

Mit dem Regierungsprogramm für die Jahre 2017-

\footnotetext{
Vgl. Europäische Kommission (2019a).

Vgl. Europäische Kommission (2018d).

Vgl. www.digitalroadmap.gv.at
} 
2022 wurde der Digitalisierung eine zentrale Bedeutung zuerkannt. Es wurde ein eigenes koordinierendes Ressort, das Bundesministerium für Digitalisierung und Wirtschaftsstandort (BMDW), geschaffen, und in jedem Ministerium eine Verantwortlichkeit für digitale Angelegenheiten über einen "Chief Digital Officer" (CDO) etabliert. Die Koordinierung der Aktivitäten im Bereich der Digitalisierung erfolgt über die interministerielle Taskforce der CDOs unter der Leitung des BMDW. Zur Unterstützung der Digitalisierungsinitiativen der Bundesregierung wurde zudem eine „Digitalisierungsagentur" gegründet, die institutionell in der Forschungsförderungsgesellschaft (FFG) angesiedelt ist.

Im Zuge einer breit angelegten Stakeholder-Befragung wurden schließlich konkrete Maßnahmen im Rahmen der Handlungsfelder Infrastruktur und Anwendungen als Grundlage für die Strategieentwicklung erarbeitet. Von 34 Maßnahmen für die Handlungsfelder Infrastruktur und Anwendungen dienen 24 konkret der Erleichterung und der deutlichen Kostensenkung des Ausbaus der digitalen Infrastruktur. Weitere zehn Maßnahmen haben das Ziel, die technischen Möglichkeiten und Potentiale von $5 \mathrm{G}$ für Wirtschaft und Gesellschaft nutzbar zu machen, indem sie eine zeitnahe Entwicklung von 5G-Diensten und -Anwendungen unterstützen. Die Entwicklung dieser Anwendungen soll mitunter durch gezielte Förderprogramme, Innovationskooperationen, Testumgebungen und die innovationsfördernde öffentliche Beschaffung (IÖB) vorangetrieben werden.

Tatsächlich schreitet der Ausbau der digitalen Infrastruktur in Österreich zügig voran. Im Rahmen der "Breitbandinitiative“ der Bundesregierung konnten bisher 3.100 Standorte in mehr als $1.100 \mathrm{Ge}$ meinden mit Glasfaser angebunden werden. Die Breitbandinitiative, die im November 2015 von der Europäischen Kommission notifiziert wurde, läuft mit dem Ausschreibungszyklus bis Ende des Jahres 2020. Die vollständige Umsetzung aller damit ver- bundenen Projekte wird voraussichtlich bis Ende 2025 andauern.

Im Bereich der öffentlichen Hand wurde des Weiteren eine Reihe von Maßnahmen, wie z.B. die Einrichtung eines elektronischen "One-Stop-Shop“ für Unternehmensgründungen zur Vereinfachung der Verfahrensschritte bei einer Gründung und zur Verkürzung der Gründungsdauer, eine komfortable digitale Plattformoberfläche für die öffentliche Auftragsvergabe, oder auch die Einführung des Programms „KMU.digital“4 zwecks Unterstützung der digitalen Transformation von Klein- und Mittelunternehmen durch die gezielte Förderung von Beratungs- und Qualifizierungsmaßnahmen mittlerweile umgesetzt. Gleichzeitig werden strategische Zukunftssektoren im Bereich der Digitalisierung gestärkt. Bereiche wie Big Data und Data Science, Cloud-Computing, Quantentechnologie oder Cybersecurity werden hierzu gezielt mit spezifischen Programmen unterstützt.

Um das Potential der Digitalisierung voll ausschöpfen zu können, liegt ein weiterer Schwerpunkt der aktuellen Bemühungen in der Verbesserung der Fähigkeiten und Qualifizierung der Bevölkerung. Folglich werden der Auf- und Ausbau digitaler Fähigkeiten und Kompetenzen durch eine Intensivierung der IKT-Fachkräfteausbildung und der beruflichen Weiterbildung angestrebt; im tertiären Sektor soll dies insbesondere durch einen Ausbau der MINT-Fächer gelingen. Auf breiterer Ebene, also alle Bevölkerungsschichten umfassend, spielt die Initiative der Bundesregierung "fit4internet" eine prominente Rol$1 e^{5}$. In diesem Zusammenhang wurde auf Basis des europäischen Referenzrahmens für digitale Kompetenzen mit DigComp 2.2 AT ein digitales Kompetenzmodell für Österreich entwickelt. Es dient der Einordnung und Vergleichbarkeit digitaler Kompetenzen. DigComp 2.2 AT gliedert das Feld der digitalen Kompetenzen in sechs Bereiche (Grundlagen und Zugang, Umgang mit Informationen und Daten, Kommunikation und Zusammenarbeit, Kreation digitaler Inhalte,

4 Vgl. https://www.wko.at/Content.Node/kampagnen/KMU-digital/index.html

5 Vgl. https://www.fit4internet.at/ 
Sicherheit, Problemlösen und Weiterlernen) und beschreibt diese näher in insgesamt 25 einzelnen Kompetenzen. Seitens des BMDW wurde auch eine interdisziplinäre österreichische Taskforce als Beratungsorgan eingerichtet. Die Taskforce arbeitet daran, dass das DigComp.at-Referenzmodell eine möglichst breit akzeptierte und kohärente Arbeitsgrundlage zur Entwicklung und Verbesserung digitaler Kompetenzen aller Bürgerinnen und Bürger ist bzw. wird. Zu diesem Zweck aktualisiert die Taskforce das Referenzmodell laufend, unterstützt die Vernetzung relevanter Stakeholder und trägt zur Qualitätssicherung von Projekten und Maßnahmen bei. ${ }^{6}$

Außerdem plant die Bundesregierung, in Zukunft Berufsrollenbilder zu monitoren und vor diesem Hintergrund insbesondere die Lehrberufe an die Anforderungen der digitalen Arbeitswelt laufend anzupassen bzw. diese an aktuelle Entwicklungen auszurichten.

Insgesamt haben sich damit die Bemühungen der österreichischen Bundesregierung um Verbesserungen der Rahmenbedingungen für die Entwicklung der digitalen Technologien spürbar intensiviert. Die Schaffung eines federführenden Ressorts und einer Taskforce für Digitalisierung, sowie die Gründung einer Digitalisierungsagentur haben die Dynamik bei der Implementierung der in der Digital Roadmap vorgesehenen Maßnahmen deutlich belebt.

\subsection{Digitale Transformation, Digitalisierung und Industrie 4.0 in Österreichs Unternehmen}

Der digitale Wandel von Wirtschaft, Gesellschaft und Verwaltung spielt für die Entwicklung des Standorts Österreich eine entscheidende Rolle. Der folgende Abschnitt gibt einen kurzen Überblick zum Status-quo der digitalen Transformation in Österreichs Unternehmen, insbesondere was KMU betrifft, und zeigt anhand der Digital Innovation Hubs (DIH) exemplarisch wie Unternehmen auf dem Weg zur Digitalisierung auf innovative Art unterstützt werden können. Ebenso birgt die Digitalisierung der Produktion (Industrie 4.0) großes Potential; hier geht es vor allem darum, Chancen rasch zu erkennen und neue technologische Möglichkeiten zu nutzen, um die Innovationsfähigkeit zu stärken. In diesem Zusammenhang werden auch Forschungsfelder aufgezeigt, die für die Technologieentwicklung in Österreich zentral sind und die als wichtige Treiber von Industrie 4.0 und Digitalisierung gesehen werden. Schließlich wird in diesem Kontext auch auf den Prozess zur Ausarbeitung einer österreichischen Robotik- und KI-Strategie eingegangen.

\section{Digitale Transformation in Österreichs}

\section{Unternehmen - Status-quo}

Der Status der Digitalisierung in der österreichischen Volkswirtschaft lässt sich unter anderem an der Frage des unternehmerischen Einsatzes bestimmter Technologien und Techniken, die im Zentrum des technologischen Wandels stehen, aufzeigen. Die 2018 veröffentlichten Ergebnisse der europaweit durchgeführten Erhebung zum IKT-Einsatz in Unternehmen ${ }^{7}$ enthält dabei auch für Österreich einige zentrale Erkenntnisse: Zunächst wird deutlich, dass Österreichs Unternehmen (welche mehr als zehn Mitarbeiterinnen und Mitarbeiter umfassen; der Finanzsektor ausgenommen) bei der Analyse so genannter Big Data im Gesamtdurchschnitt deutlich weniger aktiv sind als der Durchschnitt der EU-28: $6 \%$ der österreichischen Unternehmen führen diese Art von Analysen durch, im Unterschied zu $12 \%$ in der gesamten EU. Dieses Ergebnis lässt sich vor allem darauf zurückführen, dass Österreichs KMU deutlich hinter dem EU-Durchschnitt liegen $(6 \%$ im Vergleich zu $12 \%$ ). Unter den österreichischen Großunternehmen fällt der Abstand mit $29 \%$ zu $33 \%$ deutlich geringer aus. Dennoch kann die österreichi-

\footnotetext{
6 Vgl. BMDW (2018).

7 Vgl. https://ec.europa.eu/eurostat/web/digital-economy-and-society/data/database
} 
sche Wirtschaftsstruktur nur zum Teil als Erklärung herangezogen werden, liegt der Anteil der Unternehmen mit Big Data-Analysen doch in allen Wirtschaftsbereichen hinter dem EU-28 Wert. Dies verdeutlicht auch die Analyse der Frage, inwieweit Unternehmen in Österreich sich mit eigenen Mitteln und Beschäftigten Analysen von Big Data widmen: In Österreich ist dies nur in $4 \%$ aller Unternehmen der Fall, in der EU-28 hingegen liegt der Durchschnittswert bei $8 \%$. Wiederum gilt für KMU derselbe Wert; Großunternehmen liegen mit einem Anteil von $25 \%$ leicht hinter dem EU-Durchschnitt (29\%).

Weiters wird der Einsatz von Robotern in Unternehmen als Indikator für die Durchdringung der österreichischen Volkswirtschaft mit digitalen Technologien herangezogen. Dabei gilt es, zwischen "klassischen“ Industrierobotern, wie sie bereits seit Jahrzehnten in teilautomatisierten Wirtschaftsbereichen genutzt werden, und „intelligenten“ Robotern, wie sie für die vollautomatisierten Prozesse der Industrie 4.0 zentral sind, zu unterscheiden. Die EU selbst unterscheidet in der IKT-Erhebung allerdings nur zwischen Industrie- und so genannten Servicerobotern. Nimmt man letztere in den Fokus, in der berechtigten Annahme, dass diese eher auf dem Einsatz künstlicher Intelligenz beruhen und komplexere Aufgaben automatisieren, dann ergibt sich dadurch ein weiterer Einblick auf den Stand der Digitalisierung Österreichs: In 2018 haben 2 \% aller österreichischen Unternehmen Service-Roboter eingesetzt, was dem Durchschnitt der EU-28 entspricht. Allerdings ist der Anteil unter den $\mathrm{KMU}$ mit $1 \%$ nur halb so groß wie in der EU insgesamt. Unter den Großunternehmen liegt Österreich mit einem Anteil von $10 \%$ sogar leicht über dem europäischen Wert (9\%). Anders als im Bereich Big Data gibt es in Österreich damit Wirtschaftsbereiche, die über dem europäischen Durchschnitt (z.B. Information und Kommunikation oder Metallerzeugung und -bearbeitung, Herstellung von Metallerzeugnissen) oder unter dem europäischen Durch- schnitt (z.B. Mineralölverarbeitung, Herstellung von chemischen und pharmazeutischen Erzeugnissen, Gummi- und Kunststoffwaren, Glas und Glaswaren, Keramik, Verarbeitung von Steinen und Erde oder Verkehr und Lagerei) liegen.

Der Status der digitalen Transformation von KMU in Österreich wurde 2017 und 2018 eigens auch im Auftrag der Wirtschaftskammer Österreich untersucht. ${ }^{8}$ Die Ergebnisse dieser Studien zeigen, dass sich in Österreich die Unternehmen aus der Bankenund Versicherungsbranche zu digitalen Vorreitern entwickelt und damit auch Unternehmen aus dem engeren IKT-Bereich abgelöst haben. Den geringsten Digitalisierungsindexwert weisen KMU aus den Branchen Transport und Verkehr sowie Gewerbe und Handwerk auf. Insgesamt ist es in der Einschätzung der Unternehmen jedenfalls zu einer Intensivierung von Digitalisierung und Ausweitung auf alle Bereiche unternehmerischen Handelns (Produktion, Arbeitsbeziehungen, Marketing usw.) gekommen, was auch die Umsätze über digitale Kanäle betrifft. In der Selbsteinschätzung der KMU sieht sich allerdings eine deutliche Mehrheit als digitale Neulinge oder schätzt sich lediglich als "digital bewusst" ein (der Anteil in den beiden als "digital orientiert" bzw. "digitaler Champion" bezeichneten Kategorien ist folglich entsprechend gering bzw. liegt teilweise bei $0 \%$ ). Zentrale Herausforderungen für KMU entstehen offenbar vor allem aus Unsicherheiten im Zusammenhang mit dem Datenschutz, fehlendem Know-how, aber auch aufgrund mangelnder eigener Ressourcen für Digitalisierungsprojekte. Über alle Branchen hinweg sind es etwa $40 \%$ der österreichischen KMU, die bislang noch keinerlei Anpassung ihres Portfolios an die durch Digitalisierung veränderten Märkte vorgenommen haben.

Eine von Ernst \& Young durchgeführte und 2018 veröffentlichte Befragung zu „Industrie 4.0 im österreichischen Mittelstand “9 gibt ebenso Einblicke in Teilaspekte der Digitalisierung. Demzufolge ist bei $23 \%$ der befragten Industrieunternehmen die Ferti-

8 Vgl. Arthur D. Little (2017a); Arthur D. Little (2018).

9 Vgl. Ernst \& Young (2018). 
gung schon jetzt mithilfe digitaler Technologien ganz oder teilweise vernetzt, und in mehr als einem Drittel der befragten Unternehmen werden zumindest Teile der Produktion schon digital gesteuert. Demgegenüber nutzt jedes vierte Unternehmen derzeit keine Fertigung mithilfe digitaler Technologien und plant dies auch künftig nicht. Allerdings wird auch hier ein deutlicher Anstieg erwartet und soll dies mit entsprechenden Investitionen vorangetrieben werden, wobei sich kleinere Unternehmen deutlich weniger aktiv zeigen als Großunternehmen.

\section{Digital Innovation Hubs (DIH)}

Um von der Digitalisierung profitieren zu können, müssen Unternehmen lernen, digitale Technologien in den Unternehmensalltag und die Unternehmenstätigkeit zu integrieren. Besonders bei KMU besteht die Notwendigkeit und auch das Interesse, sich zu informieren, zu testen und zu experimentieren. Erst dann können sie grundlegende Unternehmensentscheidungen für digitale Innovationen treffen, um neue Geschäfts- und Arbeitsmodelle voranzubringen. Hierfür benötigen sie Zugang zu neuen Technologien und Expertise, den Aufbau ausreichenden Wissens auf Unternehmensebene und die Weiterbildung der eigenen Mitarbeiterinnen und Mitarbeiter.

Um die KMU bei diesen Herausforderungen entsprechend zu unterstützen, hat das BMDW die Digital Innovation Hubs (DIH) ins Leben gerufen, ein Förderprogramm, welches von der Österreichischen Forschungsförderungsgesellschaft (FFG) administriert wird. ${ }^{10}$ Ziel ist es, insbesondere KMU bei der Digitalisierung zu unterstützen, indem in Österreich mehrere DIH jeweils als Netzwerk von bereits existierenden Einrichtungen (sogenannte „Digitalzentren“) geschaffen werden, die ihre Expertise und Infrastruktur den KMU für deren Transformationsprozess zur Verfügung stellen.

Das nationale DIH-Programm verfolgt mit neuen Interventionen bzw. mit der Anknüpfung an beste- hende Maßnahmen damit eine Agenda wie folgt dargestellt und knüpft damit auch an die auf europäischer Ebene formulierte Initiative "Digitizing European Industry" (DEI) an:11

- Mobilisierung österreichischer KMU zur aktiven Teilnahme am digitalen Wandel, um Produktivitäts-, Innovations- und Wertschöpfungspotentiale zu heben sowie die Wettbewerbsfähigkeit durch den Einsatz von Digitalisierungs-Technologien zu stärken;

- Bereitstellung eines institutionalisierten Zugangs für KMU zu Expertisen und Know-how zu Digitalisierung sowie Wissenstransfer in die Unternehmen über Weiterbildungsmaßnahmen;

- Unterstützung von Digitalisierungs-Innovationen in KMU durch Zugang zu Infrastruktur, ErschlieBung neuer Geschäftsmodelle, gemeinschaftliche F\&E sowie Entwicklung von Prototypen für Digitalisierungs-Anwendungen;

- Verbesserte Einbindung der österreichischen Kompetenzträger in europäische Netzwerke und erfolgreichere Teilnahme an einschlägigen europäischen Initiativen.

Ein DIH besteht aus mindestens drei Einrichtungen mit Forschungsschwerpunkten im Digitalisierungsbereich (z.B. Universitäten, Fachhochschulen, Intermediäre, Kompetenzzentren, Forschungseinrichtungen, Unternehmen), die ein sogenanntes Digitalzentrum bilden. In Hinblick auf das Ausschreibungsziel der regionalen Abdeckung sind Konsortien explizit eingeladen, DIH-Hub-Projekte mit mehr als drei Digitalzentren einzureichen.

Im Rahmen der ersten Ausschreibung steht ein Gesamtbudget in Höhe von drei Mio. $€$ zur Verfügung, womit folgende Ziele adressiert werden:

- Große regionale Abdeckung: Ein Großteil der österreichischen KMU soll einen Knoten des Hubs („Digitalzentrum“) als ersten Ansprechpartner in ihrer direkten Umgebung haben;

- Inhaltliche Abstimmung des Angebots auf regio-

10 Vgl. https://www.ffg.at/dih/1.Ausschreibung2018

11 Vgl. Europäische Kommission (2018a). 
nale Bedürfnisse: Besonders zur Einreichung aufgerufen sind Konsortien, die einen Schwerpunkt ihres Angebots auf einen der Themenbereiche Künstliche Intelligenz (KI), Security, Blockchain und 3D-Printing legen;

- Vorbereitung der österreichischen Stakeholder auf die Beteiligung an entsprechenden europäischen Innovation-Hub-Initiativen.

\section{Zentrale Forschungsfelder - Technologie- Roadmap Industrie 4.0}

F\&E ist ein wichtiger Treiber im Kontext von Industrie 4.0 und Digitalisierung. Aus diesem Grund haben mehr als 70 Vertreterinnen und Vertreter aus Politik, Wirtschaft, Wissenschaft und Arbeitnehmerseite in der Arbeitsgruppe „Forschung, Entwicklung \& Innovation" der Plattform Industrie 4.0 Österreich ${ }^{12}$ eine Technologie-Roadmap erarbeitet, in der acht ineinandergreifende Forschungsfelder identifiziert wurden, die für die Technologieentwicklung in Österreich zukünftig zentral sind.

Die Arbeitsgruppe wurde eingerichtet, um Empfehlungen für den F\&E-Bereich zu erarbeiten und relevante Akteure zu vernetzen. Dabei sollen der Politik und Unternehmen Inputs geliefert werden, in welche Richtung die Entwicklung geht, um schließlich rechtzeitig geeignete Maßnahmen ergreifen zu können. Gemeinsam soll das Bewusstsein um die Bedeutung von Forschung, Entwicklung und Innovation gesteigert sowie Forschungseinrichtungen und Unternehmen dahingehend unterstützt werden, dass sie mit optimalen Rahmenbedingungen erfolgreich arbeiten können. Aufgrund der zu erwartenden weitreichenden Veränderungen durch Industrie 4.0 und dadurch entstehende Auswirkungen auf die Gesellschaft wurde daher ein interdisziplinärer Ansatz bei der Erarbeitung der behandelten Themen gewählt, der nicht nur technische Aspekte adressiert.
Die Roadmap stellt Gebiete dar, in denen Forschungsbestrebungen zu intensivieren sind, um für die Umsetzung von Industrie 4.0 geeignete Lösungen anbieten zu können. Sie soll als Orientierungshilfe dienen, in welchen Bereichen Weiterentwicklung sinnvoll und notwendig ist, um Österreichs Position im globalen Wettbewerb zu stärken. Konkret handelt es sich dabei um folgende Forschungsfelder ${ }^{13}$ :

- Virtualisierung: Präzise digitale Abbildungen, wie z.B. ein digitaler Zwilling, eine digitale Fabrik etc., bilden eine notwendige Voraussetzung für Industrie 4.0 und sind ohne Modellierung und Simulation undenkbar. Daher muss bereits vor der Produktion daran gearbeitet werden, anhand eines Modells Produkteigenschaften und Produktionsabläufe vorherzusagen, diese zu steuern und zu verfolgen.

- Sensorsysteme: Messsysteme liefern wichtige Informationen für die Produktion und gewinnen damit vor allem für die Qualitätskontrolle zunehmend an Bedeutung. In Zukunft müssen Sensoren intelligenter (z.B. via Selbstdiagnose, vorausschauende Instandhaltung) und energieeffizienter werden.

- Software Engineering: Die Querschnittstechnologie Software zählt zu den Schlüsseltechnologien für den Transformationsprozess im Bereich der Industrie 4.0. Mit Hilfe von Software wird erst das intelligente Kombinieren von Algorithmen, Sensoren sowie deren gemessenen Daten, physikalischer Objekte und cyber-physischer Systeme ermöglicht. Um sich den immer schneller ändernden Anforderungen in der Produktion anpassen zu können, muss die Querschnittstechnologie Software in Zukunft adaptiver werden.

- Physische Systeme: Die Vernetzung mit Informationstechnologie und Sensorik schafft neue Chancen und Herausforderungen für die physische Repräsentanz "smarter" Maschinenkonzepte. Smarte Maschinen und Roboter werden zentral für die Pro-

12 Der Verein „Industrie 4.0 Österreich“ wurde 2015 als Initiative des BMVIT sowie von Arbeitgeber- und Arbeitnehmerverbänden gegründet; vgl. https://plattformindustrie40.at/uber-den-verein-2/ und Verein Industrie 4.0 Österreich - die Plattform für intelligente Produktion (2018).

13 Vgl. https://plattformindustrie40.at/ergebnispapier-forschung-entwicklung-innovation-in-der-industrie-4-0-praesentiert/ 
duktion, durch additive Fertigung (3D-Druck) kann schneller und sicherer produziert werden. Auch neue Oberflächenbehandlungen zur Funktionalisierung und Werkstoffe (Keramiken, Kunststoffe, Metalle, Verbundstoffe) für Industrie 4.0 mit optimierten Eigenschaften halten Einzug in die Produktion. Um Smarte Logistik zu verwirklichen, bedarf es in Zukunft auch der Entwicklung autonomer, zellularer, selbststeuernder Transportsysteme.

- Cyber-Physical-Systems (CPS): Ein wesentliches Merkmal von CPS ist, mit verschiedenen Umgebungen und damit auch Sensortechnologien in Kontakt treten zu können und die (kognitive) Fähigkeit, effektiv mit verschiedenen Arten von Endbenutzern auf verschiedenen Qualifikations- und Kompetenzniveaus durch den Einsatz von MenschMaschine-Schnittstellen zu kommunizieren. Maschinen werden zukünftig häufiger mit Menschen interagieren und zusammenarbeiten. Dadurch werden neue intelligente Produktionssysteme geschaffen. Folglich besteht in diesem Zusammenhang auch Bedarf an einer neuen theoretischen Grundlage für die Erforschung und Entwicklung großer, verteilter, komplexer Systeme.

- Arbeits- und Assistenzsysteme: Die Einführung von umfassenden Industrie-4.0-Technologien wirft die Frage nach der bestmöglichen Gestaltung der Zusammenarbeit zwischen Mensch und Maschine auf. Derzeitige Entwicklungen konzentrieren sich auf die Verbesserung der Benutzerschnittstelle zwischen Mensch und Maschine mit dem Ziel der aktiven Arbeitserleichterung in Echtzeit (z.B. durch Visualisierung mit Hilfe von Augmented Reality). Das wird auch Auswirkungen auf die Arbeitsorganisation und Kompetenzen haben, wofür noch Bewusstsein und Akzeptanz geschaffen werden muss. Gerade die Frage, wie arbeitsorganisatorische Handlungsspielräume optimal genutzt werden können, eröffnet ein großes Spektrum an noch zu beantwortenden Fragen.
- Wertschöpfungsnetzwerke und Geschäftsmodelle: Industrie 4.0 verändert die Wertschöpfung und Geschäftsmodelle - reine Produzenten werden zunehmend zu produzierenden Serviceanbietern. Datengetriebene und -basierte Modelle sind der Schlüssel hierzu. Um diese Chancen zu nutzen, bedarf es einer neuen Herangehensweise in vielen Prozessen, wie der Erstellung und Bewertung von Wertemodellen sowie der Definition von benötigten Daten.

- Domänenwissen und Schlüsseltechnologien: Domänenwissen bezeichnet das bereits vorhandene Wissen in einem Anwendungsgebiet. Die erfolgreiche Einbettung von Industrie-4.0-Technologien in die Produktion erfordert eine verstärkte Berücksichtigung des erforderlichen Prozess- bzw. Domänenwissens. Technologische Veränderungen müssen daher so gestaltet werden, dass das notwendige Wissen, einschließlich des aktuellen Erfahrungswissens der Arbeitnehmerinnen und Arbeitnehmer, zur Verfügung steht.

\section{Künstliche Intelligenz (KI) und Robotik}

$\mathrm{KI}$ und Robotik ist ein Zukunftsthema, das stetig an Bedeutung zunimmt und die Industrie nachhaltig verändern wird. Aus diesem Grund hat das BMVIT bereits 2017 den „Österreichischen Rat für Robotik und künstliche Intelligenz" eingerichtet. ${ }^{14}$ Dieser präsentierte im November 2018 das Whitepaper „Die Zukunft Österreichs mit Robotik und Künstlicher Intelligenz positiv gestalten", das den Entwicklungsstand, die Chancen und Herausforderungen, sowie Handlungsfelder und -empfehlungen für Aktivitäten auf Bundesebene formuliert, insbesondere was alles im Umgang mit diesen Technologien zu berücksichtigen ist. ${ }^{15}$

Drei Eckpfeiler werden für eine österreichische Robotik- und KI-Strategie als relevant angesehen:16

1. Smart Governance: Eine breite Beteiligung aller Stakeholder, insbesondere aber auch von Bürge-

14 Vgl. https://www.acrai.at/

15 Vgl. https://www.bmvit.gv.at/innovation/forschungspolitik/robotikrat.html

16 Vgl. Österreichischer Rat für Robotik und Künstliche Intelligenz (2018). 
rinnen und Bürgern im Strategieprozess wird als notwendig erachtet, um die Akzeptanz der neuen Technologien zu steigern.

2. Smart Innovation: Es benötigt eine treffsichere Forschungs-, Entwicklungs- und Investitionspolitik, um die Potenziale von Robotik und KI-Technologien in all ihren Anwendungsbereichen auszuschöpfen und damit neue Märkte und Anwendungen zu erschließen.

3. Smart Regulation: Stabile und sichere Rahmenbedingungen sind die Voraussetzung für das Vertrauen der wirtschaftlichen Akteure und die positive Entwicklung von Märkten. Der Einsatz von Robotik und $\mathrm{KI}$ muss daher die Sicherheit der Menschen gewährleisten und hat den ethischen Standards, den Grundrechten und dem europäischen Wertegefüge zu entsprechen.

Daraus abgeleitet ergeben sich vier Handlungsfelder, die als prioritär für die Entwicklung einer smarten Strategie für Robotik und KI erachtet werden: ${ }^{17}$
- Technologie, F\&E und Wirtschaft

- Arbeitswelt und Qualifizierung

- Gesellschaft und Recht

- Bewusstseinsbildung, Kommunikation und Öffentlichkeitsarbeit

Tatsächlich sieht eine aktuelle länderübergreifende Studie der Boston Consulting Group (BCG) und der Tochterfirma BCG GAMMA zum Thema KI in der Wirtschaft für Österreich noch Handlungsbedarf. ${ }^{18}$ Wie in Abbildung 4-1 dargestellt, nutzen in Österreich aktuell lediglich $13 \%$ aller Unternehmen konkrete KI-Anwendungen, $29 \%$ entwickeln diese gerade erst. Absoluter Vorreiter im internationalen Vergleich ist China. Dort befassen sich $85 \%$ der Unternehmen aktiv mit Kl; jedes dritte Unternehmen setzt $\mathrm{KI}$ schon für Produktion oder Dienstleistungen ein. In Europa sind Deutschland und Frankreich derzeit führend, wo sich jeweils $49 \%$ aller Unternehmen aktiv mit KI beschäftigen und jeweils $20 \%$ konkrete KI-Anwendungen nutzen.

\section{Abbildung 4-1: Anteil der Unternehmen pro Land, die Künstliche Intelligenz nutzen, in \%}

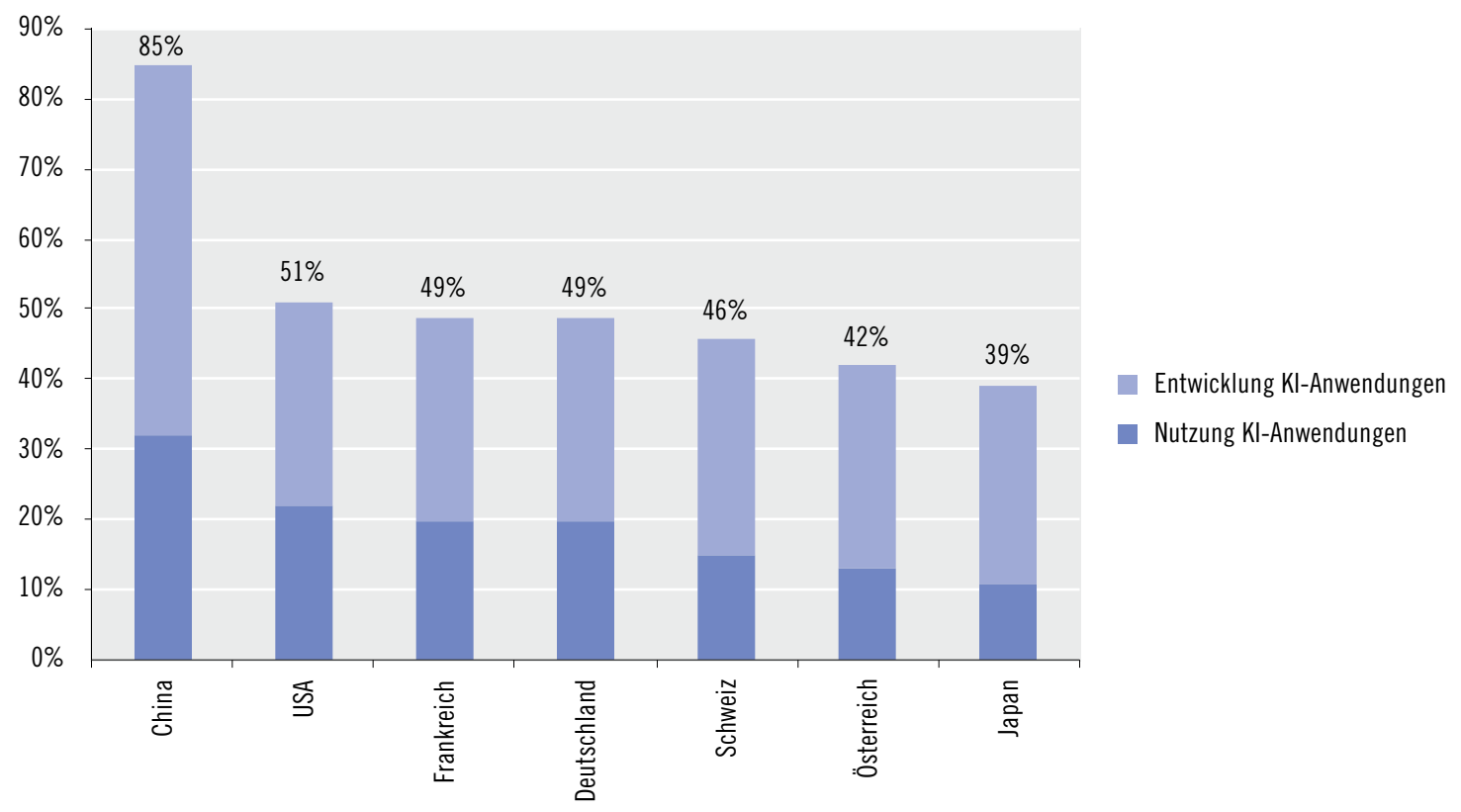

Quelle: BCG und BCG Gamma (2018), Darstellung IWI.

17 Ebenda.

18 Vgl. BCG und BCG Gamma (2018). Anm.: Für die Studie wurden 2.700 Managerinnen und Manager verschiedener Branchen aus Deutschland, China, Frankreich, Japan, Österreich, der Schweiz und den USA zu den KI-Strategien ihrer Unternehmen befragt. 
Ein Vergleich zwischen den Branchen zeigt, dass in Österreich insbesondere die Bereiche Finanzdienstleistungen sowie Konsumgüter/Handel Aufholbedarf haben. Besser sieht es bei Unternehmen mit Fokus auf Technologie, Medien und Telekommunikation und in der Energieindustrie aus. In diesen Branchen sind in Österreich mehr als zwei Drittel aller Unternehmen bereits im Bereich $\mathrm{KI}$ aktiv. ${ }^{19}$

Forschung zu KI hat in Österreich eine lange Tradition. Zu den traditionellen Schwerpunkten gehören logische Systeme und wissensbasierte Ansätze, neuronale Netze, Robotik und sprachverstehende Systeme. Neue Schwerpunkte haben sich auf dem Gebiet Produktion und Industrie 4.0 gebildet, z.B. für vorausschauende Wartung. Der Bund förderte diese Forschung zwischen 2012 und 2017 mit insgesamt 349,9 Mio. $€ \epsilon^{20}$

Um die Zukunft der $\mathrm{KI}$ in Österreich im breiten Konsens mitzugestalten, ist es notwendig, sowohl die Zivilgesellschaft als auch möglichst viele Akteure aus Wirtschaft und Forschung in den Strategieprozess einzubinden, damit Österreich im Wettbewerb um die KI-Technologie vorne mit dabei ist, aber auch gesellschaftliche und individuelle Grundrechte gewahrt werden. Zu diesem Zweck haben das BMVIT und das BMDW ein Vorbereitungspapier namens „Artificial Intelligence Mission Austria 2030" erstellt, um im Laufe des Jahres 2019 auf dieser Basis eine Strategie zu entwerfen. Neben dem White Paper des österreichischen Rates für Robotik und künstliche Intelligenz dienen internationale Studien und EU-Leitlinien als Grundlagen. Für die Ausarbeitung der Strategie unter Federführung des BMVIT werden ressortübergreifende Arbeitsgruppen gebildet. ${ }^{21}$

\section{G als infrastruktureller „Schlüssel“ für die Digitale Transformation}

Als Land mit hohem Lohnniveau kann Österreich nur durch Innovation und innovative Technologien den Industriestandort weiter ausbauen und seine Wettbewerbsfähigkeit erhalten. Dafür ist auch eine moderne und leistungsstarke digitale Infrastruktur von großer Bedeutung. Nicht zuletzt auf Grund der hohen Anzahl und geografischen Verteilung von erfolgreichen KMU ist eine flächendeckende 5G-Versorgung auch essentiell für die zukünftige wirtschaftliche Entwicklung des Landes.

Mit $5 \mathrm{G}$ wird die Basisinfrastruktur für viele nachgelagerte Industrien und Dienstleistungen geschaffen, insbesondere auch in ländlichen Regionen. Eine Studie von Arthur D. Little kalkulierte als Effekte für Österreich in der 5G-Vorreiterrolle einen BIP-Beitrag bis 2030 von 32 Mrd. €, 35.000 zusätzliche Beschäftigte und weitere indirekte Effekte durch den Zuzug von Fachkräften, ausländische Direktinvestitionen, F\&E-Wachstum und die Ansiedlung von Start-ups. ${ }^{22}$

Mit der nationalen 5G-Strategie hat die österreichische Bundesregierung ein konkretes Arbeitsprogramm für zentrale Handlungsfelder und einen Zeitplan vorgelegt, um den 5G-Standard in Österreich so rasch wie möglich flächendeckend zu ermöglichen. ${ }^{23}$ Ziel ist es, Österreich zu einem 5G-Vorreiter in Europa zu machen und in die Top-3 der DigitalisierungsLänder innerhalb der EU sowie in die Top-10-Länder weltweit zu bringen. Der geplante Weg dorthin wird in drei Phasen unterteilt:

- Phase 1: Bis Mitte 2018 sollen erste vorkommerzielle 5G-Teststellungen umgesetzt werden. ${ }^{24}$

- Phase 2: Bis Ende 2020 soll das Zwischenziel einer nahezu flächendeckenden Verfügbarkeit ult-

19 Vgl. BCG und BCG Gamma (2018).

20 Vgl. BMVIT und BMDW (2018).

21 Vgl. https://infothek.bmvit.gv.at/strategie-fuer-kuenstliche-intelligenz-auch-fuer-oesterreich/

22 Vgl. Arthur D. Little (2017b).

23 Vgl. BMVIT et al. (2018).

24 Derzeit werden 5G-Teststellungen und Pilotregionen umgesetzt. 2018 wurde bspw. eine 5G-Datenverbindung mit einem Drohnenflug in Innsbruck getestet oder auch ein Testbetrieb am Wiener Rathausplatz gestartet. 
raschneller Breitbandanschlüsse (100 Mbit/s) verwirklicht werden. Dadurch wird die Grundlage für einen flächendeckenden Ausbau von 5G geschaffen. Gleichzeitig soll auch die Markteinführung von $5 \mathrm{G}$ in allen Landeshauptstädten erfolgen.

- Phase 3: Bis Ende 2023 sollen 5G-Dienste auf den Hauptverkehrsverbindungen nutzbar sein und bis Ende 2025 soll das Ziel einer nahezu flächendeckenden Verfügbarkeit von $5 \mathrm{G}$ verwirklicht werden.

Industrie 4.0, autonomes Fahren, der fächendeckende Einsatz von Big Data und KI oder "Internet der Dinge"-Anwendungen benötigen eine leistungsfähige IT-Infrastruktur, die ubiquitäres Computing und die Übermittlung großer Datenmengen erst möglich macht. Gemeinsam mit der Breitbandinfrastruktur braucht es dafür ein extrem leistungsfähiges Mobilfunknetz. 5G hat das Potential als disruptiver Faktor in der Digitalisierung für eine weitere Beschleunigung der Prozesse zu sorgen. Das volle wirtschaftliche Potential wird sich jedoch nur im Kontext neuer und innovativer Geschäftsmodelle entfalten können. Es müssen daher parallel zum Aufbau der 5G-Infrastruktur Prozesse in Gang gesetzt werden, die Anwendungsgebiete erschließen, Akteure vernetzen sowie neue Ideen hervorbringen. Diese Bündelung von Aktivitäten sollte in Umsetzungsprojekten und Maßnahmen münden, die den Transfer von $5 G$ in die Breite fördern.

Eine Stakeholderbefragung sieht die größten Potentiale von 5G-Technologien für den Standort Österreich für Anwendungen im Bereich Mobilität und Verkehr. Beträchtliches Potential mit großer wirtschaftlicher und gesellschaftlicher Relevanz wird darüber hinaus in den Themenfeldern Wirtschaft und Industrie, Umwelt und Energie, Gesundheit, Pflege und Soziales, Politik und Verwaltung, Bildung sowie Sicherheit, Schutz und Vertrauen als Querschnittsthema gesehen. Um zeitnahe und effizient verschiedene Anwendungen entwickeln zu können, setzt die 5G-Strategie folglich auf sechs Schritte, damit Ös- terreich eine Vorreiterrolle in Europa einnehmen kann: ${ }^{25}$

1. Förderung von aktivem Austausch und Vernetzung durch Einrichtung und Etablierung einer 5G-Plattform;

2. Internationale Einbindung durch Forschungs- bzw. gezielte Wirtschaftskooperationen;

3. F\&E von 5G-Anwendungen durch 5G-Innovationsund Testlabore;

4. Umsetzung von 5G-Pilotprojekten in der öffentlichen Verwaltung;

5. Regionale Leuchtturmprojekte für 5G-Anwendungen;

6. Einrichtung von 5G-Testbeds und -Sandboxes u.a. mit experimenteller Anwendung bei Großevents.

\subsection{Fachkräfte und Ausbildungssystem für den digitalen Wandel}

Digitalisierung als Grundlage gesellschaftlichen und wirtschaftlichen Wandels ist zurzeit ein allgegenwärtiges Thema, wiewohl Veränderungen durch die Verbreitung von Mikroprozessoren zumindest seit den 1980er-Jahren diskutiert werden. Weder die elektronische Verarbeitung wie die Fernübertragung immer größer werdender Datenmengen sind für sich neue Themen, die Frage der Übernahme von Arbeitsprozessen durch Maschinen ist sogar so alt wie die Industrialisierung selbst; nicht die Technologie revolutioniert sich, sondern der Umgang mit ihr: Daten werden nicht nur massenhaft erfasst, sondern auch intelligent miteinander verbunden. Daraus ergeben sich neue Bedürfnisse an die Beschäftigten in dem Sinn, als die Digitalisierung eine zunehmend höhere Qualifikation der Beschäftigten erfordert.

Die Digital Roadmap Austria hebt hervor, wie der digitale Wandel die Struktur der Arbeit beeinflusst bzw. verändern wird. Betont wird u.a., dass neue Technologien nicht ganze Arbeitsplätze, sondern

25 Vgl. BMVIT et al. (2018). 
meist nur gewisse Tätigkeiten verändern werden. Daraus folgt, dass sich die Anforderungen an die Qualifikationsprofile der Beschäftigten ändern. In der wissenschaftlichen Literatur wird dieser Zusammenhang bereits seit Längerem über folgenden Wirkungsweg beschrieben: ${ }^{26}$ Der Bedarf an Fertigkeiten der Beschäftigten hängt vom Stand der Technologie ab, wobei technologischer Fortschritt diesen Bedarf in dem Sinn ändert, als, erstens, manche Fertigkeiten eher nachgefragt werden als andere. Die Entlohnung am Arbeitsmarkt hängt für bestimmte Tätigkeiten davon ab, in welchem Ausmaß diese von den Arbeitnehmerinnen und Arbeitnehmern auf Grundlage ihrer Fertigkeiten angeboten werden. Zweitens lässt sich ungeachtet der relativen Änderungen als Tendenz festmachen, dass technologischer Fortschritt generell den Bedarf an Fertigkeiten erhöht, d.h. es ist nötig, dass immer mehr Beschäftigte über immer mehr Kompetenzen verfügen.

Vor diesem Hintergrund wird auch in der Diskussion zwischen den Anforderungen (,tasks“) einer Tätigkeit und den dazu erforderlichen Fertigkeiten („skills“) unterschieden. ${ }^{27}$ Die Unterscheidung zwischen Aufgaben und Fertigkeiten ist vor allem dann relevant, wenn Beschäftigte mit einer bestimmten Fertigkeit mehrere Aufgaben übernehmen können. Die derzeitige Entwicklung ist dadurch geprägt, dass die Digitalisierung Anforderungen stellt, die ganz spezifische Kompetenzen erfordern. Diese sind nicht unbedingt mit dem bisherigen Weg der generell höheren Qualifikation gleichzusetzen. Die Zunahme des Bedarfs an Fertigkeiten, um mehrere Anforderungen durchführen zu können, wird auch als Ursache einer zunehmenden Polarisierung auf dem Arbeitsmarkt angesehen, indem die Arbeitsplätze sowohl für hoch qualifizierte und hoch bezahlte Arbeit als auch für niedrig qualifizierte Arbeit mit geringen Löhnen zunehmen.
Nachdem zu Beginn der 2000er-Jahre unter einem "digital divide" noch der fehlende Zugang zum Internet eines Teils der Bevölkerung an sich als Problem gesehen wurde, zeichnet sich im laufenden Jahrzehnt ein neuer „digital divide“ ab: Demnach verläuft die Kluft nun zwischen jenen, die Internet und IKT bewusst einsetzen, um Informationen zu erhalten und diese Informationen gezielt nutzen, und jenen, die bloß als Konsumenten agieren. ${ }^{28}$ Diese Kluft setzt sich im Beruflichen fort, als diejenigen, die IKT bewusst zur Informationsgewinnung einsetzen, über Kompetenzen verfügen, die auch am Arbeitsmarkt zunehmend nachgefragt werden. Die Digitalisierung erfordert damit spezifische kognitive und abstrakte Kompetenzen, die zur oben erwähnten Polarisierung führen können. Ein solche Polarisierung kann in Österreich bislang jedoch kaum beobachtet werden. Ein möglicher Grund hierfür ist, dass das Ausbildungssystem mit seiner Differenzierung im mittleren Bereich nach Lehre und Schule gerade in Österreich überaus gut mit den Bedürfnissen am Arbeitsmarkt abgestimmt ist.

Die Digitalisierung der Arbeit erfordert also, dass immer mehr Arbeitnehmerinnen und Arbeitnehmer in der Lage sind, mit ihren Fertigkeiten mehrere Anforderungen zu beherrschen. Gefordert ist nicht unbedingt ein formal höheres Ausbildungsniveau, sondern über die Kompetenzen zu verfügen, um den zukünftigen, digitalen Anforderungen gerecht zu werden. In diesem Zusammenhang zeigt der DESI-Teilindex Humankapital, dass Österreich in den Bereichen IKTFachkräfte in Prozent der Beschäftigten sowie in Prozent der MINT-Absolventinnen und -Absolventen je 1.000 Personen (20-29-Jährige) im EU-Vergleich an der sechsten bzw. fünften Stelle liegt (siehe auch Abschnitt 1.3.2). In diesem Zusammenhang verweist der DESI-Report auch auf die Relevanz der im aktuellen Regierungsprogramm genannten Ziele ${ }^{29}$ :

26 Die beschrieben Thesen gehen v.a. auf Jan Tinbergen zurück, der in den 1970er-Jahren hierzu einflussreiche Artikel veröffentlicht hat. Vgl. Tinbergen (1974) und Tinbergen (1975).

27 Diese Unterscheidung geht auf Arbeiten von David Autor und Lawrence Katz zurück und wird u.a. von Daron Acemoglu und David Autor im Handbook of Labor Economics (Volume 4b) ausgeführt.

28 Vgl. OECD (2009).

29 Vgl. Europäische Kommission (2019b). 
- Die Förderung ständiger Weiterbildung, besonders wenn es um die Unterstützung beim digitalen Lernen geht;

- Die Ausstattung der Schulen mit der passenden digitalen Infrastruktur;

- Eine digitale Erfolgsbilanz für Studierende vom Kindergarten bis zum Ende der Schulbildung;

- Die Weiterentwicklung der berufsbildenden höheren Schulen (BHS) und der höheren technischen Lehranstalt (HTL), besonders in Bezug auf MINTFächer und Digitalisierung;

- Mehr Studienplätze, um Digitalisierung und MINTFächer auf der Tertiärstufe auszubauen;

- Die Einführung einer „Digitalisierungsoffensive Bildung" als Aus- und Weiterbildungsstrategie, die ihrerseits sicherstellen soll,

- dass digitale Kompetenzen in allen Schulen unterrichtet werden sollen;

- von der Volksschule an Programmiersprachen (wie Scratch) gelehrt werden;

- digitale Kompetenzen von Lehrerinnen und Lehrern via Pflichtschulungen gestärkt werden;

- ein umfassendes Angebot an digitalen Berufsausbildungen existiert.

- Die Einführung einer österreichischen digitalen Akademie, d.h. einer Online-Plattform für Schulungsangebote und lebenslanges Lernen.

Mit dem "Digital-Dossier 2018“30 hat das Bundesministerium für Digitalisierung und Wirtschaftsstandort eine Bestandsaufnahme über den Status-quo der Digitalisierung in Österreich erstellt. Darin wird auch auf den DESI eingegangen und der „Pakt für Digitale Kompetenz" als Zusammenschluss von Wirtschaft, Bildungseinrichtungen und der öffentlichen Verwaltung mit verschiedenen Teilprogrammen präsentiert.

Mit einem umfassenden „Masterplan für Digitalisierung in der Bildung" des Bundesministeriums für Bildung, Wissenschaft und Forschung sollen die Veränderungen, die sich durch die fortschreitende
Digitalisierung ergeben, stufenweise und vor allem fächendeckend in das österreichische Bildungssystem einfließen. ${ }^{31}$ Der Masterplan sieht drei Aktionsfelder vor:

- Pädagogische Konzepte und Lehr- und Lerninhalte: Digitale Kompetenzen und Digitale Bildung sollen im Zuge einer grundlegenden Überarbeitung bestehender Lehrpläne systematisch in die Lehrpläne eingearbeitet werden. Der Digitalisierung soll im Sinne eines modernen Unterrichts methodisch und didaktisch in allen Gegenständen Rechnung getragen werden.

- Aus-, Fort-, und Weiterbildung der Pädagoginnen und Pädagogen: Digitale Kompetenzen und Digitale Bildung sollen systematisch in der Ausbildung bzw. Fort- und Weiterbildung von Pädagoginnen und Pädagogen verankert werden.

- Infrastruktur: Die infrastrukturelle Ausstattung soll verbessert werden. Es soll flächendeckend die Voraussetzung geschaffen werden, dass digitale Medien und Tools an Schulen zum Einsatz kommen können.

Weiters wurde seitens des BMDW mit DigComp 2.2 $A T$ ein digitales Kompetenzmodell für Österreich entwickelt, das der Einordnung und Vergleichbarkeit digitaler Kompetenzen dient und damit die Grundlage für lebenslanges Lernen, gesellschaftliche Inklusion und Beschäftigung in einer digitalisierten Gesellschaft bildet. ${ }^{32}$ Auf Initiative des BMDW wurde im Dezember 2018 außerdem der Verein "fit4internet" gegründet, der als Plattform - in Zusammenarbeit mit Unternehmen, Institutionen und Organisationen - die digitalen Kompetenzen in Österreich steigern und damit die Teilhabe der gesamten Gesellschaft an Digitalisierung ermöglichen soll. ${ }^{33}$

Die Maßnahmen sind dringend notwendig, um mit Aus- und Weiterbildung auf allen Stufen den digitalen Wandel in Österreich zielgerecht zu unterstützen. Bereits heute verweist die Wirtschaft, speziell

30 Vgl. https://www.bmdw.gv.at/DigitalisierungundEGovernment/Documents/DigitalDossier.pdf

31 Vgl. https://bmbwf.gv.at/presseunterlagen/masterplan-digitalisierung/

32 Vgl. BMDW (2018).

33 Vgl. https://www.fit4internet.at/ 
die IT-Branche, auf die hohe Nachfrage nach hochqualifizierten IT-Kräften, die nicht gedeckt werden kann, sowie auf einen anhaltend hohen Bedarf an qualifiziertem Humanpotenzial im Bereich Informatik. ${ }^{34}$ Die Informatik wird dabei als Schlüsselkompetenz der Unternehmen gesehen. Das betrifft nicht nur Informatik-Studien im engeren Sinn, sondern auch interdisziplinäre Informatikstudien wie Wirtschaftsinformatik, Geoinformatik oder Medizininformatik. Letztere sind dadurch charakterisiert, spezifische Geschäftsprobleme mit informatischen Methoden zu lösen. Der Bedarf bildet sich dabei weniger an reinen Programmiererinnen und Programmierern ab, die eher in ganz speziellen Unternehmen benötigt werden, sondern zunehmend über interdisziplinär Ausgebildete, die spezielle Informatiklösungen gestalten können. Dafür ist im jeweiligen Anwendungsgebiet entsprechendes Prozesswissen nötig.

Vor diesem Hintergrund werden daher zusätzlich zur reinen Programmiertätigkeit zwei Typen von Informatik-Kompetenzen unterschieden: Erstens die interdisziplinäre Entwicklung von IT-Lösungen, das betrifft v.a. Informatikerinnen und Informatiker mit Prozesswissen, wie z.B. Wirtschaftsinformatikerinnen und -informatiker, die im Produktionsbereich eingesetzt werden. Zweitens sind aber auch zunehmend Nicht-Informatikerinnen und Nicht-Informatiker an IT-Know-how für ihre jeweiligen Fachbereiche angewiesen, wie z.B. Personen, die in der Krankenpflege tätig sind und neue Methoden und Verfahren anwenden, im Gegensatz zu Personen im Bereich der Medizintechnik, die diese entwickeln und warten. Um dieser Entwicklung gerecht zu werden, hat das Bundesministerium für Digitalisierung und Wirtschaftsstandort im Bereich der Berufsausbildungen mit digitalem Schwerpunkt auch erst jüngst neue Berufsbilder für digitale Lehrberufe erlassen. Hierzu zählen „Applikationsentwicklung Coding" sowie "Informationstechnologie“ mit den zwei Schwerpunkten „Systemtechnik“ und „Betriebstechnik“, wie auch die "E-Commerce-Kauffrau“ bzw. der „E-Commerce-Kaufmann".35

Auf tertiärer Stufe hat man bereits auf die sich verändernden Umfeldveränderungen bezugnehmend auf künftige Anforderungen in der Berufs- und Arbeitswelt reagiert. So befasste sich eigens im Rahmen des Projekts "Zukunft Hochschule“ (durchgeführt vom damaligen BMWFW) eine Arbeitsgruppe "Informatik“" mit der Identifikation von sektorenspezifischen Ausbildungsprofilen, der Entwicklung neuer, mitunter auch kooperativer Ausbildungsangebote, der Entwicklung von Angeboten zur Vermittlung von IT-Kompetenzen für alle Studierende, der Entwicklung von spezifischen Angeboten für "Job-Outs“36, gemeinsamen künftigen Aktivitäten im Bereich Studieninformation und Maßnahmen zur Erhöhung des Studieninteresses für Informatik und zur Steigerung des Frauenanteils sowie zur Intensivierung des Dialogs der Hochschulen mit der Wirtschaft. ${ }^{37}$ Hinsichtlich der Studienfelder Wirtschaftswissenschaften und Informatik liegt der Fokus im Universitätsbereich auf dem Aufbau von Personalkapazitäten und der Verbesserung von Betreuungsverhältnissen, hingegen bei Fachhochschulen auf dem Ausbau von Studienplätzen und dem Angebot von interdisziplinären Studiengängen. ${ }^{38}$ Überdies soll das Studieninteresse an Informatik insgesamt erhöht werden, insbesondere jenes von Frauen.

Was das Studienangebot betrifft, so wurden im Wintersemester 2018 an zehn öffentlichen Universitäten Österreichs insgesamt 15 Bachelor- und 27 Masterstudien im Bereich Informatik ${ }^{39}$ angeboten. An den Fachhochschulen wurden im selben Studienjahr

$34 \mathrm{Vgl}$. https://bmbwf.gv.at/fileadmin/user_upload/wissenschaft/Zukunft_Hochschulen/AF_3_Informatik.pdf

35 Vgl. https://www.bmdw.gv.at/Presse/Archiv/Archiv\%202018/Seiten/Schramboeck-Lehre-wird-wieder-cool-Neue-Lehrberufestark-nachgefragt.aspx

36 Unter „Job-Outs“ werden Studienabbrecher verstanden, die aufgrund eines beruflichen Angebots ihr Studium nicht beendet haben.

37 Vgl. https://bmbwf.gv.at/fileadmin/user_upload/wissenschaft/Zukunft_Hochschulen/Management_Summary.pdf

38 Vgl. BMBWF (2017).

39 Dies umfasst „Informatik, allgemein“ (480) und „Informatik“ (481). 
Tabelle 4-1: Ordentliche Studierende im Studienfeld Informatik, Wintersemester 2013-2017

\begin{tabular}{|c|c|c|c|c|c|c|}
\hline & & 2013 & 2014 & 2015 & 2016 & 2017 \\
\hline \multirow[t]{5}{*}{ Universitäten } & Bachelorstudium & 9.770 & 10.065 & 10.701 & 10.597 & 10.481 \\
\hline & Diplomstudium & 301 & 259 & 12 & 12 & 7 \\
\hline & Masterstudium & 3.648 & 3.778 & 3.858 & 4.026 & 4.263 \\
\hline & Doktoratsstudium & 1.220 & 1.234 & 1.181 & 1.129 & 1.070 \\
\hline & Gesamt & 14.939 & 15.336 & 15.752 & 15.764 & 15.821 \\
\hline \multirow[t]{4}{*}{ Fachhochschulen } & Bachelorstudium & 3.120 & 3.076 & 3.211 & 3.400 & 3.540 \\
\hline & Diplomstudium & 10 & 9 & 3 & 0 & 0 \\
\hline & Masterstudium & 1.485 & 1.571 & 1.536 & 1.462 & 1.358 \\
\hline & Gesamt & 4.615 & 4.656 & 4.750 & 4.862 & 4.898 \\
\hline
\end{tabular}

Anmerkung: Durch die Umstellung auf die Bologna-Studienarchitektur werden heute kaum noch Diplomstudiengänge angeboten.

Quelle: BMBWF, uni:data

insgesamt 56 Bachelor- und 68 Masterstudien offeriert. Insgesamt bieten in Österreich damit zehn Universitäten und 15 Fachhochschulen Studien im Bereich Informatik an.

Tabelle 4-1 zeigt den Verlauf der Anzahl von (ordentlichen) Informatik-Studierenden für die Wintersemester 2013 - 2017 an den Universitäten und Fachhochschulen. Für die Universitäten zeigt sich, dass trotz des Bedarfs seit 2015 kein Aufwärtstrend zu erkennen ist; die Zahlen der Bachelor- und Doktorats-Studierenden sind sogar zurückgegangen, wenn auch die Zahl der Master-Studierenden zugenommen hat. Demgegenüber zeigt sich an den Fachhochschulen ein anderes Bild: Die Anzahl der Studierenden im Studienfeld Informatik weist im Zeitraum 2013 - 2017 eine steigende Tendenz auf. Dieser Aufwärtstrend ist allerdings auf die Bachelor-Studierenden zurückzuführen, bei den Master-Studierenden lässt sich ein solch positiver Trend aktuell nicht erkennen. Insgesamt jedoch, werden die Studierendenzahlen an Universitäten und Fachhochschulen im Studienfeld summiert, hat sich die Studierendenzahl sowohl auf Bachelor- als auch auf Master-Ebene von 12.890 in 2013 auf 14.021 in 2017 bzw. von 5.133 in 2012/13 auf 5.621 in 2016/17 erhöht.

Vonseiten des Bedarfs deuten verschiedene Indikatoren darauf hin, dass Absolventinnen und Absol- venten von Universitäten und Fachhochschulen stark nachgefragt werden. ${ }^{40}$ In Zukunft sollen daher mehrere Informatik-Studien an einem Standort wie auch eine Vielfalt an Studienrichtungen im Feld der Informatik angeboten werden. Angesichts dessen wird auch eine Reihe von Maßnahmen gesetzt, um die digitalen Kompetenzen in der Bevölkerung in Zukunft grundsätzlich noch stärker aus- bzw. aufzubauen. Hierzu zählen ${ }^{41}$ :

- Die Lehre von Informatik in sämtlichen Studienrichtungen, um Basis-Kompetenzen und IT-Skills zu vermitteln bzw. entsprechende Angebote bereitzustellen.

- Das Angebot von innovativen Studienformaten, die mittels ihrer Gestaltung bestimmte Zielgruppen adressieren, wie z.B. berufstätige Studierende und Job-outs. E-learning oder die Gestaltung von Fernstudien sind in diesem Kontext von besonderem Interesse.

- Die Gestaltung neuer Studiengänge in Bereichen wie etwa Big Data, Industrie 4.0, Data Engineering, Data Science etc.

Um die Dynamik systemisch zu verankern, legen auch die aktuellen Leistungsvereinbarungen mit den Universitäten für die Periode 2019-2021 einen Schwerpunkt auf Digitalisierung. Folglich soll z.B. an der Universität Innsbruck das Angebot an Freifächern im

40 Vgl. https://bmbwf.gv.at/fileadmin/user_upload/wissenschaft/Zukunft_Hochschulen/AF_3_Informatik.pdf 41 Vgl. https://bmbwf.gv.at/fileadmin/user_upload/wissenschaft/Zukunft_Hochschulen/AF_3_Informatik.pdf 
Bereich der Digitalisierung erweitert werden. An der Technischen Universität Graz ist es Ziel, neben der Weiterentwicklung des e-Learnings auch an "Learning Analytics" (darunter ist die maschinelle Bewertung des Lernverhaltens der Studierenden zu verstehen) zu arbeiten. Die Technische Universität Wien hat eigens einen Vizerektor für Digitalisierung und Infrastruktur etabliert, und die Technische Universität Graz strebt die Entwicklung zur „digitalen Universität" an.

Verschiedene Indikatoren deuten darauf hin, dass Österreich eher von einem „brain drain" als von einem „brain gain" betroffen ist; also Österreich Hochqualifizierte eher verliert als gewinnt. ${ }^{42}$ So verweist mitunter die OECD darauf, dass der Zuzug von Arbeitsmigrantinnen und -migranten von außerhalb der EU und EFTA schwierig ist, was letztlich auch die Motivation Hochqualifizierter reduziert, zuziehen. ${ }^{43}$ Ein weiteres Problem liegt darin, dass Hochqualifizierte im Bereich Informatik generell nicht in der Mangelberufsliste berücksichtigt sind. ${ }^{44}$ Allerdings gibt es seit 2019 in Österreich eine zweite Mangelberufsliste mit acht (Mangel-)Berufen, die einen Hochschulabschluss voraussetzen, darunter „Diplomingenieurinnen und Diplomingenieure für Datenverarbeitung". Das bedeutet, dass Arbeitskräfte mit diesen Ausbildungen unter erleichterten Voraussetzungen als "besonders Hochqualifizierte" zugelassen werden und eine RotWeiß-Rot-Karte erhalten.

\subsection{Digitalisierung im öffentlichen Sektor}

Für den öffentlichen Sektor bedeutet Digitalisierung zweierlei: einerseits die Übertragung analoger Informationsbestände, Prozesse und Services in digitale Formate, andererseits die Einführung völlig neuer In- teraktionsformen und der Zugang zu neuen Wissensformen durch die Nutzung von Digitaltechnik. Im Hinblick auf dieses breite Verständnis der digitalen Transformation als tiefgreifender Veränderungsprozess arbeitet der öffentliche Sektor in Österreich bereits seit vielen Jahren an der Verbesserung der Services, aber auch an der Zusammenführung von Informationen, sowie gemeinsamen Strategien. Denn erst wenn in der Verwaltung gezielt organisatorische, logistische, rechtliche und fachlich aufeinander abgestimmte Maßnahmen gesetzt werden, kann sich das Transformationspotential der Digitalisierung auch für die Gesellschaft entfalten. Der Fokus der Digitalisierungsmaßnahmen im öffentlichen Sektor liegt derzeit in Österreich auf der Bereitstellung innovativer digitaler Services, wie z.B. das Digitale Amt, E-Health Lösungen, oder Open Data. Maßnahmen im Hintergrund widmen sich verstärkt der Interoperabilität und der Integration von Infrastrukturen.

\subsubsection{Digitalisierungsstrategie, Digital Austria und Chief Digital Officers}

Auf nationaler Ebene hat Österreich bereits im Dezember 2016 eine Digitalisierungsstrategie erarbeitet: die „Digital Roadmap“45. Diese bietet einen Überblick über aktuelle und zukünftige Herausforderungen rund um das Thema Digitalisierung und bildet die Basis für die neue Digitalisierungsstrategie, die derzeit (Stand Mai 2019) ausgearbeitet wird. Im Regierungsprogramm 2017-2022 wurde die digitale Weiterentwicklung als Querschnittsthema festgeschrieben $^{46}$. Durch die Bundesministeriengesetz-Novelle 2017 (BMDG-Novelle), BGBI. Nr. 164/201747, die mit 08.01.2018 in Kraft getreten ist, ist es zu einigen Veränderungen bei den Zuständigkeiten der jeweiligen Bundesministerien gekommen. Insbesondere im Be-

42 Vgl. Wisbauer und Fuchs (2014).

43 Vgl. OECD (2014).

44 Tatsächlich scheinen Informatikerinnen und Informatiker und andere IT-Berufe trotz des nach vorliegender Faktenlage offenbar bestehenden Mangels in der „Mangelberufsliste 2019“ nicht auf.

45 Vgl. https://www.digitalroadmap.gv.at/

46 Vgl. BKA (2017).

47 Vgl. https://www.ris.bka.gv.at/eli/bgbl/I/2017/164 
reich der Digitalisierung wurden Angelegenheiten, die bisher in die Zuständigkeit des Bundeskanzleramtes, wie z.B. die Agenden des Datenschutzes, oder des Bundesministeriums für Finanzen gefallen sind, zusammengefasst und dem Bundesministerium für Digitalisierung und Wirtschaftsstandort übertragen.

Um die Innovations- und Digitalisierungsthemen zwischen den Ministerien und die Mitarbeit an einer bundesweiten Innovations- und Digitalisierungsstrategie zu koordinieren, wurde in jedem Ressort ein Chief Digital Officer (CDO) ernannt. Durch abgestimmtes Vorgehen können nun Ressort-übergreifende Ziele, wie z.B. Verfahren weiter zu automatisieren oder verbesserte digitale Services bereit zu stellen, effizienter koordiniert und realisiert werden. Die CDOs liefern weiters regelmäßig Fortschrittsberichte an die Bundesregierung. Die Leitung dieser Task Force liegt im BMDW, der "Chief Digital Officer des Bundes“ ist der Leiter der Sektion „Digitalisierung und E-Government". Schließlich umfasst E-Governance „die Unterstützung der Beziehungen, Prozesse und der politischen Partizipation innerhalb der staatlichen Stellen aller Ebenen sowie zwischen den staatlichen Stellen und all ihren Anspruchsgruppen durch die Bereitstellung geeigneter Interaktionsmöglichkeiten mittels elektronischer Medien ${ }^{448}$.

Die Koordination der Digitalisierungsmaßnahmen der gesamten Bundesregierung (Zeitplan, Schwerpunkte, Pilotierungen etc.) werden damit in Abstimmung mit der "CDO-Task Force" und weiters mit der neu gegründeten „Digitalisierungsagentur" erarbeitet. Mit der strukturellen Neuausrichtung ist auch eine Konsolidierung wesentlicher Regulative für die digitale Verwaltung verbunden. Insbesondere das 2016 novellierte E-Government-Gesetz ${ }^{49}$ sowie das IKT-Konsolidierungsgeset ${ }^{50}$, die gemeinsam zentrale Bausteine der digitalen Verwaltung sowie die Finan- zierung gemeinsamer Vorhaben regeln, liegen nun in der Kompetenz des Digitalisierungsressorts. Darüber hinaus wurde normiert, dass strategische Fragestellungen der Bundesrechenzentren (BRZ GmbH) einvernehmlich mit dem BMDW zu klären sind.

\section{Digitalisierungsagentur}

Ein zentrales Element der strukturellen Neuausrichtung ist die Digitalisierungsagentur „Digital Austria (DIA)“51, die im Rahmen der Forschungsförderungsgesellschaft als eigenständiger Bereich etabliert wurde. Mit „Digital Austria“ wurde eine Dachmarke geschaffen, unter der alle Strategien und Leuchtturmprojekte zusammengefasst werden. Inhaltlich setzt die Verwaltung hier - neben dem Ausbau der Breitband- und 5G-Versorgung - vor allem auf benutzerfreundliche, digitale Applikationen für Bürgerinnen, Bürger und die Wirtschaft.

Digitalisierung wird hierbei als Aufgabe betrachtet, welche die Zusammenarbeit der zentralen Impuls- und (Förder-)Geldgeber für Forschung, Entwicklung und Wirtschaft voraussetzt. Die Agentur hat auch im Bereich des digitalen Staates Aufgaben zu erfüllen, wie etwa die Beratung der Bundesregierung, die Unterstützung der CDOs, die weitere Ausarbeitung der Digitalisierungsstrategie oder die internationale Koordination. Im Mittelpunkt einer verantwortungsvollen digitalen Entwicklung sollen stets "die Menschen mit ihren Bedürfnissen" stehen. Die neue Digitalisierungsagentur DIA wird neben ihrer Aufgabe als zentrale Anlaufstelle und Koordinationsplattform auch für das Monitoring sämtlicher Digitalisierungsmaßnahmen zuständig sein.

Es wird erwartet, dass diese strukturelle Neuausrichtung der Digitalagenden den öffentlichen Sektor im internationalen Vergleich weiter in die Liga der führenden Digitalnationen aufsteigen lässt. Derzeit belegt Österreich im eGovernment-Benchmark 2018

48 Vgl. Gisler et al. (2011).

49 Vgl. https://www.digitales.oesterreich.gv.at/e-government-gesetz

50 Vgl. https://www.parlament.gv.at/PAKT/VHG/XXVI/I/I_00381/index.shtml

51 Vgl. https://www.digitalaustria.gv.at/ 
der Europäischen Kommission den sechsten Platz von 34 untersuchten Ländern. ${ }^{52}$ Heimische E-Government-Lösungen wie FinanzOnline oder Justiz 3.0 werden hierbei als Best Practices für ganz Europa genannt. Der Index für die digitale Wirtschaft und Gesellschaft (Digital Economy and Society Index $D E S I)$ der Europäischen Kommission weist Österreich im Bereich der digitalen öffentlichen Dienste den Rang 8 zu. ${ }^{53}$ Bisher ist Österreich hier nur im Bereich der "Online Government Services" (vorausgefüllte Formulare und Online Erledigungen) unter den Top 5 im EU-Vergleich, während andere Bereiche nur im Mittelfeld liegen (zu einer umfassenden Diskussion des Digital Economy and Society Index siehe auch Abschnitt 1.3.2).

Mit dem „Digitalen Amt“ wird nun ein verwaltungsträgerübergreifender Ansatz Realität, der alle relevanten Akteure auf Bundes-, Länder- und GemeindeEbene einbindet. Dieser breite Ansatz stellt sicher, dass die notwendigen Regelungen geschaffen werden, um die digitale öffentliche Verwaltung in Österreich fit für die neuen Anforderungen zu machen.

\subsubsection{Digitales Amt}

Die rechtlichen Rahmenbedingungen zur Fortsetzung der Digitalisierung in der Verwaltung werden in dem vom BMDW mit 31.01.2019 gestarteten Reformprojekt "Digitales Amt" zwischen Bund, Ländern und Gemeinden erarbeitet. ${ }^{54}$ Damit erhält Österreich einen verlässlichen Rahmen für die Entwicklung, Testung und Einführung elektronischer verwaltungsübergreifender Prozesse. Laut EGovernment Monitor 2018 nutzen bereits 74 \% der Österreicherinnen und Österreicher E-Government Services. ${ }^{55}$ Weiters ist die Zufriedenheit mit den Angeboten mit 72 \% zwar höher als in Deutschland oder der Schweiz, doch zeigt sich in den letzten
Jahren ein Abwärtstrend. Services des öffentlichen Sektors werden von den Nutzerinnen und Nutzern mit der Benutzerfreundlichkeit von privaten Anbietern oder sozialen Medien verglichen. Dies erfordert eine Verbesserung und Vereinfachung der Angebote der Verwaltung. Ziel des "Digitalen Amtes" ist es daher, die Behördenwege der Bürgerinnen und Bürger sowie der Unternehmen möglichst vollständig digital abzubilden und anzubieten. Dazu soll sich die gesamte Planung an den Lebenssituationen der Bürgerinnen und Bürger orientieren, während die Organisationslogik und die bestehende Kompetenzstruktur der Verwaltung in den Hintergrund rücken.

\section{Plattform oesterreich.gv.at und App}

Auf der neuen, zentralen Online-Plattform https:// www.oesterreich.gv.at/ sollen alle digitalen Behördenservices zusammengeführt werden. Bereits bestehende Plattformen wie der Amtshelfer HELP.gv.at oder das Rechtsinformationssystem RIS.gv.at werden dazu in oesterreich.gv.at eingebunden. Dort findet sich auch der direkte Zugang zum Unternehmensserviceportal usp.gv.at und damit zu zahlreichen eGovernment-Anwendungen (eGründung, Sozialversicherung etc.) sowie unternehmensrelevante Informationen. Daten von Antragstellerinnen und Antragstellern, welche die Verwaltung bereits verfügbar hat, sollen nicht mehrfach von Behörden abgefragt werden, sondern direkt zwischen den Verwaltungsstellen ausgetauscht werden („Data once only“). Neben erleichterten Zugangsmodalitäten („Single-SignOn") und verbesserter Benutzerfreundlichkeit steht vor allem die Kompatibilität mit unterschiedlichen mobilen Endgeräten im Fokus. E-Government wird in Richtung Mobile Government ausgebaut.

Der Ausbau der Plattform und der mobilen App erfolgt bis Ende 2019 in mehreren Schritten: Ab Mitte März 2019 können An- und Ummeldungen ebenso

52 Vgl. https://ec.europa.eu/digital-single-market/en/news/egovernment-benchmark-2018-digital-efforts-european-countries-arevisibly-paying

53 Vgl. https://ec.europa.eu/digital-single-market/desi

54 Vgl. https://www.bmdw.gv.at/DigitalisierungundEGovernment/DigitalisierungVerwaltung/Seiten/Das-digitale-Amt.aspx

55 Vgl. https://www.egovernment-monitor.de/die-studie/2018.html 
elektronisch erledigt werden wie Behördenwege nach der Geburt eines Kindes, das Reisepassservice, die Einsicht ins Pensionskonto, ein Steuerauszug plus Antrag auf die Pendlerpauschale und die Beantragung von Wahlkarten. Im Juli folgen der Strafregisterauszug und Verlustmeldungen von Dokumenten. Ab Dezember soll es dann auch den digitalen Führerschein, Zulassungsschein, Personalausweis und die E-Card geben.

\section{Elektronische Zustellung}

Die E-Zustellung ist ein zentrales und sicheres Postfach für elektronische Dokumente. Sobald man bei einem elektronischen Zustelldienst angemeldet ist, können mit der Handy-Signatur Schriftstücke von Behörden (Strafregisterauszug, Meldebestätigung etc.) und Unternehmen (Kaufverträge, Polizzen etc.) sicher über das kostenlose elektronische Postfach empfangen werden.

\section{Stammzahlenregisterbehörde}

Aufgaben der Stammzahlenregisterbehörde sind die Vergabe von Stammzahlen und bereichsspezifischen Personenkennzeichen, die Führung der Ergänzungsregister, die Ausstellung von Stammzahlen bei Verwendung von elektronischen Identifizierungsmitteln eines anderen Mitgliedstaats der EU, die Festlegung und Publikation der mathematischen Verfahren zur Bildung der Stammzahlen- und bereichsspezifischerPersonenkennzeichen sowie die Eintragung von Vollmachtsverhältnissen auf die Bürgerkarte der Vertreterin bzw. des Vertreters. ${ }^{56}$

\section{Elektronische Gründung}

Die elektronische Gründung über das Unternehmensserviceportal (USP) ist in Österreich eine Erfolgsgeschichte: So haben im Jahr 2018 bereits an die 750
Einzelunternehmen die vollständige elektronische Gründung ihres Betriebes vorgenommen, nahezu 1.000 Gründerinnen und Gründer haben das Service teilweise beansprucht. ${ }^{57}$ Die Errichtung einer Ein-Personen-GmbH, wo die Gesellschafterin beziehungsweise der Gesellschafter gleichzeitig die Geschäftsführung innehat, haben im selben Jahr 504 Unternehmerinnen und Unternehmer elektronisch durchgeführt. Das entspricht einer Durchdringungsrate von fast einem Drittel der möglichen Grundgesamtheit.

\section{Elektronischer Identitätsnachweis}

Seit 2017 besteht in Österreich für Bürgerinnen und Bürger sowie Unternehmen das Recht auf elektronischen Verkehr mit Behörden. Seit damals gelten e-ID (auf Chipkarte oder per Mobiltelefon) als vollwertige elektronische Identitätsnachweise. Im Mai 2018 wurde mit der millionsten Anmeldung zur Handysignatur ein Meilenstein gesetzt, heute können bereits rd. 200 Anwendungen im behördlichen und privatwirtschaftlichen Bereich erledigt werden. ${ }^{58}$

\subsubsection{Digitalisierung in der Verwaltung}

Von den CDOs werden gemeinsame Themen für eine zukunftsorientierte digitale Verwaltung weiterentwickelt - wie z.B. die Frage nach einer einheitlichen Datenstrategie des Bundes. Dabei geht es um die Fähigkeit des Bundes, Daten von Bürgerinnen und Bürgern sicher zu verwahren, Daten im Sinne des Prinzips "Once only" für Userinnen und User zu nutzen sowie qualitativ hochwertige Daten für Big Data-Auswertungen, „Predictive Analytics“ und Artificial Intelligence-Anwendungen zur Verfügung zu haben.

Die Konsolidierung der IT des Bundes ist ein weiterer wichtiger Eckstein für die digitale Verwaltung

56 Mit den „Angelegenheiten des E-Governments“ fallen aufgrund der Bundesministeriengesetz-Novelle 2017 (BMDG-Novelle), BGBI. Nr. 164/2017, die Aufgaben der Stammzahlenregisterbehörde in den Wirkungsbereich des BMDW. Diese Änderung der Zuständigkeit wurde mit der Novelle BGBI. I Nr. 104/2018 im E-Government-Gesetz verankert.

57 Vgl. https://www.usp.gv.at/

58 Vgl. https://www.ots.at/presseaussendung/OTS_20180508_OTS0021/schramboeck-elektronische-unterschrift-schreibt-erfolgsgeschichte 
und hat als programmatisches Ziel bereits zu ersten Projekten geführt. In Zentrum steht die Frage, in welchem Ausmaß es sinnvoll ist, weitere Betriebsleistungen beim Bundesrechenzentrum (BRZ) zu bündeln. Dieses Projekt, in dem das Bundeskanzleramt, das Bundesministerium für Digitalisierung und Wirtschaftsstandort sowie das Bundesministerium für Finanzen zusammenarbeiten, hat als erstes Ziel die Erhebung der Potenziale in den einzelnen Ministerien bzw. aller IT-Verfahren und Kosten. Auf der anderen Seite sind die Kapazitäten, Ressourcen und Rahmenbedingungen zu schaffen, die das BRZ in die Lage versetzen, die neuen Aufgabenstellungen optimal zu erfüllen.

Weiteres Ziel ist es, den Anteil an "Shared Services" im Bund auszubauen und die gemeinsamen Services zukunftssicher zu gestalten. Zentrale Services dabei sind die Personalservices des Bundes. Für viele Bereiche, wie etwa den elektronischen Personalakt, Personalgeschäftsprozesse oder das Bildungsmanagement, gibt es in den einzelnen Ressorts unterschiedliche Lösungen. Hier ist eine Zentralisierung der Lösungen ein wichtiger aber verhältnismäßig einfach zu erreichender Konsolidierungsschritt.

Ein weiteres zentrales Projekt ist die Erneuerung und weitergehende Vereinheitlichung des Elektronischen Aktes (ELAK). Eine zentrale Betriebsführung und eine Modernisierung der Verwaltungsregularien sollen dabei Hand in Hand mit technologischen Erneuerungen sowie dem Einsatz innovativer Lösungen (Stichwort KI und Robotik) zur Reduzierung des Verwaltungsaufwandes gehen.

Kleinere Projekte zur Vereinheitlichung sind etwa die Entwicklung eines bundesweiten Content Management Systems (CMS) für Homepages und Intranet-Seiten oder die Einrichtung einer einheitlichen E-Mail-Adresse für alle Zentralstellen der Ministerien
- ein erster Schritt in Richtung eines einheitlichen EMail-Systems für den Bund.

An der Schnittstelle zwischen öffentlichem Sektor und innovativen Start-Ups agiert das Innovation Lab des BRZ. So hostete das BRZ im Februar 2019 den European Youth Award Social Hackathon und unterstützte damit junge IT-Talente, indem es eine abgesicherte Sandbox-Umgebung, in der unter simulierten Produktiv-Bedingungen ausführlich getestet werden kann, zur Verfügung stellt. ${ }^{59}$

Der vom Bundesministerium für öffentlichen Dienst und Sport ausgelobte österreichische Verwaltungspreis 2019 wurde unter anderem auch in der Kategorie "Innovatives Servicedesign und digitale Services“ vergeben. Weiters schrieben das BMVIT und das BMDW gemeinsam den Sonderpreis für „Innovationsfördernde Öffentliche Beschaffung" aus. ${ }^{60}$

\subsubsection{Digitale Entwicklungen in der öffentlichen Beschaffung}

Öffentliche Vergaben sind ein bedeutender Wirtschaftsfaktor in Österreich. So wird jährlich ein Volumen in der Höhe von 60 Mrd. $€$ (Stand: 2015) von über 7.000 öffentlichen Auftraggebern vergeben. ${ }^{61}$ Das entspricht in etwa $18 \%$ des Bruttoinlandsproduktes. Mit dem neu in Kraft getretenen Bundesvergabegesetz 2018 müssen alle Ausschreibungen der öffentlichen Hand elektronisch abgewickelt werden. ${ }^{62} \mathrm{Um}$ diese Angebote zu finden, gab es für Unternehmerinnen und Unternehmer bisher unterschiedliche Publikationsplattformen, die oft nur gegen Entgelt genutzt werden konnten. Nun hat das Unternehmensserviceportal USP das neue Service „Ausschreibungssuche" entwickelt, damit zukünftig alle in Österreich veröffentlichten Ausschreibungen potentiellen Teilnehmern kostenlos und uneinge-

59 Vgl. https://www.brz.gv.at/wie-wir-arbeiten/veranstaltungen/social-hackathon-european-youth-award-eya.html

$60 \mathrm{Vgl}$. https://www.oeffentlicherdienst.gv.at/verwaltungsinnovation/wettbewerbe/oesterreichischer_verwaltungspreis/index. html

61 Vgl. Bröthaler und Plank (2017).

62 Vgl. https://www.ris.bka.gv.at/GeltendeFassung.wxe?Abfrage=Bundesnormen\&Gesetzesnummer=20010295 
schränkt zur Verfügung stehen. ${ }^{63}$ Vor allem für die vielen Betriebe aus Österreichs klein- und mittelständischer Wirtschaft wird es mit dieser deutlichen Verbesserung der Transparenz einfacher, schneller und unkomplizierter, an einer passenden Ausschreibung teilzunehmen und gleichzeitig Zeit und Geld zu sparen. Mit dem Service ist es ab 01.03.2019 möglich, ohne zusätzliche Anmeldung alle nach dem österreichischen Bundesvergabegesetz veröffentlichten Ausschreibungen an einer Stelle zu suchen und einzusehen. Darüber hinaus bietet das Service zahlreiche Features, wie eine Volltextsuche und verschiedene Filter- und Sortiermöglichkeiten. Datensätze zu öffentlichen Ausschreibungen werden auch am Open Data Portal veröffentlicht.

\subsubsection{Open Data}

Der öffentliche Sektor in Österreich verfügt über groBe Datenmengen - von Bildungs-, Wirtschafts- und Sozialdaten bis hin zum Kulturerbe, sowie geografischen und Wetterdaten. Einige dieser Daten sind ohne einschränkende Bedingungen bereits allgemein zugänglich. Die Weiterverwendung solcher Informationen kann von entscheidender Bedeutung für die Entwicklung neuer Technogien, wie z.B. von KI-Anwendungen sein, welche die Verarbeitung großer Mengen hochwertiger Daten erfordern.

Österreich hat mit https://www.data.gv.at/ bereits seit einigen Jahren einen zentralen „Österreich“Katalog, der die Metadaten der dezentralen Datenkataloge der Verwaltung in Österreich aufnimmt und abrufbar macht. Die auf data.gv.at bereitgestellten Datensätze beinhalten sowohl Open GovernmentInformationen (OGD) als auch Informationen nach der Public Sector Information-Richtlinie über die Weiterverwendung von Informationen des öffentlichen
Sektors (IWG). ${ }^{64}$ Durch den offenen und strukturierten Zugang soll die Weiterentwicklung der Wissensgesellschaft gefördert werden, da sich mit diesen Daten beispielsweise Softwarelösungen (wie z.B. Apps) erstellen lassen, die der Allgemeinheit dienen oder bei denen durch Verknüpfen verschiedener Daten ein informativer und auch ökonomischer Mehrwert geschaffen wird.

Seit dem Start von data.gv.at im Jahr 2012 verantwortet das Bundesrechenzentrum (BRZ) im Auftrag der Cooperation OGD Österreich ${ }^{65}$ den Betrieb der Plattform und die Umsetzung der technischen Weiterentwicklungen. Dabei kommen hauptsächlich Open Source-Komponenten zum Einsatz. data.gv.at wurde 2014 als Sieger in der Kategorie „Improving the Delivery of Public Services" von den Vereinten Nationen mit dem UN Public Service Award ausgezeichnet und hat im Durchschnitt pro Tag 1.200 Webseitenbesucher, die auf über 20.700 Datensätze und 450 Anwendungen zugreifen können. Die nächste Ausbaustufe findet im Frühjahr 2019 statt, wo die Plattform um Datensätze zu Ausschreibungen der öffentlichen Verwaltung erweitert wird. Ziel ist, die Transparenz in öffentlichen Beschaffungsvorgängen signifikant zu erhöhen.

\subsubsection{Digitalisierung von Forschungsdaten aus Archiven, Sammlungen und Museen bzw. historischen Bibliotheken}

Wissenschaftliche Einrichtungen, Archive und Gedächtnisorganisationen generieren entweder analoge oder inzwischen vermehrt digitale Forschungsdaten. Damit diese Daten breit nutzbar sind, wurden in den letzten Jahren zahlreiche Digitalisierungsinitiativen finanziert, unterstützt und angeregt. Zentral hierfür sind nationale und internationale Forschungsinfrastrukturen und übergreifendes Forschungsda-

63 Siehe hierzu Leitkonzept für eine innovationsfördernde öffentliche Beschaffung: https://www.ffg.at/sites/default/files/images/ Beschaffung/leitkonzept_ioeb-1.pdf

sowie: https://www.usp.gv.at/Portal.Node/usp/public/content/news/ueber_das_usp/407001.html

$64 \mathrm{Vgl}$. https://www.digitales.oesterreich.gv.at/public-sector-information-richtlinie

65 Das Bundeskanzleramt, die Städte Wien, Linz, Salzburg und Graz gründeten am 13.07.2011 gemeinsam die „Cooperation Open Government Data Österreich“, kurz „Cooperation OGD Österreich“. https://www.data.gv.at/infos/cooperation-ogd-oesterreich/ 
tenmanagement. So hat auch das European Strategy Forum on Research Infrastructures (ESFRI) ${ }^{\text {b6 }}$ einen langfristigen Prozess zur Etablierung von europäischen Forschungsinfrastrukturen entwickelt. Diese werden in Form von European Research Infrastructure Consortia (ERIC) auf Antrag und durch eine Entscheidung der Europäischen Kommission dauerhaft institutionalisiert. In 2019 ist Österreich an insgesamt 13 ESFRI-Infrastrukturen beteiligt (siehe hierzu auch Abschnitt 3.3.5).

Weiters nimmt das Wiener Wiesenthal Institut für Holocaust Studien am Horizon 2020 Projekt European Holocaust Research Infrastructure EHRI teil. ${ }^{67} \mathrm{EH}-$ RI bietet dabei Zugang zu fragmentierten, teils weit verstreuten und oftmals schwer zugänglichen Quellen zum Holocaust, insbesondere in Archiven.

Allgemein ermöglichen die Forschungsinfrastrukturen damit nicht nur die internationale Zusammenarbeit, sondern stellen eine wichtige Grundlage für die empirische, oftmals interdisziplinäre Forschung in den Natur-, Technik-, Lebens-, Sozial- und Geisteswissenschaften, sowie in Kunst und Design Studies dar.

\section{Digital Humanities und Digitales Kulturerbe}

Österreich verfügt über ein reichhaltiges Kulturerbe und konnte durch die Digitalisierung in den letzten Jahren in diesem Bereich internationale Anerkennung gewinnen. So hat beispielsweise die UNESCO die umfangreiche Tonbandsammlung heimischer Dialekte - viele davon sind nicht mehr existent - des Phonogramm-Archivs der Österreichischen Akademie der Wissenschaften (ÖAW) zum Weltdokumentener- be erklärt. ${ }^{68}$ Das Phonogrammarchiv ist, wie viele andere Forschungseinrichtungen im Bereich der digitalen Geisteswissenschaften, in das Netzwerk digitalhumanities.at ${ }^{69}$ eingebunden. Die ÖAW koordiniert zudem im Rahmen des Austrian Center for Digital Humanities ACDH das österreichische CLARIAH Konsortium zur Teilnahme an den Europäischen Forschungsinfrastrukturen CLARIN und DARIAH. Ein weiterer Fokus des ACDH liegt auf digitalen Editionen und den digitalen Archivierungsplattformen AR$\mathrm{CHE}^{70}$ und $\mathrm{GAMS}^{71}$.

Im März 2019 ist erstmals auch ein österreichisches Projekt aus dem Bereich Sozial- und Geisteswissenschaften als Anwärter auf ein FET-ForschungsFlaggschiff von der Europäischen Kommission in einem hoch kompetitiven Verfahren ausgewählt worden: Time Machine ${ }^{72}$. Das Konsortium wird von Österreich aus durch ICARUS ${ }^{73}$ geleitet und beteiligt weiters die Österreichische Nationalbibliothek, die TU Wien, und das niederösterreichische Landesarchiv sowie viele internationale Universitäten, Unternehmen, Archive, Bibliotheken und Forschungsinstitute. ICARUS ist ein gemeinnütziger Verein mit Sitz in Wien und besteht aus mehr als 160 archivarischen und anverwandten Institutionen aus mehr als 30 europäischen Ländern, Kanada und den USA. Im Rahmen der Time Machine sollen nun neue Formen der Digitalisierung und der künstlichen Intelligenz entwickelt und erprobt werden, um Europas außerordentliches kulturelles Erbe zu heben wie auch den offenen und kostenfreien Zugang zu Informationen zu ermöglichen. Erstmals sollen so bislang verstreute und komplexe historische Datenmengen zusammenge-

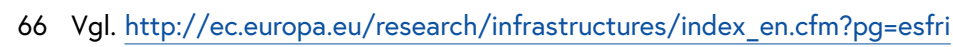

67 Vgl. https://www.ehri-project.eu/ und https://www.vwi.ac.at

68 Vgl. https://www.oeaw.ac.at/phonogrammarchiv/unesco/

69 Vgl. http://www.digital-humanities.at/ Dem Netzwerk gehören weiters an: Uni Innsbruck, TU Wien, Österreichische Nationalbibliothek, Universität Wien, TU Graz, Uni Graz, OFAl - Österreichisches Institut für Artificial Intelligence, Universität für Angewandte Kunst.

70 Vgl. https://www.oeaw.ac.at/acdh/tools/arche/

71 Vgl. http://gams.uni-graz.at/: Das Akronym GAMS steht für Geisteswissenschaftliches Asset Management System. Es dient zur Verwaltung nahezu beliebiger digitaler Ressourcen (vom Text bis zum Videofilm) und bietet Forscherinnen und Forschern die Möglichkeit, digitale Ressourcen zitabel und mit Metadaten versehen webbasiert zu speichern, zu verwalten und zu veröffentlichen.

72 Vgl. http://timemachine.eu/

73 Vgl. https://icar-us.eu/ 
führt werden. Inhalte reichen von mittelalterlichen Manuskripten bis hin zu historischen Objekten oder Smartphone- und Satellitenfotos. Ziel ist die Erstellung einer großfächigen digitalen Infrastruktur, die Europas gesamte soziale, kulturelle und geographische Evolution abbildet. In Anbetracht des schieren Ausmaßes und der Komplexität der Daten haben die für Time Machine entwickelten Künstliche Intelligenz-Technologien das Potenzial, Europa einen immensen Wettbewerbsvorteil im globalen Rennen um die Entwicklung von derartigen Technologien zu verschaffen. ${ }^{74}$

Die neuen Möglichkeiten für Synergien zwischen Kulturerbe und Wirtschaft können auch anhand des bereits seit 2008 bestehenden Projektes Europeana illustriert werden. Österreich beteiligt sich aktiv an Europeana, denn Digitalisierung von Kulturerbe bedeutet Zugang für Forschung, freie Nachnutzung für die Allgemeinheit und Möglichkeit wirtschaftlicher Nutzung beispielsweise durch Tourismus und Kreativwirtschaft. Europeana bietet mit Stand $2018 \mathrm{Zu}$ gang zu über 51 Mio. Objekten aus den Sammlungen von mehr als 3.700 Bibliotheken, Archiven, Museen, Galerien und audiovisuellen Sammlungen aus ganz Europa über das Hauptportal „Europeana Collections" (https://www.europeana.eu/) sowie über die Anwendungsprogrammierschnittstellen der Plattform. Über diese Schnittstellen können Daten auch weiterverwendet werden und es stehen Dienstleistungen für beitragende Kultureinrichtungen, Forschung, Bildung und Kreativwirtschaft, sowie interessierte Bürgerinnen und Bürger zur Verfügung. ${ }^{75}$ In Zukunft soll Europeana als kulturelles und digitales Innovationsprojekt weiter ausgebaut werden. Aus Österreich sind Inhalte speziell aus den Sammlungen der Österreichischen Nationalbibliothek, dem Kunsthistorischen Museum, dem Museum für Völkerkunde, dem Theatermuseum, der Österreichischen Media- thek von Seiten des Technischen Museums, dem Filmarchiv Austria, der Gesellschaft für Historische Tonträger, dem ORF und vieles mehr über Europeana abrufbar. $^{76}$

Die öffentliche Zugänglichkeit zu digitalem Kulturerbe ist in Österreich allerdings noch nicht ausreichend gewährleistet. In einer 2017 erschienenen Studie $^{77}$ gaben 193 österreichische Organisationen an, ihre Sammlungen zu digitalisieren, allerdings sind die Objektdatenbanken nur von 50 Einheiten auch öffentlich zugänglich. Der Mittelwert des Digitalisierungsgrades der österreichischen Museen beträgt $43 \%$. Im Vergleich zur Museumsstatistik 2014 sind die Zahlen leicht angestiegen, so werden nun auch soziale Medien vermehrt genutzt. Einige Museen (10\%) bieten ihre Inhalte auch bereits in Form einer App an, hierbei führend sind kulturgeschichtliche, historische und archäologische Museen.

Digitalisierungsstrategien variieren je nach Typus der Organisation und vor allem je nach Datenart: Archivalien, Museumssammlungen und museale Objekte, historische Bücher erfordern jeweils andere Herangehensweisen und eignen sich in unterschiedlichem Maße als Forschungsdaten. Außerdem erfordern sie jeweils andere Speicher- und Rechenleistungen. Die Frage nach der langfristigen Speicherung und damit nach der Kuratierung des digitalen Kulturerbes wird somit immer wieder aufs Neue gestellt.

\subsubsection{Resümee}

Der öffentliche Sektor in Österreich ist in einigen Digitalisierungs-Bereichen bereits federführend, mehrheitlich zeigt er sich jedoch im Mittelfeld im europäischen Vergleich. Die Bemühungen um Digitalisierung und damit einhergehend zur Bereitstellung von öffentlichen Daten, wie z.B. unserem digitalen Kulturerbe, sind stetig aber noch nicht genug. Leuchtturm-

74 Vgl. https://icar-us.eu/en/pressebereich/pressetexte/

75 Vgl. https://publications.europa.eu/en/publication-detail/-/publication/58538a59-b4aa-11e8-99ee-01aa75ed71a1/language-en

$76 \mathrm{Vgl}$. https://www.kunstkultur.bka.gv.at/europeana-und-die-digitalisierung-des-kulturellen-erbes

77 Quelle: Statistik Austria, Wirkungsanalyse/MÖ; Anmerkung: 296 Einheiten (= 372 Museen) haben die Frage nach dem Digitalisierungsgrad beantwortet. Siehe dazu: http://www.museen-in-oesterreich.at/_docs/_statistiken/de/Museumsbund_Oesterreich_Zur_Lage_der_oesterreichischen_Museen.pdf 
projekte wie Europeana oder Time Machine an der Schnittstelle zwischen den Geistes- und Sozialwissenschaften und der Gesellschaft bzw. Wirtschaft können hier Vorbildwirkung zeigen. Robuste und skalierbare Infrastrukturen sind für jegliche Digitalisierungsmaßnahmen im öffentlichen Sektor zentral, so auch für die digitalen Services im Rahmen der neu geschaffenen E-Governance Strukturen.

Der Begriff „E-Government“ steht hierbei für eine moderne und effiziente Verwaltung. Um in den TopBereich der führenden Digitalnationen aufzusteigen, wird es notwendig sein, in den nächsten Jahren den
Weg weiterhin konsequent zu verfolgen, und neben den Bürger- und Unternehmensservices vor allem die E-Demokratie und die öffentlichen Beteiligungsformen zu verbessern und zu erweitern. Neben dem Ausbau von Open Government Data und der dazugehörigen Stärkung der Transparenz in Politik und Verwaltung - beides Grundvoraussetzung für Partizipation - können nun die verstärkte Einbindung von Bürgerinnen und Bürgern in politische Entscheidungsprozesse, sowie die Bereitstellung neuer Kommunikationskanäle, wie z.B. elektronische Konsultations- und Petitionsprozesse, angestrebt werden. 
5. FTI-Evaluierungs-
kultur und -praxis 
Zur Reflexion, Bewertung und Weiterentwicklung von Maßnahmen, Instrumenten und Praktiken der FTI-Politik sind Evaluierungen ein unverzichtbarer Teil einer lernenden und strategisch orientierten FTI-Politik. Im österreichischen Forschungs- und Technologiebericht $2017^{1}$ wurde über den Stand der Evaluierungspraxis im FTI-Bereich in Österreich bereits eine umfassende Einschätzung gegeben, die auch im aktuellen Review der OECD über Österreichs FTI-Politik ${ }^{2}$ übernommen und bestätigt wurde. Zusammenfassend kann festgehalten werden, dass in den letzten 15 Jahren ein erheblicher Anstieg der FTI-Bewertungsaktivitäten zu verzeichnen war. Ebenso hat eine Professionalisierung der FTI-Evaluierungspraxis, begleitet von einem Aufbau von Kompetenzen und Kapazitäten, stattgefunden. Zusammengefasst wurde eine verantwortungsbewusstere Evaluierungskultur im FTI-Bereich entwickelt. Ausdruck dieser geteilten Verantwortung ist die Weiterentwicklung der Österreichischen Plattform für Forschungs- und Technologiepolitikevaluierung (fteval), deren Mitglieder Ministerien, Agenturen, Forschungsinstitute und Beratungsunternehmen sind. Die Plattform bietet Diskussionsforen, entwickelt Publikationen, führt Schulungen und Workshops durch und hat 2017 ein öffentlich zugängiges Repositorium über FTI-politische Studien und Evaluierungen eingerichtet.

\subsection{Aktuelle Entwicklungen}

Um den Datenzugang für Forschung zu erleichtern, wurde in Österreich 2018 die Plattform Registerforschung gegründet (Abschnitt 5.1.1). Eine weitere strukturelle Entwicklung war die Erstellung neuer Standards der Evaluierung in der Forschungs-, Technologie- und Innovationspolitik. Darüber wird im Abschnitt 5.1.2 berichtet. Abschnitt 5.1.3 bietet eine Zusammenfassung über die Österreichische Ratsprä- sidentschaftskonferenz "Impact of R\&I Policy at the Crossroads of Policy Design, Implementation and Evaluation". Wie gewohnt wird über wichtige FTIEvaluierungen, die im Jahr 2018 durchgeführt bzw. abgeschlossen wurden, im Abschnitt 5.2 berichtet.

\subsubsection{Die Österreichische Plattform Registerforschung}

Im OECD-Review wird als strukturelle Schwachstelle des österreichischen Evaluierungssystems auf die begrenzte Verfügbarkeit bzw. Zugänglichkeit und Vernetzbarkeit statistischer Daten bei öffentlichen Stellen wie Ministerien, Fördereinrichtungen sowie der Statistik Austria ausführlich Bezug genommen. Die derzeitige Praxis begrenzt u.a. den Zugriff auf unternehmensspezifische Roh- und Mikrodaten und den Spielraum für den Abgleich einzelner Datenquellen. Damit gehen methodische Beschränkungen in Bezug auf robuste Kontrollgruppenansätze einher, die Aussagen über Wirkungen FTI-politischer Interventionen erheblich erschweren.

Vor diesem Hintergrund hat sich im Jahr 2018 in Österreich die Plattform Registerforschung konstituiert, die sich dafür einsetzt, der wissenschaftlichen Forschung Zugang zu Daten aus öffentlichen Registern zu bieten und dafür die notwendigen rechtlichen, organisatorischen und ethischen Rahmenbedingungen mitzugestalten. ${ }^{3}$ Der rechtliche Rahmen für die Forschung mit Registern wird im ForschungsOrganisationsgesetz (FOG) in der novellierten Fassung von $2018^{4}$ definiert. Durch das Gesetz werden aber noch keine Register tatsächlich freigegeben, sondern es wird der Rahmen für den Zugang zu Registerdaten des öffentlichen Sektors geschaffen, um Forschungsfragen beantworten zu können. Dies ist jedoch mit einer Reihe von Herausforderungen verbunden: die Novellierung des Bundesstatistikgesetzes; die Öffnung von Registerdaten zu wissenschaft-

Vgl. BMBWF et al. (2017).

Vgl. OECD (2018a).

Vgl. https://www.registerforschung.at/mission

Vgl. https://www.ris.bka.gv.at/GeltendeFassung.wxe?Abfrage=Bundesnormen\&Gesetzesnummer=10009514 
lichen Zwecken, die im Detail zu regeln ist; und es bedarf für jeden einzelnen Datenbestand einer Verordnung der zuständigen Ministerien. Auch fehlt es weiterhin an geeigneten Werkzeugen, wie z.B. eines sicheren Online-Dienstes, der Fernzugriff auf anonymisierte Unternehmensdaten ermöglicht.

\subsubsection{Die neuen Standards der Evaluierung} in der Forschungs-, Technologie- und Innovationspolitik

Die Standards der Plattform für Forschungs- und Technologiepolitikevaluierung (fteval) wurden erstmals 2003 veröffentlicht, legen wesentliche Eckpunkte und Prozessmeilensteine für die Evaluierung von FTI-Politik dar und dienen ihren Mitgliedern als verbindliche Leitlinie ("Code of Conduct"). Sie bieten beauftragenden Einrichtungen, Evaluatorinnen und Evaluatoren sowie den Betroffenen einer Evaluierung einen Verhaltensrahmen und eine Handlungsanleitung, wie "gute" Evaluierungen geplant, gemanagt, durchgeführt und genutzt werden können. Damit tragen sie zu einer besseren Evaluierungskultur bei, die gleichsam Voraussetzung und Folge einer relevanten, effektiven, effizienten und transparenten Politik ist.

Sechs Jahre nach dem letzten Update der Standards für Forschungs- und Technologiepolitikevaluierung durch die fteval wurde 2017 beschlossen, dass eine Revision der Standards neuen Entwicklungen im FTI-Politikevaluierungsbereich Rechnung tragen soll. Dazu zählen:

- Geänderte Rahmenbedingungen, wie die Einführung der wirkungsorientierten Haushaltsführung ${ }^{5}$ (performance und gender budgeting ${ }^{6}$ ) sowie regulativer Verankerungen von Evaluierungen im FTIBereich7;

- Der in der österreichischen FTI-Praxis vollzogene Wandel von einer pionierhaften, experimentellen zu einer reifen Evaluierungskultur, die von einer hohen Zahl an Evaluierungen geprägt ist ${ }^{8}$;

- Eine zunehmende Hinwendung zu ex-post Wirkungs- und ex-ante Designevaluierungen, die Prozessevaluierungen, die in den 2000 er Jahren überwogen haben, schrittweise ergänzen;

- Eine Ausweitung der Evaluierungsgegenstände weg von Evaluierungen einzelner Programme hin zu größeren Portfolioevaluierungen (z.B. Evaluierung aller internationaler Programme des FWF) und anderen FTI-politischen Interventionen wie Institutionen (z.B. Evaluierung von aws und FFG), Instrumenten (z.B. Forschungsprämie) und Regulationen ( $z$.B. innovationsfördernde öffentliche Beschaffung);

- Höhere Anforderungen an ethische Standards in Bezug auf Transparenz, Unabhängigkeit, Unparteilichkeit, Glaubwürdigkeit, Partizipation, Schutz persönlicher Daten und Forschungsintegrität.

Ausdruck dieser Veränderungen war, dass im Zuge der gemeinschaftlichen Erstellung der neuen Evaluierungsstandards auch das Missionsstatement der fteval angepasst wurde. Während dieses früher an die Notwendigkeit der Schaffung einer Evaluierungskultur und der Durchführung von mehr, transparenteren und besseren Evaluierungen im engeren F\&E-Bereich appelliert hat, trägt das neue Missionsstatement der Selbstverständlichkeit von Evaluierung als wichtiger Bestandteil einer entwickelten und inklusiven FTIPolitik Rechnung:

„Die Mission der Österreichischen Plattform für Forschungs- und Technologiepolitikevaluierung ist es, die Qualität, die Transparenz und einen adäquaten Deckungsgrad von Evaluierungen für eine bessere strategische Planung der FTI-Politik in Österreich zu erreichen. Daher wird gemeinsam mit den österreichischen forschungs-, technologie- und innovationspolitischen EntscheidungsträgerInnen die

5 Vgl. BMF (2011).

6 Vgl. Steger (2012).

7 Zum Beispiel die überarbeiteten FTI-Richtlinien aus dem Jahr 2015 oder die institutionellen Standards des FWF: https://www.fwf. ac.at/de/forschungsfoerderung/entscheidung-evaluation/evaluationsstandards/evaluation-im-fwf/die-evaluierung-des-fwf/

8 Vgl. Tsipouri und Sidiropolous (2013). 
bestehende Evaluierungskultur stetig weiterentwickelt." "9

Zur Überarbeitung der Standards wurden seitens der fteval externe Begleitung und Expertise hinzugezogen, die in einem zweistufigen Prozess zunächst die Grundprinzipien der neuen Standards erarbeitet hat. Diese wurden von einer Begleitgruppe der fteval reflektiert und modifiziert wie auch in einem onlineGruppendiskussionsverfahren kommentiert und überarbeitet. Der zweistufige Prozess dauerte ca. sechs Monate. Danach erfolgte eine finale Qualitätssicherung, sodass die Standards im Herbst 2018 von der Generalversammlung der fteval angenommen und verabschiedet wurden. Die neuen Standards der fteval haben die Evaluierungsdefinition von der im Jahr 2016 finalisierten Überarbeitung der DeGEvalStandards übernommen ${ }^{10}$, die eine Evaluierung zusammenfasst als "systematische Untersuchung von Nutzen und/oder Güte eines Gegenstands (Evaluationsgegenstand) auf Basis von empirisch gewonnenen Daten. ([Dies] - Einfügung durch Autor) impliziert eine Bewertung anhand offengelegter Kriterien für einen bestimmten Zweck." 11

Neben der Berücksichtigung der oben genannten Entwicklungen sollten die neuen Standards auch mehr Handlungsanleitung geben, um Evaluierungsprozesse weniger störanfällig zu machen. Ein besonderer Schwerpunkt lag dabei auf Aspekten der professionellen Formulierung von Terms of References (ToRs), klaren konzeptionellen und begrifflichen Grundlagen (inkl. Glossar), einer verbesserten Arbeitsteilung zwischen Monitoring und externer Evaluierung, sowie der Erstellung von Prinzipien, die quasi als Kurzfassung der Standards Handlungsorientierung bei der Konzeptionierung, der Durchführung und der Nutzung von Evaluierungen bieten.

Erstmalig wurde in den fteval-Standards auch die besondere Bedeutung von Evaluierung in der Forschungs-, Technologie- und Innovationspolitik begründet und hergeleitet. Dabei wurden neben Aspekten des Markt-, System- und Politikversagens auch die Bedürfnisse eines zielorientierten, transformativen Wandels in Innovationssystemen zur Legitimation FTI-politischen Handelns vor dem Hintergrund großer globaler und gesellschaftlicher Herausforderungen berücksichtigt. ${ }^{12}$

Neuerungen betreffen u.a. eine verstärkte horizontale Betonung von Genderdimensionen in FTI-politischen Evaluierungsprozessen, die obligatorische Veröffentlichung von FTI-Politikevaluierungsberichten im öffentlich zugänglichen Repositorium ${ }^{13}$ der fteval nach Abnahme durch den Auftraggeber, die Anregung zur Einführung eines Management ResponseSystems, sowie zahlreiche ethische und prozedurale Hinweise zur Umsetzung eines möglichst effizienten, transparenten und effektiven Evaluierungsprozesses.

Die neuen Standards der fteval stehen kostenlos in deutscher und englischer Sprache auf der Homepage der fteval zum Download ${ }^{14}$ zur Verfügung.

\subsubsection{Die Österreichische \\ Ratspräsidentschaftskonferenz „Impact of R\&I Policy at the Crossroads of Policy Design, Implementation and Evaluation"}

\section{Verstärktes Augenmerk auf Impact-Evaluierung der FTI-Politik \\ Die meisten Wirkungsevaluierungen FTI-politischer Interventionen stellen entweder die wissenschaftlich- technischen Wirkungen oder die ökonomischen Wir- kungen in den Mittelpunkt. Dafür wurden in den letz- ten Jahrzehnten geeignete Indikatoren, Datengrund- lagen und Methoden geschaffen und laufend}

9 Vgl. https://www.fteval.at/content/home/plattform/about/

10 Die fteval ist institutionelles Mitglied der DeGEval.

11 Vgl. https://www.degeval.org/glossar-der-standards-fuer-evaluation/

12 Vgl. Boon und Edler (2018); Forray et al. (2012); Kuhlmann und Rip (2014); Mazzucato (2011); Weber und Rohracher (2012).

13 Vgl. https://repository.fteval.at/

14 Vgl. https://www.fteval.at/content/home/plattform/standards/ 
weiterentwickelt. Die Nachvollziehbarkeit und Einschätzung sozialer bzw. gesellschaftlicher Wirkungen FTI-politischer Interventionen hingegen erlebt erst seit kurzem eine neue Aufmerksamkeit. Ursache dafür ist die Beschäftigung der FTI-Politik mit den großen gesellschaftlichen Herausforderungen (sogenannte neue missionsorientierte FTI-Politik) ${ }^{15}$, um mittels geeigneter forschungs-, technologie- und innovationspolitischer Maßnahmen einen Beitrag zur Lösung derselben leisten zu können. Der von der Europäischen Kommission für „Horizon Europe“, dem 9. Europäischen Rahmenprogramm für Forschung und Innovation, vorgeschlagene Missionszugang innerhalb der zweiten Säule des nächsten Rahmenprogramms sieht explizit eine überprüfbare Zielerreichung für die noch auszuwählenden konkreten F\&E-Missionen vor. Für diese Missionen als auch für die in Horizon Europe wie schon in Horizon 2020 - postulierte Bewältigung globaler Herausforderungen ist die soziale Wirkungsdimension überaus relevant, da sie explizit mit den von der Gesellschaft festgelegten Zielen (zum Beispiel den Zielen für nachhaltige Entwicklung der Vereinten Nationen/SDGs ${ }^{16}$ ) in Beziehung steht.

Zur besseren Verfolgung und Messung der Wirkungsdimensionen von Horizon Europe wurde unmittelbar nach der Veröffentlichung des Vorschlags der Europäischen Kommission für Horizon Europe im Juli 2018 ein Expertenbericht ${ }^{17}$ vorgelegt, der die folgenden drei Wirkungsdimensionen unterscheidet: (1) wissenschaftliche Auswirkungen, (2) gesellschaftliche Auswirkungen und (3) ökonomische Auswirkungen. Schon im Jahr zuvor hat eine ERAC ad-hoc Arbeitsgruppe einen Report vorgelegt, der ebenfalls für eine Berücksichtigung unterschiedlicher Wirkungsdimensionen argumentiert ${ }^{18}$, den Fokus aber auf die Messung der Wirkungen europäischer Rahmenprogramme auf nationaler Ebene gelegt hat.

\section{Beauftragung und Durchführung einer internationalen Konferenz in Wien}

Die Entwicklungen auf europäischer Ebene antizipierend wurde im Jahr 2017 angeregt, unter der Schirmherrschaft des BMVIT und mit Unterstützung des BMBWF und des BMDW eine Veranstaltung im Rahmen der österreichischen Ratspräsidentschaft zur Messung missionsorientierter FTI-Interventionen durchzuführen. Beauftragt damit wurde die Österreichische Plattform für Forschungs- und Technologiepolitikevaluierung (fteval), die die Veranstaltung im November 2018 gemeinsam mit dem Manchester Institute of Innovation Research und dem Institut Francilien Recherche, Innovation et Société aus Paris organisiert hat. Ausgangspunkt für die inhaltliche Planung der Veranstaltung war, dass erstens nicht nur die europäische, sondern auch die nationale FTI-Politik mehr denn je dazu angehalten wird, gesellschaftliche Beiträge zu leisten und entsprechende Auswirkungen zu dokumentieren, und dass zweitens die neue Wirkungsagenda Auswirkungen auf den gesamten Politikzyklus, also auf die Politikgestaltung, die Politikumsetzung und die Politikbewertung, hat. Für die Konferenz haben sich 296 Expertinnen und Experten aus 39 Ländern angemeldet; 255 davon haben tatsächlich an der Konferenz teilgenommen.

\section{Thematischer Hintergrund}

Sowohl die Präsidentschaftsveranstaltung als auch der Expertenreport der Europäischen Kommission haben das Konzept der Wirkungspfade zur weiteren Diskussion der Messung der drei unterschiedlichen Wirkungsdimensionen gewählt, um den Gestaltungsund Prozesscharakter von Wirkungsschaffung und Wirkungsentfaltung zu betonen. Hierbei sind insbesondere die Wirkungspfade zur Messung gesellschaftlicher Wirkungen herausfordernd. Diese sehen

\footnotetext{
5 Vgl. Gassler et al. (2006).

Vgl. https://sustainabledevelopment.un.org/?menu=1300

Vgl. Van den Besselaar et al. (2018).

Vgl. ERAC (2017).
} 
sich mit grundlegenden definitorischen Problemen konfrontiert. Während "soziale Wirkung" im EU-Kontext als Überbegriff verstanden wird (z.B. im Falle der "Better Regulation Toolbox" der Europäischen Kommission), der Auswirkungen auf die Gesellschaft, Politik, Umwelt, Wirtschaft und auf andere Dimensionen impliziert, wird "gesellschaftliche Auswirkung" als spezifischeres Konzept verstanden. Auch die in der wissenschaftlichen Literatur üblicherweise verwendeten Ansätze und Modelle zur Fassung sozialer Auswirkungen von FTI-politischen Maßnahmen verweisen auf eine Vielzahl von Themen, einschließlich der Auswirkungen auf die Politik, und lassen klare Abgrenzungen vermissen ${ }^{19}$. Vorhandene Bewertungen der gesellschaftlichen Wirkung FTI-politischer Interventionen sind daher bislang oft nur kontextual und spezifisch sowie qualitativ und anekdotischer Natur.

Neben den theoretischen Abgrenzungsproblemen gibt es gravierende Defizite in Bezug auf die Indikatorik zur Bewertung gesellschaftlicher Wirkungen sowie einen Mangel an systematisch erhobenen, qualitätsgesicherten Daten. Überdies liegt häufig eine fälschliche Gleichsetzung gesellschaftlicher Wirkung mit Verbreitung (Disseminierung) oder Übertragung (Transfer) vor, auf die sich die meisten der sogenannten alternativen Metriken (,altmetrics“) fokussieren. Zu den besonderen Herausforderungen für die Entwicklung geeigneter Indikatoren zur Messung gesellschaftlicher Wirkungen zählen,

1. dass die Zeit zur Erreichung der tatsächlichen Auswirkungen auf die Gesellschaft länger ist als die Erzielung konkreter Ergebnisse;

2. dass die Zuordnung sozialer Veränderungen schwieriger ist als die Zuordnung wissenschaftlicher Referenzen oder ökonomischer Attribute;

3. dass die Verfügbarkeit und Vergleichbarkeit der Daten zur Verfolgung der Auswirkungen in Gesellschaft und Politik stark limitiert sind.
Der Literaturübersicht aus dem Expertenbericht der Europäischen Kommission ${ }^{20}$ zufolge sind spezifische und häufig verwendete Indikatoren zur Messung gesellschaftlicher Wirkung nahezu nicht vorhanden, oder wenn, dann oft nur als Vorschläge ohne systematische Anwendung ${ }^{21}$. Es ist daher auch wenig verwunderlich, dass die meisten Agenturen und Evaluierungsvorhaben gesellschaftliche Auswirkungen von FTI nicht berücksichtigen. In wenigen Fällen wird gesellschaftliche Wirkung in ex-ante Evaluierungen mitunter als zu berücksichtigendes Kriterium genannt, jedoch ohne spezifische Indikatoren dafür angeben zu können.

\section{Ausrichtung der Konferenz}

Die österreichische Ratspräsidentschaftskonferenz "Impact of Research and Innovation Policy at the Crossroads of Policy Design, Implementation and Evaluation" hat sich daher der Frage gewidmet, wie Wirkungen entlang der drei oben genannten Wirkungsdimensionen durch eine förderliche FTI-Politik besser gestaltet, konzipiert und gemessen werden können. Die Konferenz hat dabei das Thema Wirkungsevaluierung in vier Blöcken strukturiert:

1. Das Wesen wirkungsorientierter FTI-Politik;

2. Gestaltung, Implementierung und Unterstützungsmaßnahmen für eine wirkungsorientierte FTI-Politik;

3. Neuartige Konzepte, Werkzeuge und Methoden zur Einschätzung gesellschaftlicher Wirkung von FTI-politischen Maßnahmen und

4. Effekte von Wirkungsevaluierungen auf das Politiklernen.

Diese Themenblöcke wurden in fünf Keynote-Präsentationen, vier Paneldiskussionen, sieben spezifischen Fachsessionen, in denen 40 ex-ante ausgewählte Artikel präsentiert wurden, drei Workshops, einer Fallstudie über Wirkungsmessung beim französischen Nationalinstitut für landwirtschaftliche Forschung

19 Vgl. Brewer (2011) und (2013); Flecha (2018); Raua et al. (2018); Reale et al. (2017).

20 Vgl. Van den Besselaar et al. (2018).

21 Vgl. Barré (2010); Reale et al. (2017). 
und einer Postersession, in der elf Posters vorgestellt wurden, adressiert.

\section{Ergebnisse}

Das Feedback auf die Konferenz war durchwegs positiv. $93 \%$ gaben an, dass die Organisation sehr gut bzw. gut war; 97 \% würden die Konferenz weiterempfehlen.

Insgesamt hat die Konferenz die folgenden unmittelbaren Resultate erbracht:

- Der Wissensstand über Wirkungsevaluierungen im FTI-Bereich wurde breit konsolidiert.

- Aktuelle Experimente zur Beförderung wirkungsförderlicher Politiken und Maßnahmen wurden zur Diskussion gestellt.

- Methodologische Erfahrungen zur besseren Einschätzung gesellschaftlicher Auswirkungen einer missionsorientierten FTI-Politik wurden umfassend ausgetauscht.

- Indikatoren, um den Fortschritt auf den wichtigsten Wirkungspfaden oder die tatsächliche Wirkungserreichung kurz-, mittel- und langfristig zu messen, wurden vorgestellt und reflektiert.

- Die verstärkte Anwendung umfassenderer Wirkungsevaluierungsansätze im Bereich der FTI-Politik wurde angeregt.

- Die Notwendigkeit der Nutzung eindeutiger Identifikatoren und der Schaffung besserer Datengrundlagen wurde diskutiert.

- Es erfolgte eine Sensibilisierung für die Nutzung und die Auswirkungen von Big-Data-Ansätzen und künstlicher Intelligenz für Text-Mining, automatisiertes Sammeln von Daten und automatisierte Datenanalysen.

- Die Notwendigkeit eines klaren Erwartungsmanagements wurde erkannt.

- Es wurde von mehreren Seiten anerkannt, dass für die Wirkungsmessung sowohl Forschungsorganisationen als auch Agenturen selbst entsprechen- de prozedurale Vorkehrungen zur Unterstützung gesellschaftlicher Wirkungen und zur Dokumentation derselben einrichten sollen.

\subsection{Ausgewählte Evaluierungen}

Evaluierungen von Förderprogrammen, -initiativen und -instrumenten nehmen in der österreichischen FTI-Politik wie auch in der Community einen wichtigen Stellenwert ein. Standard ist, dass die Evaluierungen von unabhängigen Expertinnen und Experten mit langjähriger Evaluierungserfahrung und einschlägigem Fachwissen durchgeführt werden. Um einen Einblick in die jüngsten Evaluierungen zu erhalten, werden infolge die Ergebnisse der rezentesten Evaluierungen kurz zusammengefasst.

\subsubsection{Beyond Europe}

Das „Beyond Europe“-Programm des Bundesministeriums für Digitalisierung und Wirtschaftsstandort (BMDW), das vonseiten der österreichischen Forschungsförderungsgesellschaft (FFG) umgesetzt wird, unterstützt Forschungs- und Innovationskooperationen österreichischer Unternehmen mit außereuropäischen Partnern. Den Hintergrund des „Beyond Europe"-Programms bilden die Empfehlungen zu „Beyond Europe - Die Internationalisierung Österreichs in Forschung, Technologie und Innovation über Europa hinaus“22 der Arbeitsgruppe 7a an die Task Force FTI der Bundesregierung, die im Rahmen der Umsetzung der FTI-Strategie im Juli 2013 erarbeitet wurden. Das Programm ist thematisch wie geographisch offen, wobei in Bezug auf die Zielländer auf die Prioritätenlisten der Empfehlungen zur „Beyond Europe"-Strategie hingewiesen wird. Mit dieser spezifischen Ausrichtung (thematische Offenheit, industrienahe und unternehmensgetriebene Forschungs- 
kooperationen mit Regionen außerhalb Europas) ist das "Beyond Europe“-Programm in der österreichischen Förderlandschaft einzigartig.

Bislang fanden drei Ausschreibungen in den Jahren 2015, 2017 und 2018/1923 statt, in denen jeweils die beiden Instrumente „Sondierungen“ und „F\&E Kooperationen - experimentelle Entwicklung" angeboten wurden.

\section{Zentrale Evaluierungsergebnisse}

Das Zentrum für Soziale Innovation (ZSI) wurde im Juli 2018 mit der Durchführung einer Zwischenevaluierung ${ }^{24}$ nach der zweiten Ausschreibung betraut. Zur Beantwortung der Evaluierungsfragen wurden verschiedene Inhalte und Arbeitsschritte sowie eine Mischung aus qualitativen und quantitativen Methoden angewendet.

In Summe zeigen die durchgeführten Evaluierungsschritte und -methoden (Programmstatistik, Befragung der Teilnehmenden, Fokusgruppe, Interviews und Vergleich mit Programmen anderer europäischer Länder) ein in sich stimmiges Programm, das die ad- ressierte Zielgruppe erreicht und auf gutem Weg ist, die angestrebten Ziele zu erreichen. Abbildung 5-1 zeigt die außereuropäischen Länder, mit denen Österreich im Programm „Beyond Europe“ kooperiert im Vergleich mit Kooperationen in Horizon 2020 (dargestellt in \% aller Kooperationsprojekte).

Beim Gesamtbild ist eine Reihe von besonders positiven Ergebnissen hervorzuheben:

- Die Erfolgsquoten sind ebenso wie die Mischung aus kleinen und großen, erfahrenen und eher neuen Antragstellenden angemessen.

- Die geographisch offenen Ausschreibungen adressieren im Wesentlichen die Prioritätsländer der Empfehlungen zur „Beyond Europe“-Strategie (Ausnahmen stellen Russland und Israel dar).

- Die Initiative für die Antragstellung ging in den meisten Fällen von österreichischer Seite und dort wiederum meist von einem Unternehmen aus.

- Die Teilnehmenden äußern sich sehr zufrieden hinsichtlich des besonderen Know-hows der außereuropäischen Partner (Exzellenzziel), der Möglichkeit, neue Märkte zu erschließen (Marktziel), so-

\section{Abbildung 5-1: Kooperationsländer im Programm Beyond Europe}

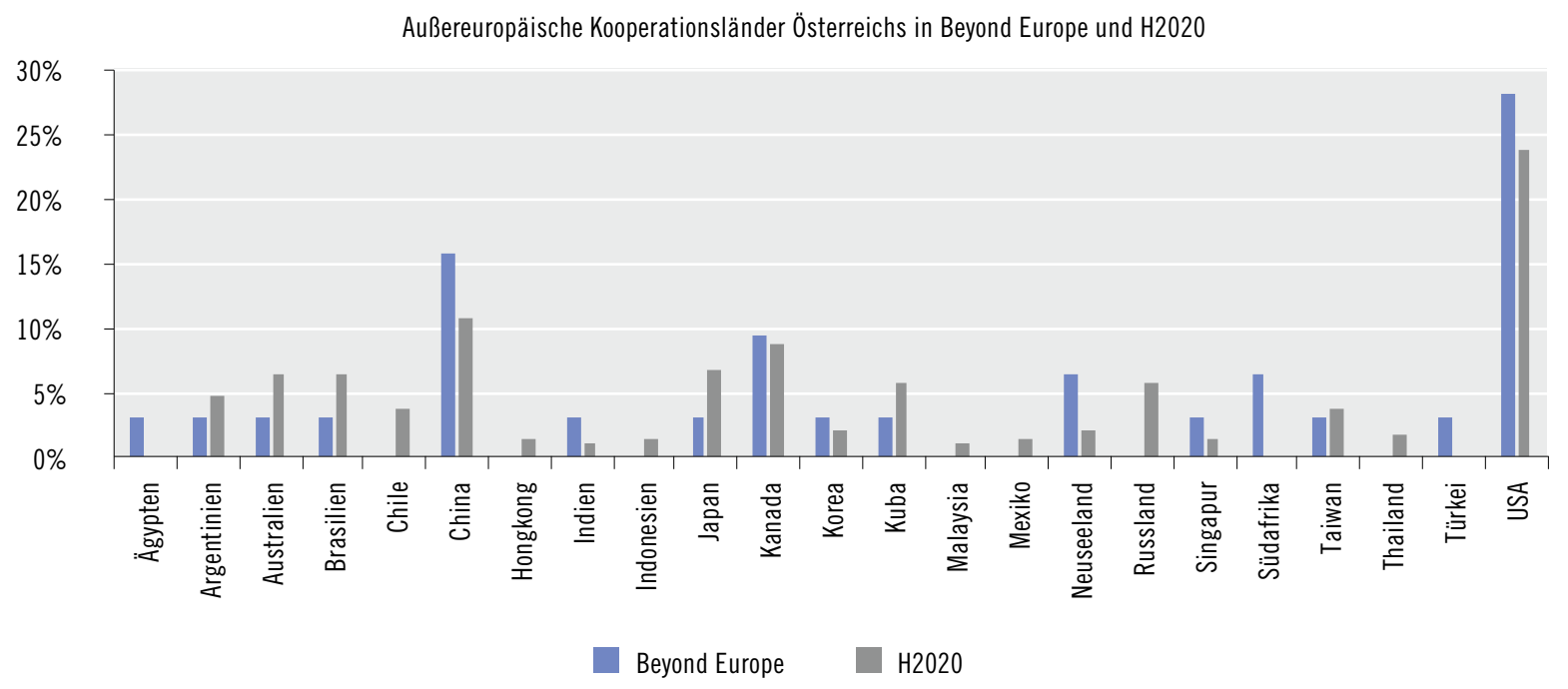

Quelle: FFG Programmstatistik, FFG EU PM Portal (Datenstand 29.9.2018).

23 Die dritte Ausschreibung war bis 13.03.2019 offen.

24 Vgl. Sturn et al. (2018). 
wie generell der Qualität der Zusammenarbeit. In einigen Fällen werden auch der Zugang zu besonderen Infrastrukturen und die Chance zum Adressieren gesellschaftlicher Herausforderungen positiv hervorgestrichen.

- Die Projekte sind sehr marktnah, in den meisten Fällen liegen bereits Ergebnisse vor.

- Die Zusammenarbeit mit den FFG-Mitarbeitenden wird sehr geschätzt.

- Die Additionalität ist sehr hoch und die Befragten (geförderte, aber auch nicht-geförderte) würden rückblickend mit überaus großer Mehrheit wieder einreichen.

Im Gegensatz dazu werden folgende Punkte kritisch gesehen:

- Ziele, Maßnahmen, erwartete Outcomes und Impacts sowie die angenommenen Wirkungszusammenhänge sind in den verschiedenen Dokumenten (Programmdokument, FFG-Homepage, verschiedene Kommunikationsformate) nicht konsistent dargestellt.

- Die Erfolgswahrscheinlichkeiten kleiner Unternehmen sind gering (allerdings nicht geringer als bei vergleichbaren FFG-Projekten); dies gilt ebenso für die Beteiligung von Frauen.

- Antragsaufwand und Bürokratie werden ebenso wie Verfahren und Management kritisch bewertet (dies betrifft die Sprache "Deutsch“ im Förderungsvertrag, lange Fristen, komplizierte Verfahren, mangelndes Feedback zum Antrag usw.). Vor allem trifft dies auf kleine Unternehmen und die Sondierungsprojekte zu.

Verbesserungen zum Feedback wurden vonseiten der FFG bereits umgesetzt. Auch wurden im Zuge der Vorbereitungen für die dritte Ausschreibung die Erfordernisse für außereuropäische Partner vereinfacht (bei den Sondierungsprojekten sind nunmehr "Letters of Intent" ausreichend). Dies sind wichtige Erleichterungen für die einreichenden Konsortien. Zusätzlich könnte überlegt werden, ob englischsprachige Förderungsverträge möglich sind, oder zumindest eine Standardvorlage, um den Übersetzungsaufwand jedes einzelnen Projektes zu verringern. In jedem Fall sollte vermieden werden, dass „Beyond Europe“ aufgrund administrativer Hürden für kleine Unternehmen unattraktiv wird. Ansonsten stünde das Programm in Gefahr, sein Alleinstellungsmerkmal als niederschwellige Alternative zu europäischen Programmen zu verlieren.

Insgesamt lassen sich aus der Bewertung der zentralen Charakteristika des Programms nur wenige Hinweise auf drängende Änderungserfordernisse ableiten, vielmehr konnten Evidenzen wie folgt für eine gesamthafte Bewertung des Programms „Beyond Europe" gefunden werden:

- Das themenoffene Format wird von allen Seiten geschätzt und auch die geographisch offenen Kooperationsmöglichkeiten werden angesichts immer weniger werdender Alternativen mehrheitlich positiv bewertet.

- Gezielte Calls mit ausgewählten Ländern oder Regionen könnten bei entsprechender Ausweitung des Programmbudgets ein Zusatzangebot darstellen. Im Rahmen bilateraler Kooperationen kann auch die Finanzierung über "Matching Funds“ intensiviert werden.

- Die Zulassung von Forschungseinrichtungen als Projektleitungen wie auch die Ermöglichung anderer Arten der FTI-Kooperation jenseits experimenteller Entwicklung wird vonseiten der Programmteilnehmenden mehrheitlich gewünscht, steht aber nicht an allererster Stelle der Änderungswünsche. Auch ist zu beachten, dass diesbezügliche Änderungen des Programmdesigns möglicherweise den in sich stimmigen Fokus auf marktnahe, unternehmensgetriebene Kooperationen verwässern.

- Eine Ausweitung der derzeit angebotenen Instrumente (beispielsweise um industrienahe Dissertationen) kann eine zusätzlich attraktive Einreichmöglichkeit darstellen, erhöht aber gleichzeitig die Komplexität des Programms. Ähnliches gilt für die Anregungen hinsichtlich eines zweistufigen Verfahrens.

- In der Programmkommunikation ist eine engere Zusammenarbeit mit WKÖ und IV nach dem Vor- 
bild der internationalen Beispiele („Business France“, "Team Sweden“, "Innovation Norway“) anzustreben, ebenso eng sollte mit den beiden OSTA-Büros in den USA und China kommuniziert werden.

- Für ergänzende multilaterale Kooperationen könnte EUREKA als flexibles Finanzierungsinstrument (auch durch die "Global Stars Initiative") vermehrt genutzt werden. Die internationalen Beispiele zeigen, dass andere Länder hier sehr gezielt vorgehen und ihre existierenden bilateralen Kooperationen in EUREKA einbringen. Eine intensivere Zusammenarbeit zwischen "Beyond Europe" und EUREKA sollte mit einer intensiveren bereichsübergreifenden Koordination innerhalb der FFG einhergehen.

Das Programm ist zu klein, um sehr große Sprünge zu wagen. Bei einer Budgetierung des Programms in der derzeitigen Höhe sollten keine Änderungen vorgenommen werden, die den Komplexitätsgrad weiter erhöhen oder den Fokus des Programms verändern. Ziel sollte eher sein, durch regelmäßige Ausschreibungen die Planungssicherheit zu erhöhen und den Aufwand der Einreichung (vor allem für die Sondierungen) zu verringern. Oder mit den Worten eines Interviewpartners: „Keep it going. Die Kontinuität ist wichtig, "Beyond Europe" sollte als dauerhaftes Element in der Förderungslandschaft etabliert werden."

Dieser Weg ist mit der dritten Ausschreibung auch eingeschlagen worden: Bei einem Budget in gleicher Höhe wie bei den vorangegangenen Ausschreibungen blieben die Regeln unverändert, allerdings gab es wichtige Vereinfachungen bei den Sondierungen.

\subsubsection{Global Incubator Network (GIN)}

Das Global Incubator Network (GIN) ist eine von der Nationalstiftung für Forschung, Technologie und Entwicklung finanzierte und von FFG und aws gemeinsam durchgeführte Initiative, die 2015 gestartet wurde, um die Positionierung Österreichs als internatio- nal attraktiven Gründungsstandort zu unterstützen. GIN umfasst sowohl Maßnahmen mit finanzieller Förderung für Start-ups (goAustria, goAsia) als auch Veranstaltungen und Vernetzungsaktivitäten, und richtet sich damit sowohl an Start-ups aus Österreich, die ihre Geschäftstätigkeit internationalisieren wollen, als auch an Start-ups aus anderen Ländern (insbesondere aus asiatischen Zielmärkten), die sich in Österreich ansiedeln und etablieren wollen. Ebenso angesprochen sind Investorinnen und Investoren wie Venture Capital Gebende und Inkubatoren. Investorinnen und Investoren bietet GIN z.B. die Internationalisierung bestehender Investments, den Zugang zu neuen Investitionsmöglichkeiten und die Vernetzung mit privaten und öffentlichen Co-Investoren. 2018 konzentrierte sich GIN dabei auf die ausgewählten Zielländer Israel, Südkorea, Japan, Hongkong und Singapur.

\section{Zentrale Evaluierungsergebnisse}

Im Frühjahr 2018 wurde das Programm „Global Incubator Network" durch die Convelop Cooperative Knowledge Design $\mathrm{GmbH}$ einer Zwischenevaluierung ${ }^{25}$ unterzogen - mit einem Schwerpunkt auf der Analyse des Zielsystems und der Interventionslogik, der GIN-Maßnahmen und ihres Beitrags zum Zielsystem sowie der Abschätzung von Effekten und Wirkungen. Die Evaluierung beruht auf einer Dokumenten- und Datenanalyse, sowie auf Interviews.

Die Interventionslogik des Programms geht davon aus, dass Start-up Unternehmen meist weder über die nötige Erfahrung, die Kontakte und Zugänge, noch über die nötigen finanziellen Mittel oder Möglichkeiten, diese zu besorgen, verfügen, um aus eigener Kraft Internationalisierungsaktivitäten (v.a. mit weit entfernten Märkten) erfolgreich umzusetzen. In Österreich existiert hierfür auch kein finanzkräftiger Private Equity Markt. Schließlich ist für Start-ups, Investorinnen und Investoren aus den Zielländern Österreich als Start-up Hub nur wenig sichtbar. Das Zielsystem von GIN ist bei näherer Betrachtung sehr vielfältig, die Ziele reichen von Vernetzung, Sichtbar-

25 Vgl. Handler et al. (2019). 
keit Österreichs als Innovations-/ Start-up Hotspot, über die Internationalisierung der Start-ups bis zur Stärkung des Entrepreneurial Eco-Systems und der Unterstützung von Wissens- und Technologietransfer. Die Vielzahl an Zielen steht in einem Spannungsfeld mit einer klaren Ausrichtung auf nachhaltige Wirkungen, die Initiative könnte davon profitieren, das aktuelle Zielsystem zu vereinfachen und hierarchisch zu gliedern.

Da GIN inklusive Konzeptions- und Anlaufphase zum Zeitpunkt der Evaluierung erst 2,5 Jahre aktiv war, prüfte die Evaluierung, ob die ersten erzielten Effekte in die gewünschte Richtung weisen, ob Strategie und Vorgehen solche Effekte erwarten lassen, wie GIN-Stakeholder die Möglichkeiten einschätzen und wie sich die Initiative im internationalen Vergleich auszeichnet. Die Evaluierung zeigt, dass ohne GIN eine Internationalisierung im Sinne verstärkter Partizipation an und Nutzung von Risikokapital sowie "Intellectual Capital“ aus den Zielländern nicht stattfinden würde. Bisherige Erfolge haben die Sichtbarkeit von GIN erhöht und erste nachhaltige Effekte generiert:

- 90 geförderte Unternehmen (62 goAustria und 28 goAsia);

- Mehr Sichtbarkeit durch eine Vervielfachung (+149\%) der Dealflows über die ersten fünf Ausschreibungen;

- Ein erfolgreiches Investment durch einen internationalen VC im Rahmen eines Co-Investment Pitchings, sowie weitere laufende Verhandlungen über solche Investitionen;

- Wirtschaftskooperationen zwischen goAustria und goAsia Start-ups und einem österreichischen Konzern;

- Ein goAsia Start-up arbeitet an seiner Hong Kong Niederlassung.

Neben goAustria, goAsia und GIN Co-Investment Pitches wurden nach und nach ergänzende Maßnahmen (wie GIN Corporate Day, GIN Ambassador Konzept, Corporation Innovation, GIN Conference) entwickelt, die bestehende GIN-Netzwerke erweitern und GIN-Netzwerkpartner untereinander verlinken, um letztlich den Wissens- und Informationsaustausch zu intensivieren und "Selbstläufereffekte" zu generieren (wie z.B. bei VC-Investitionen oder Kooperationen zwischen Start-ups und großen Unternehmen).

Schwachstellen der Initiative sehen GIN-Stakeholder vor allem darin, dass die Zielunternehmen nicht reif genug sind und die Betreuungsphase der Unternehmen nicht lang genug dauert. Zur Weiterentwicklung sollte GIN daher seine Beziehungen mit Inkubatoren und Akzeleratoren intensivieren und gemeinsam mit diesen follow-on Maßnahmen initiieren, um so die Betreuungsphase für die Startups zu verlängern und zu verstärken. Diese verstärkte Zusammenarbeit mit Inkubatoren und Akzeleratoren, wie auch ein verstärkter Fokus auf Investorinnen und Investoren, wurde Anfang 2019 in Angriff genommen. Darüber hinaus regt die Evaluierung an, das Zielsystem von GIN und die Rollenverteilung seiner Stakeholder zu überarbeiten und die Datenlage und Dokumentation der GIN-Maßnahmen zu verbessern, um ein laufendes Monitoring und nachfolgernde Evaluierungen zu erleichtern. Dies wird insofern auch als erforderlich angesehen, als im Regierungsprogramm explizit der Ausbau des GINProgramms festgehalten ist.

\subsubsection{Frontrunner-Initiative}

Die Frontrunner-Initiative wurde ausgehend von der FTI-Strategie 2011 der österreichischen Bundesregierung mit dem übergeordneten Ziel, Österreich als „Innovation Leader" zu etablieren, entwickelt. Dabei wird eine ganz besondere Gruppe von Unternehmen adressiert, nämlich jene, die bereits „FrontrunnerUnternehmen" sind bzw. das Potential aufweisen, sich zu einem solchen zu entwickeln. Unter „Frontrunner-Unternehmen" werden international aktive, besonders exportintensive Unternehmen verstanden, die in einem hoch kompetitiven Marktumfeld operieren und den Wettbewerb als Technologie- bzw. Innovationsführer prägen. Vor diesem Hintergrund bietet die Frontrunner-Initiative zwei unabhängige Schie- 
nen an, die zum einen von der FFG und zum anderen von der aws abgewickelt werden.

So fördert die FFG umfangreiche F\&E-Projekte (bis zu 3 Mio. $€$ mit einer Laufzweit von mindestens 24 und maximal 36 Monaten), die von Unternehmen zur Erreichung (bzw. Absicherung) einer Frontrunner-Position dienen und deren Rolle klar in einer einschlägigen Unternehmensstrategie verankert ist. Die Förderung erfolgt in Form eines nicht rückzahlbaren Zuschusses und beträgt bis zu $25 \%$ bei Großunternehmen, $35 \%$ bei mittleren Unternehmen und $45 \%$ bei Kleinunternehmen.

Bei der Frontrunner-Schiene der aws erfolgt die Förderung durch einen Zuschuss (bis zu 0,5 Mio. $€$ ) zusätzlich zu einem zinsengünstigen aws erp-Kredit. Gefördert werden Investitionen in Prototypen, Demonstrationsanlagen sowie in den Aufbau und die Erweiterung von Produktionskapazitäten für die Umsetzung von Produkt- und Verfahrensinnovationen.

\section{Zentrale Evaluierungsergebnisse}

Ziel der unter dem Lead von Technopolis und mit Beteiligung des Zentrums für Soziale Innovation und des Zentrums für Europäische Wirtschaftsforschung in Mannheim 2018-2019 durchgeführten Evaluierung ${ }^{26}$ war es, den Programmverlauf (Programmstart war im zweiten Halbjahr 2013) bis 2017 nachzuzeichnen, wobei die Aufgabenstellung der Evaluierung neben der Analyse der Wirkungen der durch die Programme ge- förderten Projekte auch eine Einschätzung hinsichtlich der Relevanz der ursprünglichen Ziele im nunmehr gegebenen Umfeld beinhaltete. Zu beachten ist, dass die geförderten Projekte eine längere Laufzeit aufweisen (bis zu drei Jahre) und daher die im Jahr 2016 und danach bewilligten Projekte vielfach noch im Laufen sind. Eine endgültige Wirkungsanalyse, die vor allem auch weitergehende Impacts auf die betreffenden Unternehmen (sowie deren Umfeld, wie z.B. deren Zulieferer, Kooperationspartner etc.) miteinschließt, konnte daher zum gegenwärtigen Zeitpunkt noch nicht vorgenommen werden.

In Tabelle 5-1 sind die Eckdaten der FrontrunnerInitiative dargestellt. Insgesamt wurden bis einschließlich 201795 Projekte mit einem Barwert von in Summe 93,3 Mio. $€$ von der FFG gefördert und 60 Projekte mit einem Barwert von 24,3 Mio. $€$ von der aws unterstützt. Pro Jahr wurden damit Mittel in der Höhe von etwa 20 Mio. $€$ ausgeschüttet.

Die empirischen Grundlagen für die Evaluierung bestanden in der Analyse der Monitoringdaten, einem umfangreichen Online-Survey bei den geförderten Unternehmen (wobei jeweils die verantwortlichen Projektleitungen befragt wurden) sowie in einer ökonometrischen Kontrollgruppenanalyse. Ergänzt wurden diese quantitativen Methoden durch qualitative Ansätze, indem in Form von „Fallvignetten“ fünf ganz unterschiedliche Förderfälle näher beleuchtet wurden.

Tabelle 5-1: Kennzahlen zur Frontrunner-Initiative

\begin{tabular}{l|c|c|c|c|c|c}
\multicolumn{2}{c}{ Jahr } & \multicolumn{2}{c}{ FFG } & \multicolumn{2}{c}{ aws } \\
\cline { 2 - 6 } & Projekte & $\begin{array}{c}\text { Gesamtkosten } \\
\text { (in 1.000 } € \text { ) }\end{array}$ & $\begin{array}{c}\text { Barwert } \\
\text { (in 1.000 } € \text { ) }\end{array}$ & Projekte & $\begin{array}{c}\text { Bemessungsgrundlage } \\
\text { (in 1.000 } € \text { ) }\end{array}$ & $\begin{array}{c}\text { Zuschuss } \\
\text { (in 1.000 } € \text { ) }\end{array}$ \\
\hline Summe & 95 & 350.189 & 93.283 & 60 & 483.869 & 24.257 \\
\hline 2013 & 26 & 65.589 & 17.485 & 14 & 176.888 & 7.041 \\
\hline 2014 & 19 & 66.288 & 16.976 & 10 & 76.406 & 4.141 \\
\hline 2015 & 18 & 63.280 & 16.864 & 14 & 84.971 & 4.946 \\
\hline 2016 & 13 & 59.800 & 16.823 & 12 & 77.904 & 4.668 \\
\hline 2017 & 9 & 57.675 & 15.528 & 10 & 67.701 & 3.461 \\
\hline 2017 (Nationalfonds) & 10 & 37.558 & 9.607 & - & - & -
\end{tabular}

Quelle: FFG.

26 Vgl. Warta et al. (2019). 
Die Ergebnisse zeigen, dass vor allem die Zielgruppe der bestehenden Frontrunner-Unternehmen sehr gut erreicht wurde. Die Zielgruppe der Frontrunner-Position anstrebenden Unternehmen war anfangs (noch) gering vertreten. Erst in jüngsten Jahren finden sich auch vermehrt solche Unternehmen. Insgesamt zeigt sich, dass die geförderten Projekte für die Unternehmen einen strategisch hohen Stellenwert aufweisen und sie in diesen Projekten aufgrund der Förderhöhe und der längeren Laufzeit vermehrt auch höhere technologische Risiken eingehen. Vielfach werden die Projekte auch zur "Verbreiterung“ (im Sinne einer Diversifizierung) der Frontrunner-Position eingesetzt.

Durch einen ökonometrischen Kontrollgruppenansatz wurde analysiert, inwieweit die Wirkung der Förderung bei den österreichischen Unternehmen im Vergleich zu einer Kontrollgruppe eingeschätzt werden kann. Als Kontrollgruppe wurden dabei deutsche Unternehmen mit einer jeweils ähnlichen Frontrunner-Position herangezogen, wobei als Datengrundlage hierfür der Mannheimer Innovationspanel (MIP) des Zentrums für Europäische Wirtschaftsforschung (ZEW) diente. Dabei zeigte sich, dass durch die Förderung eine hohe Umsatz- und Beschäftigungswirkung (v.a. auf die F\&E-Beschäftigten) im Vergleich zu den Kontrollunternehmen gegeben ist.

Insgesamt zeigt sich, dass die Ziele der Frontrunner-Initiative an Relevanz gewonnen haben, dass jedoch neben den zwei ursprünglich formulierten Zielen ein drittes und durchaus relevantes Ziel, nämlich die Etablierung neuer Frontrunner-Positionen etablierter Frontrunner-Unternehmen, zu nennen ist. Durch eine derartige Erweiterung der Zielebene würde ein zentrales Ziel der FTI-Strategie, nämlich die laufende Modernisierung der österreichischen Wirtschaftsstruktur sicherzustellen, in größerem Ausmaß als bisher betont werden.

\subsubsection{Umsetzung von H2020, EUREKA, COSME, EEN und ERA in Österreich}

Der Bereich „Europäische und Internationale Programme" der FFG (FFG-EIP) wurde vom Bund (vertreten durch mehrere Ministerien) und WKO für den Zeitraum 2014-2020 mit der Leistung von Informations-, Beratungs- und Vernetzungsservices beauftragt, um eine optimale Beteiligung österreichischer FTI-Akteure in $\mathrm{H} 2 \mathrm{O} 2 \mathrm{O}$ und in multilateralen FTI-Initiativen zu erreichen. Darüber hinaus ist FFG-EIP die Nationale Kontaktstelle (NCP) der Europäischen Kommission für das Rahmenprogramm und alle Initiativen und Programme in Verbindung mit H2O2O. Kernzielgruppen sind Akteure aus den Bereichen Wissenschaft und Wirtschaft. Die Beauftragung von FFG-EIP verfolgte vier übergeordnete Ziele, die Teil der nationalen FTIStrategie Österreichs sind: (1) Halten des bisherigen Niveaus der Beteiligung der österreichischen Forschungsorganisationen und Forschenden an H2O2O und am Europäischen Forschungs- und Innovationsraum: Die ausgezahlten Rückfüsse sollen weiterhin über dem Wert der österreichischen Eigenmittelzahlungen zum EU-Haushalt liegen, (2) Stärkung der österreichischen F\&E durch Internationalisierung, (3) Abstimmung der Information und Beratung über nationale FFG-Programme, europäische und multilaterale Förderprogramme, (4) Stärkung des österreichweiten Interessensaustausches im Bereich Forschung und Innovation im EU-Kontext.

Ein besonderes Augenmerk wird auf eine systemorientierte Unterstützung und eine Selbstbefähigung der Akteure gelegt. Zur Umsetzung der Beratungs- und Betreuungsleistungen hat FFG-EIP eine Toolbox mit spezifizierten Leistungen entwickelt, die alle Forschenden an Unternehmen, Universitäten, Fachhochschulen und außeruniversitären Forschungsorganisationen in Österreich adressiert. Die zum Einsatz kommenden Instrumente lassen sich in drei Funktionsgebiete gliedern: ${ }^{27}$

27 Vgl. https://www.ffg.at/europa/service 
1. Bewusstseinsbildung und Informationsvermittlung zur Förderung der Bekanntheit und Reputation europäischer und multilateraler Programme,

2. Programm- und Projektberatung entlang aller Projektphasen und

3. Strategische Beratung und ERA-Orientierungswissen zur Unterstützung der optimalen Nutzung vorhandener Förderprogramme.

Für die Integration der einzelnen Aufgabenstellungen im Bereich der Governance der EU-FTI-Politik in Österreich dient das ERA-Observatorium. ${ }^{28}$ Das übergeordnete Ziel der Instrumente des ERA-Observatoriums ist die Unterstützung der eigenverantwortlichen Entscheidungsfindung insbesondere von politiknahen FTI-Akteuren im EU-Kontext. Die konkreten Zielsetzungen des ERA-Observatoriums sind (1) die Bereitstellung von Informationen und Austauschmöglichkeiten über die relevanten EU-Politiken (ERA Portal Austria, Europa Forum Forschung), (2) eine bedarfsgerechte Beratung und Betreuung der verschiedenen ministeriellen Ressorts für H2O2O, (3) eine strategische Beratung der Politik (ERA Council Forum Austria) und von intelligenten Prozessen für den Strukturwandel im Politikbereich (ERA-Policy Forum, ERA-Roundtable, Arbeitsgruppen zu EIT, JPI etc.), sowie (4) die Durchführung eines wirkungsorientierten Monitorings der österreichischen Teilnahme an H2O2O und ERA (EU-Performance Monitoring, ERA Reporting Board).

\section{Zentrale Evaluierungsergebnisse}

Ziel der Evaluierung der Umsetzung von Horizon 2020 in Österreich ${ }^{29}$ war eine systematische Gesamtbetrachtung der Umsetzung von $\mathrm{H} 2 \mathrm{O} 20$ und ERA in Österreich sowie von EUREKA, COSME und EEN. Die Evaluierung wurde in 2017 und 2018 unter dem Lead des Austrian Institute of Technology gemeinsam mit Joanneum Research, der KMU Forschung Austria und dem Zentrum für Soziale Innovation durchgeführt. Schwerpunkte waren hierbei die
Beauftragung der FFG mit der Beratung und Betreuung von $\mathrm{H} 2020$ und ERA (EIP-Beauftragung), die Beauftragung der FFG mit dem EU-Performance Monitoring (EU-PM-Beauftragung) sowie das ERA-Observatorium Austria. Darüber hinaus wurden ergänzend die Beauftragungen zu EUREKA, COSME und dem EEN analysiert. Im Fokus der Evaluierung standen die Ausrichtung und Umsetzung der vorhandenen Formate und Unterstützungsleistungen mit Blick auf die intendierten Wirkungen und Handlungsbedarfe bzw. Handlungsoptionen.

Die Evaluierung kommt zu dem Schluss, dass die Relevanz der Leistung der FFG-EIP in den drei Bereichen Bewusstseinsbildung und Information, Programm- und Projektberatung sowie strategische Beratung und ERA-Orientierungswissen in Bezug auf den Bedarf der FFG-Kundinnen und Kunden generell hoch ist. Dies trifft insbesondere auf die persönlichen Beratungsleistungen und die Angebote zur Weiterbildung im Rahmen der FFG-Akademie zu. Das individuelle Dienstleistungsspektrum von FFG-EIP wird insgesamt auch als effektiv bewertet, insbesondere trägt es zur Steigerung der Antrags-Kompetenz der Forscherinnen und Forscher bei und erhöht die Erfolgswahrscheinlichkeit in der Antragstellung. Die Evaluierung identifiziert jedoch auch Potenziale zur Erhöhung der Effektivität, die durch zielgruppengerechte Anpassungen im Leistungsportfolio erzielt werden können. Hinsichtlich der zentralen Zielgruppe Wissenschaft und der insbesondere in diesem Bereich angestrebten Selbstbefähigung der Akteure sind der Evaluierung zufolge deutliche Fortschritte erzielt worden, die von den Universitäten bereitgestellten Kapazitäten an den jeweiligen Forschungsservicestellen sind jedoch sehr heterogen. Gleichzeitig steigt die Bedeutung von europäischen FTI-Netzwerken und strategischen Partnerschaften in den europäischen Rahmenprogrammen, und es besteht sowohl für den außeruniversitären als auch für den universitären Bereich ein Bedarf, verstärkt strate-

28 Vgl. https://era.gv.at/directory/166

29 Vgl. Biegelbauer et al. (2018). 
gisch auf internationaler Ebene zu agieren. Die Evaluierung folgert daraus eine entsprechende Anpassung der Unterstützungsleistungen der FFG-EIP betreffend die Weitergabe an sämtlichen Informationen relevanter Entwicklungen (z.B. Call-Inhalte). Neben den unmittelbaren Unterstützungsleistungen kommt der Weiterentwicklung der "Selbstermächtigung“ (Empowerment) der Forschungsorganisationen eine Schlüsselrolle zu. Hierfür sind die Forschungsservicestellen und Vizerektorate der Universitäten die zentralen Ankerpunkte. Der Evaluierung zufolge verfügt die FFG über eine gute Basis an Beratungsleistungen, um Forschungsservicestellen und Vizerektorate anzusprechen (ERA Dialog), es besteht jedoch Bedarf, die Professionalisierung der Servicestellen und die strategische Selbstermächtigung weiter voranzutreiben.

Hinsichtlich der zentralen Zielgruppe Wirtschaft zeigt sich der Evaluierung zufolge eine allgemeine Anhebung des Informationsniveaus für das "Ökosystem" via H2O20-Unterstützung ebenso wie die Erfüllung spezifischer Beratungsbedarfe. Optimierungspotenzial wird im Bereich des „Erwartungsmanagements“, d.h. der klareren Vermittlung über Möglichkeiten und Grenzen der angebotenen Leistungen, geortet. Ein besonderes Element im Beratungs- und Betreuungsangebot der FFG-EIP für die Zielgruppe Wirtschaft stellt das C3-Konzept (Core Customer Concept) dar, das speziell auf die - mit Blick auf ihr Potenzial der Teilnahme an EU-Projekten - herausragenden Unternehmen abgestimmt ist. Die Evaluierung wirft diesbezüglich die Frage auf, inwiefern die Erfahrungen dieser Gruppe von Unternehmen auch für andere nutzbar gemacht werden könnten.

Die Evaluierung spricht sich auch für eine Fortführung des EU-Performance Monitorings aus. Diesem kommt eine zentrale Position als „Informations-Broker" für die nationalen Policy-Akteure in Bezug auf ERA zu.

Hinsichtlich des ERA Observatoriums kommt die Evaluierung zu dem Schluss, dass die Aktivitäten mehrheitlich gut und sinnvoll wahrgenommen wer- den. Ein wichtiges Instrument, das Europa Forum Forschung, sollte neben seiner bisherigen Informationsfunktion zukünftig verstärkt Dialogelemente beinhalten und einen erweiterten Kreis an Akteuren einbeziehen. In Bezug auf die Funktion der Beratung und Betreuung ministerieller Ressorts wird in der Evaluierung auch das gegenwärtig diskutierte Verbindungsbüro in Brüssel eingebracht, das relevante Unterstützungsarbeit leisten könnte. Die Evaluierung spricht sich dahingehend für eine Klärung der Zielsetzungen eines solchen Verbindungsbüros mit der FTI-Community aus. Hinsichtlich einer Neuaufsetzung der FTI-politischen Ratslandschaft in Österreich empfiehlt die Evaluierung eine starke europäische Dimension in den Aufgaben und bei der Besetzung des neu zu konstituierenden Gremiums sowie eine regelmäßige Information über die Aktivitäten dieses Gremiums zu gewährleisten. Zudem soll dem Querschnittscharakter der FTI-Politik Rechnung getragen werden. Die ERA-Formate sollen gebündelt und durch eine aktivere Koordination zwischen den Bundesministerien gestärkt werden.

Im Sinne eines Ausblicks formuliert die Evaluierung abschließend drei mögliche Szenarien zur Positionierung Österreichs in Horizon Europe bzw. den entsprechenden Unterstützungsstrukturen:

1. Szenario "Enhancement in Continuity" (Inkrementelle Weiterentwicklung): Der Leitgedanke dieses Szenarios besteht darin, dass der gut etablierte und in weiten Teilen auch gut funktionierende Status-quo weiterentwickelt wird und im Zuge dessen Anpassungen der FTI-Politik an die Erfordernisse neuer Instrumente und Initiativen auf europäischer Ebene vorgenommen werden, ohne dass wesentliche Veränderungen in Österreich, z.B. im Bereich Governance, vorgenommen würden.

2. Szenario "Smart and Proactive Alignment" (FTIPolitik als Impulsgeberin im europäischen Mehrebenensystem): In diesem Szenario strebt die österreichische Politik einen Ausbau ihrer Rolle hin zur (pro)aktiven Mitgestalterin europäischer FTIPolitik an, um die sich daraus ergebenden Chancen der EU FTI-Politik gezielter und effektiver für 
Österreich zu nutzen. Die Festlegung eines solchen Konzepts als Leitlinie für eine kohärente österreichische Position zur EU FTI-Politik wäre ein zentraler Bestandteil der neuen österreichischen FTI-Strategie.

3. Szenario "Distributed Empowerment" (Stärkung und Vernetzung der FTI-Community): In diesem Szenario erfolgt die Ausweitung der Mitgestaltung und Beteiligung an der europäischen FTI-Politik vor allem über die Stärkung und bessere Vernetzung der FTI-Akteure selbst, die seitens der österreichischen Politik bei diesem Prozess der Selbstermächtigung (Empowerment) unterstützt werden.

\subsubsection{FIT-IT und IKT der Zukunft}

Die Förderung von Informations- und Kommunikationstechnologien zählt zu den thematischen Kernbereichen der Forschungsförderung im BMVIT. Im Jahr 2011 startete die Neukonzeption und -gestaltung der Themeninitiative "IKT der Zukunft" (IKT der Zukunft, ECSEL, benefit und AAL), die dem Impulsprogramm FIT-IT (2002-2012) nachfolgte. Unter dem neuen Programmschirm wurde die nationale IKT-Förderung stärker anwendungsfeldbezogen ausgerichtet und mit der Förderung der österreichischen Beteiligung an den Gemeinsamen Technologieinitiativen ARTEMIS und ENIAC sowie seit 2014 auch mit den nationalen und transnationalen Programmen für die Bewältigung des demographischen Wandels (benefit und $A A L)$ zusammengefasst.

\section{Zentrale Evaluierungsergebnisse}

Im August 2018 wurde eine umfassende Evaluierung $^{30}$ von inspire research vorgelegt, die zum einen die ex-post Evaluierung des Impulsprogramms FIT-IT (2002-2012) umfasst, zum anderen die Zwischenevaluierung der nachfolgenden Themeninitiative „IKT der Zukunft" (IKT der Zukunft, ECSEL, benefit und AAL) beinhaltet. Die Evaluierung basiert auf einer umfang- reichen Sekundärdatenanalyse (Förderdaten der FFG, SCOPUS-Publikationsdaten, amtliche F\&E-Statistik, Patentdatenbank, PREDICT - Prospective Insights in ICT R\&D Research), einer Online-Befragung aller Fördernehmerinnen und Fördernehmer, Fallstudien zu den Langzeitwirkungen der Förderung und einem Überblick über bisherige Evaluierungen sowie Programmbeispielen zur IKT-Forschung in anderen Ländern.

Insgesamt wurden über die Laufzeit des Impulsprogramms FIT-IT in den einzelnen Programmen und Programmlinien mehr als 1.000 Projektanträge mit einem beantragten Gesamtvolumen von 663 Mio. $€$ eingereicht. $67 \%$ der Anträge entfielen dabei auf die verschiedenen Programmlinien des Impulsprogramms FIT-IT, $9 \%$ der Anträge wurden für österreichische Beteiligungen an den Gemeinsamen Technologieinitiativen ARTEMIS und ENIAC eingereicht, $24 \%$ der Anträge beinhalteten Projekte zum Demographischen Wandel. Mehr als 470 Anträge wurden genehmigt, der Barwert der Förderungen machte 165 Mio. $€$ aus, davon gingen 25 Mio. $€$ an Projekte zum Demographischen Wandel und rd. 45 Mio. $€$ an ARTEMIS- und ENIAC-Projekte.

Auf den nationalen Förderschwerpunkt IKT der Zukunft entfiel seit der Neugestaltung des Programms nur mehr ein Viertel der insgesamt für die Ausschreibungen im Zeitraum 2012-2016 genehmigten Fördermittel. Mehr als die Hälfte der Mittel wurde für die Förderung österreichischer Partner in Projekten der Gemeinsamen Technologieinitiative ECSEL (bzw. deren Vorgängerinitiativen ARTEMIS und ENIAC) verwendet (siehe Abbildung 5-2).

Die Ergebnisse der Evaluierung zeigen, dass es dem Impulsprogramm FIT-IT insgesamt sehr gut gelungen ist, die gesetzten Ziele zu erreichen. Ein sehr hoher Anteil von $81 \%$ der ehemals beteiligten wissenschaftlichen Partner und $67 \%$ der ehemals beteiligten Unternehmen sehen heute noch positive Nachwirkungen der geförderten Projekte in ihren Einrichtungen. Vor allem die Qualifizierung von Mit-

30 Vgl. Geyer und Good (2018). 
Abbildung 5-2: Impulsprogramm FIT-IT und Themeninitiative „IKT der Zukunft": Summe der genehmigten Barwerte verteilt auf die Projektlaufzeiten nach Programmen/Förderthemen, in Mio. $€$

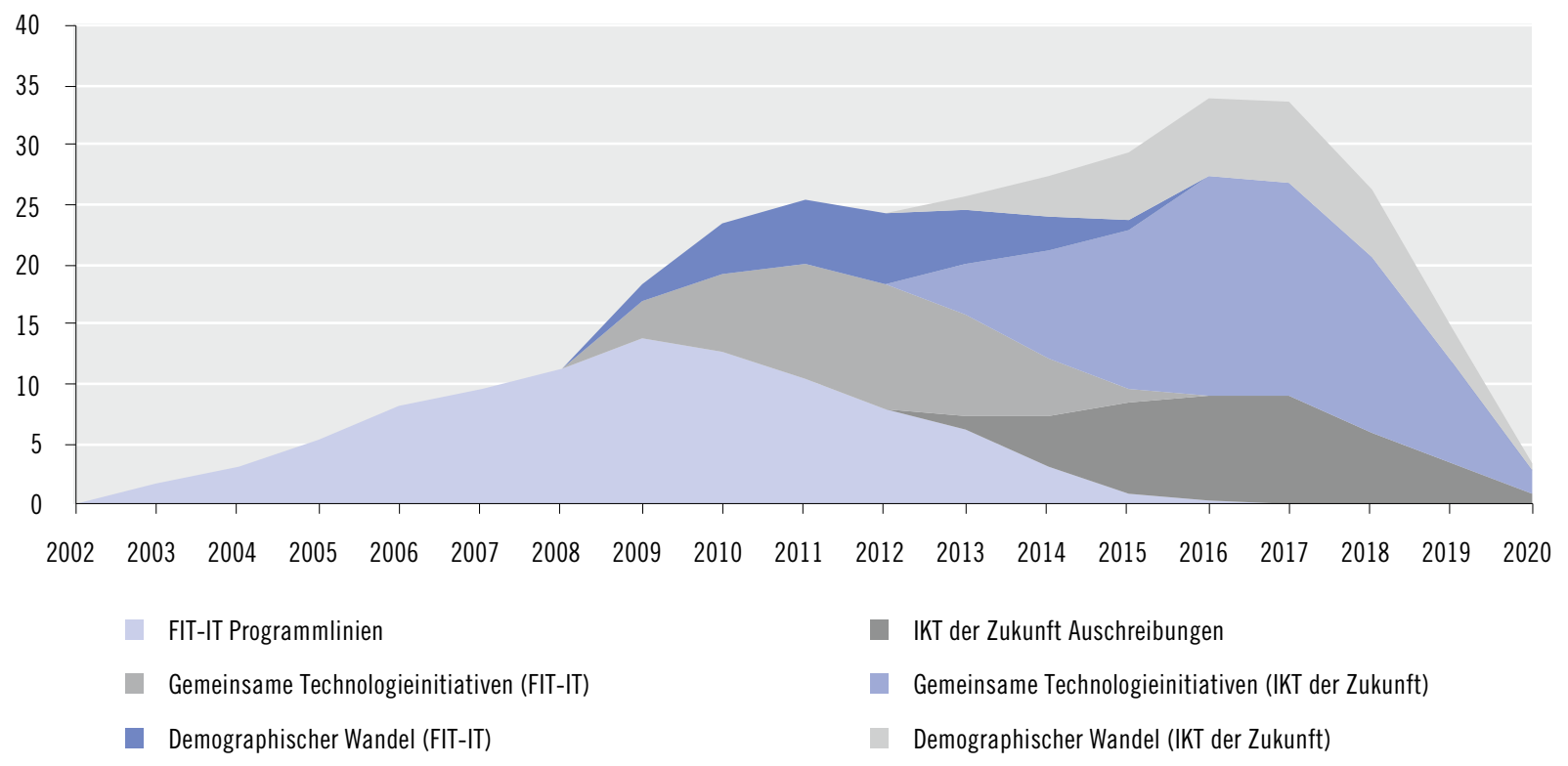

Quelle: FFG; Auswertung und Darstellung: Geyer und Good (2018).

arbeiterinnen und Mitarbeitern und der mit den Projekten erfolgte interne Know-how-Aufbau, aber auch die entstandenen Netzwerke und Kooperationen für die F\&E-Zusammenarbeit im IKT-Bereich wurden als langfristiger Nutzen der FIT-IT Förderung genannt. Nach Einschätzung der Programmteilnehmerinnen und -teilnehmer hat FIT-IT maßgeblich zur Stärkung der Leistungs- und Wettbewerbsfähigkeit der österreichischen IKT-Forschung und zur Förderung der Kooperationen zwischen Wirtschaft und Wissenschaft beigetragen. Die Programmkonzeption, die Programmumsetzung und das Programmmanagement von FIT-IT werden von den ehemals Programmbeteiligten auch heute noch durchwegs als exzellent bewertet.

Die bibliometrischen Auswertungen zeigen eine intensive Publikationstätigkeit der wissenschaftlichen Partner, aber auch der beteiligten Unternehmen während und nach der Laufzeit der FIT-IT Projekte. Die Auswertung der PATSTAT-Datenbank über die Patentanmeldungen der an den FIT-IT Projekten beteiligten Unternehmen zeigt eine sehr deutliche Zunahme der Patentierungsaktivitäten über die Laufzeit des FIT-IT-Programms, die mit einem deutlich steigenden Anteil der Bedeutung von Patenten in IKTTechnologiefeldern und einer Internationalisierung der Patentanmeldungen verbunden war.

Die überaus positive Einschätzung und Bewertung der Ergebnisse und Wirkungen des Impulsprogramms FIT-IT durch die ehemaligen Förderempfänger steht jedoch in einem Spannungsverhältnis mit den Ergebnissen einer im Zuge der Evaluierung durchgeführten Sonderauswertung der F\&E-Erhebung von Statistik Austria zur Entwicklung der internen F\&E-Ausgaben und der F\&E-Beschäftigen von geförderten und nicht-geförderten Unternehmen. Demnach kann aus den Daten der F\&E-Erhebung nur für KMU und nur für die ersten Programmjahre von FIT-IT in der Gruppe der geförderten Unternehmen eine stärkere Entwicklung der internen F\&E-Ausgaben und der Zahl an F\&E-Beschäftigen festgestellt werden. Die generelle Ausweitung der Angebote der direkten und indirekten (d.h. der steuerlichen) Forschungsförderung für Unternehmen seit Mitte der 2000er Jahre dürften hier die Auswertungsergebnisse beeinflusst haben. 
Durch die thematische Neuausrichtung des nationalen Förderschwerpunkts IKT der Zukunft mit der Festlegung von spezifischen IKT-Themenfeldern, Anwendungsfeldern und Querschnittszielen hat sich der fachlich-inhaltliche Charakter der Förderung im Vergleich zum Impulsprogramm FIT-IT deutlich verändert. Während die neuen IKT-Themenfelder bei den Programmbeteiligten insgesamt auf hohe Akzeptanz stoßen, trifft die nunmehrige verpflichtende Ausrichtung der Projekte an spezifischen Anwendungsfeldern nicht auf ausschließliche Zustimmung. Durch die Anwendungsfelder könnten stärker generisch ausgerichtete IKT-Projekte nicht mehr im Programm eingereicht werden. Außerdem wären die Alleinstellungsmerkmale des IKT-Programms im Vergleich zu anderen thematischen Förderprogrammen des BMVIT geringer geworden. Das IKT-Förderprogramm des BMVIT habe damit an eigenständigem Profil verloren, da anwendungsfeldorientierte IKT-Forschung auch in anderen thematischen Programmen adressiert werden würde.

Weiterhin als ausgezeichnet bewerten die Programmteilnehmerinnen und -teilnehmer die Qualität der Programmdurchführung von IKT der Zukunft. Die Förderempfänger sind mit der Abwicklung des Programms überaus zufrieden. Hervorgehoben werden insbesondere die Informationsveranstaltungen zum Programm und den neuen Ausschreibungen, die Einreichmodalitäten sowie die Kompetenz und Unterstützung durch die FFG-Mitarbeiterinnen und Mitarbeiter bei administrativen und inhaltlichen Fragen.

Im nationalen Förderschwerpunkt IKT der Zukunft kam mit den Leitprojekten auch ein neues Förderinstrument zum Einsatz, mit dem national vorhandenes Know-how zusammengeführt und durch Erarbeiten von modellhaften Lösungen für bedeutende gesellschaftliche Herausforderungen ein Sektor bzw. eine Branche spezifisch gestärkt werden soll. Die bisher vorliegenden Erfahrungen zeigen, dass es dem Instrument Leitprojekt durchaus gelingt, die angestrebte Plattformfunktion wirksam auszufüllen und über das geförderte Projektkonsortium hinaus neue Partner für die Entwicklung von innovativen Lösungen zu mobilisieren.

Bezüglich der Förderung österreichischer Partner in den Projekten der Gemeinsamen Technologieinitiative ECSEL (bzw. der Vorgängerinitiativen ARTEMIS und ENIAC) zeigt sich, dass Österreich mit 29,5 Mio. $€$ bewilligter EU-Beiträge für diese Projekte auf Rang sechs unter den beteiligten EU-Staaten liegt. Nur die Niederlande und Belgien erhielten bezogen auf ihre Wirtschaftsleistung höhere EU-Beiträge als Österreich. Die Evaluierungsergebnisse legen jedoch nahe, dass trotz bereits gesunkener Förderintensität nach wie vor substanzielle Mitnahmeeffekte bei der Unternehmensförderung auftreten.

Die Zielgruppe des Impulsprogramms FIT-IT bzw. nunmehr jene der Themeninitiative IKT der Zukunft umfasst nicht nur den IKT-Sektor im engeren Sinne, sondern adressiert nicht zuletzt aufgrund der Digitalisierung eine zunehmend breite Schnittmenge unterschiedlicher Wirtschaftsbereiche. Von den Antragstellern im Impulsprogramm FIT-IT sind nur rd. $20 \%$ der einreichenden produzierenden Unternehmen dem IKT-Sektor im engeren Sinn zuzurechnen, jedoch rd. $60 \%$ der einreichenden Dienstleistungsunternehmen. Diese Verteilung spiegelt sich auch in den Auswertungen der für den IKT-Sektor zur Verfügung stehenden Daten über F\&E des JRC (PREDICT). Sie zeigen, dass in den Jahren 2006-2015 die staatlichen F\&E-Mittel für IKT insgesamt um durchschnittliche $6,6 \%$ pro Jahr gestiegen sind, wobei die Steigerung für industrielle Forschung (+ $182 \%$ über zehn Jahre) besonders hoch ausfiel. In absoluten Zahlen haben die staatlichen F\&E-Mittel, die Unternehmen des IKTSektors zugutekamen, von 27,4 Mio. € im Jahr 2006 auf 81,1 Mio. $€$ im Jahr 2015 zugenommen. Damit geht nur zum Teil eine entsprechende Zunahme der F\&E-Ausgaben der Unternehmen selbst einher. Einen sehr starken Zuwachs der Forschungsausgaben der Unternehmen hat in den Jahren 2002-2016 der IKTDienstleistungssektor verzeichnet, von 138 Mio. $€$ auf 457 Mio. $€$ (bzw. einer jährlichen Steigerung von 8,9 \%), während sich It. PREDICT die F\&E-Ausgaben der Unternehmen im Produktionsbereich im selben 
Zeitraum von 648 Mio. $€$ auf 459 Mio. $€$ verringerten (durchschnittlich 2,4 \% jährlich).

Der Förderschwerpunkt Demographischer Wandel ist unter den drei Förderschwerpunkten der Themeninitiative IKT der Zukunft nach wie vor der strukturell und organisatorisch ambitionierteste. Durch die Einbeziehung von nicht-klassischen Akteuren und den Fokus auf Lösungen für (End-)Anwenderinnen und Anwender unterscheiden sich diese Projekte sehr deutlich von klassischen F\&E-Projekten. Die Kombination von nationalen Ausschreibungen (benefit) und transnationalen Ausschreibungen (AAL) hat sich bewährt, die Vermarktung von Lösungen für die beteiligten Unternehmen stellt jedoch nach wie vor eine große Herausforderung dar. Die nicht-klassischen Partnerstrukturen machen auch die Aufrechterhaltung der Zusammenarbeit nach Projektende schwieriger als in klassischen kooperativen F\&E-Projekten. Gleichzeitig wird aber auch eines der Hauptziele der Förderung erreicht, nämlich bei den Unternehmen das Verständnis über die Anforderungen und Bedürfnisse der Nutzerinnen und Nutzer und Endanwenderinnen und -anwender zu erhöhen.

Welche Empfehlungen wurden abgegeben? Um die Abgrenzung des nationalen Förderschwerpunkts IKT der Zukunft zu anderen thematischen Programmen zu stärken, sollte diese Initiative bevorzugt besonders ambitionierte Projekte der industriellen Forschung fördern, die auch einen entsprechend hohen Beitrag an wissenschaftlicher Forschung vor allem aus dem Bereich der Hochschulen erfordern. So können die Ziele „Spitzentechnologien weiterentwickeln“ und "Spitzenkräfte bereitstellen und gewinnen" noch besser unterstützt werden. Kooperative F\&E-Projekte der experimentellen Entwicklung sowie Vorhaben, die überwiegend durch die internen Forschungsleistungen der Unternehmen getragen werden, sollten hingegen bevorzugt in anderen (thematischen) Programmen gefördert werden.

Bei der Programmumsetzung empfiehlt die Evaluierung auf einen Mix an Förderinstrumenten zu setzen, wobei neben kooperativen F\&E-Projekten verstärkt auch Leitprojekte ausgeschrieben werden können. Voraussetzung ist allerdings, dass die Themen der Leitprojekte ausreichend inhaltlich spezifiziert werden können und ein Wettbewerb zwischen potenziellen Einreichern möglich ist. Sondierungsprojekte sollten weiterhin zur Unterstützung spezifischer Programmziele oder auch als Begleitmaßnahmen für Leitprojekte eingesetzt werden. Ausschließlich durch Unternehmen getragene Projekte bzw. Vorhaben, in denen Forschungseinrichtungen nur in untergeordneter Rolle (z.B. im Unterauftrag) vertreten sind, sollten an das FFG-Basisprogramm verwiesen werden.

Die inhaltlichen Anforderungen und die Größe kooperativer F\&E-Projekte in der Themeninitiative IKT der Zukunft sollten jedenfalls die Beschäftigung von Doktorandinnen und Doktoranden ermöglichen, wobei ein entsprechendes Konzept bereits im Antrag eingefordert werden könnte. Der Fokus von IKT der Zukunft auf wissenschaftlich besonders ambitionierte Forschung ließe sich damit auch sehr gut mit strukturell wirksamen Maßnahmen wie Stiftungsprofessuren kombinieren. Schließlich wird empfohlen, die Programmmonitoringstrukturen weiter zu verbessern. Zur Unterstützung der Steuerung und kontinuierlichen Weiterentwicklung des Programms sollten Wirkungs- und Zielindikatoren festgelegt werden, die mit möglichst einfachen Mitteln, vergleichbar, regelmäßig bzw. zeitnah erhoben werden können. Als zentrale Datenquelle bietet sich dazu die jährlich durchgeführte Befragung der Projektpartnerinnen und -partner abgeschlossener Projekte zum FFG-Wirkungsmonitoring an. Die Evaluierung regt an, die Nutzung der Daten aus der FFG-Wirkungserhebung programmbezogen und über den Zeitverlauf der Programme hinweg möglich zu machen.

\subsubsection{Produktion der Zukunft}

Das BMVIT initiierte 2011 die FTI-Initiative „Produktion der Zukunft", um die Erfolgsgeschichte der Produktionsforschung in Österreich nachhaltig für den Wirtschaftsstandort Österreich zu sichern und auszubauen. Die Initiative zielt auf die Förderung der 
Kooperation von Wissenschaft und Wirtschaft, den Humanressourcenaufbau sowie den Aufbau von Forschungsinfrastruktur ab. Die Herstellung konkurrenzfähiger Produkte und die Steigerung der Produktivität zur Sicherung des Wirtschaftswachstums in Österreich stehen dabei im Vordergrund. ${ }^{31}$

Die FTI-Initiative „Produktion der Zukunft“ verfolgt im Wesentlichen vier strategische Zielsetzungen:

1. Stärkung der Wettbewerbsfähigkeit und Nachhaltigkeit der österreichischen Industrie;

2. Steigerung der Innovationsleistung in der Sachgüterproduktion durch:

a. eine verbesserte Nutzung der Kooperationsoption;

b. eine Verbreitung der Innovationsbasis zur Mobilisierung bisher wenig innovativer Unternehmen;

c. einen verbesserten Zugang zur Forschungskompetenz von Forschungseinrichtungen und Unternehmen;

3. Gezielter Aufbau von Forschungskompetenz in Forschungseinrichtungen zu ausgewählten Themenstellungen;

4. Verstärkung europäischer und internationaler Kooperationen und Netzwerke.

\section{Zentrale Evaluierungsergebnisse}

Ziel der von Convelop und inspire research in 20182019 gemeinsam durchgeführten Zwischenevaluierung $^{32}$ war es, das Programmdesign, die bisherige Abwicklung sowie die damit erzielten Wirkungen bei den Fördernehmenden zu analysieren, um das Maß der Zielverfolgung und die Effektivität der FTI-Initiative einschätzen und Hinweise für etwaige Anpassungen und die Weiterentwicklung der Initiative geben zu können. Um die Evaluierungsfragen zu beantworten, wurden u.a. Förderdaten der FFG und öffentlich verfügbare Daten genutzt sowie der nationale und internationale Kontext zur FTI-Initiative analysiert. Des Weiteren wurden Interviews mit För- dernehmenden, Vertreterinnen und Vertretern abgelehnter Förderanträge, Programmverantwortlichen und anderen Stakeholdern sowie eine Fragebogenerhebung (Online-Befragung der Fördernehmenden und der abgelehnten Förderprojekte) durchgeführt.

In den insgesamt 24 Ausschreibungen der FTI-Initiative von 2011-2017, die Gegenstand der Evaluierung waren, wurden acht Förderinstrumente der FFG eingesetzt, um diese Zielsetzungen zu verfolgen. Tabelle 5-2 zeigt, wie sich die Teilnehmenden und die eingereichten und durchgeführten Projekte auf diese Instrumente verteilten. Tabelle 5-3 beschreibt die Projektkosten und Fördervolumina.

Insgesamt präsentiert sich die FTI-Initiative „Produktion der Zukunft" als erfolgreiches Programm, das seine Ziele gemäß der Evaluierung auf vorbildliche Weise verfolgt. Es adressiert die Zielgruppe präzise und schöpft das noch offene Zielgruppenpotential seit 2011 stetig aus. Die Analyse zeigt, dass praktisch alle im Themenfeld "Engineering" in den Jahren 2011-2017 top-publizierenden österreichischen Einrichtungen als Forschungspartner an der FTI-Initiative teilnehmen. Bei den Wirtschaftspartnern erreicht die Initiative rd. 12 \% der Zielgruppe. Das offene Potential umfasst vor allem KMU mit einer geringeren Technologieorientierung und wird seit 2011 kontinuierlich ausgeschöpft, sodass auf jedes genehmigte Projekt rd. ein „Neuling“ entfällt. Auf Basis der vorliegenden Daten lässt sich aktuell noch keine Verlangsamung der Ausschöpfungsgeschwindigkeit feststellen. Die Auswahl der Fördernehmenden durch das Programm erfolgt in einer Art und Weise, dass die eingereichten Projekte weitgehend ausgewogen und ohne nennenswerte Selektionsverzerrung gefördert werden.

Die überwiegende Zahl der Wirtschafts- (75\%) und Forschungspartner (85\%) kann ihre selbstgesteckten F\&E-Ziele erreichen. Die Wirkungen aus der Förderung von kooperativen F\&E-Projekten, die für fast $70 \%$ des gesamten Fördervolumens der FTI-Ini-

31 Vgl. https://www.ffg.at/produktion-der-zukunft-das-foerderprogramm

$32 \mathrm{Vgl}$. Jud et al. (2019). 
Tabelle 5-2: Programmteilnahme nach Instrumenten: Eingereichte Projekte und teilnehmende Organisationen

\begin{tabular}{|c|c|c|c|c|}
\hline Instrument & $\begin{array}{r}\text { Eingereichte } \\
\text { Projekte (Anzahl) }\end{array}$ & Erfolgsrate & $\begin{array}{r}\text { Teilnehmende } \\
\text { Organisationen } \\
\text { (Anzahl) }\end{array}$ & $\begin{array}{r}\text { davon in } \\
\text { genehmigten } \\
\text { Projekten }\end{array}$ \\
\hline Sondierung & 120 & $30 \%$ & 209 & $33 \%$ \\
\hline Einzelprojekte Industrielle Forschung & 20 & $0 \%$ & 20 & $0 \%$ \\
\hline Kooperative F\&E-Projekte & 628 & $30 \%$ & 2.459 & $31 \%$ \\
\hline Kooperative F\&E-Projekte transnational & 81 & $36 \%$ & 171 & $46 \%$ \\
\hline Leitprojekte & 7 & $29 \%$ & 138 & $38 \%$ \\
\hline F\&E-Dienstleistungen & 43 & $26 \%$ & 92 & $28 \%$ \\
\hline Stiftungsprofessuren & 11 & $36 \%$ & 11 & $36 \%$ \\
\hline F\&E-Infrastruktur & 2 & $50 \%$ & 24 & $96 \%$ \\
\hline Innovationslabore & 2 & $100 \%$ & 30 & $100 \%$ \\
\hline Summe & 914 & $30 \%$ & 3.154 & $33 \%$ \\
\hline
\end{tabular}

Quelle: FFG; Berechnung: convelop.

Tabelle 5-3: Programmteilnahme nach Instrumenten: Projektkosten und Fördervolumen

\begin{tabular}{|c|c|c|c|c|c|}
\hline Instrument & $\begin{array}{r}\text { Projektkosten } \\
\text { It. Antrag } \\
\text { (in } € \text { ) }\end{array}$ & $\begin{array}{r}\text { Genehmigte } \\
\text { Projektkosten } \\
\text { (in } € \text { ) }\end{array}$ & $\begin{array}{r}\text { Fördervolumen } \\
\text { (in } € \text { ) }\end{array}$ & Förderquote & $\begin{array}{l}\text { Fördermittel- } \\
\text { verteilung }\end{array}$ \\
\hline Sondierung & 6.361 .780 & 6.339 .981 & 4.711 .660 & $74 \%$ & $3 \%$ \\
\hline Einzelprojekte Industrielle Forschung & 0 & 0 & 0 & - & $0 \%$ \\
\hline Kooperative F\&E-Projekte & 177.894 .102 & 171.650 .244 & 114.480 .274 & $67 \%$ & $77 \%$ \\
\hline Kooperative F\&E-Projekte transnational & 12.507 .730 & 12.829 .684 & 9.836 .236 & $77 \%$ & $7 \%$ \\
\hline Leitprojekte & 13.451 .431 & 11.952 .332 & 7.645 .568 & $64 \%$ & $5 \%$ \\
\hline F\&E-Dienstleistungen & 1.101.183 & 1.101.183 & 1.101.183 & $100 \%$ & $1 \%$ \\
\hline Stiftungsprofessuren & 12.110 .000 & 12.110 .000 & 5.298 .700 & $44 \%$ & $4 \%$ \\
\hline F\&E-Infrastruktur & 3.537 .556 & 2.992 .106 & 1.496 .000 & $50 \%$ & $1 \%$ \\
\hline Sondierung & 8.047 .513 & 8.047 .513 & 4.000 .000 & $50 \%$ & $3 \%$ \\
\hline Summe & 235.011.295 & 227.023 .043 & 148.569 .621 & $65 \%$ & $100 \%$ \\
\hline
\end{tabular}

Quelle: FFG; Berechnung: convelop.

tiative verantwortlich sind, zeigen sich hauptsächlich in drei Punkten:

1. Die Fördernehmenden erreichen nicht nur ihre selbst gesteckten F\&E-Ziele, sondern auch ihre strategischen Ziele, wie z.B. Verbesserung der Produkt- und Leistungsqualität, Verbesserung des Zugangs zu Forschungseinrichtungen und zu Unternehmen, sowie Publikation der Ergebnisse in wissenschaftlichen Fachzeitschriften.

2. Kooperationen und Netzwerke, die aus der Durchführung der Förderprojekte entstehen, spielen bei der Zielverfolgung eine wesentliche Rolle.

3. Kooperationen und Netzwerke sind nicht nur für die Durchführung der F\&E-Aktivitäten wichtig, sondern erschließen darüber hinaus noch neue Wirtschaftskooperationen, Kundenbeziehungen und Marktzugänge.

Leitprojekte erzielen einen zusätzlichen Mehrwert, weil sie zu bestimmten Leitthemen eine besonders große Zahl unterschiedlicher Kooperationspartner zusammenbringen und dadurch die Möglichkeiten zum Zugriff auf komplementäres Know-how sowohl in qualitativer als auch in quantitativer Hinsicht erhöhen. Internationale Projektkooperationen werden eingegangen, um Marktzugänge zu erhalten und geeignete Partnerinnen und Partner mit komplementärem Know-how zu finden, die international gut vernetzt sind. 
In Summe erzielt die FTI-Initiative Förderwirkungen, mit denen sie all ihre strategischen Zielsetzungen verfolgt. Die Wettbewerbsfähigkeit der am Programm teilnehmenden Wirtschaftspartner wird gesteigert und es werden nachhaltige Effekte erzielt. Kooperationen sind nicht nur auf die geförderten Projekte beschränkt, sondern führen in vielen Fällen zu Folgekooperationen und dauerhaften Netzwerkbeziehungen bei den Partnern. Forschungskompetenz wird in Forschungseinrichtungen gezielt aufgebaut, ihre internationale Sichtbarkeit erhöht und Folgeprojekte erleichtert. Die Förderung von Stiftungsprofessuren führt zur gezielten strategischen Weiterentwicklung des Hochschulprofils und zum Aufbau von hochqualifiziertem Humankapital. Pilotfabriken verstärken diese Effekte mit ihrer spezifischen thematischen Ausrichtung weiter. Sowohl europäische als auch internationale Kooperationen werden forciert. Mit Hilfe von M-ERA.NET und den bilateralen Vereinbarungen mit der Chinese Academy of Science (CAS) und der Shanghai University (SHA) konnten Projekte durchgeführt werden, die auf Basis einfacher kooperativer F\&E-Projekte nicht möglich gewesen wären. Internationale Kooperationen wurden dadurch verstärkt. Darüber hinaus haben die Projekte geholfen, Know-how-Lücken zu füllen, Partner mit spezifischen Fertigkeiten zu identifizieren und neue Märkte zu erschließen, wodurch sie zusätzlichen und nachhaltigen Mehrwert gestiftet haben.

Nicht nur der inhaltliche Teil der FTI-Initiative (Auswahl der Förderinhalte und Förderinstrumente, Entwicklung von Themenvorgaben) ist erfolgreich, auch ihre Umsetzung funktioniert gut. Mit der Antrags- und Programmabwicklung durch die FFG ist der überwiegende Teil der Fördernehmenden zufrieden. Für die Informationsbereitstellung zu den Ausschreibungen sowie die Beratung und Unterstützung durch die FFG gilt das in noch höherem Maße. Kritik an der Umsetzung gibt es nur vereinzelt und in wenigen Punkten. So beschreiben manche Interviewpartnerinnen bzw. -partner den Themenfindungsprozess als nicht ausreichend zugänglich und die Lerneffekte zwischen Ausschreibungen als nicht ausreichend, oder üben diese Kritik an Detailaspekten des E-Call Systems der FFG oder der Informationsdissemination zu den Ausschreibungen und ihren geförderten Projekten.

Die Evaluierung identifizierte abschließend auch einige noch nicht ausgeschöpfte Wirkungspotentiale sowie Anpassungs- und Ergänzungsbedarfe im Leistungsangebot. Aufbauend auf diesen Ergebnissen wurden Vorschläge und Anregungen zur Anpassung, Ergänzung und Weiterentwicklung der FTI-Initiative erarbeitet, die unter folgenden Stichworten zusammengefasst werden können:

- Fühlungsvorteile regionaler Agenturen nutzen und bundesländerübergreifende Kooperationen forcieren;

- Umfassenden Überblick über Kooperationsmöglichkeiten schaffen;

- Internationale Kooperationsmöglichkeiten (geeignete Partnerländer) ausloten.

Darüber hinaus sollten die FFG-Vertragsvorlagen auf Optimierungsmöglichkeiten untersucht, das Instrument der Stiftungsprofessuren weiter gestärkt und realistische Betriebsperspektiven für Pilotfabriken nach der Förderung aufgezeigt werden.

\subsubsection{Mobilität der Zukunft}

„Mobilität der Zukunft" (MdZ, Laufzeit von 20122020) ist das nationale Forschungs-, Technologieund Innovationsförderungsprogramm für Mobilitätsund Verkehrstechnologie. Es geht davon aus, dass neue Technologien und Innovationen eingebettet im sozioökonomischen systemischen Kontext einen wichtigen Beitrag zur Gestaltung einer zukunftsfähigen Mobilität leisten können. MdZ liegt in der Verantwortung des Bundesministeriums für Verkehr, Innovation und Technologie (BMVIT) und wird von der Österreichischen Forschungsförderungsgesellschaft (FFG) administriert. Jährlich steht bis 2020 ein Förderbudget von 15-20 Mio. € zur Verfügung; insgesamt wurden im Zeitraum 2012-2016 28 Einzelausschreibungsrunden durchgeführt, für die Maßnahmen wurden knapp 108 Mio. € vergeben. 


\section{Zentrale Evaluierungsergebnisse}

Die Zwischenevaluierung ${ }^{33}$, durchgeführt in 2018 von der KMU Forschung Austria in Kooperation mit dem deutschen Wuppertal Institut, hat einen Schwerpunkt auf der Überprüfung des Designs und der Prozesse des FTI-Programms "Mobilität der Zukunft" (MdZ). Eine detaillierte Wirkungsanalyse des Programms war im Rahmen dieser Evaluierung nicht vorgesehen, dennoch werden Zwischenergebnisse und bisherige Wirkungen sowie die bisherige Zielerreichung im Programm analysiert. Darüber hinaus sind die Wirkungsindikatoren im Programm und in der Wirkungsorientierten Folgenabschätzung (WFA) Gegenstand der Evaluierung. Die Evaluierung erstreckt sich auf den Programmzeitraum 2012-2017.

Die Evaluierung sieht MdZ als ein nach den wesentlichen Prinzipien neuer missionsorientierter Politik aufgebautes und organisiertes Programm (d.h. inklusive Berücksichtigung der Verbindung sozialer und technologischer Innovation, Nutzerfokus, Einbindung von sozialen und gesellschaftlichen Zielen etc.). Das System der drei strategischen Zielcluster (sozial, umweltbezogen und wirtschaftlich) im Programm wird insgesamt als konsistent und als einem missionsorientierten Programm angemessen bewertet. Hinsichtlich des gesamten Zielsystems bestehen der Evaluierung zufolge einige Optimierungspotentiale. Grundsätzlich wird die Integration von systemischen und technologischen Themenfeldern im Programm MdZ vor allem aufgrund einer notwendigen Konkretisierung und Fokussierung innerhalb des weiten Feldes „Mobilität", limitierter Ressourcen, einer bestmöglichen Adressierung und Mobilisierung der Community, sowie einer leichteren Anknüpfung an übergeordneten Strategien als legitim und sinnvoll beurteilt. Die Evaluierung sieht die gegenwärtigen Themenfelder auch als umfassend und nachvollziehbar abgeleitet an; auch sind die Themenfelder explizit mit operativen Zielen verknüpft. Die Einführung von Roadmaps zur weiteren Spezifizierung der Forschungsagenden in den Themenfeldern und als wesentliches Steuerungsinstrument im Programm wird in der Evaluierung als ein zentraler Fortschritt im Programmdesign gegenüber dem Vorgängerprogramm „IV2Splus" hervorgehoben.

Ebenso thematisiert die Evaluierung Hinweise auf fallweise zu eng empfundene Themensetzungen im Programm und eine sich daraus ergebende potenzielle Gefahr, Innovationen oder Lösungen vorschnell auszuschließen, die bspw. durch andere Ansätze einen Zielbeitrag liefern könnten. Der Evaluierung zufolge hat MdZ einen relativ hohen Bedarf an Vorbereitung, Begleitung und Dissemination von Ergebnissen geförderter Projekte und stellt diesbezüglich unterschiedliche Bedeutsamkeiten und Vorgehensweisen wie auch gewisse Unschärfen in der Rollenund Aufgabenteilung innerhalb des Programms bezüglich der Implementierung von Begleitmaßnahmen (z.B. Vernetzungsveranstaltungen, Community-Workshops und ähnliches) fest. Der Darstellung bzw. dem Austausch und der Verbreitung von Ergebnissen zwecks verbesserter Möglichkeiten für Anschlussprojekte sowie einem Aufgreifen der Ergebnisse durch Nutzerakteure wird auch aus Sicht aller Befragtengruppen in der Evaluierung ein hoher Stellenwert beigemessen, zugleich wird eine noch zu geringe Aktivität hinsichtlich der Ergebnisdissemination konstatiert. Auch stellt die Evaluierung fest, dass MdZ grundsätzlich den selbst auferlegten Anspruch erfüllt, ein „lernendes Programm“ zu sein. Einige Lernoder Adaptationsimpulse scheiterten jedoch an strategischen und auch operativen Rahmenbedingungen und waren daher bislang nicht umsetzbar. Tatsächlich umgesetzte Lern- oder Adaptationsimpulse kamen bisher vorwiegend von außen bzw. durch singuläre Impulse. Ein systematisch organisierter, regelmäBiger Lernprozess innerhalb des Programms ist bislang weniger etabliert.

Als eine zentrale Herausforderung in MdZ sieht die Evaluierung die Optimierung von Themenfeld übergreifenden Aktivitäten. Die vielfach historisch gewachsenen Strukturen und Praktiken im Programm

33 Vgl. Fischl et al. (2018). 
sind vornehmlich auf Themenfeldebene angesetzt bzw. finden dort statt. Diese funktionieren zwar durchaus gut, jedoch wäre eine Weiterentwicklung der bisher eher themenfeldspezifischen Herangehensweise nicht zuletzt einer Hebung bisher noch nicht ausgeschöpfter Potenziale zuträglich. Die Evaluierung formuliert auch einige Möglichkeiten und Anknüpfungspunkte für eine Stärkung der Gesamtprogrammebene, die gleichzeitig aber nicht ausschließen, dass den Spezifika der einzelnen Themenfeld-Communities Rechnung getragen wird. Zur besseren Themenfeld übergreifenden Abstimmung der Roadmaps wird etwa die Option eines strategischen Beirats auf Programmebene thematisiert; hinsichtlich der Begleitmaßnahmen wird eine systematischere Herangehensweise empfohlen wie auch anhand einiger Beispiele erläutert; und auch die internen Koordinationsprozesse sollten der Evaluierung zufolge in Zukunft gestärkt werden.

Die im Rahmen der Evaluierung auf Programmebene durchgeführte Zielerreichungsanalyse zeigt, dass die Programmziele sowie die Ziele der Wirkungsfolgenabschätzung (WFA) zum Programm großteils bereits vollständig erreicht bzw. - mit einer Ausnahme - im vorgesehenen Zeithorizont erreicht werden. Hinsichtlich der Programmindikatorik wird empfohlen, Zielwerte hinsichtlich Realisierbarkeit zu überprüfen, sowie Zielindikatoren zwecks Abbildung gesellschaftlicher Zieldimensionen zu überarbeiten bzw. diese ggf. zu ergänzen.

\subsubsection{ERP-Kreditprogamm}

Der ERP-Fonds trägt durch spezifische Maßnahmen der direkten Wirtschaftsförderung zur Strukturverbesserung der österreichischen Wirtschaft bei. Er wurde 1962 aus den Mitteln der US-MarshallplanHilfe gegründet und ist mit der aws in Personalunion verbunden. Damit bündelt die aws sämtliche unternehmensbezogenen Wirtschaftsförderungen des Bundes und ist als One-Stop-Shop in der Lage, Un- ternehmen in sämtlichen Entwicklungsphasen - von „vor der Gründung" bis hin zur Internationalisierung - zielgerichtet und effizient zu begleiten. ${ }^{34}$

Die jährliche Dotation des ERP-Fonds beträgt rd. 600 Mio. $€$. Gemäß $\S 1$ Abs. (2) ERP-Fonds-Gesetz hat der ERP-Fonds die Aufgabe, den Ausbau, die Rationalisierung und die Produktivität der österreichischen Wirtschaft insbesondere durch Unterstützung und Anregung der produktiven Tätigkeit und des Warenaustausches zu fördern. Die Unterstützung technologisch anspruchsvoller Projekte gibt Impulse für Innovation, nachhaltiges Wachstum und für Beschäftigung. In den letzten Jahren hat sich als übergeordnetes Ziel "Wachstum und Innovation konsequent fördern" herauskristallisiert. Konkretere Zielsetzungen und Richtungen des ERP-Fonds sind in den einzelnen Jahresprogrammen bzw. in weiterer Folge innerhalb der einzelnen Programmrichtlinien festgelegt.

ERP-Kredite sind primär ein Finanzierungsinstrument für Wachstums- und Innovationsprojekte, die im Verhältnis zu Größe und Finanzierungskraft der Unternehmen hohe Volumina erreichen. Die Möglichkeiten der Mittelverwendung spannt das EU-Beihilfenrecht auf: Im Vordergrund stehen materielle Investitionen, aber auch immaterielle Investitionen und Ausgaben für Forschung, technologische Entwicklung und Innovation sind zulässige Verwendungsmöglichkeiten.

In den letzten Jahren war es erklärtes Ziel des Managements, innerhalb der Programme eine Simplifizierung vorzunehmen und die Übersichtlichkeit zu erhöhen. So kann nunmehr zwischen dem Wachstumskredit für Gründerinnen und Gründer und kleine Unternehmen, dem Wachstums- und Innovationsprogramm, dem Tourismusprogramm, dem Programm für Land- und Forstwirtschaft und dem Programm für Verkehrswirtschaft unterschieden werden. Kredite aus diesen Programminitiativen können auch mit anderen Instrumenten kombiniert werden, bspw. mit dem EFRE-Programm, dem Frontrunner-Programm,

34 Vgl. https://www.aws.at/historie/ 
dem Programm aws Industrie 4.0 oder der Absicherung durch aws Garantien.

\section{Zentrale Evaluierungsergebnisse}

Im Zentrum der vom Austrian Economics Center und des Industriewissenschaftlichen Instituts in 2018 durchgeführten Evaluierung stand die Bedeutung des ERP-Kreditprogramms der Austria Wirtschaftsservice Gesellschaft mbH sowohl auf Unternehmensebene als auch auf gesamtwirtschaftlicher Ebene. ${ }^{35}$ Ziel der Evaluierung war es, eine fundierte Informationsgrundlage für das Monitoring und die Strategieentwicklung bereitzustellen. Neben einer Analyse des Programmmanagements sowie der Effizienz in der Programmabwicklung wurde im ersten Teil der Evaluierung im Rahmen einer Unternehmensbefragung eine branchenspezifische Impactanalyse des ERP-Kreditprogrammes auf Mikroebene vorgenommen.

Die Unternehmensbefragung (Online-Fragebogenerhebung) wurde mit Unterstützung der aws im Sommer 2018 durchgeführt. Es wurden insgesamt 3.013 Unternehmen zur Befragung eingeladen, wovon 497 einen gültigen Fragebogen retourniert haben. Die regionale sowie größenspezifische Verteilung der Stichprobe spiegelt die Struktur der Grundgesamtheit wider. Schwerpunktthemen waren die Informationskanäle der Antragsteller, die Gründe und Motivation hinter dem ERP-Kreditantrag sowie eine Zufriedenheitsanalyse. Die Ergebnisse der Fragebogenerhebung wurden durch Tiefeninterviews in Unternehmen ergänzt und reflektiert.

Aufbauend auf den Ergebnissen auf Ebene der Unternehmen wurde im zweiten Teil der Evaluierung eine gesamtwirtschaftliche Impactanalyse durchgeführt. Mittels Input-Output-Analyse wurden einerseits die volkswirtschaftlichen Effekte von mittels ERP-Krediten gestützten Investitionen ermittelt, andererseits jene volkswirtschaftlichen Effekte identifiziert, welche wiederkehrend durch den laufenden Betrieb der investitionsdurchführenden Unternehmen generiert werden.
Grundlage der Unternehmensbefragung und volkswirtschaftlichen Berechnungen waren alle Unternehmen, die ein ERP-gefördertes Investitionsprojekt in den Jahren 2015, 2016 und 2017 bzw. im ersten Halbjahr 2018 abgeschlossen hatten. Die Ergebnisse zeigen, dass die aws mit dem ERP-Kreditprogramm bei den Fördernehmerinnen und Fördernehmern hohe Zufriedenheitswerte erreicht. Die Effektivität der Programmabwicklung ist hoch, die Programmgestaltung auf die Zielgruppe(n) gut zugeschnitten.

Mit den wesentlichen Bedingungen des ERP-Kredits (Höhe des Finanzierungsvolumens, Laufzeit, Kombinationsmöglichkeiten mit anderen aws-Produkten) zeigen sich die Befragten sehr zufrieden. Von Seiten des Fördergebers ist eine hohe Bereitschaft zur stetigen Verbesserung des Instruments zu erkennen, u.a. durch die Berücksichtigung von Kundenfeedback. Weitere Verbesserungen in der Ausgestaltung des Instruments können teils durch Feinjustierungen (wie z.B. durch eine höhere Flexibilität bei Laufzeiten und Tilgungsfristen) erreicht werden.

Mit der Programmabwicklung erzielt die aws sowie ihre Haus-/Treuhandbanken ebenso eine hohe Kundenzufriedenheit. Die Haus-/Treuhandbanken spielen sowohl beim Zugang zur Förderung (als Informationskanal) als auch im Rahmen der Antragstellung und Abwicklung eine zentrale Rolle. In diesem Zusammenhang ist die Qualitätssicherung seitens der aws weiterhin von hoher Bedeutung. Es ist eine anhaltend intensive Auseinandersetzung zwischen aws und Haus-/Treuhandbank im Rahmen von Betreuungs- und Abwicklungsprozessen notwendig. Dies stellt eine große organisatorische Herausforderung dar, ist jedoch für die Qualität der Programmabwicklung und die Kundenzufriedenheit von grundlegender Bedeutung. Ansatzpunkte für Verbesserungen in diesem (Schnittstellen-)Bereich sind (branchenspezifisch betrachtet) in einer teils schnelleren Bearbeitungsdauer von Anträgen im Tourismus zu lokalisieren.

35 Vgl. Kolm et al. (2018a); Kolm et al. (2018b). 
In den letzten Jahren wurden signifikante Umsätze und Arbeitsplätze geschaffen. Betrachtet man die bis dato eingetretene Wirkung der mithilfe des ERPKredits umgesetzten Investitionen in den Unternehmen, so beobachten die Befragten hohe positive Effekte. $67 \%$ der Unternehmen sehen eine steigende Umsatzentwicklung, weitere $25 \%$ eine Stabilisierung des Umsatzes aufgrund der mit dem ERP-Kredit umgesetzten Investitionen. $48 \%$ sehen des Weiteren eine Steigerung bei den Beschäftigten, zusätzliche $31 \%$ eine Stabilisierung. Weitere Effekte der Investitionen auf Unternehmensebene sind vor allem in einer Stärkung der Marktposition und Wettbewerbsfähigkeit der Unternehmen sowie im Gewinn neuer Kundinnen und Kunden zu sehen. Auch aus regionalpolitischer Sicht führen Investitionen mittels ERPKredit zu positiven Struktureffekten - es lassen sich Vernetzungs- und Netzwerkeffekte beobachten.

Rd. zwei Drittel der Unternehmen nennen Modernisierung bzw. die Erweiterung des Unternehmens als Grund für ihren ERP-Kreditantrag, insbesondere für Kleinunternehmen (fast $90 \%$ ) ist dies relevant. Weitere häufig genannte Motive sind der Auf- und Ausbau neuer Dienstleistungen und Geschäftsfelder sowie Neugründungen und Betriebsansiedelungen. Selbst in Zeiten der Hochkonjunktur bzw. niedriger Zinsen ist ein Instrument wie der ERP-Kredit attraktiv. Rund $90 \%$ der Befragten sind der Ansicht, dass dieser auch in Niedrigzinszeiten ein wichtiges Instrument der KMU-Finanzierung ist und bleibt. Die Sicherheit durch einen fixen Zinssatz ist dabei ein ebenso wichtiger Faktor wie Konstanz und Planbarkeit oder auch lange tilgungsfreie Zeiten.

Die Evaluierung zeigt, dass die den Unternehmen für Investitionen zur Verfügung stehenden Mittel des ERP-Fonds auch aus volkswirtschaftlicher Sicht bedeutend sind. Durch die mit Mitteln des ERPFonds ermöglichten Investitionen wurde in Österreich in den Jahren 2015-2017/18 ein mittel- wie unmittelbarer gesamtwirtschaftlicher Produktionswert von 4,83 Mrd. € ausgelöst. Dabei generieren sämtliche Investitionen im Beobachtungszeitraum in Österreich eine gesamtwirtschaftliche Wertschöpfung von 2,40 Mrd. €. Insgesamt wurden durch die Investitionstätigkeiten in den dreieinhalb Jahren mehr als 32.300 Arbeitsplätze in Österreich gesichert, das entspricht rd. 27.400 Vollzeitäquivalenten. Eine sektorenspezifische Analyse sowie zahlreiche vom IWI durchgeführte Wertschöpfungsstudien zeigen, dass im Zuge von Investitionstätigkeiten insbesondere Industrieunternehmen überdurchschnittlich hohe volkswirtschaftliche Effekte erzielen. In diesem Bereich haben die Mittel des ERPFonds einen besonders hohen gesamtwirtschaftlichen Impact.

Neben den Investitionseffekten wirken die ERPKredite auch positiv wie stabilisierend auf den laufenden Betrieb der Unternehmen. Ein Vergleich des Produktionszuwachses des Unternehmenssamples mit der Gesamtwirtschaft zeigt hier für den Bereich der Industrie, aber auch für die Dienstleistungen und den Handel ein überdurchschnittlich hohes Produktionswachstum. Im Zuge des laufenden Betriebs ist es dann vor allem wieder die heimische Industrie, die über ihre hochwertige Nachfrage in weitläufigen Wertschöpfungsprozessen beachtliche Multiplikatoreffekte auf die gesamte heimische Volkswirtschaft auslöst. 


\section{Abbildungs- und Tabellenverzeichnis}

\subsection{Abbildungsverzeichnis}

Abbildung 1-1: Finanzierung der in Österreich durchgeführten Forschung und experimentellen Entwicklung sowie Forschungsquote, 2008-2019 . . . . . . . . . . . . . . . . . . . . . . . . . . . . . . . . . . . 18

Abbildung 1-2: Entwicklung der Finanzierung der in Österreich durchgeführten Forschung und experimentellen Entwicklung, 2008-2019 (Index, 2008=100) . . . . . . . . . . . . . . . . . . . . . . . . . . . . . . . . . . . .7.

Abbildung 1-3: Anteile der Finanzierung der in Österreich durchgeführten Forschung und experimentellen Entwicklung, 2008-2019 . . . . . . . . . . . . . . . . . . . . . . . . . . . . . . . . . . . . 20

Abbildung 1-4: Forschungsquote in Österreich nach Finanzierung, 2008-2019 . . . . . . . . . . . . . . . . . . . . . 21

Abbildung 1-5: Forschungsquote und Finanzierung in OECD-Ländern, 2017 . . . . . . . . . . . . . . . . . . . . . . . . . 22

Abbildung 1-6: Österreichs F\&E-Quote im internationalen Vergleich, 2010 und 2017 . . . . . . . . . . . . . . . . . . . . . . 26

Abbildung 1-7: Patentintensität nach Herkunftsland (Triade-Patente, 2012 und 2016). . . . . . . . . . . . . . . . . . . 27

Abbildung 1-8: Anzahl der Artikel im Bereich Wissenschaft und Technik, 2010 und 2017 . . . . . . . . . . . . . . . . . 28

Abbildung 1-9: Anzahl der Artikel im Bereich Wissenschaft und Technik normiert mit der Länderpopulation, 2010 und 2017.29

Abbildung 1-10: Zitationsraten mit und ohne Selbstzitation, 2017 . . . . . . . . . . . . . . . . . . . . . . . . . . . . 30

Abbildung 1-11: Innovationsindikator „Gesamt", 2017 . . . . . . . . . . . . . . . . . . . . . . . . . . . . . . . . . . . . . . 32

Abbildung 1-12: Index für die digitale Wirtschaft und Gesellschaft (DESI, 2018) . . . . . . . . . . . . . . . . . . . . . . . 34

Abbildung 1-13: Indexwert „Nutzung von Informations- und Kommunikationstechnologien” im Vergleich, 2018 . . . . . . . 34

Abbildung 1-14: Indexwert „Verfügbarkeit von Informations- und Kommunikationstechnologien“ im Vergleich, 2018 . . . . 36

Abbildung 1-15: Position Österreichs im Ländervergleich - Übersicht über Ergebnisse im Global Innovation Index . . . . . . 36

Abbildung 1-16: Dimensionen des Global Competitiveness Report: Position Österreichs im Vergleich zum

Durchschnitt der 28 EU-Mitgliedsstaaten . . . . . . . . . . . . . . . . . . . . . . . . . . . . . $\quad . \quad 38$

Abbildung 1-17: Prozentualer Anteil 25 bis 64-Jähriger mit einem Abschluss im Tertiärbereich, 2017 . . . . . . . . . . . . 40

Abbildung 1-18: Absolventinnen und Absolventen des Tertiärbereichs in MINT- und IKT-Fachbereichen, 2016 . . . . . . . 41

Abbildung 1-19: Ökonomische Komplexität - Position Österreichs im internationalen Vergleich, 2016. . . . . . . . . . 42

Abbildung 1-20: Indikator „Lernförderliche Arbeitsorganisation“, 2018. . . . . . . . . . . . . . . . . . . . . . . . . . . . . . . 43

Abbildung 1-21: Grad der Kooperationen zwischen Unternehmen und Hochschulen . . . . . . . . . . . . . . . . . . . . . . . 44

Abbildung 1-22: Grad der Kooperationen zwischen Unternehmen und Forschungseinrichtungen. . . . . . . . . . . . . . . . 45

Abbildung 2-1: FFG-Förderung nach Technologiefeldern, 2018 (Barwerte in Mio. $€$ ) . . . . . . . . . . . . . . . . . . . . . . . . 81

Abbildung 3-1: Die FTI-Strategie 2020 sowie weitere nationale Teilstrategien im Kontext europäischer Entwicklungen . . . 92

Abbildung 3-2: Entscheidungsmatrix für die Aufnahme in die erste Schulstufe . . . . . . . . . . . . . . . . . . 103

Abbildung 3-3: Anteil der Incomings und Outgoings an den ordentlichen Studierenden an Universitäten, 2001-2017. . . . 106

Abbildung 3-4: Erasmus Studienaufenthalte und Praktika, 1992/93-2017/18 . . . . . . . . . . . . . . . . . . . . . 107

Abbildung 3-5: Ablauf eines strukturierten Doktoratsstudiums an der Universität Wien . . . . . . . . . . . . . . . . 110

Abbildung 3-6: Alle Drittmittelerlöse aus F\&E-Projekten und Erlöse aus Projekten des FWF, 2017 . . . . . . . . . . . . . . 121

Abbildung 3-7: Herkunft der universitären Einnahmen aus F\&E-Projekten sowie Projekten der Entwicklung und Erschließung der Künste 2017 .

Abbildung 3-8: Anteil Österreichs an EU-Forschungsgeldern (Horizon 2020), Stand 06/2018 . . . . . . . . . . . . . . . 129

Abbildung 3-9: Österreich: Anzahl der eingeworbenen ERC Grants für Pionierforschung (kumuliert seit 2007) . . . . . . . . 130

Abbildung 3-10: Anzahl F\&E-aktiver Unternehmen und deren F\&E-Umsatzintensität nach der Technologie- und Wissensintensität der Wirtschaftszweige (OECD-Definition) im Zeitverlauf. . . . . . . . . . . . . . . . . 142

Abbildung 3-11: Anteil innovationsaktiver Unternehmen nach der Technologie- und Wissensintensität der Wirtschaftszweige (OECD-Definition) im Zeitverlauf . . . . . . . . . . . . . . . . . . . . . . . . . . . . . . . . . 143

Abbildung 3-12: Unternehmen mit Produktinnovationen und Marktneuheiten. . . . . . . . . . . . . . . . . . . . . . . . 144

Abbildung 3-13: Entwicklung der F\&E-Finanzierung aus dem Ausland . . . . . . . . . . . . . . . . . . . . . . . . . . . 145

Abbildung 3-14: Anteil der Produkt- und Prozessinnovatoren mit Innovationskooperation mit Universitäten, Fachhochschulen oder anderen höheren Bildungseinrichtungen, in \% . . . . . . . . . . . . . . . . 152

Abbildung 3-15: Wagniskapital-Investitionen in ausgewählten Ländern nach Standort des Portfolio-Unternehmens, 2007-2017, in Promille des BIP . . . . . . . . . . . . . . . . . . . . . . . . . 157 
Abbildung 3-16: Wagniskapital-Investitionen in ausgewählten Ländern nach Standort der Beteiligungsfirma,

2007-2017, in Promille des BIP

Abbildung 3-17: Staatliche Forschungsausgaben für F\&E nach sozioökonomischen Zielsetzungen, 2016 . . . . . . . . . 172

Abbildung 3-18: Die Bereiche offener Wissenschaft. . . . . . . . . . . . . . . . . . . . . . . . . . . . . . . . . 186

Abbildung 4-1: Anteil der Unternehmen pro Land, die Künstliche Intelligenz nutzen, in \% . . . . . . . . . . . . . . . . 204

Abbildung 5-1: Kooperationsländer im Programm Beyond Europe . . . . . . . . . . . . . . . . . . . . . . . . . . . 227

Abbildung 5-2: Impulsprogramm FIT-IT und Themeninitiative „IKT der Zukunft“: Summe der genehmigten Barwerte

verteilt auf die Projektlaufzeiten nach Programmen/Förderthemen, in Mio. $€$.

\subsection{Tabellenverzeichnis}

Tabelle 1-1: Internationale Position Österreichs anhand einschlägiger Innovationsindikatoren

Tabelle 1-2: Österreichs Performance in Horizon 2020 nach Säulen, Projektteilnahmen, Projekten, Koordinationen und Budget . . . . . . . . . . . . . . . . . . . . . . . . . . . . . . . . 53

Tabelle 1-3: Informations- und Beratungsinstrumente des FFG-EIP nach Aufgabenbereichen. . . . . . . . . . . . . . . . 55

Tabelle 1-4: Übersicht über den Aufbau von Horizon Europe gemäß dem Vorschlag der Europäischen Kommission . . . . . . 57

Tabelle 2-1: Anzahl der Förderungen 2017-2018 . . . . . . . . . . . . . . . . . . . . . . . . . . . . . . . . . . . . 75

Tabelle 2-2: Durch den FWF finanziertes Forschungspersonal 2017-2018 . . . . . . . . . . . . . . . . . . . . . . . . . . . . 75

Tabelle 2-3: Förderungssummen in Mio. €, 2017-2018 . . . . . . . . . . . . . . . . . . . . . . . . . . . . . . . . . . . . . . 76

Tabelle 2-4: Neue Initiativen und Förderungsprogramme 2018 . . . . . . . . . . . . . . . . . . . . . . . . . . . . . . . . . . 77

Tabelle 2-5: FFG-Förderungsaufkommen, ohne Breitband, 2018. . . . . . . . . . . . . . . . . . . . . . . . . . . . 79

Tabelle 2-6: FFG-Fördersummen nach Bereich, ohne Breitband, 2017 und 2018 (in Mio. €) . . . . . . . . . . . . . . . . . 79

Tabelle 2-7: FFG-Förderungen nach Organisationstyp (Kosten, Förderungen und Barwert in Mio. €), ohne Breitband, 2018 . $\quad 80$

Tabelle 2-8: Neue Entwicklungen im Instrumentenportfolio . . . . . . . . . . . . . . . . . . . . . . . . . . . 83

Tabelle 2-9: Anzahl der Förderungen und Fördersummen in Mio. €, 2018 . . . . . . . . . . . . . . . . . . . . . . . . . . . . . 85

Tabelle 3-1: Betreuungsrelation auf Studienfeld-Ebene, Studienjahr 2015/16 . . . . . . . . . . . . . . . . . . . . . . 99

Tabelle 3-2: Laufzeit Wissenschafts-Wirtschaftskooperationen . . . . . . . . . . . . . . . . . . . . . . . . . 135

Tabelle 3-3: Anzahl von Unternehmen mit eigener Forschung in Österreich, 2009-2015 . . . . . . . . . . . . . . . . . . 141

Tabelle 3-4: Anzahl der Erstfördernehmer (Unternehmen) und deren Anteil an den Fördernehmern . . . . . . . . . . . . . 141

Tabelle 3-5: Maßnahmen zur Förderung von Wissenschaft-Wirtschaftskooperationen in der FTI-Strategie und Förderinstrumente von BMVIT, BMDW und BMBWF. . . . . . . . . . . . . . . . . . . . . . . 150

Tabelle 3-6: Indikatoren zu Wissenschaft-Wirtschaftskooperationen des European Innovation Scoreboard (letztverfügbare Zahlen nach EIS 2018), Österreich, EU und die Innovation Leader. . . . . . . . . . . . . . 151

Tabelle 3-7: Maßnahmen der FTI-Strategie zur Setzung strategischer, thematischer Schwerpunkte. . . . . . . . . . . . 170

Tabelle 3-8: Revealed Technological Advantage nach ausgewählten Technologiefeldern, 2002-2005 und 2012-2015. . . . . . . . . . . . . . . . . . . . . . . . . . . . . . . . 171

Tabelle 3-9: Maßnahmen der FTI-Strategie zur Optimierung des Fördersystems. . . . . . . . . . . . . . . . . . . . 173

Tabelle 3-10: Performance in den Rahmenprogrammen FP6 bis H2020 . . . . . . . . . . . . . . . . . . . . . . . 176

Tabelle 4-1: Ordentliche Studierende im Studienfeld Informatik, Wintersemester 2013-2017 . . . . . . . . . . . . . . 210

Tabelle 5-1: Kennzahlen zur Frontrunner-Initiative . . . . . . . . . . . . . . . . . . . . . . . . . . . . . 231

Tabelle 5-2: Programmteilnahme nach Instrumenten: Eingereichte Projekte und teilnehmende Organisationen . . . . . . . 240

Tabelle 5-3: Programmteilnahme nach Instrumenten: Projektkosten und Fördervolumen . . . . . . . . . . . . . . . . 240 


\section{Literatur}

Acemoglu, D. und Autor, D. (2011): Skills, tasks and technologies: implications for employment and earnings, in: Card, D. und Ashenfelter, O. (Hg.), Handbook of Labor Economics Volume 4 Part B, Elsevier, 1043-1171.

ACR - Austrian Cooperative Research (2018): Brücken bauen: Jahresbericht 2017, Wien. https://www.acr.ac.at/filead$\mathrm{min} /$ documents/Publikationen/ACR_Jahresbericht_2017 web.pdf

Aiginger, K., Falk, R. und Reinstaller, A. (2009): Reaching Out to the Future Needs Radical Change: Towards a New Policy for Innovation, Science and Technology in Austria, Synthesebericht der Systemevaluierung der österreichischen Forschungsförderung und -finanzierung, Wien. https://www. bmvit.gv.at/service/publikationen/innovation/forschungspolitik/endbericht_syseval.html

AIT (2011): Jahresabschluss 2010, Wien. https://www.ait.ac.at/ fileadmin/cmc/downloads/New_Presse/AIT_2010_Jahresabschluss.pdf

AIT (2018): Jahresabschluss 2017, Wien. https://www.ait.ac.at/ fileadmin/cmc/downloads/New_Presse/AIT_Jahresabschluss_2017.pdf

All European Academies (2017): The European Code of Conduct for Research Integrity, Revised Edition, Berlin. https:// ec.europa.eu/research/participants/data/ref/h2020/ other/hi/h2020-ethics_code-of-conduct_en.pdf

Arthur D. Little (2017a): Digitale Transformation von KMU in Österreich 2017, Wien.

Arthur D. Little (2017b): Österreich als 5 G Vorreiter, Wien.

Arthur D. Little (2018): Digitale Transformation von KMU in Österreich 2018, Wien.

Austria Wirtschaftsservice (2019): Über die aws. https://www. aws.at/ueber-die-aws/

Barré, R. (2010): Towards Socially Robust S\&T Indicators - Indicators as Debatable Devices, Enabling Collective Learning, Research Evaluation, 19/3, 227-31.

Bauer, B., Ferus, A., Gorraiz, J., Gründhammer, V., Gumpenberger, C., Maly, N., Mühlegger, J.M., Preza, J.L., Sánchez-Solís, B., Schmidt N. und Steineder, C. (2015): Forschende und ihre Daten: Ergebnisse einer österreichweiten Befragung, Report 2015. 10.5281/zenodo.32043

BCG und BCG Gamma (2018): Mind the (AI) Gap.

Ben-David, J. (1991): The profession of science and its powers, Scientific growth: Essays on the social organization and ethos of science, Berkeley: University of California Press, 187-209.

Biegelbauer, P., Dinges, M., Wang, A., Weber, M., Ploder, M., Polt, W., Streicher, J., Unger, M., Fischl, I., Kaufmann, P., Gassler, H., Konzett-Smoliner, S. und K. Schuch (2018): Evaluierung der Umsetzung von H2O20, EUREKA, COSME, EEN und ERA in Österreich, Endbericht, Wien. https://repository.fteval. at/333/1/FFG_EIP_Endbericht_barrierefrei__FINAL.pdf

Binder, D., Thaler, B., Unger, M., Ecker, B., Mathä, P. und Zaussinger, S. (2017): MINT an öffentlichen Universitäten, an Fachhochschulen sowie am Arbeitsmarkt: Eine Bestandsaufnahme, IHS-Forschungsbericht, Studie im Auftrag des BMWFW, Wien.
Brewer, J. (2011): The impact of impact, Research Evaluation, Volume 20, Issue 3, 1 September 2011, 255-256. https:// doi.org/10.3152/095820211X12941371876869

Brewer, J. (2013): The public value of the social sciences, Bloomsbury: London, New Delhi, New York, Sidney.

Birke, B. und Fettelschoß, K. (2013): Evaluierung des Förderprogrammes "Sparkling Science“, Wien. http:// repository.fteval.at/38/1/Evaluierung $\% 20$ des\%20 F\%C3\%B6rderprogrammes\%20Sparkling\%20Science.pdf

BKA (2017): Zusammen. Für unser Österreich, Regierungsprogramm 2017-2022, Wien. https://www.bundeskanzleramt. gv.at/documents/131008/569203/Regierungsprogramm 2017\%E2\%80\%932022.pdf/b2fe3f65-5a04-47b6-913d$\overline{2 f e 512 f f 4 c e 6}$

BKA, BMF, BMUKK, BMVIT, BMWFJ und BMWF (2011): Der Weg zum Innovation Leader: Strategie der Bundesregierung für Forschung, Technologie und Innovation, Wien. https:// www.bmvit.gv.at/innovation/publikationen/forschungspolitik/downloads/fti_strategie.pdf

BKA, BMF, BMVIT, BMWFW und RFTE (2014): Österreichischer Forschungsinfrastruktur-Aktionsplan 20142020, Wien. https://rio.jrc.ec.europa.eu/en/file/7386/ download?token=jPANRXup

BMB (2016): Bildungsstandards: ein Beitrag zur Unterrichts- und Schulentwicklung, Wien. https://bildung.bmbwf.gv.at/ schulen/unterricht/ba/bildungsstandards.pdf?6kdmdx

BMBWF (2017): Universitätsbericht 2017, Wien. https://www. bmbwf.gv.at/fileadmin/user_upload/Publikationen/Universitätsbericht_2017_barrierefrei.pdf

BMBWF (2018a): Deutschförderklassen und Deutschförderkurse: Leitfaden für Schulleiterinnen und Schulleiter, Wien. https://bildung.bmbwf.gv.at/schulen/unterricht/ba/ deutschfoerderklassen.pdf?6hwybc

BMBWF (2018b): Dossier: Angaben zur Wirkungsorientierung aus den Bundesfinanzgesetzen 2018 und 2019, Wien. https://bmbwf.gv.at/fileadmin/user_upload/Publikationen/2018/Dossier_WiOr_2018_19_kopie___Kopie.pdf

BMBWF, BMDW und BMVIT (2018): Zukunftsoffensive für Forschung, Technologie und Innovation, Vortrag an den Ministerrat 25/63, Wien. https://www.bundeskanzleramt.gv.at/ documents/131008/972899/25_63_mrv.pdf/2b02f3d809cd-45ee-b9f2-f3cfd8244990

BMDW (2018): Digitales Kompetenzmodell für Österreich - DigComp 2.2 AT, Wien. https://www.bmdw.gv.at/DigitalisierungundEGovernment/DigitalisierungGesellschaft/Documents/DigComp_2.2_AT_barrierefrei_V14.pdf

BMDW und BMVIT (2018): Österreichischer Forschungs- und Technologiebericht 2018, Bericht der Bundesregierung an den Nationalrat gem. § 8 (2) FOG über die Lage und Bedürfnisse von Forschung, Technologie und Innovation in Österreich. https://www.bmbwf.gv.at/ftb

BMF (2011): Handbuch Wirkungsorientierte Haushaltsführung, Wien. https://www.bmf.gv.at/budget/haushaltsrechtsreform/Handbuch_Wirkungsorientierte_Haushaltsfuehrung. pdf?67rujw 
BMF (2018): Bundesfinanzgesetz 2018, Wien. https://service. bmf.gv.at/BUDGET/Budgets/2018_2019/bfg2018/Bundesfinanzgesetz_2018.pdf

BMNT, BMBWF und BMVIT (2019): Bioökonomie - Eine Strategie für Österreich, Wien. https://www.bmnt.gv.at/dam/ jcr:47f45fc8-91ff-41fd-9c71-4fc9e12fb591/190313_Boes Online_04x.pdf

BMUKK (2013): Empfehlungen für Diversität und Mehrsprachigkeit in Organisation, Personal, Curricula, Forschung, Lehre der Pädagog/inn/enausbildung (neu). https://bildung. bmbwf.gv.at/schulen/unterricht/ba/sprachen_diversitaet_empf_26214.pdf?61ed9c

BMVIT und BMDW (2018): AIM AT 2030 Artificial Intelligence Mission Austria 2030: Die Zukunft der Künstlichen Intelligenz in Österreich gestalten, Wien. https://www.bmvit. gv.at/innovation/publikationen/ikt/downloads/aimat_ ua.pdf

BMVIT und BMDW (2019): Bundesstrategie für Künstliche Intelligenz - Erarbeitung einer Strategie für Künstliche Intelligenz (KI) zur Umsetzung der Ziele der Digitalisierungsstrategie, Vortrag an den Ministerrat 37/29, Wien. https://www.bundeskanzleramt.gv.at/documents/131008/1101773/37_29 mrv.pdf/9f18fb12-ced7-4ba8-93eb-ffd650cf178b

BMVIT, BMF und BMDW (2018): 5G-Strategie - Österreichs Weg zum 5G-Vorreiter in Europa, Wien. https://www. bmvit.gv.at/service/publikationen/telekommunikation/ downloads/5Gstrategie_ua.pdf

BMVIT, BMWF, BMWFJ und BMeiA (2013): Beyond Europe - Die Internationalisierung Österreichs in Forschung, Technologie und Innovation über Europa hinaus, Wien. https:// era.gv.at/directory/160/attach/FTI_AG7a_Brosch_re_Ansicht.pdf

BMWF (2008): Österreichischer Forschungsdialog, Ergebnisdokumentation, Wien. https://bmbwf.gv.at/fileadmin/ user_upload/forschung/forschungsdialog/OESTERR_FORSCHUNGSDIALOG_Ergebnisdokumentation_0808bmwf. pdf

BMWF (2011): Universitätsbericht 2011, Wien. https://bmbwf. gv.at/fileadmin/user_upload/wissenschaft/publikationen/ Universitaetsbericht2011.pdf

BMWFJ, BMVIT, BBG und AIT (2012): Leitkonzept für eine innovationsfördernde öffentliche Beschaffung (IÖB) in Österreich, Wien. https://www.bmvit.gv.at/innovation/publikationen/forschungspolitik/downloads/ioeb_leitkonzept.pdf

BMWFW (2014): Universitätsbericht 2014, Wien. https:// bmbwf.gv.at/fileadmin/user_upload/Universitaetsbericht_2014.pdf

BMWFW (2015a): Aktionsplan für einen wettbewerbsfähigen Forschungsraum: Maßnahmen des Bundesministeriums für Wissenschaft, Forschung und Wirtschaft zur verstärkten Umsetzung der FTI-Strategie der Bundesregierung in ausgewählten Themenfeldern, Wien. https://www.bmbwf. gv.at/fileadmin/user_upload/wissenschaft/publikationen/ forschung/Forschungsaktionsplan_web.pdf

BMWFW (2015b): Der Gesamtösterreichische Universitätsentwicklungsplan 2016-2021, Wien. https://bmbwf.gv.at/ fileadmin/user_upload/wissenschaft/publikationen/ guep/2016_2021_GUEP.pdf
BMWFW (2017a): Der Gesamtösterreichische Universitätsentwicklungsplan 2019-2024, Wien. https://bmbwf.gv.at/ fileadmin/user_upload/wissenschaft/publikationen/ guep/2019-2024_GUEP_Langversion.pdf

BMWFW (2017b): Geisteswissenschaften, Sozialwissenschaften, Kulturwissenschaften: Beitrag zur strategischen Weiterentwicklung der Rahmenbedingungen, Wien. https:// www.bmbwf.gv.at/fileadmin/user_upload/forschung/ GSK-Bericht_Beitrag_zur_strategischen_Weiterentwicklung_der_Rahmenbedingungen.pdf

BMWFW (2017c): WBV-Arbeitsbehelf: Erläuterungen zur Erstellung der Wissensbilanz gemäß der Verordnung über die Wissensbilanz BGBL. II Nr. 97/2017, Wien. www.bmbwf. gv.at/unidata/rechtlichegrundlagen

BMWFW und BMVIT (2016): Österreichischer Forschungs- und Technologiebericht 2016, Lagebericht gem. § 8 (1) über die aus Bundesmitteln geförderte Forschung, Technologie und Innovation in Österreich. https://www.bmbwf.gv.at/ftb

BMWFW und BMVIT (2017): Österreichischer Forschungs- und Technologiebericht 2017, Lagebericht gem. § 8 (1) über die aus Bundesmitteln geförderte Forschung, Technologie und Innovation in Österreich. https://www.bmbwf.gv.at/ftb

BMWFW und OeAD (2016): Sparkling Science Ausschreibung 2016, Ausschreibungstext, Wien.

Boon, W. und Edler, J. (2018): Demand, challenges and innovation: Making sense of new trends in innovation policy, Science and Public Policy, 45/4, 435-447.

Böheim, M. (2008): Reformoptionen zur Wettbewerbspolitik in Österreich, WIFO Monatsberichte 6/2008.

Brechelmacher, A., Park, E., Ates, G. und Campbell, D.F.J. (2015): The Rocky Road to Tenure - Career Paths in Academia, in: Fumasoli, T., Goastellec, G., Kehm, B.M (Hg.): Academic Work and Careers in Europe: Trends, Challenges, Perspectives, Dordrecht: Springer, 13-40.

Bröthaler, J. und Plank, L. (2017): Öffentliche Vergaben in Österreich - Methodische Grundlagen und empirische Ermittlung des gesamten Beschaffungsvolumens und öffentlicher Auftragsvergaben, im Auftrag des ANKÖ - Auftragnehmerkataster Österreich, Wien. https://www.ankoe.at/fileadmin/images/news/ANKOE_Oeffentliche-Vergaben-inOesterreich_Studie_TU-Wien.pdf

Bundesgesetz über die Organisation der Universitäten und ihre Studien (Universitätsgesetz 2002 - UG), BGBI. I Nr. 3/2019.

Bundesgesetz, mit dem das $\mathrm{GmbH}-\mathrm{Gesetz}$ und die Notariatsordnung geändert werden (Elektronische NotariatsformGründungsgesetz - ENG), BGBI. I Nr. 71/2018.

Bundesgesetz, mit dem die Neugründung von Betrieben und die Übertragung von Klein- und Mittelbetrieben gefördert wird (Neugründungs-Förderungsgesetz - NeuFöG), BGBI. I Nr. 40/2017.

Bundesverband der Deutschen Industrie e.V. (BDI) (2018): Innovationsindikator 2018, Schwerpunkt: Offenheit der Innovationssysteme. http://www.innovationsindikator.de/ fileadmin/content/2018/pdf/ausgaben/Innovationsindikator_2018.pdf

Bundesverband der Deutschen Industrie e.V. (BDI) (2018): Innovationsindikator 2018. http://www.innovationsindikator. de/mein-indikator/ 
Bundeswettbewerbsbehörde (2018): Tätigkeitsbericht 2017, Wien. https://www.bwb.gv.at/fileadmin/user_upload/ Downloads/taetigkeitsbereich/Taetigkeitsbericht_2017. $\underline{\text { pdf }}$

Charlotte Bühler Institut (2009): Bundesländerübergreifender BildungsRahmenPlan für elementare Bildungseinrichtungen in Österreich, Wien. https://bildung.bmbwf.gv.at/schulen/ sb/bildungsrahmenplan.pdf?6wber5

Citizen Science Network Austria (2018): Leistungsbericht 2017, Wien. https://www.citizen-science.at/images/CSNA_Leistungsbericht2017.pdf

Convelop Cooperative Knowledge Design GmbH (2015): Evaluierung der Zielvereinbarung des BMWFW mit der ACR, Graz, Wien.

Cornell Collage, INSEAD und World Intellectual Property Organization (WIPO) (2018): Global Innovation Index 2018: Energizing the world with innovation, 11. Auflage, World Intellectual Property Organization (WIPO).

Council of the European Union (2015): Review of the European Research Area advisory structure - Council conclusions (adopted on 01/12/2015), 14458/15 RECH 285 COMPET 537 ,Brüssel, 01.12.2015.

Council of the European Union (2018): Outcome of the Council Meeting, 3655th Council meeting, 14406/18. Brussels, 29-30.12.2018.

Ecker, B., Fink, N., Sardadvar, S., Kaufmann, P., Sheikh, S., Wolf, L., Brandl, B., Lorentz S. und Sellner, R. (2017): Evaluierung der Forschungsprämie, Wien. https://www.bmf.gv.at/budget/aktuelle-berichte/BMF_Evaluierung_der_Forschungspraemie_Endbericht.pdf?67ruop

Ecker, B., Reiner, C. und Gogola, G. (2018): Case study on the policy mix for science-industry knowledge transfer in Austria: Contribution to the OECD TIP Knowledge Transfer and Policies Project. https://www.innovationpolicyplatform. org/system/files/imce/PolicyMix\%20Austria_TIPKnowledgeTransferCaseStudy2019.pdf

Egerth, H. und Pseiner, K. (2019): Wirtschaft im Umbruch: "Chancen erarbeiten, Chancen nutzen", Jahrespressekonferenz der FFG, Wien, 6. März 2019, mimeo.

EHEA Paris 2018 Ministerial Conference (2018): Paris Communiqué (Paris, May 25th 2018). http://ehea.info/Upload/ document/ministerial_declarations/EHEAParis2018_Communique_final_952771.pdf

ERA Observatorium Austria und BMWFW (2016): Österreichische ERA Roadmap, Wien. https://era.gv.at/object/document/2581/attach/oesterreichische_ERA_Roadmap.pdf

ERAC (2015): ERAC Opinion on the European Research Area Roadmap 2015-2020. ERAC 1208/15. Brüssel, 20.04.2015.

ERAC (2017): Final Report of the ERAC Ad-hoc Working Group on Measuring the Impact of EU Framework Programmes for Research and Innovation at National Level, ERAC 1206/17.

ERAC (2018): ERAC Opinion on the 2018 review of the ERA advisory structure, ERAC 1209/18, Brüssel, 21.09.2018.

Ernst \& Young (2017): Digitalisierung in österreichischen Mittelstandsunternehmen, Wien.

Ernst \& Young (2018): Industrie 4.0 im österreichischen Mittelstand, Wien.
ESFRI (2018): Roadmap 2018: Strategy Report on Research Infrastructures, European Strategy Forum on Research Infrastructures, December 2018.

Europäische Kommission (2012): A Reinforced European Research Area Partnership for Excellence and Growth, Communication from the Commission to the European Parliament, the Council, the European Economic and Social Committee and the Committee of the Regions. Brussels, 17.7.2012, $\operatorname{COM(2012)~} 392$ final.

Europäische Kommission (2014): Special Eurobarometer 419: Public perceptions of science, research and innovation. http://ec.europa.eu/commfrontoffice/publicopinion/archives/ebs/ebs_419_en.pdf

Europäische Kommission (2016): Open Innovation, Open Science, Open to the World: a vision for Europe. http://ec.europa. eu/newsroom/dae/document.cfm?doc_id=16022

Europäische Kommission (2017): LAB - FAB - APP — Investing in the European future we want, Report of the independent High Level Group on maximising the impact of EU Research \& Innovation Programmes, Generaldirektion Forschung und Innovation, Brüssel. http://ec.europa.eu/research/ evaluations/pdf/archive/other_reports_studies_and_documents/hlg_2017_report.pdf

Europäische Kommission (2018a): Digitizing European Industry, Progress so far, 2 years after the launch, Luxembourg.

Europäische Kommission (2018b): European Innovation Scoreboard, Luxembourg. https://ec.europa.eu/docsroom/documents/30183

Europäische Kommission (2018c): Europe is back: Accelerating breakthrough innovation - Full set of recommendations from the Independent High-Level Group of Innovators on establishing a European Innovation Council, Publications Office of the European Union, Luxembourg.

Europäische Kommission (2018d): Österreich - Country Profile Digital Economy and Society Index 2017. http://ec.europa. eu/newsroom/document.cfm?doc_id=42989

Europäische Kommission (2018e): Proposal for a Council Regulation laying down the multiannual financial framework for the years 2021 to 2027 , COM/2018/322 final - 2018/0132 (APP), Brussels, 2.5.2018.

Europäische Kommission (2018f): Proposal for a Regulation of the European Parliament and of the Council establishing Horizon Europe - the Framework Programme for Research and Innovation, laying down its rules for participation and dissemination, COM/2018/435 final, Brüssel. https://eurlex.europa.eu/legal-content/EN/TXT/?qid=15 $\overline{4038763151}$ 9\&uri=CELEX\%3A52018PC0435

Europäische Kommission (2019a): Index für die digitale Wirtschaft und Gesellschaft (DESI) 2018 - Länderbericht Österreich. http://ec.europa.eu/newsroom/dae/document. cfm?doc_id =52342

Europäische Kommission (2019b): The Digital Economy and Society Index (DESI). https://ec.europa.eu/digital-singlemarket/en/desi

European Research Erea and Innovation Committee (2015): European Research Area Roadmap 2015-2020, Brüssel. https://era.gv.at/object/document/1845/attach/ERA_Roadmap_st01208_en15.pdf 
Eurostat (2019): Digitale Wirtschaft und Gesellschaft. https:// ec.europa.eu/eurostat/web/digital-economy-and-society/ data/database

Felt, U. und Fochler, M. (2018): Der gesellschaftliche Impact sozialwissenschaftlichen Wissens in Österreich: Wirkungswege, Messung, Potentiale, Wien.

FFG (2018): Laura Bassi 4.0 Programmdokument (01.06.201830.06.2021). https://www.ffg.at/sites/default/files/programmdokument_lbc_4.0_final.pdf

FFG und BMDW (2018): Digital Innovation Hubs, Ausschreibungsleitfaden, 1. Ausschreibung, Wien.

Fischl, I., Kaufmann, J., Oberholzner, Th. und Th. Koska (2018): Zwischenevaluierung des FTI-Programms "Mobilität der Zukunft" (MdZ), Wien. https://repository.fteval.at/376/1/ Endbericht_Eval_MdZ_2018_FINAL.pdf

Flecha, R. (2018): A Note on Key Concepts for Social Impact of Research Assessment. https://archive.org/details/ANoteOnKeyConceptsForSociallmpactOfResearchAssessment

Fornahl, D., Zellner C. und Audretsch, D.B. (Hg.) (2005): The Role of Labour Mobility and Informal Networks for Knowledge Transfer, Springer.

Forray, D., Mowery, D.C. und Nelson, R. (2012): Public R\&D and Social Challenges: What Lessons from Mission R\&D Programs, Research Policy, 41/10, 163-183.

Frauenhofer und KMU Forschung Austria (2017): Evaluierung der Förderungsgesellschaften Austria Wirtschaftsservice $\mathrm{GmbH}$ (aws) und Forschungsförderungsgesellschaft $\mathrm{mbH}$ (FFG), Wien.

Frietsch, R. Rammer, Ch., Astor, M., Berger, M., Daimer, St., Hud, M., Klaus, C., Lerch, Ch., Limbers, J. und Neuhäusler, P. (2019): Schrittweise Erhöhung der FuE-Quote auf bis zu 3,5\% des BIP - Instrumente und Auswirkungen auf volkswirtschaftliche Kennzahlen, Abschlussbericht Juni 2017, Aktualisierung: Januar 2019, Berlin.

FWF (2018): FWF-Strategie zu Gleichstellung und Diversität von Forscherinnen und Forschern (2019-2020). https:// fwf.ac.at/fileadmin/files/Dokumente/Ueber_den_FWF/ Publikationen/FWF-relevante_Publikationen/FWF_Strategie_Gender_Diversitaet_2018.pdf

Gassler, H., Polt, W., Rammer, Ch. (2006): Schwerpunktsetzungen in der Forschungs- und Technologiepolitik - eine Analyse der Paradigmenwechsel seit 1945, Österreichische Zeitschrift für Politikwissenschaft (ÖZP), 35. Jg., H. 1, 7-23.

Gesetz vom 6. März 1906, über Gesellschaften mit beschränkter Haftung (GmbH-Gesetz - GmbHG), BGBI. I Nr. 71/2018.

Gewerbeordnung 1994 - GewO 1994, BGBI. I Nr. 112/2018.

Geyer, A. und Good, B. (2018): Evaluierung des Impulsprogramms FIT-IT und der Themeninitiative "IKT der Zukunft", Endbericht, Wien. https://repository.fteval.at/364/1/ EVAL_BMVIT-IKT-Bericht_180824_final.pdf

Gisler, M., Günter, M. und Spahni, D. (2001): eGovernment, Proceedings of the 34th Annual Hawaii International Conference on System Sciences (HICSS-34), IEEE Computer Society, Volume 5, 5002.

Handler, R., Jud, Th. und S. Kupsa (2019): Global Incubator network - GIN, Bericht zur Zwischenevaluierung, Wien. https://repository.fteval.at/374/1/GINEval_Endbericht_gesamt.pdf
Harvard University (Hg.) (2018): The Atlas of Economic Complexity: Global Rankings \& Projections. http://atlas.cid. harvard.edu/rankings/

Heigl, F., Dörler, D., Bartar, P., Brodschneider, R., Cieslinski, M., Ernst, M. und Ziegler, D. (2018): Qualitätskriterien für Citizen Science auf Österreich forscht (Version 1.0), Zenodo.

Institut für Innovation und Technik (iit) (2018): Innovationsfähigkeitsindikator: Länderanalyse. https://www.iit-berlin.de/ de/indikator/laenderanalyse

Janger, J., Firgo, M., Hofmann, K., Kügler, A., Strauss, A., Streicher, G. und Pechar, H. (2017a): Wirtschaftliche und gesellschaftliche Effekte von Universitäten, Wien.

Janger, J., Schubert, T., Andries, P., Rammer, C. und Hoskens, M. (2017b): The EU 2020 innovation indicator: A step forward in measuring innovation outputs and outcomes? Research Policy, 46(1), 30-42. https://doi.org/10.1016/j.respol.2016.10.001

Jud, Th., Handler, R., Kupsa, S. und Pohn-Weidinger, S. (2017): Evaluierung der Innovationsscheck-Programme, Wien-Graz. https://www.bmvit.gv.at/innovation/publikationen/evaluierungen/downloads/evaluierung_innovationsscheck_programme.pdf

Jud, Th., Kupsa, S., Handler, R., Geyer, A. und Good, B. (2019): Zwischenevaluierung der FTI-Initiative „Produktion der Zukunft", vorläufiger Endbericht, Wien.

Keuschnigg, C. und Sardadvar, S. (2019): Mobilisierung und Einsatz von Wagniskapital im Rahmen von Innovations- und Wachstumsfinanzierung, Studie im Auftrag von RFTE und AVCO, Wien.

Knappik, M. (2013): Wege zur wissenschaftlichen Textkompetenz - Schreiben für reflexive Professionalisierung: Ein förderdiagnostisches Instrument zur Unterstützung von Studierenden bei der Aneignung wissenschaftlicher Textkompetenz, Wien. https://bildung.bmbwf.gv.at/schulen/unterricht/ba/sprachen_sfrp_broschuere_26215. pdf?4dzgm2

Kolm, B., Schneider H. W., Brunner, Ph., Lengauer, S. D. und Welbich, S. (2018a): Branchenspezifische Impactanalyse des ERP-Kreditprogammes auf Unternehmensebene, Abschlussbericht, Teil 1, Wien.

Kolm, B., Schneider H. W., Brunner, Ph., Luptacik, P. und Welbich, S. (2018b): Qualitative Untersuchung und gesamtwirtschaftliche Impactanalyse des ERP-Kreditprogrammes, Abschlussbericht, Teil 2, Wien.

Kornberg, R., Ball, J., Eichler, R., Liskov, B., Neher, E. und Schekman, R. (2015): Evaluierung des Institute of Science and Technology Austria (IST Austria), Klosterneuburg. https://ist.ac.at/fileadmin/user_upload/pdfs/Basic_documents/2015_EvalReport_IST_Austria.pdf

Kratz, F. und Netz, N. (2018): Which mechanisms explain monetary returns to international student mobility? Studies in Higher Education; 43(2), 375-400.

Kuhlmann, S. und Rip, A. (2014): The Challenge of Addressing Grand Challenges, Brussels: EU Commission. 
Lambert, R., Masson, A., Mogenson, J., Sandberg, O., van der Zwan, A., Jahn, S. und Guy, K. (2008): Policy Mix Peer Reviews: Country Report Austria on the design and implementation of national policy mixes. https://bmbwf.gv.at/ fileadmin/user_upload/forschung/forschungsdialog/ CREST_Austrian_Policy_Mix_Report_-_September_2008. $\underline{\mathrm{pdf}}$

Lasinger, D., Stampfer, M., Strassnig, M., Bartik, H., Hofinger, J. und Lutter, J. (2019): Motive für die Ansiedlung von F\&EEinheiten in Wien: Ein Vergleich von ausgewählten Standorten mit Wien. https://www.wien.gv.at/wirtschaft/standort/pdf/fe-ansiedlung.pdf

Leitner, K-H., Zahradnik, G., Dömötör, R., Raunig, M., Pardy, M. und Mattheiss, E. (2018): Austrian Startup Monitor 2018, Wien. http://austrianstartupmonitor.at/wp-content/uploads/2018/10/Austrian-StartUpMonitor2018_12MB.pdf

Manahl, C., Wagner, I. und Schuch, K. (2016): Evaluierung des wissenschaftlichen Impacts von „Sparkling Science“, Wien. https://repository.fteval.at/206/1/2016_Evaluierung\%20 des\%20wissenschaftlichen\%20Impacts\%20von\%20 \%E2\%80\%9ESparkling\%20Science\%E2\%80\%9C.pdf

Mazzucato, M. (2011): The Entrepreneurial State, Soundings, 49/49, 131-142.

Mazzucato, M. (2018): Mission-Oriented Research \& Innovation in the European Union: A problem-solving approach to fuel innovation-led growth, Europäische Kommission, Brüssel. https://ec.europa.eu/info/sites/info/files/mazzucato_report_2018.pdf

Mitterauer, B. und Birke, B. (2009): Bericht zur Evaluation des Förderprogramms Sparkling Science, Wien. https://www. aq.ac.at/de/analysen-berichte/dokumente-analysenberichte/Endbericht-Sparkling-Science--2009-11-04. pdf?m=1458205145

OECD (2009): PIAAC Problem Solving in Technology-Rich Environments: A Conceptual Framework, OECD Education Working Papers No. 36. https://doi.org/10.1787/19939019

OECD (2010): OECD Science, Technology and Industry Outlook 2010, OECD Publishing, Paris. https://doi.org/10.1787/ sti_outlook-2010-en

OECD (2014): Recruiting Immigrant Workers: Austria 2014, Recruiting Immigrant Workers, OECD Publishing, Paris. https://doi.org/10.1787/9789264226050-en

OECD (2015): Frascati Manual 2015: Guidelines for Collecting and Reporting Data on Research and Experimental Development, The Measurement of Scientific, Technological and Innovation Activities, OECD Publishing, Paris. https://doi. org/10.1787/9789264239012-en

OECD (2017): Digital platforms for facilitating access to research infrastructures, OECD Science, Technology and Industry Policy Papers, No. 49, OECD Publishing, Paris. https://doi.org/10.1787/8288d208-en

OECD (2018a): OECD Reviews of Innovation Policy: Austria 2018, OECD Publishing, Paris. https://doi. org/10.1787/9789264309470-en

OECD (2018b): Main Science and Technology Indicators. http:// www.oecd.org/sti/msti.htm
Österreichische Hochschulkonferenz (2015): Empfehlung der Hochschulkonferenz zur qualitativen Weiterentwicklung der Doktoratsausbildung in Österreich. http://www.hochschulplan.at/wp-content/uploads/2015/07/2015-06-12 HSK-Empfehlung-Doktoratsausbildung.pdf

Österreichische Universitätenkonferenz (2015): Positionspapier der Österreichischen Universitätenkonferenz zum Doktorat, Wien. https://uniko.ac.at/modules/download. php?key=10897_DE_O\&cs=3D3C

Österreichischer Rat für Robotik und Künstliche Intelligenz (2018): Die Zukunft Österreichs mit Robotik und Künstlicher Intelligenz positiv gestalten, White Paper des Österreichischen Rats für Robotik und Künstliche Intelligenz, Wien.

Österreichischer Wissenschaftsrat (2018): Ein Exzellenzprogramm für Österreich: Stellungnahme und Empfehlungen, Wien. https://www.wissenschaftsrat.ac.at/downloads/ Endversion_OEWR_Ein_Exzellenzprogramm_fuer_Oesterreich.pdf

Pichler, R., Stampfer, M. und Hofer, R. (2007): Forschung, Geld und Politik: Die staatliche Forschungsförderung in Österreich 1945-2005, Studienverlag: Innsbruck, Wien und Bozen.

Rat der Europäischen Union (2019): Proposal for a Regulation of the European Parliament and of the Council establishing Horizon Europe - the Framework Programme for Research and Innovation, laying down its rules for participation and dissemination - Common understanding, Brüssel.

Raua, H., Gogginsb, G. und Fahyb, F. (2018): From invisibility to impact: Recognising the scientific and societal relevance of interdisciplinary sustainability research, Research Policy, 47, 266-276. 10.1016/j.respol.2017.11.005

Reale, E., Avramov, D., Canhial, K., Donovan, C., Flecha, R., Holm, P. und Van Horik, R. (2017): A review of literature on evaluating the scientific, social and political impact of social sciences and humanities research, Research Evaluation, January, 1-11. 10.1093/reseval/rvx025

Rechnungshof (2018): Entwicklung ausgewählter Forschungsprogramme des Bundes, Bericht des Rechnungshofes, Bund 2018/12, Wien. https://www.rechnungshof.gv.at/rh/ home/home/Entwicklung_ausgew._Forschungsprogramme_Bundes.indd.pdf

RFTE (2009): Strategie 2020, Wien. https://www.rat-fte.at/ files/rat-fte-pdf/090824_FINALE\%20VERSION_FTI-Strategie2020.pdf

RFTE (2015): Lange Nacht der Forschung 2008 bis 2014: Geschichte, Analyse, Potenziale, Wien. https://www.ratfte.at/files/rat-fte-pdf/publikationen/2016/11092015 LNF\%20lmactanalyse.pdf

RFTE (2018a): Bericht zur wissenschaftlichen und technologischen Leistungsfähigkeit Österreichs 2018, Wien. https:// www.rat-fte.at/files/rat-fte-pdf/leistungsberichte/Leistungsbericht\%202018.pdf

RFTE (2018b): Empfehlung zur Einrichtung einer österreichweiten Datenbank zur Darstellung des Forschungsinputs und -outputs, Wien. https://www.rat-fte.at/files/rat-ftepdf/einzelempfehlungen/2018/180322_Empfehlung_Forschungsfoerderungsdatenbank_final.pdf 
RFTE (2018c): Exzellenzprogramm zur Förderung der Wissenschaft als Schlüssel für mehr Kooperation und Wettbewerb in der Grundlagen- und Spitzenforschung, Wien. https://www.rat-fte.at/files/rat-fte-pdf/einzelempfehlun$\overline{\text { gen/2018/180322_Empfehlung_Exzellenzprogramm_final. }}$ pdf

Royal Society (1985): The Public Understanding of Science, London. https://royalsociety.org/topics-policy/publications/1985/public-understanding-science/

Ruhland, S., Kaufmann, J. und Rastegar, K. (2017): Analyse der Rahmenbedingungen, Hemmnisse und Hindernisse für innovative Unternehmensgründungen in Österreich, Wien.

Ruhland, S., Kaufmann, J., Ploder, M. und Zinke, G. (2018): Evaluierung der Umsetzung des Leitkonzepts für eine innovationsfördernde öffentliche Beschaffung in Österreich, Wien. https://www.ioeb.at/fileadmin/ioeb/Dokumente/IOEB_ allgemein/IOEB-Evaluierung_Endbericht-Langfassung_____. pdf

Sardadvar, S. (2018): Is a company's access to private equity and venture capital affected by location or management diversity? Some empirical evidence from Austria, International Journal of Business and Management, 13(7), 45-57.

Schiefer, A. (2017): Forschung und experimentelle Entwicklung (F\&E) im Unternehmenssektor 2015 - Teil 1, Statistische Nachrichten, 10/2017, 883-909.

Schuch, K. und Testa, G. (2018): RIO Country Report 2017: Austria, Research and Innovation Observatory country report series, Publications Office of the European Union: Luxembourg.

Science Europe (2018): Practical Guide to the International Alignment of Research Data Management. https://www. scienceeurope.org/wp-content/uploads/2018/12/SE_ RDM_Practical_Guide_Final.pdf

Scopus (Hg.): Scimago Journal \& Country Rank. https://www. scimagojr.com/

Shapiro, C. (2002): Competition Policy and Innovation, OECD Science, Technology and Industry Working Papers, 2002/11, OECD Publishing, Paris. https://www.oecd-ilibrary.org/ science-and-technology/competition-policy-and-innovation_037574528284

Statistik Austria (2015): Erhebung über Forschung und experimentelle Entwicklung 2009-2015, Wien.

Statistik Austria (2018): Kindertagesheimstatistik, Wien.

Statistik Austria (2019): IKT-Einsatz in Haushalten 2018, Wien.

Steger, G. (2012): Making Public Finance Management Systems gender responsive: The GRB Experience in Austria, United Nations Commission on the Status of Women, Fifty-sixth session, 27 February - 9 March 2012, New York. https://www.bmf.gv.at/budget/haushaltsrechtsreform/ United_Nations_Commission_on_the_Status_of_WomenGerhard-_2.pdf?67ruij

Sturn, D., Dall, E., Degelsegger-Márquez, A., Lampert, D. und Schuch, K. (2018): Zwischenevaluierung des Programms „Beyond Europe“, Wien. https://www.zsi.at/de/object/ publication/5158

Tiefenthaler, B und Warta, K. (2016): Begleitende Analyse der Citizen-Science-Pilotprojekte im Rahmen des Programms Sparkling Science, Wien.

Tiefenthaler, B. (2018): Analyse der institutionellen Wirkungen von Sparkling Science, Wien.
Tinbergen, J. (1974): Substitution of graduate by other labor, Kyklos 27, 217-226.

Tinbergen, J. (1975): Income Difference: Recent Research, NorthHolland Publishing Company, Amsterdam.

Tsipouri, L., und Sidiropolous, N. (2013): Public procurement in SEE innovation evaluations: a comparative and needs assessment study, Journal for Research and Technology Policy Evaluation, 38, 6-19.

United Nations (2015): Transforming our world: the 2030 Agenda for Sustainable Development: Resolution adopted by the General Assembly on 25.09.2015, A/RES/70/1.

Van den Besselaar, P., Flecha, R. und Radauer, A. (2018): Monitoring the Impact of EU Framework Programmes, Expert Report, Publications Office of the European Union: Luxembourg.

Verein Industrie 4.0 Österreich - die Plattform für intelligente Produktion (2018): Ergebnispapier Forschung, Entwicklung \& Innovation in der Industrie 4.0, Wien.

Warta, K., Dudenbostel, Gassler, H., Rammer, C. und Köhler, M. (2019): Evaluierung der Frontrunner-Initiative, Wien.

Weber, K.M. und Rohracher, H. (2012): Legitimizing Research, Technology and Innovation Policies for Transformative Change, Research Policy, 41(6), 1037-1047.

Weltwirtschaftsforum (2017): The Global Competitiveness Report 2017-2018, Cologny/Geneva: World Economic Forum (Insight Report).

Weltwirtschaftsforum und A. T. Kearney Incorporated (2018): Readiness for the future of production report 2018, Cologny/Geneva: World Economic Forum (Insight report). https://www.weforum.org/reports/readiness-for-the-futureof-production-report-2018

Wisbauer, A. und Fuchs, R. (2014): Außenwanderungen 2012, Statistische Nachrichten, 3/2014, Wien. http://www.statistik.at/web_de/static/aussenwanderungen_2012_statistische_nachrichten_heft_32014____076135.pdf 
8.1 Länderkürzel

\begin{tabular}{|c|c|c|c|c|c|}
\hline Land & Kürzel & Land & Kürzel & Land & Kürzel \\
\hline Albanien & $\mathrm{AL}$ & Frankreich & $\mathrm{FR}$ & Nigeria & NG \\
\hline Argentinien & $A R$ & Hongkong & HK & Niederlande & $\mathrm{NL}$ \\
\hline Österreich & AT & Kroatien & $\mathrm{HR}$ & Norwegen & NO \\
\hline Australien & $\mathrm{AU}$ & Ungarn & $\mathrm{HU}$ & Neuseeland & $\mathrm{NZ}$ \\
\hline Belgien & $\mathrm{BE}$ & Irland & $\mathrm{IE}$ & Polen & PL \\
\hline Bulgarien & BG & Indien & IN & Portugal & PT \\
\hline Brasilien & BR & Israel & IL & Rumänien & RO \\
\hline Kanada & $\mathrm{CA}$ & Island & IS & Serbien & RS \\
\hline Schweiz & $\mathrm{CH}$ & Italien & IT & Russland & RU \\
\hline Chile & $\mathrm{CL}$ & Japan & $J P$ & Schweden & SE \\
\hline China & $\mathrm{CN}$ & Südkorea & $\mathrm{KR}$ & Singapur & SG \\
\hline Zypern & $\mathrm{CY}$ & Liechtenstein & $\mathrm{LI}$ & Slowenien & $\mathrm{SI}$ \\
\hline Tschechische Republik & $\mathrm{CZ}$ & Litauen & LT & Slowakei & SK \\
\hline Deutschland & $\mathrm{DE}$ & Luxemburg & LU & Türkei & TR \\
\hline Dänemark & DK & Lettland & LV & Taiwan & TW \\
\hline Estland & $\mathrm{EE}$ & Montenegro & ME & Ukraine & UA \\
\hline Griechenland & EL & Mazedonien & MK & Vereinigtes Königreich & UK \\
\hline Spanien & ES & Malta & MT & Vereinigte Staaten von Amerika & US \\
\hline Finnland & $\mathrm{FI}$ & Mexiko & $M X$ & Südafrika & ZA \\
\hline
\end{tabular}

\subsection{Abkürzungsverzeichnis}

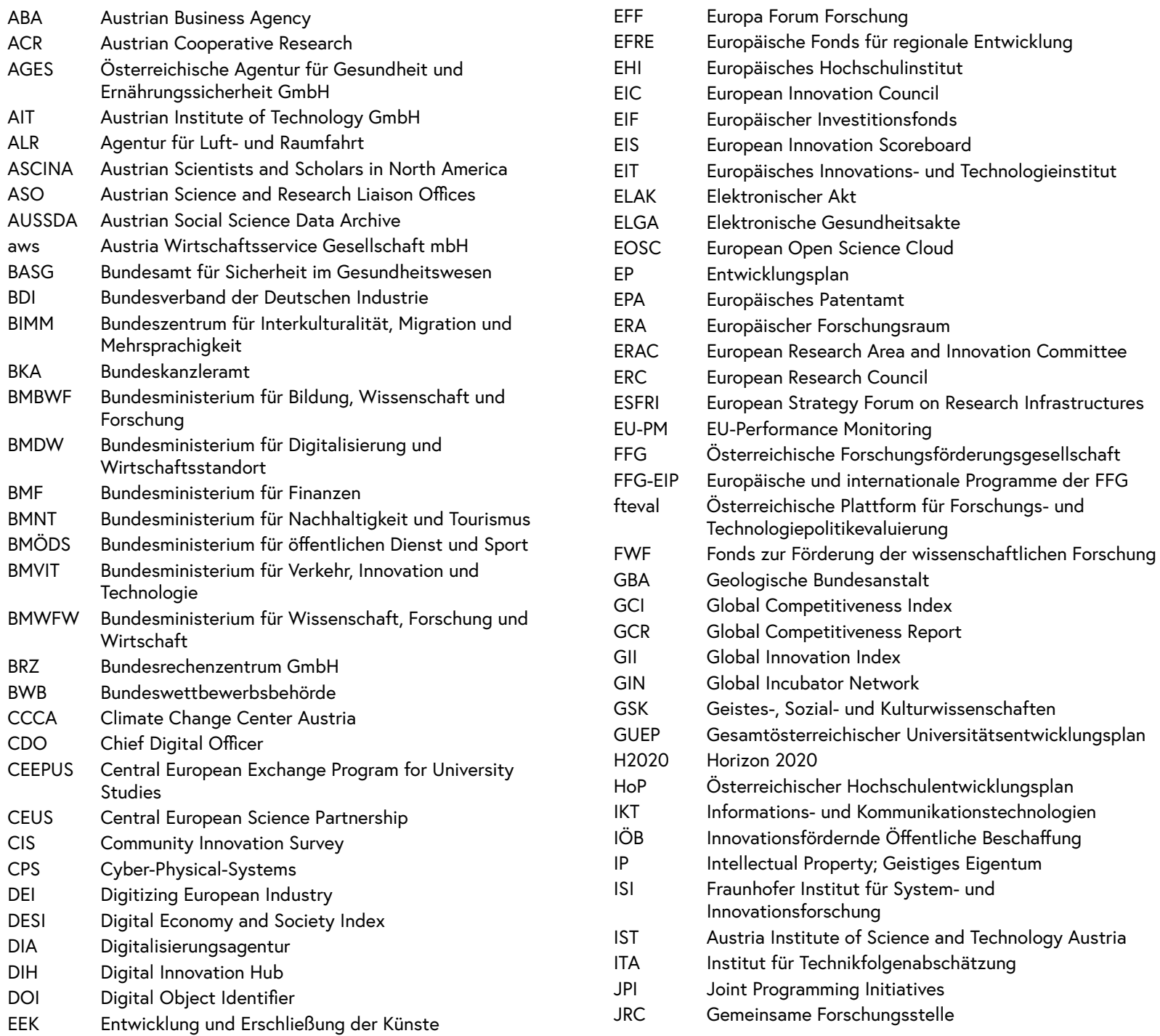


KEF

Künstliche Intelligenz

LBG Ludwig Boltzmann Gesellschaft - Österreichische Vereinigung zur Förderung der wissenschaftlichen Forschung

LISA Life Science Austria GmbH

LNF Lange Nacht der Forschung

LV Leistungsvereinbarung

MdZ Mobilität der Zukunft

MSCA Marie-Skłodowska-Curie-Maßnahmen

NARIC National Academic Recognition Information Centre

NCP National Contact Point, Nationale Kontaktstelle

NCP-IP Nationale Kontaktstelle für Wissenstransfer und Geistige Eigentumsrechte

NFTE Nationalstiftung für Forschung, Technologie und Entwicklung

OA Open Access

OANA Open Access Network Austria

ÖAW Österreichische Akademie der Wissenschaften

ÖAWI Österreichischen Agentur für Wissenschaftliche Integrität

OeAD Österreichische Austauschdienst $\mathrm{GmbH}$

OECD Organisation für wirtschaftliche Zusammenarbeit und Entwicklung

ÖGMBT Österrreichische Gesellschaft für Molekulare Biowissenschaften und Biotechnologie

Ol-Strategie Open Innovation Strategie
ÖPA Österreichisches Patentamt

ÖPPM Österreichische Plattform für Personalisierte Medizin

OSTA Offices of Science and Technology Austria

PGA Partial General Approach, partielle allgemeine Ausrichtung

QIBB Qualitätsinitiative Berufsbildung

RFTE Rat für Forschung und Technologieentwicklung

RINA Research and Innovation Network Austria

SAL Silicon Austria Labs $\mathrm{GmbH}$

SDG Sustainable Development Goal

SIC CORDIS Subject Index Classification Codes

SQA Qualitätsinitiative Schulqualität Allgemeinbildung

TRC Translational Research Center

UNIKO Österreichische Universitätenkonferenz

USP Unternehmensserviceportal

VBCF Vienna Biocenter Core Facilities $\mathrm{GmbH}$

$\mathrm{VCl}$ 256WEF Weltwirtschaftsforum

WFA Wirkungsorientierte Folgenabschätzung

w-fFORTE Wirtschaftsimpulse von Frauen in Forschung und Technologie

WIPO Weltorganisation für geistiges Eigentum

WTZ Wissenstransferzentrum

ZAMG Zentralanstalt für Meteorologie und Geodynamik

ZEW Leibniz-Zentrum für Europäische Wirtschaftsforschung

GmbH Mannheim

ZH Zukunft Hochschule

ZSI Zentrum für Soziale Innovation $\mathrm{GmbH}$

\subsection{Aktivitäten im Rahmen der EU-Ratspräsidentschaft}

\section{Tabelle 8-1: Treffen auf Ministerebene}

\begin{tabular}{l|l}
\hline Datum & Veranstaltung \\
\hline 10.07.2018 & $\begin{array}{l}\text { Anhörung von Minister Faßmann im Ausschuss für Industrie, Forschung und Energie (ITRE) des Europäischen Parla- } \\
\text { ments }\end{array}$ \\
\hline 16.-17.07.2018 & $\begin{array}{l}\text { Informelles Treffen der Forschungsminister in Wien und erster Meinungsaustausch zu Horizon Europe-Vorschlag auf } \\
\text { politischer Ebene }\end{array}$ \\
\hline 27.-28.09.2018 & Sitzung des Rates für Wettbewerbsfähigkeit in Brüssel und Verhandlungen über den Vorschlag zu Horizon Europe \\
\hline 30.11.2018 & $\begin{array}{l}\text { Sitzung des Rates für Wettbewerbsfähigkeit in Brüssel und Verhandlungen über den Horizon Europe-Vorschlag, die } \\
\text { zur Annahme des „partiellen allgemeinen Ansatzes“ sowie der Schlussfolgerungen des Rates zum Europäischen For- } \\
\text { schungsraum geführt haben }\end{array}$
\end{tabular}

Tabelle 8-2: Veranstaltungen der österreichischen Präsidentschaft mit Fokus auf „Horizon Europe“

\begin{tabular}{|c|c|c|}
\hline Datum & Veranstaltung & Ergebnis \\
\hline 17.07.2018 & Informal Meeting of Research Ministers & Outcome Informal COMPET Research \\
\hline 13.09.2018 & FFG FORUM 2018 & Outcome of FFG Forum 2018 \\
\hline 17.-18.09.2018 & ERAC Plenary/Working Party on Research Meeting & $\begin{array}{l}\text { Outcome of ERAC Plenary/Working Party on Research } \\
\text { Meeting }\end{array}$ \\
\hline 01.-02.10.2018 & Marie Skłodowska-Curie Actions beyond 2020 & Outcome of MSCA Conference \\
\hline 29.10 .2018 & Quantum Flagship Kick-Off Conference & Quantum Flagship Conference - Outcome \\
\hline 30.-31.10.2018 & $\begin{array}{l}\text { Industrial Technologies } 2018 \text { - innovative industries for } \\
\text { smart growth }\end{array}$ & IND-TECH Conference Outcome \\
\hline 06. -08.11 .2018 & COST CSO-Meeting \& Awareness Day & COST event - outcome \\
\hline 21.-22.11.2018 & Innovative Enterprise Vienna 2018 & Innovative Enterprise 2018 - Outcome \\
\hline 28.-29.11.2018 & $\begin{array}{l}\text { Impact of Social Sciences and Humanities for a European } \\
\text { Research Agenda }\end{array}$ & Impact of SSH conference - Outcome \\
\hline 04.-06.12.2018 & Imagine Digital - Connect Europe (ICT 2018) & \\
\hline
\end{tabular}


Tabelle 8-3: Veranstaltungen des österreichischen Ratsvorsitzes mit Fokus auf die Governance des ERA

\begin{tabular}{|c|c|c|}
\hline Datum & Veranstaltung & Ergebnis \\
\hline 10.09.2018 & ESFRI Meeting & \\
\hline 11.09.2018 & ESFRI Roadmap Meeting & Outcome of ESFRI Roadmap Meeting \\
\hline 10.-14.09.2018 & $\begin{array}{l}\text { ICRI } 2018 \text { (International Conference on Research Infra- } \\
\text { structures) }\end{array}$ & Outcome of ICRI 2018 \\
\hline 17.-18.09.2018 & ERAC Plenary/RWP Meeting & Outcome of ERAC Plenary/RWP Meeting \\
\hline 19.-20.09.2018 & $\begin{array}{l}10 \text { Years Joint Programming - Achievements and the Way } \\
\text { Forward }\end{array}$ & Outcome of 10 Years Joint Programming event \\
\hline 22.10 .2018 & The Role of Competitive Research Funding in Science & Outcome of Competitive Research Funding Conference \\
\hline 30.10 .2018 & $\begin{array}{l}\text { The European Open Science Cloud (EOSC): Austria takes } \\
\text { the initiative }\end{array}$ & EOSC Conference Outcome - presentations \\
\hline 05.-06.11.2018 & $\begin{array}{l}\text { Impact of R\&I Policy at the Crossroads of Policy Design, } \\
\text { Implementation and Evaluation }\end{array}$ & Impact of R\&I Policy Conference Outcome \\
\hline 23.11.2018 & $\begin{array}{l}\text { Launch of the European Open Science Cloud (EOSC) } \\
\text { Governance Structure }\end{array}$ & EOSC Launch event - Outcome \\
\hline \multicolumn{3}{|l|}{ Höhere Bildung } \\
\hline 20.-21.09.2018 & $\begin{array}{l}\text { The New Student: Flexible learning paths and future } \\
\text { learning environments }\end{array}$ & Outcome of the conference \\
\hline 27.-28.09.2018 & Meeting of the Bologna Follow-Up Group & Outcome of BFUG meeting \\
\hline 15.-17.10.2018 & Asia-Europe Meeting/Senior Officials' Meeting & ASEM Meeting - Outcome \\
\hline 18.-19.10.2018 & Meeting of DGs for Higher Education & Meeting of DGs for Higher Education - Outcome \\
\hline 15.-17.11.2018 & 13th European Quality Assurance Forum 2018 & European Quality Assurance Forum - Outcome \\
\hline
\end{tabular}

Tabelle 8-4: FTI-Veranstaltungen der österreichischen Präsidentschaft mit Fokus auf sektorale Politiken

\begin{tabular}{|c|c|c|}
\hline Datum & Veranstaltung & Ergebnis \\
\hline 11.-12.07.2018 & High Level Group Meeting on Education and Training & Outcome of HLG Meeting \\
\hline 04.-06.07.2018 & WIRE 2018 - 9th Week of Innovative Regions in Europe & Outcome WIRE 2018 \\
\hline 05.-07.09.2018 & IIASA-JRC Evidence for Policy Summer School 2018 & Outcome of IIASA-JRC Summer School \\
\hline 08.-12.09.2018 & BE OPEN - Science \& Society Festival & Outcome of BE OPEN \\
\hline 28.09.2018 & $\begin{array}{l}\text { Human Biomonitoring in Europe - science and policy for } \\
\text { healthy citizens }\end{array}$ & Outcome of Human Biomonitoring in Europe conference \\
\hline 02.10 .2018 & $\begin{array}{l}\text { High Level EU Conference: "The European Defence Fund - } \\
\text { Driving Factor for Defence Research and Innovation" }\end{array}$ & Outcome of EDF Conference \\
\hline 03.-05.10.2018 & International Sustainable Energy Conference 2018 & Outcome of ISEC 2018 \\
\hline 04.10 .2018 & $\begin{array}{l}\text { European Creative Industries Summit 2018: BEYOND I } \\
\text { Cross-Innovation as Driver for Growth in the European } \\
\text { Digital Single Market }\end{array}$ & $\begin{array}{l}\text { "Vienna Declaration on Cross-Innovation for an Inclusive } \\
\text { and Creative Society" }\end{array}$ \\
\hline 11.-12.10.2018 & Risk Data Hub \& Austrian Disaster Network Days & Outcome of Austrian Disaster Network Days \\
\hline 23.-25.10.2018 & Enterprise Europe Network Annual Conference & Outcome of EEN Conference \\
\hline 05.-06.11.2018 & Space Conference & \\
\hline 12.-14.11.2018 & European Big Data Value Forum & Outcome of European Big Data Value Forum \\
\hline 13.11.2018 & $\begin{array}{l}\text { Smart specialisation (RIS3): European workshop on univer- } \\
\text { sities as regional lead institutions }\end{array}$ & Outcome of RIS3 Workshop on Universities \\
\hline 14.-15.11.2018 & Europe's Transformation: Where People Matter & Outcome of „Europe's Transformation“ event \\
\hline 20.-21.11.2018 & SET Plan Conference & SET Plan Conference - Outcome \\
\hline 26.-27.11.2018 & ECOVATION 2018 & ECOVATION 2018 - Outcome \\
\hline 26.-27.11.2018 & The Future of Mobility in European Cities (Urban Mobility) & Future of Mobility - Outcome \\
\hline $05 .-06.12 .2018$ & Security Research Event (SRE) 2018 & SRE - Outcome \\
\hline 06.-07.12.2018 & Informal Meeting of the Education Committee & \\
\hline
\end{tabular}




\subsection{Open-Innovation-Maßnahmen und Beispiele für dazugehörige Umsetzungsinitiativen}

\begin{tabular}{|c|c|c|c|c|c|c|c|}
\hline & & & & \\
\hline & Maßnahme 1 & Maßnahme 2 & Maßnahme 3 & Maßnahme 4 & Maßnahme 5 & Maßnahme 6 \\
\hline & & $\begin{array}{l}\text { Errichtung von } \\
\text { offenen Innova- } \\
\text { tions- und Experi- } \\
\text { mentierräumen }\end{array}$ & $\begin{array}{l}\text { Verankerung von } \\
\text { Open Innovaton- } \\
\text { Elementen in } \\
\text { Kindergärten und } \\
\text { Schulen sowie } \\
\text { in der Aus- und } \\
\text { Weiterbildung von } \\
\text { Pädagoginnen und } \\
\text { Pädagogen }\end{array}$ & $\begin{array}{l}\text { Weiterentwicklung } \\
\text { der öffentlichen } \\
\text { Verwaltung mittels } \\
\text { Open Innovation } \\
\text { und stärkerer } \\
\text { Einbindung von } \\
\text { Bürgerinnen und } \\
\text { Bürgern }\end{array}$ & $\begin{array}{l}\text { Aufbau und Betrieb } \\
\text { einer Open Inno- } \\
\text { vation-Plattform } \\
\text { für soziale/gesell- } \\
\text { schaftliche Innova- } \\
\text { tion und als Beitrag } \\
\text { zur Lösung globaler } \\
\text { Herausforderungen }\end{array}$ & $\begin{array}{l}\text { Aufbau und Betrieb } \\
\text { einer Innovations- } \\
\text { landkarte samt } \\
\text { Matchmaking-Platt- } \\
\text { form für Innovati- } \\
\text { onsakteurinnen und } \\
\text {-akteure }\end{array}$ & $\begin{array}{l}\text { Aufbau von For- } \\
\text { schungskompetenz } \\
\text { für die Anwendung } \\
\text { von Open Innova- } \\
\text { tion in der Wissen- } \\
\text { schaft }\end{array}$ \\
\hline $\begin{array}{l}\text { Handlungs- } \\
\text { feld } 1\end{array}$ & $\begin{array}{l}\text { Aufbau einer Kultur } \\
\text { für Open Innovation } \\
\text { und Vermittlung von } \\
\text { Open Innovation- } \\
\text { Kompetenzen in } \\
\text { allen Altersgruppen }\end{array}$ & & $\begin{array}{l}\text { BMVIT - Massive } \\
\text { Open Online Cour- } \\
\text { ses „Smart Cities“ } \\
\text { FFG, BMVIT - Talen- } \\
\text { te Regional } \\
\text { BMBWF - MINT-3D- } \\
\text { Druck }\end{array}$ & $\begin{array}{l}\text { BMVIT - Offene } \\
\text { Konsultationen im } \\
\text { Zuge der Erarbei- } \\
\text { tung der Energiefor- } \\
\text { schungsstrategie }\end{array}$ & & & $\begin{array}{l}\text { LBG - Open Inno- } \\
\text { vation in Science } \\
\text { Research and } \\
\text { Competence Center } \\
\text { (OIS) }\end{array}$ \\
\hline $\begin{array}{l}\text { Handlungs- } \\
\text { feld } 3\end{array}$ & $\begin{array}{l}\text { Mobilisierung von } \\
\text { Ressourcen und } \\
\text { Schaffung von Rah- } \\
\text { menbedingungen } \\
\text { für Open Innovation }\end{array}$ & $\begin{array}{l}\text { ÖBB - Open Innova- } \\
\text { tion Lab \& Service } \\
\text { Design Center } \\
\text { FFG, BMVIT - Inno- } \\
\text { vationswerkstätten } \\
\text { \& Innovationslabore } \\
\text { FFG - Bildungs- } \\
\text { LABs }\end{array}$ & $\begin{array}{l}\text { FFG - Bildungs- } \\
\text { LABs }\end{array}$ & $\begin{array}{l}\text { BBG - Projekt } \\
\text { Ecolnnovation }\end{array}$ & & $\begin{array}{l}\text { BMVIT - Open4In- } \\
\text { novation-Plattform }\end{array}$ & \\
\hline
\end{tabular}




\begin{tabular}{|c|c|c|c|c|c|c|c|}
\hline Maßnahme 7 & Maßnahme 8 & Maßnahme 9 & Maßnahme 10 & Maßnahme 11 & Maßnahme 12 & Maßnahme 13 & Maßnahme 14 \\
\hline \multirow[t]{2}{*}{$\begin{array}{l}\text { Verankerung } \\
\text { von Anreizme- } \\
\text { chanismen für } \\
\text { Forschungs- } \\
\text { partnerschaften } \\
\text { mit unüblichen } \\
\text { Akteurinnen und } \\
\text { Akteuren in der } \\
\text { wissenschaftlichen } \\
\text { Forschungsförde- } \\
\text { rung zur Stärkung } \\
\text { von Open Inno- } \\
\text { vation }\end{array}$} & $\begin{array}{l}\text { Verstärkte Einbin- } \\
\text { dung von Usern } \\
\text { und Bürgerinnen } \\
\text { und Bürgern in FTI- } \\
\text { Förderprogrammen }\end{array}$ & $\begin{array}{l}\text { Entwicklung von } \\
\text { fairen Sharing- und } \\
\text { Abgeltungsmodel- } \\
\text { len für Crowdwork }\end{array}$ & $\begin{array}{l}\text { Weiterentwicklung } \\
\text { und Zurverfü- } \\
\text { gungstellung von } \\
\text { Open Innovation- } \\
\text { Methoden und } \\
\text {-Instrumenten } \\
\text { spezifisch für } \\
\text { Klein- und Mittel- } \\
\text { betriebe (KMU) }\end{array}$ & $\begin{array}{l}\text { Entwicklung und } \\
\text { Durchführung von } \\
\text { Co-Creation und } \\
\text { Open Innovation- } \\
\text { Trainingsprogram- } \\
\text { men für Interes- } \\
\text { sierte }\end{array}$ & $\begin{array}{l}\text { Verankerung von } \\
\text { Open Data- und } \\
\text { Open Access- } \\
\text { Prinzipien in der } \\
\text { Forschung }\end{array}$ & $\begin{array}{l}\text { Ausrichtung der } \\
\text { IP- und Verwer- } \\
\text { tungsstrategien } \\
\text { von Unternehmen, } \\
\text { Hochschulen, } \\
\text { Forschungsein- } \\
\text { richtungen und } \\
\text { Intermediären an } \\
\text { Open Innovation } \\
\text { zur Optimierung } \\
\text { des Innovationspo- } \\
\text { tenzials }\end{array}$ & $\begin{array}{l}\text { Realisierung } \\
\text { einer umfassenden } \\
\text { Kommunikationsof- } \\
\text { fensive über Open } \\
\text { Innovation zur } \\
\text { Bewusstseins- und } \\
\text { Netzwerkbildung }\end{array}$ \\
\hline & $\begin{array}{l}\text { OeAD, BMBWF } \\
\text { - Citizen Science } \\
\text { Award }\end{array}$ & & $\begin{array}{l}\text { Salzburg - Kom- } \\
\text { petenzzentrum für } \\
\text { Open Innovation } \\
\text { (KOI) }\end{array}$ & $\begin{array}{l}\text { Österreichisches } \\
\text { Patentamt - Trai- } \\
\text { nings und Veran- } \\
\text { staltungen }\end{array}$ & $\begin{array}{l}\text { Österreichisches } \\
\text { Patentamt - Open } \\
\text { Data Initiative } \\
\text { FWF - Plan S } \\
\text { - Making Open } \\
\text { Access a reality } \\
\text { by } 2020\end{array}$ & $\begin{array}{l}\text { Österreichisches } \\
\text { Patentamt - Be- } \\
\text { wusstseinsbildung } \\
\text { für Verwertungs- } \\
\text { strategien }\end{array}$ & $\begin{array}{l}\text { BMBWF \& BMVIT } \\
\text { - Informations- \& } \\
\text { Kommunikations- } \\
\text { arbeit über die } \\
\text { offizielle Open } \\
\text { Innovation Websi- } \\
\text { te (www.openinno- } \\
\text { vation.gv.at) } \\
\text { BMBWF \& BMVIT - } \\
\text { Schwerpunktmäßi- } \\
\text { ge Vernetzung zu } \\
\text { OI im Rahmen von } \\
\text { Workshops } \\
\text { IHS - RiConfigure } \\
\text { - Social Lab zu } \\
\text { Quadruple Helix } \\
\text { Innovation gemein- } \\
\text { sam mit ÖBB Open } \\
\text { Innovation Lab }\end{array}$ \\
\hline $\begin{array}{l}\text { ZAMG - Hacka- } \\
\text { thon zu innovati- } \\
\text { ven Lösungen für } \\
\text { Big Data Problems }\end{array}$ & $\begin{array}{l}\text { BMVIT - AAL } \\
\text { Testregionen }\end{array}$ & & $\begin{array}{l}\text { FFG - Open } \\
\text { Innovation Schwer- } \\
\text { punkt bei COIN- } \\
\text { Programmlinie } \\
\text { Netzwerke }\end{array}$ & & $\begin{array}{l}\text { BMVIT - Open } \\
\text { Content-Plattform } \\
\text { "e-genius" } \\
\text { BMVIT - Austausch } \\
\text { von Open FTI Data } \\
\text { Pionieren }\end{array}$ & & $\begin{array}{l}\text { BMVIT - Informa- } \\
\text { tions- \& Kommu- } \\
\text { nikationsarbeit } \\
\text { im Rahmen der } \\
\text { Open4Innovation- } \\
\text { Plattform }\end{array}$ \\
\hline $\begin{array}{l}\text { FFG - Ideen Lab } \\
4.0 \\
\text { CDG - Partnership } \\
\text { in Research }\end{array}$ & $\begin{array}{l}\text { FFG - Impact } \\
\text { Innovation }\end{array}$ & $\begin{array}{l}\text { aws (ncp-ip) - } \\
\text { Arbeitsgruppe } \\
\text { zu Abgeltungs- } \\
\text { mechanismen bei } \\
\text { Open Innovation }\end{array}$ & $\begin{array}{l}\text { Salzburg - Kom- } \\
\text { petenzzentrum für } \\
\text { Open Innovation } \\
\text { (KOI) } \\
\text { Österreichisches } \\
\text { Patentamt - KMU } \\
\text { Recherchedienst- } \\
\text { leistungsangebot } \\
\text { FFG - Impact } \\
\text { Innovation }\end{array}$ & & $\begin{array}{l}\text { Österreichisches } \\
\text { Patentamt- Patent } \\
\text { Scan } \\
\text { FWF-Plan S - Ma- } \\
\text { king Open Access } \\
\text { a reality by } 2020 \\
\text { Universitäten, } \\
\text { BMBWF - Umset- } \\
\text { zung der OANA- } \\
\text { Empfehlungen zu } \\
\text { Open Access } \\
\text { BMVIT - Zurverfü- } \\
\text { gungstellung von } \\
\text { Forschungsergeb- } \\
\text { nissen geförderter } \\
\text { Projekte (Open4In- } \\
\text { novation - Platt- } \\
\text { form) }\end{array}$ & & \\
\hline
\end{tabular}




\section{Forschungsförderungen und -aufträge des Bundes It. Bundesforschungsdatenbank}

Die Datenbank zur Erfassung der Forschungsförderungen und -aufträge des Bundes (B_f.dat) ${ }^{1}$ existiert bereits seit dem Jahr 1975 und wurde im damaligen Bundesministerium für Wissenschaft und Forschung als "Faktendokumentation des Bundes" eingerichtet. Heute wird die Datenbank vom Bundesministerium für Bildung, Wissenschaft und Forschung betreut. Die Meldepflicht der Ressorts gegenüber der jeweiligen Wissenschaftsministerin bzw. dem jeweiligen Wissenschaftsminister findet sich im Forschungsorganisationsgesetz FOG, BGBI. Nr. 341/1981, zuletzt geändert durch BGBI. I Nr. 31/2018. Im Jahr 2008 erfolgte die Umstellung auf eine Datenbank, zu der alle Ressorts Zugang haben und selbstständig forschungsrelevante Förderungen und Aufträge eintragen. Jedes Ressort ist in seinem jeweiligen Wirkungsbereich für die Validität und Vollständigkeit der Daten verantwortlich. Seit 01.06.2016 ist die Bundesforschungsdatenbank öffentlich zugänglich und bietet eine aktuelle Übersicht über die finanzierten Projekte der Bundesministerien. Als Dokumentationsdatenbank dient die B_f.dat auch dazu, kurze inhaltliche Informationen über die F\&E-Projekte zu erfassen. Bezogen auf das jeweilige Berichtsjahr umfasst die Datenbank laufende, neu bewilligte und auch bereits abgeschlossene F\&E-Projekte, sowie deren Gesamtfinanzierungsvolumen und die tatsächlich im Berichtsjahr jeweils ausbezahlten Mitteln. Insgesamt ergibt sich somit ein aktuelles Bild von beauftragten F\&E-Projekten und deren Finanzierung seitens des Bundes.

Die Abbildung 9-1 bis 9-4 geben jeweils einen Überblick über die in der Bundesforschungsdatenbank B_f.dat von den seitens der Ressorts eingetragenen F\&E-Projekten und deren Finanzierung im Jahr 2018. Die Daten in der B_f.dat zeigen, dass das Ge- samtfinanzierungsvolumen der 493 F\&E-Projekte in 2018 438,43 Mio. € beträgt. Knapp 84 \% der Fördermittel in 2018 wurden als Globalförderung an Forschungsinstitutionen ${ }^{2}$ ausbezahlt. Darin enthalten ist auch die globale Institutionenförderung; zieht man diese von den ausbezahlten Teilbeträgen $a b$, so beträgt die Fördersumme 71,39 Mio. €. Dies sind um 3,75 Mio. $€$ bzw. 5,5 \% mehr als im Jahr 2017. Anzumerken ist, dass es sich bei dieser Fördersumme je Berichtsjahr meist um Teilbeträge eines laufenden oder beendeten Projekts handelt, die - abhängig vom jeweiligen Projektfortschritt - auch jährlichen Schwankungen unterworfen sein können.

Bei der Differenzierung nach dem Hauptstandort der Antragstellerinnen und Antragssteller zeigt sich, dass das Bundesland Wien mit 70,4\% der laufenden und abgeschlossenen F\&E-Projekte und mit 82,7\% der ausbezahlten F\&E-Mittel mit Abstand den größten Anteil an Förderungen aufweist (siehe Abbildung 9-1). Dahinter folgt Niederösterreich mit 4,5\% der Projekte und einem Anteil von $8 \%$ am Fördervolumen. Für Vorarlberg ist hingegen im Jahr 2018, wie bereits in den Jahren zuvor, kein Projekt in der $B_{-} f$. dat dokumentiert. Bei den 7,7\% der Finanzierungsbeträge, die ins Ausland fließen, handelt es sich vorwiegend um Mitgliedsbeiträge.

Die Zahl der laufenden und abgeschlossenen F\&EProjekte mit Universitäten als Auftragnehmer ist nach einem Rückgang im Jahr 2017 - von 97 auf 107 Projekte in 2018 (plus $10 \%$ ) angestiegen. Der Finanzierungsbetrag hat sich im Berichtszeitraum ebenso um rd. $9 \%$ auf 5,95 Mio. $€$ erhöht. Damit entspricht die Projektanzahl an Universitäten 21,7\% der gesamten laufenden und abgeschlossenen Projekte und $1,4 \%$ der ausbezahlten Mittel. Unter den Universitäten liegt die Universität für Bodenkultur Wien mit 32 laufenden und abgeschlossenen Projekten an der Spitze und weist mit 1,97 Mio. $€$ in 2018 die höchsten Finanzierungsbeträge auf (siehe Abbildung 9-2). All-

1 Vgl. www.bmbwf.gv.at/bfdat-public

2 In der Darstellung berücksichtigt wurden Institutionenförderungen mit Förderbeträgen von jeweils über $500.000 €$. 
gemein unterscheiden sich die Anzahl der Projekte und die Teilbeträge je Universität im Vergleich zum Vorjahr recht deutlich. Demnach lukrieren nicht immer dieselben Universitäten gleich große bzw. gleich viele Projekte.
Nach Wissenschaftszweigen aufgeschlüsselt blieben die Anteile im Vergleich zu den Vorjahren dagegen recht konstant. Wie in Abbildung 9-3 dargestellt entfallen mit 30,3\% der Projekte der Großteil auf die Sozialwissenschaften; in 2017 waren es 35,1\%. Den

\section{Abbildung 9-1: Anteil der laufenden und abgeschlossenen F\&E-Projekte und Finanzierungsbeträge nach}

Hauptstandort der Auftragnehmerin bzw. des Auftragnehmers, 2018
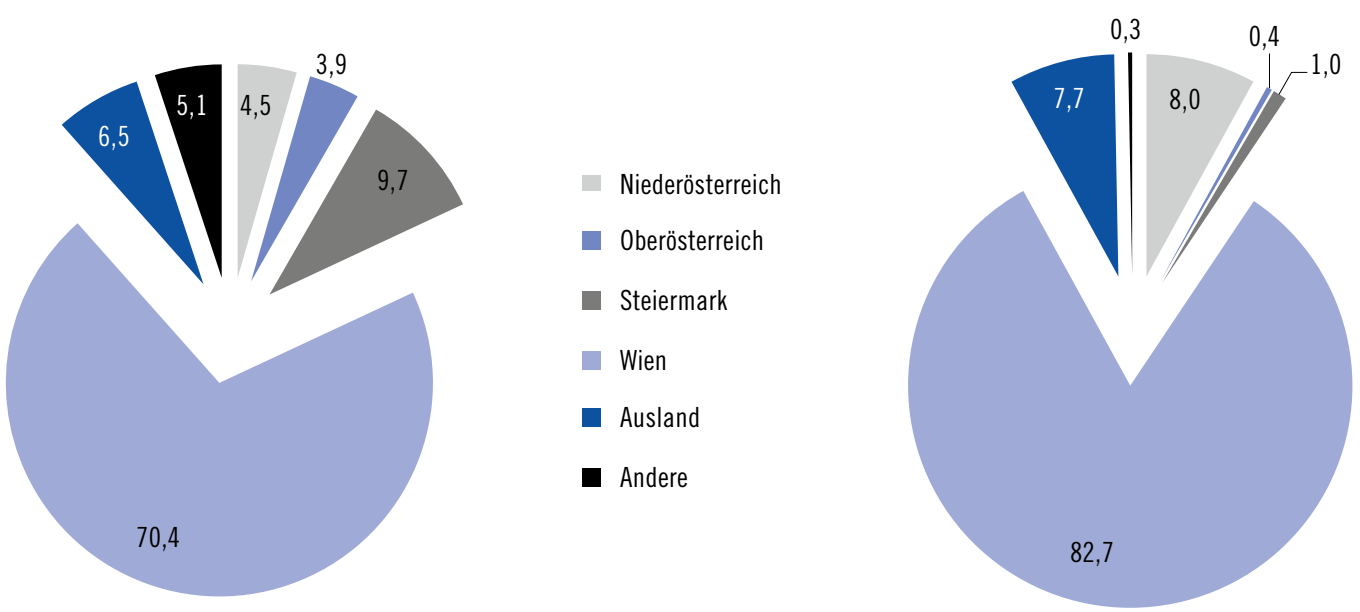

Quelle: BMBWF, Bundesforschungsdatenbank B_f.dat (inkl. „großer“ Globalförderungen für Forschungsinstitutionen und FWF), Vorarlberg 2018 ohne laufendem bzw. abgeschlossenem Projekt. Anm.: Die Kategorie „Andere“ beinhaltet alle Bundesländer mit einem jeweils weniger als 2,5\%-Anteil an laufenden und abgeschlossenen F\&E-Projekten (Burgenland, Kärnten, Salzburg, Tirol).

\section{Abbildung 9-2: Laufende und abgeschlossene F\&E-Projekte und Finanzierungsbeträge 2018 nach Universität}

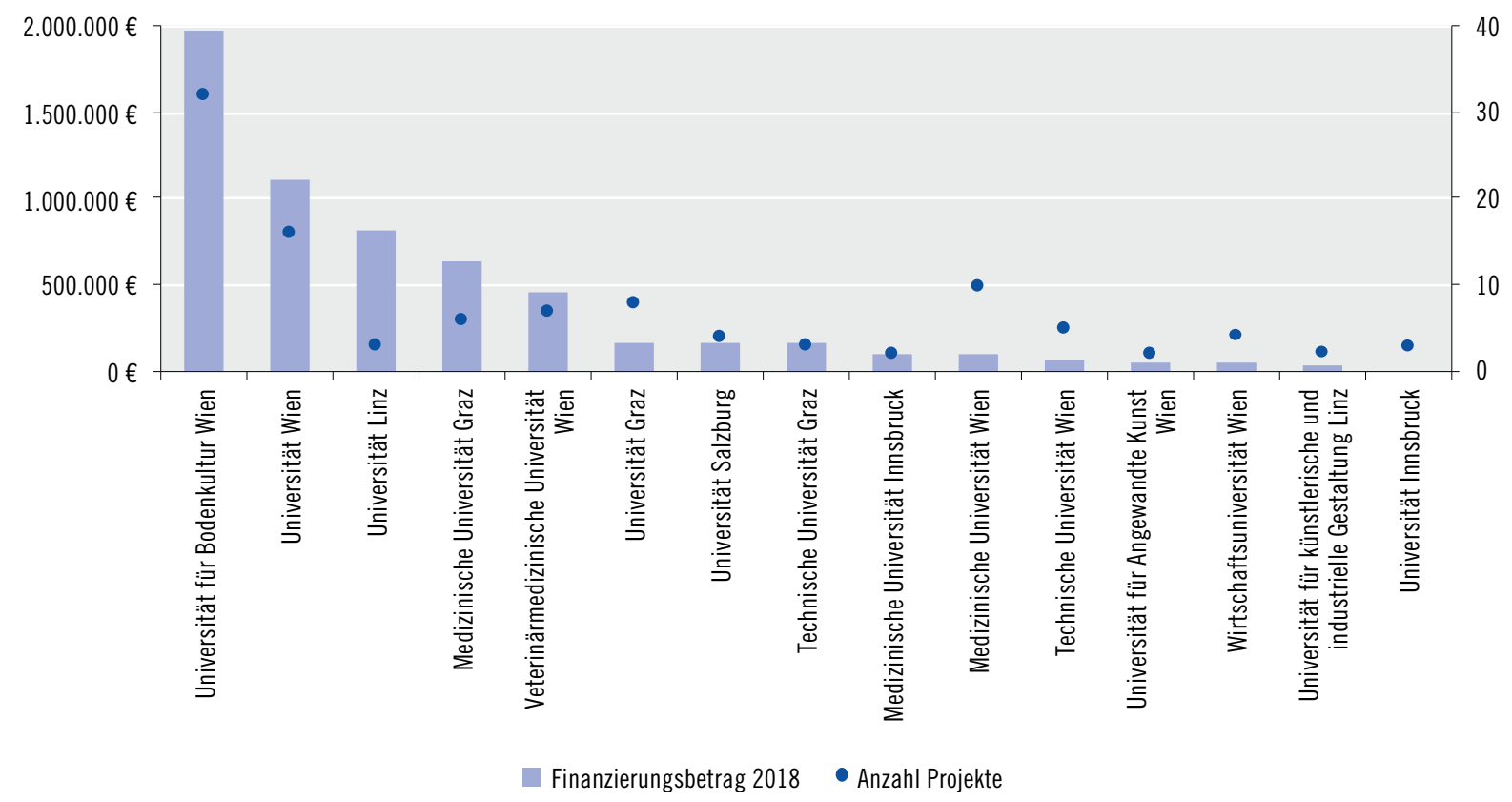

Quelle: BMBWF, Bundesforschungsdatenbank B_f.dat, Darstellung WPZ Research. 
größten Anteil an F\&E-Mitteln weisen hingegen die Naturwissenschaften mit 21,2\% auf; in 2017 waren es $19,8 \%$.
Die Bundesforschungsdatenbank zeigt auch, dass das Bundesministerium für Bildung, Wissenschaft und Forschung (BMBWF) den größten Anteil an Pro-

Abbildung 9-3: Laufende und abgeschlossene F\&E-Projekte und Finanzierungsbeträge 2018 nach

Wissenschaftszweig, in \%

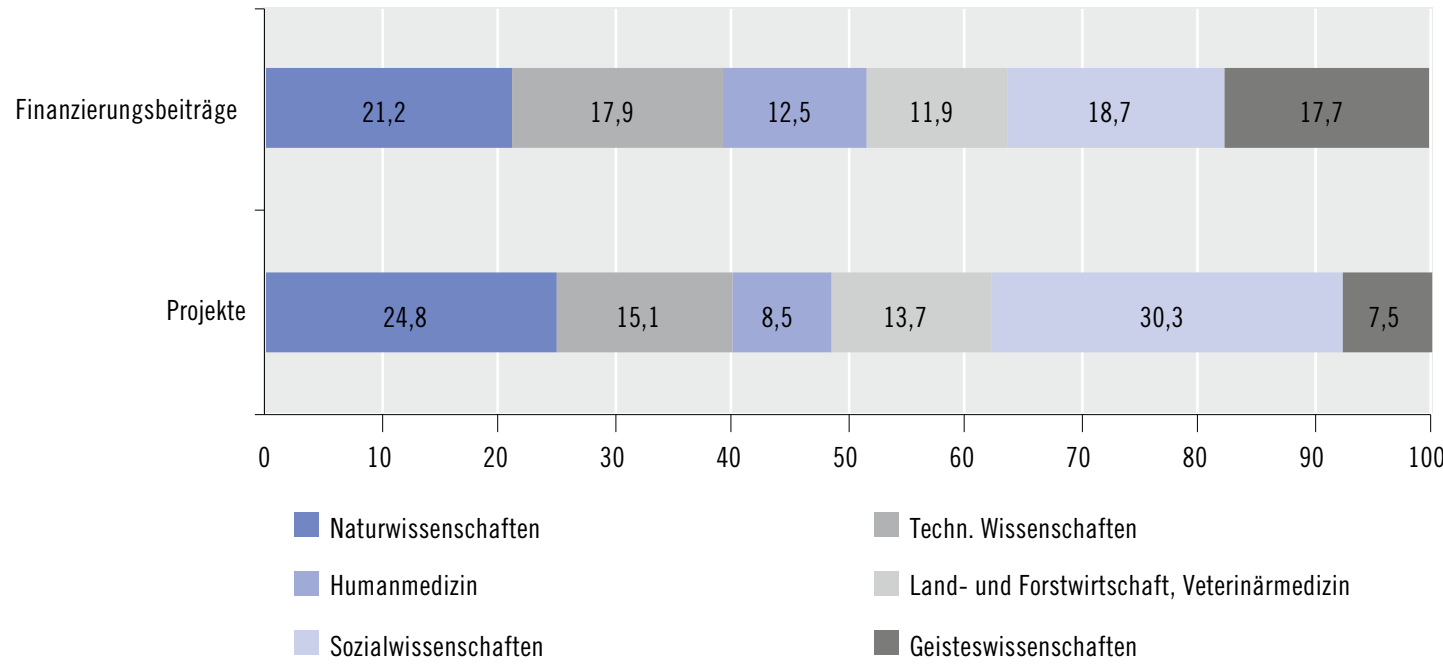

Quelle: BMBWF, Bundesforschungsdatenbank B_f.dat, Darstellung WPZ Research.

Abbildung 9-4: Anteil der laufenden und abgeschlossenen F\&E-Projekte und Finanzierungsbeträge 2018 nach Ressort, in \%
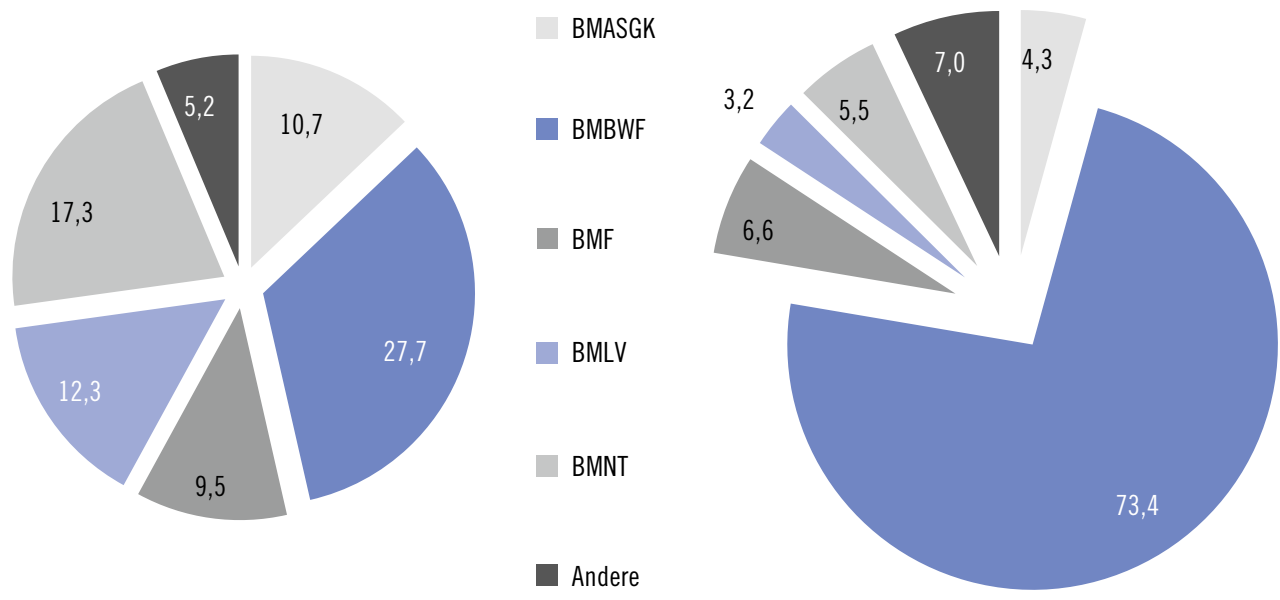

Quelle: BMBWF, Bundesforschungsdatenbank B_f.dat (exkl. „große“ Globalförderungen mit Förderbeiträgen über $500.000 €$ ). Anm.: Die Kategorie „Andere“ beinhaltet alle Ressorts mit mit einem jeweils geringeren als $5 \%$-Anteil an laufenden und abgeschlossenen F\&E-Projekten (BKA, BMASK, BMEIA, BMGF, BMI, BMLVS, BMVIT, BMDW, BMVRDJ, BMWFW). 
jekten und Finanzierungsbeträgen unter den Ressorts aufweist (siehe Abbildung 9-4). Es entfallen 27,7 \% der F\&E-Projekte ${ }^{3}$ bzw. 73,4 \% der Beträge (ohne Globalförderungen) auf das BMBWF. Gemessen an der Anzahl der Projekte folgt danach das Bundesministerium für Nachhaltigkeit und Tourismus
(BMNT), gemessen an den Finanzierungsbeträgen das Bundesministerium für Finanzen (BMF). Der vergleichsweise geringe Prozentsatz des BMVIT (1,7\%) lässt sich darauf zurückführen, dass die Abwicklung des Großteils der F\&E-Mittel an die Förderagenturen FFG und aws ausgelagert ist.

3 Aufgrund von Kombinationsprojekten zwischen den Ministerien kann es bei dieser Form der Darstellung zu Doppelzählungen kommen. 
10. Statistik

(1)




\subsection{Finanzierung der Bruttoinlands- ausgaben für F\&E (Tabellen 1 und 2)}

Im Jahr 2019 werden nach einer Schätzung von Statistik Austria in Österreich rd. 12,8 Mrd. € für Forschung und Entwicklung (F\&E) aufgewendet. Damit steigt die Forschungsquote - also der Anteil der F\&E-Aufwendungen am nominellen Bruttoinlandsprodukt (BIP) - von 3,17 \% im Jahr 2018 auf 3,19\%. Der nominelle Anstieg der gesamtösterreichischen F\&E-Aufwendungen von 2018 auf 2019 wird auf $4,5 \%$ geschätzt und ist damit höher als der prognostizierte Anstieg des Bruttoinlandsprodukts von 3,8\%. Die österreichische Forschungsquote liegt bereits seit 2014 über den von der EU bis 2020 angestrebten $3 \%$, aber unter dem österreichischen Zielwert von $3,76 \%$. In den vergangenen zwei Jahrzehnten sind die heimischen Ausgaben für Forschung und Entwicklung stark angestiegen: 2009 betrug die Forschungsquote noch 2,60\%; vor 20 Jahren lag sie bei $1,85 \%$.

2019 werden die heimischen Unternehmen voraussichtlich rd. 6,3 Mrd. € für Forschung aufwenden und damit fast die Hälfte der F\&E-Ausgaben finanzieren $(49,0 \%)$. Auf den öffentlichen Sektor wird mit rd. 4,5 Mrd. € ein Anteil von 34,9\% der gesamten F\&EAusgaben entfallen, wobei der Bund mit annähernd 3,8 Mrd. $€(29,6 \%)$ die mit Abstand wichtigste Finanzquelle darstellt. Die F\&E-Finanzierung des Bundes beinhaltet auch die Ausschüttungen durch die Forschungsprämie, die für 2019 vom Bundesministerium für Finanzen auf 670 Mio. $€$ geschätzt werden. Mit einer Steigerung von 3,6 \% gegenüber 2018 wird der Anstieg der öffentlichen F\&E-Finanzierung knapp unter dem Anstieg des Bruttoinlandsprodukts liegen. Rd. 547 Mio. $€$ werden von den Bundesländern finanziert. Weitere öffentliche Einrichtungen - wie etwa
Gemeinden, Kammern oder Sozialversicherungsträger - werden rd. 132 Mio. $€$ beitragen. Das Ausland wird nach wie vor eine wichtige Finanzierungsquelle für die heimischen F\&E-Ausgaben sein: Voraussichtlich werden rd. 2 Mrd. $€$ für Forschung nach Österreich fließen; hauptsächlich von multinationalen Unternehmen, deren Tochterunternehmen in Österreich Forschung betreiben. Die F\&E-Finanzierung durch den privaten gemeinnützigen Sektor wird rd. 77 Mio. $€$ betragen.

Im EU-Vergleich für 2017 (das jüngste Jahr, für das Vergleichsdaten verfügbar sind) weist Österreich die zweithöchste Forschungsquote der EU-28 auf. Nur in Schweden ist die Forschungsquote mit 3,40\% deutlich höher. Auch Dänemark (3,05\%) und Deutschland (3,02 \%) erfüllen das $3 \%$-Ziel der EU bereits. Darüber hinaus sind Finnland (2,76\%), Belgien (2,58 \%) und Frankreich $(2,19 \%)$ die einzigen weiteren Länder, deren F\&E-Quote 2017 über dem EU-Durchschnitt von $2,06 \%$ lag.

Für Europa wichtige Vergleichsländer haben teilweise bedeutend höhere Aufwendungen für F\&E als die EU. Innerhalb der europäischen Nicht-EU-Länder erreicht die Schweiz mit 3,37\% (2015) einen sehr hohen Wert, die Forschungsquoten Islands und Norwegens lagen mit 2,10\% und 2,09\% ebenfalls über dem EU-Schnitt.

Unter den großen außereuropäischen Volkswirtschaften ist der F\&E-Aufwand in Südkorea mit 4,55\% am BIP mehr als doppelt so hoch wie in der Europäischen Union. Auch Japan (3,20\%) und die USA $(2,79 \%)$ erreichen bedeutend höhere Forschungsquoten als Europa. Seit 2015 liegt die Forschungsquote Chinas ebenfalls über jener der EU-28: 2017 erreichte diese $2,13 \%$ (EU: $2,06 \%$ ).

In die Schätzung der österreichischen Bruttoin-

1 Auf der Grundlage der Ergebnisse der F\&E-statistischen Vollerhebungen sowie sonstiger aktuell verfügbarer Unterlagen und Informationen, insbesondere der F\&E-relevanten Voranschlags- und Rechnungsabschlussdaten des Bundes und der Bundesländer, wird von Statistik Austria jährlich die „Globalschätzung der österreichischen Bruttoinlandsausgaben für F\&E“ erstellt. Im Rahmen der jährlichen Erstellung der Globalschätzung erfolgen, auf der Basis von neuesten Daten, jeweils auch rückwirkende Revisionen bzw. Aktualisierungen. Den Definitionen des weltweit (OECD, EU) gültigen und damit die internationale Vergleichbarkeit gewährleistenden Frascati-Handbuchs entsprechend wird die Finanzierung der Ausgaben der in Österreich durchgeführten Forschung und experimentellen Entwicklung dargestellt. Gemäß diesen Definitionen und Richtlinien ist die ausländische Finanzierung von in Österreich durchgeführter F\&E sehr wohl einbezogen, hingegen österreichische Zahlungen für im Ausland durchgeführte F\&E sind ausgeschlossen (Inlandskonzept). 
landsausgaben für F\&E 2019 wurden Voranschlagsund Rechnungsabschlussdaten des Bundes und der Bundesländer, aktuelle Konjunkturprognosen sowie vorläufige Teilergebnisse der F\&E-Erhebung über das Berichtsjahr 2017 im Unternehmenssektor einbezogen.

\subsection{F\&E-Ausgaben des Bundes 2019}

Die in Tabelle 10-1 ausgewiesenen Ausgaben des Bundes für im Jahr 2019 in Österreich durchgeführte F\&E setzen sich wie folgt zusammen: Gemäß der Methodik der F\&E-Globalschätzung ist das Kernstück die Gesamtsumme der vom Bund im Inland finanzierten F\&E auf Basis der „Detailübersicht Forschungswirksame Mittelverwendungen des Bundes" der F\&E-Beilage zum Bundesfinanzgesetz (BFG) 2019; Teil b. Zusätzlich wurden die nach derzeitigem Informationsstand im Jahr 2019 voraussichtlich im Wege der Nationalstiftung für Forschung, Technologie und Entwicklung zur Auszahlung gelangenden Mittel sowie die 2019 voraussichtlich zur Auszahlung gelangenden Forschungsprämien einbezogen (Quelle: jeweils BMF).

Zusätzlich zu den Ausgaben des Bundes für in Österreich durchgeführte F\&E wird der Bund im Jahr 2019 Beitragszahlungen an internationale Organisationen, die Forschung und Forschungsförderung als Ziel haben, in Höhe von 103,3 Mio. € leisten (siehe „Detailübersicht Forschungswirksame Mittelverwendungen des Bundes" der F\&E-Beilage zum BFG 2019; Teil a), die jedoch gemäß dem Inlandskonzept nicht in die österreichischen Bruttoinlandsausgaben für $F \& E$ eingerechnet werden.

In den Tabellen „Ausgaben des Bundes für Forschung und Forschungsförderung" wurden die gesamten forschungswirksamen Ausgaben des Bundes, welche die forschungswirksamen Anteile an den Beitragszahlungen an internationale Organisationen (s.o. Pkt. 2.2) einschließen, auf Basis der „Detailübersicht Forschungswirksame Mittelverwendungen des Bundes" der F\&E-Beilage zum BFG 2019 (Teil a und Teil b) ausgewertet. Diese entsprechen dem von
OECD und EU angewendeten „GBARD“-Konzept ${ }^{2}$, welches sich primär auf die Budgets des Zentralbzw. Bundesstaates bezieht, im Gegensatz zum Inlandskonzept die forschungsrelevanten Beitragszahlungen an internationale Organisationen einschließt und die Grundlage der Klassifizierung von F\&E-Budgetdaten nach sozioökonomischen Zielsetzungen für die Berichterstattung an EU und OECD bildet.

2019 entfallen auf folgende sozioökonomischen Zielsetzungen die stärksten Anteile an den Ausgaben des Bundes für Forschung und Forschungsförderung: - Förderung der allgemeinen Erweiterung des Wissens: $30,9 \%$

- Förderung von Handel, Gewerbe und Industrie: $24,5 \%$

- Förderung des Gesundheitswesens: 22,1\%

- Förderung der sozialen und sozioökonomischen Entwicklung: 4,9\%

- Förderung der Erforschung der Erde, der Meere, der Atmosphäre und des Weltraumes: 4,3\%

- Förderung der Erzeugung, Speicherung und Verteilung von Energie: 3,5\%

\subsection{F\&E-Ausgaben der Bundesländer}

Die als Teilsumme in Tabelle 10-1 ausgewiesene Forschungsfinanzierung durch die Bundesländer beruht auf den von den Ämtern der Landesregierungen gemeldeten F\&E-Ausgaben-Schätzungen auf Basis der jeweiligen Landesvoranschläge. Die F\&E-Ausgaben der Landeskrankenanstalten werden gemäß einer mit den Ämtern der Landesregierungen vereinbarten Methodik von Statistik Austria jährlich geschätzt.

\subsection{F\&E-Ausgaben 2016 im internationalen Vergleich}

Die Übersichtstabelle zeigt anhand der wichtigsten F\&E-relevanten Kennzahlen die Position Österreichs im Vergleich zu den anderen Mitgliedstaaten der Europäischen Union bzw. der OECD (Quelle: OECD, MSTI 2018-2).

2 GBARD: Government Budget Allocations for Research and Development = „Staatliche Mittelzuweisungen oder Ausgaben für Forschung und Entwicklung" (EU-Übersetzung). 


\section{Tabellenübersicht des statistischen Anhangs}

Tabelle 10-1: Globalschätzung 2019: Bruttoinlandsausgaben für F\&E Finanzierung der in Österreich durchgeführten Forschung und experimentellen Entwicklung 2005-2019 .

Tabelle 10-2: Globalschätzung 2019: Bruttoinlandsausgaben für F\&E Finanzierung der in Österreich durchgeführten Forschung und experimentellen Entwicklung 2005-2019 in Prozent des BIP . . . . . . . . . . . . . 269

Tabelle 10-3: Ausgaben des Bundes für Forschung und Forschungsförderung 2016 bis 2019 . . . . . . . . . . . . . . 270

Tabelle 10-4: Detailübersicht Forschungswirksame Mittelverwendungen des Bundes, 2017-2019 . . . . . . . . . . . . 271

Tabelle 10-5: Ausgaben des Bundes für Forschung und Forschungsförderung nach sozioökonomischen Zielsetzungen, 2004-2019. . . . . . . . . . . . . . . . . . . . . . . . . . . . . . . . . 281

Tabelle 10-6: Ausgaben des Bundes für Forschung und Forschungsförderung nach sozioökonomischen

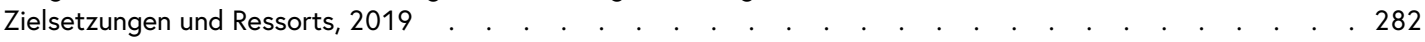

Tabelle 10-7: Allgemeine forschungswirksame Hochschulausgaben des Bundes („General University Funds“), $2000-2019$. . 283

Tabelle 10-8: Forschungsförderungen und Forschungsaufträge der Bundesdienststellen nach Durchführungssektoren / -bereichen und vergebenden Ressorts, 2018 . . . . . . . . . . . . . . . . . . . . . 284

Tabelle 10-9: Forschungsförderungen und Forschungsaufträge der Bundesdienststellen nach sozio-ökonomischen Zielsetzungen und vergebenden Ressorts, 2018 . . . . . . . . . . . . . . . . . . . . . 285

Tabelle 10-10: Forschung und experimentelle Entwicklung (F\&E) im internationalen Vergleich, 2016 . . . . . . . . . . . 286

Tabelle 10-11: Österreichs Teilnahme vom 4. Europäischen Rahmenprogramm bis Horizon 2020 . . . . . . . . . . . . . 287

Tabelle 10-12: Österreichische Projekte, Beteiligungen und Koordinationen in Horizon 2020 differenziert nach Organisationstypen und Bundesländern . . . . . . . . . . . . . . . . . . . . . . . . . . . . . . . . . 287

Tabelle 10-13: Österreichische Beteiligungen und Projekte in Horizon 2020 im Vergleich zur Summe aller Staaten differenziert nach Säulen und horizontalen Programmlinien . . . . . . . . . . . . . . . . . . . . . 288

Tabelle 10-14: FWF: Anteile der Neubewilligungen nach Fachgebiet (ÖFOS 2012 3-Steller), 2018. . . . . . . . . . . . . 289

Tabelle 10-15: FWF: Anteile der Neubewilligungen nach Organisationstyp, 2016-2018 . . . . . . . . . . . . . . . . . . . 290

Tabelle 10-16: FFG: Anteile der Neubewilligungen nach Themenfeld der Förderung, 2016-2018 . . . . . . . . . . . . 290

Tabelle 10-17: FFG: Förderungen nach Bundesland, 2016-2018 . . . . . . . . . . . . . . . . . . . . . . . . . . . . . . . 290

Tabelle 10-18: FFG: Projektkosten und Förderung nach Subject Index Code, 2018 . . . . . . . . . . . . . . . . . . . . . . . . 291

Tabelle 10-19: aws: Anteile der Neubewilligungen nach Themenfeld der Förderung (Branche), 2016-2018 . . . . . . . . 292

Tabelle 10-20: aws: Anteile der Neubewilligungen nach Unternehmensgröße, 2016-2018 . . . . . . . . . . . . . . . . 292

Tabelle 10-21: aws: Leistungsüberblick nach regionaler Verteilung, 2017-2018 . . . . . . . . . . . . . . . . . . . . . . . 292

Tabelle 10-22: CDG: CD-Labors nach Universitäten/Forschungseinrichtungen, 2018 . . . . . . . . . . . . . . . . . . . . . . 293

Tabelle 10-23: CDG: JR-Zentren nach Fachhochschulen, 2018 ...$\quad$. . . . . . . . . . . . . . . . . . . . . . . . . . . . . . . 293

Tabelle 10-24: CDG: Entwicklung der CDG 1989-2018 bzw. JR-Zentren 2012-2018 . . . . . . . . . . . . . . . . . . . . . 294

Tabelle 10-25: CDG: CD-Labors nach Thematischen Clustern, 2018 . . . . . . . . . . . . . . . . . . . . . . . . . . . . . . 295

Tabelle 10-26: CDG: JR-Zentren nach Thematischen Clustern, 2018 . . . . . . . . . . . . . . . . . . . . . . . . . . . . . . 295 

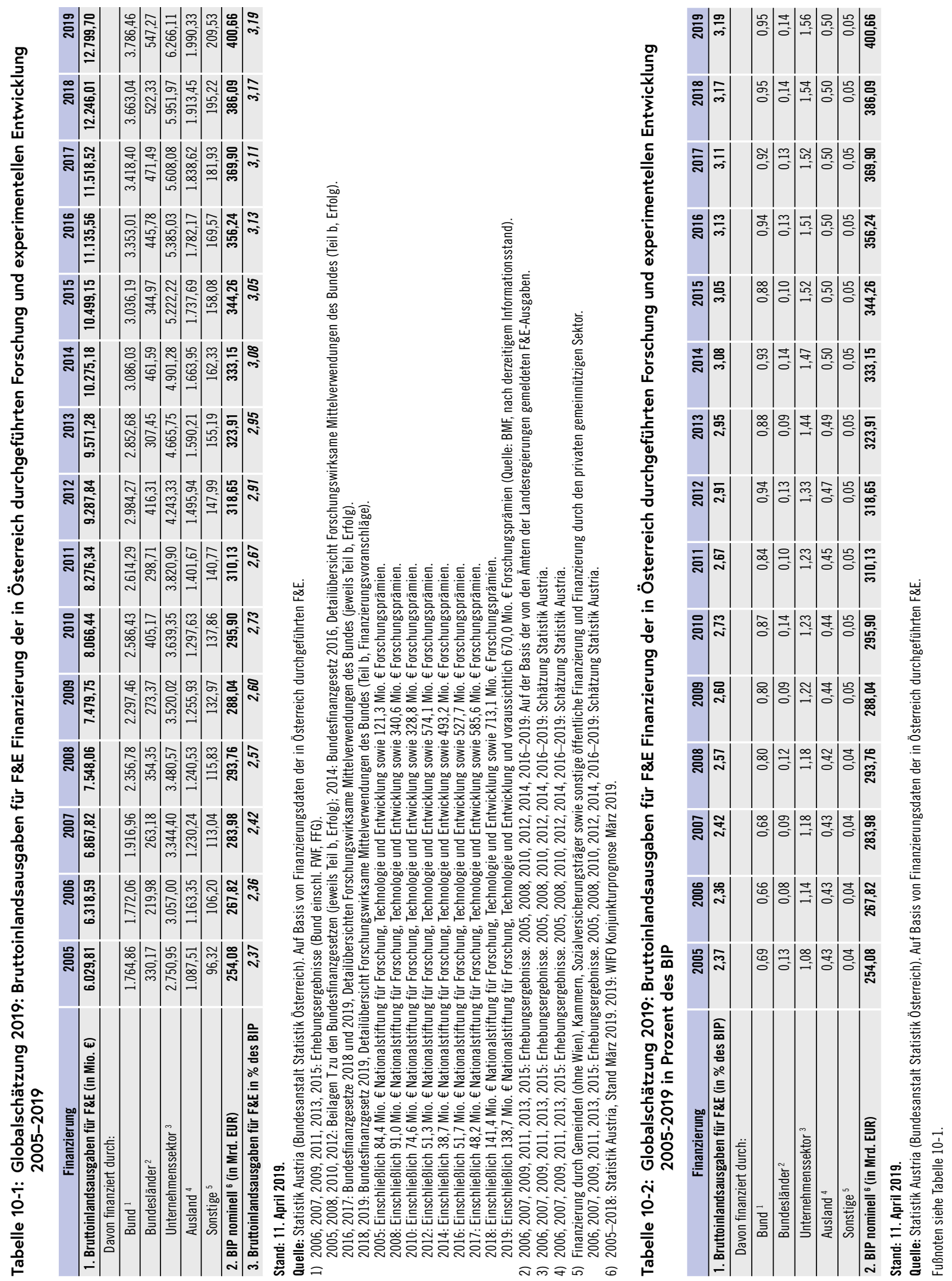
Tabelle 10-3: Ausgaben des Bundes für Forschung und Forschungsförderung 2016 bis 2019

\begin{tabular}{|c|c|c|c|c|c|c|c|c|}
\hline \multirow{3}{*}{ Ressorts $^{1}$} & \multicolumn{4}{|c|}{ Erfolg } & \multicolumn{4}{|c|}{ Finanzierungsvoranschlag } \\
\hline & \multicolumn{2}{|c|}{$2016^{2}$} & \multicolumn{2}{|l|}{$2017^{3}$} & \multicolumn{2}{|l|}{$2018^{3}$} & \multicolumn{2}{|l|}{$2019^{3}$} \\
\hline & in Mio. $€$ & in $\%$ & in Mio. € & in $\%$ & in Mio. $€$ & in $\%$ & in Mio. $€$ & in $\%$ \\
\hline Bundeskanzleramt ${ }^{4}$ & 40,289 & 1,4 & 43,112 & 1,5 & 44,255 & 1,5 & 44,069 & 1,4 \\
\hline Bundesministerium für Familien und Jugend & 1,095 & 0,0 & 1,138 & 0,0 & & & & \\
\hline Bundesministerium für öffentlichen Dienst und Sport & & . & & & - & - & - & - \\
\hline Bundesministerium für Europa, Integration und Äußeres & 2,152 & 0,1 & 2,232 & 0,1 & 2,765 & 0,1 & 3,007 & 0,1 \\
\hline Bundesministerium für Arbeit, Soziales und Konsumentenschutz & 5,747 & 0,2 & 7,111 & 0,2 & . & & & . \\
\hline Bundesministerium für Gesundheit und Frauen & 5,764 & 0,2 & 5,649 & 0,2 & & & & \\
\hline $\begin{array}{l}\text { Bundesministerium für Arbeit, Soziales, Gesundheit und } \\
\text { Konsumentenschutz }\end{array}$ & & . & & & 12,860 & 0,4 & 13,064 & 0,4 \\
\hline Bundesministerium für Bildung & 39,927 & 1,4 & 34,304 & 1,2 & & & & \\
\hline Bundesministerium für Wissenschaft, Forschung und Wirtschaft & $2.213,521$ & 77,0 & $2.265,857$ & 78,5 & & & & \\
\hline Bundesministerium für Bildung, Wissenschaft und Forschung & & . & & & $2.197,742$ & 75,5 & $2.361,438$ & 76,7 \\
\hline Bundesministerium für Digitalisierung und Wirtschaftsstandort & & . & & & 101,120 & 3,5 & 99,570 & 3,2 \\
\hline Bundesministerium für Finanzen & 30,683 & 1,1 & 31,714 & 1,1 & 32,307 & 1,1 & 32,026 & 1,0 \\
\hline Bundesministerium für Inneres & 1,234 & 0,0 & 1,327 & 0,0 & 1,447 & 0,0 & 1,428 & 0,0 \\
\hline Bundesministerium für Landesverteidigung und Sport & 2,352 & 0,1 & 3,202 & 0,1 & & & & \\
\hline Bundesministerium für Landesverteidigung & & . & . & & 4,684 & 0,2 & 4,688 & 0,2 \\
\hline $\begin{array}{l}\text { Bundesministerium für Land- und Forstwirtschaft, Umwelt und } \\
\text { Wasserwirtschaft }\end{array}$ & 44,373 & 1,5 & 43,989 & 1,5 & & & & \\
\hline Bundesministerium für Nachhaltigkeit und Tourismus & &. & & & 38,948 & 1,3 & 39,191 & 1,3 \\
\hline Bundesministerium für Justiz & 0,082 & 0,0 & 0,063 & 0,0 & & & & \\
\hline Bundesministerium für Verfassung, Reformen, Deregulierung und Justiz & & . & & & 0,019 & 0,0 & 0,059 & 0,0 \\
\hline Bundesministerium für Verkehr, Innovation und Technologie & 488,487 & 17,0 & 450,081 & 15,6 & 477,134 & 16,4 & 482,547 & 15,7 \\
\hline Insgesamt & $2.875,706$ & 100,0 & $2.889,779$ & 100,0 & $2.913,281$ & 100,0 & $3.081,087$ & 100,0 \\
\hline
\end{tabular}

\section{Stand: 11. April 2019.}

Quelle: Statistik Austria (Bundesanstalt Statistik Österreich).

1) Entsprechend der im jeweiligen Jahr gültigen Fassung des Bundesministeriengesetzes 1986 (2016, 2017: BGBI. I Nr. 49/2016; 2018, 2019: BGBI. I Nr. 164/2017).

2) Bundesfinanzgesetz 2018, Detailübersicht Forschungswirksame Mittelverwendungen des Bundes.

3) Bundesfinanzgesetz 2019, Detailübersicht Forschungswirksame Mittelverwendungen des Bundes.

4) Einschließlich oberste Organe. 
Tabelle 10-4: Detailübersicht Forschungswirksame Mittelverwendungen des Bundes, 2017-2019

\author{
Detailübersicht \\ Forschungswirksame Mittelverwendungen des Bundes
}

\title{
Forschungswirksame Ausgaben des Bundes 2017 bis 2019 nach Ressorts
}

Die nachfolgenden Übersichten sind gegliedert nach:

1. Beitragszahlungen aus Bundesmitteln an internationale Organisationen, die Forschung und Forschungsförderung (mit) als Ziel haben (Teil a)

2. Bugetierte Ausgaben des Bundes für Forschung und Forschungsförderung in Österreich (Teil b, Bundesbudget Forschung)

Für die Aufstellung dieser Ausgaben ist in erster Linie der Gesichtspunkt der Forschungswirksamkeit maßgebend, beruhend auf dem Forschungsbegriff des Frascati-Handbuches der OECD, der auch im Rahmen der Erhebungen über Forschung und experimentelle Entwicklung (F\&E) von Statistik Austria zur Anwendung gelangt. 
BUNDESVORANSCHLAG 2019

Detailübersicht Forschungswirksame Mittelverwendungen des Bundes

(Beträge in Millionen Euro)

\begin{tabular}{|c|c|c|c|c|c|c|c|c|c|c|c|c|c|}
\hline \multicolumn{14}{|c|}{ a) Beitragszahlungen an internationale Organisationen - Finanzierungsvoranschlag } \\
\hline \multirow{3}{*}{ VA-Stelle } & \multirow{3}{*}{ Konto } & \multirow{3}{*}{ Ugl } & \multirow{3}{*}{ Bezeichnung } & \multirow{3}{*}{$\begin{array}{l}A \\
n \\
m\end{array}$} & \multicolumn{3}{|c|}{ Finanzierungsvoranschlag 2019} & \multicolumn{3}{|c|}{ Finanzierungsvoranschlag 2018} & \multicolumn{3}{|c|}{ Erfolg 2017} \\
\hline & & & & & \multirow{2}{*}{ Insgesamt } & \multicolumn{2}{|r|}{ hievon } & \multirow{2}{*}{ Insgesamt } & \multicolumn{2}{|r|}{ hievon } & \multirow{2}{*}{ Insgesamt } & \multicolumn{2}{|r|}{ hievon } \\
\hline & & & & & & $\%$ & Forschung & & $\%$ & Forschung & & $\%$ & Forschung \\
\hline & & & Bundeskanzleramt & & & & & & & & & & \\
\hline & & & UG10 & & & & & & & & & & \\
\hline 10010100 & 7800 & 100 & $\begin{array}{l}\text { Mitgliedsbeiträge an Institutionen im } \\
\text { Ausland }\end{array}$ & & 0,113 & 100 & 0,113 & 0,113 & 100 & 0,113 & 0,180 & 100 & 0,180 \\
\hline 10010100 & 7800 & 101 & Mitgliedsbeitrag für OECD & & & & & & & & 3,755 & 20 & 0,751 \\
\hline 10010100 & 7800 & 102 & $\begin{array}{l}\text { OECD-Energieagentur } \\
\text { (Mitgliedsbeitrag) }\end{array}$ & & & & & & & & 0,225 & 20 & 0,045 \\
\hline 10010100 & 7800 & 103 & OECD-Beiträge zu Sonderprojekten & & & & & & & & & & \\
\hline 10010100 & 7800 & 110 & Mitgliedsbeitrag AV-Infostelle & & 0,032 & 5 & 0,002 & 0,032 & 5 & 0,002 & 0,030 & 5 & 0,002 \\
\hline \multirow[t]{5}{*}{10010200} & 7800 & 100 & $\begin{array}{l}\text { Mitgliedsbeiträge an Institutionen im } \\
\text { Ausland }\end{array}$ & & 0,006 & 30 & 0,002 & 0,006 & 30 & 0,002 & 0,006 & 30 & 0,002 \\
\hline & & & Summe UG10 & & 0,151 & & 0,117 & 0,151 & & 0,117 & 4,196 & & 0,980 \\
\hline & & & Summe Bundeskanzleramt & & 0,151 & & 0,117 & 0,151 & & 0,117 & 4,196 & & 0,980 \\
\hline & & & $\begin{array}{c}\text { BM für Europa, Integration und } \\
\text { Äußeres }\end{array}$ & & & & & & & & & & \\
\hline & & & UG12 & & & & & & & & & & \\
\hline 12020200 & 7800 & 101 & Mitgliedsbeitrag für OECD & $*$ & 3,675 & 20 & 0,735 & 3,115 & 20 & 0,623 & & & \\
\hline 12020200 & 7800 & 102 & $\begin{array}{l}\text { OECD-Energieagentur } \\
\text { (Mitgliedsbeitrag) }\end{array}$ & * & 0,225 & 20 & 0,045 & 0,225 & 20 & 0,045 & & & \\
\hline 12020200 & 7840 & 000 & Laufende Transfers an Drittländer & $*$ & 3,194 & 35 & 1,118 & 3,144 & 35 & 1,100 & 3,144 & 35 & 1,100 \\
\hline 12020200 & 7840 & 002 & $\begin{array}{l}\text { Organisation der VN für } \\
\text { industr.Entwicklung(UNIDO) }\end{array}$ & & 0,822 & 46 & 0,378 & 0,605 & 46 & 0,278 & 0,897 & 46 & 0,413 \\
\hline 12020200 & 7840 & 003 & $\begin{array}{l}\text { Org. VN } \\
\text { Erziehung, Wissensch.u.Kultur(UNES } \\
\text { CO) }\end{array}$ & & 2,165 & 30 & 0,650 & 2,131 & 30 & 0,639 & 2,131 & 30 & 0,639 \\
\hline \multirow[t]{5}{*}{12020200} & 7840 & 056 & $\begin{array}{l}\text { Drogenkontrollprogramm der VN } \\
\text { (UNDCP) }\end{array}$ & & 0,406 & 20 & 0,081 & 0,400 & 20 & 0,080 & 0,400 & 20 & 0,080 \\
\hline & & & Summe UG12 & & 10,487 & & 3,007 & 9,620 & & 2,765 & 6,572 & & 2,232 \\
\hline & & & $\begin{array}{l}\text { Summe BM für Europa, } \\
\text { Integration und Äußeres }\end{array}$ & & 10,487 & & 3,007 & 9,620 & & 2,765 & 6,572 & & 2,232 \\
\hline & & & BM für Finanzen & & & & & & & & & & \\
\hline & & & UG15 & & & & & & & & & & \\
\hline \multirow[t]{5}{*}{15010100} & 7800 & 000 & $\begin{array}{l}\text { Laufende Transferzahlungen an das } \\
\text { Ausland }\end{array}$ & & 0,151 & 100 & 0,151 & 0,151 & 100 & 0,151 & 0,387 & 26 & 0,101 \\
\hline & & & Summe UG15 & & 0,151 & & 0,151 & 0,151 & & 0,151 & 0,387 & & 0,101 \\
\hline & & & Summe BM für Finanzen & & 0,151 & & 0,151 & 0,151 & & 0,151 & 0,387 & & 0,101 \\
\hline & & & $\begin{array}{l}\text { BM für Bildung, Wissenschaft und } \\
\text { Forschung }\end{array}$ & & & & & & & & & & \\
\hline & & & UG30 & & & & & & & & & & \\
\hline 30010300 & 7800 & 104 & OECD-Schulbauprogramm & & 0,031 & 100 & 0,031 & 0,031 & 100 & 0,031 & 0,023 & 100 & 0,023 \\
\hline \multirow[t]{3}{*}{30010400} & 7800 & 000 & $\begin{array}{l}\text { Laufende Transferzahlungen an das } \\
\text { Ausland }\end{array}$ & * & 0,037 & 100 & 0,037 & 0,037 & 100 & 0,037 & 0,039 & 100 & 0,039 \\
\hline & & & Summe UG30 & & 0,068 & & 0,068 & 0,068 & & 0,068 & 0,062 & & 0,062 \\
\hline & & & UG31 & & & & & & & & & & \\
\hline 31030100 & 7800 & 000 & $\begin{array}{l}\text { Laufende Transferzahlungen an das } \\
\text { Ausland }\end{array}$ & & 0,750 & 100 & 0,750 & 0,750 & 100 & 0,750 & 0,729 & 100 & 0,729 \\
\hline 31030100 & 7800 & 066 & $\begin{array}{l}\text { Forschungsvorhaben in } \\
\text { internationaler Kooperation }\end{array}$ & & 0,802 & 100 & 0,802 & 0,802 & 100 & 0,802 & 0,237 & 100 & 0,237 \\
\hline 31030100 & 7800 & 200 & $\begin{array}{l}\text { Beiträge an internationale } \\
\text { Organisationen }\end{array}$ & & 1,570 & 50 & 0,785 & 1,570 & 50 & 0,785 & 1,389 & 50 & 0,695 \\
\hline 31030204 & 7800 & 062 & ESO & & 6,730 & 100 & 6,730 & 6,520 & 100 & 6,520 & 6,309 & 100 & 6,309 \\
\hline 31030204 & 7800 & 063 & $\begin{array}{l}\text { Europ. Zentrum für mittelfristige } \\
\text { Wettervorhersage }\end{array}$ & & 1,300 & 100 & 1,300 & 1,300 & 100 & 1,300 & 1,103 & 100 & 1,103 \\
\hline 31030204 & 7800 & 064 & $\begin{array}{l}\text { Molekularbiologie - Europäische } \\
\text { Zusammenarbeit }\end{array}$ & & 3,000 & 100 & 3,000 & 2,900 & 100 & 2,900 & 2,859 & 100 & 2,859 \\
\hline
\end{tabular}




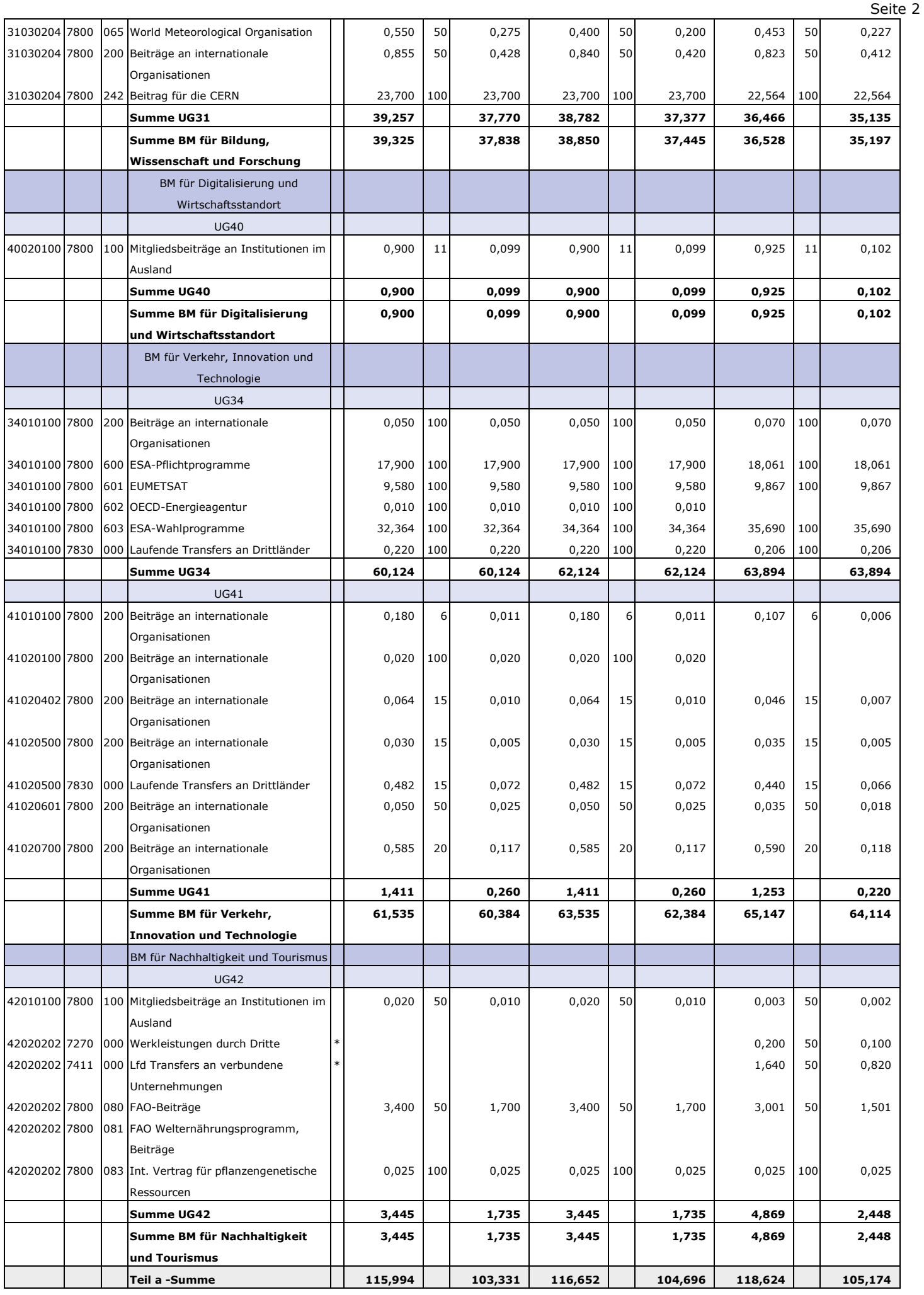




\begin{tabular}{|c|c|c|c|c|c|c|c|c|c|c|c|c|c|}
\hline \multicolumn{14}{|c|}{$\begin{array}{l}\text { b) Bundesbudget Forschung - Finanzierungsvoranschlag } \\
\text { (ausgen. die bereits im Abschnitt a) ausgewiesen sind) }\end{array}$} \\
\hline \multirow{3}{*}{ VA-Stelle } & \multirow{3}{*}{ Konto } & \multirow{3}{*}{ Ugl } & \multirow{3}{*}{ Bezeichnung } & \multirow{3}{*}{ A } & \multicolumn{3}{|c|}{ Finanzierungsvoranschlag 2019} & \multicolumn{3}{|c|}{ Finanzierungsvoranschlag 2018} & \multicolumn{3}{|c|}{ Erfolg 2017} \\
\hline & & & & & \multirow{2}{*}{ Insgesamt } & \multicolumn{2}{|c|}{ hievon } & \multirow{2}{*}{ Insgesamt } & \multicolumn{2}{|r|}{ hievon } & \multirow{2}{*}{ Insgesamt } & \multicolumn{2}{|c|}{ hievon } \\
\hline & & & & & & $\%$ & Forschung & & $\%$ & Forschung & & $\%$ & Forschung \\
\hline & & & Parlamentsdirektion & & & & & & & & & & \\
\hline & & & UG02 & & & & & & & & & & \\
\hline \multirow[t]{5}{*}{02010500} & 7330 & 086 & $\begin{array}{l}\text { Nationalfonds für Opfer des } \\
\text { Nationalsozialismus }\end{array}$ & * & 3,858 & 1 & 0,039 & 3,726 & 2 & 0,075 & 3,723 & 3 & 0,097 \\
\hline & & & Summe UG02 & & 3,858 & & 0,039 & 3,726 & & 0,075 & 3,723 & & 0,097 \\
\hline & & & Summe Parlamentsdirektion & & 3,858 & & 0,039 & 3,726 & & 0,075 & 3,723 & & 0,097 \\
\hline & & & Bundeskanzleramt & & & & & & & & & & \\
\hline & & & UG10 & & & & & & & & & & \\
\hline 10010100 & 7260 & 000 & $\begin{array}{l}\text { Mitgliedsbeiträge an Institutionen im } \\
\text { Inland }\end{array}$ & & 0,002 & 28 & 0,001 & 0,002 & 28 & 0,001 & 0,785 & 50 & 0,393 \\
\hline 10010100 & 7270 & 000 & Werkleistungen durch Dritte & & 0,651 & 4 & 0,026 & 0,672 & 4 & 0,027 & 1,887 & 4 & 0,075 \\
\hline 10010200 & 7260 & 000 & $\begin{array}{l}\text { Mitgliedsbeiträge an Institutionen im } \\
\text { Inland }\end{array}$ & & 0,001 & 50 & 0,001 & 0,001 & 50 & 0,001 & & 50 & \\
\hline 10010200 & 7270 & 000 & Werkleistungen durch Dritte & & 9,495 & 4 & 0,380 & 9,386 & 4 & 0,375 & 2,649 & 4 & 0,106 \\
\hline 10010401 & 7340 & 001 & $\begin{array}{l}\text { Pauschalabgeltung gem. § } 32 \text { Abs. } 5 \\
\text { BStatG }\end{array}$ & & 49,891 & 1 & 0,499 & 50,891 & 1 & 0,509 & 50,630 & 1 & 0,506 \\
\hline \multirow[t]{3}{*}{10010402} & & & Österr. Staatsarchiv & & 15,490 & 3 & 0,465 & 14,865 & 3 & 0,446 & 14,333 & 3 & 0,430 \\
\hline & & & Summe UG10 & & 75,530 & & 1,372 & 75,817 & & 1,359 & 70,284 & & 1,510 \\
\hline & & & UG25 & & & & & & & & & & \\
\hline 25010500 & 7270 & 006 & Werkleistungen durch Dritte (zw) & $*$ & 0,676 & 42 & 0,284 & 0,732 & 61 & 0,447 & 0,333 & 89 & 0,295 \\
\hline 25010500 & 7420 & 113 & $\begin{array}{l}\text { Familie und Beruf Management } \\
\text { GesmbH. (zw) }\end{array}$ & $*$ & 2,140 & 33 & 0,706 & 2,140 & 33 & 0,706 & 2,140 & 38 & 0,804 \\
\hline 25020100 & 7270 & 000 & Werkleistungen durch Dritte & * & 0,947 & 1 & 0,009 & 0,947 & 1 & 0,009 & 0,700 & 2 & 0,012 \\
\hline 25020200 & 7270 & 000 & Werkleistungen durch Dritte & $*$ & 1,787 & 2 & 0,036 & 1,787 & 2 & 0,036 & 1,327 & 2 & 0,027 \\
\hline & & & Summe UG25 & & 5,550 & & 1,035 & 5,606 & & 1,198 & 4,500 & & 1,138 \\
\hline & & & UG32 & & & & & & & & & & \\
\hline 32010300 & & & Denkmalschutz & & 38,343 & 18 & 6,902 & 38,343 & 18 & 6,902 & 35,596 & 18 & 6,407 \\
\hline 32030100 & & & Bundesmuseen & & 128,162 & 27 & 34,604 & 128,162 & 27 & 34,604 & 126,362 & 27 & 34,118 \\
\hline & & & Summe UG32 & & 166,505 & & 41,506 & 166,505 & & 41,506 & 161,958 & & 40,525 \\
\hline & & & Summe Bundeskanzleramt & & 247,585 & & 43,913 & 247,928 & & 44,063 & 236,742 & & 43,173 \\
\hline & & & BM für Inneres & & & & & & & & & & \\
\hline & & & UG11 & & & & & & & & & & \\
\hline 11010100 & 7278 & 020 & $\begin{array}{l}\text { Konzepte, Analysen und Gutachten } \\
\text { (ADV) }\end{array}$ & * & & & & & & & 0,009 & 100 & 0,009 \\
\hline 11010200 & 7270 & 900 & Werkleistungen durch Dritte & * & & & & 0,012 & 100 & 0,012 & 0,012 & 100 & 0,012 \\
\hline 11020600 & & & Bundeskriminalamt & * & 13,904 & 8 & 1,112 & 14,182 & 8 & 1,135 & 12,664 & 8 & 1,013 \\
\hline 11020600 & 7270 & 900 & Werkleistungen durch Dritte & $*$ & & & & & & & 0,009 & 100 & 0,009 \\
\hline 11020800 & 7270 & 900 & Werkleistungen durch Dritte & $*$ & & & & & & & 0,121 & 100 & 0,121 \\
\hline 11030100 & 7660 & 900 & $\begin{array}{l}\text { Zuschüsse f. Ifd. Aufwand an private } \\
\text { Institutionen }\end{array}$ & * & & & & & & & 0,088 & 100 & 0,088 \\
\hline 11030100 & 7672 & 009 & Projekte des AMIF (Kofinanzierung) & $*$ & & & & & & & 0,028 & 100 & 0,028 \\
\hline 11030500 & 7270 & 900 & Werkleistungen durch Dritte & * & & & & & & & 0,047 & 100 & 0,047 \\
\hline & & & Summe UG11 & & 13,904 & & 1,112 & 14,194 & & 1,147 & 12,978 & & 1,327 \\
\hline & & & UG18 & & & & & & & & & & \\
\hline 18010100 & 7660 & 900 & $\begin{array}{l}\text { Zuschüsse f. Ifd. Aufwand an private } \\
\text { Institutionen }\end{array}$ & * & & & & 0,017 & 100 & 0,017 & & & \\
\hline 18010100 & 7672 & 009 & Projekte des AMIF (Kofinanzierung) & $*$ & 0,316 & 100 & 0,316 & 0,283 & 100 & 0,283 & & & \\
\hline & & & Summe UG18 & & 0,316 & & 0,316 & 0,300 & & 0,300 & & & \\
\hline & & & Summe BM für Inneres & & 14,220 & & 1,428 & 14,494 & & 1,447 & 12,978 & & 1,327 \\
\hline & & & $\begin{array}{c}\text { BM für Verfassung, Reformen, } \\
\text { Deregulierung und Justiz }\end{array}$ & & & & & & & & & & \\
\hline & & & UG13 & & & & & & & & & & \\
\hline 13010100 & 7271 & 900 & Werkleistungen (durch Dritte) & $*$ & 0,088 & 50 & 0,044 & 0,038 & 50 & 0,019 & 0,045 & 50 & 0,023 \\
\hline 13030101 & 7271 & 900 & Werkleistungen (durch Dritte) & $*$ & 0,030 & 50 & 0,015 & & & & 0,079 & 50 & 0,040 \\
\hline & & & Summe UG13 & & 0,118 & & 0,059 & 0,038 & & 0,019 & 0,124 & & 0,063 \\
\hline & & & $\begin{array}{l}\text { Summe BM für Verfassung, } \\
\text { Reformen, Deregulierung und } \\
\text { Justiz }\end{array}$ & & 0,118 & & 0,059 & 0,038 & & 0,019 & 0,124 & & 0,063 \\
\hline
\end{tabular}




\begin{tabular}{|c|c|c|c|c|c|c|c|c|c|c|c|c|c|}
\hline & & & BM für Landesverteidigung & & & & & & & & & & \\
\hline & & & UG14 & & & & & & & & & & \\
\hline 14040100 & & & Heeresgeschichtliches Museum & $*$ & 3,509 & 15 & 0,526 & 3,479 & 15 & 0,522 & 3,417 & 20 & 0,683 \\
\hline 14050100 & 7270 & 000 & Werkleistungen durch Dritte & $*$ & 0,258 & 58 & 0,150 & 0,258 & 58 & 0,150 & 0,103 & 58 & 0,060 \\
\hline 14050100 & 7270 & 900 & Werkleistungen durch Dritte & $*$ & 1,000 & 100 & 1,000 & 1,000 & 100 & 1,000 & 2,417 & 100 & 2,417 \\
\hline 14050100 & 7411 & 028 & FFG - Verteidigungsforschung & & 3,000 & 100 & 3,000 & 3,000 & 100 & 3,000 & & & \\
\hline \multirow[t]{5}{*}{14050202} & 4691 & 000 & $\begin{array}{l}\text { Versuche und Erprobungen auf } \\
\text { kriegstechn. Gebiet }\end{array}$ & & 0,120 & 10 & 0,012 & 0,120 & 10 & 0,012 & 0,424 & 10 & 0,042 \\
\hline & & & Summe UG14 & & 7,887 & & 4,688 & 7,857 & & 4,684 & 6,361 & & 3,202 \\
\hline & & & $\begin{array}{l}\text { Summe BM für } \\
\text { Landesverteidigung }\end{array}$ & & 7,887 & & 4,688 & 7,857 & & 4,684 & 6,361 & & 3,202 \\
\hline & & & BM für Finanzen & & & & & & & & & & \\
\hline & & & UG15 & & & & & & & & & & \\
\hline 15010100 & 6430 & 001 & Arbeiten des WIIW & & 0,846 & 50 & 0,423 & 0,829 & 50 & 0,415 & 0,809 & 50 & 0,405 \\
\hline 15010100 & 6430 & 002 & Arbeiten des WSR & & 1,371 & 50 & 0,686 & 1,371 & 50 & 0,686 & 1,371 & 50 & 0,686 \\
\hline 15010100 & 6430 & 003 & Arbeiten des Wifo & & 4,250 & 52 & 2,210 & 4,167 & 52 & 2,167 & 4,085 & 50 & 2,043 \\
\hline 15010100 & 7270 & 000 & Werkleistungen durch Dritte & $*$ & 1,473 & 18 & 0,265 & 1,444 & 18 & 0,260 & 1,526 & 100 & 1,526 \\
\hline 15010100 & 7661 & 002 & $\begin{array}{l}\text { Institut für Finanzwissenschaft und } \\
\text { Steuerrecht }\end{array}$ & & & & & & & & & & \\
\hline 15010100 & 7662 & 002 & $\begin{array}{l}\text { Institut für höhere Studien und wiss. } \\
\text { Forschung }\end{array}$ & $*$ & 3,672 & 56 & 2,056 & 3,600 & 56 & 2,016 & 3,573 & 53 & 1,892 \\
\hline 15010100 & 7663 & 005 & Forum Alpbach & & & & & & & & & & \\
\hline 15010100 & 7666 & 020 & $\begin{array}{l}\text { Europ.Zentrum f. Wohlfahrtspolit. u. } \\
\text { Sozialforsch. }\end{array}$ & & & & & & & & & & \\
\hline \multirow[t]{6}{*}{15010100} & 7669 & 020 & Sonstige Förderungsbeiträge & $*$ & 0,300 & 100 & 0,300 & 0,300 & 100 & 0,300 & 0,826 & 27 & 0,226 \\
\hline & & & $\begin{array}{l}\text { Forschungswirksamer } \\
\text { Lohnnebenkostenanteil }\end{array}$ & & 25,935 & 100 & 25,935 & 26,312 & 100 & 26,312 & 24,835 & 100 & 24,835 \\
\hline & & & Summe UG15 & & 37,847 & & 31,875 & 38,023 & & 32,156 & 37,025 & & 31,613 \\
\hline & & & Summe BM für Finanzen & & 37,847 & & 31,875 & 38,023 & & 32,156 & 37,025 & & 31,613 \\
\hline & & & $\begin{array}{c}\text { BM für Arbeit, Soziales, Gesundheit } \\
\text { und Konsumentenschutz }\end{array}$ & & & & & & & & & & \\
\hline & & & UG20 & & & & & & & & & & \\
\hline 20010101 & 7340 & 302 & $\begin{array}{l}\text { Überweisung an das AMS gem. § } 41 \\
\text { (2) (zw) }\end{array}$ & $*$ & 496,610 & 1 & 4,966 & 471,610 & 1 & 4,716 & 469,610 & 1 & 3,242 \\
\hline 20010201 & 7270 & 006 & Werkleistungen durch Dritte (zw) & $*$ & 314,021 & & 0,471 & 335,145 & & 0,503 & 387,099 & & 0,646 \\
\hline \multirow[t]{3}{*}{20010201} & 7668 & 901 & $\begin{array}{l}\text { Nicht einzeln anzuführende } \\
\text { Subventionen (zw) }\end{array}$ & $*$ & & & & & & & 138,202 & 1 & 0,701 \\
\hline & & & Summe UG20 & & 810,631 & & 5,437 & 806,755 & & 5,219 & 994,911 & & 4,589 \\
\hline & & & UG21 & & & & & & & & & & \\
\hline 21010100 & 7270 & 000 & Werkleistungen durch Dritte & & 5,854 & 3 & 0,176 & 5,927 & 3 & 0,178 & 3,822 & 3 & 0,115 \\
\hline 21010300 & 7270 & 000 & Werkleistungen durch Dritte & & 1,026 & 16 & 0,164 & 0,894 & 16 & 0,143 & 0,894 & 16 & 0,143 \\
\hline 21010300 & 7660 & 900 & $\begin{array}{l}\text { Zuschüsse f. Ifd. Aufwand an private } \\
\text { Institutionen }\end{array}$ & & 2,500 & 2 & 0,050 & 3,094 & 2 & 0,062 & 4,705 & 2 & 0,094 \\
\hline 21010400 & 7262 & 001 & $\begin{array}{l}\text { Beitrag Europ. Zentrum } \\
\text { Wohlfahrtspol.u.Sozialfor. }\end{array}$ & & 0,587 & 50 & 0,294 & 0,587 & 50 & 0,294 & 0,587 & 50 & 0,294 \\
\hline 21010400 & 7270 & 000 & Werkleistungen durch Dritte & & 1,972 & 4 & 0,079 & 2,153 & 4 & 0,086 & 1,446 & 4 & 0,058 \\
\hline \multirow[t]{3}{*}{21010400} & 7270 & 304 & Werkleistungen EU-SILC & & 1,128 & 100 & 1,128 & 1,128 & 100 & 1,128 & 1,818 & 100 & 1,818 \\
\hline & & & Summe UG21 & & 13,067 & & 1,891 & 13,783 & & 1,891 & 13,272 & & 2,522 \\
\hline & & & UG24 & & & & & & & & & & \\
\hline 24010200 & 7420 & 012 & $\begin{array}{l}\text { Transferzahlungen, } \\
\text { Ernährungsagentur (Ges.m.b.H) }\end{array}$ & & 49,878 & 11 & 5,487 & 49,878 & 11 & 5,487 & 49,878 & 11 & 5,487 \\
\hline 24030100 & 7270 & 000 & Werkleistungen durch Dritte & & 3,657 & 4 & 0,146 & 4,004 & 4 & 0,160 & 1,630 & 4 & 0,065 \\
\hline \multirow[t]{5}{*}{24030200} & 7270 & 000 & Werkleistungen durch Dritte & & 5,165 & 2 & 0,103 & 5,165 & 2 & 0,103 & 4,830 & 2 & 0,097 \\
\hline & & & Summe UG24 & & 58,700 & & 5,736 & 59,047 & & 5,750 & 56,338 & & 5,649 \\
\hline & & & $\begin{array}{l}\text { Summe BM für Arbeit, Soziales, } \\
\text { Gesundheit und } \\
\text { Konsumentenschutz }\end{array}$ & & 882,398 & & 13,064 & 879,585 & & 12,860 & $1.064,521$ & & 12,760 \\
\hline & & & $\begin{array}{l}\text { BM für Bildung, Wissenschaft und } \\
\text { Forschung }\end{array}$ & & & & & & & & & & \\
\hline & & & UG30 & & & & & & & & & & \\
\hline 30010400 & & & Qualitätsentwicklung und -steuerung & $*$ & 32,395 & 8 & 2,592 & 41,277 & 8 & 3,302 & 35,206 & 8 & 2,816 \\
\hline 30010400 & 7340 & 000 & $\begin{array}{l}\text { Transferzahlungen an sonst. Träger } \\
\text { öffentl.Rechtes }\end{array}$ & & 0,261 & 100 & 0,261 & 0,247 & 100 & 0,247 & 0,119 & 100 & 0,119 \\
\hline 30010400 & 7340 & 003 & Basisabgeltung (BIFIE) & & 11,200 & 80 & 8,960 & 11,600 & 80 & 9,280 & 11,549 & 80 & 9,239 \\
\hline
\end{tabular}


Seite 5

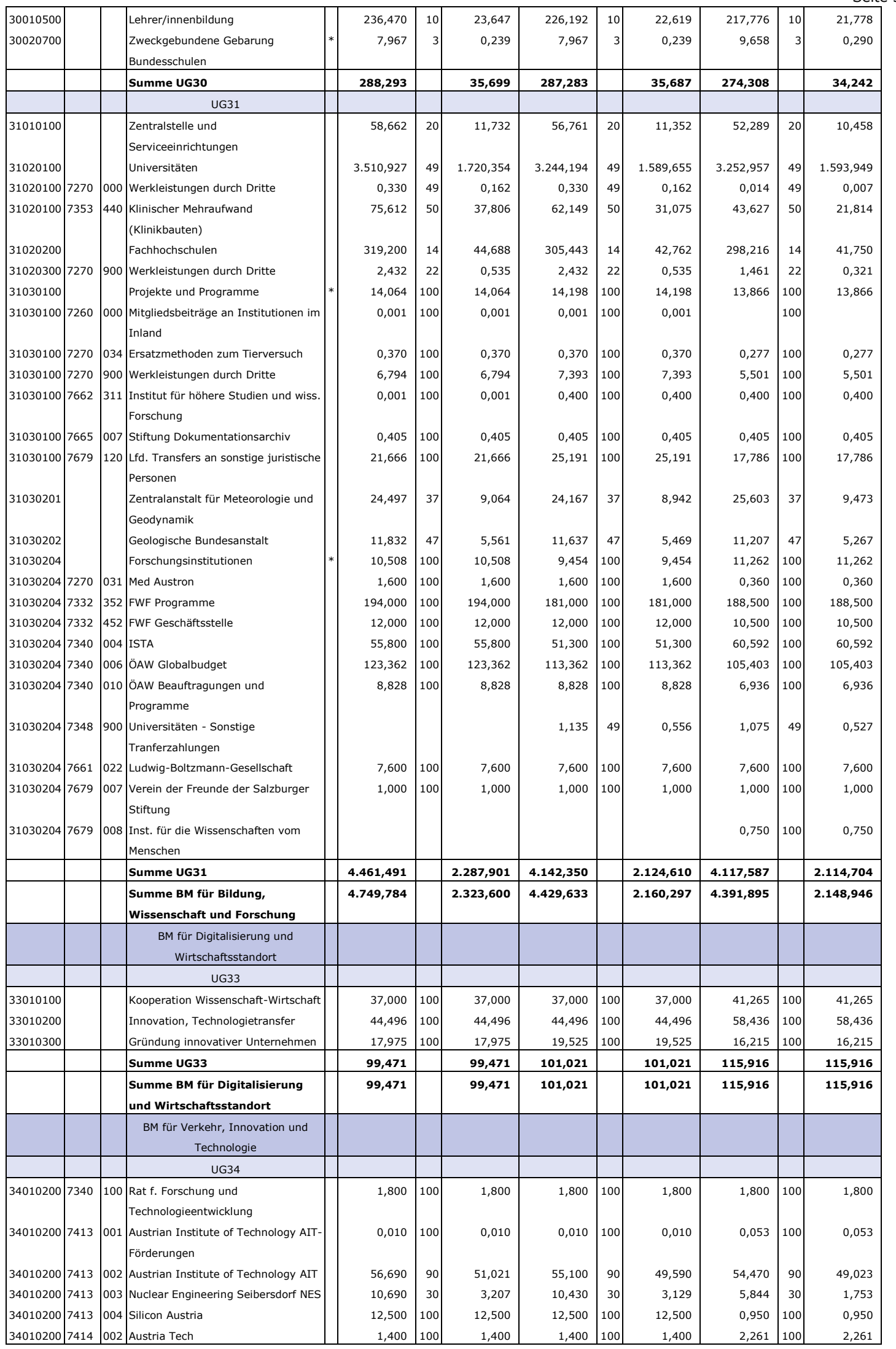




\begin{tabular}{|c|c|c|c|c|c|c|c|c|c|c|c|c|c|}
\hline 34010200 & 7660 & 075 & F\&T-Förderung & & 0,300 & 100 & 0,300 & 0,300 & 100 & 0,300 & 0,233 & 100 & 0,233 \\
\hline 34010200 & 7661 & 030 & $\begin{array}{l}\text { Österreichische } \\
\text { Computergesellschaft }\end{array}$ & & 0,040 & 100 & 0,040 & 0,040 & 100 & 0,040 & 0,053 & 100 & 0,053 \\
\hline 34010200 & 7662 & 341 & $\begin{array}{l}\text { Joanneum Research } \\
\text { Forsch.ges.m.b.H(Techn.schwerp) }\end{array}$ & & 2,350 & 100 & 2,350 & 2,350 & 100 & 2,350 & 2,239 & 100 & 2,239 \\
\hline 34010200 & 7666 & 005 & $\begin{array}{l}\text { Österreichisches Institut für } \\
\text { Nachhaltigkeit }\end{array}$ & & 0,030 & 100 & 0,030 & 0,030 & 100 & 0,030 & 0,050 & 100 & 0,050 \\
\hline 34010200 & 7667 & 006 & $\begin{array}{l}\text { Sonstige gemeinnützige } \\
\text { Einrichtungen }\end{array}$ & & 1,610 & 100 & 1,610 & 1,610 & 100 & 1,610 & 1,668 & 100 & 1,668 \\
\hline 34010200 & 7668 & 040 & Salzburg Research & & 0,410 & 100 & 0,410 & 0,410 & 100 & 0,410 & 0,376 & 100 & 0,376 \\
\hline 34010200 & 7668 & 050 & Profactor & & 0,500 & 100 & 0,500 & 0,500 & 100 & 0,500 & 0,315 & 100 & 0,315 \\
\hline 34010200 & 7690 & 002 & Preisverleihungen & & 0,010 & 100 & 0,010 & 0,010 & 100 & 0,010 & 0,010 & 100 & 0,010 \\
\hline 34010300 & 7260 & 000 & $\begin{array}{l}\text { Mitgliedsbeiträge an Institutionen im } \\
\text { Inland }\end{array}$ & & 0,160 & 100 & 0,160 & 0,160 & 100 & 0,160 & 0,156 & 100 & 0,156 \\
\hline 34010300 & 7270 & 000 & Werkleistungen durch Dritte & & 2,000 & 100 & 2,000 & 5,500 & 100 & 5,500 & 5,841 & 100 & 5,841 \\
\hline 34010300 & 7280 & 030 & $\begin{array}{l}\text { FTI-Projekte, Beauftragungen an } \\
\text { Dritte }\end{array}$ & & 1,700 & 100 & 1,700 & 1,700 & 100 & 1,700 & 1,476 & 100 & 1,476 \\
\hline 34010300 & 7330 & 352 & Translational research (F\&E) & & & 100 & & 0,095 & 100 & 0,095 & 0,430 & 100 & 0,430 \\
\hline 34010300 & 7330 & 652 & Fonds wissensch./Programmabw. & & & 100 & & 0,005 & 100 & 0,005 & 0,020 & 100 & 0,020 \\
\hline 34010300 & 7411 & 001 & FFG - Basisprogramme & & 95,000 & 100 & 95,000 & 95,000 & 100 & 95,000 & 77,000 & 100 & 77,000 \\
\hline 34010300 & 7411 & 002 & FFG - FTI-Programme, Förderungen & & 159,655 & 100 & 159,655 & 147,905 & 100 & 147,905 & 152,933 & 100 & 152,933 \\
\hline 34010300 & 7411 & 003 & $\begin{array}{l}\text { FFG - FTI-Programme (F\&E- } \\
\text { Dienstleist.,Sonst.WV) }\end{array}$ & & 10,000 & 100 & 10,000 & 10,000 & 100 & 10,000 & 9,627 & 100 & 9,627 \\
\hline 34010300 & 7411 & 004 & FFG - Administrative Kosten & & 16,700 & 100 & 16,700 & 16,700 & 100 & 16,700 & 14,552 & 100 & 14,552 \\
\hline 34010300 & 7412 & 001 & $\begin{array}{l}\text { Austria Wirtschaftsservice GmbH } \\
\text { AWS - Förderungen }\end{array}$ & & 11,254 & 100 & 11,254 & 13,373 & 100 & 13,373 & 11,548 & 100 & 11,548 \\
\hline 34010300 & 7412 & 003 & $\begin{array}{l}\text { Austria Wirtschaftsservice GmbH } \\
\text { AWS - Admin.Kost. }\end{array}$ & & 0,250 & 100 & 0,250 & 0,250 & 100 & 0,250 & 0,734 & 100 & 0,734 \\
\hline \multirow[t]{3}{*}{34010300} & 7432 & 030 & FTI-Projekte, Förderungen & & 0,350 & 100 & 0,350 & 0,350 & 100 & 0,350 & 0,610 & 100 & 0,610 \\
\hline & & & Summe UG34 & & 385,409 & & 372,257 & 377,528 & & 364,717 & 345,249 & & 335,711 \\
\hline & & & UG41 & & & & & & & & & & \\
\hline 41010200 & 7330 & 080 & $\begin{array}{l}\text { Transferzahlungen an Klima- und } \\
\text { Energiefonds }\end{array}$ & $*$ & 47,000 & 95 & 44,650 & 47,000 & 95 & 44,650 & 49,500 & 95 & 47,025 \\
\hline 41020100 & 7270 & 000 & Werkleistungen durch Dritte & & 1,726 & 50 & 0,863 & 1,726 & 50 & 0,863 & 1,782 & 40 & 0,713 \\
\hline 41020100 & 7270 & 800 & Elektromobilität & & 0,200 & 60 & 0,120 & 0,400 & 60 & 0,240 & 0,038 & 60 & 0,023 \\
\hline 41020100 & 7270 & 801 & $\begin{array}{l}\text { E-Mobilität für alle: Urbane } \\
\text { Elektromobilität }\end{array}$ & & 0,001 & 20 & & 0,001 & 20 & & & & \\
\hline 41020100 & 7411 & 002 & FFG - FTI-Programme, Förderungen & & 1,000 & 100 & 1,000 & 1,000 & 100 & 1,000 & 1,383 & 100 & 1,383 \\
\hline 41020100 & 7411 & 003 & $\begin{array}{l}\text { FFG - FTI-Programme (F\&E- } \\
\text { Dienstleist.,Sonst.WV) }\end{array}$ & & 0,010 & 100 & 0,010 & 0,010 & 100 & 0,010 & & & \\
\hline 41020100 & 7411 & 004 & FFG - Administrative Kosten & & 0,010 & 100 & 0,010 & 0,010 & 100 & 0,010 & & & \\
\hline 41020100 & 7480 & 501 & $\begin{array}{l}\text { Progr.Kombinierter } \\
\text { Güterverk.Straße-Schiene-Schiff }\end{array}$ & & 3,300 & 50 & 1,650 & 3,300 & 50 & 1,650 & 0,399 & 50 & 0,200 \\
\hline 41020100 & 7660 & 000 & $\begin{array}{l}\text { Zuschüsse f. Ifd. Aufwand an private } \\
\text { Institutionen }\end{array}$ & & 1,030 & 95 & 0,979 & 1,030 & 95 & 0,979 & 0,360 & 95 & 0,342 \\
\hline 41020100 & 7668 & 055 & Technisches Museum Wien & & 0,601 & 80 & 0,481 & 0,601 & 80 & 0,481 & 0,500 & 80 & 0,400 \\
\hline 41020300 & 7270 & 000 & Werkleistungen durch Dritte & & & & & & & & & & \\
\hline 41020300 & 7411 & 002 & FFG - FTI-Programme, Förderungen & & 0,001 & 50 & 0,001 & 0,001 & 50 & 0,001 & & & \\
\hline 41020300 & 7411 & 004 & FFG - Administrative Kosten & & 0,001 & 50 & 0,001 & 0,001 & 50 & 0,001 & & & \\
\hline 41020300 & 7489 & 001 & Breitbandinitiative (admin. Aufwand) & & 0,001 & 50 & 0,001 & 0,001 & 50 & 0,001 & & & \\
\hline 41020300 & 7489 & 002 & Breitband - Förderungen & & 0,001 & 50 & 0,001 & 0,001 & 50 & 0,001 & 0,012 & 50 & 0,006 \\
\hline 41020402 & 7270 & 000 & Werkleistungen durch Dritte & & 1,029 & 5 & 0,051 & 1,159 & 5 & 0,058 & 0,552 & 5 & 0,028 \\
\hline \multirow[t]{5}{*}{41020402} & 7270 & 006 & Werkleistungen durch Dritte (zw) & & 1,750 & 5 & 0,088 & 1,750 & 5 & 0,088 & 2,725 & 5 & 0,136 \\
\hline & & & Summe UG41 & & 57,661 & & 49,906 & 57,991 & & 50,033 & 57,251 & & 50,256 \\
\hline & & & $\begin{array}{l}\text { Summe BM für Verkehr, } \\
\text { Innovation und Technologie }\end{array}$ & & 443,070 & & 422,163 & 435,519 & & 414,750 & 402,500 & & 385,967 \\
\hline & & & BM für Nachhaltigkeit und Tourismus & & & & & & & & & & \\
\hline & & & UG42 & & & & & & & & & & \\
\hline 42010100 & & & Zentralstelle & $*$ & 0,200 & 100 & 0,200 & 0,200 & 100 & 0,200 & 0,190 & 100 & 0,190 \\
\hline 42010200 & 7411 & 000 & $\begin{array}{l}\text { Lfd Transfers an verbundene } \\
\text { Unternehmungen }\end{array}$ & $*$ & 37,303 & 33 & 12,310 & 37,303 & 33 & 12,310 & 37,302 & 33 & 12,310 \\
\hline 42020300 & & & Forschung und Sonstige Maßnahmen & * & 2,000 & 100 & 2,000 & 2,000 & 100 & 2,000 & 1,964 & 100 & 1,964 \\
\hline 42020401 & & & Landwirtschaftliche Schulen & $*$ & 45,065 & 21 & 9,464 & 43,731 & 21 & 9,184 & 47,174 & 21 & 9,907 \\
\hline 42020402 & & & Landwirtschaftliche Hochschule & & 5,172 & 3 & 0,155 & 5,100 & 3 & 0,153 & 4,749 & 3 & 0,142 \\
\hline
\end{tabular}


Seite 7

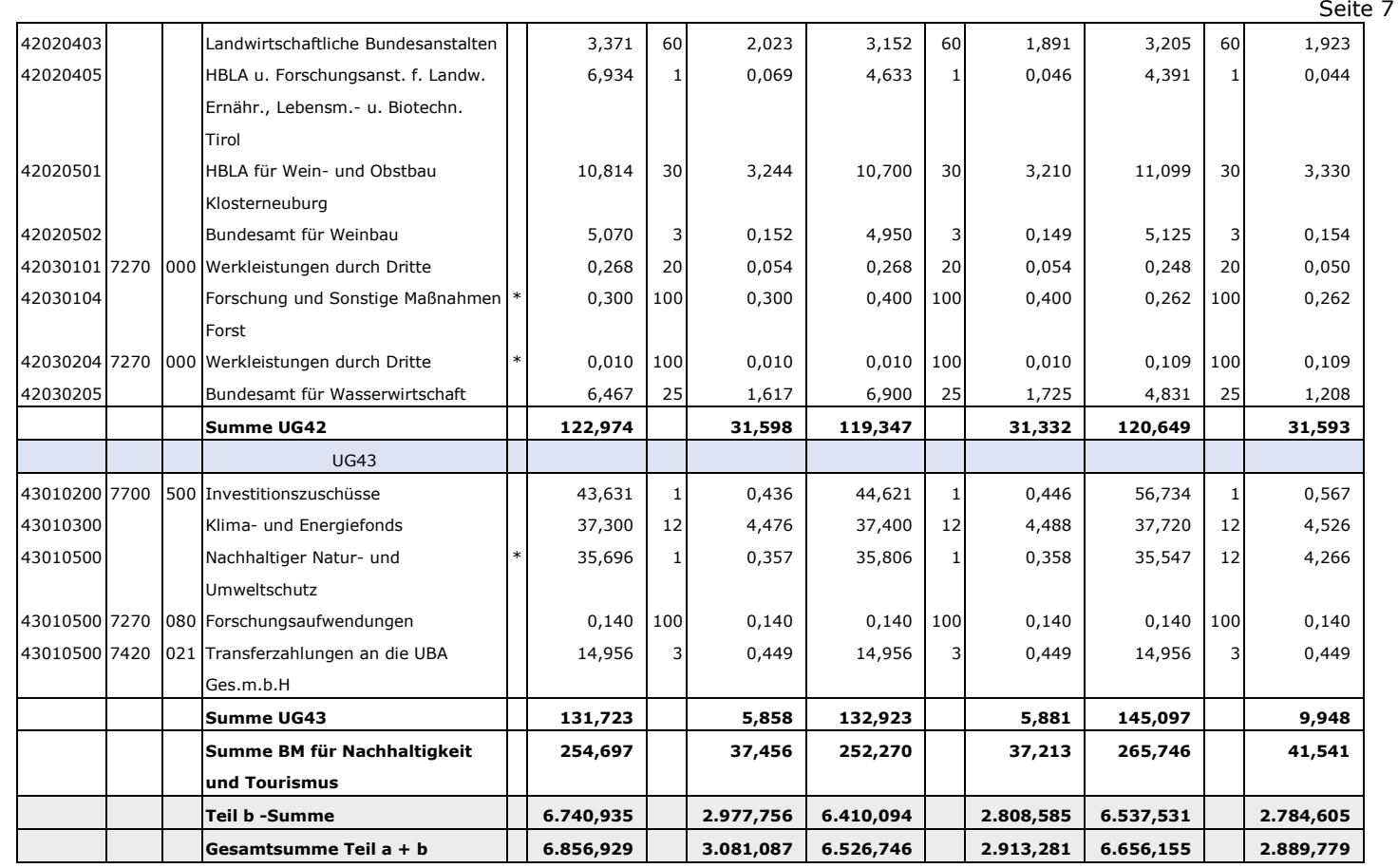


BUNDESVORANSCHLAG 2019

Detailübersicht Forschungswirksame Mittelverwendungen des Bundes

Anmerkungen

\begin{tabular}{|c|c|c|c|}
\hline \multicolumn{4}{|c|}{ Allgemeine Anmerkungen } \\
\hline \multicolumn{4}{|c|}{ *) F\& E Koeffizienten geschätzt } \\
\hline \multicolumn{4}{|c|}{ a) Beitragszahlungen aus Bundesmitteln an internationale Organisationen, die Forschung und Forschungsförderung (mit) als Ziel haben, } \\
\hline \multicolumn{4}{|c|}{ b) Bundesbudget-Forschung - Finanzierungsvorschlag (ausgen. die bereits im Abschnitt a) ausgewiesen sind) } \\
\hline \multicolumn{4}{|c|}{ Für die Aufstellung dieser Ausgaben ist in erster Linie der Gesichtspunkt der Forschungswirksamkeit maßgebend, der inhaltlich über den Aufgabenbereich 99} \\
\hline \multirow{4}{*}{\multicolumn{4}{|c|}{$\begin{array}{l}\text { "Grundlagen-, angewandte Forschung und experimentelle Entwicklung" hinausgeht und auf dem Forschungsbegriff des Fascati-Handbuches der OECD beruht, wie er } \\
\text { im Rahmen der forschungsstatistischen Erhebungen der STATISTIK AUSTRIA zur Anwendung gelangt. } \\
\text { Forschungswirksame Anteile bei den Bundesausgaben finden sich daher nicht nur bei den Ausgaben des Aufgabenbereiches } 99 \text { "Grundlagen-, angewandte Forschung } \\
\text { und experimentelle Entwicklung" sondern auch in zahlreichen anderen Aufgabenbereichen. }\end{array}$}} \\
\hline & & & \\
\hline & & & \\
\hline & & & \\
\hline \multicolumn{4}{|c|}{ Finanzierungsvoranschlag } \\
\hline \multirow[t]{2}{*}{ VA-Stelle } & Konto & Ugl & Anmerkung \\
\hline & & & Parlamentsdirektion \\
\hline \multirow[t]{2}{*}{02010500} & 7330 & 086 & *) Forschungsanteil liegt bei 2,600 \% (System rundet). \\
\hline & & & Bundeskanzleramt \\
\hline 25010500 & 7420 & 113 & *) Forschungsanteil liegt bei $37,570 \%$ (System rundet). \\
\hline 25010500 & 7270 & 006 & *) Forschungsanteil liegt bei $88,589 \%$ (System rundet). \\
\hline 25020100 & 7270 & 000 & *) Forschungsanteil liegt bei $1,714 \%$ (System rundet). \\
\hline \multirow[t]{2}{*}{25020200} & 7270 & 000 & *) Forschungsanteil liegt bei 2,035 \% (System rundet). \\
\hline & & & BM für Inneres \\
\hline 11010100 & 7278 & 020 & *) Teilbetrag der Voranschlagsstelle. \\
\hline 11010200 & 7270 & 900 & *) Teilbetrag der Voranschlagsstelle. \\
\hline 11020600 & 7270 & 900 & *) Teilbetrag der Voranschlagsstelle. \\
\hline 11020600 & & & * Teilbetrag der Voranschlagsstelle \\
\hline 11020800 & 7270 & 900 & *) Teilbetrag der Voranschlagsstelle. \\
\hline 11030100 & 7660 & 900 & $\begin{array}{l}\text { *) Teilbetrag der Voranschlagsstelle. Aufgrund der Budgetstrukturänderung wurde die Voranschlagsstelle } 11030100 \text { ab } 2018 \text { in } \\
\text { die Voranschlagsstelle } 18010100 \text { überführt. }\end{array}$ \\
\hline 11030100 & 7672 & 009 & $\begin{array}{l}\text { *) Teilbetrag der Voranschlagsstelle. Aufgrund einer Budgetstrukturänderung wurde die Voranschlagsstelle } 11030100 \text { ab } 2018 \text { in } \\
\text { die Voranschlagsstelle } 18010100 \text { überführt. }\end{array}$ \\
\hline 11030500 & 7270 & 900 & *) Teilbetrag der Voranschlagsstelle. \\
\hline 18010100 & 7672 & 009 & *) Teilbetrag der Voranschlagsstelle \\
\hline \multirow[t]{3}{*}{18010100} & 7660 & 900 & $\begin{array}{l}\text { *) Aufgrund einer Budgetstrukturänderung wurde die Voranschlagsstelle } 11030100 \text { ab } 2018 \text { in die Voranschlagsstelle } 18010100 \\
\text { überführt. }\end{array}$ \\
\hline & & & *) Teilbetrag der Voranschlagsstelle. \\
\hline & & & BM für Europa, Integration und Äußeres \\
\hline \multirow[t]{2}{*}{12020200} & 7840 & 000 & Beiträge an die IAEO (Internationale Atomenergieorganisation) zur Förderung der internationalen Bemühungen um nukleare \\
\hline & & & Sicherheit und Nichtverbreitung von Kernwaffen sowie zum Atomteststopp. \\
\hline 12020200 & 7800 & 101 & *) BMG-Novelle \\
\hline \multirow[t]{2}{*}{12020200} & 7800 & 102 & *) BMG-Novelle . \\
\hline & & & BM für Verfassung, Reformen, Deregulierung und Justiz \\
\hline \multirow[t]{3}{*}{13010100} & 7271 & 900 & *) Studie zum "Umgang mit Misshandlungsvorwürfen gegen Exekutivbedienstete" (Auftragnehmer:ALES) Auftragsvolumen 2018: \\
\hline & & & 50.158 Euro + Studie des Instituts für Konfliktforschung zum Thema "Schutz der sexuellen Integrität" Auftragsvolumen 76.500 \\
\hline & & & Euro (davon 2018: 38250 Euro und 2019: 38.250 Euro) \\
\hline \multirow[t]{3}{*}{13030101} & 7271 & 900 & *) Reduktion um $€ 33.100,00$ für Studie De-Radikalisierung im Gefängnis, Erhöhung um gesamt $€ 79.440,79$ für Projekt \\
\hline & & & Suizidprävention im Strafvollzug \\
\hline & & & BM für Landesverteidigung \\
\hline \multicolumn{2}{|l|}{14040100} & & *) Teilbetrag (eigene Fistl); \\
\hline 14050100 & 7270 & 900 & *) Teilbetrag der Voranschlagsstelle. \\
\hline \multirow[t]{2}{*}{14050100} & 7270 & 000 & *) Teilbetrag der Voranschlagsstelle. \\
\hline & & & BM für Finanzen \\
\hline 15010100 & 7662 & 002 & *) Forschungsanteil liegt bei 52,939 (System rundet) \\
\hline 15010100 & 7669 & 020 & *) Teilbetrag der Voranschlagsstelle. Forschungsanteil liegt bei 27,361 \% (System rundet). \\
\hline \multirow[t]{2}{*}{15010100} & 7270 & 000 & *) Teilbetrag der Voranschlagsstelle. \\
\hline & & & BM für Arbeit, Soziales, Gesundheit und Konsumentenschutz \\
\hline 20010101 & 7340 & 302 & *) Forschungsanteil liegt bei $0,69 \%$ (System rundet auf $1 \%$ ) \\
\hline 20010201 & 7668 & 901 & *) Forschungsanteil liegt bei 0,51 \% (System rundet auf $1 \%$ ) \\
\hline 20010201 & 7270 & 006 & *) Forschungsanteil im Erfolg 2017 liegt bei 0,17 \% (System rundet auf $0 \%$ ) \\
\hline
\end{tabular}




\begin{tabular}{|c|c|c|c|}
\hline & & & *) Forschungsanteil im BVA 2019 liegt bei 0,15 \% (System rundet auf $0 \%$ ) \\
\hline & & & BM für Bildung, Wissenschaft und Forschung \\
\hline 30010400 & 7800 & 000 & *) Teilbetrag der VA-Stelle. \\
\hline 30010400 & & & Teilbetrag der Voranschlagsstelle \\
\hline 30020700 & & & Teilbetrag der Voranschlagsstelle \\
\hline 31030100 & & & *) Der Restbetrag ergibt sich rechnerisch bei dieser VA-Stelle. \\
\hline 31030204 & & & $\begin{array}{l}\text { *) Der Restbetrag ergibt sich rechnerisch bei dieser VA-Stelle. } \\
\text { BM für Verkehr, Innovation und Technologie }\end{array}$ \\
\hline 41010200 & 7330 & 080 & $\begin{array}{l}\text { * KLIEN: ab } 2016 \text { werden bei dieser Post nur mehr F\&E-Projekte finanziert; daher die Erhöhung von } 39 \text { auf } 95 \% \text {. } \\
\text { BM für Nachhaltigkeit und Tourismus }\end{array}$ \\
\hline 42010100 & & & *) PSP-Element 42P101010001, 42P101010002 und 42P101020002. \\
\hline 42010200 & 7411 & 000 & Finanzstellen 90306 (AGES) und 90309 (BFW). \\
\hline 42020202 & 7411 & 000 & *) Austrian Development Agency (ADA) \\
\hline 42020202 & 7270 & 000 & *) Austrian Development Agency (ADA), Aufwandsentschädigung \\
\hline 42020300 & & & PDP-Element 42P101010001 und 42P10102001 \\
\hline 42020401 & & & *) Finanzstellen 22010 (Francisco-Josephinum), 22013 (Raumberg-Gumpenstein), 22016 (Gartenbau). \\
\hline 42030104 & & & *) PSP-Element 42P101020002 \\
\hline 42030204 & 7270 & 000 & *) Finanzstelle 701 (Nat. u. int. Wasserwirtschaft), Teilbetrag von 7270.000. \\
\hline 43010500 & & & *) Teilbetrag der VA-Stelle. \\
\hline \multicolumn{4}{|c|}{ Ergebnisvoranschlag } \\
\hline VA-Stelle & Konto & Ugl & Anmerkung \\
\hline
\end{tabular}




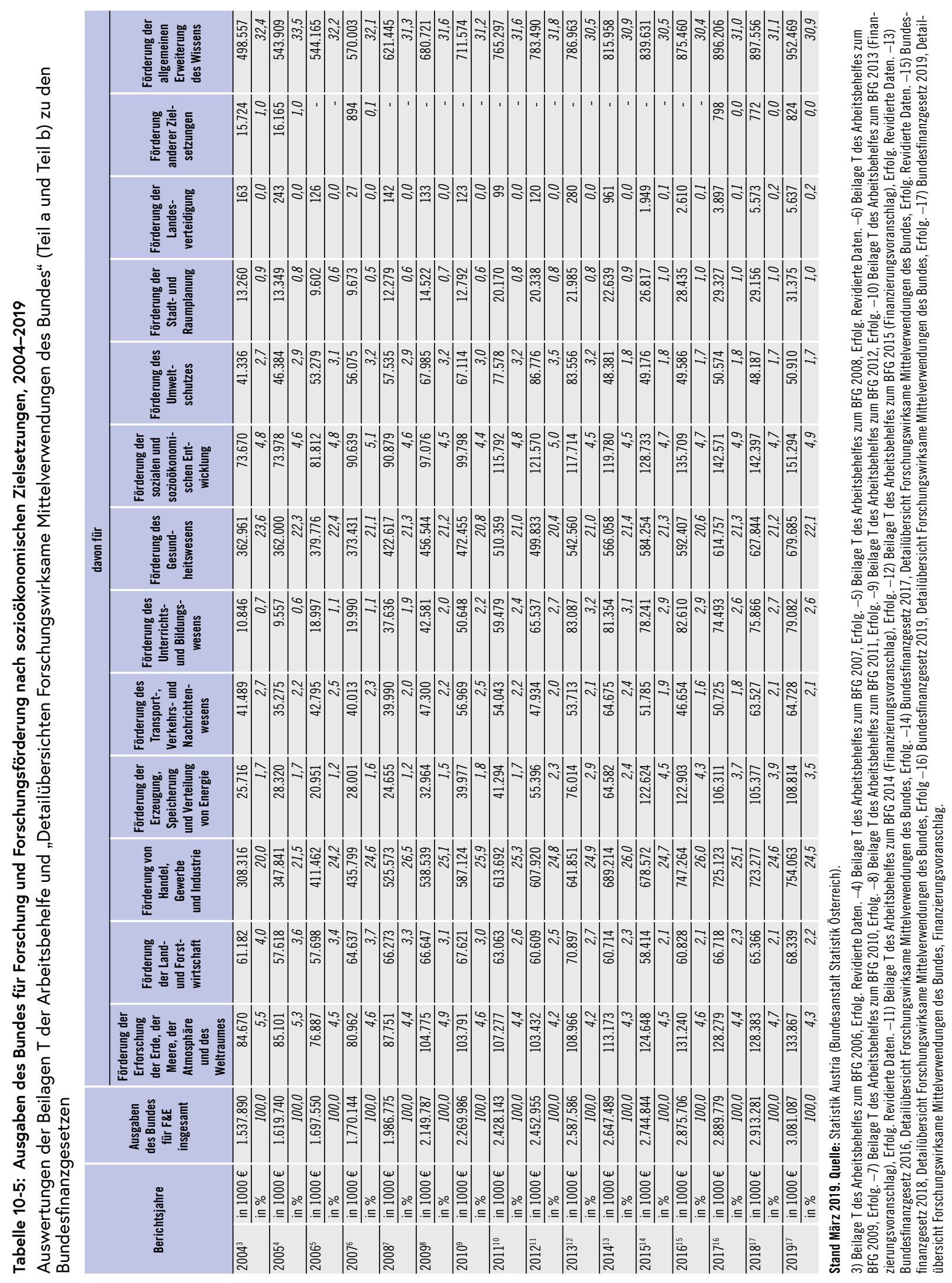




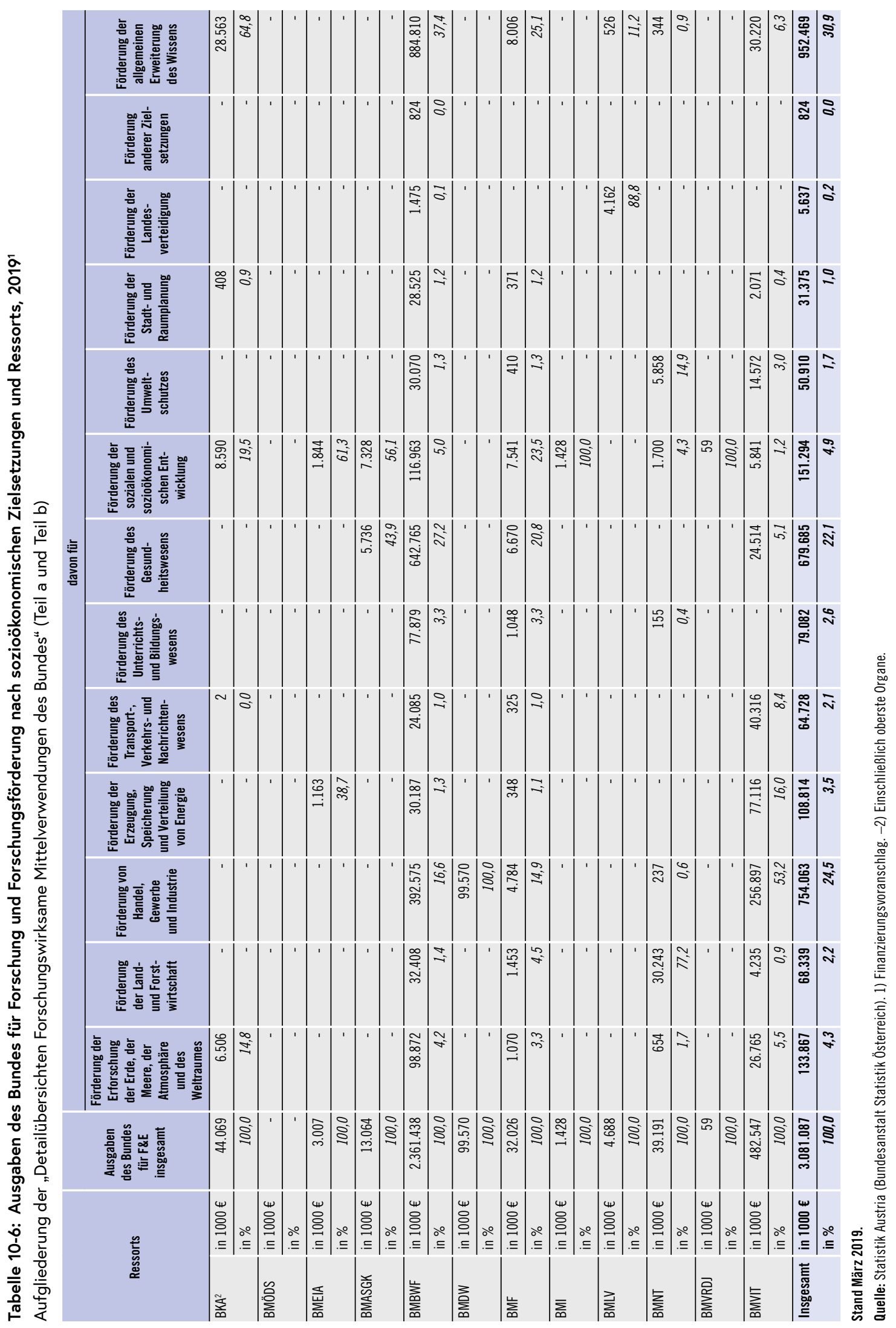


Tabelle 10-7: Allgemeine forschungswirksame Hochschulausgaben des Bundes („General University Funds“), 2000-20191

\begin{tabular}{|c|c|c|}
\hline \multirow{3}{*}{ Jahre } & \multicolumn{2}{|c|}{ Allgemeine Hochschulausgaben } \\
\hline & insgesamt & F\&E \\
\hline & \multicolumn{2}{|c|}{ in Mio. $€$} \\
\hline 2000 & $1.956,167$ & 842,494 \\
\hline 2001 & $2.008,803$ & 866,361 \\
\hline 2002 & $2.104,550$ & 918,817 \\
\hline 2003 & $2.063,685$ & 899,326 \\
\hline 2004 & $2.091,159$ & 980,984 \\
\hline 2005 & $2.136,412$ & $1.014,543$ \\
\hline 2006 & $2.157,147$ & $1.027,270$ \\
\hline 2007 & $2.314,955$ & $1.083,555$ \\
\hline 2008 & $2.396,291$ & $1.133,472$ \\
\hline 2009 & $2.626,038$ & $1.236,757$ \\
\hline 2010 & $2.777,698$ & $1.310,745$ \\
\hline 2011 & $2.791,094$ & $1.388,546$ \\
\hline 2012 & $2.871,833$ & $1.395,130$ \\
\hline 2013 & $3.000,004$ & $1.453,596$ \\
\hline 2014 & $3.059,949$ & $1.481,744$ \\
\hline 2015 & $3.117,320$ & $1.509,576$ \\
\hline 2016 & $3.262,376$ & $1.610,742$ \\
\hline 2017 & $3.319,288$ & $1.638,460$ \\
\hline 2018 & $3.330,311$ & $1.644,530$ \\
\hline 2019 & $3.610,048$ & $1.781,501$ \\
\hline
\end{tabular}

\section{Stand März 2019.}

Quelle: Statistik Austria (Bundesanstalt Statistik Österreich).

1) 2000-2019: Auf Basis der Beilagen T der Arbeitsbehelfe und „Detailübersichten Forschungswirksame Mittelverwendungen des Bundes“ zu den Bundesfinanzgesetzen. 


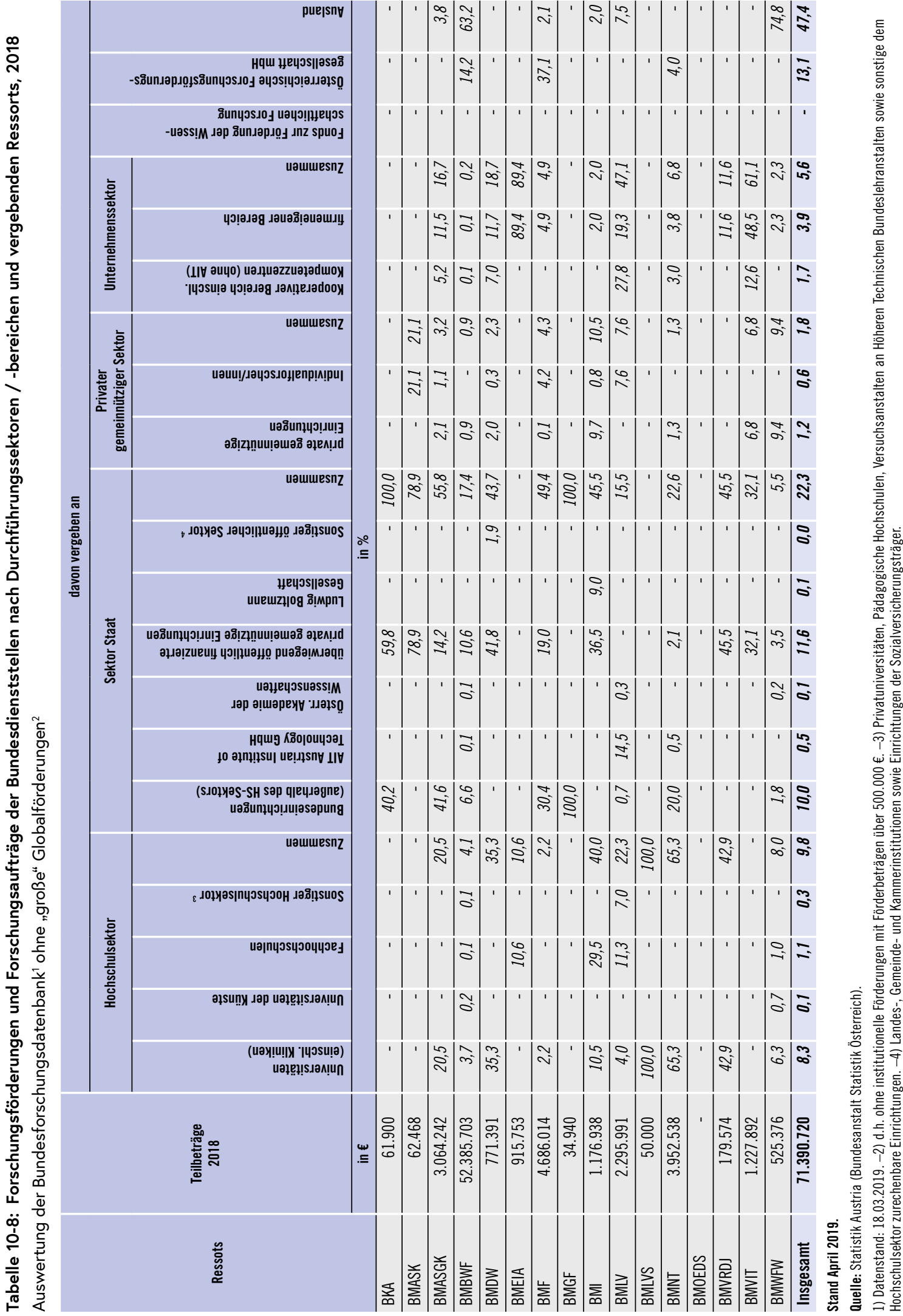




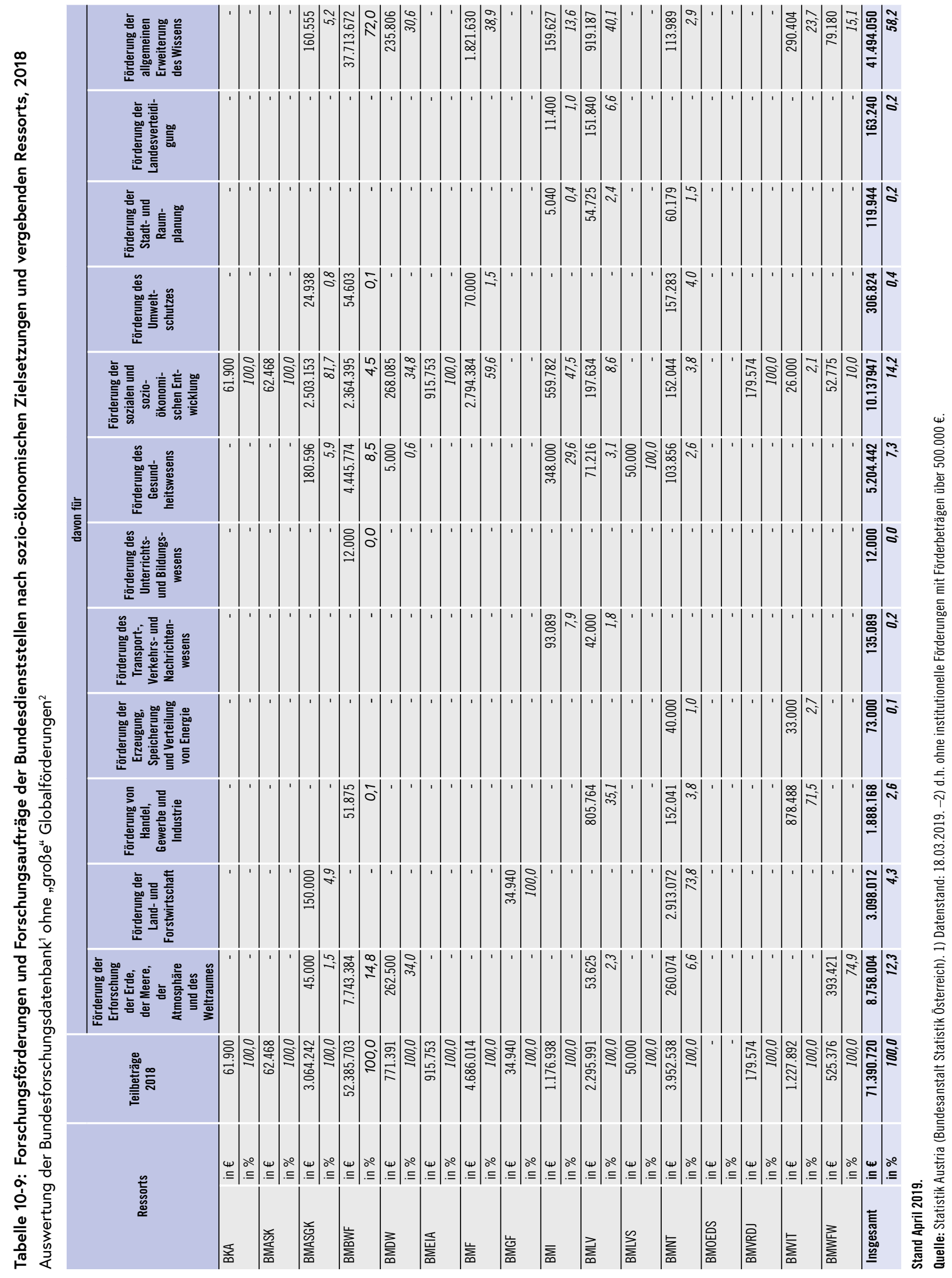


Tabelle 10-10: Forschung und experimentelle Entwicklung (F\&E) im internationalen Vergleich, 2016

\begin{tabular}{|c|c|c|c|c|c|c|c|c|}
\hline \multirow{4}{*}{ Länder } & \multirow{4}{*}{$\begin{array}{l}\text { Brutto- } \\
\text { inlands- } \\
\text { ausgaben } \\
\text { für F\&E } \\
\text { in \% } \\
\text { des BIP }\end{array}$} & \multirow{2}{*}{\multicolumn{2}{|c|}{$\begin{array}{l}\text { Finanzierung der } \\
\text { Bruttoinlandsausgaben } \\
\text { für F\&E durch }\end{array}$}} & \multirow{4}{*}{$\begin{array}{l}\text { Beschäftigte } \\
\text { in F\&E } \\
\text { in Vollzeit- } \\
\text { äquivalenten }\end{array}$} & \multicolumn{4}{|c|}{ Bruttoausgaben für F\&E des } \\
\hline & & & & & \multirow{2}{*}{$\begin{array}{l}\text { Unter- } \\
\text { nehmens- } \\
\text { sektors }\end{array}$} & \multirow{2}{*}{$\begin{array}{l}\text { Hochschul- } \\
\text { sektors }\end{array}$} & \multirow{2}{*}{$\begin{array}{l}\text { Sektors } \\
\text { Staat }\end{array}$} & \multirow{2}{*}{$\begin{array}{l}\text { privaten } \\
\text { gemein- } \\
\text { nützigen } \\
\text { Sektors }\end{array}$} \\
\hline & & Staat & Wirtschaft & & & & & \\
\hline & & \multicolumn{2}{|c|}{ in $\%$} & & \multicolumn{4}{|c|}{ in \% der Bruttoinlandsausgaben für F\&E } \\
\hline Belgien & $2,55^{\text {e) }}$ & $22,5^{5)}$ & $58,6^{5)}$ & 79.109 е) & 70,1 е) & 20,5 e) & 8,9 е) & $0,6^{\text {e) }}$ \\
\hline Dänemark & 3,12 & $30,2 \mathrm{~d}(5)$ & $59,1^{5)}$ & 62.869 & 64,8 & 32,7 & 2,2 & 0,3 \\
\hline Deutschland & 2,92 & $28,5^{d)}$ & 65,2 & 657.894 & 68,2 & 18,0 b) & $13,8 \mathrm{~d}$ & . \\
\hline Finnland & 2,74 & 28,9 & 57,0 & 47.429 & 65,8 & 25,1 & 8,2 & 0,9 \\
\hline Frankreich & $2,25 \mathrm{pl}$ & $34,8^{5)}$ & $54,0^{5)}$ & $428.643^{5)}$ & 63,6 p) & 22,0 p) & 12,9 p) & $1,6^{\mathrm{p})}$ \\
\hline Griechenland & 0,99 & 42,6 & 40,2 & $41.790 \mathrm{~d})$ & 42,2 & 31,9 & 25,0 & 0,9 \\
\hline Irland & $1,16^{\mathrm{e})}$ & $25,8^{\text {e) }}$ & 49,0 e) & 29.849 & 72,2 e) & $23,6^{\text {e) }}$ & 4,2 e) & . \\
\hline Italien b) & 1,37 & 35,2 & 52,1 & 290.040 & 60,8 & 24,2 e) & 12,6 & 2,5 \\
\hline Luxemburg & 1,30 & $47,7^{5)}$ & $47,1^{5)}$ & 5.312 & 54,1 & 19,7 e) & 26,3 d)e) & . \\
\hline Österreich & $3,13^{6)}$ & $35,1^{6)}$ & $48,4^{6)}$ & $74.897^{\text {е) }}$ & 70,2 ble) & 22,2 b)e & $7,1^{\text {bee) }}$ & $0,5^{\mathrm{e})}$ \\
\hline Portugal & 1,28 & 42,6 & 44,4 & 50.406 & 48,4 & 44,7 & 5,3 & 1,6 \\
\hline Schweden & 3,27 p) & $28,3^{\mathrm{e} / 4)}$ & $57,3^{5)}$ & 90.690 p) & $69,6^{\mathrm{p})}$ & $26,8^{\mathrm{pl}}$ & $3,4^{\mathrm{p})}$ & $0,2^{\mathrm{p})}$ \\
\hline Spanien & 1,19 & 40,0 & 46,7 & 205.873 d) & 53,7 & 27,5 & 18,5 & 0,2 \\
\hline Vereinigtes Königreich ${ }^{\text {e) }}$ & 1,68 & 26,3 & 51,8 & 417.390 & 67,1 & 24,3 & 6,6 & 2,1 \\
\hline EU $15^{\text {e) }}$ & 2,10 & $31,4^{5)}$ & $55,9^{5)}$ & 2.618 .029 & 65,0 & 22,7 & 11,2 & 1,0 \\
\hline Estland & 1,25 & 37,6 & 48,2 & 5.772 & 51,5 & 35,5 & 11,4 & 1,5 \\
\hline Lettland & 0,44 & 47,7 & 21,6 & 5.120 d) & 24,5 & 43,8 & 31,8 & . \\
\hline Litauen & 0,84 & 39,2 & 39,0 & 10.924 & 35,0 & 38,9 & 26,1 & . \\
\hline Polen & 0,96 & 38,9 & 53,1 & 111.789 & $65,7 \mathrm{~b}$ & 31,4 & $2,5^{\mathrm{b})}$ & 0,4 \\
\hline Slowakische Republik & 0,79 & 41,0 & 46,2 & 17.768 & 50,4 & 27,7 & 21,4 & 0,5 \\
\hline Slowenien & 2,01 & 20,2 & 69,2 & 14.403 & 75,7 & 10,8 & 13,4 & 0,0 \\
\hline Rumänien & 0,48 & 39,6 & 49,4 & 32.232 & 55,2 & 11,3 & 33,3 & 0,2 \\
\hline EU-28 e) & 1,94 & $31,8^{5)}$ & $54,6^{5)}$ & 2.957 .034 & 64,9 & 22,8 & 11,4 & 1,0 \\
\hline Australien & $1,88^{\mathrm{e}) 5)}$ & $34,6^{1)}$ & $61,9^{11}$ & 147.809 е)2) & 53,4 e,5) & $30,6^{\mathrm{e} \mid 5)}$ & 12,7 e)5) & 3,2 e)5) \\
\hline Chile $^{\text {p) }}$ & $0,36^{b)}$ & $46,4^{\text {b) }}$ & $35,8^{\text {b) }}$ & $16.633^{\text {d) }}$ & $38,5^{b)}$ & $41,8^{\mathrm{b})}$ & $13,2^{\mathrm{b})}$ & $6,5^{\text {b) }}$ \\
\hline Island & 2,03 & 34,2 & 35,0 & 3.247 & 63,0 d) & 32,1 & 4,9 & . \\
\hline Israel d)e & 4,39 & 13,5 & 34,7 & $77.143^{3)}$ & 85,6 & 11,8 & 1,6 & 1,0 \\
\hline Japan & 3,14 & $15,0^{\text {e) }}$ & 78,1 & 872.340 d) & 78,8 & 12,3 & 7,5 & 1,4 \\
\hline Kanada & 1,70 & 31,3 e) & 42,2 & 223.146 & 52,6 & 40,2 & 6,8 & 0,5 \\
\hline Korea & 4,23 & 22,7 & 75,4 & 447.408 & 77,7 & 9,1 & 11,5 & 1,6 \\
\hline Mexiko & 0,49 e)p) & $67,4^{\mathrm{epp} p}$ & 20,7 elp) & $59.073^{4)}$ & 30,6 elp) & $26,8^{\mathrm{elp} p}$ & $36,5^{\mathrm{elp} p}$ & $6,2^{\mathrm{e}) \mathrm{p})}$ \\
\hline Neuseeland $^{5 \text { ) }}$ & 1,23 & 37,1 & 43,8 & 26.400 & 51,1 & 28,0 & 20,9 & . \\
\hline Norwegen & 2,03 & 45,7 & 43,2 & 43.918 & 53,3 & 32,6 & 14,2 & . \\
\hline Schweiz $^{5)}$ & 3,37 & 24,4 & 63,5 & 81.451 & 71,0 & 26,7 & $0,9 \mathrm{~d})$ & 1,5 \\
\hline Türkei & 0,94 & 35,1 & 46,7 & 136.953 & 54,2 & 36,3 & 9,5 d) & . \\
\hline Vereinigte Staaten ${ }^{\mathrm{d} p \mathrm{p})}$ & 2,76 & 23,6 & 63,2 & . & 72,6 & 13,1 & 10,2 & $4,1^{\mathrm{e})}$ \\
\hline OECD insgesamt ${ }^{\mathrm{e})}$ & 2,34 & 25,8 & 61,4 & . & 69,9 & 17,5 & 10,2 & 2,4 \\
\hline Volksrepublik China & 2,11 & 20,0 & 74,7 & 3.878 .057 & 77,5 & 6,8 & 15,7 & . \\
\hline
\end{tabular}

Quelle: OECD (MSTI 2018-2), Statistik Austria (Bundesanstalt Statistik Österreich).

b) Bruch in der Zeitreihe. -d) Abweichende Definition. -e) Geschätzte Werte. -p) Vorläufige Werte.

1) 2008. -2) 2010. -3) 2012. -4) 2013. -5) 2015. -6) Statistik Austria; entsprechend F\&E-Globalschätzung 2019.

Vollzeitäquivalent $=$ Personenjahr. 
Tabelle 10-11: Österreichs Teilnahme vom 4. Europäischen Rahmenprogramm bis Horizon 2020

\begin{tabular}{|c|c|c|c|c|c|}
\hline & FP4 & FP5 & FP6 & FP7 & H2O20 \\
\hline & $1994-1998$ & 1998-2002 & 2002-2006 & 2007-2013 & 2014 bis $03 / 2019$ \\
\hline Anzahl der bewilligten Projekte mit österr. Beteiligung & 1,444 & 1,384 & 1,324 & 2,452 & 1,894 \\
\hline Anzahl der bewilligten österr. Beteiligungen & 1,923 & 1,987 & 1,972 & 3,589 & 2,919 \\
\hline Anzahl der bewilligten Projekte mit österr. Koordination & 270 & 267 & 213 & 676 & 439 \\
\hline Bewilligtes Budget in Mio. $€$ & 194 & 292 & 425 & 1,192 & 1,108 \\
\hline $\begin{array}{l}\text { Anteil der bewilligten österr. Beteiligungen an allen bewilligten } \\
\text { Beteiligungen }\end{array}$ & $2,3 \%$ & $2,4 \%$ & $2,6 \%$ & $2,6 \%$ & $2,8 \%$ \\
\hline $\begin{array}{l}\text { Anteil der bewilligten österr. Koordinationen an allen bewilligten } \\
\text { Koordinationen }\end{array}$ & $1,7 \%$ & $2,8 \%$ & $3,3 \%$ & $2,7 \%$ & $2,6 \%$ \\
\hline Anteil Österreichs am bewilligten Budget & $1,99 \%$ & $2,38 \%$ & $2,56 \%$ & $2,63 \%$ & $2,81 \%$ \\
\hline
\end{tabular}

Quelle: Proviso Überblickreport vom Herbst 2013 (FP4-FP6); EK 11/2015 (FP7) sowie FFG (mit Datenstand 03/2019 für Horizon 2020).

Tabelle 10-12: Österreichische Projekte, Beteiligungen und Koordinationen in Horizon 2020 differenziert nach Organisationstypen und Bundesländern

\begin{tabular}{|c|c|c|c|c|c|c|c|c|c|c|c|}
\hline & Alle Staaten & Österreich & B & Ktn & NÖ & $00 ̈$ & Sbg & St & $\mathrm{T}$ & V & W \\
\hline Projekte & 21.472 & 1.894 & 18 & 79 & 200 & 190 & 60 & 455 & 131 & 22 & 1.151 \\
\hline Beteiligungen & 104.427 & 2.919 & 20 & 106 & 220 & 248 & 67 & 611 & 150 & 24 & 1.473 \\
\hline HES & 34.443 & 813 & 4 & 16 & 51 & 40 & 28 & 157 & 77 & & 440 \\
\hline REC & 21.799 & 660 & 9 & 5 & 36 & 61 & 11 & 189 & & & 349 \\
\hline PRC & 36.229 & 1.109 & 7 & 75 & 117 & 121 & 22 & 240 & 65 & 20 & 442 \\
\hline PUB & 6.138 & 154 & & 6 & & 7 & 2 & 6 & 8 & 3 & 122 \\
\hline OTH & 5.818 & 183 & & 4 & 16 & 19 & 4 & 19 & & 1 & 120 \\
\hline nicht KMU-deklariert & 83.114 & 2.205 & 14 & 74 & 122 & 191 & 59 & 395 & 114 & 20 & 1.216 \\
\hline KMU deklariert & 21.313 & 714 & 6 & 32 & 98 & 57 & 8 & 216 & 36 & 4 & 257 \\
\hline
\end{tabular}

\begin{tabular}{|c|c|c|c|c|c|c|c|c|c|c|c|}
\hline & Alle Staaten & Österreich & B & Ktn & NÖ & $00 ̈$ & Sbg & St & $T$ & V & W \\
\hline Koordinatoren gesamt & 21.472 & 558 & 1 & 28 & 56 & 33 & 8 & 112 & 31 & 1 & 288 \\
\hline HES & 10.243 & 229 & & 2 & 35 & 6 & 4 & 29 & 23 & & 130 \\
\hline REC & 4.512 & 120 & 1 & & 6 & 11 & 2 & 28 & & & 72 \\
\hline PRC & 5.879 & 182 & & 26 & 14 & 15 & $\overline{2}$ & 55 & 7 & 1 & 62 \\
\hline PUB & 456 & 16 & & & & & & & 1 & & 15 \\
\hline OTH & 382 & 11 & & & 1 & 1 & & & & & 9 \\
\hline nicht KMU-deklariert & 16.287 & 401 & & 10 & 41 & 18 & 8 & 57 & 25 & & 242 \\
\hline KMU deklariert & 5.185 & 157 & 1 & 18 & 15 & 15 & & 55 & 6 & 1 & 46 \\
\hline
\end{tabular}

Diese Darstellungen beruhen auf Vertragsdaten. (Das ist ein Unterschied zu den Meldungen der Vorjahre, die auf den Bewilligungsangaben beruhen.) Vertragsdaten weisen durch den durch die Kommission überprüften Organisationstyp Zuweisungen auf.

Quelle: FFG auf Basis der EC 03/2019 Vertragsdaten. 
Tabelle 10-13: Österreichische Beteiligungen und Projekte in Horizon 2020 im Vergleich zur Summe aller Staaten differenziert nach Säulen und horizontalen Programmlinien

\begin{tabular}{|c|c|c|c|}
\hline & $\begin{array}{r}\text { Bewilligte Beteiligungen } \\
\text { (alle Staaten) }\end{array}$ & $\begin{array}{r}\text { Bewilligte österr. } \\
\text { Beteiligungen }\end{array}$ & $\begin{array}{r}\text { Anteil Österreich an allen } \\
\text { Staaten [in \%] }\end{array}$ \\
\hline H2O2O & 104.427 & 2.919 & 2,8 \\
\hline EC Treaty & 103.302 & 2.907 & 2,8 \\
\hline Excellent Science & 34.492 & 784 & 2,3 \\
\hline Industrial Leadership & 23.613 & 726 & 3,1 \\
\hline Societal Challenges & 42.327 & 1.287 & 3,0 \\
\hline Spreading Excellence and widening Participation & 844 & 25 & 3,0 \\
\hline Science with and for Society & 1.381 & 75 & 5,4 \\
\hline Cross-theme & 645 & 10 & 1,6 \\
\hline \multirow[t]{2}{*}{ Euratom } & 1.125 & 12 & 1,1 \\
\hline & $\begin{array}{r}\text { Bewilligte Projekte (alle } \\
\text { Staaten) }\end{array}$ & $\begin{array}{r}\text { Bewilligte Projekte mit } \\
\text { österr. Beteiligung }\end{array}$ & $\begin{array}{r}\text { Anteil Projekte mit } \\
\text { Österreich an allen } \\
\text { Projekten [in \%] }\end{array}$ \\
\hline H2O2O & 21.472 & 1.894 & $8,8 \%$ \\
\hline EC Treaty & 21.421 & 1.889 & $8,8 \%$ \\
\hline Excellent Science & 11.887 & 583 & $4,9 \%$ \\
\hline Industrial Leadership & 4.008 & 416 & $10,4 \%$ \\
\hline Societal Challenges & 5.064 & 803 & $15,9 \%$ \\
\hline Spreading Excellence and widening Participation & 204 & 22 & $10,8 \%$ \\
\hline Science with and for Society & 129 & 56 & $43,4 \%$ \\
\hline Cross-theme & 129 & 9 & $7,0 \%$ \\
\hline Euratom & 51 & 5 & $9,8 \%$ \\
\hline
\end{tabular}

Quelle: FFG auf Basis der EC 03/2019 Vertragsdaten. 
Tabelle 10-14: FWF: Anteile der Neubewilligungen nach Fachgebiet (ÖFOS 2012 3-Steller), 2018

\begin{tabular}{|c|c|c|c|c|c|c|}
\hline \multirow{2}{*}{ Fachgebiet } & \multicolumn{2}{|c|}{2016} & \multicolumn{2}{|c|}{2017} & \multicolumn{2}{|c|}{2018} \\
\hline & in $\%$ & in Mio. € & in $\%$ & in Mio. $€$ & in $\%$ & in Mio. € \\
\hline $101 *$ Mathematik & 14,14 & 25,99 & 11,51 & 25,02 & 8,30 & 19,17 \\
\hline $102 *$ Informatik & 4,41 & 8,11 & 5,68 & 12,33 & 4,93 & 11,38 \\
\hline 103*Physik, Astronomie & 10,85 & 19,94 & 10,8 & 23,47 & 11,85 & 27,35 \\
\hline $104 *$ Chemie & 4,36 & 8,02 & 4,52 & 9,82 & 4,42 & 10,21 \\
\hline $105^{*}$ Geowissenschaften & 3,35 & 6,15 & 3,49 & 7,59 & 3,20 & 7,39 \\
\hline $106 *$ Biologie & 20,04 & 36,84 & 19,94 & 43,33 & 23,43 & 54,07 \\
\hline $107 *$ Andere Naturwissenschaften & 0,31 & 0,57 & 0,24 & 0,51 & 0,09 & 0,22 \\
\hline $201 *$ Bauwesen & 0,46 & 0,85 & 0,42 & 0,92 & 1,37 & 3,16 \\
\hline 202*Elektrotechnik, Elektronik, Informationstechnik & 0,82 & 1,51 & 0,58 & 1,25 & 1,35 & 3,11 \\
\hline $203 *$ Maschinenbau & 0,05 & 0,09 & 0,27 & 0,59 & 0,28 & 0,64 \\
\hline 204*Chemische Verfahrenstechnik & 0,03 & 0,06 & 0,14 & 0,31 & 0,03 & 0,07 \\
\hline $205 *$ Werkstofftechnik & 0,57 & 1,05 & 0,37 & 0,8 & 0,32 & 0,73 \\
\hline 206*Medizintechnik & 0,2 & 0,37 & 0,5 & 1,09 & 0,61 & 1,40 \\
\hline 207*Umweltingenieurwesen, Angewandte Geowissenschaften & 0,24 & 0,45 & 0,48 & 1,04 & 0,53 & 1,21 \\
\hline $208 *$ Umweltbiotechnologie & 0,05 & 0,1 & 0,06 & 0,13 & 0,03 & 0,08 \\
\hline 209*Industrielle Biotechnologie & 0,22 & 0,4 & 0,45 & 0,99 & 0,10 & 0,23 \\
\hline $210 *$ Nanotechnologie & 1,04 & 1,92 & 0,55 & 1,19 & 0,74 & 1,71 \\
\hline $211 *$ Andere Technische Wissenschaften & 0,16 & 0,3 & 0,19 & 0,41 & 0,22 & 0,51 \\
\hline 301*Medizinisch-theoretische Wissenschaften, Pharmazie & 11,87 & 21,82 & 10,8 & 23,47 & 11,15 & 25,73 \\
\hline 302*Klinische Medizin & 4,43 & 8,13 & 4,07 & 8,85 & 3,40 & 7,84 \\
\hline $303 *$ Gesundheitswissenschaften & 0,86 & 1,58 & 0,91 & 1,97 & 1,24 & 2,85 \\
\hline 304*Medizinische Biotechnologie & 0,2 & 0,36 & 0,1 & 0,22 & 0,11 & 0,26 \\
\hline 305*Andere Humanmedizin, Gesundheitswissenschaften & 0,04 & 0,08 & 0,12 & 0,27 & 0,37 & 0,86 \\
\hline 401*Land- und Forstwirtschaft, Fischerei & 0,29 & 0,54 & 0,54 & 1,17 & 0,39 & 0,9 \\
\hline 402*Tierzucht, Tierproduktion & 0,4 & 0,73 & 0,03 & 0,06 & 0,03 & 0,06 \\
\hline 403*Veterinärmedizin & 0,5 & 0,92 & 0,26 & 0,57 & 0,05 & 0,12 \\
\hline 404*Agrarbiotechnologie, Lebensmittelbiotechnologie & 0,05 & 0,09 & & & 0,04 & 0,09 \\
\hline $405 *$ Andere Agrarwissenschaften & & & 0,14 & 0,31 & 0,14 & 0,32 \\
\hline 501*Psychologie & 1,46 & 2,69 & 1,29 & 2,8 & 1,61 & 3,72 \\
\hline 502*Wirtschaftswissenschaften & 3,16 & 5,81 & 3,12 & 6,79 & 1,86 & 4,29 \\
\hline $503 *$ Erziehungswissenschaften & 0,42 & 0,76 & 0,15 & 0,33 & 0,26 & 0,59 \\
\hline $504 *$ Soziologie & 1,39 & 2,56 & 1,74 & 3,78 & 2,62 & 6,04 \\
\hline 505*Rechtswissenschaften & 0,82 & 1,51 & 0,47 & 1,02 & 0,004 & 0,01 \\
\hline $506 *$ Politikwissenschaften & 0,4 & 0,73 & 0,4 & 0,86 & 0,58 & 1,35 \\
\hline 507*Humangeographie, Regionale Geographie, Raumplanung & 0,51 & 0,95 & 0,27 & 0,58 & 0,44 & 1,00 \\
\hline $508 *$ Medien- und Kommunikationswissenschaften & 0,2 & 0,37 & 0,44 & 0,96 & 0,18 & 0,41 \\
\hline 509*Andere Sozialwissenschaften & 0,3 & 0,54 & 0,24 & 0,51 & 0,93 & 2,15 \\
\hline $601 *$ Geschichte, Archäologie & 3,35 & 6,16 & 3,71 & 8,07 & 2,93 & 6,75 \\
\hline $602 *$ Sprach- und Literaturwissenschaften & 2,89 & 5,32 & 4,03 & 8,75 & 3,49 & 8,05 \\
\hline 603*Philosophie, Ethik, Religion & 2,37 & 4,35 & 2,68 & 5,83 & 2,41 & 5,57 \\
\hline 604*Kunstwissenschaften & 2,09 & 3,84 & 2,53 & 5,5 & 3,37 & 7,78 \\
\hline $605^{*}$ Andere Geisteswissenschaften & 0,66 & 1,22 & 1,77 & 3,85 & 0,62 & 1,44 \\
\hline Gesamt & 100,00 & 183,80 & 100,00 & 217,34 & 100,00 & 230,82 \\
\hline
\end{tabular}


Tabelle 10-15: FWF: Anteile der Neubewilligungen nach Organisationstyp, 2016-2018

\begin{tabular}{|c|c|c|c|c|c|c|}
\hline \multirow{2}{*}{ Organisationstyp } & \multicolumn{2}{|c|}{2016} & \multicolumn{2}{|c|}{2017} & \multicolumn{2}{|c|}{2018} \\
\hline & in $\%$ & in Mio. $€$ & in $\%$ & in Mio. $€$ & in $\%$ & in Mio. $€$ \\
\hline Universitäten $^{1}$ & 82,97 & 152,5 & 85,14 & 185 & 83,30 & 192,3 \\
\hline Fachhochschulen & 1,33 & 2,4 & 0,46 & 1 & 0,37 & 0,9 \\
\hline Privatuniversitäten & 1,13 & 2,1 & 0,56 & 1,2 & 1,12 & 2,6 \\
\hline Akademie der Wissenschaften & 7,83 & 14,4 & 7,81 & 17 & 7,79 & 18,0 \\
\hline Außeruniv. Forschungsstätten ${ }^{2}$ & 6,74 & 12,4 & 6,03 & 13,1 & 7,42 & 17,1 \\
\hline Gesamt & 100,00 & 183,8 & 100,00 & 217,3 & 100,00 & 230,8 \\
\hline
\end{tabular}

Quelle: FWF.

1 Inkl. Universität für Weiterbildung Krems, 2 Beinhalten Forschungsstätten im Ausland.

Tabelle 10-16: FFG: Anteile der Neubewilligungen nach Themenfeld der Förderung, 2016-2018

\begin{tabular}{|c|c|c|c|c|c|c|}
\hline & & 2016 & & 2017 & & 2018 \\
\hline & in $\%$ & Gesamtförderung [in Mio. $€$ ] & in $\%$ & Gesamtförderung [in Mio. $€$ ] & in $\%$ & Gesamtförderung [in Mio. $€$ ] \\
\hline Energie/Umwelt & 16,9 & 88,0 & 15,0 & 84,2 & 17,98 & 111,0 \\
\hline IKT & 20,3 & 105,7 & 20,9 & 117,8 & 19,93 & 123,1 \\
\hline Mobilität & 11,6 & 60,4 & 12,5 & 70,2 & 10,63 & 65,6 \\
\hline Produktion & 22,8 & 118,7 & 23,1 & 129,9 & 25,73 & 158,9 \\
\hline Life Sciences & 10,7 & 56,0 & 10,6 & 59,5 & 12,19 & 75,3 \\
\hline Sicherheit & 1,6 & 8,1 & 1,5 & 8,4 & 1,28 & 7,9 \\
\hline Weltraum & 1,5 & 7,6 & 1,4 & 8,0 & 1,20 & 7,4 \\
\hline Sonstige & 14,8 & 77,0 & 15,0 & 84,4 & 11,06 & 68,3 \\
\hline Gesamt & 100,0 & 521,5 & 100,0 & 562,5 & 100,00 & 617,6 \\
\hline
\end{tabular}

Quelle: FFG.

Tabelle 10-17: FFG: Förderungen nach Bundesland, 2016-2018

\begin{tabular}{|c|c|c|c|c|c|c|}
\hline \multirow{2}{*}{ Bundesland } & \multicolumn{2}{|r|}{2016} & \multicolumn{2}{|r|}{2017} & \multicolumn{2}{|r|}{2018} \\
\hline & in $\%$ & Gesamtförderung [in Mio. $€$ ] & in $\%$ & Gesamtförderung [in Mio. $€$ ] & in $\%$ & Gesamtförderung [in Mio. $€$ ] \\
\hline Burgenland & 1,3 & 6,7 & 1,3 & 7,6 & 1,0 & 6,0 \\
\hline Kärnten & 4,6 & 23,7 & 4,6 & 25,6 & 5,0 & 30,9 \\
\hline Niederösterreich & 8,9 & 46,6 & 7,3 & 40,9 & 8,6 & 52,8 \\
\hline Oberösterreich & 19,8 & 103,2 & 19,5 & 109,5 & 22,0 & 136,0 \\
\hline Salzburg & 3,7 & 19,1 & 3,3 & 18,4 & 3,0 & 18,6 \\
\hline Steiermark & 23,3 & 121,4 & 29,9 & 168,0 & 28,2 & 174,0 \\
\hline Tirol & 5,9 & 31,0 & 7,2 & 40,4 & 4,8 & 29,6 \\
\hline Vorarlberg & 3,2 & 16,8 & 3,2 & 18,2 & 2,8 & 17,1 \\
\hline Wien & 28,1 & 146,6 & 22,9 & 128,9 & 23,2 & 143,4 \\
\hline Ausland & 1,2 & 6,3 & 0,9 & 4,9 & 1,5 & 9,2 \\
\hline Gesamt & 100,0 & 521,5 & 100,0 & 562,5 & 100,0 & 617,6 \\
\hline
\end{tabular}

Quelle: FFG. 
Tabelle 10-18: FFG: Projektkosten und Förderung nach Subject Index Code, 2018

\begin{tabular}{|c|c|c|c|}
\hline & $\begin{array}{r}\text { Gesamtkosten } \\
\text { [in Mio. €] }\end{array}$ & $\begin{array}{r}\text { Gesamtförderung [in } \\
\text { Mio. €] }\end{array}$ & $\begin{array}{r}\text { Barwert } \\
\text { [in Mio. €] }\end{array}$ \\
\hline SIC gesamt & $1.244,59$ & 617,57 & 500,84 \\
\hline Industrielle Fertigung & 196,06 & 89,19 & 64,93 \\
\hline Oberlächenverkehr und -technologien & 160,83 & 69,47 & 48,72 \\
\hline Elekronik, Mikroelekronik & 117,96 & 65,70 & 56,46 \\
\hline Werkstofftechnik & 131,80 & 56,23 & 48,01 \\
\hline IKT-Anwendungen & 99,76 & 49,75 & 40,14 \\
\hline Informationsverarbeitung, Informationssysteme & 82,15 & 37,88 & 33,61 \\
\hline Energieeinsparung, -umwandlunge und -transport & 49,29 & 28,15 & 27,14 \\
\hline Biowissenschaften & 66,36 & 27,46 & 21,50 \\
\hline Automatisierung & 39,52 & 19,03 & 13,46 \\
\hline Energieeinsparung & 32,57 & 16,95 & 13,10 \\
\hline Medizin, Gesundheit & 26,09 & 16,43 & 12,14 \\
\hline Medizinische Biotechnologie & 33,48 & 15,38 & 12,84 \\
\hline Bautechnik & 21,84 & 13,39 & 13,12 \\
\hline Regenerative Energieträger & 18,58 & 12,74 & 11,23 \\
\hline Nachhaltige Entwicklung & 14,65 & 9,44 & 9,30 \\
\hline Sicherheit & 15,82 & 9,09 & 5,55 \\
\hline Weltraum & 13,23 & 7,28 & 3,66 \\
\hline Sonstige Technologie & 10,13 & 7,15 & 4,61 \\
\hline Mathematik, Statistik & 9,50 & 7,02 & 7,02 \\
\hline Abfallwirtschaft & 9,19 & 6,98 & 6,98 \\
\hline Messverfahren & 10,43 & 6,94 & 5,16 \\
\hline Lebensmitte & 5,57 & 3,70 & 3,56 \\
\hline Nanotechnologie und Nanowissenschaften & 5,84 & 3,55 & 2,92 \\
\hline Luftverkehr und -technologien & 7,42 & 3,38 & 3,38 \\
\hline Landwirtschaftliche Biotechnologie & 7,99 & 3,35 & 2,57 \\
\hline Sonstige Energiethemen & 7,46 & 3,00 & 2,75 \\
\hline Umwelt & 4,08 & 2,68 & 2,33 \\
\hline Robotik & 4,21 & 2,59 & 2,59 \\
\hline Unternehmensaspekte & 3,72 & 2,18 & 1,61 \\
\hline Landwirtschaftliche Biotechnologie & 3,36 & 1,53 & 1,13 \\
\hline Quantentechnologie & 6,15 & 1,52 & 1,52 \\
\hline Geowissenschaften & 1,76 & 1,41 & 1,41 \\
\hline Information, Medien & 2,01 & 1,36 & 1,27 \\
\hline Soziale Aspekte & 1,68 & 0,96 & 0,96 \\
\hline Netzwerktechnologien & 1,22 & 0,74 & 0,65 \\
\hline Wirtschaftliche Aspekte & 1,00 & 0,66 & 0,66 \\
\hline Beschäftigung & 0,79 & 0,56 & 0,49 \\
\hline Koordinierung, Zusammenarbeit & 0,63 & 0,50 & 0,38 \\
\hline Industrielle Biotechnologie & 0,36 & 0,36 & 0,36 \\
\hline Meteorologie & 0,58 & 0,36 & 0,36 \\
\hline Forschung zu Klimawandel und Kohlenstoffkreislauf & 0,26 & 0,26 & 0,26 \\
\hline Forschungsethik & 0,57 & 0,18 & 0,18 \\
\hline Telekommunikation & 0,04 & 0,03 & 0,03 \\
\hline Normen & 0,01 & 0,01 & 0,01 \\
\hline Regionalentwicklung & 0,01 & 0,01 & 0,01 \\
\hline Wasserressourcen und -bewirtschaftung & 0,00 & 0,00 & 0,00 \\
\hline Ohne Zuordnung & 18,60 & 11,06 & 10,80 \\
\hline
\end{tabular}

Quelle: FFG. 
Tabelle 10-19: aws: Anteile der Neubewilligungen nach Themenfeld der Förderung (Branche), 2016-2018

\begin{tabular}{|c|c|c|c|c|c|c|}
\hline \multirow{2}{*}{ Fachgebiet, Themenfelder oder Branche } & \multicolumn{2}{|c|}{2016} & \multicolumn{2}{|c|}{2017} & \multicolumn{2}{|c|}{2018} \\
\hline & in $\%$ & in Mio. € & in $\%$ & in Mio. $€$ & in $\%$ & in Mio. $€$ \\
\hline Dienstleistungen & 19,3 & 156,6 & 24,7 & 282,4 & 13,3 & 291,8 \\
\hline Energie- und Wasserversorgung, Abwasser & 0,2 & 1,4 & 0,8 & 9,0 & 0,6 & 13,7 \\
\hline Handel, Instandhaltung, Reparatur & 14,9 & 121,2 & 13,3 & 152,5 & 10,3 & 226,3 \\
\hline Nahrungs- und Genussmittel, LW, FW & 12,8 & 104,0 & 12,9 & 147,6 & 6,1 & 133,9 \\
\hline Sachgüterproduktion & 37,7 & 306,0 & 36,1 & 413,9 & 29,8 & 651,9 \\
\hline Sonstige Branchen & 0,7 & 6,0 & 1,7 & 18,9 & 1,1 & 24,6 \\
\hline Tourismus & 9,8 & 79,7 & 6,5 & 74,5 & 2,8 & 61,8 \\
\hline Verkehr und Nachrichtenübermittlung & 2,0 & 15,9 & 1,8 & 20,1 & 1,8 & 39,1 \\
\hline Nicht zugeordnet & 2,5 & 20,1 & 2,3 & 26,4 & 34,1 & 746,5 \\
\hline Gesamt & 100,0 & 810,9 & 100,0 & $1.145,4$ & 100,0 & $2.189,5$ \\
\hline
\end{tabular}

Quelle: aws.

Tabelle 10-20: aws: Anteile der Neubewilligungen nach Unternehmensgröße, 2016-2018

\begin{tabular}{|c|c|c|c|c|c|c|}
\hline \multirow{2}{*}{ Organisationstyp } & \multicolumn{2}{|c|}{2016} & \multicolumn{2}{|c|}{2017} & \multicolumn{2}{|c|}{2018} \\
\hline & in $\%$ & in Mio. $€$ & in $\%$ & in Mio. $€$ & in \% & in Mio. $€$ \\
\hline EPU & 7,7 & 62,8 & 8,5 & 97,3 & 9,3 & 203,5 \\
\hline Kleinstunternehmen & 17,3 & 140,2 & 17,8 & 204,3 & 14,8 & 324,9 \\
\hline Kleinunternehmen & 15,1 & 122,8 & 28,0 & 320,2 & 22,2 & 486,5 \\
\hline Mittelunternehmen & 29,7 & 241,0 & 29,1 & 333,5 & 18,7 & 409,1 \\
\hline Großunternehmen & 27,6 & 223,6 & 14,4 & 165,0 & 30,6 & 670,6 \\
\hline Nicht zugeordnet & 2,5 & 20,6 & 2,2 & 25,2 & 4,3 & 95,0 \\
\hline Gesamt & 100,0 & 810,9 & 100,0 & $1.145,4$ & 100,0 & $2.189,5$ \\
\hline
\end{tabular}

Quelle: aws.

Tabelle 10-21: aws: Leistungsüberblick nach regionaler Verteilung, 2017-2018

\begin{tabular}{|c|c|c|c|c|c|c|c|c|c|c|}
\hline \multirow{2}{*}{ Region } & \multicolumn{2}{|c|}{ Zusage } & \multicolumn{2}{|c|}{$\begin{array}{l}\text { Finanzierungsleistung } \\
\text { [in Mio. €] }\end{array}$} & \multicolumn{2}{|c|}{$\begin{array}{l}\text { Finanzierungsbarwert } \\
\text { [in Mio. €] }\end{array}$} & \multicolumn{2}{|c|}{$\begin{array}{l}\text { Gesamtprojektkosten } \\
\text { [in Mio. €] }\end{array}$} & \multicolumn{2}{|c|}{ Neue Arbeitsplätze } \\
\hline & 2017 & 2018 & 2017 & 2018 & 2017 & 2018 & 2017 & 2018 & 2017 & 2018 \\
\hline Burgenland & 92 & 379 & 13,4 & 48,8 & 3,5 & 24,5 & 45,6 & 187,0 & 131 & 1.321 \\
\hline Kärnten & 414 & 980 & 72,1 & 154,9 & 8,3 & 49,4 & 273,9 & 697,9 & 528 & 3.428 \\
\hline Niederösterreich & 790 & 2.538 & 206,7 & 404,9 & 45,2 & 230,4 & 662,5 & 966,4 & 1.841 & 16.710 \\
\hline Oberösterreich & 1.634 & 3.632 & 434,8 & 587,8 & 74,1 & 284,6 & $1.092,3$ & $1.573,5$ & 2.887 & 18.890 \\
\hline Salzburg & 346 & 1.062 & 56,7 & 148,1 & 13,1 & 98,0 & 191,1 & 663,9 & 381 & 7.210 \\
\hline Steiermark & 652 & 2.218 & 96,0 & 246,5 & 29,5 & 171,2 & 548,7 & 777,9 & 1.095 & 11.223 \\
\hline Tirol & 390 & 1.505 & 82,2 & 153,6 & 21,8 & 91,1 & 319,4 & 452,6 & 643 & 6.118 \\
\hline Vorarlberg & 160 & 641 & 38,2 & 68,6 & 9,4 & 50,6 & 315,9 & 335,5 & 502 & 3.458 \\
\hline Wien & 958 & 3.260 & 118,8 & 331,1 & 43,8 & 265,9 & 487,3 & $1.091,7$ & 1.380 & 16.842 \\
\hline Ausland \& nicht zugeordnet & 46 & 90 & 26,5 & 45,2 & 9,3 & 11,7 & 54,2 & 70,2 & 63 & 176 \\
\hline Gesamt & 5.482 & 16.305 & $1.145,4$ & $2.189,5$ & 258,0 & $1.277,4$ & $3.990,9$ & $6.816,6$ & 9.451 & 85.376 \\
\hline
\end{tabular}

Quelle: aws. 
Tabelle 10-22: CDG: CD-Labors nach Universitäten/Forschungseinrichtungen, 2018

\begin{tabular}{|c|c|c|}
\hline Universität/Forschungseinrichtung & Anzahl der CD-Labors 2018 & Budget 2018 [in €] \\
\hline Donau-Universität Krems & 1 & $186.000,00$ \\
\hline Medizinische Universität Graz & 1 & $220.000,00$ \\
\hline Medizinische Universität Innsbruck & 6 & $1.583 .000,67$ \\
\hline Medizinische Universität Wien & 10 & $3.454 .469,00$ \\
\hline Montanuniversität Leoben & 10 & $2.902 .435,08$ \\
\hline Technische Universität Graz & 10 & $3.586 .636,48$ \\
\hline Technische Universität Wien & 17 & $5.797 .743,05$ \\
\hline Universität für Bodenkultur Wien & 7 & $3.507 .646,22$ \\
\hline Universität Graz & 1 & $27.105,00$ \\
\hline Universität Innsbruck & 1 & $37.500,00$ \\
\hline Universität Linz & 6 & $2.397 .900,66$ \\
\hline Universität Salzburg & 1 & $443.301,77$ \\
\hline Universität Wien & 6 & $1.494 .869,36$ \\
\hline Veterinärmedizinische Universität Wien & 4 & $1.171 .114,75$ \\
\hline Wirtschaftsuniversität Wien & 1 & $81.634,53$ \\
\hline Österreichische Akademie der Wissenschaften & 1 & $267.279,00$ \\
\hline Forschungszentrum Jülich GmbH & 1 & $446.175,00$ \\
\hline University of Cambridge & 1 & $322.700,00$ \\
\hline Gesamt & 85 & $27.927 .510,57$ \\
\hline
\end{tabular}

Quelle: CDG, Anm.: Budgetdaten 2018 sind Plan-Daten per 31.12.2018.

Tabelle 10-23: CDG: JR-Zentren nach Fachhochschulen, 2018

\begin{tabular}{l|r}
\hline Fachhochschule & Anzahl der JR-Zentren 2018 \\
\hline Fachhochschule Joanneum Gesellschaft mbH & 2 \\
\hline Fachhochschule Kärnten - gemeinnützige Privatstiftung & 1 \\
\hline Fachhochschule St. Pölten GmbH & 1 \\
\hline Fachhochschule Technikum Wien & 2 \\
\hline Fachhochschule Vorarlberg GmbH & 2 \\
\hline FH 0Ö Forschungs und Entwicklungs GmbH & 3 \\
\hline IMC Fachhochschule Krems GmbH & 1 \\
\hline Gesamt & 12
\end{tabular}

\begin{tabular}{r|r}
\hline 18 & Budget 2018 [in €] \\
\hline 1 & $337.769,00$ \\
\hline 1 & $340.306,00$ \\
\hline 2 & $319.261,02$ \\
\hline 2 & $154.021,99$ \\
\hline 3 & $367.783,91$ \\
\hline 1 & $844.958,00$ \\
\hline 12 & $330.220,43$ \\
\hline
\end{tabular}

Quelle: CDG, Anm.: Budgetdaten 2018 sind Plan-Daten per 31.12.2018. 
Tabelle 10-24: CDG: Entwicklung der CDG 1989-2018 bzw. JR-Zentren 2012-2018

\begin{tabular}{|c|c|c|c|c|}
\hline Jahr & $\begin{array}{r}\text { Ausgaben der CD-Labors und } \\
\text { JR-Zentren [in } € \text { ] }\end{array}$ & $\begin{array}{r}\text { Aktive } \\
\text { CD-Labors }\end{array}$ & $\begin{array}{r}\text { Aktive } \\
\text { JR-Zentren }\end{array}$ & $\begin{array}{r}\text { Aktive } \\
\text { Mitgliedsunternehmen }\end{array}$ \\
\hline 1989 & 247.088 & 5 & & \\
\hline 1990 & 1.274 .682 & 7 & & \\
\hline 1991 & 2.150 .389 & 11 & & \\
\hline 1992 & 3.362 .572 & 16 & & \\
\hline 1993 & 2.789 .910 & 17 & & \\
\hline 1994 & 3.101 .677 & 18 & & \\
\hline 1995 & 2.991 .214 & 14 & & \\
\hline 1996 & 2.503 .325 & 14 & & 6 \\
\hline 1997 & 2.982 .793 & 15 & & 9 \\
\hline 1998 & 3.108 .913 & 18 & & 13 \\
\hline 1999 & 3.869 .993 & 20 & & 15 \\
\hline 2000 & 3.624 .963 & 18 & & 14 \\
\hline 2001 & 4.707 .302 & 20 & & 18 \\
\hline 2002 & 7.295 .957 & 31 & & 40 \\
\hline 2003 & 9.900 .590 & 35 & & 47 \\
\hline 2004 & 10.711 .822 & 37 & & 63 \\
\hline 2005 & 11.878 .543 & 37 & & 66 \\
\hline 2006 & 12.840 .466 & 42 & & 79 \\
\hline 2007 & 14.729 .108 & 48 & & 82 \\
\hline 2008 & 17.911 .784 & 58 & & 99 \\
\hline 2009 & 17.844 .202 & 65 & & 106 \\
\hline 2010 & 19.768 .684 & 61 & & 110 \\
\hline 2011 & 20.580 .208 & 61 & & 108 \\
\hline 2012 & 22.167 .259 & 64 & 1 & 114 \\
\hline 2013 & 23.666 .522 & 73 & 4 & 131 \\
\hline 2014 & 25.634 .725 & 71 & 5 & 129 \\
\hline 2015 & 24.954 .856 & 73 & 7 & 145 \\
\hline 2016 & 23.967 .799 & 72 & 9 & 136 \\
\hline 2017 & 26.196 .507 & 76 & 11 & 147 \\
\hline 2018 & 30.621 .831 & 85 & 12 & 158 \\
\hline
\end{tabular}

Quelle: CDG, Anm.: Budgetdaten 2018 sind Plan-Daten per 31.12.2018. 
Tabelle 10-25: CDG: CD-Labors nach Thematischen Clustern, 2018

\begin{tabular}{lrr}
\hline Thematischer Cluster & Anzahl der CD-Labors 2018 & Budget 2018 [in €] \\
\hline Chemie & 8 & $2.867 .655,91$ \\
\hline Life Sciences und Umwelt & 17 & $6.975 .680,99$ \\
\hline Maschinen- und Instrumentenbau & 7 & $2.338 .898,81$ \\
\hline Mathematik, Informatik, Elektronik & 18 & $6.305 .235,34$ \\
\hline Medizin & 16 & $\mathbf{1 6}$ \\
\hline Materialien und Werkstoffe & 3 & $4.205 .169,67$ \\
\hline Wirtschafts-, Sozial- und Rechtswissenschaften & $\mathbf{8 5}$ & $4.862 .621,07$ \\
\hline Gesamt & $372.248,78$ \\
\hline
\end{tabular}

Quelle: CDG, Anm.: Budgetdaten 2018 sind Plan-Daten per 31.12.2018.

Tabelle 10-26: CDG: JR-Zentren nach Thematischen Clustern, 2018

\begin{tabular}{l|rr}
\hline Thematischer Cluster & Anzahl der JR-Zentren $\mathbf{2 0 1 8}$ & Budget 2018 [in €] \\
\hline Chemie & - & - \\
\hline Life Sciences und Umwelt & 1 & $200.000,00$ \\
\hline Maschinen- und Instrumentenbau & 1 & 7 \\
\hline Mathematik, Informatik, Elektronik & 1 & $216.958,00$ \\
\hline Medizin & 2 & $1.689 .006,32$ \\
\hline Materialien und Werkstoffe & $230.220,43$ \\
\hline Wirtschafts-, Sozial- und Rechtswissenschaften & -12 & $258.135,60$ \\
\hline Gesamt & $\mathbf{1 2}$ & - \\
\hline
\end{tabular}

Quelle: CDG, Anm.: Budgetdaten 2018 sind Plan-Daten per 31.12.2018. 
